\title{
Green algae in soil: \\ assessing their biodiversity and biogeography with molecular-phylogenetic methods based on cultures
}

\author{
Dissertation \\ zur Erlangung des mathematisch-naturwissenschaftlichen Doktorgrades \\ "Doctor rerum naturalium" \\ der Georg-August-Universität Göttingen \\ im Promotionsprogramm Biologie \\ der Georg-August University School of Science (GAUSS)
}

vorgelegt von

Ladislav Hodač

aus Mladá Boleslav (Tschechien)

Göttingen, 2015 


\section{Betreuungsausschuss}

Prof Dr. Thomas Friedl, Experimentelle Phykologie und Sammlung von Algenkulturen, Albrecht-von-Haller-Institut für Pflanzenwissenschaften

Prof. Dr. Rolf Daniel, Genomische und Angewandte Mikrobiologie und Göttinger Genomlabor, Institut für Mikrobiologie und Genetik

\section{$\underline{\text { Mitglieder der Prüfungskommission }}$}

Referent: Prof Dr. Thomas Friedl, Experimentelle Phykologie und Sammlung von Algenkulturen, Albrecht-von-Haller-Institut für Pflanzenwissenschaften

Korreferent: Prof. Dr. Rolf Daniel, Genomische und Angewandte Mikrobiologie und Göttinger Genomlabor, Institut für Mikrobiologie und Genetik

\section{Weitere Mitglieder der Prüfungskommission:}

Prof. Dr. Elvira Hörandl, Systematik, Biodiversität und Evolution von Pflanzen (mit Herbarium), Albrecht-von-Haller-Institut für Pflanzenwissenschaften

PD Dr. Gernot Arp, Abteilung Geobiologie, Geowissenschaftliches Zentrum

PD Dr. Michael Hoppert, Abteilung für Allgemeine Mikrobiologie, Institut für Mikrobiologie und Genetik

Prof. Dr. Hermann Behling, Abteilung Palynologie und Klimadynamik, Albrecht-von-HallerInstitut für Pflanzenwissenschaften

Tag der mündlichen Prüfung: 13.01.2016 


\section{Contents}

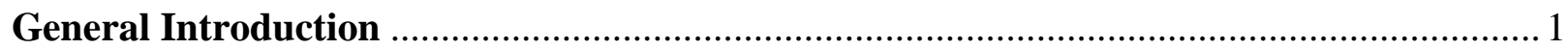

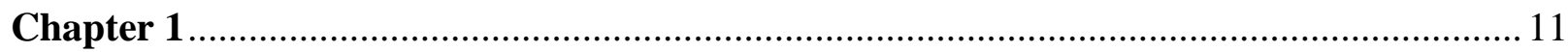

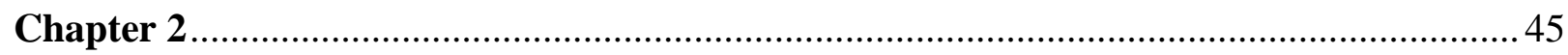

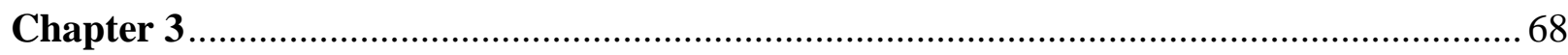

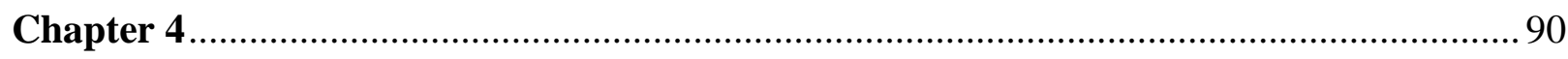

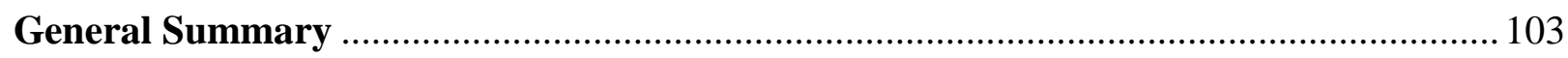

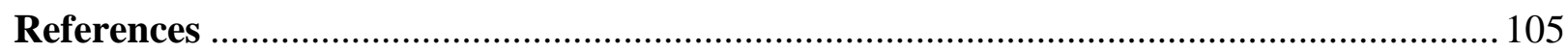

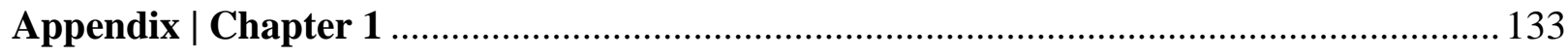

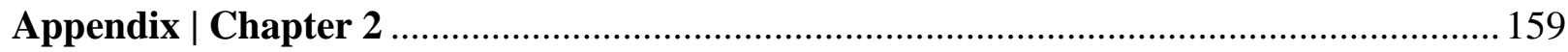

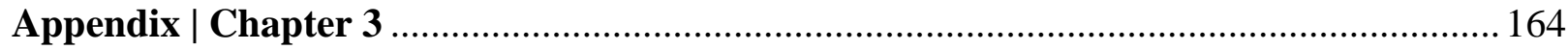

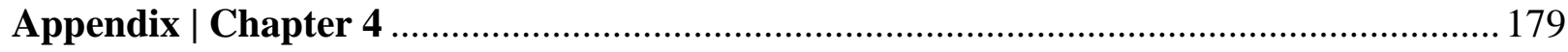

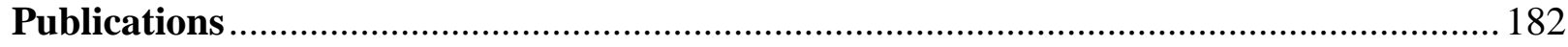

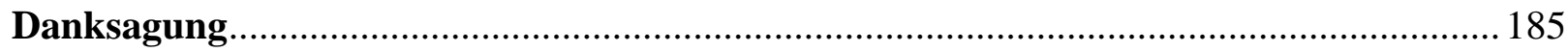




\section{General Introduction}

\section{Importance of soil microalgae}

Terrestrial eukaryotic microalgae were recognized as a part of soil microflora in the late $19^{\text {th }}$ century and were introduced into soil ecology during the second half of the $20^{\text {th }}$ century (Gollerbach and Shtina 1969; Shtina and Gollerbach 1976; Metting 1981; Starks et al. 1981). As photoautotrophs, microalgae constitute the basis of soil food webs and play a key role in soil development (Zenova and Shtina 1990; Zenova et al. 1995; Hu et al. 2002; Hu et al. 2003b; Rahmonov and Piątek 2007; Langhans et al. 2009; Viles 2012). The role of terrestrial eukaryotic microalgae in geomorphic processes was discussed by Viles (2012). Changes in algal abundance and diversity enable biological monitoring of soil recovery after anthropogenic pollution and contamination (Megharaj et al. 1986; Dahlin et al. 1997; Megharaj et al. 2000; Bérard et al. 2004; Krivorotov and Bukareva 2005; Nagy et al. 2005; Novakovskaya and Patova 2007; Kalinowska and Pawlik-Skowrońska 2010; Temraleeva et al. 2011; Hastings et al. 2014), or during postpyrogene soil development (Scherbina et al. 2014). Some authors recognized the importance of algal diversity in quality assessment of agricultural ecosystems (Shields and Durrell 1964; Gollerbach and Shtina 1969; Kabirov 1993; Hastings et al. 2014) and of land usage (King and Ward 1977). Particular species of soil microalgae are considered to be biological indicators (Balezina 1974; Shtina 1990; Megharaj et al. 2000; Bérard et al. 2005; Krivorotov and Bukareva 2005; Zancan et al. 2006; Dotsenko 2008), classified as a part of ecologically meaningful algocenoses (Gollerbach and Shtina 1969; Zenova et al. 1995; Kuzyakhmetov 1998; Şalaru et al. 2008; Kabirov et al. 2010). The interest in soil microalgae resulted in a series of comprehensive reviews on algal taxonomy, diversity, ecology and geographic distribution (Reisigl 1964; Gollerbach and Shtina 1969; Shtina and Gollerbach 1976; Metting 1981; Starks et al. 1981; Shtina 1990; Ettl and Gärtner 1995; Ettl and Gärtner 2014).

\section{Distribution of soil microalgae: a microscopy-based approach}

Our knowledge of algal species diversity in soils is still based mostly upon morphological observations accomplished via light microscopy of mixed or monoclonal algal cultures. Since the major fraction of algal species is invisible by direct microscopy of soil particles, algal growth should be first triggered via enrichment culturing techniques (Starks et al. 1981; Ettl and Gärtner 1995). The growth of particular species of microalgae is differentially favored according to the chemical composition of culturing media, or whether algae are cultured in liquid or agarized 
media. The most widely used medium for culturing of terrestrial microalgae, the Bold's Basal Medium (BBM; Bischoff and Bold 1963), is selective in favor of easy-growing (generalist) rather than rare species (Broady 1996). Our picture of the algal diversity in soils is incomplete, not only due to selective culturing techniques but also because of the demanding morphological determination of similarly looking species.

Research on diversity of edaphic microalgae has a long tradition particularly in Europe, beginning in the $19^{\text {th }}$ century (Kützing 1843; Hansgirg 1888). In fact, most of the early recognized microscopic algae are also known from soils, e.g., Stichococcus (Nägeli 1849), Chlorella (Beijerinck 1893), Hormidium/Klebsormidum (Kützing 1843), Chlamydomonas (Ehrenberg 1833), Coccomyxa (Schmidle 1901), Scenedesmus (Meyen 1829) or Pleurastrum/Leptosira (Borzì 1895). Apart from several studies from British Isles (Bristol 1920; 1927; Lund 1945; Lund 1947), the most biodiversity research on soil microalgae was conducted in continental Europe. Considering terrestrial ecosystems of Central and Western Europe, species lists of edaphic microalgae were revealed from forests (Trenkwalder 1975; Komáromy 1983; Kostikov et al. 2001a; Hoffmann et al. 2007; Škaloud 2009), grasslands (Komaromy 1976; Komáromy 1983; Neustupa 2001), agricultural fields (Lukešová 1993; Zancan et al. 2006), urban regions (Perútková 2014), contaminated (e.g., post-mining) areas (Lukešová and Komárek 1987; Lukešová and Hoffmann 1996; Frouz et al. 2001; Lukešová 2001; Neustupa and Škaloud 2004; Kalinowska et al. 2008; Trzcińska and Pawlik-Skowrońska 2008), subalpine soils (Rosa and Lhotský 1971; Lukešová et al. 2010), alpine soils (Reisigl 1958; 1964; Türk and Gärtner 2001; Peer et al. 2010) and xeric sand soils (Hoppert et al. 2004; Rahmonov and Piątek 2007; Langhans et al. 2009). Particular attention was paid to the edaphic microalgae in Eastern Europe and Russia, focusing on characteristic habitats including steppe grasslands (Musabaeva 2009), steppe forests (Kostikov et al. 2001b; Maltseva 2005b; 2005a; 2007; Maltsev 2013; Scherbina et al. 2014), Carpathian and other forests (Chornevych et al. 2008; Iljushenko 2008; Posrednikova et al. 2009; Nikorych and Chornevych 2012; Maltsev and Negrulja 2013), Russian tundra (Novakovskaya and Patova 2008; Patova and Dorokhova 2008), agricultural ecosystems (Asfandijarova 2008; Şalaru et al. 2008; Maltseva et al. 2009), disturbed soils (e.g., post-mining) (Novakovskaya and Patova 2007; Bachura and Khramchenkova 2008), urban areas (Soare and Dobrescu 2010; Shekhovtseva 2014; Dorokhova et al. 2015), or islands in the Black Sea (Vinogradova and Darienko 2008; Darienko 2012).

The best sampled region outside Europe is North America, where the investigators focused on algal communities of mountainous regions (Bischoff and Bold 1963; Khaybullina et al. 2010), deserts (Flechtner et al. 1998; Flechtner et al. 2005; Flechtner et al. 2008), xeric sandy soils 
(Smith et al. 2004), contaminated soils (Maxwell 1991; Nagy et al. 2005), serpentine soils (Terlizzi and Karlander 1979) and soils of agricultural landscapes (Fairchild and Willson 1967; Hunt et al. 1979; Metting and Rayburn 1979; Metting 1981). Microalgae of desert soil crusts were further studied in Africa (Büdel et al. 2009; Mansour and Shaaban 2010) and Asia (Islam 1960; Friedmann et al. 1967; Novichkova-Ivanova 1972; Chunxiang and Yongding 2003; Hu et al. 2003b; Al-Fredan and Fathi 2007; Bhatnagar et al. 2008). A lot of attention was paid to the edaphic microflora of the extremely cold and dry polar regions including both the Arctic (Androsova 1964; Broady 1978; Elster et al. 1999; Kaštovská et al. 2005; Stibal et al. 2006; Kaštovská et al. 2007; Matula et al. 2007; Kim et al. 2008; Patova and Dorokhova 2008) and the Antarctic (Broady 1979a; Broady 1984; Broady 1989; 1996; Mataloni et al. 2000; Cavacini 2001; Fermani et al. 2007). The least known is the algal diversity in humid tropical soils and so far we are aware of investigations from South and Central America (Archibald 1972; Archibald and Bold 1975; Tell 1976; Büdel 2003; Spitzer et al. 2014), Asia (MacEntee et al. 1977; Watanabe 1983; Reynaud and Lumpkin 1988; Ray and Thomas 2012; Lin et al. 2013), Australia and Oceania (Arvik and Willson 1974; Carson and Brown 1976; MacEntee et al. 1977; Carson and Brown 1978; Watanabe 1983). The edaphic microflora of small and isolated islands is almost unknown, except for e.g. St. Paul's Rocks in the equatorial Atlantic Ocean (Smith et al. 1974).

\section{Phylogenetic diversity of soil microalgae}

Considering the modern system of Eukaryotes, soil microalgae comprise photoautotrophic microorganisms that are phylogenetically nested within five kingdoms (Archeplastida, Excavata, Stramenopila, Alveolata and Cryptophyceae; Fig. 1). Archaeplastida (Adl et al. 2005)—mainly Chlorophyta and Streptophyta (Friedl and Rybalka 2012; Leliaert et al. 2012)-represents a dominant group of the most soil types (Broady 1979b; Metting 1981; Hoffmann 1989; Megharaj et al. 2000; Lukešová 2001; Zancan et al. 2006; Trzcińska and Pawlik-Skowrońska 2008; Freeman et al. 2009; Lin and Wu 2014). Stramenopila (Patterson 1989) are as well abundant in soils, represented by the classes Bacillariophyceae (Lukešová and Komárek 1987; Fermani et al. 2007; Darienko 2012; Stanek-Tarkowska and Noga 2012), Xanthophyceae (Broady 1979b; Maltseva 2005b; 2005a; Posrednikova et al. 2009; Novis et al. 2015) and Eustigmatophycae (Neustupa 2001; Khaybullina et al. 2010; Darienko 2012; Scherbina et al. 2014). Three further groups were sporadically recorded from soils: Excavata-Euglenophyta (Ashley et al. 1985; Maltseva 2005a; Şalaru et al. 2008; Lukešová et al. 2010; Lang et al. 2011), Alveolata-Dinophyta (Ettl and Gärtner 1995; Kutovaya et al. 2012) and Cryptophyceae-incertae sedis (Paulsen et al. 1992; Ettl and Gärtner 1995). Across the temperate zone, particularly the Green algae 
(Chlorophyta lineage) are highly abundant in soils (Sumbali and Mehrotra 2009), whereas in tropics Cyanobacteria dominate (Roger and Reynaud 1982; Lin et al. 2013). It is widely accepted that variation of key physico-chemical parameters in soils ( $\mathrm{pH}$, organic carbon, nitrogen) affect green algae less than other microalgae (Metting 1981; Starks et al. 1981; Hoffmann 1989). Examples from temperate forests and grasslands show, that alkaline and nutrient rich soils favor higher group diversity comprising Cyanobacteria, Diatoms, Xanthophytes and Green algae, whereas rather acidic and nutrient poor soils are often inhabited only by Green algae (Sumbali and Mehrotra 2009; Nikorych and Chornevych 2012). Compared to different terrestrial microhabitats (hard natural or artificial substrates, tree bark, deadwood), the diversity of microalgae in soils is much higher and rarely dominated by a single group (Hallmann et al. 2011b; Kulichová et al. 2014). In soils of temperate regions, usually several groups of microalgae codominate.

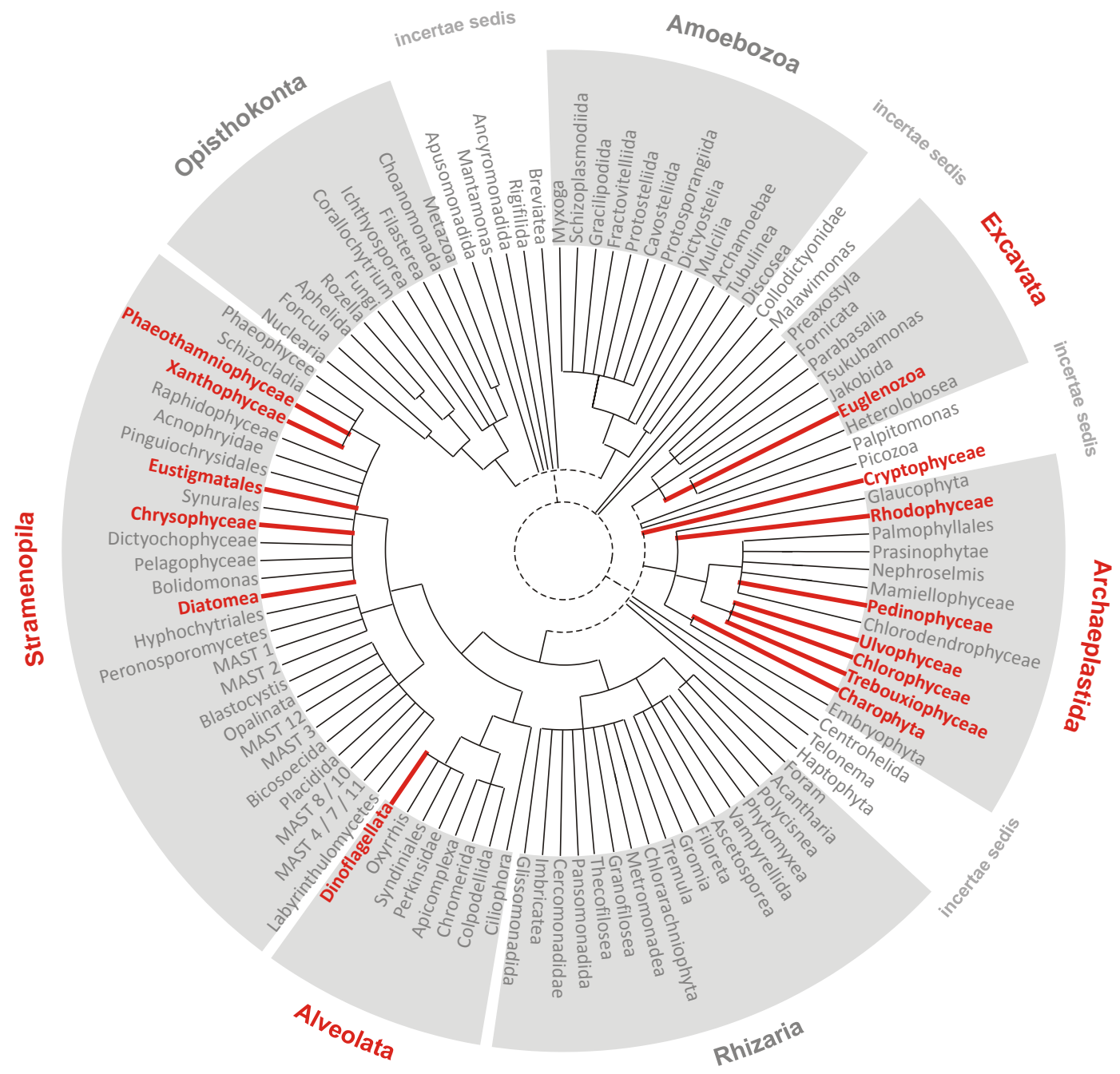

Figure 1. Schematic tree of Eukaryotes and their major lineages. Red branches represent algal lineages which include species living in soils. Adopted and modified after del Campo et al. (2014). 


\section{Molecular diversity of soil Green microalgae}

Considering modern systematics of Green algae, we recognize 20 lineages, which comprise terrestrial species (Rindi et al. 2009; Friedl and Rybalka 2012; Leliaert et al. 2012), Fig. 2. The most terrestrial green microalgae belong to the Chlorophyta lineage, which has an ancestor in common with the Streptophyta (the estimated split of both lineages is in Neoproterozoic; Becker 2013). Whereas the Streptophyta (= primarily freshwater lineage) colonized terrestrial habitats at first, Chlorophyta (= primarily marine lineage) colonized land later on (Becker and Marin 2009). The molecular evidence of green algal diversity in terrestrial habitats is still scant. The most molecular data were so far retrieved from soils, e.g., Alpine glacier forefields (Frey et al. 2013), Siberian permafrost (Vishnivetskaya 2009), Himalaya (Schmidt et al. 2011; Schmidt and Darcy

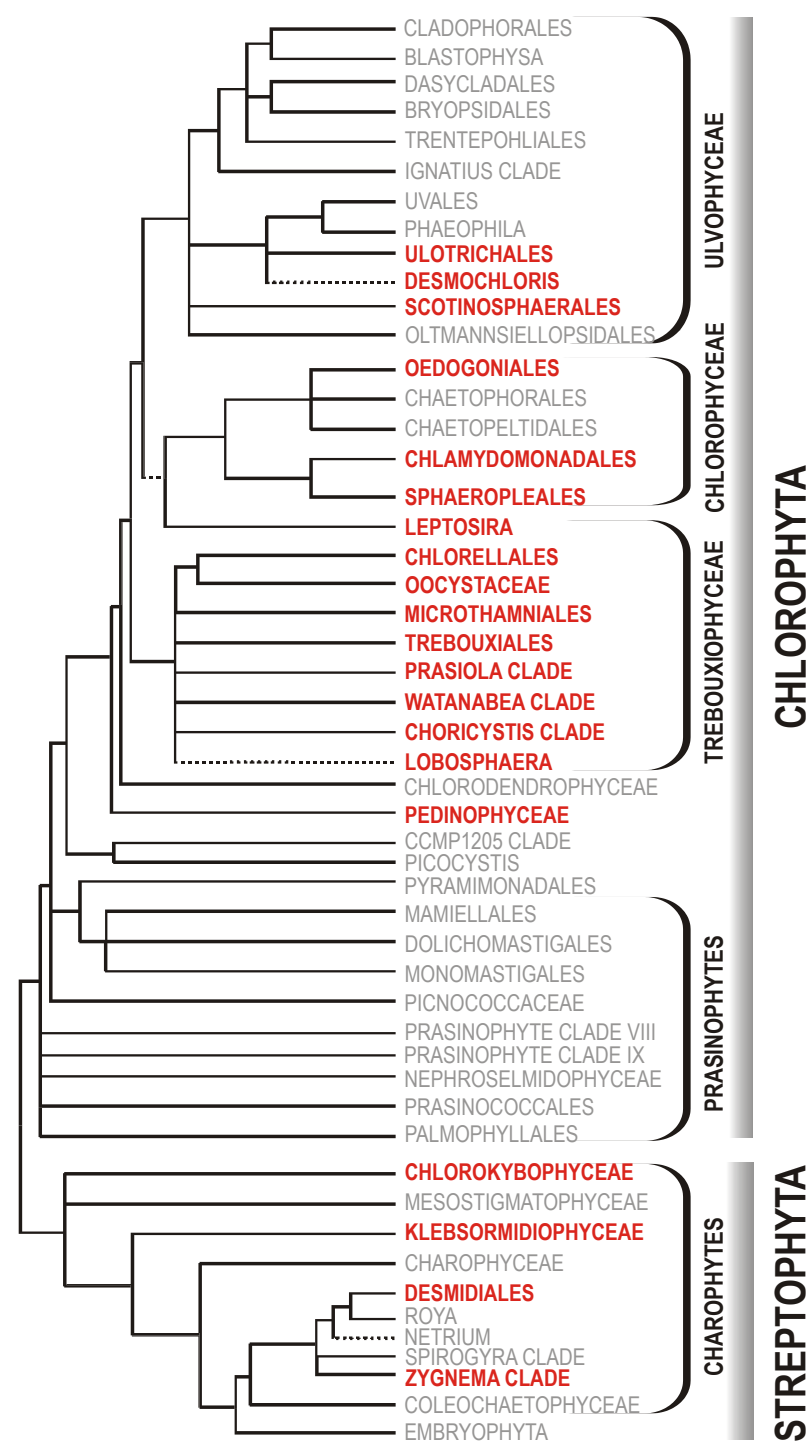

Figure 2. Schematic tree of Chlorophyta, Streptophyta and their major lineages. Red letters represent algal lineages which include soil dwelling species. Adopted and modified after Leliaert et al. (2012). 2014), North-American desert crusts (Lewis and Flechtner 2002; Lewis and Lewis 2005; Flechtner et al. 2013) and mountains (Freeman et al. 2009), Atacama desert volcano (Costello et al. 2009), Ecuadorean rainforest (Faßhauer et al. 2011; Spitzer et al. 2014) and Antarctica (Lawley et al. 2004; Schmidt et al. 2011). A few molecular diversity studies recovered green microalgae in different aero-terrestrial habitats, e.g., European tree barks (Kulichová et al. 2014; Hallmann et al. in prep.-a) and epilithic biofilms (Horath and Bachofen 2009; Hallmann et al. 2011b; Ragon et al. 2012; Hallmann et al. 2013a), Ecuadorean rainforest tree barks and tree leaves (Faßhauer et al. 2011; Spitzer et al. 2014) or South and Central American sloth hairs (Suutari et al. 2010). Central European soils were intensively studied by microscopy-based approach, however, no molecular diversity studies were published so far. Consequently, molecular taxonomy studies on soil Green algae are scant as well. A few genera of Green 
algae, which underwent taxonomic revisions, comprise species confirmed in European soils, e.g., Bracteacoccus (Fučíková et al. 2012), Chlorella (Luo et al. 2010; Bock et al. 2011; Treves et al. 2013), Coccomyxa (Darienko et al. 2015), Chloropyrula (Gaysina et al. 2013), Chlamydomonas (Pröschold et al. 2001), Chloroidium (Darienko et al. 2010), Chromochloris (Fučíková and Lewis 2012), Dictyococcus (Fučíková et al. 2011a), Interfilum (Mikhailyuk et al. 2008), Klebsormidium (Škaloud et al. 2014a), Neocystis (Eliáš et al. 2013), Pseudomuriella (Fučíková et al. 2011a; Fučíková et al. 2011b) and Scenedesmus-relatives (Lewis and Flechtner 2004; Hegewald et al. 2013; Kaufnerová and Eliáš 2013). Some terrestrial microalgae were described elsewhere and redetected in European soils, but are still in need of taxonomic revisions, e.g., Stichococcus (Neustupa et al. 2007; Hodač et al. subm.), Jenufa (Němcová et al. 2011; Hodač et al. 2012), Xylochloris (Neustupa et al. 2011; Hodač et al. 2012). Unique Green microalgae were so far recorded from North American desert soils, e.g., Bracteamorpha, Rotundella, Tumidella (Fučíková et al. 2013), Desertella, Eremochloris, Xerochlorella (Fučíková et al. 2014), Flechtneria (Sciuto et al. 2015) and Tetraflagellochloris (Barsanti et al. 2013). Green algal species descriptions from African or polar soils are still extremely rare and include Desmochloris from Namib Desert (Darienko et al. 2009) or Pabia from the Antarctic (Friedl and O'Kelly 2002).

\section{Biogeography of soil Green microalgae}

In Green microalgae, unambiguous recognition of the most species is not yet possible, due to a lack of morphological features and because molecular taxonomy do not follow any consensual species concept. Microalgae in terrestrial habitats exhibit morphological convergence (LópezBautista et al. 2007), i.e., a tendency to evolve simple globular or filamentous forms throughout unrelated phylogenetic groups (Fig. 3). Demanding morphological determination of similarly looking species lead to general underestimation of Green algal diversity and to putative misinterpretations of their distribution patterns (Hoffmann 1989; Rindi et al. 2009; Sharma and Rai 2010; Rindi et al. 2011; Guiry 2012; Škaloud et al. 2015). Whereas in aquatic protists biogeography is widely accepted (Finlay and Clarke 1999; Šlapeta et al. 2006; Řezáčová and Neustupa 2007; De Wever et al. 2009; Naselli-Flores and Padisák 2015), biogeography of terrestrial Green microalgae is still far from well explored (Lawley et al. 2004; Sharma et al. 2007; Rindi et al. 2009; Leliaert et al. 2012; Bates et al. 2013; Ryšánek et al. 2014). Terrestrial Green microalgae can be encountered nearly everywhere around the globe. Particularly soil species are capable of survival after long periods of desiccation (Trainor 1970; Buzer et al. 1985; Trainor 1985; Trainor and Gladych 1995) and can photosynthesize even in cave darkness (Kol 1967). Their resting cysts may easily survive transportation over long distances in the air being 
resistant to drought, high as well as low light intensities and high UV radiation, e.g., due to the presence of thickened cell walls or light protection pigments ( Rezanka et al. 2004; Karsten et al. 2005; Häubner et al. 2006; Gustavs et al. 2010). Early morphology-based attempts comparing geographic distributions of soil microalgae, e.g. Feher (1948), as well as later studies concordantly suggested that a couple of soil algae might be cosmopolitan (Metting 1981; Starks et al. 1981; Hoffmann 1989), Fig. 3. The putative cosmopolitanism of some microalgae was in accordance with the neutral dispersal model (Finlay and Clarke 1999; Finlay 2002; Fenchel and Finlay 2004), which claimed that protists generally do not have biogeography. This view changed rapidly with the era of molecular phylogenies (Sharma and Rai 2010). Molecular markers enabled description of a plethora of morphologically indistinguishable species (Boenigk et al. 2005; Rindi et al. 2008; Dal Grande et al. 2014). As a result, microalgal biogeography seemed to be supported by cryptic species mainly observed within a restricted area. The biogeographic evidence comprises multiple algal lineages such as (1) Stramenopila, e.g., Spumella (Boenigk et al. 2005), Xanthonema (Rybalka et al. 2009; Rybalka et al. 2013), Synura (Škaloud et al. 2014b), Frustulia

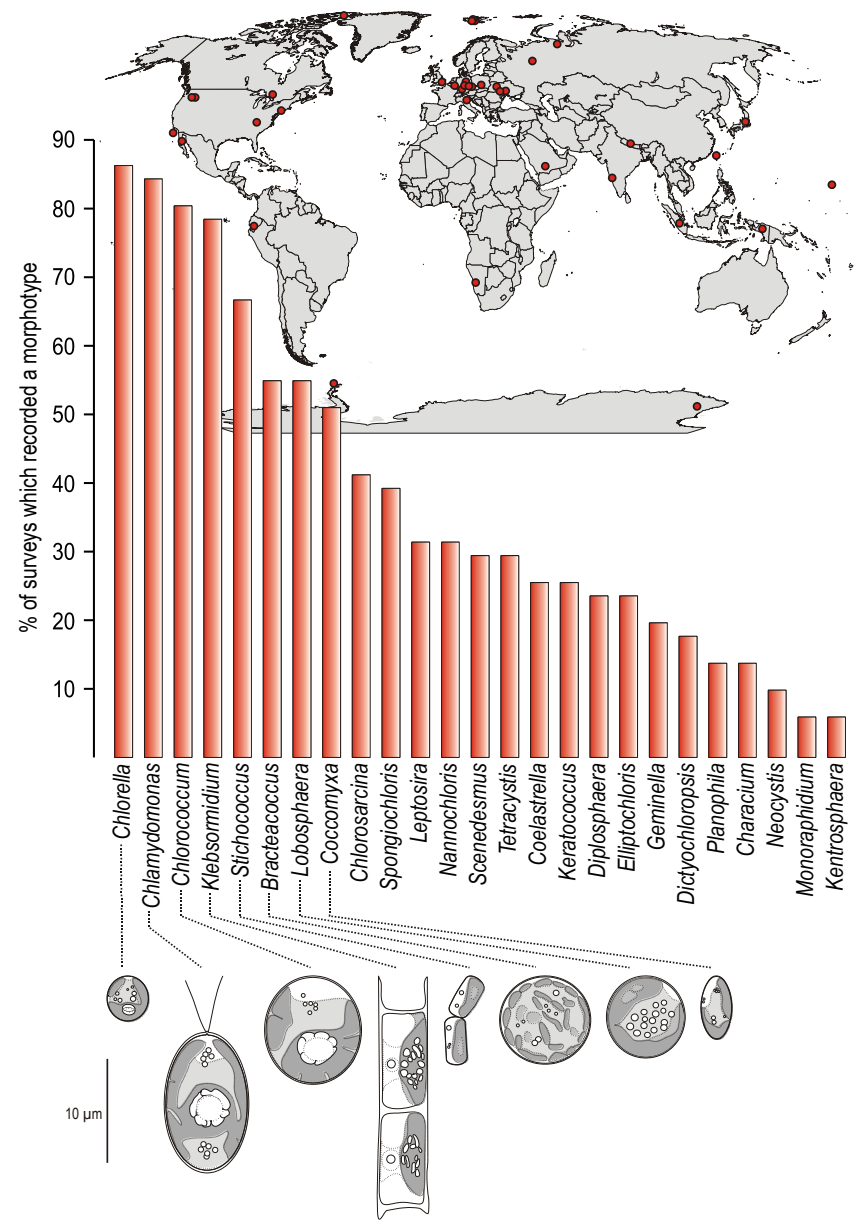

Figure 3. Bar chart summary of the most frequently recorded Green algal morphospecies/morphotypes. Species occurrences were derived from 46 floristic studies (shown in the world map and mentioned on pages 2-3).
(Veselá et al. 2012); (2) Streptophyta, e.g., Micrasterias (Jurdíková et al. 2014), Klebsormidium, (Ryšánek et al. 2014; Škaloud et al. 2014a) and (3) Chlorophyta, e.g., Asterochloris (Řídká et al. 2014; Škaloud et al. 2015). However, more extensive samplings of molecular data from geographically remote regions already confirmed that at least some species of terrestrial Green microalgae are cosmopolitan. A wide geographic distribution might apply for free living species of Coccomyxa (Darienko et al. 2015) and Klebsormidium (Ryšánek et al. 2014), as well as for lichenized species of Dictyochloropsis (Dal Grande et al. 2014) and Diplosphaera (Fontaine et al. 2013). In conclusion, biogeography studies on Green microalgae still have to cope with immense lack of molecular diversity data, since only 
a few regions have been sampled so far (Novis et al. 2008; De Wever et al. 2009; Kulichová et al. 2014). Species richness of eukaryotic microorganisms is generally poorly understood and diversities revealed from environmental samples significantly differ when using culturedependent or culture-independent approaches (Moon-van der Staay et al. 2006; Doherty et al. 2007; Lara et al. 2007; Hallmann et al. 2009; Hallmann et al. 2013a; Spitzer 2013). Particularly in Green microalgae, the link between culture-independent and culture-dependent diversities has been rarely studied. To establish a link between the both approaches, more monoclonal cultures/strains need to be sequenced and phylogenetically characterized. Although there is no universal barcoding marker for identification of microalgae, plastid and mitochondrial markers are widely used, along with the nuclear SSU (e.g. V4/V9 hypervariable regions) and the internal transcribed spacers ITS1-5.8S-ITS2 (Hall et al. 2010; Bock et al. 2011; Buchheim et al. 2011; Fučíková et al. 2011b; Krienitz and Bock 2012; Wolf et al. 2013; Darienko et al. 2015; Darienko and Pröschold 2015; Heeg and Wolf 2015; Lutz et al. 2015; Sciuto et al. 2015).

\section{General aims}

\section{(1) Biodiversity of terrestrial Green microalgae | Chapters 1-2}

Traditional morphospecies-based studies revealed a considerable diversity of Green microalgae in terrestrial habitats of Central Europe. Terrestrial Green algae in temperate climate zones, however, are still poorly explored using molecular techniques, in contrast to those from less favorable environments, e.g. hot desert soils. We expect molecular methods to reveal high algal diversities in moist soils of temperate grasslands and forests as well as in submerged algal biofilms. By focusing on cultures of Green microalgae from Central European soils and semiterrestrial biofilms, we aim to investigate genetic diversity of microscopically hardly distinguishable morphospecies.

\section{(2) Biogeography of terrestrial Green microalgae | Chapters 3-4}

Terrestrial Green microalgae are able of long-distance dispersal by resisting harsh environmental stresses. Such 'airborne' microalgae might dwell in soils and terrestrial biofilms across distant geographic regions and climate zones. However, there is little existing molecular evidence to address the question of microbial biogeography in terrestrial Green algae. With focusing on a few exemplar morphogenera, we aim to investigate the widely accepted idea of cosmopolitan distribution of terrestrial Green microalgae. 


\section{Summary of the Chapters}

Within the frame of this thesis, terrestrial samplings from two main sources were examined. Soil samples originating from 27 grassland and 30 forest sites of the German Biodiversity Exploratories were investigated in Chapters 1, 3-4. Semi-terrestrial biofilm samples originating from two karstic streams in Germany were studied in Chapter 2. Culturing and sequencing approach was applied consistently throughout the Chapters. Environmental samples were cultivated in standard enrichment media favoring the growth of terrestrial Green algae. After isolating the algae into monoclonal cultures, they were subjected to combined microscopical and molecular phylogenetic analyses. New sequence data were complemented by accessions from GenBank and additional algal strains were kindly provided by the SAG Culture Collection of Algae and by further projects of the Department of Experimental Phycology (Lepka 2007; Spitzer 2013).

Chapter 1 | Molecular diversity of microscopic Green algae isolated from German soils

Here we examined the diversity of culturable Green microalgae dwelling in forest and grassland soils. We asked whether our cultured strains genetically match SSU-amplicons (clones, pyrotag reads) from published culture-independent diversity surveys. Our approach enables to link operational taxonomic units from soil metagenomes with physical organisms. We contribute an extensive dataset of sequenced isolates from Central Europe (57 sampling sites, 188 sequenced monoclonal cultures, 61 monophyletic species of Green microalgae). We further inform about new species of soil microalgae and one novel lineage witihn the class Trebouxiophyceae. By putting geographic data for identical ITS2-ribotypes on the world map, we suggest long-distance dispersal of Stichococcus, Chlorella and Klebsormidium.

Chapter 2 | Diversity of microscopic Green algae (Chlorophyta) in calcifying biofilms of two karstic streams in Germany

Green algal biofilms cover periodically desiccating stromatolites in karstic streams in Germany. We investigated this unusual semi-terrestrial habitat for the first time using a combined morphological and molecular approach. Thirty-four species were inferred from 18S rDNA sequence analyses. Among the closest relatives are species known from aquatic and terrestrial habitats. We isolated filamentous Green algae morphologically resembling Gongrosira Kützing, often reported from freshwater tufa-stromatolites. Molecular analysis suggests that the taxon 
represents a collective morphotype, which encompasses several genera phylogenetically nested within the Ulvophyceae (Chlorophyta).

Chapter 3 | Phylogenetic analysis of polar Chlorella and Stichococcus suggests biogeography of airborne microalgae

Here we aim to uncover biogeographic patterns in cosmopolitan morphospecies Chlorella and Stichococcus. Already published $18 \mathrm{~S}$ rDNA sequences from terrestrial and aquatic habitats worldwide, provided a basis for biogeographic analyses. We expanded existing data by new sequences from terrestrial habitats of Germany, Ecuador, the Arctic and Antarctica. We found out that particular Stichococcus clades exhibit either temperate-polar or temperate-tropical distribution, but not both. The clades of Chlorella-like microalgae which include polar and hot desert strains (e.g., C. vulgaris and Muriella terrestris) were so far never uncovered in the tropics. Our data provide evidence that airborne microalgae might exhibit biogeography.

Chapter $4 \mid$ Molecular evidence for the wide distribution of two lineages of terrestrial Green algae (Chlorophyta) from tropics to temperate zone

Terrestrial microalgae Jenufa and Xylochloris were originally described from the Southeast Asia. Our discovery of similar $18 \mathrm{~S}$ rDNA sequences in terrestrial samples originating from temperate Europe and South and Central America suggests temperate-pantropic distribution of Jenufa and Xylochloris. Both lineages occur in soils, but differ in subaerial growth; whereas Jenufa growths on rocks and artificial hard substrates, Xylochloris was so far detected on organic substrates (tree barks). 


\title{
Chapter 1
}

\section{Molecular diversity of microscopic Green algae isolated from German soils}

\author{
Ladislav Hodač, Christine Hallmann, Karolin Spitzer and Thomas Friedl
}

University of Göttingen, Experimental Phycology and Culture Collection of Algae (SAG), Göttingen, Germany

(Manuscript draft)

\begin{abstract}
Microscopic Green algae are highly diversified in soils of temperate forests and grasslands. The morphology-based literature counts hundreds of (morpho)species, however, only a minor fraction of this diversity has been verified via DNA sequencing of monoclonal cultures. Therefore, SSUbased operational taxonomic units in soil algal metagenomes can hardly be linked with physical organisms. Here we extended the sampling of sequenced algal isolates and cultivated soils from 57 sampling sites in Central European forests and grasslands. We isolated and sequenced 188 monoclonal cultures and retrieved 61 species of Green microalgae. More than a half of them match conspecific cultures isolated elsewhere in Europe, predominantly from terrestrial habitats, but also from freshwaters. Furthermore, $90 \%$ of our new cultures match environmental clones retrieved from the same sampling sites and from different terrestrial and aquatic habitats. We contribute new ITS2-based evidence of the long-distance dispersal of Stichococcus, Chlorella and Klebsormidium. On the other hand, at least seven novel species were detected, suggesting that soils in Central Europe might veil algal diversity hardly accessible without culturing.
\end{abstract}

Keywords: soil biodiversity; Chlorophyta; monoclonal cultures, phylogeny, biogeography, morphology

Abbreviations: MB, Bayesian inference; ML, maximum likelihood; ITS2, internal transcribed spacer 2; OTU, operational taxonomic unit; GBE, German Biodiversity Exploratories; HAI, Hainich; ALB, Schwäbische Alb; SCH, Schorfheide-Chorin 


\section{Introduction}

Molecular diversity of soil microorganisms is far from well understood (Daniel 2005; Mocali and Benedetti 2010; Shade et al. 2012). Nonetheless, investigations of soil prokaryotes successfully target ecological questions (Lauber et al. 2009; Nacke et al. 2011; Leff et al. 2015; Prober et al. 2015), accelerated by modern diversity acquisition techniques. In contrast, less attention was paid to the molecular diversity of soil eukaryotic photoautotrophs - microalgae abundant in the topsoil horizon (Starks et al. 1981; Hoffmann et al. 2007; Wojtuń et al. 2013) and generally recognized as primary producers and soil stabilizers (Lukešová 2001; Hu et al. 2002; Hu et al. 2003a; Hu et al. 2003b). Unlike in the case of bacteria (Preston-Mafham et al. 2002; Prosser 2002) and fungi (Bailly et al. 2007; Prober et al. 2015) functional diversity of eukaryotic microalgae in soils remains poorly understood. The prevalent morphology-based concept of ecologically meaningful algocenoses (Gollerbach and Shtina 1969; Zenova et al. 1995; Kuzyakhmetov 1998; Şalaru et al. 2008; Kabirov et al. 2010) urgently needs molecular re-evaluation. Similar to prokaryotes and non-photoautotrophic protists (Boenigk et al. 2012; Caron et al. 2009; Couradeau et al. 2011), molecular diversity of microalgae is underestimated (Guiry 2012; Škaloud et al. 2015). Despite considerable sequencing efforts focused on microalgae and other protists in aquatic ecosystems (Moon-van der Staay et al. 2001; Chariton et al. 2010; Cheung et al. 2010; Medinger et al. 2010), molecular diversity of soil microalgae remains poorly explored (Lawley et al. 2004; Bates et al. 2013). Estimates of species richness in soils significantly differ between culture-dependent and culture-independent approaches. This applies for prokaryotes (Rondon et al. 2000; Daniel 2005; Shade et al. 2012), non-photoautotrophic protists (Moon-van der Staay et al. 2006; Doherty et al. 2007; Lara et al. 2007) and terrestrial microalgae (Hallmann et al. 2009; Hallmann et al. 2013a; Spitzer 2013). Guiry (2012) estimates that there are approximately 72500 algal species, 13000 of these species represent Chlorophyta with 8000 described (cultured) species and 5000 species to be discovered. Even though this estimate relies on Algaebase - the largest online database of information on algae (Guiry and Guiry 2015) — species were verified through molecular methods in a minority of cases.

18S rDNA (SSU) marker once changed our view of microbial diversity (Moreira and LopezGarcia 2002), was substantially criticized (Forney et al. 2004; Richards and Bass 2005; Škaloud et al. 2015), but still holds its significance due to application universality (Lesaulnier et al. 2008; Bråte et al. 2010; Cheung et al. 2010; Bates et al. 2013; Lutz et al. 2015). However, cultureindependent diversity studies often miss a deeper taxonomic resolution and treat Green microalgae as undifferentiated group-Chlorophyta (Lawley et al. 2004; Brown et al. 2009; 
Bates et al. 2013; Lutz et al. 2015). Number of detected species (or operational taxonomic units, OTUs) directly depends from sequence-similarity threshold-value and sequence length (Caron $e t$ al. 2009; Grattepanche et al. 2014). Eukaryote-wide next generation sequencing (NGS) studies prefered SSU hypervariable regions SSU-V4 (Cheung et al. 2010; Taib et al. 2013) and SSU-V9 (Amaral-Zettler et al. 2009; Taib et al. 2013). Considering SSU-V4, 97\% sequence similarity is a widely accepted minimum cutoff-value, applied in e.g. Ion Torrent sequencing of snow Chlorophyta (Lutz et al. 2015), 454-pyrosequencing of Chlorophyta in an estuary reservoir (Sun et al. 2014) or from saltern ponds (Filker et al. 2015). The maximum cutoff-value is usually $99 \%$, used in e.g. 454-pyrosequencing of aquatic Chlorophyta (Zhan et al. 2013). In biodiversity acquirements, next generation sequencing outperform any traditional approach by means of achieved species/OTU quantity and detection of extremely rare and (so far) uncultivable species (Shi et al. 2009; Santoferrara et al. 2014; Xiao et al. 2014). On the other hand, Xiao et al. (2014) noticed limits of NGS for microalgal species resolution and affirmed importance of microscopic species identification. The same recognized Bazin et al. (2014) applying 98\% cutoff and Viprey et al. (2008), who proposed a more stringent threshold for species identification (98.5-99.5\%), both cloning marine Green microalgae.

In the taxonomy of Green microalgae, cultures are crucial for precise species characterization (Lukešová 1993; Ettl and Gärtner 1995; Lukešová and Hoffmann 1996; Lukešová 2001). Fine scale identification of microorganisms is essential for understanding their role in ecosystems (Rastogi and Sani 2011) and urgently required for reproducible comparisons of biodiversity shifts between environments (Richards and Bass 2005). Cultures of microalgae are not only valuable for original species characterization, but also for preservation of genetic material (Müller et al. 2007a; Day et al. 2010). This applies particularly in times of accelerated biodiversity loss (Pimm and Raven 2000). Monitoring of microbial/protistan biodiversity shifts correlated with climate change will hardly be possible without preservation of the current status, e.g., by maintaining cultures or environmental samples (Cary and Fierer 2014; Fierer and Cary 2014). Leading morphology-based identification key for terrestrial algae counts 785 species of (mostly cultured) Green microalgae (Ettl and Gärtner 1995; Ettl and Gärtner 2014). It is not clear how the (morpho)species diversity correlates with OTU-based diversities reported from terrestrial ecosystems via culture-independent techniques. This is due to scarcity of biodiversity surveys combining morphological observations and molecular techniques. Such comparative studies so far focused mainly on phytoplankton (Krienitz and Bock 2012), semi-terrestrial biofilms (Brinkmann et al. 2015; Hodač et al. 2015) and aerophytic biofilms (Lin et al. 2012; Kulichová et al. 2014). Regarding soils - the most species-rich terrestrial habitats-polyphasic data are 
available so far for arid lands (Lin and Wu 2014; Flechtner et al. 2013; Fučíková et al. 2014) or subtropical farmlands (Lin et al. 2013).

In the present study, for the first time we investigate molecular diversity of culturable Green microalgae in soils from Central Europe. The soils of this region remain almost untouched by culture-dependent molecular diversity surveys targeting Green microalgae, despite the fact that significant fraction of them were described from here (Ettl and Gärtner 1995). Expecting differences in cultured and uncultured microbial diversities, we hypothesize that extensive sampling and culturing would retrieve new species, not yet submitted to GenBank from cultureindependent surveys. We investigate 188 new monoclonal cultures of soil Green microalgae sampled from 57 sites covering deciduous and needle forests and meadows and pastures in Germany (Fischer et al. 2010). To achieve comparability with culture-independent studies, we sequenced SSU marker and conducted species identification following monophyletic species concept (Johansen and Casamatta 2005; Mallet 2005; Vyverman et al. 2010; Škaloud and Rindi 2013; Leliaert et al. 2014). Allowing minimal sequence divergence-using high sequence similarity threshold of $\geq 99.90 \%$ - we aim to evaluate how our data match already published cultured species. In a second step we test whether our cultured species match OTU-based diversities acquired by cloning or NGS techniques, hereby applying a similarity threshold of $97 \%$ for pyrotags (Sun et al. 2014; Lutz et al. 2015) and 99\% for clones (Viprey et al. 2008; Zhan et al. 2013). Since soil is a semi-aquatic habitat, it presumably hosts an assemblage of terrestrial and aquatic species. Though morphological literature suggest cosmopolitanism of microalgae in temperate soils (Ettl and Gärtner 1995; Lukešová 2001), the molecular evidence is missing. This we aim to prove by comparing our isolates with cultures sampled elsewhere, analyzing the highly variable ITS2 marker (Müller et al. 2007b; Coleman 2009; Buchheim et al. 2011; Keller 2011; Caisová et al. 2013; Wolf et al. 2013).

\section{Material and Methods}

\section{Sampling sites}

Soil samples were collected from defined plots within large-scale and long-term research sites termed Biodiversity Exploratories (Hainich-Dün, Abbr. HAI; Schorfheide-Chorin, Abbr. SCH; Schwäbische Alb; Abbr. ALB; Fig. 1a). The sampling covers plots in forests (Figs. 1b, 1c, 1d) and grasslands (Figs. 1e, 1f), which represent a gradient of land-use intensities, described in detail by Fischer et al. (2010). We analyzed samples from two soil horizons: 1) drill core (A-horizon) samples were collected in HAI, SCH, ALB in April 2008 from 27 grassland and 30 forest plots 
and multiple samples from within each plot were pooled, 2) soil surface (O-horizon, upper $3 \mathrm{~cm})$ samples were taken in autumn (September 2010) and spring (March 2011, June 2011) from two forest and three grassland plots within the HAI exploratory (using a sterile spoon and transported in sterile Petri dishes. In the latter case, three different sampling points were defined within each plot (middle, north-east corner, south-west corner; Fig. S5e). These subplot samples were analyzed in two ways, i.e., separately and pooled together.
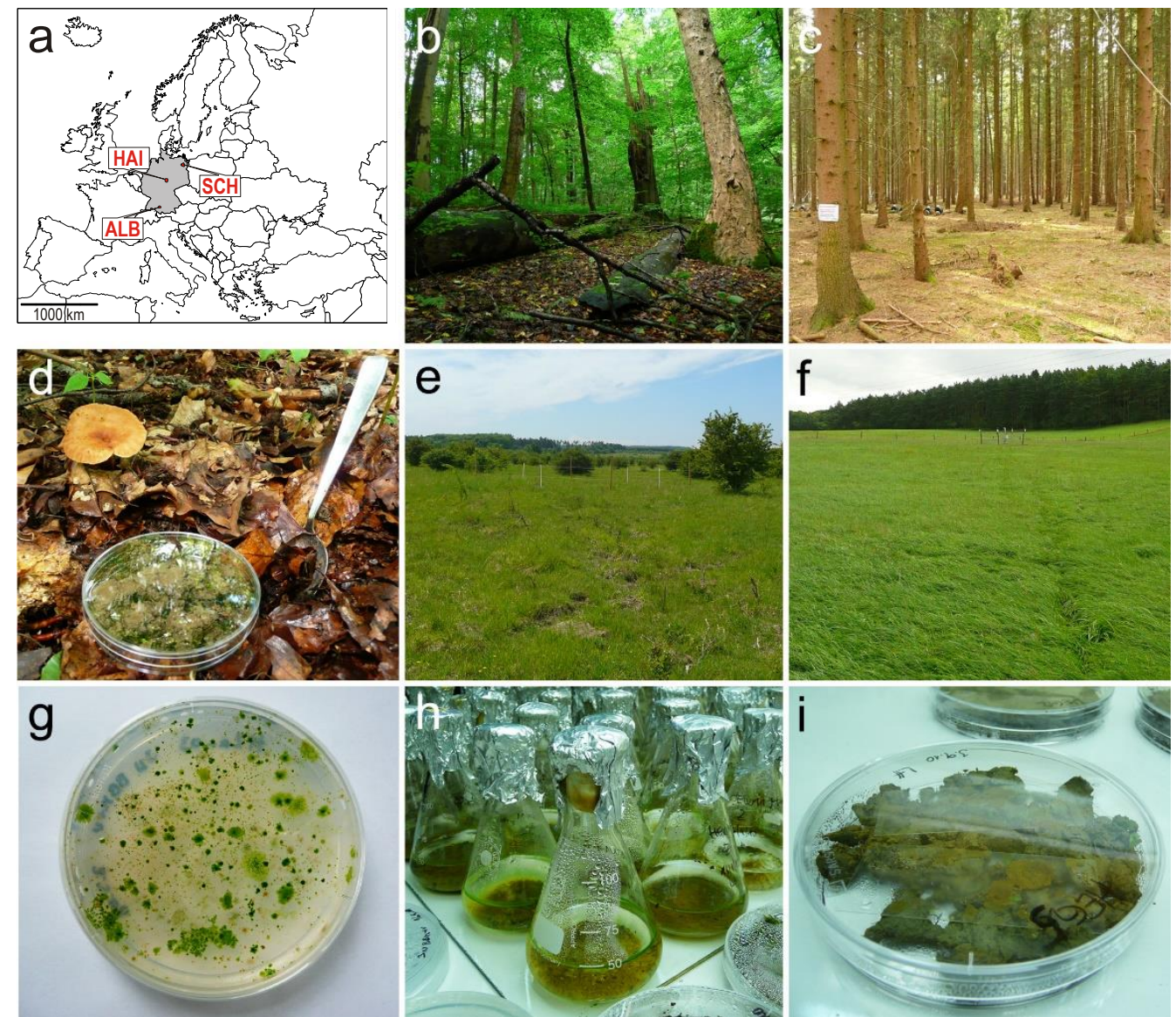

Figure 1. Soil sampling in the German Biodiversity Exploratories. (a) Localization of the three sampling areas (highlighted in red):

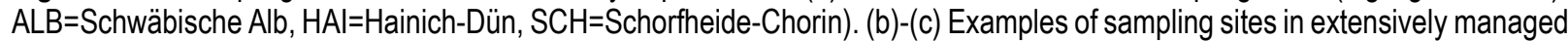
(b) and intensively managed (c) forests (Hainich). (d) Soil sample taken by a spoon from topsoil. (e)-(f) Examples of sampling sites in extensively managed (e) and intensively managed ( $f$ ) grasslands (Hainich). (g)-(i) Culturing techniques-establishment of mixed cultures. (g) Algal colonies on agar plate with spread soil suspension); (h) Algal growth in soil suspended in liquid BBM medium (Bischoff and Bold 1963); (i) Culturing of microalgae on coverslips put on the soil surface.

\section{Soil culturing, isolation and light microscopy of monoclonal cultures}

Two grams of each soil sample were suspended and cultured in liquid media and spread on agarized media (Fig. 1g). The composition of liquid (in $100 \mathrm{ml}$ Erlenmeyer flasks; Fig. 1h) and $1.5 \%$ agarized (Petri dishes) media was the same: 3NBBM+V and BG11 (EPSAG; https://www.uni-goettingen.de/de/list-of-media-and-recipes/186449.html). Both liquid and 
agarized cultures were kept for at least six weeks at $18{ }^{\circ} \mathrm{C}$ under a light : dark regime of $14: 10$ hours at a light intensity of about $25 \mu \mathrm{E}$ from a white fluorescent bulb. After three, four and six weeks of growth in liquid media, algal suspension was inspected using a light microscope. The algal colonies growing on the agarized plates were transferred with sterile inoculating loops onto new fresh plates with the same medium and final monoclonal cultures were kept on agar slants $(1.5 \%)$ under the same growth conditions. The soil surface samples were treated in the same way as described above. The only modification concerns the BG11 medium which was replaced by Z medium. In addition to the culturing techniques on media, cover slips method was used (Ettl and Gärtner 1995; Lukešová 2001). Sterile glass cover slips were put directly on wet soil samples (stored in sterile Petri dishes; Fig. 1i) and after 2-3 weeks of algal growth on the contact surface, the covers slips were analyzed by light microscopy. The light microscopy of the mixed raw cultures and unialgal isolates was accomplished using an Olympus BX60 microscope (Tokyo, Japan) with Nomarski DIC optics with an attached ColorView III camera (Soft Imaging System, Münster, Germany). The observations were documented by micrographs, processed using the Cell^D image software (Soft Imaging System, Münster, Germany). Cell size measurements (diameter of spherical cells; length/width of elongated cells) were conducted were conducted in program ImageJ (Schneider et al. 2012) and were based on 100 cells per culture. and the summary statistics including maximum and minimum values were computed in PAST 2.12 (Hammer et al. 2001).

\section{DNA isolation, PCR and sequencing}

DNA was extracted from 188 monoclonal isolates (Table S1) using the Invisorb Spin Plant Kit (Stratec molecular, Berlin, Germany) as recommended by the manufacturer. 18S rDNA marker was amplified using primers NS1 and 18L (Hamby et al. 1988). For selected isolates 18S and ITS2 rDNA gene region was amplified using primers NS1 and LR1850 (Friedl 1996). Conditions for PCR and sequencing reactions and the standard set of sequencing primers were described previously by Mikhailyuk et al. (2008). The newly determined sequences will be deposited in GenBank. Two additional isolates from polar regions (Shukla et al. 2011), and two from Ecuador (Spitzer 2013; Faßhauer in prep.) were sequenced for 18S rDNA.

\section{Phylogenetic analysis}

The newly obtained 80 full to almost full $18 \mathrm{~S}$ rDNA sequences $(\sim 1600-1800$ nucleotides in length; Tables S2a, S2b) were compared to NCBI database using BLAST queries (Altschul et al. 
1997) in order to download all closest relatives available from GenBank (Benson et al. 2012). All sequences were checked for chimeras by Bellerophon (Huber et al. 2004). Two alignments were established, one for Green algae (Chlorophyta + Streptophyta) and another for Stramenopiles including Xanthophyceae and Eustigmatophyceae. Both alignments were further processed in the same way. Alignments were computed by the program MAFFT-Q (Katoh and Toh 2008) and inspected in BioEdit (Hall 1999) for possibly misaligned nucleotide positions. The alignment of Green algae consisted of 169 sequences with 1817 positions (711/564 variable/parsimony informative). The alignment of Stramenopiles consisted of 72 sequences with 1895 positions (613/434 variable/parsimony informative). In both cases, the $G T R+\Gamma+I$ model was selected as the best fitting substitution model using AIC criterion in jModelTest 0.1.1 (Posada 2008). Phylogenetic trees were calculated using maximum likelihood method in the program RAxML 7.0.4 (Stamatakis et al. 2008). The Bayesian phylogenetic trees were calculated using MrBayes 3.2.1 x64 (Ronquist et al. 2012). For the latter, two MCMC runs for three million generations each were employed with one cold and three heated chains with trees sampled every 100 generations. Internal edge support values were inferred from the rapid bootstrapping algorithm (100 replicates) as implemented in RAxML (Stamatakis et al. 2008) and from Bayesian posterior probabilities using MrBayes 3.2 .1 x64 (Ronquist et al. 2012). Pairwise sequence similarities were derived from p-distances based on Kimura-2-parameter model computed in the program MEGA5 (Tamura et al. 2011). Pairwise similarities among our isolates and their closest GenBank relatives are listed in Table S3. The same method was used to identify species represented by partial $18 \mathrm{~S}$ rDNA sequences (Tables S4a, S4b).

\section{Delimitation of morphotypes and statistical analysis of their distribution}

The morphological diversity of monoclonal cultures is summarized in Table S5. Observations on morphotypes occurring in mixed cultures (agar plates, liquid media and cover slips) were documented as binary presence/absence matrices (Table S6a; Table S6b) and summarized in Table S7. Delimitation of morphotypes was accomplished by identification literature (Reisig1 1964; Trenkwalder 1975; Metting 1981; Huber-Pestalozzi et al. 1983; Ettl and Gärtner 1995; Andreyeva 1998; Komárek and Anagnostidis 1998; Komárek and Anagnostidis 2005; Hoffmann et al. 2007; Škaloud 2009; Komárek 2013; Ettl and Gärtner 2014). Morphotype representatives are documented as drawings of monoclonal cultures and microphotographs of monoclonal cultures and mixed cultures. Where it was possible, taxa were morphologically determined at the genus level. Otherwise, assignation at higher taxonomic level was preferred. Taxonomically 
ambiguous taxa predominantly occurring as similar living forms were combined into collective morphotypes. For example, sarcinoid forms traditionally named Chlorosarcina, Chlorosarcinopsis and Desmotetra represented one collective morphotype and named Chlorosarcinaceae. The resulting binary presence/absence matrix was further used for multivariate statistical analysis. Prior to the analysis, we pooled the presence/absence data of each three replicates representing the same land-use intensity, e.g., AEG1+AEG2+AEG3=AEG (intensively managed grassland plots within ALB). Redundancy analysis (RDA) (Hill 1979; Jaarsma et al. 2006) was conducted to test for correlations between important soil physicochemical parameters, i.e., $\mathrm{pH}$, organic carbon/ $\mathrm{C}_{\text {org }}$, total nitrogen $/ \mathrm{N}_{\text {tot }}$ (Metting 1981; Marschner et al. 2003) and the composition of algal morphotypes at the sampling sites. The original measurement data were obtained from Nadine Herold in the frame of the German Biodiversity Exploratories project. Because the diversity data were pooled within each land-use category (Fischer et al. 2010), in the ordination analysis we used corresponding arithmetic means of each physico-chemical parameter. For statistical support the Monte Carlo test with 2000 permutations under reduced model was used (Hope 1968). The morphotype composition of the soil surface samples was analyzed using Non-metric Multidimensional Scaling (NMDS) using Jaccard distance measure. All statistical analyses were performed in PAST version 2.12 (Hammer et al. 2001) and CANOCO version 4.5 (ter Braak and Šmilauer 2002). Hence Green algae (Chlorophyta, Streptophyta) and stramenopiles (Xanthophyceae, Eustigmatophycae) were present in almost all samples and many taxa are relatively easy to cultivate (compared to e.g. Diatoms or Cyanobacteria) we focused on them in further molecular studies.

\section{Results}

\section{Molecular diversity and morphology of the monoclonal isolates}

We analyzed a total of 188 18S sequences; 146 sequences were longer (1600-1800 bp) and 42 shorter (300-1600 bp). Within this dataset we distinguished different 73 phylotypes corresponding to species at $\geq 99.90 \%$ sequence similarity level. The species belong mainly to Green algae: Chlorophyta (59 spp.) and Streptophyta (2 spp.) and to Stramenopiles: Xanthophyceae (11 spp.) and Eustigmatophyceae (1 sp.).

\section{Green algae}

We detected 61 species assigned of 21 lineages from four classes of Chlorophyta (Chloro-, Trebouxio-, Ulvo- and Prasinophyceae) and one class of Streptophyta (Klebsormidiophyceae). 
The Chlorophyceae are represented by ten lineages: Oogamochlamydinia, Chloromonadinia, Tatrensinia, Reinhardtinia, Desmotetra, Stephanosphaerinia, Jenufa, Scenedesmaceae, Pseudomuriellaceae, Bracteacoccaceae). The Trebouxiophyceae are represented by eight lineages: Prasiola/Stichococcus, Neocystis, Botryococcus, Watanabea, Lobosphaera, Xylochloris/Dictyochloropsis, 'Navichloris', Chlorellaceae. The Ulvophyceae are represented by Ulotrichales and Pedinophyceae by Pedinomonas. The Klebsormidiophyceae is represented by Klebsormidium.

\section{Streptophyta; Klebsormidiophyceae}

The Streptophyta were represented by two different species of Klebsormidium (Fig. 2), common terrestrial algae with unbranched filaments and a single band-shaped chloroplast (with a pyrenoid) per cell. (I) Klebsormidium flaccidum (e.g., isolate LH10HG2056; Fig. 3a) from grasslands (HAI, SCH) and forest (HAI) is identical to K. flaccidum SAG 7.91 (EU434019) from freshwater (Fig. 2). Klebsormidium cf. dissectum/elegans (isolate LH08HW9106; Fig. 3b) from grasslands (ALB) and forests (HAI) is identical to authentic strain K. dissectum SAG 2155 (from soil) and to authentic strain $K$. elegans SAG 7.96 (from tree bark). The whole clade including species of Klebsormidium and Interfilum is highly supported.

\section{Chlorophyta; Pedinophyceae}

Prasinophyte algae were represented by one isolate belonging to Pedinomonas (Fig. 2), exhibiting small Chlamydomonas-like cells containing one pyrenoid and basal stigma. Pedinomonas cf. minor (isolate LH08SG2033; Fig. 3c) from grassland (SCH) is closely related to P. minor SAG 1965-3 (HE610132) from freshwater (Fig. 2). The clade comprising both accessions is highly supported.

\section{Chlorophyta; Ulvophyceae}

The class Ulvophyceae is here represented by the Ulvales/Ulotrichales lineage (Watanabe and Nakayama 2007) and one genus Pseudendocloniopsis (Fig. 2), with spherical to ovoid (slightly acuminate) cells with a cup-shaped chloroplast and prominent pyrenoids. Pseudendoclonium cf. botryoides (isolate LH08HW9058; Fig. 3d) from forest (HAI) was closely related to authentic strain $P$. botryoides SAG 465-1 (AJ416103) from freshwater. The clade comprising both accessions is highly supported. 


\section{Chlorophyta; Chlorophyceae; Sphaeropleales.}

Scenedesmaceae (Hegewald and Hanagata 2000) comprised three species (Fig. 2) with spherical to symmetrically elliptic cells with slightly acuminate poles. (I) Coelastrella multistriata (e.g., isolate LH10HG7083; Fig. 3e), identical to terrestrial C. multistriata Hanagata C6-2 (AB012846), was found in grasslands (HAI, ALB) and forest (ALB). (II) Coelastrella sp. (e.g., isolates LH10HG2098, LH10HG7018; Fig. 3f) occurs in grasslands (HAI) and is identical to Scenedesmus sp. KGU Y002 (AB742453). (III) Acutodesmus rubescens (e.g., isolate LH08SG8041; Fig. 3g) from grassland (SCH) is identical to 'Scenedesmus' (Acutodesmus) rubescens CCAP 232/1 (X74002). The Scenedesmaceae clade is highly supported.

Members of Pseudomuriellaceae and Bracteacoccaceae (Fučíková et al. 2013) exhibit spherical cells with several to multiple parietal chloroplasts without pyrenoids. Pseudomuriella aurantiaca (isolates LH10HG9038, LH10HG2039; Fig. 3h) from grasslands (HAI) is identical to the authentic strain P. aurantiaca SAG 249-1 (X91268) isolated from soil. Bracteacoccus cohaerens (isolate LH10HG9034; Fig. 3i) from grassland (HAI) is identical to the authentic strain B. cohaerens UTEX 1272 (GQ985406) from soil (USA). Another isolate from grassland (SCH), Bracteacoccus cf. cohaerens (isolate LH08SG2015), is slightly different from the same GenBank accession but might be as well conspecific. Both Pseudomuriella and Bracteacoccus clades were highly supported.

\section{Chlorophyta; Chlorophyceae; Chlamydomonadales}

Isolates assigned to Chlorophyceae were nested in two major lineages Sphaeropleales and Chlamydomonadales, and Jenufa clade incertae sedis (Fig. 2). The Chlamydomonadales isolates were characteristic for monadoid/flagellate Chlamydomonas-like and/or coccal Chlorococcumlike morphologies (Fig. 4a-k). The Sphaeropleales were either coccal colonial Scenedesmus-like, or non-colonial Bracteacoccus-like (Fig. 4a-e).

Oogamochlamydinia clade (Nakada et al. 2008). The clade comprises monadoid isolates belonging to two species of similar morphology differing in cell size. (I) Oogamochlamys sp.(I) (isolate LH08SG8047; Fig. 4a) from grassland (SCH) is less closely related to O. ettlii UTEX 2218 and O. gigantea SAG 21.72. (II) Oogamochlamys sp.(II) strain SAG 2476 (= isolate LH08AW 1069); Fig. 4b) from forest (ALB) is less closely related to freshwater Chlamydomonas sp. CCAP 11/159. Both species are nested in well supported clade including Oogamochlamys and Lobochlamys accessions.

Chloromonadinia clade (Nakada et al. 2008). One monadoid species, Chlamydomonas cf. gerloffii (isolate LH08SW5031; Fig. 4c), found in forest (SCH), is closely related to freshwater 
C. gerloffii CCAP 11/72 (FR865610). The species is nested in a highly supported clade including authentic strains $C$. gerloffii CCAP 11/72 and Chloromonas rosae UTEX 1337 accessions.

Reinhardtinia clade (Nakada et al. 2008). Monadoid isolates of Chlamydomonas-like morphology clustered in four different species. (I) Chlamydomonas rapa (isolates LH08SG1077, LH08SG9055) detected in grasslands ( $\mathrm{SCH})$, is identical to freshwater authentic strain $C$. rapa SAG 48.72 (U70790). (II) Chlamydomonas cf. rapa (isolate LH10HG1027; Fig. 4d) from grassland (HAI), is very closely related to C. rapa SAG 48.72 and might be conspecific as well. These three accessions create a highly supported clade, a sister group to C. reinhardtii (M32703). Two isolates from grasslands $(\mathrm{ALB} / \mathrm{SCH})$ were nested in another highly supported subclade within Reinhardtinia clade. (III) Heterochlamydomonas sp. (isolate LH08AG2004; Fig. 4e) closely related to freshwater H. rugosa SAG 45.86 (AF367859) and authentic strain H. inaequalis UTEX 1705 (AF367857). (IV) Chlamydomonas cf. typica (isolate LH08SG9022; Fig. 4f) is closely related to soil $C$. typica NIES-2246 (AB701557). The clade including the four species from Germany is highly supported.

Desmotetra clade (Nakada et al. 2008). Desmotetra Deason \& Floyd detected in this study is characterized by pairs of widely elliptic non-motile cells remaining attached after division. $D$. stigmatica (isolate LH08SG2049; Fig. 4g) from grassland (SCH) is identical to authentic strain D. stigmatica UTEX B 962 (DQ009760) in a highly supported clade - a sister group of the Reinhardtinia clade. The species was further found in grassland (HAI; isolate LH10HG6P18).

Tatrensinia clade (Nakada et al. 2008). The clade comprises two Chlorococcum-like species from grasslands (HAI/SCH). (I) 'Tatrensinia' ('Chlorococcum') sp.1 (LH08SW7115; Fig. 4h) is closely related to soil Haematococcaceae clone Amb_18S_582 (EF023273). (II) 'Tatrensinia' ('Chlorococcum') sp.2 (isolates LH10HG7016, LH10HG9131; Fig. 4i) is closely related to 'Chlorococcum' cf. tatrense (= Chlamydocapsa sp.) CCCryo 101-99 (AF514407) from Spitsbergen permafrost. Both species are nested in a highly supported clade.

Stephanosphaerinia clade (Nakada et al. 2008). Isolates exhibiting monadoid Chlamydomonas-like morphology clustered in four different species. (I) Chlorococcum sphacosum ('Neospongiococcum' gelatinosum) (isolate LH10HG3113; Fig. 4j) is identical to authentic strain C. sphacosum SAG 66.80 (JN968580) and authentic strain N. gelatinosum SAG 64.80 (JN968584), both isolated from soils. (II) Chlamydopodium vacuolatum (isolate LH10HG1013) identical to 'Chlorococcum' robustum Kr 1986/30 (AY122332) from freshwater. (III) 'Stephanosphaerinia' ('Chlorococcum') sp. (isolate LH10HG6108), is closely related to freshwater isolate Chlorococcales sp. VII3 (FJ946904) from Antarctica. The three species are nested in a highly supported clade. Another highly supported clade consisted of (IV) 
Chlorococcum cf. minutum SAG 2479 (= LH08AW5056; Fig. 4k) and closely related C. minutum SAG 21.95 (JN968585) from soil. Chlorococcum cf. minutum was detected in grassland and forest (ALB).

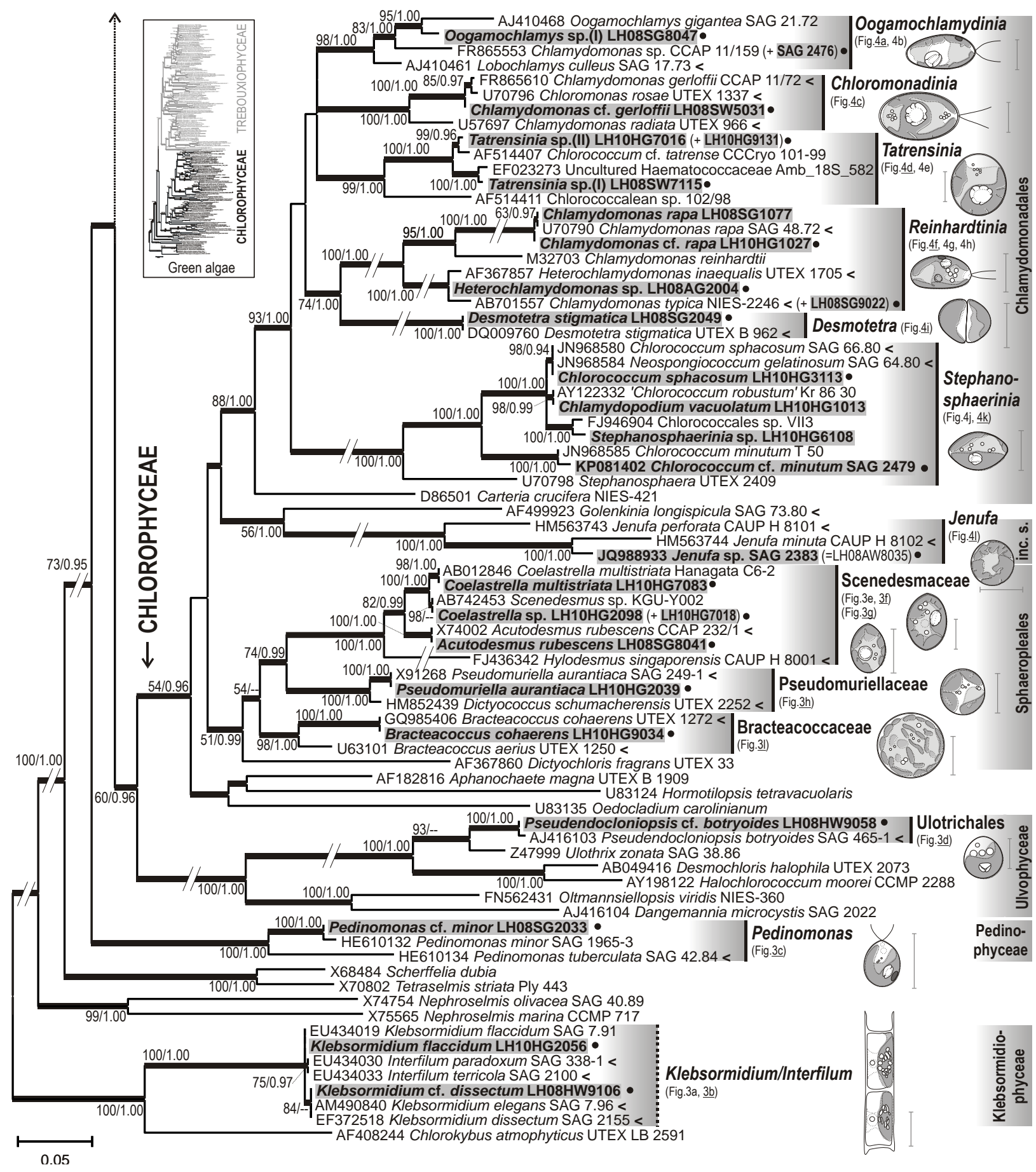

Figure 2. $18 \mathrm{~S}$ rDNA phylogeny of Green algae, part 1 including Chlorophyceae, Ulvophyceae, Pedinophyceae and Klebsormidiophyceae. New accessions are written in bold and grey underlaid. Black dots mark references to microphotographs (Fig. 3, Fig. 4) sorted in top down order. Original drawings of representative morphologies are shown for each clade (scale bars $=5 \mu \mathrm{m})$. Sequences of authentic strains are marked by $a^{\prime}<'$ sign. Numbers next to branches indicate statistical support values (maximum-likelihood bootstraps/Bayesian posterior probabilities). 


\section{Chlorophyta; Chlorophyceae; Jenufa clade}

Jenufa clade (Němcová et al. 2011) comprises coccid species with spherical cells with an extensively lobed parietal chloroplasts without pyrenoids. Jenufa sp. SAG 2383 (= LH08AW8035; Fig. 4l; JQ988933) and (identical) isolate LH08AW8098, found in forest (ALB), are related to authentic strain J. minuta CAUP H 8102 (HM563744) from tree bark in Singapore. The Jenufa clade, incertae sedis within the Chlorophyceae, consists of J. minuta CAUP H 8102, J. perforata CAUP H 8101 and Jenufa sp. SAG 2383 (= LH08AW8035) and is highly supported.
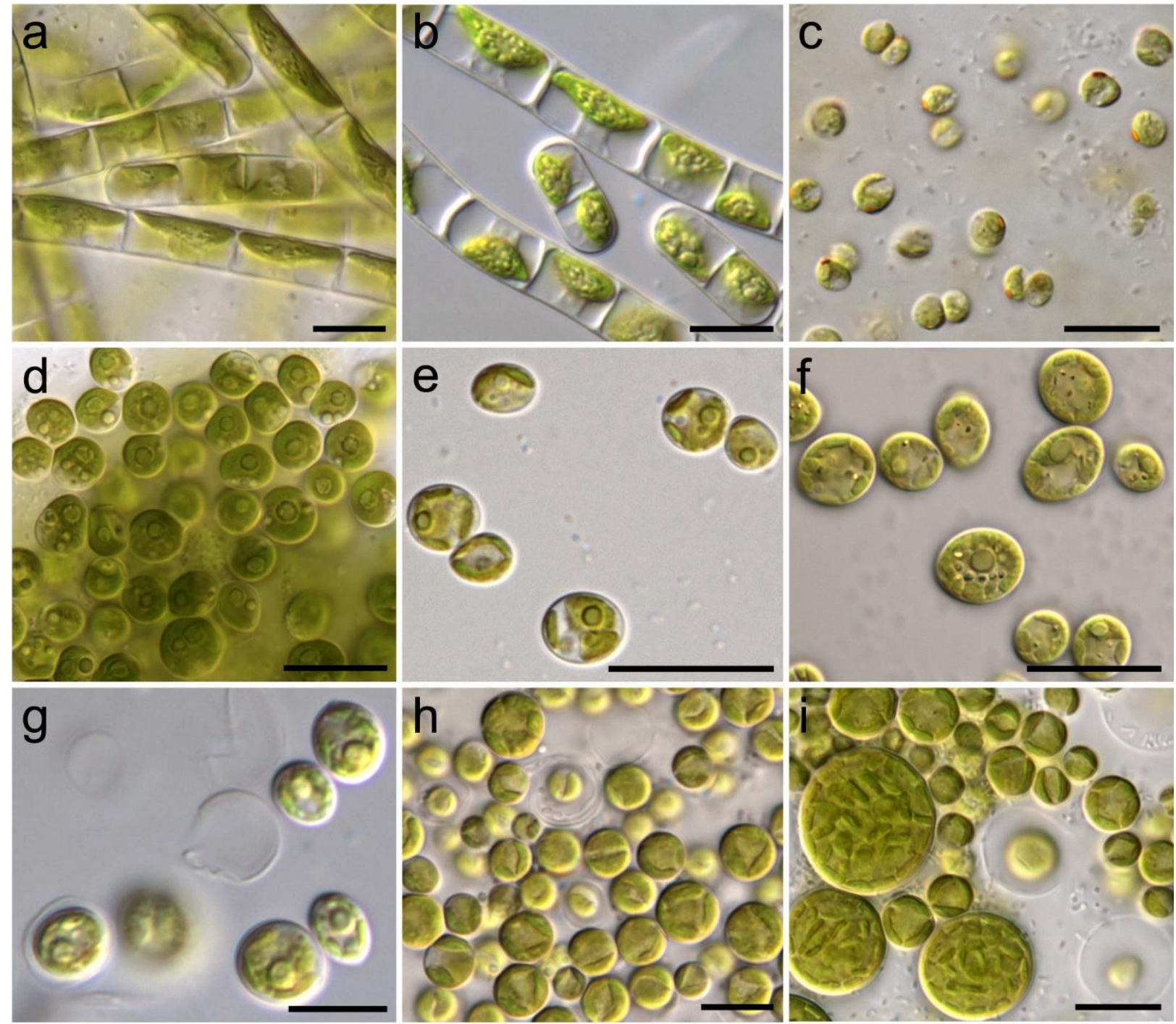

Figure 3. Microphotographs of monoclonal cultures of Green algae (Klebsormidiophyceae, Prasinophyceae, Ulvophyceae and Chlorophyceae/Sphaeropleales). (a) Klebsormidium cf. flaccidum LH10HG2056 (l=6.4-19.7 $\mu \mathrm{m}, \quad w=4.5-7.0 \mu \mathrm{m}) ;(\mathrm{b})$ Klebsormidium cf. dissectum LH08HW9106; (c) Pedinomonas minor LH08SG2033 (l=2.9-5.6 $\mu$ m, w=2.5-4.8 $\mu \mathrm{m})$; (d) Pseudendocloniopsis botryoides LH08HW9058 ( $\varnothing=4.7-9.5 \mu \mathrm{m}) ;(\mathrm{e})$ Coelastrella multistriata LH10HG7083 ( $\varnothing=3.5-7.0 \mu \mathrm{m}) ;(\mathrm{f})$ Coelastrella sp. LH10HG7018; (g) Acutodesmus rubescens LH08SG8041; (h) Pseudomuriella aurantiaca LH10HG2039 ( $\varnothing=3.6-$ $8.2 \mu \mathrm{m})$; (i) Bracteacoccus cohaerens LH10HG9034 ( $\varnothing=5.4-12.3 \mu \mathrm{m})$. Scale bars $=10 \mu \mathrm{m}$. 

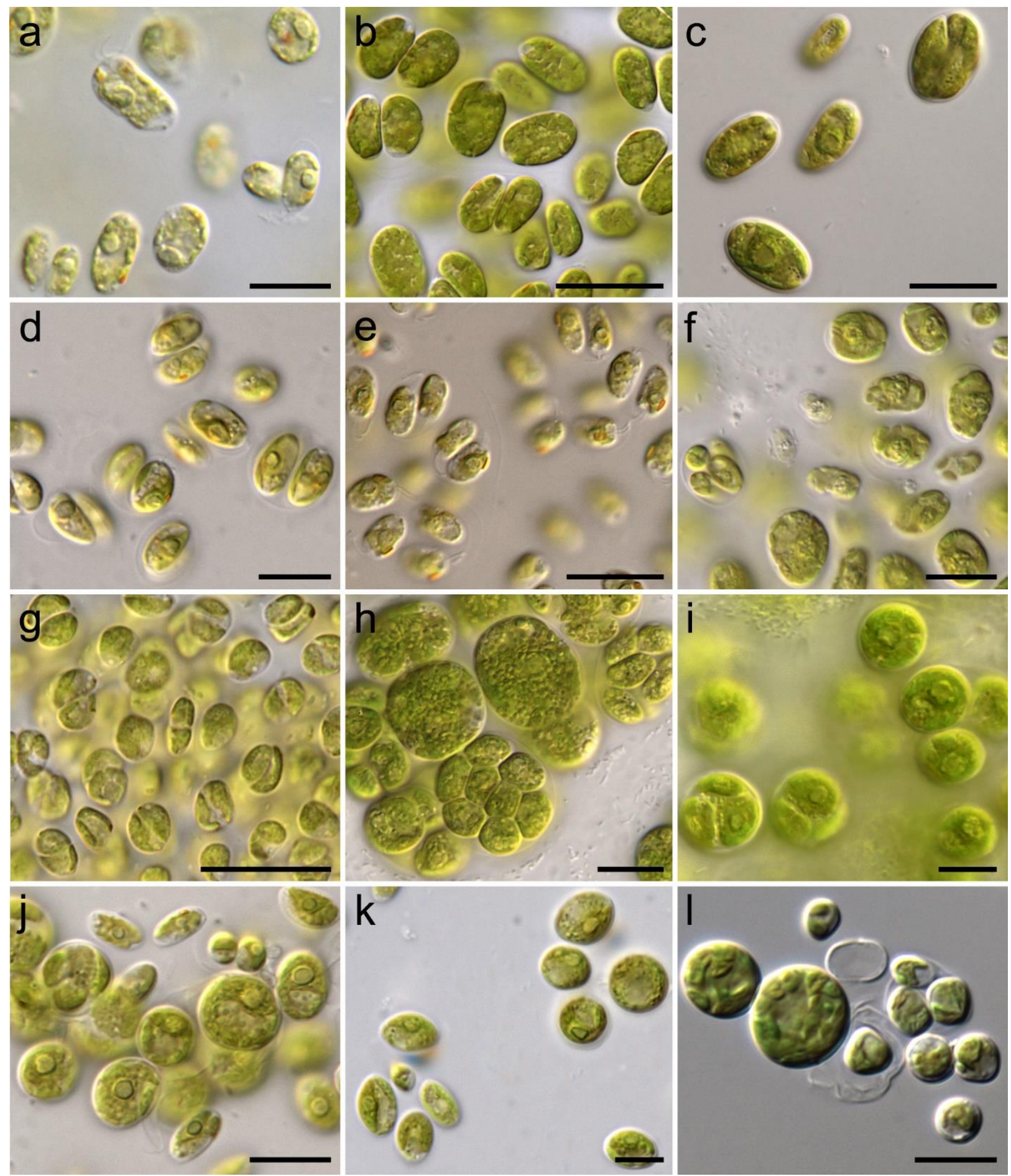

Figure 4. Microphotographs of monoclonal cultures of Green algae (Chlorophyceae/Chlamydomonadales). (a) Oogamochlamys sp.(I) LH08SG8047; (b) Oogamochlamys sp.(II) SAG 2476 (= LH08AW1069; I=9.1-14.5 $\mu$ m, w=5.1-10.0 $\mu \mathrm{m}$ ); (c) Chlamydomonas cf. gerloffii LH08SW5031 (I=10.9-16.4 $\mu \mathrm{m}, \mathrm{w}=4.3-10.1 \mu \mathrm{m})$; (d) Chlamydomonas cf. rapa LH10HG1027 (I=6.1$9.9 \mu \mathrm{m}, \mathrm{w}=2.7-5.1 \mu \mathrm{m}) ;(\mathrm{e})$ Heterochlamydomonas sp. LH08AG2004 (I=6.0-10.5 $\mu \mathrm{m}, \mathrm{w}=3.1-7.5 \mu \mathrm{m}) ;$ (f) Chlamydomonas cf. typica LH08SG9022 (l=6.7-12.2 $\mu \mathrm{m}, \mathrm{w}=4.0-8.0 \mu \mathrm{m}) ;(\mathrm{g})$ Desmotetra stigmatica LH08SG2049 $(\varnothing=3.5-6.6 \mu \mathrm{m}) ;(\mathrm{h})$ Tatrensinia sp.(I) LH08SW7115 (I=8.3-17.8 $\mu \mathrm{m}, \mathrm{w}=5.2-13.3 \mu \mathrm{m})$; (i) Tatrensinia sp.(II) LH10HG9131 ( $\varnothing=7.9-13.1 \mu \mathrm{m})$; (j) Chlorococcum sphacosum LH10HG3113 (l=5.8-9.3 $\mu \mathrm{m}, \mathrm{w}=2.6-6.3 \mu \mathrm{m}) ;(\mathrm{k})$ Chlorococcum cf. minutum SAG 2479 (= LH08AW5056; l=5.6-9.5 $\mu \mathrm{m}, \mathrm{w}=5.6-9.5 \mu \mathrm{m})$; (I) Jenufa sp. SAG 2383 (= LH08AW8035; $\varnothing=2.9-7.3 \mu \mathrm{m})$. Scale bars $=10 \mu \mathrm{m}$. 


\section{Chlorophyta; Trebouxiophyceae}

Isolates assigned to Trebouxiophyceae are phylogenetically nested in eight different clades (Fig. 5) and comprised rod-shaped, spherical and elliptic morphologies (Fig. 6).

Stichococcus-like rod-shaped isolates were spread across the Prasiola/Stichococcus clade (Sluiman and Guihal 2008) and we distinguished at least nine different species. (I) 'Stichococcus' sp. (isolate LH08AW8025; Fig. 6a) from forest (ALB) is identical to 'Stichococcus' sp. SAG 2059 (AY762604) from a building façade. (II) 'Stichococcus' sp. (isolate LH08SG5057; Fig. 6b), occurring in grasslands (HAI, SCH, ALB), is identical to 'Stichococcus' sp. SAG 2060 (AY762606) from a building façade. These species clustered near to authentic strain Stichococcus deasonii UTEX 1706 (DQ275460) from soil, yet the clade is not supported. (III) 'Stichococcus' sp. (isolates LH08AG7010, LH10HG6110) from grasslands (HAI, ALB) is closely related to authentic strain Stichococcus deasonii UTEX 1706 (DQ275460). (IV) 'Stichococcus' sp. (isolate LH08SG1073) from grassland (SCH) is identical to 'Stichococcus' sp. SAG 2406 (= WB47; KF144240) from Westerhöfer creek. (V) 'Stichococcus' sp. (isolate LH08SW1099) from forest (SCH) clustered near to the aforementioned species (IV) in an unsupported clade. (VI) 'Stichococcus' sp. SAG 2482 (= LH08AW8023; Fig. 6c) from forest (ALB) is identical to 'Stichococcus' sp. D4-2A (KF144238) from Deinschwanger creek. (VII) Stichococcus sp. SAG 2481 (= LH08AW8002) from forest (ALB) is identical to Stichococcus sp. K4-4 (AB055866) of unknown origin (probably isolated in Japan). Pseudostichococcus is represented by two species exhibiting rod-shaped Stichococcus-like morphology. (I) Pseudostichococcus cf. monalantoides (e.g., isolate LH10HG3045), detected in grasslands (HAI, ALB), is closely related to authentic strain P. monallantoides SAG 380-1 (JX185690). (II) Pseudostichococcus sp. (isolate LH08SW8044) from forest (SCH) is identical to 'Stichococcus' mirabilis CCAP 379/3 (AJ311638). The clade comprising both Pseudostichococcus species and 'Stichococcus' mirabilis CCAP 379/3 consistently occurred in both maximum-likelihood and Bayesian phylogenies, yet without statistical support. Diplosphaera is represented by two species exhibiting spherical to slightly cylindrical (shortly rod-shaped) cells which characteristically remain in doublets after cell division. (I) 'Chlorella' cf. sphaerica (isolate LH08HW8075) from forest (HAI) is very closely related to authentic strain C. sphaerica SAG 11.88 (AJ416105) from New Zealand. Both specimens might be conspecific. Another isolate (LH08AG9089) from grassland (ALB) is as well closely related to the same authentic strain with a slightly higher genetic distance. The clade including both species (Diplosphaera clade) is well supported.

Elliptic morphotypes. Elliptic cell forms were characteristic for phylogenetically unrelated lineages Neocystis (Eliáš et al. 2013), Coccomyxa (Darienko et al. 2015), Chloroidium (Darienko 
et al. 2010) and 'Navichloris' (this study). The isolates from grasslands (HAI, SCH, ALB) and forests (HAI, ALB) belonging to Neocystis brevis (e.g., LH10HG9080; Fig. 6d) are identical to authentic strain N. brevis CAUP D 802 (JQ920360) from soil (the Neocystis clade was highly supported). Coccomyxa was represented by three species. (I) C. simplex (isolate LH08SG9051; Fig. 6e) from grassland $(\mathrm{SCH})$ is identical to authentic strain C. rayssiae SAG 216-8 (= UTEX 273; HQ317304) from freshwater. The clade further includes authentic strain C. chodatii SAG 216-2 (FJ648512) and is highly supported. (II) Coccomyxa viridis SAG 2483 (= LH08AW8039; Fig. 6f), from forest (ALB), is identical to lichenized C. glaronensis CCALA 306 (AM167525). The clade comprises as well authentic strain C. mucigena SAG 216-4 (FJ648513) and is highly supported. (III) Coccomyxa sp. (isolate LH08AW1017) from forest (ALB) is very closely related to lichenized Coccomyxa sp. KN-2011-T3 (HE586515) from Indonesia. The clade additionally includes Uncultured Trebouxiophyceae RL75K2 (HE617184) from an acidic mining lake in Germany, and is highly supported. The strain SAG 2477 (= LH08AW3007; Fig. 6g; KP081399) detected in forest (ALB), exhibits symmetrically elliptic cells resembling Coccomyxa Schmidle. The species is neither phylogenetically related to Coccomyxa nor to other known genera and clades of Trebouxiophyceae. The closest BLAST-hit Leptochlorella sp. clone Qe17 (FJ790649) from China exhibits low similarity (=95.54\%). In both maximum-likelihood and bayesian phylogenies, SAG 2477 consistently clusters near to the Leptochlorella clade (including authentic strain Leptochlorella corticola CAUP H8401; HE984579), yet without statistical support. The isolates of Chloroidium, exhibiting widely elliptic cells with a band-shaped lobed chloroplast with a pyrenoid, were assigned to two species. (I) C. saccharophilum (e.g., isolate LH10HG7062; Fig. 6h), detected in grassland (HAI) and forest (ALB), is identical to authentic strain $C$. saccharophilum SAG 211-9a (FM946000) from tree sap. Chloroidium cf. ellipsoideum (e.g., isolate LH10HG9105; Fig. 6i), detected in grasslands (HAI, ALB), is closely related to authentic strain C. ellipsoideum SAG 3.95 (FM946012). The clade comprising both species is highly supported.

Coccoid isolates exhibiting predominantly spherical vegetative cells comprised phylogenetically divergent lineages Lobosphaera (Neustupa et al. 2011), Xylochloris (Neustupa et al. 2011), Dictyochloropsis (Dal Grande et al. 2014), Prasiola clade ('Chlorella' mirabilis) and members of the Chlorellaceae (Krienitz et al. 2004). The isolates assigned to Lobosphaera exhibited spherical cells with one parietal chloroplast without a pyrenoid and could be phylogenetically classified in two species. (I) Lobosphaera cf. irregularis (isolates LH08SW5063, LH08AW3064; KP081398) from forests (ALB, SCH) is identical to 'Myrmecia' (= Lobosphaera) irregularis CCAP 221/8 (HQ902935). (II) Lobosphaera cf. bisecta (isolate 
LH10HG3P15; Fig. 6j) from grassland (HAI) is identical to authentic strain Myrmecia (= Lobosphaera) bisecta SAG 2043 (Z47209) from soil (Italy). The Lobosphaera clade (including as well 'Parietochloris' cohaerens UTEX 1707; EU878372) is highly supported. Xylochloris sp. SAG 2382 (= LH08AG7024; Fig. 6k; JQ988942) from grassland (ALB), with characteristic parietal star-like lobed chloroplasts, is less closely related (similarity $=96.47 \%$ ) to authentic strain X. irregularis CAUP H7801 (EU105209) from tree bark (Singapore). The clade including both Xylochloris accessions is only partly supported and the new Xylochloris sp. SAG 2382 is likely to represent new taxon on at least species or even genus level. Closely related genus Dictyochloropsis comprises D. splendida (isolate LH08AW3050; Fig. 6l; JQ988930) from forest (ALB), identical to D. splendida CAUP H8601 (GU017662) from soil near to a fumarole (Czech Republic). Dictyochloropsis and Xylochloris species built a well-supported clade. Chlorella-like isolates from the Prasiola clade, phylogenetically assigned to 'Chlorella' mirabilis, were characterized by spherical cells with one parietal chloroplast containing characteristic small starch granules over the pyrenoid circumference. The isolates from grasslands in ALB (isolate LH08AG9040; Fig. 7a) and HAI (isolate LH10HG6139) are closely related to 'Chlorella' mirabilis Andreyeva 748-I (X74000) from soil and might represent two different species. The clade including both isolates and 'Chlorella' mirabilis Andreyeva 748-I is highly supported.

Chlorellaceae were represented by four different morphological types, i.e., Chlorella-like (spherical to slightly elliptic cells with cup-shaped chloroplast with one pyrenoid), Auxenochlorella-like (spherical cells with facultatively degraded chloroplasts; heterotrophic), Muriella-like (bigger spherical cells with single parietal chloroplast without a pyrenoid), Nannochloris-like (smaller spherical cells with single parietal chloroplast without a pyrenoid). Chlorella vulgaris isolates were sampled in grasslands (HAI, SCH) and forests (HAI). For example, C. vulgaris isolates LH08SG3006 and LH08HG1081 (Fig. 7b) were identical $($ similarity $=99.94-100 \%)$ to authentic strain of C. vulgaris SAG 211-11b (FM205832) from freshwater (Netherlands). Chlorella cf. vulgaris isolate LH10HG2081 (Fig. 7c) is closely related to C. vulgaris SAG 211-11b and identical to isolates L1 and L4 from soil (King George Island, Antarctica). The Antarctic strains (and isolate LH10HG2081) constitute a well-supported clade and might represent a new Chlorella species. Muriella cf. terrestris isolates LH08SG3009 and LH10HG7118 (Fig. 7d) from grasslands (HAI, SCH) are almost identical (similarity = 99.94\%) to Muriella terrestris ASIB V38 (AB012845) from soil (Italy), within a highly supported clade. Nannochloris-like isolates clustered as four different species. (I) Nannochloris sp. isolates from grasslands (e.g., LH08SG8030, Fig. 7e; LH10HG6095, Fig. 7f) are almost identical to $N$. bacillaris (AB080300) within a highly supported clade. The remaining Nannochloris-like isolates 
clustered within the highly supported Unidentified Chlorellaceae clade, a sister group to $N$. bacillaris (AB080300) and comprises three different (yet undescribed) species. One species comprises isolates from grasslands of HAI and ALB (e.g., isolate LH08AG1034; Fig. 7g), identical to 'Marvania' sp. WB67 (KF144207) from Westerhöfer creek within a highly supported clade. Second species from grasslands (HAI, SCH) (e.g., isolate LH10HG9020; Fig. 7h) is identical to Nannochloris sp. JL-4-6 (AY195983) from freshwater (USA). Third species from grassland (SCH) (e.g., LH08SG3093; LH08SG3078, Fig. 7i) is identical to Nannochloris sp. Ant1 (EF440182) from permafrost (Antarctica). Auxenochlorella-like colorless isolates represent two different taxa on either species or even genus level. (I) A. protothecoides (e.g., isolate LH10HG6096; Fig. 7k) from grasslands (HAI) is identical to authentic strain A. protothecoides SAG 211-7a (X56101) isolated from tree sap (Germany). This species clusters together with Chlorella sp. CCAP 211/61 (AB206551) in a highly supported clade. (I) Auxenochlorella sp. SAG 2478 (=LH08AW4103; Fig. 7l; KP081390) from forest (ALB), is less closely (similarity = $97.46 \%$ ) related to $A$. protothecoides SAG 211-7a, without any known closer relatives, probably representing either new species or genus. The whole clade including Auxenochlorella sp. and A. protothecoides is highly supported. Another highly supported clade, consisting of isolate LH10HG709K (Fig. 7j) from grassland soil (HAI) and clone HEW1B K3342 from forest soil (HAI), does not include other known relatives (not shown in phylogenetic trees in this Chapter, but in Fig. 2 Chapter 3). The species might represent a new genus with basal placement among the Nannochloris-like lineages within the Chlorellaceae. 


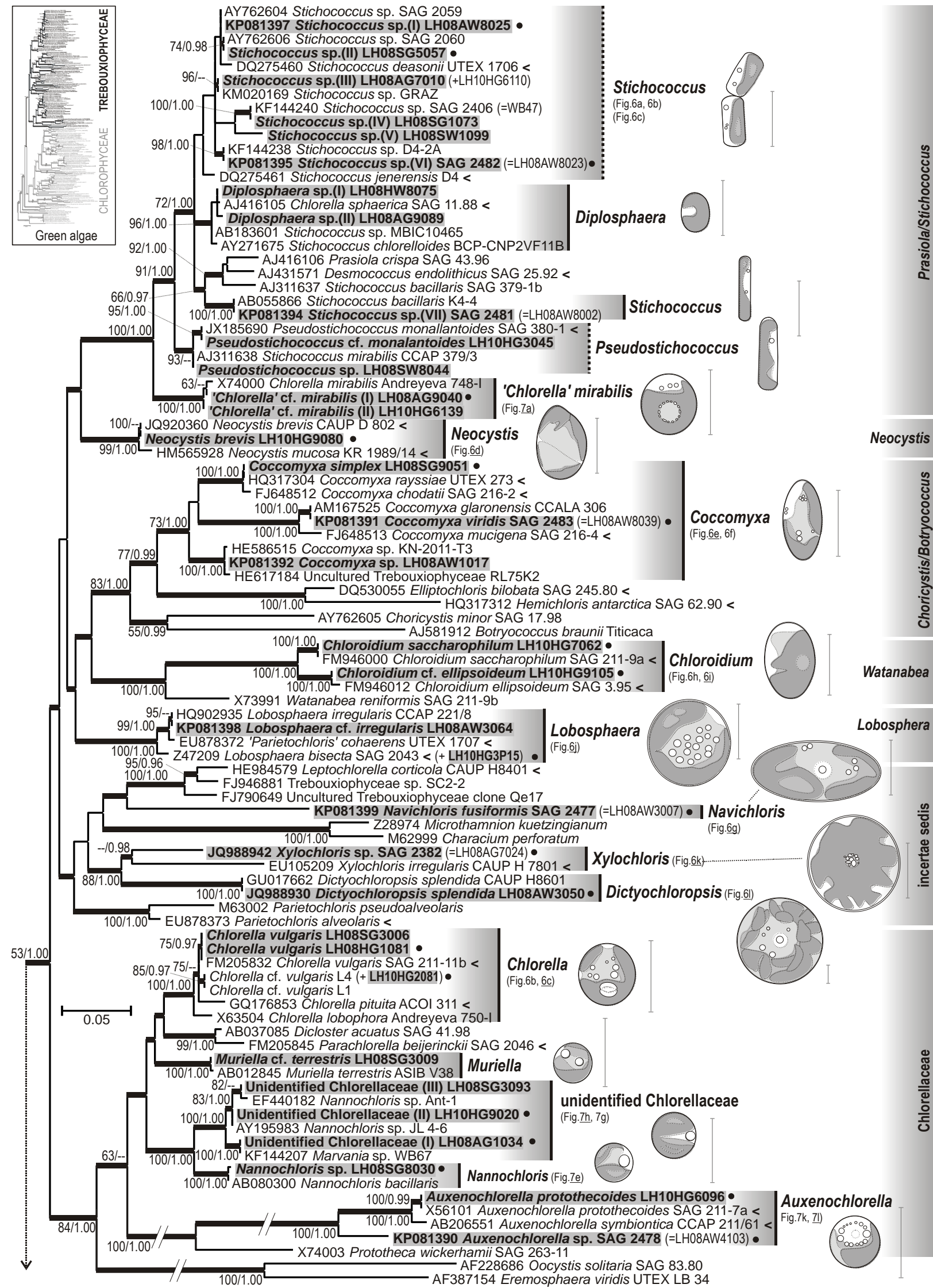

Figure 5. 18S rDNA phylogeny of Green algae, part 2 including Trebouxiophyceae. New accessions are written in bold and grey underlaid. Black dots mark references to microphotographs (Fig. 6, Fig. 7) sorted in top down order. Original drawings of representative morphologies are shown at each clade (scale bars $=5 \mu \mathrm{m})$. Sequences of authentic strains are marked by '>'. Numbers next to branches indicate statistical support values (maximum-likelihood bootstraps/Bayesian posterior probabilities). 


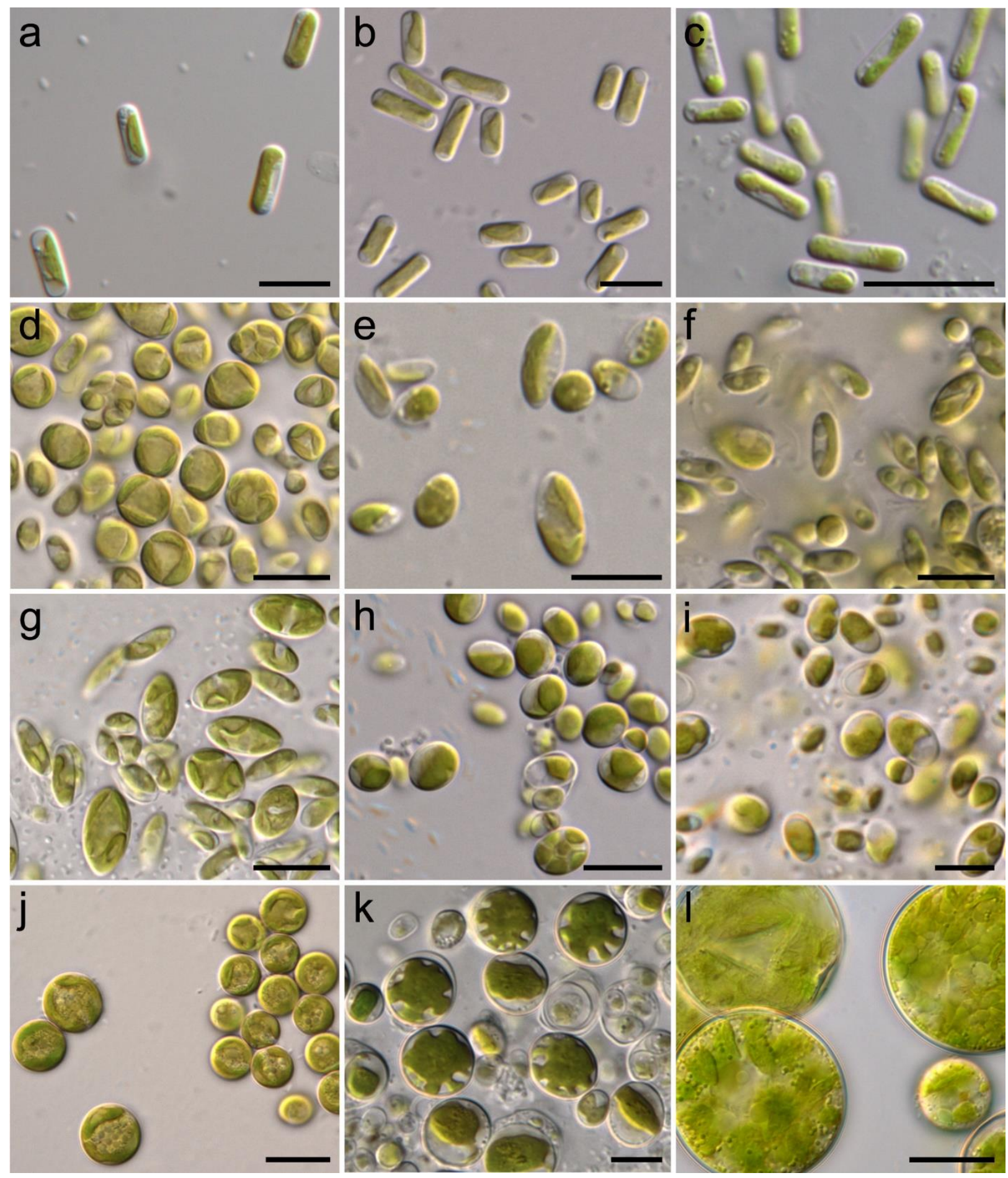

Figure 6. Microphotographs of monoclonal cultures of Green algae (Trebouxiophyceae). (a) Stichococcus sp.(I) LH08AW8025

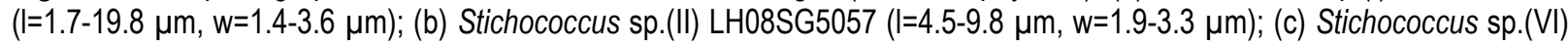
SAG 2482 (= LH08AW8023; I=3.8-7.2 $\mu \mathrm{m}, \mathrm{w}=1.9-6.3 \mu \mathrm{m}$ ); (d) Neocystis brevis LH10HG9080 (l=4.8-8.7 $\mu \mathrm{m}, \mathrm{w}=2.7-6.6 \mu \mathrm{m}) ;(\mathrm{e})$ Coccomyxa simplex LH08SG9051 (l=4.4-7.8 $\mu \mathrm{m}, \mathrm{w}=2.0-4.3 \mu \mathrm{m}) ;(\mathrm{f})$ Coccomyxa viridis SAG 2483 (= LH08AW8039; I=4.7-8.4 $\mu \mathrm{m}, \mathrm{w}=1.8-3.6 \mu \mathrm{m}$ ); (g) Navichloris fusiformis SAG 2477 (= LH08AW3007; I=8.3-15.3 $\mu \mathrm{m}, \mathrm{w}=3.6-7.8 \mu \mathrm{m}$ ); (h) Chloroidium saccharophilum LH10HG7062 (I=5.6-9.1 $\mu \mathrm{m}, \mathrm{w}=4.0-6.2 \mu \mathrm{m})$; (i) Chloroidium cf. ellipsoideum LH10HG9105 (I=4.8-7.8 $\mu \mathrm{m}$, $\mathrm{w}=3.3-5.3 \mu \mathrm{m})$; (j) Lobosphaera bisecta LH10HG3P15 $(\varnothing=6.2-9.6 \mu \mathrm{m}) ;(\mathrm{k})$ Xylochloris sp. SAG 2382 (= LH08AG7024; I=4.7$12.9 \mu \mathrm{m}, \mathrm{w}=3.1-10.6 \mu \mathrm{m})$; (I) Dictyochloropsis splendida LH08AW3050 $(\varnothing=7.7-39.4 \mu \mathrm{m})$. Scale bars $=10 \mu \mathrm{m}$. 

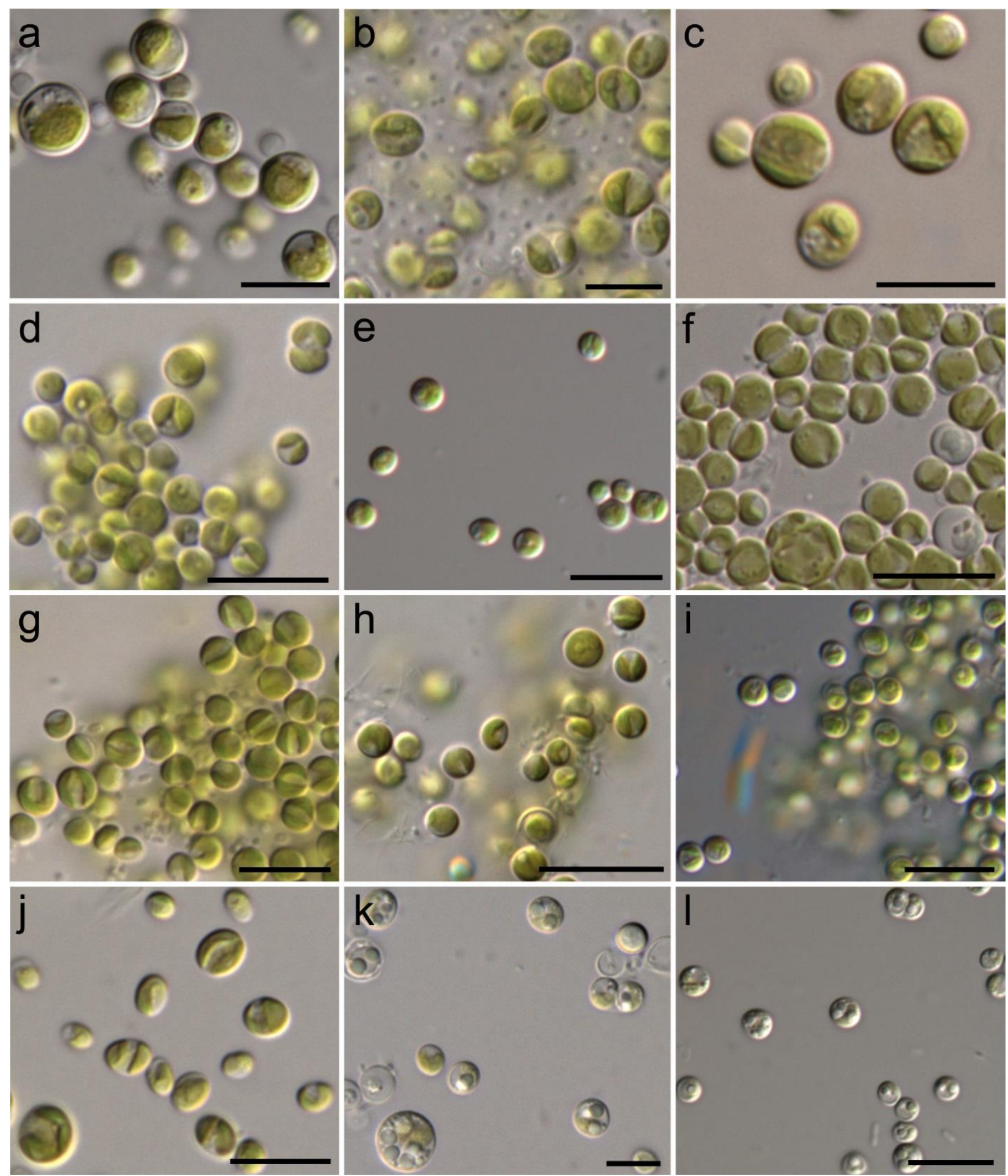

Figure 7. Microphotographs of monoclonal cultures of Green algae (Trebouxiophyceae). (a) 'Chlorella' cf. mirabilis LH08AG9040 $(\varnothing=3.0-6.4 \mu \mathrm{m}) ;(\mathrm{c})$ Chlorella cf. vulgaris LH10HG2081 ( $\varnothing=2.5-6.2 \mu \mathrm{m}) ;(\mathrm{d})$ Muriella terrestris LH10HG7118; (e) Nannochloris sp. LH08SG8030 ( $\varnothing=2.2-3.9 \mu \mathrm{m}) ;(\mathrm{f})$ Nannochloris sp. LH10HG6095 ( $\varnothing=2.1-3.9 \mu \mathrm{m}) ;(\mathrm{g})$ Unidentified Chlorellaceae (I) LH08AG1034 ( $\varnothing=2.4-4.5 \mu \mathrm{m})$; (h) Unidentified Chlorellaceae (II) LH10HG9020 ( $\varnothing=2.4-4.8 \mu \mathrm{m}) ;$ (i) Unidentified Chlorellaceae (III) LH08SG3078 $(\varnothing=2.1-3.7 \mu \mathrm{m}) ;$ (j) Unidentified Chlorellaceae LH10HG709K (I=3.1-5.1 $\mu \mathrm{m}, \quad w=2.0-3.7 \mu \mathrm{m}) ;(\mathrm{k})$ Auxenochlorella protothecoides LH10HG6096 ( $\varnothing=2.9-6.5 \mu \mathrm{m}) ;(\mathrm{I})$ Auxenochlorella sp. SAG 2478 (= LH08AW4103; $\varnothing=2.2-5.2$ $\mu \mathrm{m})$. Scale bars $=10 \mu \mathrm{m}$. 


\section{Geographic dispersal and taxonomic 'novelty' of the isolated Green algae}

In total, we identified 61 species of Green algae. Thirty-nine (53\%) species were detected once and $34(47 \%)$ species were detected more than once (e.g., Chlorella vulgaris; 19 times; Table S4a). Thirty (41\%) species were retrieved from more than one plot and 18 (25\%) species were retrieved from more than one exploratory (Fig. 8; Table S4b). There were only two species which we detected in all three exploratories, i.e., Stichococcus sp. (II) and Neocystis brevis (Table S4b). Almost all clades included both forest and grassland species, with the only exception of the Chlorellaceae with most species detected only in grasslands (Fig. 8). More than a half of all detected species $(61 \%)$ exhibited $>99.90 \%$ sequence similarity to accessions known from previous studies. But only less than a half of these accessions belong to validly named (authentic) strains (Fig. 9a). Many next relatives to our species originate from Europe (54\%), most of them from soil $(30 \%)$ or various aquatic habitats $(27 \%)$. Species less closely related to allready published accessions (<99.98\% sequence similarity) mostly originate from grassland plots (62\%; Fig. 9a). ITS2 sequence comparisons revealed occurrences of identical ribotypes separated by considerable distances, e.g., 'Chlorella' mirabilis, Chlorella vulgaris, Pseudostichococcus monallantoides and Klebsormidium dissectum (Fig. 9b). Other notably high similarities were detected in Chlamydopodium vaculolatum (99\%) and Muriella terrestris (98\%) (Fig. 9b). 


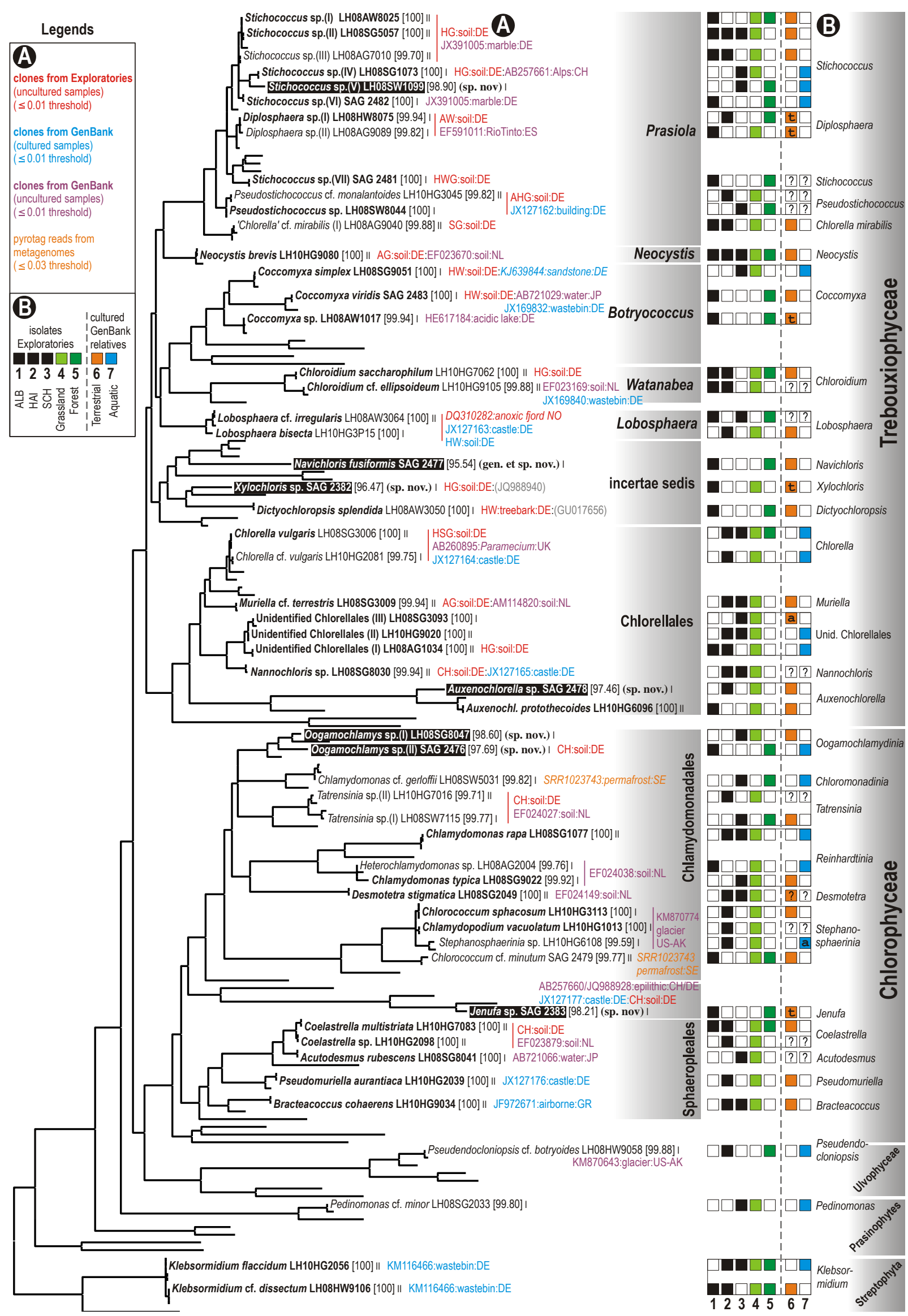

Figure 8. Diversity and distribution of all green microalgae isolated within this study. See further explanations on next page. 
Figure 8. Diversity and distribution of all Green microalgae isolated within this study. The $18 \mathrm{~S}$ phylogenetic tree gives an overview of all detected species (each species is represented by one sequence in the tree; due to a lack of space, names of reference sequences were removed from the figure; the comprehensive taxon samplings are shown in Fig. 2, Fig. 5). Numbers in brackets give percentage sequence similarity to the closest relatives. 'I' or 'Il' indicate whether a species was found only once or multiple times within the Exploratories. Colored squares to the right of the tree summarize the distribution of each species across the three Exploratories (ALB, HAl, SCH) and across the two different habitats (grassland, forest). This evidence is based on sequenced isolates listed in Table S4. Further two squares indicate whether the closest GenBank-relative originate from terrestrial or aquatic habitats. Numbers in brackets behind isolate identifiers indicate (18S-based) sequence similarity to the closest cultured relatives. Algal clones, which are closely related to our isolates, are differentiated by colors due to their source (red: environmental clones obtained from the same sampling sites (Hallmann et al. in prep.-b); purple: GenBank accessions from culture-independent surveys; blue: GenBank accessions from culture-dependent studies).

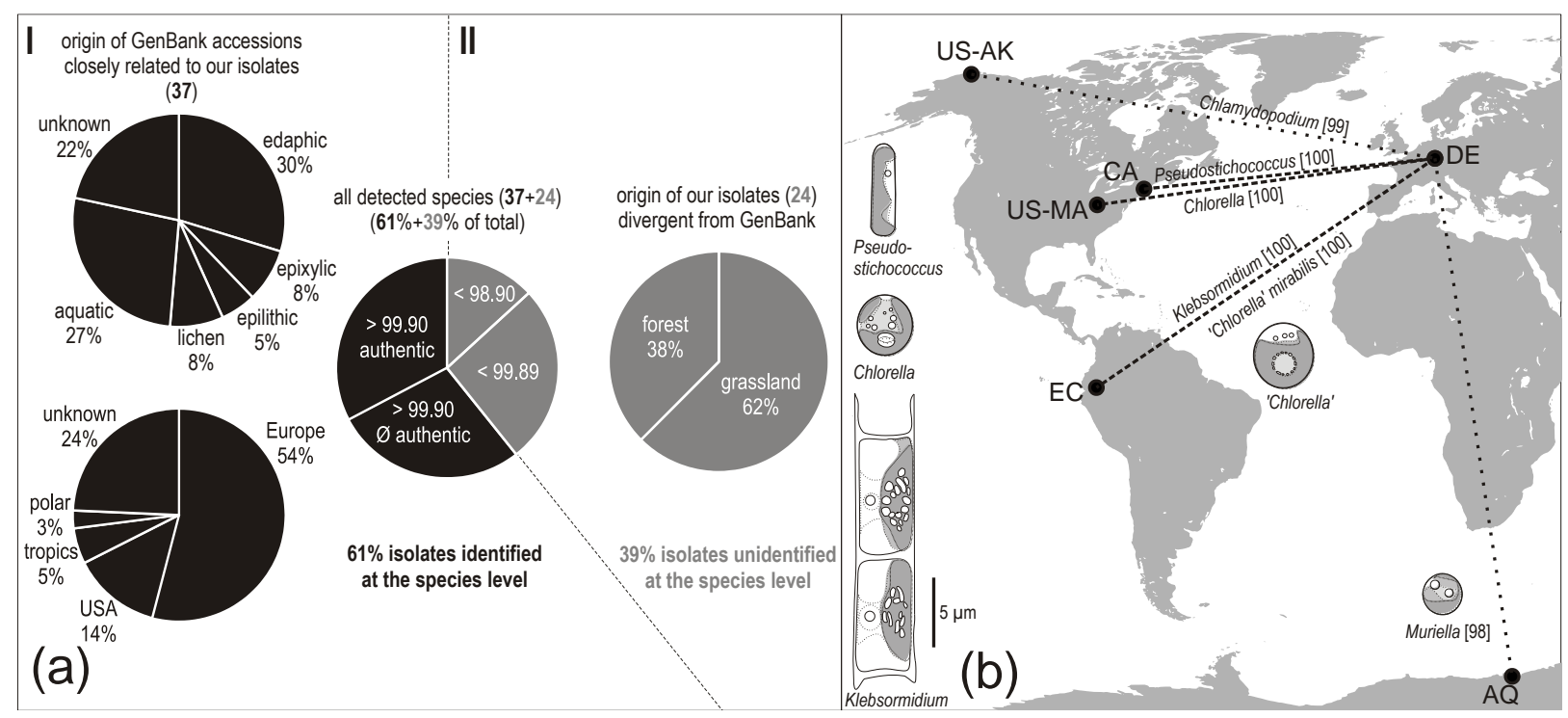

Figure 9. Geographic distribution of Green algal species isolated from soils. (a) Pie charts derived from 18S rDNA sequence similarities illustrate (I) how many isolates could be identified at species level (and where their closest relatives originated from), (II) how many isolates represent undescribed species (and from which habitats these undescribed species originated from); (b) Map showing places where identical or highly similar ITS2 rDNA sequences were found. Evidence for 100\%-identity: Stichococcus LH08SW8044 (DE) and SAG 379-4 (CA), Chlorella LH08SG3006 (DE) and FN298918 (US-MA), Klebsormidium LH08AG1113 (DE) and KS164CL6L (EC), 'Chlorella' mirabilis LH08AG9040 (DE) and KFFB12-1 (EC); Evidence for high similarity [98-99\%]: Chlamydopodium LH10HG1013 (DE) and FR865591 (US-AK), Muriella LH08SG3009 (DE) and JN653521

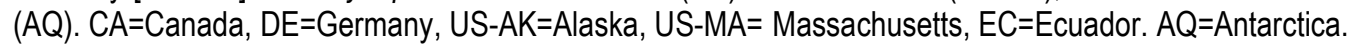

\section{Stramenopiles: Xanthophyceae}

Phylogenetic analysis of the Stramenopiles (Fig. 10a), assigned isolates to the classes Xanthophyceae (six clades) and Eustigmatophyceae (one clade) (Fig. 10b).

Tribonematalean lineage (Maistro et al. 2009). Tribonematalean isolates with unbranched filaments could be assigned to Xanthonema (three species) and Heterothrix (two species). (I) Xanthonema sp. (e.g., isolates LH10HG7029, LH10HG9058; Fig. 11a), detected in grassland (HAI) and forest (HAI), is closely related to X. exile PAB 395 (AM491615). (I) Xanthonema cf. exile (isolate LH10HG7078; Fig. 11b) from grassland (HAI) is almost identical to $X$. exile PAB 395 (AM491615). (III) Xanthonema cf. bristolianum (isolates LH10HG6059, LH08HW9018; 
Fig. 11c) from grassland (HAI) and forest (HAI), is identical to terrestrial $X$. bristolianum CCALA 516 (AM490819) isolated from snow (Slovakia). The Xanthonema clade is well supported. (IV) Heterothrix sp. (isolates LH10HG7061, Fig. 11d; LH08SG5052, Fig. 11e) from grasslands (HAI, SCH) is related to Heterothrix sp. ACOL A1 (AM491612) and might represent a new or undescribed species. (V) H. sessile (e.g., isolate LH10HG5079) from grassland (HAI) is identical to H. sessile IBSG-V28 (AM490818). The Heterothrix clade is highly supported.

Chlorellidialean lineage (Maistro et al. 2009). Chlorellidialean heterotrichal isolates clustered in three different species of Heterococcus. (I) Heterococcus sp. (isolates LH10HG2140, LH10HG9085; Fig. 11f) from grasslands (HAI) is related to freshwater Xanthophyceae sp. IX3 (FJ946906) from Antarctica and might represent a new species. (II) Heterococcus cf. chodatii (e.g., isolate LH10HG9111; Fig. 11g, 11h) from grasslands (HAI) is identical to authentic strain H. chodatii SAG 835-3 (AM490822) from a subaerial habitat (Switzerland). The clade including H. chodatii (and H. pleurococcoides PAB 380; AJ579335) is well supported. (III) Heterococcus cf. caespitosus (isolate LH08AG2020) from grassland (ALB) is almost identical to authentic strain $H$. caespitosus SAG 835-2a (AM490820) from soil (Germany) and at the same to $H$. protonematoides SAG 835-9 (AJ579334) from soil (Switzerland). The clade including both species is highly supported, as well as the whole Heterococcus clade.

Botrydiopsalean lineage (Maistro et al. 2009). Botrydiopsalean isolates (two species) exhibited either cell packages or solitary spherical cells with numerous discoidal chloroplasts. (I) Botrydiopsalean sp. (e.g., isolate LH08AW1076; Fig. 11i) from grassland (SCH) and forest (ALB) is related to Chlorellidium pyrenoidosum PAB 785 (AJ579338) from soil (Antarctica). The clade including both species is not supported and the isolate LH08AW1076 might represent a new species. (II) Botrydiopsis sp. (isolate LH08AW4043; Fig. 11j) from forest (ALB) is related to $B$. callosa SAG 30.83 (AJ579340) from soil (Italy). The clade including both species is well supported and the isolate LH08AW4043 might represent a new species.

The thallous genus Asterosiphon Dangeard is represented by Asterosiphon sp. (isolate LH10HG3064; Fig. 11k) from grassland (HAI), which is less closely related (similarity = 98.54\%) to A. dichotomus UTEX LB 2066 (AM490829). Asterosiphon sp. LH10HG3064 exhibited filamentous and coccal stages in the culture. The Asterosiphon clade is well supported and the isolate $\mathrm{LH} 10 \mathrm{HG} 3064$ probably represents a new species.

\section{Stramenopiles: Eustigmatophyceae}

The genus Eustigmatos Hibberd is represented by Eustigmatos sp. (isolates LH10HG5036, LH10HG9133; Fig. 111) from grasslands (HAI) is closely related to E. magna CCMP 387 
(U41051) from soil (New Zealand). Both isolates exhibit sphaerical cells with characteristic angular shaped 'false pyrenoid'. The clade including both isolates and E. magna CCMP 387 is supported.

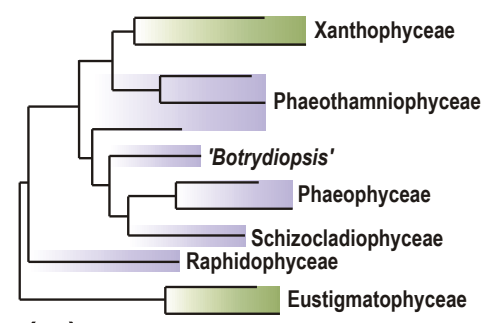

\section{3/-- [ Xanthonema sp. LH10HG9058 •}

$73 /--$

73/0.98 Xanthonema cf. exile LH10HG7078 •

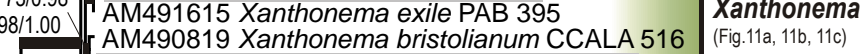

Xanthonema cf. bristolianum LH08HW9018 •

100/ 43277 Xanthonema hormidioides SAG 836-

100/1.00 F Heterothrix sp. LH10HG7061 •

Heterothrix sp. LH08SG5052 •

100/1.00 AM491612 Heterothrix sp.1 ACOL A1 Heterothrix

85/-- Heterothrix sessile LH10HG5079 (Fig.11d, 11e)

AM490818 Heterothrix sessile IBSG-V28

(a)

Eustigmatophyceae

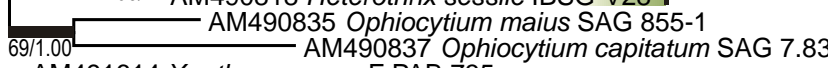

AM491614 Xanthonema sp. F PAB 735

- AM5280826 Tribonema utriculosum SAG 22.94

$95 / 1.00$

$92 / 1.00 \sqrt{10}$

AM491617 Pseudobumilleriopsis sp. SAG 58.94

AJ579332 Pseudobumilleriopsis pyrenoidosa SAG 69.90

99/1.00

AM490828 Botrydium granulatum SAG 805-4

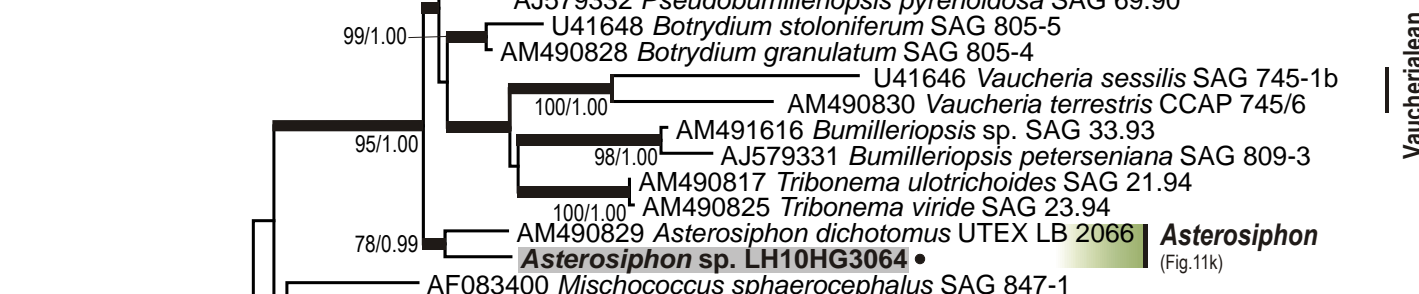

- AF083400 Mischococcus sphaerocephalus SAG 847-1

AF109729 Pseudopleurochloris antarctica SAG B 39.98

AM490833 Phaeobotrys solitaria SAG 15.95

AM490831 Pleurochloridella botrydiopsis CCMP 1665

AJ579333 Sphaerosorus composita SAG 53.91
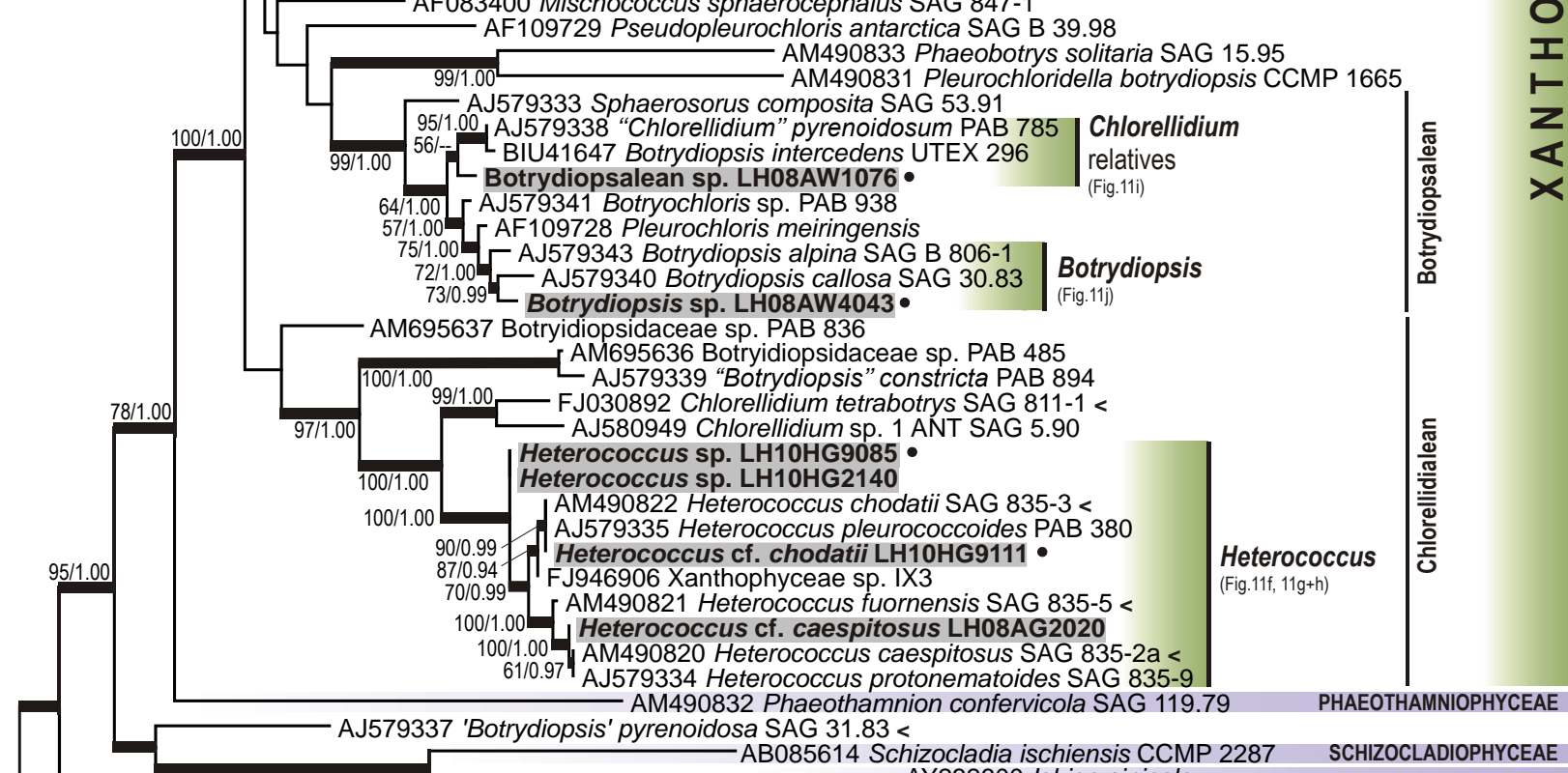

PHAEOTHAMNIOPHYCEAE

chizocladia ischiensis CCMP 228 AY232600 Ishige sinicola

SCHIZOCLADIOPHYCEAE

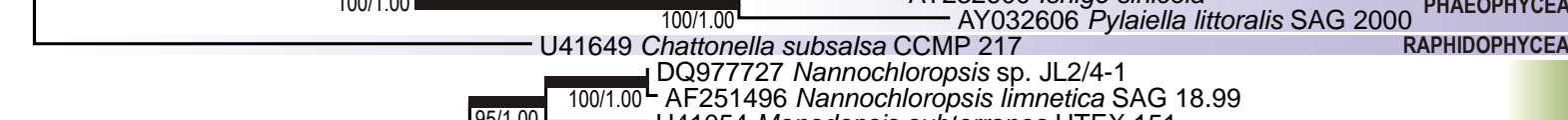

(b)

$100 / 1.00$

AF251496 Nannochloropsis limnetica SAG 18.99

U41054 Monodopsis subterranea UTEX 151

U41052 Pseudocharaciopsis minuta UTEX 2113
AF045051 Vischeria helvetica SAG 876-1 <

100/1.00, U41051 Eustigmatos magna CCMP

\begin{tabular}{l|l|l}
$69 / 0.95$ & Eustigmatos sp. LH10HG9133 • & Eustigmatos
\end{tabular}

$89 / 100$ Eustigmatos sp. LH10HG5036

Figure 10. Phylogeny of some Stramenopiles, including Xanthophyceae and Eustigmatophyceae. (a) Schematic multi-gene phylogeny (modified after Maistro et al. (2009)) of the same Stramenopile groups which were used for the 18S phylogeny. (b) 18S rDNA phylogenetic tree showing placement of the new isolates (grey underlaid; black dots mark isolates which are shown on microphotographs (Fig. 11). References to the microphotographs (Fig. 11, listed below clade names) correspond to black dots in top down order. Sequenced authentic strains are marked by a ' $<$ ' sign. Numbers next to branches indicate statistical support values (maximum-likelihood bootstraps/Bayesian posterior probabilities). 

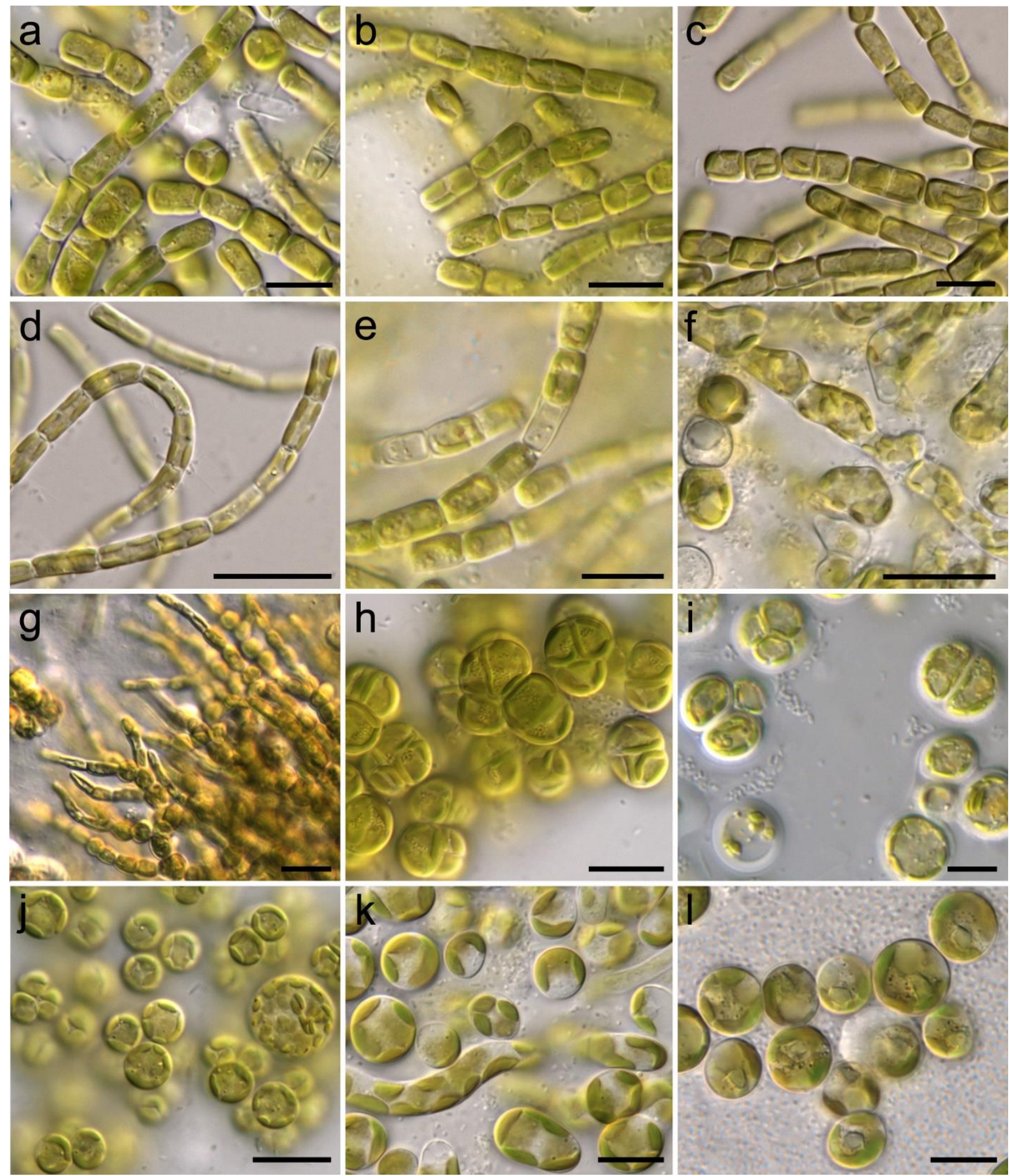

Figure 11. Microphotographs of monoclonal cultures (Trebouxiophyceae, Xanthophyceae, Eustigmatophyceae). (a) Xanthonema sp. LH10HG9058; (b) Xanthonema exile LH10HG7078; (c) Xanthonema cf. bristolianum LH08HW9018; (d) Heterothrix sp. LH10HG7061; (e) Heterothrix sp. LH08SG5052; (f) Heterococcus sp. LH10HG9085; (g) Heterococcus cf. chodatii LH10HG9111; (h) Heterococcus cf. chodatii LH10HG9111; (i) Botrydiopsalean sp. LH08AW1076; (j) Botrydiopsis cf. callosa LH08AW4043; (k) Asterosiphon sp. LH10HG3064; (I) Eustigmatos sp. LH10HG9133. Scale bars $=10 \mu \mathrm{m}$. 


\section{Morphological diversity observed in cultured soil samples}

Microscopic investigation of the cultured soil samples (liquid cultures, agar plates, cover slips; Fig. 1g-i) revealed 45 distinct morphotypes (Figs. S1, S2, S3, S4). We compared the diversities among the Exploratories and between the two soil horizons (Fig. S5a-b). The A-horizon counts lower morphotype diversity (22 morphotypes; 57 plots/57 samples) than the O-horizon (46 morphotypes; 5 plots/20samples; Fig. S5b). The highest diversity exhibited Chlorophyta/Streptophyta (24), followed by Bacillariophyceae (8), Cyanobacteria (7), Xanthophyceae/Eustigmatophyceae (5), Rhodophyta (1) and Cryptophyta (1) (Table S6, S7). In general, the number of morphotypes is nearly the same in grasslands and forests (Fig. S5b-c), irrespective of the sampling technique (drill cores, soil surface samples). The A-horizon sampling revealed negligible differences in alpha-diversity between the three regions. In average, intermediately managed plots were slightly more diverse than extensively and intensively managed plots (Fig. S5f). The same applies for forests and grasslands, yet without statistical support. However, the morphotype composition differed between forest and grassland plots, explained through differences in physico-chemical parameters (Fig. S5d). The statistically significant ordination model $(P<0.05)$ based on Redundancy Analysis (RDA) revealed separation of forest and grassland replicate samples along the first ordination axis which is correlated with $\mathrm{pH}$ values $(R=0.74 ; P<0.05)$. The contribution of total nitrogen $(R=0.26 ; P<$ $0.05)$ and organic carbon $(R=0.11 ; P<0.05)$ is lower. The difference between soil communities in forests and grasslands can be explained by higher diversity of Cyanobacteria, Diatoms and Xanthophytes in grasslands. Considering the O-horizon (soil surface samples from HAI), multiple samples within a single plot exhibited different morphotype compositions (Fig. S5e). The ordination based on NMDS analysis (Shepard stress $=0.11$ ) separates forest and grassland samples (axis 1) and points out higher heterogeneity of the forest plots (axis 2).

\section{Discussion}

Culturing of soil samples from 30 forest and 27 grassland sites retrieved 188 monoclonal cultures of Green microalgae. We inferred conspecifity of the sequenced isolates based on $\leq 0.001 \mathrm{SSU}$ dissimilarity threshold. In multiple cases, our isolates were divergent from already sequenced authentic strains available from GenBank. Therefore, to denominate detected species, we relied on the phylogenetic species concept (Leliaert et al. 2009; Leliaert et al. 2014), not strictly following the taxonomical nomenclature (Cantino and de Queiroz 2007; McNeill et al. 2012). This approach distinguished 61 species covering five classes of Green algae: Chloro-, 
Klebsormidio-, Pedino-, Trebouxio- and Ulvophyceae. In the following we focus mainly on two classes accounting for the most detected species - Trebouxiophyceae (36 spp.; 8 lineages) and Chlorophyceae (21 spp.; 10 lineages). We discuss whether our soil isolates can be recognized in already denominated algal strains deposited in public culture collections (e.g., SAG, UTEX, CCAP). Then we trace the distribution of our species by analysing records in GenBank and discuss their putative detectability in different environments by culture-independent sequencing.

\section{New soil isolates and their cultured relatives}

More than $50 \%$ of Green microalgae we isolated from soils are putatively conspecific with isolates from previous studies (Fig. 8; Fig. 9a). The most of these conspecific cultures originate from Central and Western Europe (54\%) and from the USA (14\%), corresponding to strains deposited in the Culture Collection of Algae in Göttingen (SAG) incl. their derivatives in UTEX or CCAP. Exotic counterpart cultures were few, e.g., Coccomyxa sp. (HE586515) from Indonesia or Nannochloris sp. from Antarctica (EF440182; Gilichinsky et al. 2007). Sequenced strainsconspecific with our isolates - originate from terrestrial (edaphic, epilithic, epixylic/lichenized) and aquatic environments. So far, there are no estimates of total diversity of microscopic Green algae in soils. However, the number of cultured species which match environmental clones, suggests that the uncultured fraction is not as extensive as in bacteria (99\%; Hirsch et al. 2010). All 61 isolated species grow easily on agarized (solid) media, which are selective and might favor so called 'weedy' or generalists species (Škaloud et al. 2014a). Additional enrichment techniques (growth in liquid media or on coverslips), indeed, retrieved additional species, which we did not retrieved as monoclonal cultures for sequencing (e.g., Macrochloris, Spongiochloris, Keratococcus, Podohedra, Scotinosphaera, Pseudendoclonium, Interfilum or Cosmarium; Fig. S1, S2). The identification literature by Ettl and Gärtner (1995) covers about 500 morphospecies of terrestrial Green microalgae-partially documented as sequenced strains deposited in culture collections. We re-detect some of them in the present study (via SSU-comparisons) and could either support or reject their ecological preference (so far inferred via morphospecies-based approaches). Among such sequenced Green microalgae, which were documented by particular algal strains in (Ettl and Gärtner 1995), we confirmed characteristic grassland species: Bracteacoccus cohaerens UTEX 1272 (Bischoff and Bold 1963), 'Chlorococcum' gelatinosum SAG 64.80 (Archibald and Bold 1970), Chlamydomonas typica NIES-2246 (Deason and Bold 1960), 'Chlorella' mirabilis Andreyeva 748-I (Andreyeva 1973), Desmotetra stigmatica UTEX B 962 (Deason and Floyd 1987), Muriella terrestris ASIB V38 (Ettl and Gärtner 1995) and Pseudomuriella aurantiaca SAG 249-1 (Hanagata 1998). A few species were repeatedly verified 
in forests, e.g., Chloromonas gerloffii and C. rosae (Ettl 1963) or in soils of both forests and grasslands, e.g., Lobosphaera bisecta SAG 2043 (Trenkwalder 1975), Chlorococcum minutum ASIB T50 (Trenkwalder 1975) and Heterochlamydomonas inaequalis UTEX 1705 (Cox and Deason 1969). Finally, in our soil samples we recognized well documented terrestrial generalists such as: Chloroidium saccharophilum SAG 211-9a (Darienko et al. 2010), Dictyochloropsis splendida CAUP H8601 (Škaloud et al. 2005) and 'Chlorella' sphaerica SAG 11.88 (TschermakWoess 1988). Despite the sampling efforts in forests, we did not retrieve species characteristic for temperate tree-bark biofilms or lichens, e.g., Apatococcus (Hallmann et al. 2009; Hallmann et al. in prep.-a), Elliptochloris (Eliáš et al. 2008), Kalinella (Neustupa et al. 2009), Leptochlorella (Neustupa et al. 2013a), Parachloroidium (Neustupa et al. 2013b), Trebouxia (Friedl and Rokitta 1997; Kroken and Taylor 2000) or Asterochloris (Škaloud et al. 2015). This might be due to selectivity of the used culturing medium, reflecting nutrient demands of soil but not corticolous species.

\section{New taxa detected in soils}

Seven isolates are considerably divergent from all GenBank clones or cultures, presumably representing novel taxa. The novel Chlorophyceae are: Jenufa sp. SAG 2383, Oogamochlamys sp.(I) LH08SG8047, Oogamochlamys sp.(II) SAG 2476. The novel Trebouxiophyceae are: Auxenochlorella sp. SAG 2478, Stichococcus sp.(V) LH08SW1099, 'Navichloris fusiformis' SAG 2477, Xylochloris sp. SAG 2382. Jenufa Němcová, Eliáš, Škaloud \& Neustupa represents a coccoid genus and at the same time a lineage incertae sedis within the class Chlorophyceae. Two terrestrial species were described from Southeast Asia (Němcová et al. 2011) and multiple congeneric accessions were acquired also from Neotropics (Hodač et al. 2012), the Alps (Horath and Bachofen 2009) and finally in the frame of this study (Jenufa sp. SAG 2383). The only European isolate Jenufa sp. SAG 2383 (Fig. 41) might represent commonly occurring terrestrial species detected in forest soils and epilithic biofilms (Hodač et al. 2012).

Oogamochlamys Pröschold, Marin, Schlösser \& Melkonian is a monadoid genus within the Oogamochlamydinia clade (Chlamydomonadales). The three species described by Pröschold et al. (2001) genetically differ from our two congeneric isolates provisionally denominated Oogamochlamys sp.(I) LH08SG8047 (Fig. 4a) and O. sp.(II) SAG 2476 (Fig. 4b). Distribution and ecology of both species remains unclear, as they were detected only once, Oogamochlamys sp.(I) in one forest site and $O$. sp.(II) in one grassland site. Remarkably, all other described Oogamochlamys species were isolated from soils in Africa and North America (Pröschold et al. 2001). And at least one another verifiably congeneric isolate-Chlamydomonas sp. CCAP 
11/159 — was detected in a lake in North America (GenBank acc. no. FR865591).

Auxenochlorella (Shihira \& Krauss) Kalina \& Punčochářová is a genus within the Chlorellaceae comprising three facultatively heterotrophic species (Darienko and Pröschold 2015; Rodó and Molinari-Novoa 2015). The new facultatively heterotrophic isolate Auxenochlorella sp. SAG 2478 (Fig. 7l) is verifiably congeneric with all described Auxenochlorella species but is still considerably divergent from all accessions available in GenBank. Auxenochlorella sp. SAG 2478 is the first species of this genus which was isolated from soil. Other Auxenochlorella species were isolated from different terrestrial and aquatic habitats, e.g., tree sap and Hydra viridis endosymbiont (Darienko and Pröschold 2015).

Stichococcus Nägeli is a common denomination of rod-shaped Green algae from the Prasiola clade, counting 50 described species (Karbovska and Kostikov 2012a). Only two Stichococcus species were verified by genetic data and the polyphyletic taxon awaits taxonomical revision. All seven Stichococcus-like species detected in the frame of this study represent new taxa, however, six of them are closely related with already existing cultures or clones. Except for the isolate Stichococcus sp. LH08SW1099 (Fig. 8; Fig. 5i Chapter 3).

Xylochloris Neustupa, Eliáš \& Škaloud is a genus incertae sedis within the Trebouxiophyceae with one described terrestrial species $X$. irregularis isolated in Southeast Asia (Neustupa et al. 2011). Our soil isolate Xylochloris sp. SAG 2382 (Fig. 6k) is putatively congeneric with $X$. irregularis, together with clones from European soils and tree-barks in South America (Hodač et al. 2012; Spitzer et al. 2014). The genus is probably widely distributed in Europe, as recently reported from tree-bark in the Southern Europe (Kulichová et al. 2014).

Navichloris fusiformis L.Hodač et al., ad interim (Fig. 6g) is a new species and genus of an undescribed lineage within the class Trebouxiophyceae. The existence of this novel lineage was already proposed by Lewis and Lewis (2005), who phylogenetically analyzed the strain Chlorella sp. BC4VF9 from Baja California, Mexico, without affiliation to any known trebouxiophycean clade. Costello et al. (2009) pointed out close relationship of Chlorella sp. BC4VF9 and two clones sampled from cold-fumarole soil on the Socompa volcano, Atacama, Chile. However, the obviously novel trebouxiophycean clade remained undescribed, since the only existing culture Chlorella sp. BC4VF9 died (Fučíková et al. 2014). Phylogenetic analysis (Fig. 12) show that the three desert accessions cluster with the strain SAG 2477 isolated in the frame of this study from a forest soil in Schwäbische Alb, Germany. The clade is statistically supported and represents a phylogenetically unique lineage of terrestrial trebouxiophycean microalgae. The strain SAG 2477 as well exhibits a unique fusiform/elliptic morphology, generally resembling Coccomyxa (Schmidle 1901). However, phylogenetically it is not related to any member of the recently 
revisited genus Coccomyxa (Darienko et al. 2015). The characteristic feature of the strain SAG 2477 are lens-like light zones in the chloroplast lobes (Fig. 6g), similar to Elliptochloris incisiformis Hoffmann \& Kostikov described from forest soil in Belgium (comp. Fig. 27, Hoffmann et al. 2007), but molecular marker are not available. Notably, the fusiform morphology shared by SAG 2477 and Chlorella sp. BC4VF9 (http://pediastrum.eeb.uconn.edu/isolates /detail/411) further supports the relatedness of both species.

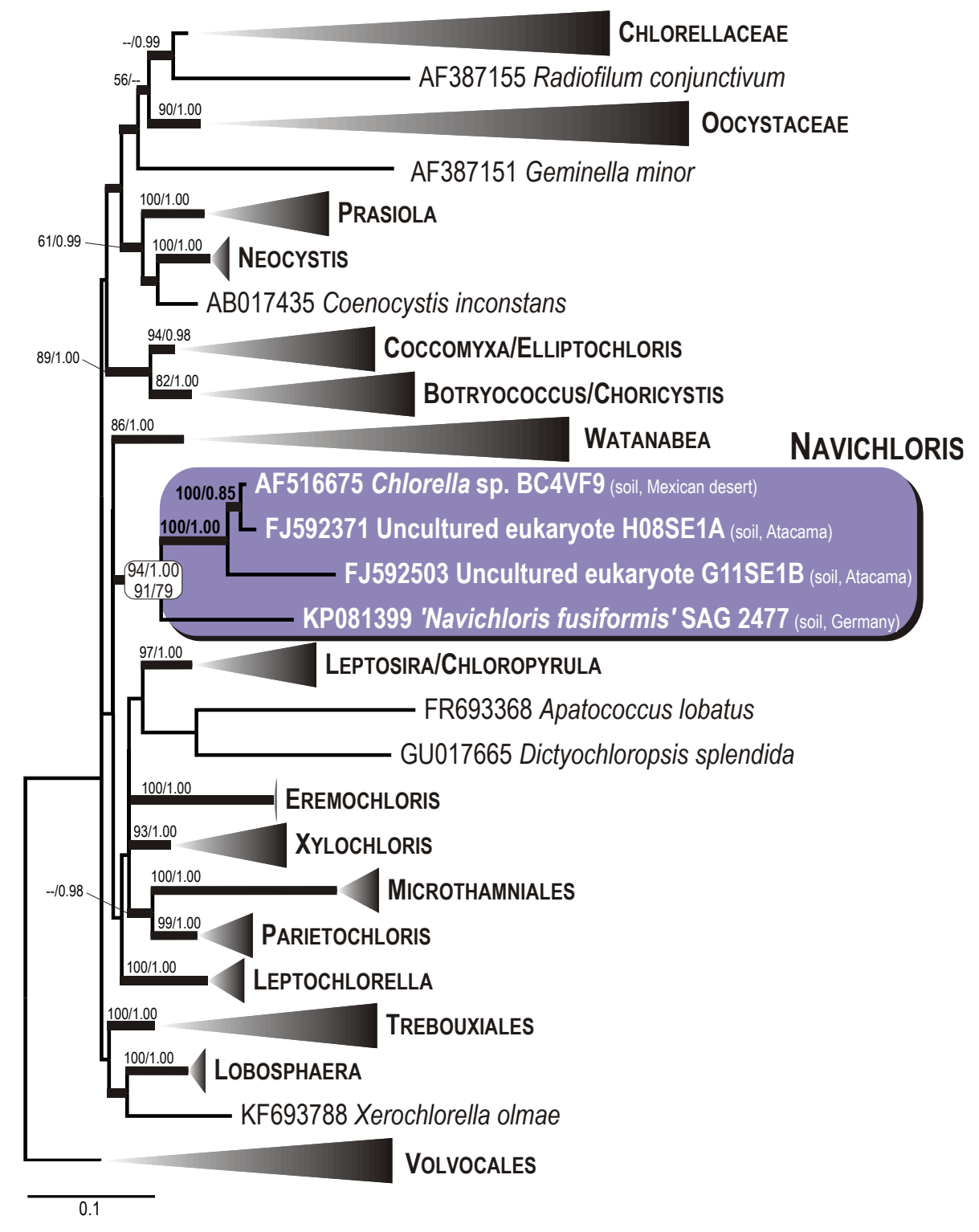

Figure 12. Schematic 18S rDNA phylogeny of the class Trebouxiophyceae. The newly proposed Navichloris lineage is highlighted in a violet box. The full 18S rDNA dataset used for the tree computation is identical as in Fučíková et al. (2014), Fig. 3. We complemented the dataset by adding three sequences, i.e., KP081399 (SAG 2477; retrieved in this study), FJ592371, FJ592503 (Costello et al. 2009); the resulting 18S rDNA dataset comprised 135 sequences. The phylogeny was inferred using RAxML (Stamatakis et al. 2008) and statistical support values for the most branches (maximum likelihood/posterior probabilities were computed in the same program and in MrBayes (Ronquist et al. 2012), respectively). Additional statistical support values (maximum parsimony and bio-neighbor-joining) for the Navichloris lineage were computed in the program SeaView ver. 4 (Gouy et al. 2010). 


\section{Cultured Green microalgae re-detected in environmental clones}

Almost $90 \%$ of our cultured species match clones amplified from environmental samples, when considering a $\leq$ 0.010 SSU-similarity threshold. Even though this similarity level might lack accuracy for species discrimination, it enables to detect species which are closely related; in comparison, Achtman and Wagner (2008) indicates a $\leq 0.013$ SSU-threshold for conspecifity in eubacteria. Culture-independent cloning of microalgae from identical sampling sites, conducted by Hallmann et al. (in prep.-b), allows to recognize those species, which are re-detectable by both approaches. Due to their frequent occurrence in soils, they might reach high population densities, being key players of soil algal communities (Büdel et al. 2009). This applies for several trebouxiophytes (e.g., Stichococcus spp., Coccomyxa spp., Chlorella vulgaris, Muriella-like species) and chlorophytes (Oogamochlamydinia, Tatrensinia, Jenufa, Scenedesmaceae). Even though comparable cloning studies on soil microalgae are rare, our isolates additionally matched clones from North American soils (Neocystis, Chloroidium, Reinhardtinia, Desmotetra; Lesaulnier et al. 2008) and from Dutch historical soil samples (Muriella; Moon-van der Staay et al. 2006). In multiple cases, clones closely related to our isolates, were amplified from either much dryer or even aquatic habitats, such as Stichococcus on marble monuments (Hallmann et al. 2013b) and on rocks in the Alps (Horath and Bachofen 2009), Diplosphaera in Rio Tinto river (Aguilera et al. 2007); Coccomyxa (HE617184) in an acidic lake and purification plant in Japan (GenBank acc. no. AB721066), Lobosphaera in sulfidic water (Behnke et al. 2006) or Stephanosphaerinia and Pseudendocloniopsis in glacier debris of Alaska (Schmidt and Darcy 2014). Other isolates matched clones amplified from cultured environmental material, such as building stone (Hallmann et al. 2013a) and even from aeroplankton in Greece (Genitsaris et al. 2011). Remaining cultures were more divergent from all available environmental clones; hence we queried them against selected soil metagenomes deposited in Sequence Read Archive (Wheeler et al. 2007). Here we achieved only a $\leq 0.03$ match of Chlorococcum cf. minutum SAG 2479 and Chlamydomonas cf. gerloffii LH08SW5031 with SSU-V4 pyrotag reads from permafrost metagenome in Sweden (Mondav et al. 2014).

\section{Conclusive remarks}

Our results suggest that Green microalgae in soils of Central Europe are far from known, being less intensively studied than e.g. desert soil crusts (Lewis and Lewis 2005; Fučíková et al. 2014). Even the choice of standard culturing media - supposedly not favoring demanding species (Lukešová 2001; Hoffmann et al. 2007)—retrieved a plethora of (phylogenetic) species. The most of them are re-detectable in environmental clone libraries, but some would remain 
undiscovered without culturing approach. Particularly high phylogenetic diversity hide in morphospecies known as Stichocococcus (Nägeli 1849) or Nannochloris-like (Henley et al. 2004) - consisting of unrelated species which exhibit morphological convergence characteristic for terrestrial Green microalgae (Sharma and Rai 2010). Simplistic morphology of microscopic algae favor their fast dispersal (Sharma et al. 2007), however, cosmopolitanism of Green microalgae remains questionable (Lawley et al. 2004; De Wever et al. 2009). Traditional morphology-based surveys generally proclaim ubiquity of soil Green microalgae (Feher 1948; Starks et al. 1981; Ettl and Gärtner 1995). A summary of morphospecies inventory lists collected worldwide (Fig. S6) suggests that Green algal communities might share a general morphological composition (i.e., consist of Stichococcus-, Chlorella-, Chlamydomonas-, Chlorococcum- and Klebsormidium-like morphospecies). Our cultures contribute DNA-based hints indirectly supporting the above mentioned observations-we detected identical ITS2 signatures of Stichococcus, Chlorella and Klebsormidium originating from two different continents (Fig. 9b). The ITS2 data further supported the wide distribution of Muriella (Kochkina et al. 2014) and Chlorococcum (GenBank acc. no. FR865591). It remains a major challenge of metagenomics to explore phylogenetic structure and geographic distribution of microscopic algae (Marande et al. 2009; Raven 2012; Lie et al. 2014). However, not less important is to link OTUs from environmental metagenomes with physical cultures of well characterized species (Richards and Bass 2005; Richards et al. 2005; Dickie 2010).

\section{Acknowledgements}

We thank the managers of the three Exploratories, Kirsten Reichel-Jung, Swen Renner, Katrin Hartwich, Sonja Gockel, Kerstin Wiesner, and Martin Gorke for their work in maintaining the plot and project infrastructure; Christiane Fischer and Simone Pfeiffer for giving support through the central office, Michael Owonibi for managing the central data base, and Markus Fischer, Eduard Linsenmair, Dominik Hessenmöller, Jens Nieschulze, Daniel Prati, Ingo Schöning, François Buscot, Ernst-Detlef Schulze, Wolfgang W. Weisser and the late Elisabeth Kalko for their role in setting up the Biodiversity Exploratories project. The work has been (partly) funded by the DFG Priority Program 1374 "Infrastructure-Biodiversity-Exploratories" (DFG-FR 905/161 and FR 905/17-1). Field work permits were issued by the responsible state environmental offices of Baden-Württemberg, Thüringen, and Brandenburg (according to $§ 72$ BbgNatSchG). 


\title{
Chapter 2
}

\section{Diversity of microscopic green algae (Chlorophyta) in calcifying biofilms of two karstic streams in Germany}

\author{
Ladislav Hodač ${ }^{1}$, Nicole Brinkmann², Kathrin I. Mohr ${ }^{3}$, Gernot Arp², Christine Hallmann ${ }^{1}$, Jessica Ramm ${ }^{4}$, Karolin \\ Spitzer ${ }^{1}$, Thomas Friedl ${ }^{1}$ \\ ${ }^{1}$ University of Göttingen, Experimental Phycology and Culture Collection of Algae (SAG), Göttingen, Germany \\ ${ }^{2}$ University of Göttingen, Geoscience Centre, Göttingen, Germany \\ ${ }^{3}$ Helmholtz Centre for Infection Research, Braunschweig, Germany \\ ${ }^{4}$ Brandenburg University of Technology, Department of Freshwater Conservation, Bad Saarow, Germany
}

(Published in Geomicrobiology Journal; doi:10.1080/01490451.2013.878418)

\begin{abstract}
For the first time the diversity of microscopic green algae (Chlorophyta) from calcified biofilms of karstic streams was analyzed using a combined approach based on pure cultures, i.e. 18S rRNA gene sequencing and microscopic analyses. Our study focused on two creeks in Germany. A considerable diversity of 34 species of green microalgae comprising three classes, the Trebouxiophyceae, Chlorophyceae and Ulvophyceae, was recovered. The biofilms of both streams were rather different in their species compositions which may reflect that they are exposed to differed hydrochemical conditions. The shallow Westerhöfer creek harbored predominantly Trebouxiophyceae and exhibited higher $\mathrm{Mg}^{2+}$ and $\mathrm{SO}_{4}{ }^{2-}$ concentrations. In contrast, the deeper, longer and spatially more heterogeneous Deinschwanger creek harbored numerous species of Chlorophyceae. A lower number of species from the Ulvophyceae were spread on both studied streams. The closest relatives of the identified species were from other freshwater habitats, but mostly from phytoplankton. However, also several species we recovered from freshwater for the first time; so far they have been known from terrestrial habitats only. Less than half of the recovered species could be identified with names at the species level based on high sequence identities with available sequences from named reference strains. Most recovered species could not be identified with names to species level, i.e. they may represent species for which no 18S rRNA gene sequence have become available yet or which have been retrieved for
\end{abstract}


the first time. The genus Gongrosira Kützing, often reported from freshwater tufa-stromatolites, was found to represent most likely a collective morphotype formed by several genera nested within the Ulvophyceae.

Keywords: Chlorophyta; Trebouxiophyceae; Chlorophyceae; Ulvophyceae; 18S rRNA gene phylogeny; karst-water creeks; cultures

\section{Introduction}

Many karstic streams in Europe and elsewhere are characterized by calcium carbonate deposits which veneer macrophytes as well as biofilm-covered rock surfaces at the stream bed. These deposits are termed tufa stromatolites, defined as macroscopically laminated benthic microbial deposits produced by precipitation of minerals on organic tissue (Riding 1991). Biomineralization (biological processes) and inorganic precipitation may act together (Ford and Pedley 1996) or photosynthetic $\mathrm{CO}_{2}$ assimilation by cyanobacteria, eukaryotic algae, and plants may be the primary cause for the carbonate precipitation (Pia 1926; Wallner 1934; Hepperle and Krienitz 1996). Indeed, microsensor studies have demonstrated a photosynthetic control of $\mathrm{CaCO}_{3}$ precipitation for biofilm-covered surfaces, while inorganically driven precipitation prevails e.g. at moss surfaces (Shiraishi et al. 2008a; Shiraishi et al. 2008b). Microscopic studies revealed the dominance of filamentous cyanobacteria in the calcified biofilms of freshwater karst creeks (Freytet and Plet 1996; Garcia-Pichel et al. 2004; Brinkmann et al. 2015). But also diatoms, xanthophytes, red algae and other microscopic algae as well as bryophytes and microscopic fungi occur being associated with freshwater stromatolites (Winsborough and Golubić 1987; Heath et al. 1995; Freytet and Verrecchia 1998; Bilan and Usov 2001; Brinkmann et al. 2015).

In an ongoing larger study the possible roles of photosynthesis and respiration in calcification processes are being studied in detail at two exemplar karstic streams with prominent $\mathrm{CO}_{2}$ degassing along their course, the Westerhöfer creek and the Deinschwanger creek in Germany. Both tufa-forming streams attain high calcite supersaturation during their course downstream. The Westerhöfer creek, located in Middle Germany in the westerly Harz-foreland $\left(51^{\circ} 45^{\prime} \mathrm{N}\right.$, $10^{\circ} 5^{\prime} \mathrm{E}$ ), is $325 \mathrm{~m}$ long and less than $2 \mathrm{~m}$ wide and has its source in limestones and evaporites of the Middle Triassic Muschelkalk Group. The Deinschwanger creek is located in southern Germany at the western rim of the Franconian Alb $\left(49^{\circ} 23^{\prime} \mathrm{N}, 11^{\circ} 28^{\prime} \mathrm{E}\right)$. It is fed by three main springs and a number of side springs, most of them discharging from the Upper Jurassic 
Weißjura-Group aquifer. Compared to the Deinschwanger creek the Westerhöfer creek is rich in $\mathrm{Mg}^{2+}$ and $\mathrm{SO}_{4}{ }^{2-}$. Microscopy of biofilm samples from both creeks revealed cyanobacteria and diatoms as the dominant algae (Brinkmann et al. 2015), but other micro-algae were found only rarely or not at all. Concurrently with a study on the biodiversity of the cyanobacteria and diatoms from both creeks (Brinkmann et al. 2015), cultures of green algae were developed. Interestingly, in the enrichment cultures an unexpected variety of green micro-algae appeared besides numerous cyanobacteria and diatoms. It is not known yet whether there are green algal taxa with strict or even any preference for calcifying biofilms. Their presence in calcifying biofilms may even be entirely given by accident. There is an expectation that the most green algae in calcifying biofilms could originate from soils and other aerial and subaerial habitats. Here we report about the phylogenetic and morphological diversity of these green microalgae.

\section{Materials and Methods}

\section{Sampling, culturing and microscopy}

Biofilms from both, the Westerhöfer and Deinschwanger creeks, were collected in the spring or early summer (May/June) in 2005-2007. Five sites of the Westerhöfer creek (abbreviated as WB; Figure A) and eight sites from Deinschwanger creek (abbreviated as DB) were selected for sampling of apparently algae-dominated biofilms (Table 1). For starting enrichment cultures all

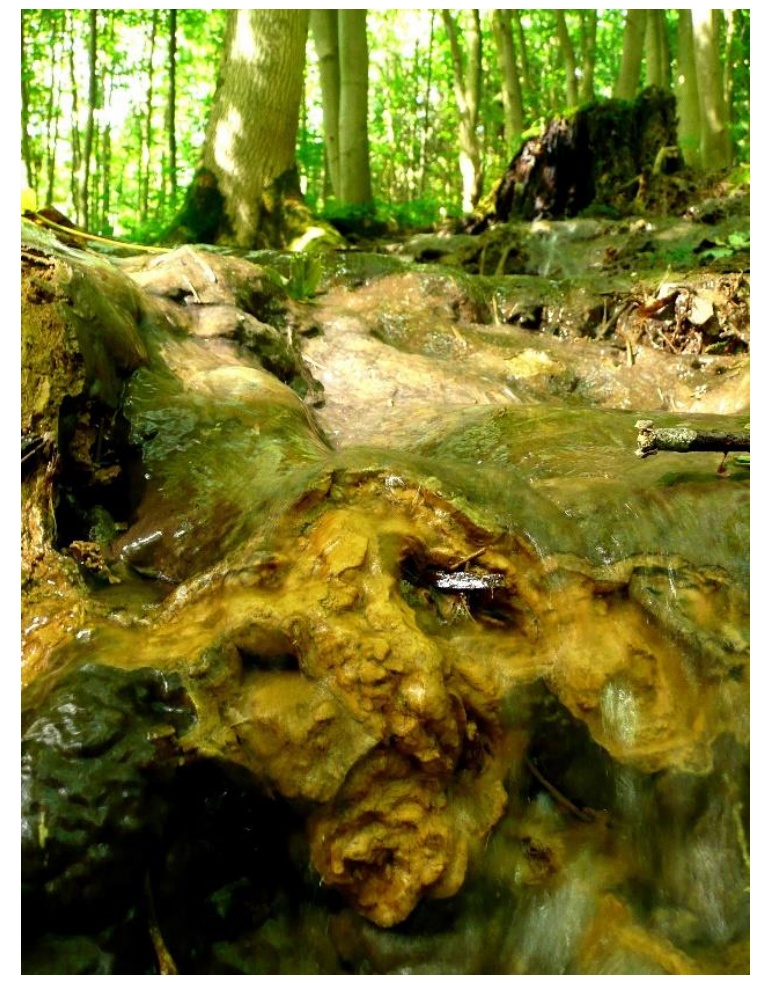

Figure A. Freshwater stromatolites (Westerhöfer Bach) samples from the WB were pooled together because it is rather short and only a short segment (about $350 \mathrm{~m}$ ) of the creek was investigated, whereas for the DB the samples from different locations were analysed separately to better reflect its higher habitat heterogeneity. The biofilms were scratched off from stone surfaces using an ethanol-sterilized knife or spatula and transferred to $1.5 \mathrm{ml}$ reaction tubes which were cooled until further processing in the laboratory the following day. A spatula-full of biofilm material was then transferred into different standard liquid growth media. Because our initial main focus was to establish cultures of cyanobacteria and diatoms, growth media 
provoking the development of these algae were used, i.e. BG11, BG11 without citrate, Z, Z 45/4, and ES (http://www.uni-goettingen.de/de/186449.html). Apart from the expected growth of cyanobacteria and diatoms, also intensive green algal growth was observed after about 5-10 days of cultivation. Putative green algal colonies were transferred on $1.5 \%$ agar plates with $3 \mathrm{~N}$ BBM+V medium (http://www.uni-goettingen.de/de/186449.html). After incubation for another 4-8 weeks, all green algal morphotypes were isolated into unialgal cultures by several rounds of streaking on fresh agar plates of $3 \mathrm{~N} \mathrm{BBM}+\mathrm{V}$ medium using sterile platinum needles. Finally, the unialgal isolates were microscopically checked for purity and further maintained on agar slants at $18^{\circ} \mathrm{C}$ under a light/dark regime of $14-\mathrm{h}: 10$-h and a photon flow rate of about $25 \mu$ mol photons $\mathrm{m}^{-2} \mathrm{~s}^{-1}$ from white fluorescent bulbs. A total of 77 pure cultures was established, out of which 45 were studied in more detail (Table S1). Nineteen of them were made publicly available, i.e. accessioned by the SAG culture collection (Göttingen University, Germany; www.epsag.unigoettingen.de). For microscopy an Olympus BX60 microscope (Tokyo, Japan) with Nomarski DIC optics and an attached ColorView III camera (Soft Imaging System, Münster, Germany) was used. Micrographs were processed using Cell^^D image software (Soft Imaging System, Münster, Germany).

\section{DNA extraction, PCR and sequencing}

DNA was extracted from all unialgal isolates (Table S1) using the Invisorb Spin Plant Kit (Stratec, Berlin, Germany) as recommended by the manufacturer. Nuclear-encoded 18S rRNA genes were amplified using primers NS1 and 18L (Hamby et al. 1988). For some strains the 18S and ITS1-5.8S-ITS2 rRNA gene region was amplified using primers NS1 and LR1850 (Friedl 1996). If a culture was suspected to be contaminated by fungi or bacteria, PCR primer 1650R (3'TCACCAGCACAYYCAAT-5'; pos. 1652-1636 of the 18S rRNA gene sequence of Chlorella vulgaris SAG 211-11b, FM205832) which preferentially binds to members of Chlorophyta, was used as the reverse PCR primer. Conditions for PCR and cycle sequencing reactions and the standard set of sequencing primers were as described earlier (Mikhailyuk et al. 2008). The newly determined sequences were deposited in GenBank under the accession numbers KF144164KF144240 (Table S1). In addition also the 18S rRNA gene sequences for the following strains were determined as references: Chlorococcum vacuolatum Starr SAG 213-8 (KF144189), Dilabifilum printzii (Vischer) Bourelly SAG 467-1 (KF144198), Scotinosphaera gibberosa (Vodenicarov \& Benderliev) Wujek \& R.H.Thompson SAG 75.80 (KF144229) and S. lemnae (Punčochářová) Wujek \& R.H.Thompson SAG 240-1 (KF144230). 
Table 1. Distribution of the recovered green algal species at the sampling sites.

\begin{tabular}{|c|c|c|c|c|c|c|c|c|c|c|}
\hline & & DB1 & DB2 & DB3 & $\overline{\mathrm{DB} 4}$ & DB5 & $\overline{\mathrm{DB} 6}$ & DB9 & DBS & WB \\
\hline & & 1.19 & 1.15 & 1.11 & 1.09 & 1.09 & 1.11 & 0.45 & 0.01 & $0.28-0.99$ \\
\hline Class & Species & 3 & 3 & 2 & 1 & 1 & 11 & 2 & 4 & 19 \\
\hline \multirow[t]{14}{*}{ Trebouxiophyceae } & Chlorella sp.* & + & + & + & - & - & + & - & + & - \\
\hline & Coccomyxa cf. pringsheimii & - & - & - & - & - & - & - & + & + \\
\hline & Coccomyxa cf. simplex & - & - & - & - & - & - & - & - & + \\
\hline & Elliptochloris subsphaerica* & - & - & - & - & - & - & - & - & + \\
\hline & Marvania sp. & - & - & - & - & - & - & - & - & + \\
\hline & Muriella terrestris ${ }^{\star *}$ & - & + & - & - & - & - & - & - & + \\
\hline & Neocystis cf. mucosa & - & - & - & - & - & - & - & - & + \\
\hline & Stichococcus bacillaris ${ }^{* *}$ & - & - & - & - & - & - & - & - & + \\
\hline & Stichococcus cf. deasonii & - & - & - & - & - & - & - & - & + \\
\hline & Stichococcus mirabilis ${ }^{* *}$ & - & - & - & - & - & - & - & - & + \\
\hline & Stichococcus sp.1** & - & - & - & - & - & + & - & - & + \\
\hline & Stichococcus sp.2 & - & - & - & + & - & - & - & - & - \\
\hline & Stichococcus sp.3 & - & - & - & - & - & - & - & - & + \\
\hline & Stichococcus sp.4 & - & - & - & - & - & - & - & - & + \\
\hline \multirow[t]{15}{*}{ Chlorophyceae } & Acutodesmus obliquus ${ }^{* *}$ & - & - & - & - & - & + & - & - & - \\
\hline & Bracteacoccus aerius-relative & - & - & - & - & - & - & - & - & + \\
\hline & Bracteacoccus sp. & - & - & - & - & - & - & + & - & - \\
\hline & Chlamydomonas sp. & - & - & - & - & - & - & - & - & + \\
\hline & Chlamydopodium sp. & - & - & - & - & - & + & - & - & - \\
\hline & Chlorococcum minutum-relative & - & - & + & - & - & - & - & - & - \\
\hline & Chlorococcum sphacosum ${ }^{*}$ & + & - & - & - & - & - & - & - & - \\
\hline & Chlorococcum ellipsoideum-relative1 & - & - & - & - & + & - & - & - & - \\
\hline & Chlorococcum ellipsoideum-relative2 & - & - & - & - & - & + & - & - & + \\
\hline & Desmodesmus cf. armatus & - & - & - & - & - & + & - & - & - \\
\hline & Monoraphidium terrestre cf. dybowskii & - & - & - & - & - & + & - & - & - \\
\hline & Mychonastes cf. homosphaera & - & - & - & - & - & + & - & - & - \\
\hline & Mychonastes sp.* & - & - & - & - & - & + & - & - & - \\
\hline & Pseudomuriella cf. schumacherensis & - & - & - & - & - & - & - & - & + \\
\hline & Scenedesmaceae sp. & - & - & - & - & - & + & - & - & - \\
\hline \multirow[t]{5}{*}{ Ulvophyceae } & Desmochloris cf. halophila & + & - & - & - & - & - & - & - & - \\
\hline & Dilabifilum printzii ${ }^{* *}$ & - & - & - & - & - & - & - & + & + \\
\hline & Hazenia mirabilis* & - & - & - & - & - & - & + & - & - \\
\hline & Pseudendocloniopsis botryoides* & - & + & - & - & - & + & - & + & + \\
\hline & Pseudendoclonium akinetum* & - & - & - & - & - & - & - & - & + \\
\hline
\end{tabular}

Legend to Table 1. The recovered species were distributed at eight sampling sites of the Deinschwanger creek (DB) and the pooled sample from the Westerhöfer creek (WB, from five sites). Numbers below sampling sites are the corresponding values [log IAP/KT] of calcite saturation index (Slcalcite, for explanation see Arp et al. 2010) and the total number of species recovered per site. Two asterisks next to a species name indicate $100 \%$, a single asterisk $99.9 \%$ sequence identity with a reference strain.

\section{Phylogenetic analyses}

To search for the closest neighboring relatives of our isolates their sequences were compared to those from reference strains at NCBI (http://www.ncbi.nlm.nih.gov) using BLASTn queries (Altschul et al. 1997). Only almost full neighboring 18S rRNA gene sequences were downloaded together with a selection of reference sequences to better represent the green algal classes Trebouxiophyceae, Chlorophyceae and Ulvophyceae as well as additional green algal lineages and aligned using MAFFT, ver. 6 (Katoh and Toh 2008) available online at http://mafft.cbrc.jp/alignment/server/index.html. Three sequence data sets were constructed after 
the alignments were manually refined using BioEdit (Hall 1999). The sequence data set of Trebouxiophyceae (Fig. 1) contained 162 sequences and was 1766 positions long with 781/562 variable/parsimony informative sites, that of Chlorophyceae (Fig. 2, Fig. 3) contained 238 sequences and was 1797 positions long with 938/653 variable/parsimony informative sites, and that of Ulvophyceae (Fig. 4) 88 sequences and was 1785 positions long with 953/813 variable/parsimony informative sites. The GTR $+\Gamma+\mathrm{I}$ model was selected as the best fitting model of nucleotide substitution for all three sequence data sets as based on the AIC criterion using jModelTest 0.1.1 (Posada 2008). Phylogenetic trees were calculated using maximum likelihood with the program RAxML 7.0.4 (Stamatakis et al. 2008) and Bayesian phylogenetic inference with MrBayes 3.2.1 x64 (Ronquist et al. 2012). For the latter, two MCMC runs for three million generations each were employed with one cold and three heated chains with trees sampled every 100 generations. Confidence values for the obtained groups (internal edge support) were inferred from the rapid bootstrapping algorithm (100 replicates) as implemented in RAxML (Stamatakis et al. 2008) and from Bayesian posterior probabilities using MrBayes 3.2.1 x64 (Ronquist et al. 2012). Pairwise sequence similarities from p-distances were determined as an additional measure of the relatedness of our isolates to certain reference strains/sequences under the Kimura 2parameter model with the program MEGA5 (Tamura et al. 2011).

\section{Statistical analysis}

The distribution of all major clades of green algae at studied sampling sites was investigated using multivariate ordination by Non-metric Multidimensional Scaling (NMDS); the analysis was conducted in PAST 2.06 (Hammer et al. 2001). The input data corresponded to a matrix similar as in Tables 1 and $\mathbf{2}$ summarizing the presence/absence of isolates at particular sampling sites as identified by $18 \mathrm{~S}$ rRNA gene sequence comparisons.

Table 2. Distribution of the recovered phylogenetic clades of Green algae.

\begin{tabular}{|c|c|c|c|c|c|c|c|c|c|}
\hline & & $\overline{D B 1}$ & $\overline{\mathrm{DB} 2}$ & DB3 & $\overline{\text { DB4 }}$ & DB5 & $\overline{D B 6}$ & $\overline{\text { DB9 }}$ & $\overline{D B S}$ \\
\hline & & 1.19 & 1.15 & 1.11 & 1.09 & 1.09 & 1.11 & 0.45 & 0.01 \\
\hline & & 2 & 2 & 2 & 2 & 2 & 5 & 2 & 4 \\
\hline \multirow[t]{3}{*}{ Trebouxiophyceae } & Chlorellaceae clade & + & + & + & - & - & + & - & + \\
\hline & Choricystis/Botryococcus clade & - & - & - & - & - & - & - & + \\
\hline & Prasiolales & - & - & - & + & - & + & - & - \\
\hline \multirow[t]{2}{*}{ Chlorophyceae } & Chlamydomonadales & + & - & + & - & + & + & - & - \\
\hline & Sphaeropleales & - & - & - & - & - & + & + & - \\
\hline \multirow[t]{2}{*}{ Ulvophyceae } & Ulotrichales & - & + & - & - & - & + & + & + \\
\hline & Ulvales & - & - & - & - & - & - & - & + \\
\hline
\end{tabular}

Legend to Table 2. Seven phylogenetic clades of Chlorophyta were distributed at the eight sampling sites of the Deinschwanger creek (DB). Numbers below sampling sites are values [log IAP/KT] of calcite saturation index (SI Calcite, for explanation see Arp et al. 2010) and the total number of clades recovered per site ("phylogenetic diversity"). 


\section{Results}

A total of 77 isolates have been obtained from both creeks (Table S1). Phylogenetic analyses of 18S rRNA gene sequences determined for each isolate distinguished 34 distinct lineages supposedly corresponding to species. They were distributed on three classes of green algae, the Trebouxiophyceae (14 species; Fig. 1), the Chlorophyceae (15 species; Fig. 2, Fig. 3) and the Ulvophyceae (5 species; Fig. 4). Forty-five isolates have been selected for phylogenetic and microscopic analyses in this paper (Figs. 1-7, Table S1, Table S2). The other isolates shared identical (partial) sequences and, therefore, were suspected to represent the same species as the selected 45 isolates. Morphological features of the 34 species (77 isolates) were investigated and are summarized in Table S2.

\section{Diversity of Trebouxiophyceae}

The recovered Trebouxiophyceae isolates were distributed on four major clades of the class (Fig. 1). Those isolates with a Stichococcus-like rod shaped morphology (Fig. 5a, Fig. 5b) were distributed in seven distinct lineages within the Prasiola clade. Based on high sequence similarities (99.9 or $100 \%$ as determined from p-distances) and short genetic distances with named reference sequences in the phylogenetic analyses (Fig. 1) two closely related isolates, WB13 and WB74 (Fig. 5a), were identified as S. bacillaris and isolate WB69 as S. mirabilis (Table 1, Table S3). For the other Stichococcus-like isolates sequence similarities with named references were lower or no closer named references were available at all. Isolate WB38 was named $S$. cf. deasonii as its next named reference was $S$. deasonii. For six isolates which were distributed on four independent lineages of the Prasiola clade (Fig. 1) no close named relatives were available and, therefore, they were named Stichococcus sp. 1 (isolates DB6-27, WB65, and SAG 2407), S. sp. 2 (isolate D4-2A), S. sp. 3 (isolate SAG 2408) and S. sp. 4 (isolate SAG 2406; Fig. 5b). Based on morphology all seven lineages may be assigned to Stichococcus, but in the $18 \mathrm{~S}$ rRNA gene phylogenies the genus was paraphyletic with many other coccoid and filamentous members of the Prasiola clade (Fig. 1). The recovered seven lineages of Stichococcus-like Trebouxiophyceae may correspond to seven distinct species, but their assignment to a single genus Stichococcus is not adequate. The genus as currently circumscribed may in fact encompass several distinct genera.

The unicellular spherical trebouxiophytes (Figs. 5c-5f) represented three distinct lineages within the Chlorellaceae clade (Fig. 1). The two isolates RK52 (Fig. 5c) and D11-2 (Fig. 5d) exhibited relatively large rounded cells with cup-shaped chloroplast and a single pyrenoid with 
starch grains attached to it, i.e. the Chlorella morphotype (Table S2). Both isolates had sequences highly similar to each other and their closest neighbors (99.9\% sequence similarity) were several unidentified Chlorella sp. strains. Both isolates represented the most frequently recovered green alga in our study, i.e. there was a total of 12 strains with almost identical 18S rRNA sequences and identical morphology representing the species (Table S1, Table S2). Resolution within the clade corresponding to the Chlorellales in the 18S rRNA phylogeny was low (Fig. 1). Both Chlorella-like isolates were distinct from the authentic (type) strain of the genus, C. vulgaris SAG 211-11b, by a relatively large genetic distance (Fig. 1), but a closer assignment to any genus of Chlorellales was impossible. Hindakia was the closest relative (Fig. 1), but with a relatively low sequence similarity of $99.7 \%$.

Two other trebouxiophyte species with spherical cells differed from the Chlorella morphotype by their smaller cells with a single band-shaped chloroplast without pyrenoid (Table S2; Fig. 5e, Fig. 5f). They were distributed in two distinct lineages outside of Chlorellales, but within the clade representing the Chlorellales (Fig. 1). Two isolates (D6-DB2 and SAG 2390) were assigned to Muriella terrestris (Fig. 5e) due to low p-distances/high sequence similarities (99.9 and 100 \%) with strain ASIB V38 (acc. no. AB012845) which has been isolated from soil (Gärtner 1996). Both our isolates also had the same high sequence similarity with an unidentified strain of Muriella AS2-4 from freshwater phytoplankton, acc. no. AY195969 (Fawley et al. 2004). Therefore, we assume that both our isolates as well as strains ASIB V38 and AS2-4 represent the same species, M. terrestris. The other species, represented by isolate WB67 (Fig. 5f), shared high sequence similarities with unidentified Nannochloris-like strains which together shared a wellsupported monophyletic origin with Marvania geminata. Because of the relatively low sequence similarity $(97.8 \%)$ with the reference sequence of $M$. geminata our isolate probably represents another yet still undescribed species of Marvania.

Other isolated trebouxiophytes exhibited reniform (Neocystis-like; Fig. 5g), elliptic to nearly spherical (Elliptochloris-like; Fig. 5h) or elongated to elliptic (Coccomyxa-like; Fig. 5i) cell shapes. The next relative to isolate SAG 2405, characterized by its mucilaginous colonies and reniform cell shape (Fig. 5g), was Neocystis mucosa strain KR 1989/14 (acc. no. HM565928), therefore it was termed Neocystis cf. mucosa. Another Neocystis strain, Neocystis brevis CAUP D802 (acc. no. HQ287929), was slightly more distant to SAG 2405 and the whole clade of Neocystis-like trebouxiophytes was highly supported. Isolate WB5-D1e (Fig. 5h) was identified as Elliptochloris subsphaerica based on its high sequence similarity of $99.9 \%$ with $E$. subsphaerica strain SAG 2202 (acc. no. FJ648518). The same short p-distance/high sequence similarity was with unidentified Elliptochloris strain SAG 2117 (acc. no. FJ648515). The whole 
clade comprising the three Elliptochloris sequences was well supported (Fig. 1). Two more species exhibited a typical Coccomyxa-like morphology (Fig. 5i) and were nested within a wellsupported clade consisting of several Coccomyxa strains. The one species, represented by the single isolate WB40, was most closely related to strain Pseudococcomyxa simplex UTEX 274 (acc. no. FJ648514). The other species was represented by four isolates, retrieved from both creeks (Table S1). Out of them for isolate WB28 an almost full 18S rRNA gene sequence was determined and strain Coccomyxa pringsheimii SAG 69.80 (acc. no. AY762603) represented its closest neighboring sequence.

\section{Diversity of Chlorophyceae}

Isolates assigned to the class Chlorophyceae were distributed in two clades representing the orders Chlamydomonadales and Sphaeropleales (Fig. 2, Fig. 3). The isolated members of Chlamydomonadales exhibited two morphological types, i.e. Chlorococcum-like large spherical cells with a single large chloroplast (Figs. 6a-6e), Chlamydomonas-like monadoid biflagellated cells (Fig. 6f), those of Sphaeropleales four morphological types, i.e. Scenedesmus-like elongated cells with acute ends (Figs. 6h-6i), Bracteacoccus-like spherical cells with numerous discoidal parietal chloroplasts without a pyrenoid (Figs. 7a, 7b), Mychonastes- or Nannochloris like small spherical cells with a single chloroplast without a pyrenoid (Figs. 7c, 7d), and Monoraphidiumlike fusiform cells.

\section{Chlamydomonadales}

The Chlorococcum-like isolates were distributed in four distinct clades within the larger and highly supported Stephanosphaerinia clade in 18S rRNA gene phylogenies (Fig. 2). One clade was formed by isolate RK50 and strain SAG 2402 and their named relative Chlamydopodium vacuolatum (acc. no. M63001) and, therefore, both were assigned to the genus Chlamydopodium. Another sequence, but from an unidentified strain, Chlamydomonadaceae sp. KMMCC:EC-34, was even closer with the former two strains (Fig. 2). A second well supported clade included the isolates GRK6-DB5, GRK6-DB6, SAG 2400 and SAG 2401. Except for the latter two, which had almost identical sequences, they were all distant to each other and had sequences from unidentified strains as their next closest relatives. The next named closest relative was Chlorococcum ellipsoideum strain UTEX 972 (acc. no. U70586) and, therefore, we named our isolates Chlorococcoum ellipsoideum-relatives (Fig. 3, Figs. 6c-e). C. ellipsoideum-relative1, isolate GRK6-DB5, was closer related to C. ellipsoideum UTEX 972 than the other three isolates of the clade. A third clade contained a single isolate, SAG 2398, which was within a well- 
supported clade together with several named closest relatives (Fig. 2). Except for Chlorococcum sphacosum SAG 66.80 (which corresponds to the authentic strain of the type of the species), our isolate was morphologically rather distinct from them and, therefore, we assigned isolate SAG 2398 to C. sphacosum (Fig. 6a; Table 1, Table S2). Both strains, SAG 2398 and SAG 66.80 (acc. no. JN968580), also shared 99.9\% sequence similarity (Table 1, Table S3). Finally, strain SAG 2399 (Fig. 6b) was nested within another distinct and highly supported clade with $C$. vacuolatum SAG 213-8 (acc. no. KF144189) and C. minutum ASIB T50 (acc. no. JN968585) as the closest next named relatives (Fig. 2). Because it appeared more similar in its morphology to C. minutum (Table S2) we named strain SAG 2399 Chlorococcum minutum-relative. The Chlamydomonas-like monadoid isolates RK68 (Fig. 6f) and DB6-shared identical (partial) sequences and isolate RK68 was within the well-supported Reinhardtinia clade in the phylogenetic analyses (Fig. 2). Its closest named next relatives were three species of Chlamydomonas and Volvox carteri. Because it exhibited solitary cells with a typical Chlamydomonas morphology we assigned it to that genus, but could not identify it to species level.

\section{Sphaeropleales}

The Scenedesmus-like isolates were distributed in three independent lineages (species) of the Scenedesmaceae clade (Sphaeropleales; Fig. 3). Desmodesmus cf. armatus was with six isolates among the most frequently recovered green algal species of our study (Table S1). Both isolates, RK43 (Fig. 6g) and strain SAG 2395, for which almost full 18S rRNA gene sequences were determined, formed a highly supported clade together with named reference strain, Desmodesmus armatus CCAP 276/4A (acc. no. FR865727) and, therefore, were assigned the isolates to this species (Fig. 3). Isolate D22-6-2B (Fig. 6h) shared an identical sequence with reference strain Acutodesmus obliquus CCAP 276/49 (acc. no. FR865726) and was assigned to this species. Finally, isolate RK49 (Fig. 6i) could not be assigned to a certain genus of the Scenedesmaceae clade because it was distinct from all named reference strains of that clade, albeit there was week support for a closer relationship with species of Acutodesmus (Fig. 3). The three Bracteacoccuslike isolates were distributed in two independent clades of the Sphaeropleales representing the genera Bracteacoccus and Pseudomuriella (Fig. 3). The next closest named reference for isolate RK3 (Fig. 7a) was Pseudomuriella schumacherensis SAG 2137 (acc. no. HQ292768) but with a sequence similarity $<99.9 \%$ and, therefore, we named our isolate $P$. cf. schumacherensis. Despite their morphological similarities with the $P$. cf. schumacherensis isolate the two other Bracteacoccus-like isolates, SAG 2403 and DB9-3 (Fig. 7b), were in a distinct clade of the 18S 
rRNA gene phylogeny, i.e. the Bracteococcaceae clade. Strain SAG 2403 had a close named relative, Bracteococcus aerius UTEX 1250 (Fig. 3), but shared less than $99.9 \%$ sequence similarity from p-distances with the latter and, thus, was named Bracteacoccus cf. aerius. In contrast, isolate DB9-3 had no named closest relative; it was most closely to an unidentified Bracteacoccus strain (KF-2011f), but rather distant from B. cf. aerius (Fig. 3). Both Mychonastes/Nannochloris-like isolates, RK48 and DB6-29 (Figs. 7c-d), were within a single well supported clade, representing the genus Mychonastes within the Chlorophyceae (Fig. 3). Isolate RK48 had Mychonastes homosphaera CAUP H6501 (which represents the authentic strain of the species) as its closest named relative, but with a sequence similarity of less than 99.9\% (Table S3) and, therefore, was named Mychonastes cf. homosphaera. Mychonastes-like isolate DB6-29 was distant from RK48 and had an unidentified Mychonastes strain, Itas 9/21 148w (acc. no. AY543066) as closest relative (with 99.9\% sequence similarity) and, therefore, was named Mychonastes sp. (Fig. 3). Several species of Mychonastes have recently been described as common members of freshwater phytoplankton (Krienitz et al. 2011), but our isolates were more distant to those and, therefore, were not included in our phylogenetic analyses. The Monoraphidium-like creek biofilm isolate, strain SAG 2393, was most closely related (with sequence similarity $<99.9 \%$ ) to two reference strains named $M$. dybowskii within the Selenastraceae clade and, therefore, was named M. cf. dybowskii. 


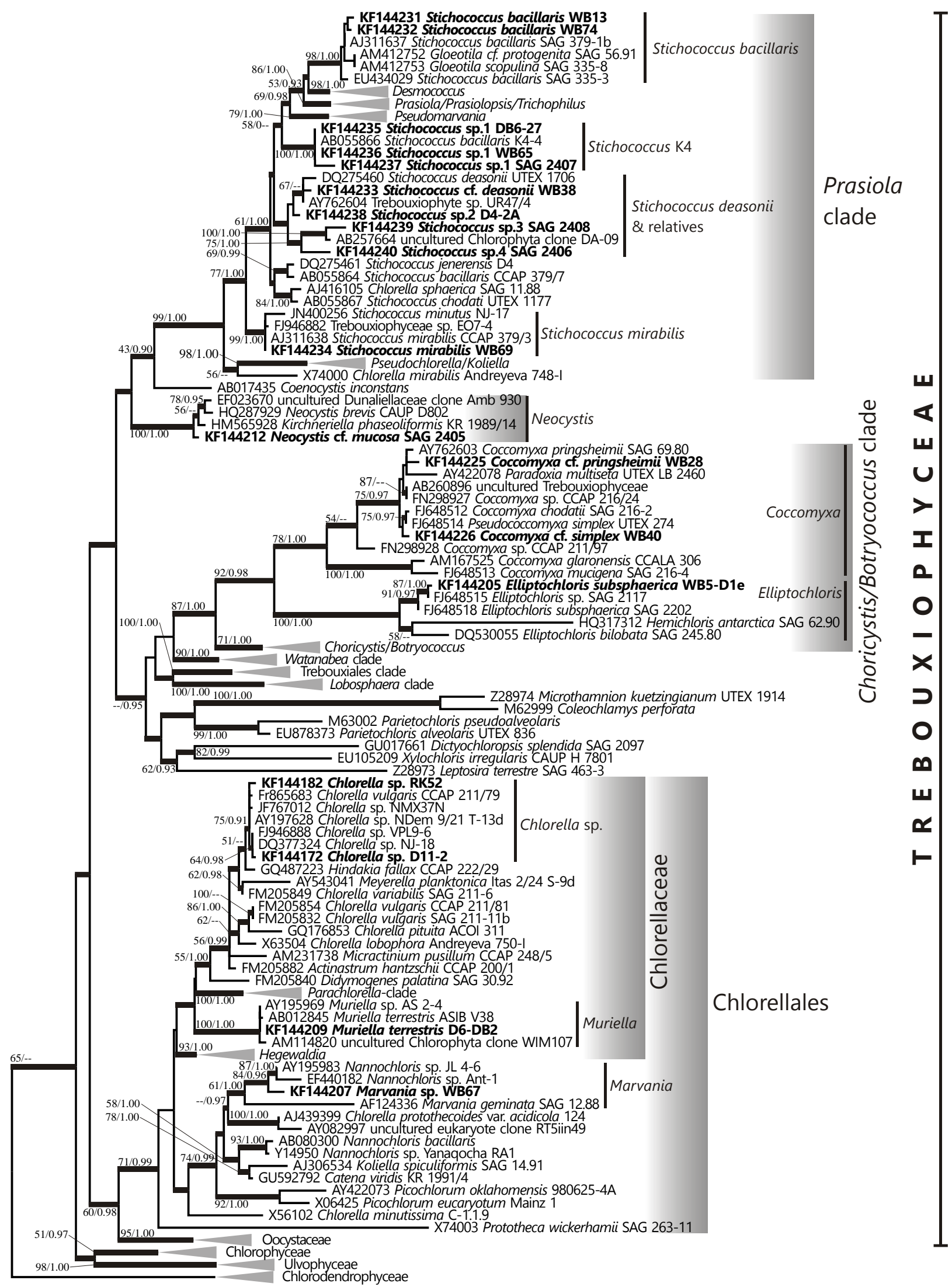

Figure 1. Maximum-likelihood (ML) phylogeny of $18 \mathrm{~S}$ rRNA gene sequences of green algal species from the class Trebouxiophyceae isolated from biofilms of two freshwater creeks (highlighted sequence names) and other members of the Trebouxiophyceae as references. Additional members of Oocystaceae (Trebouxiophyceae) as well as other classes of the Chlorophyta, the Chlorophyceae, Ulvophyceae and Chlorodendrophyceae (outgroup), are included for comparisons and to root the tree (for their accession numbers see Table S4). Additional outgroups including Picocystis salinarum and Nephroselmidophyceae were removed from the figure due to lack of space (all outgroups were the same as in Fig. 2, for accession numbers see Table S4). Numbers at internal branches indicate support from bootstrap tests using $\mathrm{ML}$ (left) and posterior probabilities from Bayesian analysis (BI; right). Thick lines mark internal branches that were resolved by both, the ML and the BI tree topologies. Grey boxes highlight the clades comprising the new isolates. 
Figure 2. Maximum-likelihood phylogeny of $18 \mathrm{~S}$ rRNA gene sequences of green algal species from the class Chlorophyceae, Chlamydomonadales clade, isolated from biofilms of two freshwater creeks (highlighted sequence names) and other members of the Chlorophyceae, Chlamydomonadales clade, as references. Additional members of Chlorophyceae as well as other classes of the Chlorophyta, the Trebouxiophyceae, Ulvophyceae, Chlorodendrophyceae; Picocystis salinarum and Nephroselmidophyceae (outgroup), are included for comparisons and to root the tree (for their accession numbers see Table S4). Numbers at internal branches indicate support from bootstrap tests using ML (left) and posterior probabilities from Bayesian analysis (Bl; right). Thick lines mark internal branches that were resolved by both, the ML and the $\mathrm{BI}$ tree topologies. Grey boxes highlight the clades comprising the new isolates.

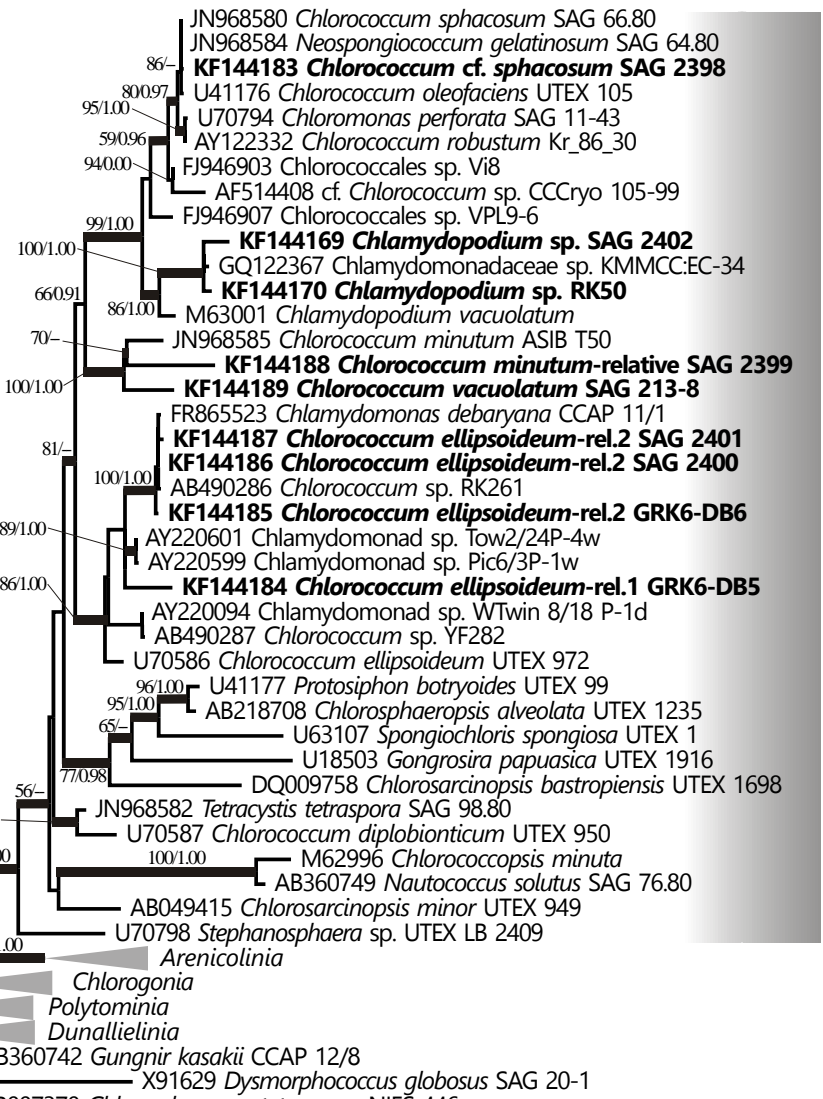

$0.1 \overline{\text { substitutions}}$ /site

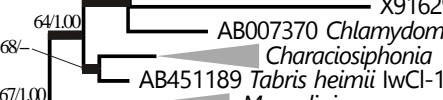

Monadinia 1 AY614714 Pseudotetracystis sp. NB1-VF11

100/1.00 Moewusinia

mydomonas chlamydogama UTEX 103

M32703 Chlamydomomas reinhardtii

961.00. EF023396 uncultured Dunaliellaceae clone Amb 733

85/- L JN903974 Chlamydomonas reinhardtii SAG 18.79

92/.00. AY781664 Chlamydomonas incerta SAG 7.73

$1001.00 \quad$ X53904 Volvox carteri UTEX 1885

KF144168 Chlamydomonas sp. RK68

100000 - AF008240 Chlamydomonas debaryana SAG 26.72 [ 100000 AY220086 Chlamydomonad sp. Pic 8/18 P-1w

99/.00 — U83121 Tetraspora sp.

971.00 U70781 Chlamydomonas baca SAG 24.87
781.00
Z AB218697 Neochlorosarcina deficiens UTEX 1700

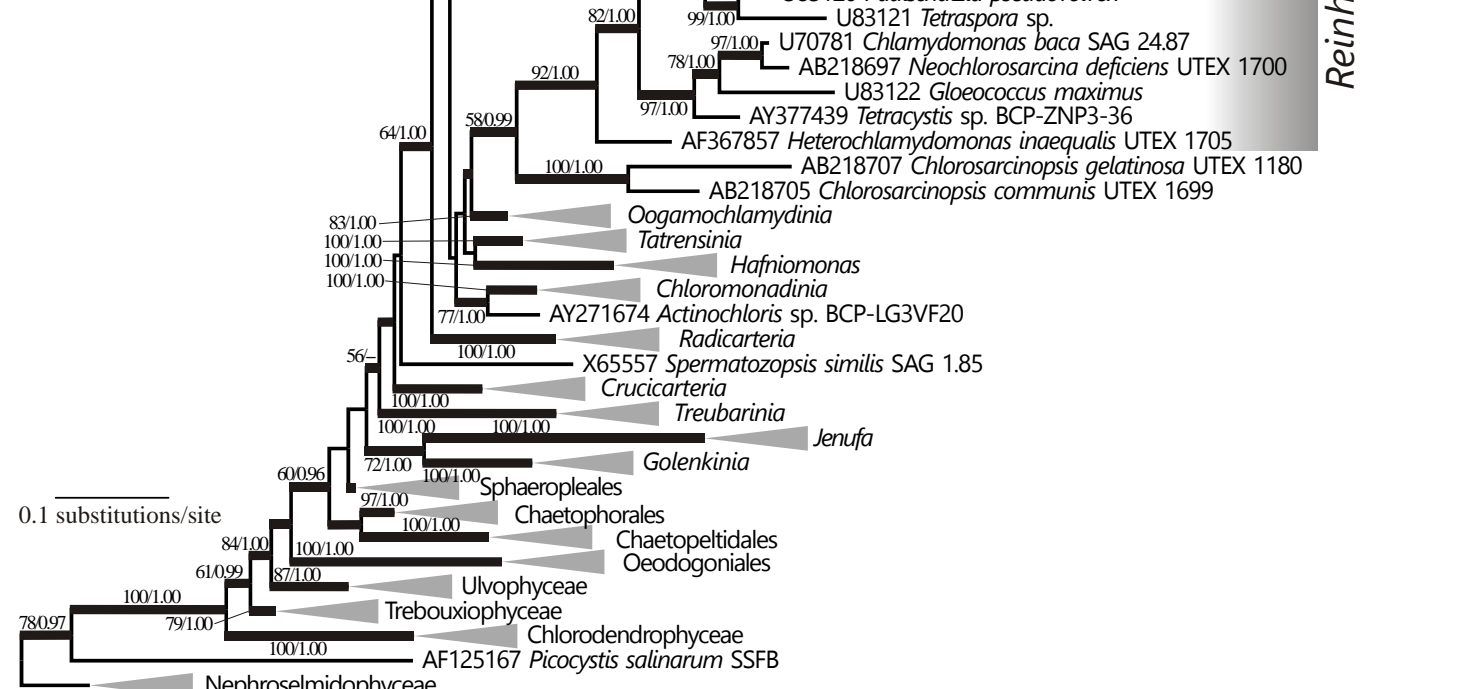

Nephroselmidophyceae 
Figure 3. Maximum-likelihood phylogeny of $18 \mathrm{~S}$ rRNA gene sequences of green algal species from the class Chlorophyceae, Sphaeropleales clade, isolated from biofilms of two freshwater creeks (highlighted sequence names) and other members of the Chlorophyceae, Sphaeropleales clade, as references. Additional members of Chlorophyceae as well as other classes of the Chlorophyta, the Trebouxiophyceae, Ulvophyceae, Chlorodendrophyceae; Picocystis salinarum and Nephroselmidophyceae (outgroup), are included for comparisons and to root the tree (for their accession numbers see Table S4). Numbers at internal branches indicate support from bootstrap tests using ML (left) and posterior probabilities from Bayesian analysis (Bl; right). Thick lines mark internal branches that were resolved by both, the ML and the $\mathrm{BI}$ tree topologies. Grey boxes highlight the clades comprising the new isolates.

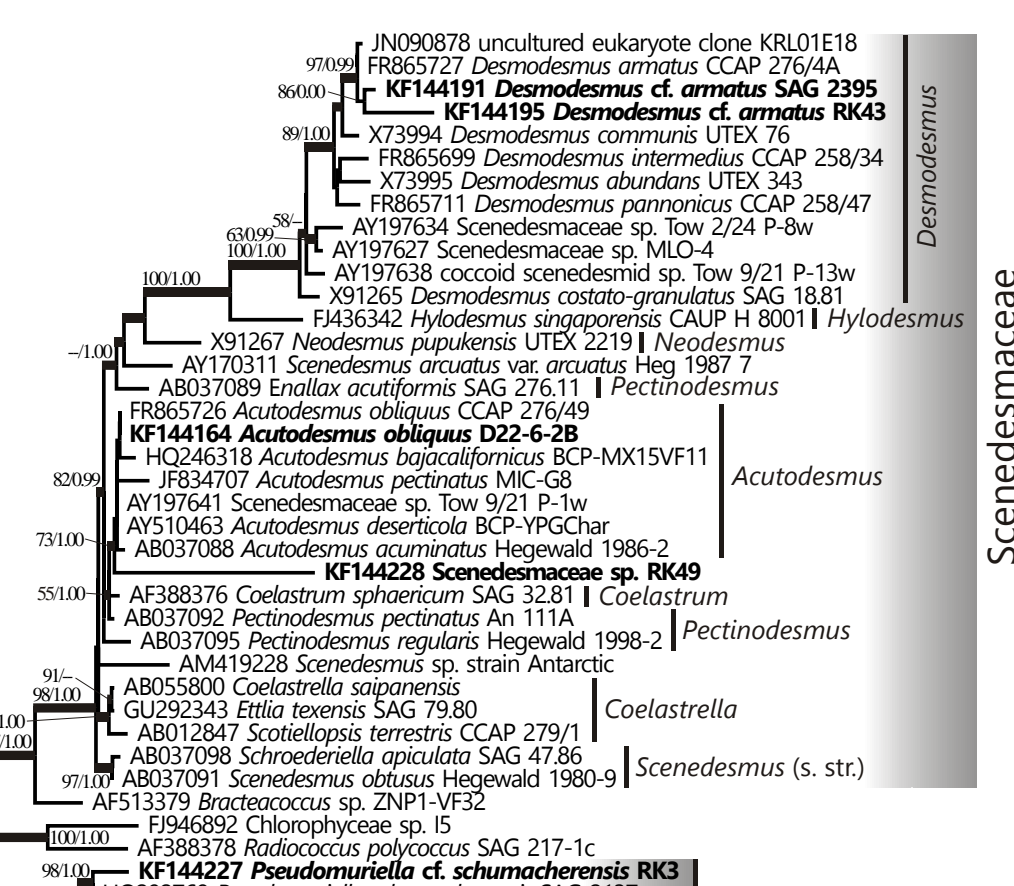

97099 JN090878 uncultured eukaryote clone KRL01E18 861000 - KF144191 Desmodesmus of armatus SAG 2395 KF144195 Desmodesmus cf. arme

X7399. Desmodesmus commis UTEX 76

- X73995 Desmodesmus intermedius CCAP 258/34

FR865711 Desmodesmus pannonicus CCAP

58/- - AY197634 Scenedesmaceae sp. Tow 2/24 P-8w

9/21 P-13w 


\section{Diversity of Ulvophyceae}

Five species from creek biofilms were members of the Ulvophyceae. Interestingly, out of them four were of pseudo-filamentous organization, i.e. formed only short filaments and also coccoid stages, whereas only one exhibited a purely coccoid vegetative stage. This was in contrast to our isolates of Trebouxiophyceae and Chlorophyceae which were all of coccoid or monadoid organization. The pseudo-filamentous isolates exhibited characteristic cup-shaped chloroplasts with one or two prominent pyrenoids (Figs. 7h-i). Three filamentous species were members of the order Ulotrichales, one belonged together with the coccoid isolate to the Ulvales (Fig. 4). Filamentous strain SAG 2396 (Fig. 7e) had a rather small genetic distance to Hazenia mirabilis UTEX LB 846 (acc. no. AF387156) and, therefore, was assigned to this species (Fig. 4.) Pseudendoclonium basiliense UTEX 2593 and two species of Gloeotilopsis formed up a clade with the former two strains which, however, received only insignificant support values (Fig. 4). Another pseudo-filamentous species we recovered was Pseudendocloniopsis botryoides; it was with nine isolates (with identical partial sequences and morphology) the second most frequently retrieved green algal species (Table S1). As represented by isolate SAG 2394 the close relationship with two available strains of Pseudendocloniopsis botryoides was well supported and, therefore, SAG 2394 was assigned to this species (Fig. 4). SAG 2394 may also form coccoid stages (Fig. 7f), a feature shared with Planophila laetevirens SAG 2008 which also was its next closest relative in the $18 \mathrm{~S}$ rRNA gene phylogeny (Fig. 4). Morphology of P. botryoides was studied at isolate DB6-19 in more detail (Fig. 7g; Table S2). The filamentous isolate SAG 2404 (Fig. 7h) was a very closely relative to Pseudendoclonium akinetum UTEX 1912 and also two species of Trichosarcina were their next closest relatives, but these relationships did not receive significant support values (Fig. 4). Finally, for another pseudo-filamentous species, Dilabifilium printzii, a total of seven isolates was established (Table S1); it was the third most frequently recovered green algal species in our study. Unfortunately, we failed to obtain an almost full $18 \mathrm{~S}$ rRNA gene sequence for these isolates. The partial sequence of isolate WB41 shared 99.9\% similarity with the sequence (acc. no. KF144198) of D. printzii strain SAG 467-1 and, therefore, we assigned our isolate to this species. Morphological features of isolate WB41 are presented in Fig. 7i and Table S2. D. printzii SAG 467-1 had an independent position within the clade representing the Ulvales in our 18S rRNA gene phylogeny (Fig. 4). The single coccoid Ulvophyceae isolate SAG 2397 clearly fell within a subclade of the Ulvales comprising the coccoid genera Chlorocystis, Desmochloris and Halochlorococcum (Fig. 4). Strain SAG 2397 had short genetic distances with two strains of Desmochloris halophila and, therefore, we 
provisionally assigned our isolate to the genus Desmochloris. However, a close relationship of strain SAG 2397 with both D. halophila strains was not supported in significance tests and Desmochloris was paraphyletic in our 18S rRNA gene phylogeny (Fig. 4). Consequently, assignment of strain SAG 2397 to a certain species within the subclade of Ulvales remained uncertain.

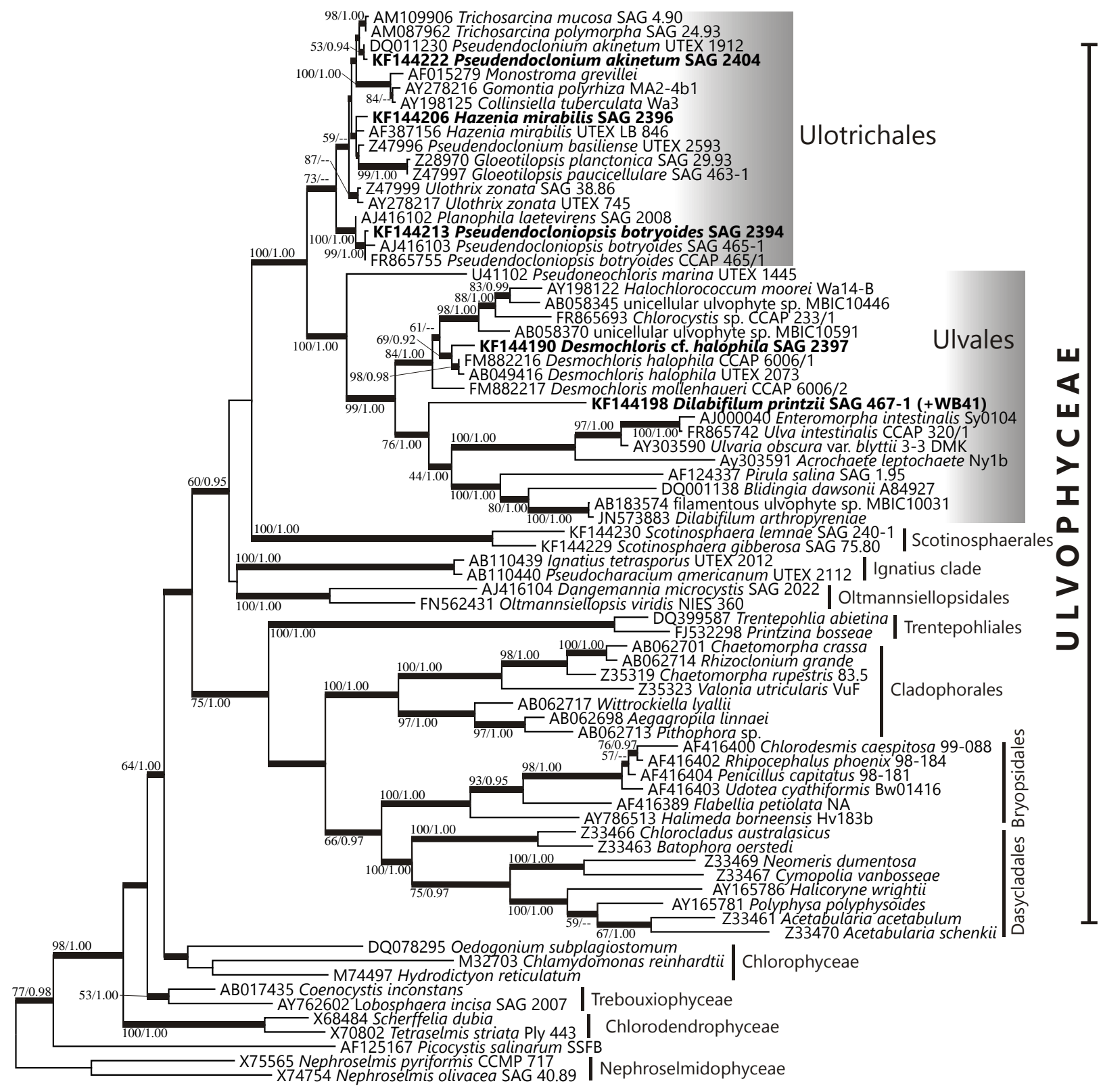

Figure 4. Maximum-likelihood (ML) phylogeny of $18 \mathrm{~S}$ rRNA gene sequences of green algal species from the class Ulvophyceae, isolated from biofilms of two freshwater creeks (highlighted sequence names) and other members of the Ulvophyceae as references. Additional members of other classes and lineages of the Chlorophyta are included for comparisons. The phylogeny was rooted with two members of the Nephroselmidophyceae. Numbers at internal branches indicate support from bootstrap tests using ML (left) and posterior probabilities from Bayesian analysis (Bl; right). Thick lines mark internal branches that were resolved by both, the $\mathrm{ML}$ and the $\mathrm{BI}$ tree topologies. Grey boxes highlight the clades comprising the new isolates. 

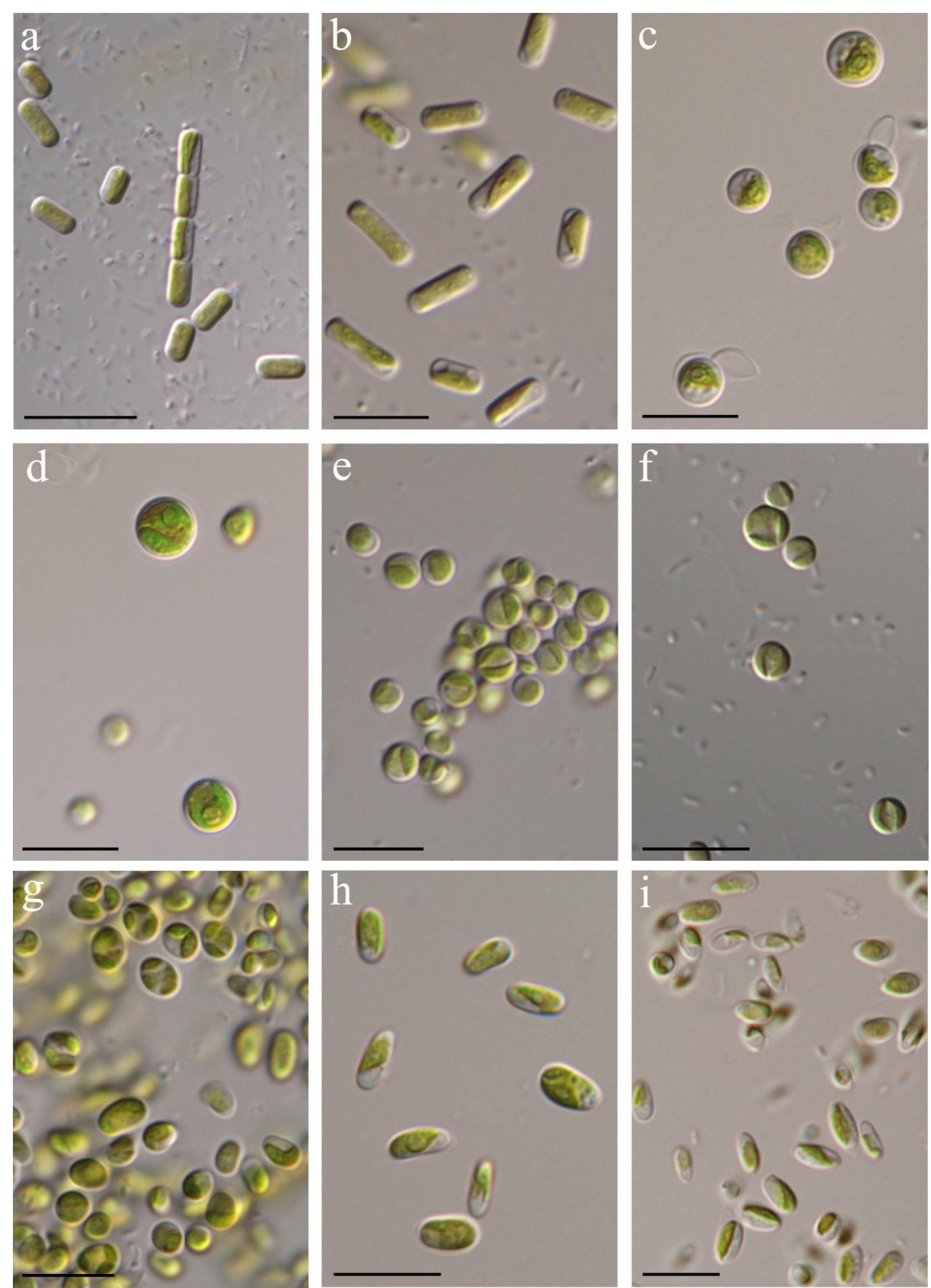

Figure 5. Morphology of green algal isolates of the class Trebouxiophyceae. Stichococcus bacillaris isolate WB74 (a), Stichococcus sp.4 SAG 2406 (b), Chlorella sp. RK52 (c), Chlorella sp. D11-2 (d), Muriella terrestris D6-DB2 (e), Marvania sp. WB67 (f), Neocystis cf. mucosa SAG 2405 (g), Elliptochloris subsphaerica WB5-D1e (h), Coccomyxa cf. pringsheimii WB32 (i). Scale bars $=10 \mu \mathrm{m}$. 

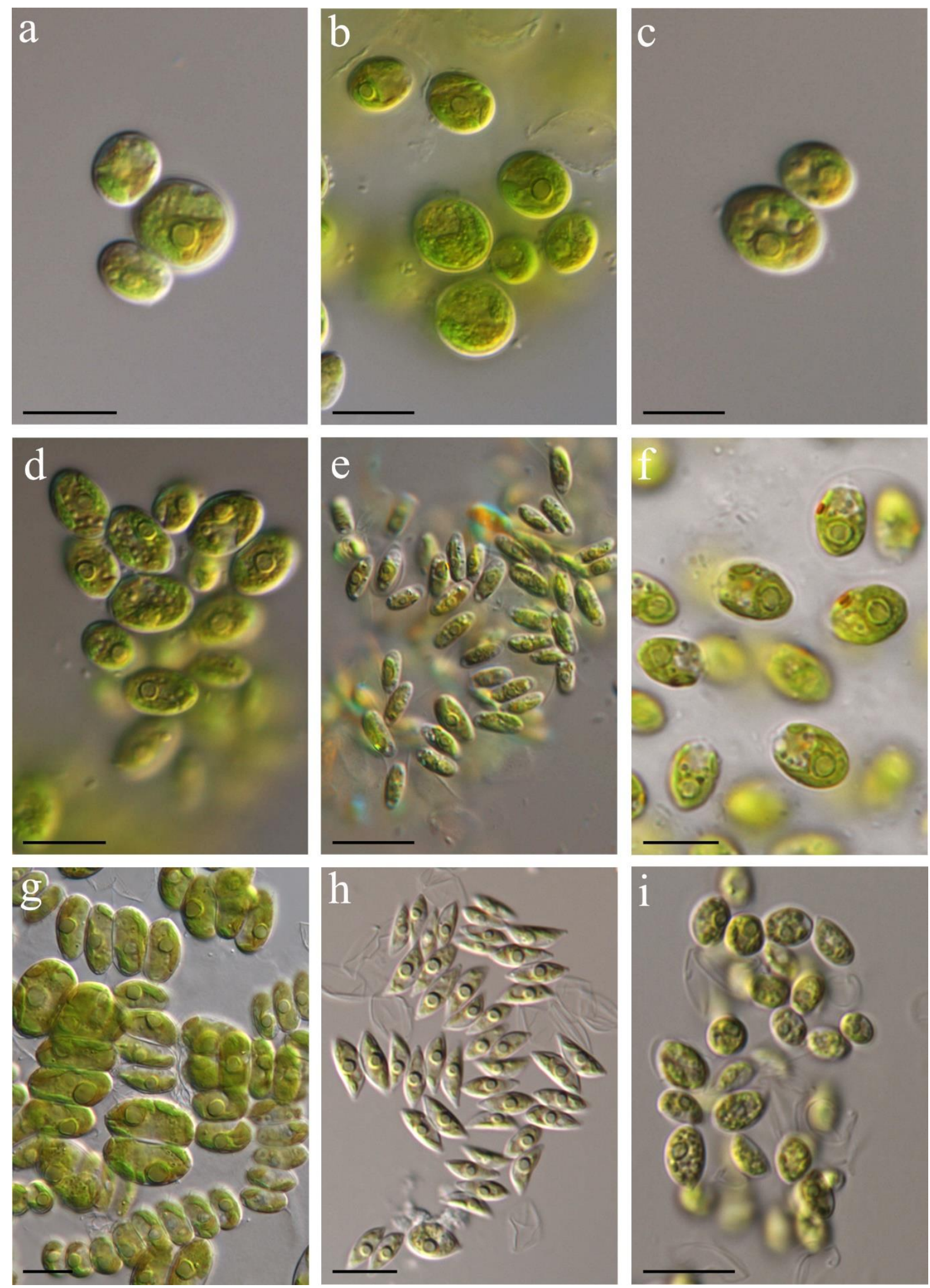

Figure 6. Morphology of green algal isolates of the class Chlorophyceae. Chlamydomonadales (a) - (f); Sphaeropleales (g) (h). Chlorococcum sphacosum SAG 2398 (a), Chlorococcum minutum-relative SAG 2399 (b), Chlorococcum ellipsoideumrelative1 GRK6-DB5 (c), Chlorococcum ellipsoideum-relative2 SAG 2400 (d), Chlorococcum ellipsoideum-relative2 GRK6-DB6, zoospores (e), Chlamydomonas sp. isolate RK68 (f), Desmodesmus cf. armatus isolate RK43 (g), Acutodesmus obliquus isolate D22-6-2B (h), Scenedesmaceae sp. isolate RK49 (i). Scale bars $=10 \mu \mathrm{m}$. 

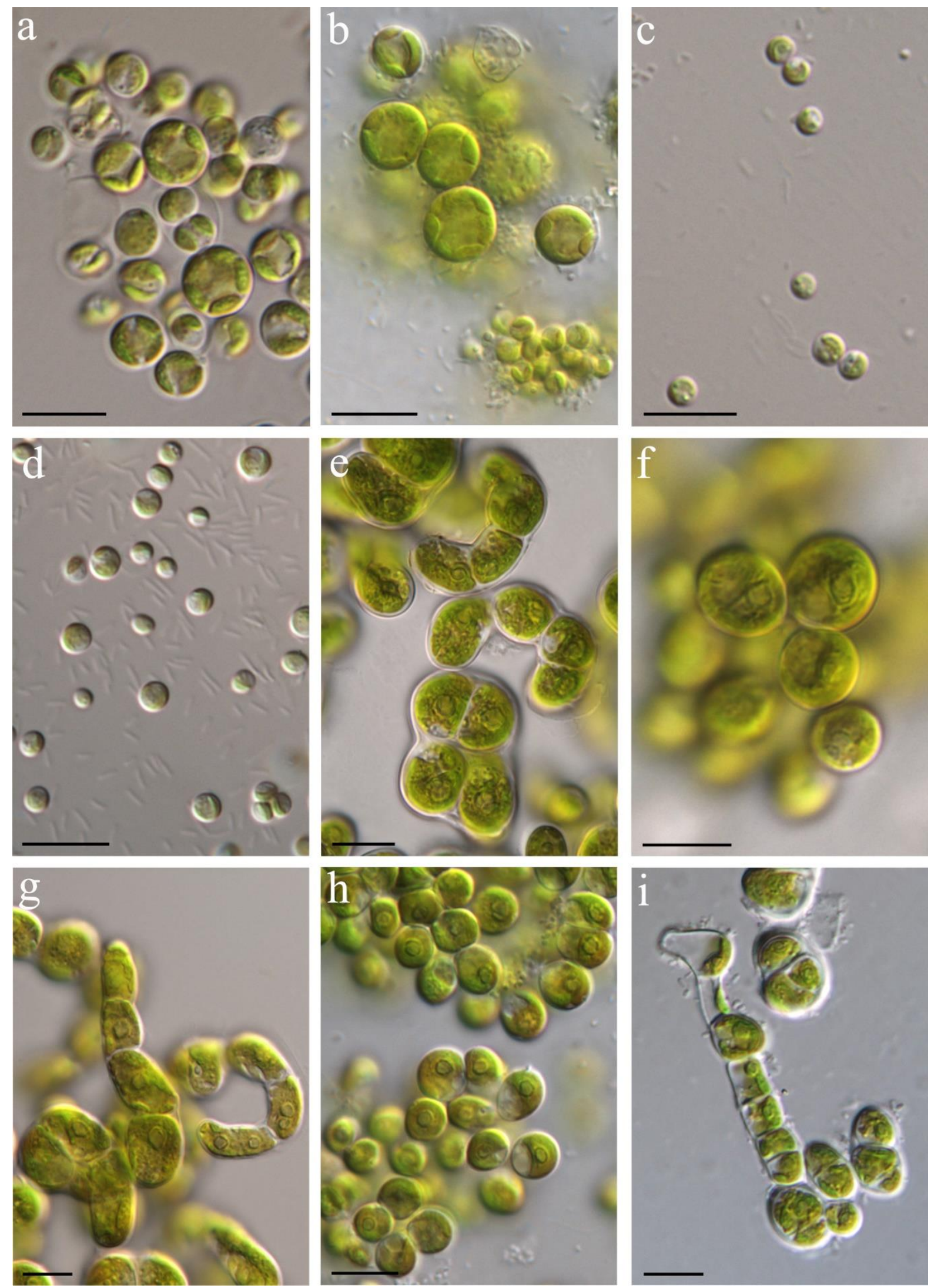

Figure 7. Morphology of green algal isolates of the classes Chlorophyceae and Ulvophyceae isolates. Chlorophyceae, Sphaeropleales (a) - (d); Ulvophyceae (e) - (i). Pseudomuriella cf. schumacherensis RK3 (a), Bracteacoccus sp. DB9-3 (b), Mychonastes cf. homosphaera RK48 (c), Mychonastes sp. isolate DB6-29 (d), Hazenia mirabilis strain SAG 2396 (e), Pseudendocloniopsis botryoides SAG 2394, coccoid stage (f), Pseudendocloniopsis botryoides DB6-19, filamentous stage (g), Pseudendoclonium akinetum SAG 2404 (h), Dilabifilum printzii isolate WB41 (i). Scale bars $=10 \mu \mathrm{m}$. 


\section{Discussion}

\section{Distribution of the isolates at the sampling sites}

In total, we detected nearly the same numbers of green algal species in the Deinschwanger (DB; 21) and Westerhöfer (WB; 18) creeks, regardless of the unequal number of sites sampled per creek (8 at the DB, 5 at the WB; Table 1). With respect to calcification the various sampling sites of the DB did not cluster according to high or low calcification when using their species compositions (Fig. 8). We conclude that we observed a rather accidental distribution of the biofilm microalgae which is not or only very little influenced by calcification. This is contrast to findings of Brinkmann et al. (2015) (this issue) who reported the diversity of cyanobacteria and diatoms of the same creek biofilms as studied here was clearly influenced by $\mathrm{SI}_{\text {calcite }}$ and $p \mathrm{CO}_{2}$ which are reciprocally linked. The highest diversity per site (11 species) was detected at site DB6 which was highly calcified (Table 1). However, from the other calcified sampling sites (DB1DB5) only 1-3 species per site could be retrieved in culture. At the spring site DBS with calcification almost absent also just four species were recovered. Two of them, Coccomyxa cf. pringsheimii and Dilabifilum printzii were retrieved only at the DB spring site, but D. printzii was recovered from the highly-calcified sites of the WB as well (Table 1). In addition, we compared the various DB sites for their "phylogenetic diversity", i.e. whether the species recovered from a certain DB site belong to a single or several phylogenetically distinct clades. With respect to the number of phylogenetic clades retrieved per site, DB6 again was the most diverse site and the spring site DBS was the second most diverse site (Table 2). The Chlamydomonadales clade (Chlorophyceae; Fig. 2) seemed to have a preference towards the highly calcified DB sites, whereas the Chlorellales (Trebouxiophyceae; Fig. 1) and the

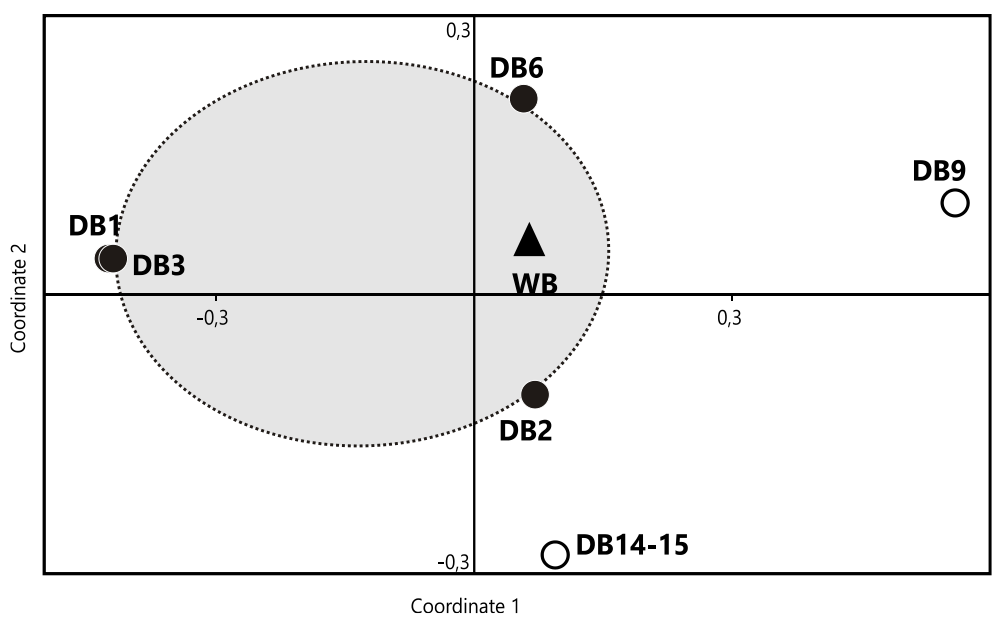

Figure 8. Ordination diagram. Nonmetric Multidimensional Scaling (NMDS) plot showing the grouping of high-calcified sampling sites (black symbols) based on presence/absence of the green algal lineages.
Ulotrichales (Ulvophyceae; Fig. 4) clades were found at the highly calcified sites as well as the spring site of the DB (Table 2). With respect to the three different green algal classes detected in both karst-water creeks, the WB was dominated by members of Trebouxiophyceae (12 out of the total of 18 species detected there belonged to Trebouxiophyceae), 


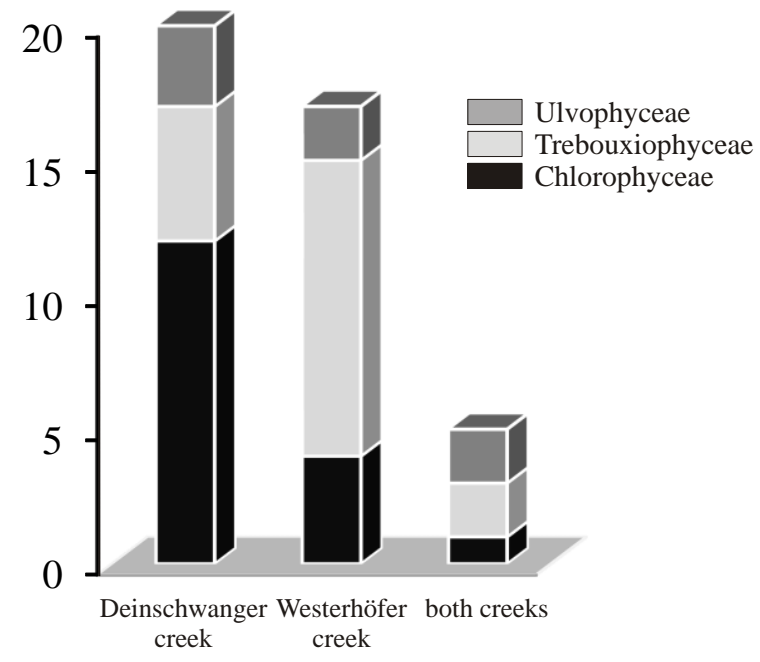

Figure 9.Bar chart showing the relative representation of the three classes of Chlorophyta at the both investigated creeks. Black, Chlorophyceae; light grey, Trebouxiophyceae; dark grey, Ulvophyceae. whereas the DB predominately harbored members of Chlorophyceae (12 out of the total of 21 species detected there belonged to Chlorophyceae). For the few members of Ulvophyceae no preference to any of the both creeks was found. Interestingly, out of the 34 detected green algal species only six were retrieved from both creeks, i.e. Coccomyxa cf. pringsheimii, Muriella terrestris and Stichococcus sp.1 of the Trebouxiophyceae, Chlorococcum ellipsoideum-relative2 of the Chlorophyceae, and Dilabifilum printzii and Pseudendocloniopsis botryoides of the Ulvophyceae (Fig. 9). It follows that the species richness of green algae at both creeks was rather different (Fig. 9).

\section{Species identification and origins}

The molecular phylogenetic diversity of inland freshwater green algae from running waters has been studied for the first time here. In particular, for estimating the diversity of microscopic green algae in epilithic biofilms of running freshwaters, studies are rare and all were exclusively based on morphology so far (for review see Lindstrøm et al. (2004) and Veselá (2006)). The majority of existing evidence about the molecular diversity of freshwater green microalgae refers to communities rather different from algal biofilms, e.g. phytoplankton (Fawley et al. 2004; Krienitz and Bock 2012). Other molecular data for the diversity of green algae from of running freshwaters have mainly been available from habitats with extreme environmental variables or artificial impact (e.g., Dorigo et al. 2002; Aguilera et al. 2007; Palacios et al. 2008; Baker et al. 2009; Aguilera et al. 2010). For example, molecular data are available for the periphyton of the extremely cold and remote Antarctica (De Wever et al. 2009; Vyverman et al. 2010). Compared to freshwater creeks, terrestrial habitats have more extensively been studied by a combined morphological/molecular approach to date (Lewis and Lewis 2005; Flechtner et al. 2013).

Sequence comparisons of $18 \mathrm{~S}$ rRNA genes enable the unambiguous comparisons of new isolates to those previously isolated from other localities and habitats world-wide (De Wever et al. 2009; Němcová et al. 2011; Hodač et al. 2012). Using sequence comparisons most of our 
isolates were closely related to those of various other inland freshwaters (Table S3). Within the Trebouxiophyceae, these were members of the Chlorellales clade, i.e. Chlorella (Bock et al. 2010; Bock et al. 2011), Muriella (Fawley et al. 2004) and Marvania (Fawley et al. 2004; Yamamoto et al. 2007). Within the Chlorophyceae, at least the Scenedesmaceae, Selenastraceae and Mychonastes, which we also retrieved in our study, are well known from freshwaters (Krienitz et al. 2011; Krienitz and Bock 2012). However, all these genera and families of green algae are known predominantly from major water bodies like lakes or ponds where they inhabit free-floating communities (phytoplankton) and, therefore, were not expected to occur in biofilm assemblages as well. While Trebouxiophyceae and many Chlorophyceae are predominately found in terrestrial and freshwater habitats, Ulvophyceae is a green algal class with a main divergence in marine habitats; it represents the only lineage of Chlorophyta that includes macroscopic sea-weeds (Leliaert et al. 2012). In contrast, our isolates were nested within the orders Ulotrichales and Ulvales which are known to occur also in freshwaters and terrestrial habitats as well. Interestingly, the characteristically branched filamentous thalli of the majority of our ulvophycean isolates strongly resembles the morphology of Gongrosira which has frequently been reported from tufa formations (Pentecost 1988; Pentecost and Spiro 1990; Freytet and Plet 1991; Johnson and John 1992). Therefore, the name Gongrosira may represent a collective morphotype whose members are distributed at least on several distant lineages within the Ulotrichales (Ulvophyceae). Moreover, the only so far sequenced strain of Gongrosira, G. papuasica UTEX 1916, has been identified as a member of Chlorophyceae (López-Bautista and Chapman 2003).

Using 18S rRNA gene sequence comparisons only for six out of the total of 34 species retrieved in our study had $100 \%$ and $99.9 \%$ identities with already available sequences from reference strains (Table 1, Table S3). Therefore, we suspect to have retrieved the same species as previous studies in these cases. However, for none of these species authentic strains were available and, therefore, our identification (species names) may be only as correct as the closest named neighbors (reference strains) of our isolates have been correctly named to species. Except for two reference strains where their origin is not known and Elliptochloris subsphaerica, all reference strains for these species have been isolated from various freshwater habitats (Table S3) and from a broad range of geographical regions, e.g. Europe, United States and Antarctica. Consequently, our findings support the world-wide distribution of these species. However, it is important to note that most of these freshwater species have been reported from open water bodies as phytoplankton so far, but are recovered by our study from submerged biofilms attached to stones in running waters for the first time. In other cases closest relatives to our isolates were 
from terrestrial habitats. Our finding of E. subsphaerica is the first record of this species from a freshwater habitat; previously it has been reported only from terrestrial habitats (Ettl and Gärtner 1995). Similarly, our still unidentified isolates of Stichococcus, WB38 (S. cf. deasonii), SAG 2408 (S. sp.3) and SAG 2406 (S. sp.4), have their closest relatives from terrestrial habitats. Species of Coccomyxa have mostly been reported from terrestrial habitats (e.g. tree bark or lichen symbiosis) so far, but were recovered from creek biofilm in our study. The latter three examples indicate that at least parts of the creek biofilm algal communities were probably driven into their habitat through (rain-) flushes from the neighboring soils. For most other biofilm species which so far were reported from phytoplankton only it is still not known whether they can be found in terrestrial habitats as well. Muriella terrestris may be an example for a green microalgal species that is known from phytoplankton as well as soil; it was frequently found in the creek biofilms as well.

For 24 species we recovered no identification at the species level was possible, i.e. they were left unnamed despite they could be assigned to a certain genus in most cases. These represent species for which no 18S rRNA gene sequence have become available yet or which have been retrieved for the first time. In this respect we see the importance of molecular-phylogenetic investigations of inland freshwater algal biodiversity, i.e. to extend our knowledge about the distribution of species of green microalgae across various freshwater and terrestrial habitats.

\section{Acknowledgements}

We are indebted to Dr. Anke Behnke and Ms.Viviane Kipp who initiated this study through their culturing efforts and developed green algal PCR primer LR1650. This work was supported by the German Science Foundation (DFG) by a grant extended to T.F. (Fr 905/13-2). Parts of this work were also supported by the German Federal Ministry of Education and Research, BMBF (AlgaTerra project, grant 01 LC 0026) within the BIOLOG program. 


\title{
Chapter 3
}

\section{Phylogenetic analysis of polar Chlorella and Stichococcus suggests biogeography of airborne microalgae}

\author{
Ladislav Hodač ${ }^{1}$, Christine Hallmann ${ }^{1}$, Karolin Spitzer ${ }^{1}$, Josef Elster ${ }^{2,3}$, Fabian Faßhauer ${ }^{1}$, Nicole Brinkmann ${ }^{4}$, \\ Daniela Lepka ${ }^{1}$, Vaibhav Diwan ${ }^{1}$ and Thomas Friedl ${ }^{1}$
}

\author{
${ }^{1}$ Experimental Phycology and Culture Collection of Algae (SAG), University of Göttingen, Germany \\ ${ }^{2}$ Centre for Polar Ecology, University of South Bohemia, České Budějovice, Czech Republic \\ ${ }^{3}$ Institute of Botany, Phycology Centrum, Academy of Sciences of the Czech Republic, Třeboň, Czech Republic \\ ${ }^{4}$ Department of Forest Botany, University of Göttingen, Germany
}

(Submitted to FEMS Microbiology Ecology; FEMSEC-15-11-0606)

\begin{abstract}
Chlorella and Stichococcus are morphologically simple airborne microalgae, omnipresent in terrestrial and aquatic habitats. The minute cell size and resistance against environmental stress facilitate their long-distance dispersal. However, the actual distribution of Chlorella- and Stichococcus-like species was so far inferred from ambiguous morphology-based evidence. Here we contribute a phylogenetic analysis of an expanded SSU and ITS2 rDNA dataset including new accessions from terrestrial habitats of polar regions, the temperate zone and the tropics in order to determine whether related species of airborne microalgae show similar patterns in geographic distribution. We found that psychrotolerant strains of Chlorella and Stichococcus are without exception conspecific (or closely related) with strains originating from the temperate zone. Seven phylogenetic species of polar Chlorellaceae have been uncovered so far with five of them being our new psychrotolerant strains. Chlorella vulgaris and Muriella terrestris show the widest distribution, including polar and hot desert environments. The morphologically more diversified Stichococcus-like lineages were so far detected not only in polar regions and hot deserts, but also in tropical rainforests. However, distinct Stichococcus clades exhibit either temperate-polar or temperate-tropical distributions. Our data suggest that terrestrial airborne microalgae might exhibit biogeography and are not distributed everywhere.
\end{abstract}

Keywords: biogeography, Chlorella, Stichococcus, polar strains 


\section{Introduction}

Terrestrial species of Chlorella (Beijerinck 1893) and Stichococcus (Nägeli 1849) are true survivalists among the green microalgae (Chlorophyta). They inhabit biofilms covering natural and artificial subaerial substrates and dwell in soils (Carson and Brown 1976; 1978; Ettl and Gärtner 1995; Sharma et al. 2007; Rindi et al. 2009). Chlorella and Stichococcus have been reported by microscopical observations from nearly all soil types, including desert soil crusts in Namibia (Büdel et al. 2009), humid tropical soils of India (Ray and Thomas 2012), Central America (Archibald 1972), Oceania (Arvik and Willson 1974; MacEntee et al. 1977; Carson and Brown 1978) and polar desert soils in Antarctica (Cavacini 2001; Fermani et al. 2007) and the Arctic (Elster et al. 1999; Kaštovská et al. 2005; Kaštovská et al. 2007; Patova and Dorokhova 2008). Langhans et al. (2009) recognized species of Chlorella and Stichococcus as key players for monitoring the succession of biological soil crust formation. Particular attention has been paid to the metabolic facilities of psychrotolerant terrestrial strains of Chlorella vulgaris and Stichococcus bacillaris (Kvíderová and Lukavský 2005; Shukla et al. 2011; Chen et al. 2012; Hong et al. 2015); both species include strains with the potential for use in biotechnology (Lang et al. 2011; Olivieri et al. 2011; Cadoret et al. 2012; Barreiro et al. 2013; Olivieri et al. 2013; Goiris et al. 2014; Mudimu et al. 2014; Safi et al. 2014; Sivakumar et al. 2014; Slocombe et al. 2015). The resistance against environmental stresses connected with metabolic versatility is a common feature of polar microalgae, which have to cope with low temperatures and shortages of nutrients and liquid water (Elster 1999; Elster and Benson 2004; Kvíderová and Lukavský 2005; Sharma et al. 2007). An extreme multi-stress resistance was detected in a psychrotolerant cryptoendolithic strain of Stichococcus (isolated from Antarctica), which survived an experimental exposure to an Earth orbital space environment (Scalzi et al. 2012).

Systematically, 'Chlorella' is the name for minute coccoid microalgae phylogenetically nested within the Chlorellaceae clade, class Trebouxiophyceae (Huss et al. 2002; Krienitz et al. 2003; Luo et al. 2010; Pröschold et al. 2010; Leliaert et al. 2012; Krienitz et al. 2015). Whereas planktonic Chlorellaceae evolved into distinct forms, e.g., Micractinium (Pröschold et al. 2010; Krienitz and Bock 2012) or Actinastrum (Luo et al. 2010; Krienitz and Bock 2012), terrestrial members exhibit morphological convergence, which is characteristic for "true" Chlorella (Chlorella clade, Bock et al. 2011) and for multiple Nannochloris-like clades (Henley et al. 2004). Morphologically simple Chlorella- and Nannochloris-like species were repeatedly uncovered in the Antarctic (Gilichinsky et al. 2007; Hu et al. 2008; De Wever et al. 2009). 
'Stichococcus' can be as well encountered in the harsh terrestrial and freshwater environments of the Antarctic and Arctic (De Wever et al. 2009; Vishnivetskaya 2009; Khan et al. 2011).

'Stichococcus' is the common name for multiple rod-shaped species phylogenetically nested within the Prasiola clade (Handa et al. 2003; Neustupa et al. 2007; Novis et al. 2008; Eliáš and Neustupa 2009). The Prasiola clade represents a trebouxiophyte lineage with the highest morphological diversity within the class (Leliaert et al. 2012), which is widely distributed in freshwaters, marine and terrestrial ecosystems (Karsten et al. 2005; Rindi et al. 2007). Despite their morphological simplicity, Stichococcus species veil a considerable phylogenetic diversity, which is still in need of taxonomic revision (Neustupa et al. 2007; Sluiman and Guihal 2008; Karbovska and Kostikov 2012a). The rod-shaped Stichococcus species are scattered across the whole Prasiola clade, but are not intermixed with morphologically distinct lineages such as Pseudomarvania (Eliáš and Neustupa 2009) or the thallous Prasiolales (Handa et al. 2003; Rindi et al. 2004; Rindi et al. 2007). SSU-based indications, that the Prasiola clade and the Chlorellaceae might represent sister lineages (Handa et al. 2003; Fučíková et al. 2014), appear to be questionable, while the Prasiola clade is undoubtedly nested within the core Trebouxiophyceae (Leliaert et al. 2012; Lemieux et al. 2014; Turmel et al. 2015), plastid genome phylogenies place Chlorellaceae outside the core Trebouxiophyceae (Lemieux et al. 2014; Leliaert and Lopez-Bautista 2015; Turmel et al. 2015).

Chlorella, Stichococcus and a handful of other green algal morphospecies are counted among the so called airborne algae (Sharma et al. 2007; Sharma and Rai 2010), which are supposed to be ubiquitous in terrestrial ecosystems (Rindi et al. 2009; Rindi et al. 2011). However, the existence of limitedly dispersed cryptic species which are morphologically hard to determine, became the rule since the introduction of molecular techniques into phycology (Boenigk et al. 2005; Rindi et al. 2008; Dal Grande et al. 2014; Ř́dká et al. 2014; Ryšánek et al. 2014; Škaloud et al. 2014a; Škaloud et al. 2015). Nevertheless, some species might be true cosmopolitans, as recently affirmed within some lineages of terrestrial green microalgae, e.g., Coccomyxa (Darienko et al. 2015), Klebsormidium (Ryšánek et al. 2014) and Diplosphaera (Fontaine et al. 2012). Remote polar regions like Antarctica and the Arctic provide an opportunity for investigating isolation-by-distance and speciation of microorganisms (Martiny et al. 2006; Hahn et al. 2015). Pioneering molecular studies on algal diversity in Antarctica found genetic divergence between polar and non-polar species; some detected Chlorella and Stichococcus species were supposed to be Antarctic endemics (Lawley et al. 2004; De Wever et al. 2009). Some five years later, the amount of sequenced algal strains and environmental clones has dramatically increased, encouraging us to reconsider whether the Antarctic endemism might be 
simply due to a sampling effect. We avoid the difficulty of the algal species concept (Leliaert $e t$ al. 2012; Leliaert et al. 2014); instead, we infer distribution patterns based on monophyletic clades of closely related sequences. By focusing on the polar strains of Chlorella and Stichococcus, we aim to show that related species of airborne microalgae have similar patterns of geographic distribution.

\section{Materials and Methods}

\section{Data sampling and microscopy of the strains}

We analyzed new SSU and ITS2 rDNA sequences obtained from the strains, isolates and clones listed in Table S1 (Chlorella-like sequences, accession numbers XY000000-XY000000) and Table S2 (Stichococcus-like sequences, accession numbers XY000000-XY000000). The geographic origin of new sequences from polar/temperate/tropical regions is shown in Fig. 1. Isolation of the algal strains and sequencing were described in Hodač et al. (2015) while PCRcloning of the algal sequences from environmental samples was described in Hallmann et al. (2013a). Observations of the cultures were conducted using an Olympus BX60 microscope (Tokyo, Japan) with Nomarski DIC optics and an attached ColorView III camera (Soft Imaging System, Münster, Germany). Cell size measurements (diameter of spherical cells; length/width of elongated cells) were conducted in ImageJ (Schneider et al. 2012) and were based on a set of 50-100 cells per culture.

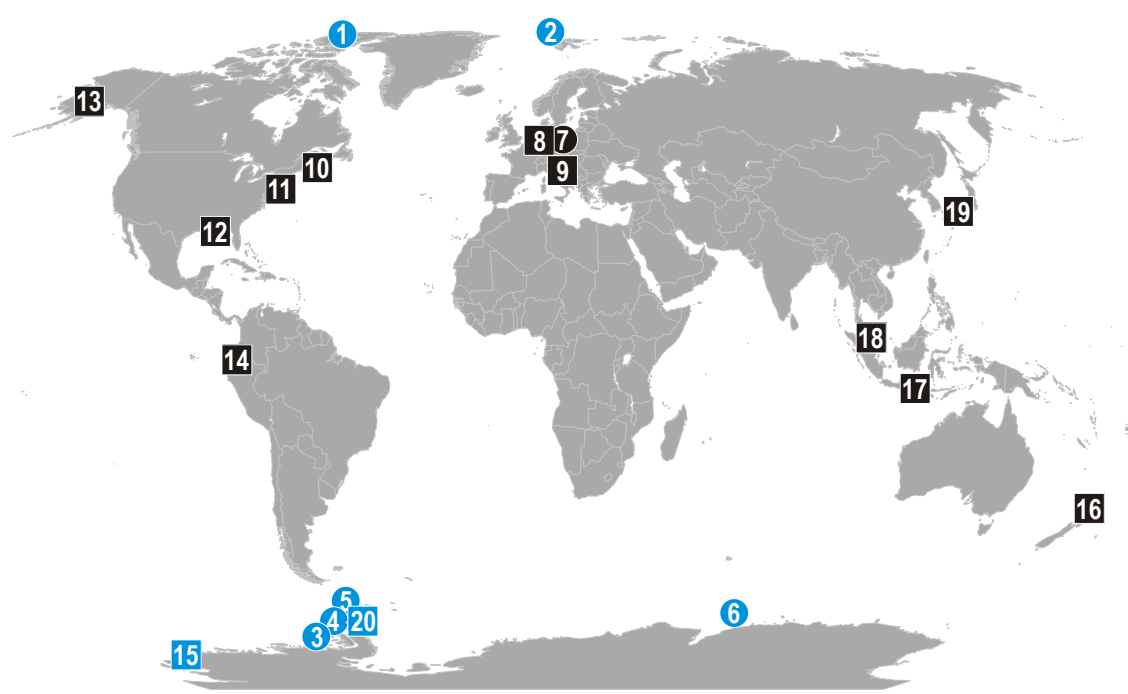

Figure 1. Geographic origin of the new Chlorella and Stichococcus 18S and/or ITS2 sequences. 1-5: Chlorella-like accessions (circles); 1-Canada (Ellesmere Island), 2-Svalbard, 3-Antarctica (Killingbeck Island), 4-Antarctica (Adelaide Island), 5-Antarctica (King George Island), 6-Antarctica (Anchorage Island), 7-German Biodiversity Exploratories. 8-20: Stichococcus-like accessions (squares); 8-German Biodiversity Exploratories, 9-Croatia, 10-Canada (New Scotia), 11-USA (Massachusetts), 12-USA (Alabama, Dauphin Island), 13-USA (Alaska), 14-Ecuador (Podocarpus National Park), 15-Antarctica (Victoria Land), 16-New Zealand (Waweira Scenic Res.), 17-Indonesia (Bali, Lake Bratan), 18-Malaysia (Kampong Kuala Jenera), 19-Japan (Taishakukyo Gorge), 20-Antarctica (Marie Bird Land). Blue symbols represent polar accessions. Detailed information on sampling sites is listed in Tables S1, S2. 


\section{S rDNA phylogenetic analyses}

The closest relative sequences to the new accessions were searched for in GenBank using the BLAST algorithm (Altschul et al. 1997). New and the GenBank accessions were checked for chimeras using Bellerophon (Huber et al. 2004). Two separate 18S rDNA alignments were created, one for Stichococcus-like accessions (Prasiola clade) and one for Chlorella-like (Chlorellaceae) accessions. Both 18S rDNA sequence alignments were computed using MAFFT v.6 (Katoh and Toh 2008) available online. The aligned sequences were checked for possible misaligned positions in BioEdit 7.0.9.0 (Hall 1999). The 18S rDNA alignment, which included the new Stichococcus accessions and the closest relatives from GenBank (Prasiola clade), contained 100 sequences/1709 positions (276 variable, 149 parsimony informative). Based on the AIC criterion in jModelTest 0.1.1 (Posada 2008), the GTR $+\Gamma+\mathrm{I}$ nucleotide substitution model was selected as the best fitting the Prasiola clade. A maximum likelihood phylogeny was computed in RAxML 7.0.4 (Stamatakis et al. 2008) under the proposed model and statistical support values were derived from rapid bootstrapping (1000 replicates) in the same program. An alternative maximum likelihood tree search procedure, i.e., quartet puzzling, was applied for the same 18S alignment using Tree-Puzzle 5.2 (Strimmer and Von Haeseler 1996) available at the Mobyle-Pasteur webserver (Néron et al. 2009). For this, the GTR $+\Gamma$ substitution model was applied. To assess the putative age of dichotomies within the Prasiola clade, relative node ages were estimated using BEAST v1.8.2 (Drummond and Rambaut 2007; Drummond et al. 2012) with 5000000 steps (after 500000 burn-in) under the relaxed molecular clock option. Absolute ages were estimated following De Wever et al. (2009) by setting the minimum and maximum age of the Chlorophyta-Streptophyta split at 700 and $1500 \mathrm{Ma}$. A tree was visualized using FigTree (Rambaut 2007). The 18S rDNA alignment of Chlorellaceae comprised 82 sequences/1668 positions (350 variable, 226 parsimony informative). The J2:G:5 substitution model was proposed by Treefinder (Jobb et al. 2004), which was subsequently used for maximum-likelihood tree reconstruction. Confidence values were inferred from 1000 bootstrap replicates in the same program. The resulting tree was visualized using MEGA5 (Tamura et al. 2011). For additional statistical support, Bayesian posterior probabilities were computed in MrBayes 3.2.1 x64 (Ronquist et al. 2012). We carried out two MCMC runs for three million generations each with one cold and three heated chains under the GTR $+\Gamma+\mathrm{I}$ evolutionary model (parameters were estimated from the data); trees were sampled every 100 generations. To summarize the genetic similarities within the alignments, the sequences were clustered into operational taxonomic units (OTUs) in the program MOTHUR v.1.13.0 (Schloss et al. 2009) implementing the 0.00 and 0.01 thresholds under exclusion of the gap positions. For sequence comparisons, p-distances were 
computed in MEGA6 (Tamura et al. 2013).

\section{ITS2 rDNA phylogeny and secondary structure analysis}

First, 63 new ITS2 rDNA sequences were submitted to the BLAST search (Altschul et al. 1997) in order to obtain their closest relatives for phylogenetic comparison. Subsequently, an online ITS2 database (Schultz et al. 2006; Selig et al. 2008; Koetschan et al. 2010; Koetschan et al. 2012) was used for ITS2 rRNA annotation (Keller et al. 2009). Minimum energy secondary structure models were computed from the annotated sequences using RNAstructure 5.3 (Reuter and Mathews 2010). For very closely related sequences, a few were folded in RNAstructure and the rest modelled using homology modelling in the ITS2 database (Wolf et al. 2005). Ambiguous models were compared to those computed in the mfold webserver (Zuker 2003). The ITS2 rRNA secondary structures were visualized by Varna 3.8 (Darty et al. 2009). Subsequently, two separate alignments of Chlorella vulgaris and Stichococcus-like sequences and structures were built using 4SALE 1.7. (Seibel et al. 2006; Seibel et al. 2008). In the same program, compensatory base changes (CBC) were computed between pairs of sequences. The Chlorellaceae ITS2 alignment contained 39 sequences/321 positions (58 variable, 39 parsimony informative). A statistical parsimony analysis was conducted with the very closely related ITS2 sequences of Chlorella vulgaris and C. pituita using TCS v.1.21 (Clement et al. 2000) to generate ribotype networks. The connection limit was set to $95 \%$. The Prasiola clade ITS2 alignment contained 56 sequences/584 positions (252 variable, 189 parsimony informative). Based on sequence-structure alignment, a neighbor-joining tree was computed in ProfDist (Müller et al. 2004; Friedrich et al. 2005; Rahmann et al. 2006; Wolf et al. 2008) using the GTR $+\Gamma$ model and bootstrapping (1000 replicates). A splitting structure among closely related ITS2-ribotypes was reconstructed by neighbor-net analysis in SplitsTree4 4.10 (Huson and Bryant 2006), applying uncorrected pdistances and with ambiguities handled as averages. Bootstrap support values for internal splits were calculated with 1000 replicates. The assignation of sequences into ribotypes was computed in DnaSP v.5 (Librado and Rozas 2009).

\section{Results}

\section{Polar Chlorellaceae and allies}

The analyzed polar Chlorella-like strains exhibit two different gross morphologies: 1) Chlorellalike spherical to slightly elliptical cells $(\varnothing=2.8-8.4 \mu \mathrm{m})$ bearing one cup-shaped chloroplast with one (Fig. 2a) or two (Fig. 2b) pyrenoids; 2) Nannochloris- and Muriella-like spherical cells (Ø 
$=2.2-7.3$ ) with a simple parietal chloroplast without a pyrenoid (Figs. 2d-f). According to the 18S-based phylogenetic analysis, the polar strains are nested in four different operational taxonomic units (OTUs; corresponding to genera) of the Chlorellaceae (Fig. 3). The OTUs 1-4 include further GenBank accessions, which are closely related to our polar strains at the $\geq 99.5 \%$ sequence similarity level.
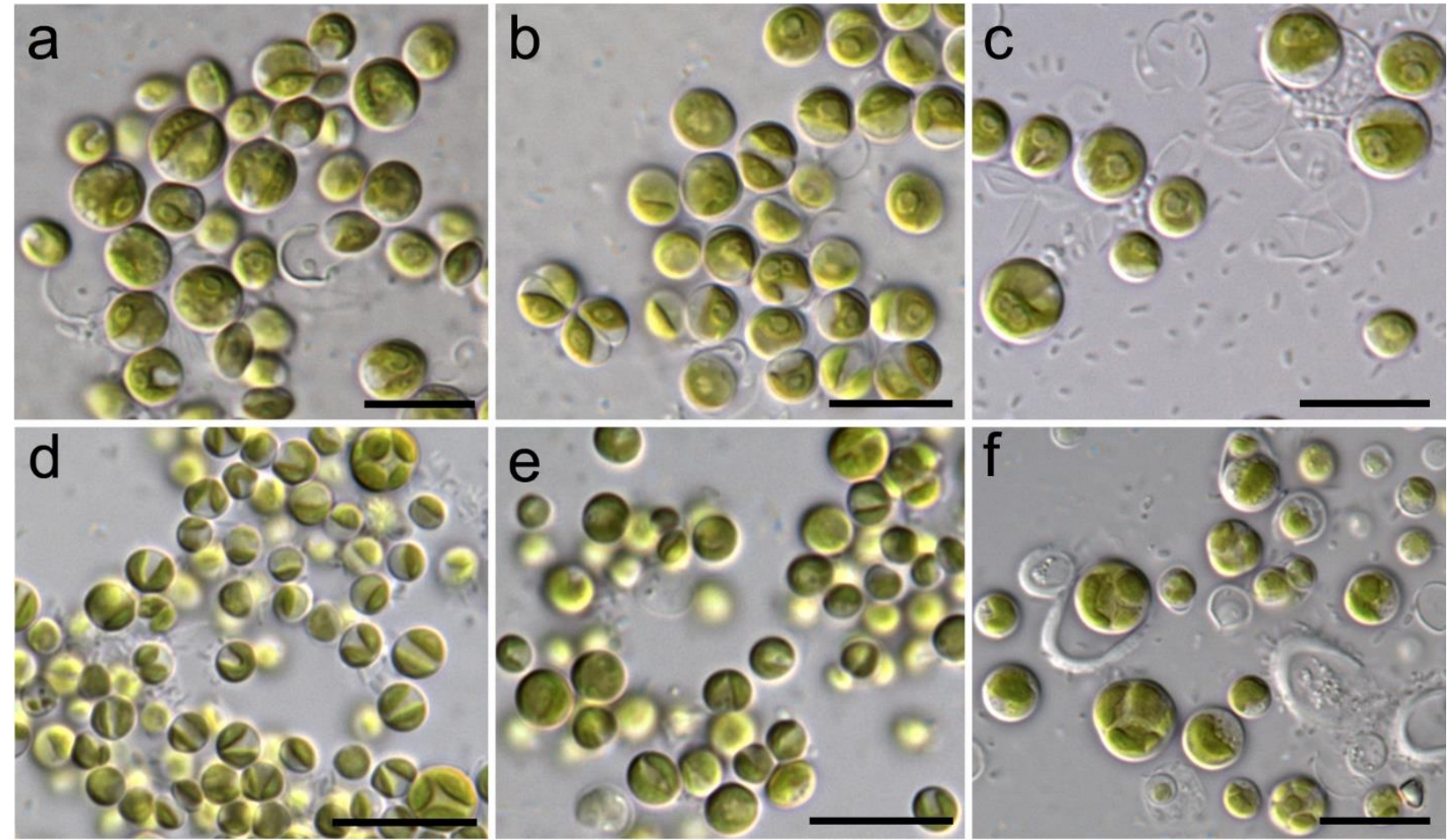

Figure 2. Microphotographs of polar strains of the Chlorellaceae. (a) Chlorella cf. vulgaris $L 5(\varnothing=2.8-5.7 \mu \mathrm{m})$; (b) Chlorella sp.1 N9 $(\varnothing=2.9-5.4 \mu \mathrm{m}) ;$ (c) Chlorella sp.2 L3 ( $\varnothing=3.4-8.4 \mu \mathrm{m}) ;(\mathrm{d})$ Muriella sp. L20 ( $\varnothing=2.2-6.3 \mu \mathrm{m}) ;(\mathrm{e})$ Marvania relative L15 $(\varnothing$ $=2.3-5.8 \mu \mathrm{m})$; (f) Unidentified Chlorellaceae L24 $(\varnothing=2.6-7.3 \mu \mathrm{m})$. Scale bars $=10 \mu \mathrm{m}$.

\section{Chlorella clade}

The Antarctic strains L1 and L4 (King George Island) and the Arctic strains L5 (Fig. 2a) and L6 (Ellesmere Island) show $\geq 99.5 \%$ sequence similarity to the authentic strain C. vulgaris SAG 211-11b. The $18 \mathrm{~S}$ phylogeny suggests divergence between the polar strains and the remaining mostly temperate sequences within OTU3 (Fig. 3). We analyzed the observed differences between the polar and temperate strains of C. vulgaris using more variable ITS2 (Fig. 4a), assigning them into 13 ribotypes (A, B, E-O), exhibiting a total variability of 54 polymorphic nucleotide positions Fig. S1a). The ribotypes A-K differ from C. vulgaris SAG 211-11b by a few nucleotide polymorphisms (Fig. S1a). In contrast, ribotypes L-O are more divergent from $C$. vulgaris SAG 211-11b; they differ from both SAG 211-11b and ribotypes A-K by sharing some identical nucleotide sites with C. pituita ACOI 311 (Fig. S1a). The ITS2 secondary structure 
model (Fig. S1b) shows that a considerable amount of nucleotide positions are conserved across all C. vulgaris ribotypes (including the polar ribotypes) and C. pituita (Fig. S1b). Moreover, none of the ribotypes (not even the polar ones) shows any compensatory base changes (CBCs) against C. vulgaris SAG 211-11b, but all show at least three CBCs against C. pituita ACOI 311 (Fig. S2a). Notably, the Antarctic strain L4 (ribotype M) differs in only a single nucleotide from the German isolate LH10HG2067 (ribotype L). Otherwise, the same ribotypes were detected from both aquatic and terrestrial habitats (i.e., ribotypes B, H, I; Fig. 4b). Ribotype B is the most common ITS2 variety of $C$. vulgaris in GenBank, including strains isolated from freshwaters in Europe, USA and China.

The Arctic strain N9 (Fig. 2b) and the Antarctic strain L3 (Fig. 3c) morphologically resemble members of the Chlorella clade. Considering the 18S phylogeny, both strains are nested within an unnamed clade (OTU1), which comprises closely related strains from freshwaters of the temperate northern hemisphere and Antarctica. The $18 \mathrm{~S}$ inference points out the relatedness to the strain Hindakia fallax CCAP 222/29. However, the ITS2 phylogeny does not support the close relationship (Fig. 4a; Fig. S2b), since both strains exhibit considerable genetic divergence from both Hindakia species. The ITS2 inference further suggests that N9 and L3 represent two different species, provisionally denominated as Chlorella sp.1 and C. sp.2, respectively. We detected a remarkably high ITS2 similarity between the Chlorella sp.1 strain N9 from the Arctic and the strain KNUA034 from Antarctica, differing by three nucleotide positions (and a one-sided/hemi compensatory base change/hCBC in the helix IV, Fig. 4c). The Chlorella sp.2 strain L3 has no known close relatives.

\section{Nannochloris-like clades}

The Nannochloris-like Antarctic strains L20 (Fig. 2d) and N5 are nested within OTU3, comprising Muriella terrestris ASIB V38 and other terrestrial and aquatic strains of almost identical 18S sequences (Fig. 3). The high 18S similarity of the strains L20 and N5 contrasts with their considerable genetic dissimilarity in the ITS2 marker (Fig. 4; Fig. S2c). The Antarctic Muriella sp.1 strain L20 exhibits high ITS2 similarity with the Antarctic clone Ant 8/104 and the isolate LH08SG3009 from a German soil. Strain L20 differs from the isolate LH08SG3009 by one hCBC in the helix III of the ITS2 secondary structure (Fig. 4d).

The Antarctic strains L13 and L15 (Fig. 2e) clustered within the OTU4 comprising further $18 \mathrm{~S}$ accessions from Antarctica and the temperate regions (Fig. 3). The phylogenetically closest named species are Marvania geminata SAG 12.88 and Nannochloris coccoides CCAP 251/1b. However, any close relationship to OTU4 (provisionally denominated as Marvania relative1) is 
supported neither in the 18S nor the ITS2 phylogeny (Fig. 4a, Fig. S2d).

Strain L24 (King George Island) represents a phylogenetically isolated lineage within the Chlorellaceae, so far without known close relatives. The strain L24 (Fig. 2f) exhibits morphology resembling Nannochloris-like lineages rather than the true Chlorella described above.

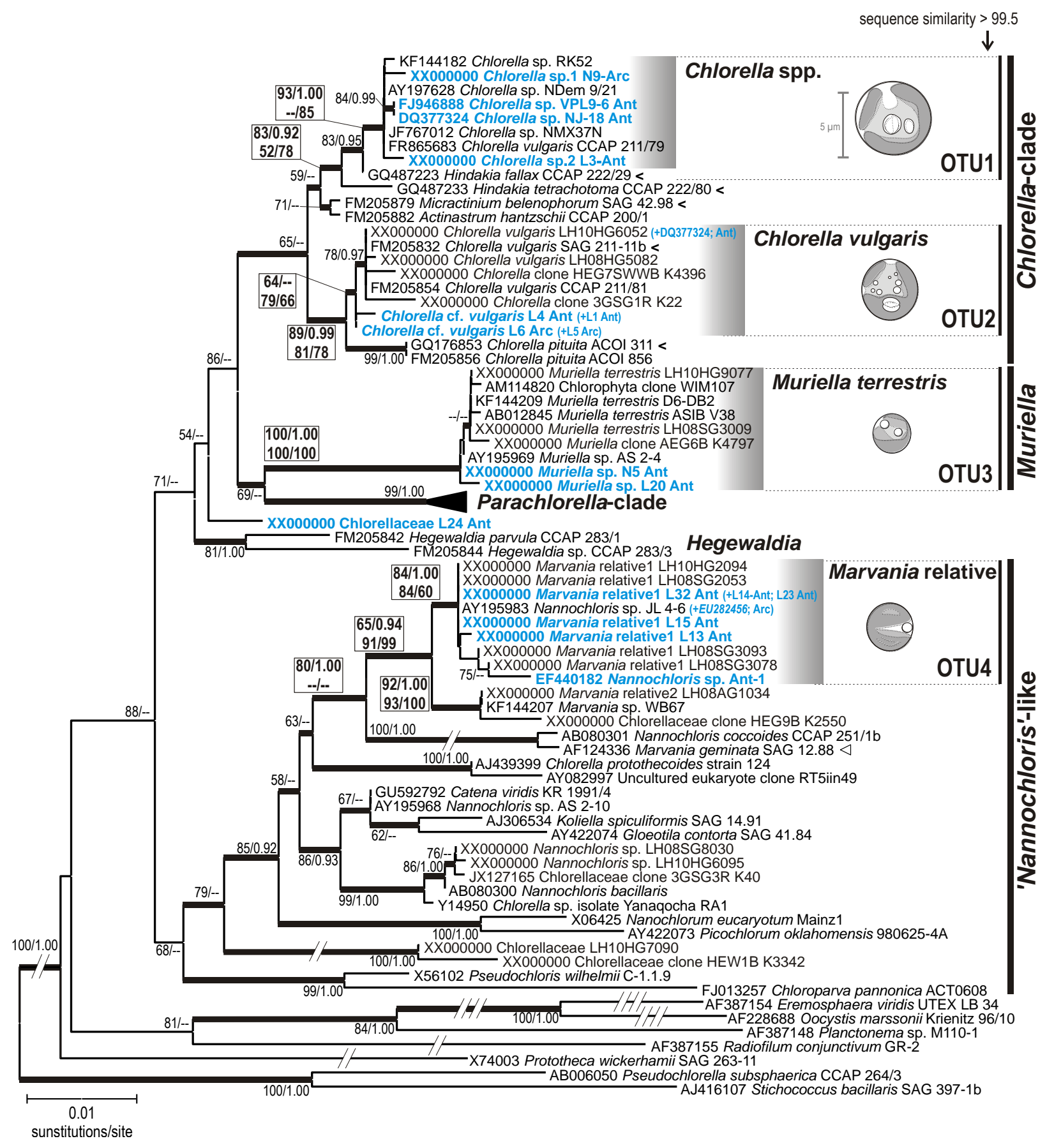

Figure 3. 18S ML-phylogeny of the polar Chlorella-like strains and relatives. All polar accessions are highlighted in blue and authentic strains are marked by a '<' sign. The numbers next to branches indicate statistical support values (maximum-likelihood bootstraps (ML)/Bayesian posterior probabilities $(B \mathrm{I})$ ); the clades of particular interest were additionally tested via maximum parsimony (MP) and bio-neighbor-joining (NJ) and the statistic support values are given in the following order: ML/BI/MP/NJ. Black bars to the right side give sequence assignations into OTUs at $\geq 99.5 \%$ similarity level. Drawings show morphology characteristic for each clade. 


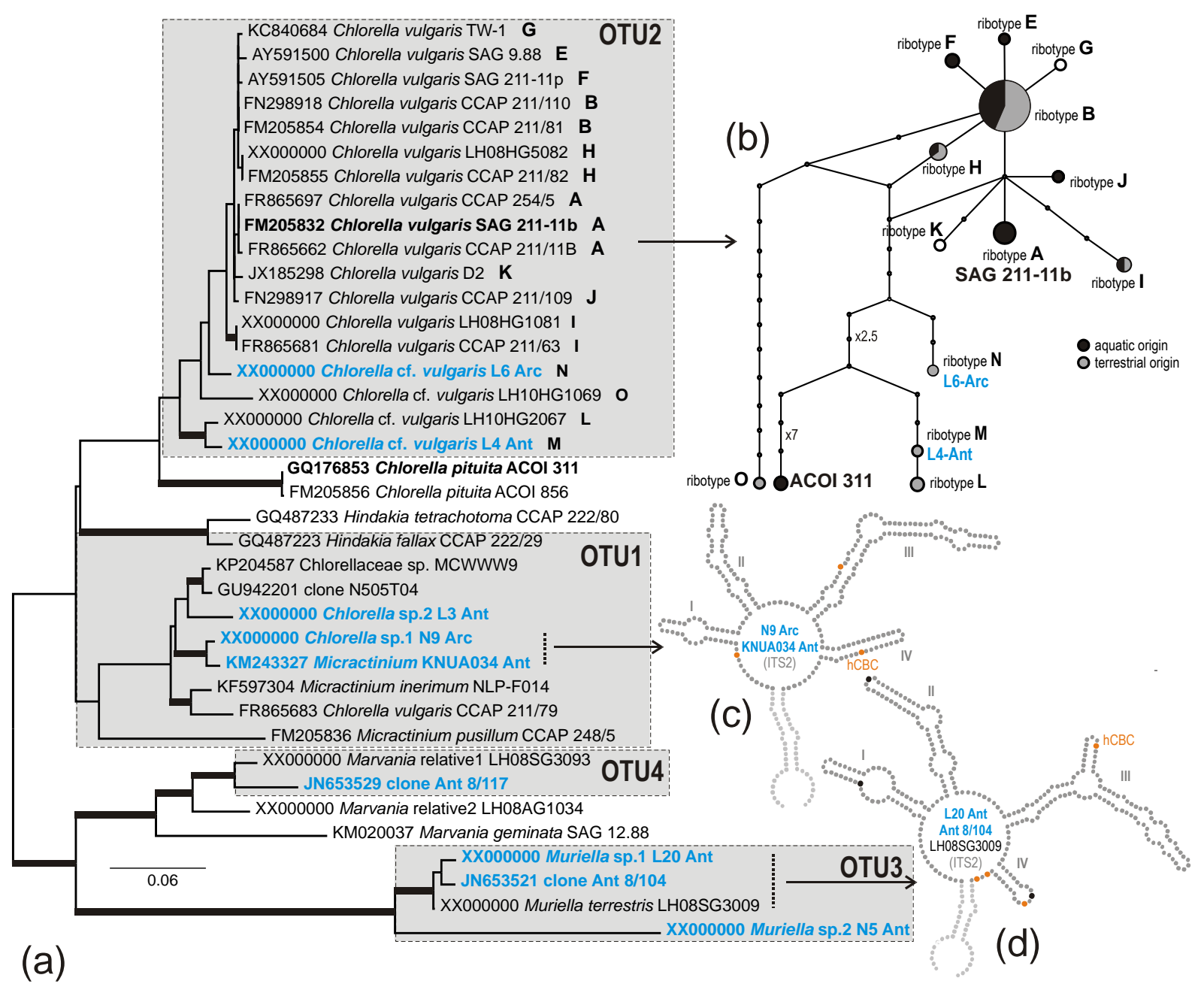

Figure 4. Analysis of ITS2 sequences obtained from polar Chlorella-like strains and relatives. (a) An ITS2-based neighbor-joining tree computed from sequences and secondary structures. (b) Ribotype network of Chlorella vulgaris accessions assigned to ribotypes A-O. Each circle represents a distinct ITS2 ribotype and its size reflects the number of identical sequences. (c)-(d) Consensual ITS2 secondary structures of closely related accessions: (c) Chlorella sp.1, (d) Muriella sp. Nucleotide positions within the ITS2 secondary structures are colored as follows: grey dots=conserved positions, orange dots=nucleotide substitutions and black dots=nucleotide deletions.

\section{Stichococcus-like lineages}

The newly obtained strains of Stichococcus-like species all exhibited characteristic rod-shaped morphology (Fig. 5a-l) and varied in both cell length (1.3-10.0 $\mu \mathrm{m})$ and width $(1.0-4.4 \mu \mathrm{m})$. Deviations from the rod-shape morphotype-Diplosphaera-like cell packages-were observed in the isolate LH08SW1099 (Fig. 5j). The Stichococcus strains do not substantially differ in chloroplast shape and the detectability of pyrenoids using light microscope is ambiguous. The $18 \mathrm{~S}$ analysis of all Stichococcus-like accessions (Table S2) revealed five monophyletic Stichococcus-like OTUs of highly similar sequences ( $\geq 99.5 \%$ ), i.e., OTU1, OTU3-6 (Fig. 6; Fig S3) and a further four Stichococcus-like OTUs of lower resolution $\geq 99.0 \%$, i.e., OTU2, OTU79 (Fig. 6; Fig S3). The OTUs could be better resolved using more polymorphic ITS2 sequences (Fig. 7; Fig. S4a) and analyzing their secondary structures (Fig. 7; Fig. S4b; Fig. S5); this approach revealed a total of 12 unambiguously distinguishable monophyletic lineages (Tab. S3). 


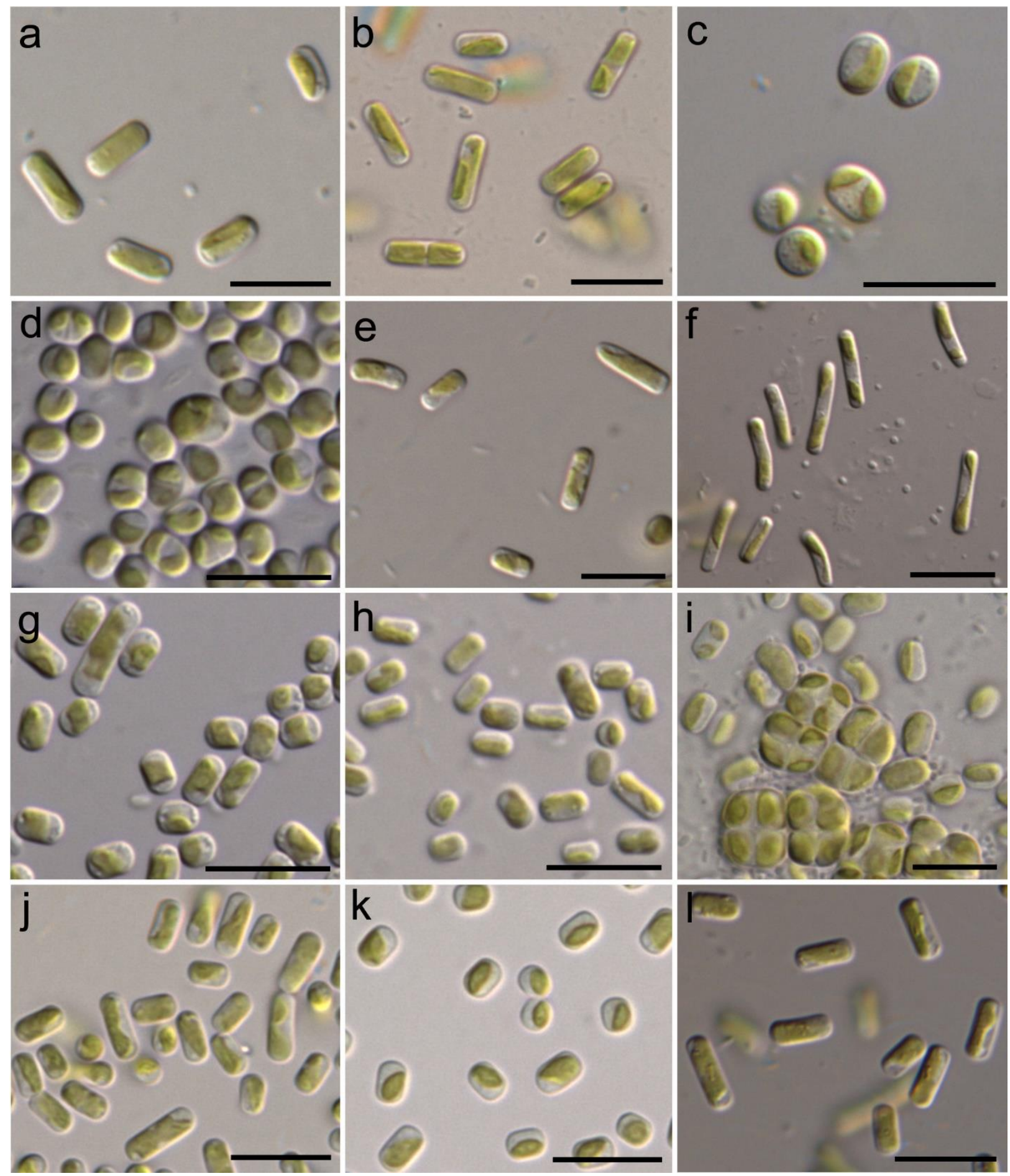

Figure 5. Microphotographs of the Stichococcus-like strains isolated from Germany and Ecuador. (a) Stichococcus clade2

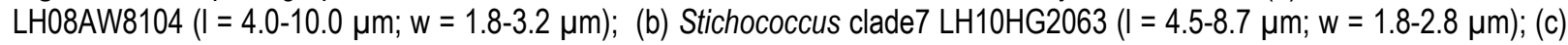

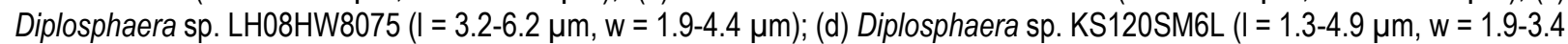
$\mu \mathrm{m}) ;(\mathrm{e})$ Pseudostichococcus sp. LH08SW8044 (I = 3.4-9.8 $\mu \mathrm{m} ; \mathrm{w}=1.6-3.3 \mu \mathrm{m}) ;(\mathrm{f})$ Stichococcus clade1 SAG 2481 (I = 3.1-8.7 $\mu \mathrm{m} ; \mathrm{w}=1.6-2.9 \mu \mathrm{m}) ;(\mathrm{g})$ Stichococcus clade1 KS075SM6T $(\mathrm{I}=2.9-6.3 \mu \mathrm{m} ; \mathrm{w}=1.5-3.0 \mu \mathrm{m}) ;(\mathrm{h})$ Stichococcus clade3

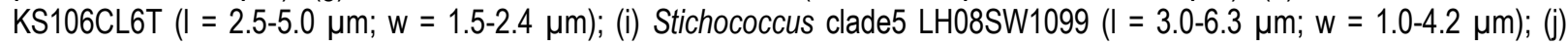
Stichococcus clade5 KS305SM6L (I = 3.3-6.8 $\mu \mathrm{m} ; \mathrm{w}=1.4-3.2 \mu \mathrm{m}) ;(\mathrm{k})$ Stichococcus jenerensis KS126SM6L $(\mathrm{I}=1.9-5.0 \mu \mathrm{m}$; $\mathrm{w}=1.6-3.2 \mu \mathrm{m}) ;(\mathrm{I})$ Stichococcus clade4 SAG $2406(\mathrm{I}=4.5-8.2 \mu \mathrm{m} ; \mathrm{w}=1.7-2.7 \mu \mathrm{m})$. Scale bars $=10 \mu \mathrm{m}$. 


\section{Stichococcus-like clades including polar species}

The polar Stichococcus-like accessions (obtained from GenBank; Table S2) represent at least three different phylogenetic lineages, here provisionally designed as "OTU2 Stichococcus deasonii \& allies", "OTU8 Diplosphaera” and "OTU9 Pseudostichococcus" (Fig. 8; Fig. S3).

OTU2 consists of the authentic strain Stichococcus deasonii SAG 2139 (Alabama, USA) and further accessions originating from terrestrial habitats (Table S2). OTU2 is poorly resolved in the 18S phylogenies (Fig. 6; Fig. S3). The ITS2 analyses suggest that, apart from Stichococcus deasonii, OTU2 consists of another three different species, here provisionally named as Stichococcus clade2 (Fig. 5a), S. clade6, and $S$. clade7 (Fig. 5b). S. clade2 includes accessions from Antarctica (e.g., HM490287), differing from its European relatives (e.g., SAG 2482) by 69 nucleotide positions in ITS2 (Fig. S5). Another two Antarctic strains cluster within OTU2: S. bacillaris NJ-10 and S. bacillaris s3, but the $18 \mathrm{~S}$ data do not provide sufficient resolution to assign them into any known species. Stichococcus deasonii is the only lineage within OTU2 which comprises accessions from the tropics (e.g., clone KSK870SM6T; Fig. S5).

OTU8 Diplosphaera is a phylogenetically heterogeneous clade consisting of multiple lineages of Stichococcus-like (S. chodati UTEX 1177), Diplosphaera-like (D. mucosa SAG 48.86) and Chlorella-like (C. sphaerica SAG 11.88) species, recognizable in the $18 \mathrm{~S}$ and/or ITS2 phylogenies, yet without sufficient resolution or statistical support. The Antarctic strain Diplosphaera mucosa SAG 48.86 is the only known polar member within OTU8. Further accessions within the clade originate from Central Europe (isolate LH08HW8075; Fig. 5c; Fig. 6) the tropics (isolate KS120SM6L; Fig. 5d; Fig. 6) and North-American deserts (e.g., Stichococcus chlorelloides BCP-CNP2-VF11B). OTU8 is the only known Stichococcus-like clade that includes species from hot deserts (Fig. 8).

OTU9 Pseudostichococcus represents a phylogenetically isolated clade of Stichococcus-like (S. mirabilis CCAP 379/3, Pseudostichococcus monallantoides SAG 380-1) and Desmococcuslike (D. spinocystis SAG 2067) species (Fig. 7; Fig. 8; Fig. S3; Fig. S5). The clade consists of two strains isolated from Antarctica: Trebouxiophyceae sp. EO7-4 and Stichococcus minutus NJ17. All other accessions within OTU9 originate from terrestrial (e.g., Pseudostichococcus sp. LH08SW8044; Fig. 5e) and aquatic habitats of the temperate zone while no tropical relatives are known so far.

\section{Stichococcus-like clades including tropical species not related to polar allies}

We identified four clades consisting of tropical and temperate species and designed them provisionally as: "OTU1 Stichococcus clade1", "OTU3 Stichococcus clade3", "OTU5 
Stichococcus clade5" and "OTU7 Stichococcus jenerensis" (Table S2).

OTUs 1, 3 and 5 are statistically highly supported in both the 18S (Fig. 6) and ITS2 (Fig. 7; Fig. S5) phylogenies and represent three undescribed Stichococcus-like species. Few nucleotide differences in the ITS2 within the clades and no compensatory base changes further support close relationships among the tropical and European accessions. OTU1 Stichococcus clade1 consists of strains isolated from Germany (SAG 2481; Fig. 5f) and Ecuador (KS075SM6T; Fig. 5g). Another German strain KS075SM6T differs from KS075SM6T by only 10 nucleotide positions within ITS2 and exhibits no compensatory base changes (Fig. S5). OTU3 Stichococcus clade3 includes the European strain SAG 2408 which differs by only three ITS2 nucleotide positions from the tropical isolate KS106CL6T (Fig. 5h). OTU3 further consists of 18S accessions sampled in the Swiss Alps and Yellowstone NP (Fig. 6; Table S2). OTU5 Stichococcus clade5 is a terrestrial lineage consisting of the European isolate LH08SW1099 (exhibiting both Stichococcus- and Diplosphaera-like morphology; Fig. 5i) and the Ecuadorian isolate KS305SM6L (Fig. 5j). The tropical isolate differs by four ITS2 nucleotide substitutions from the isolate KP09AW1004 originating from Germany. OTU5 might be distributed as well in Hawaii, since the accession KM462543 shows a high $18 \mathrm{~S}$ sequence similarity (99.46\%) to the members of Stichococcus clade5.

OTU7 Stichococcus jenerensis contains the authentic strain S. jenerensis SAG 2138, which is the only Stichococcus-like species described from the tropics. The ITS2 data suggest a pantropical distribution of the clade: the Ecuadorean isolate KS126SM6L (Fig. 5k) differs by 13 ITS2 nucleotides from the Southeast-Asian strain SAG 2138, and exhibiting no CBCs (Fig. S5). OTU7 further consists of temperate terrestrial and even marine accessions (Fig. 6; Table S2).

\section{Stichococcus-like clades from the temperate zone}

Two highly supported clades of terrestrial and aquatic Stichococcus-like species are known so far only from the temperate zone: "OTU4 Stichococcus clade4" and "OTU6 Stichococcus bacillaris". OTU4 contains the Stichococcus-like strain SAG 2406 (Fig. 5l) and further accessions (sampled in Germany; Table S2) with almost identical 18S sequences (Fig. 6). However, the ITS2 data point out a cryptic species diversity, since we detected one CBC between strain SAG 2406 and the isolate LH08SG1073 (Fig. S5). The strain CCALA 906 from Svalbard (KF355941) is the closest polar relative of OTU4, yet with low 18S similarity (98.7\%). OTU6 includes multiple Stichococcus strains (e.g., S. bacillaris SAG 379-1b; S. chloranthus SAG 3792) and strains morphologically assigned to Gloeotila (Gloeotila scopulina SAG 335-8; Gloeotila cf. protogenita SAG 56.91). OTU6 accessions originate from freshwaters (Table S2), soil (clone 
HEW1B K3375; Fig. 6) and even the Southeast Pacific (KF899844). The ITS2 data suggest a close relationship between the OTU6 Stichococcus bacillaris and the Desmococcus clade (Fig. 7; Fig. S5), which consists of cell packages-forming/filamentous terrestrial species (distributed in the polar and temperate regions; Fig. 8). OTU6 and Desmococcus are sister lineages in both the $18 \mathrm{~S}$ and ITS2 phylogenies and differ by two CBCs in the ITS2 secondary structures.

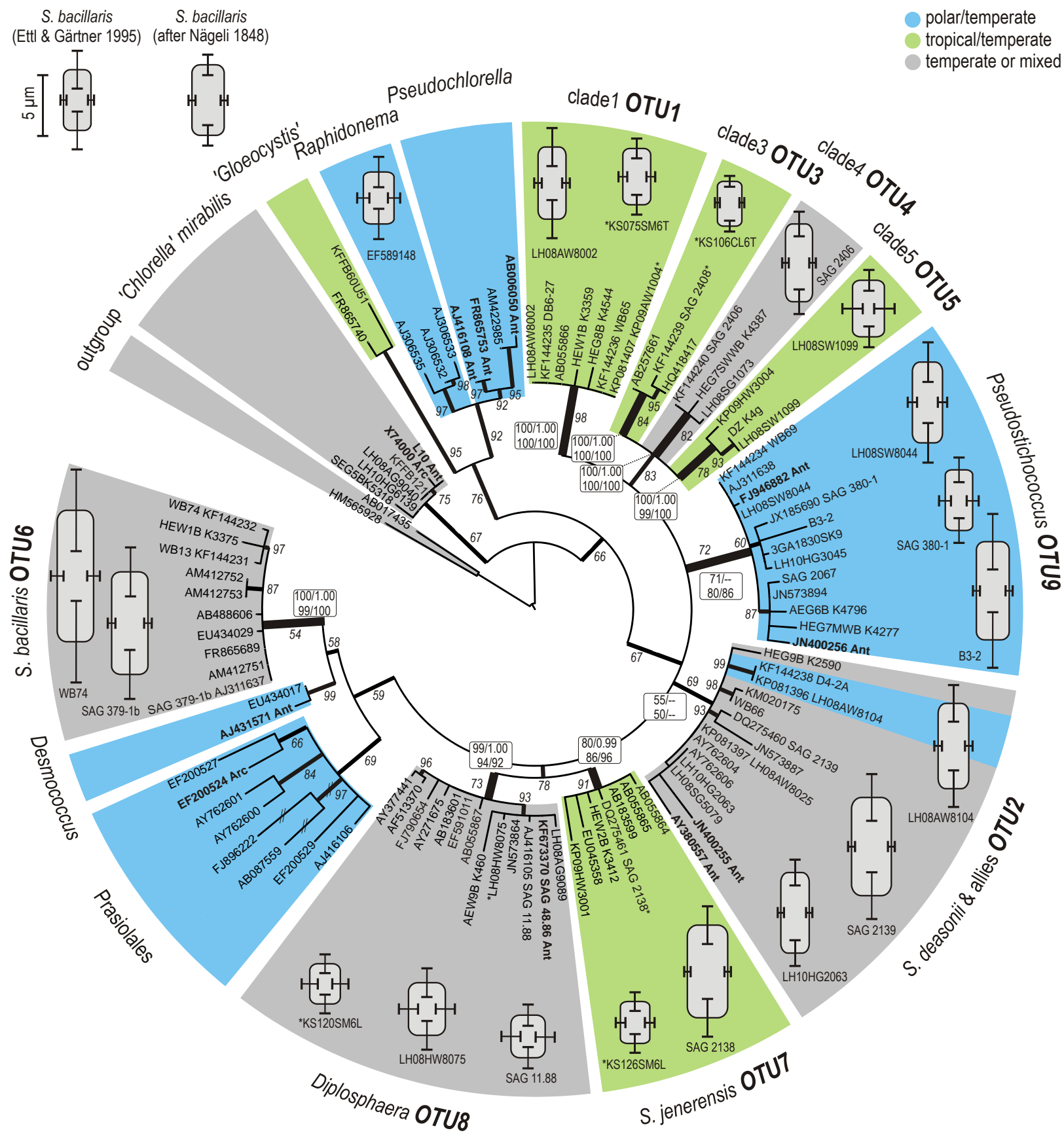

Figure 6. 18S ML (quartet puzzling) phylogeny of Stichococcus-like species and relatives. The numbers next to branches (italic letters) indicate bootstrap support values inferred from 1000 replicates. The numbers in white boxes close to clades studied in detail correspond to statistical values in the following order: ML/BI/MP/NJ. Schematic drawings within clades show cell size of representative Stichococcus-like strains as compared to Stichococcus bacillaris morphospecies after Nägeli (1849) and Ettl \& Gärtner (1995). Assignations into operational taxonomic units (OTUs) are based on sequence similarities $\geq 99.5 \%$ (= OTU) or $\geq 99.0 \%$ (= OTU). The clades are colored according to geographic origin of the sequences: blue=polar/temperate, green=tropical/temperate, grey=only temperate or temperate/polar/tropical. 


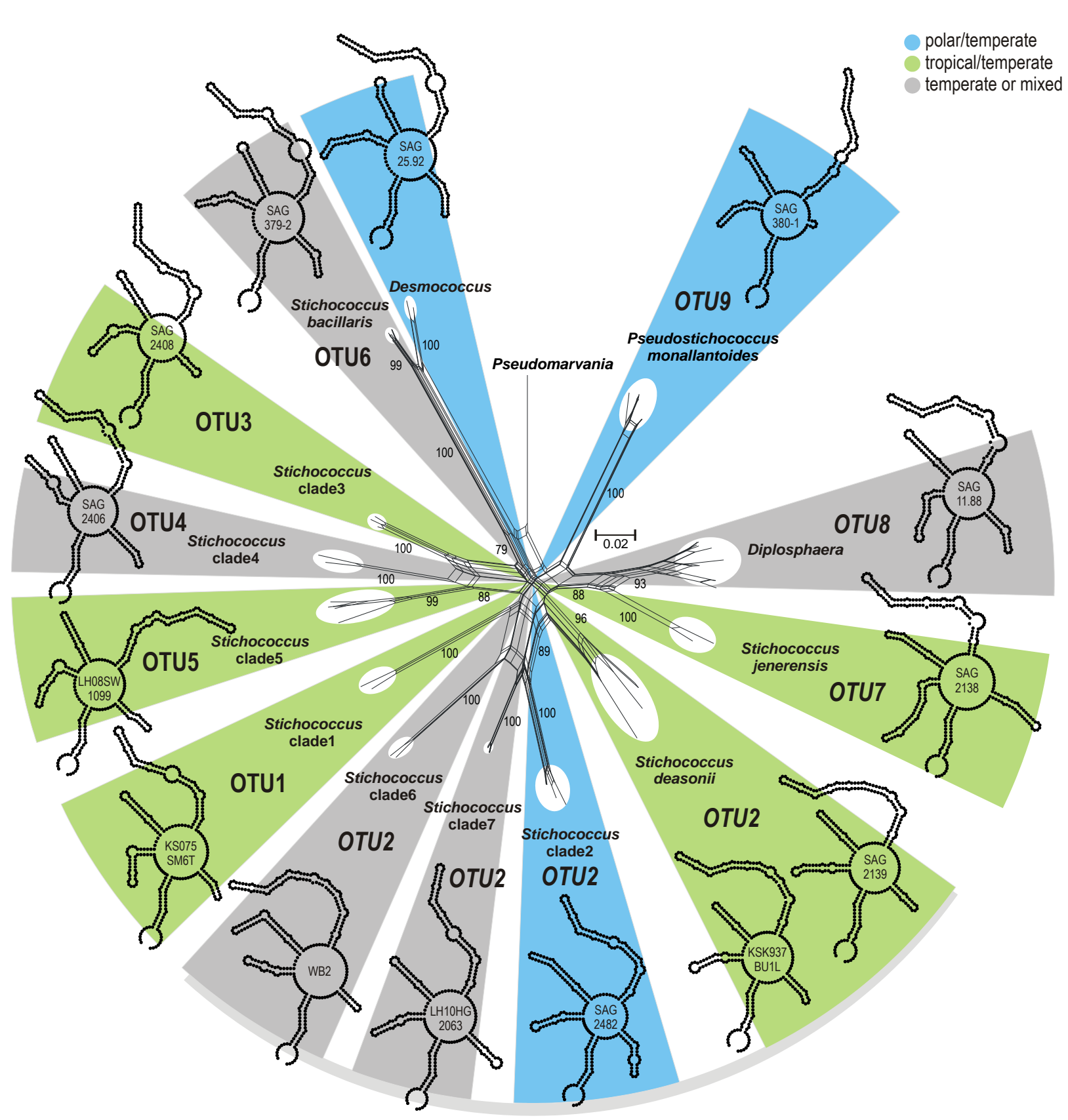

Figure 7. ITS2 phylogenetic network of Stichococcus-like species and relatives. Neighbor-net analysis of Stichococcus clades. The numbers near to splits correspond to bootstrap support values. Schematic ITS2 secondary structures are shown for representative strains. Assignations into operational taxonomic units (OTUs) are based on $18 \mathrm{~S}$ inference (Fig. 6; Table S2). The comprehensive dataset is shown in Fig. S5. The clades are colored according to geographic origin of the sequences: blue=polar/temperate, green=tropical/temperate, grey=only temperate or temperate/polar/tropical. 


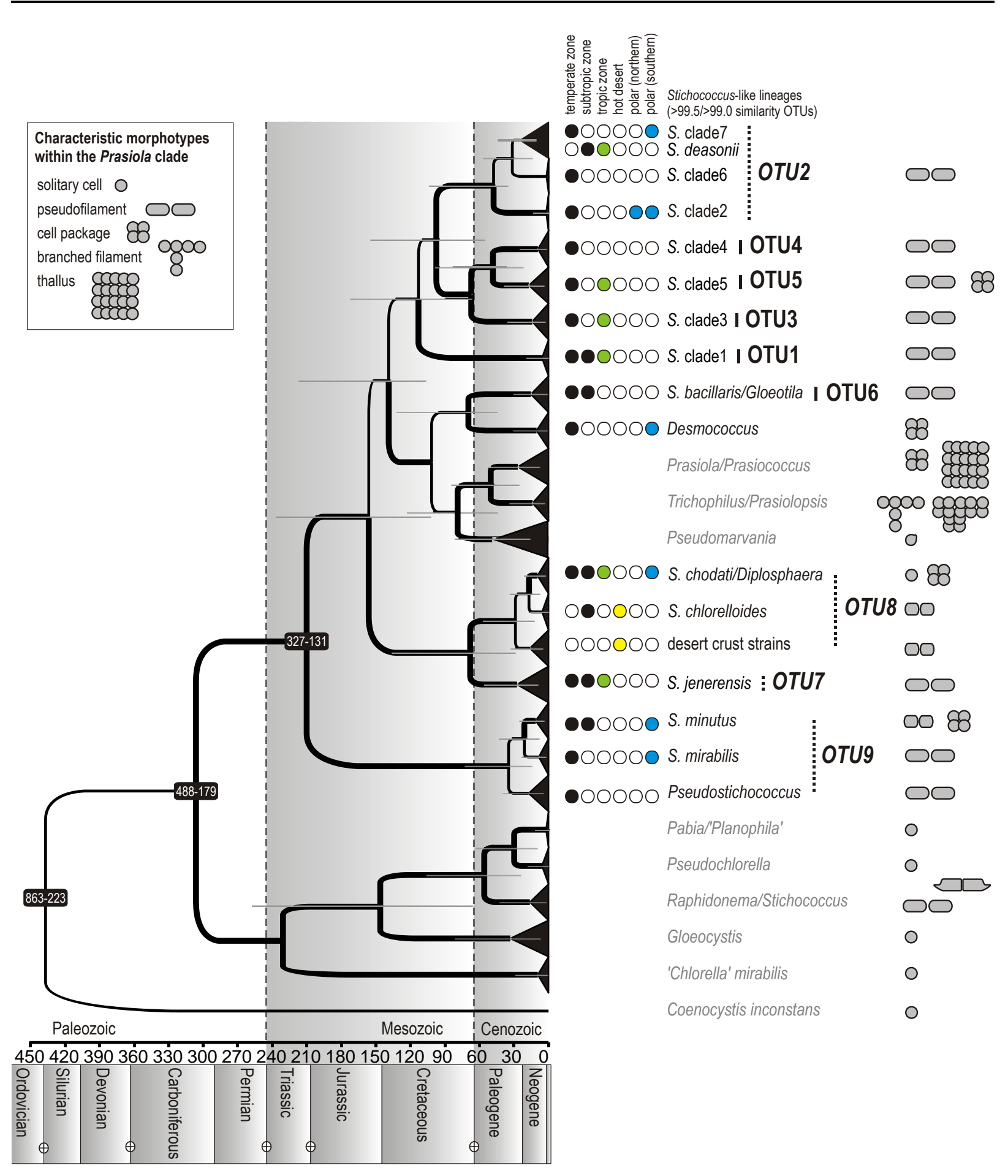

Figure 8. Calibrated 18S phylogeny of Stichococcus-like species and relatives using relaxed molecular clock. Bayesian phylogenetic tree (BEAST) computed from $18 \mathrm{~S}$ dataset identical to Fig. $\mathbf{6}$. Colored dots to the right of the tree summarize information on the geographic distribution of Stichococcus-like species and relatives (based on molecular data listed in Table S2). Schematic drawings illustrate the multiple occurrence of rod-shaped (Stichococcus-like) morphotypes throughout the Prasiola clade. Assignations into operational taxonomic units are based on sequence similarities $\geq 99.5 \%$ (= OTU) and $\geq 99.0 \%$ (= OTU), Fig. 6. 


\section{Discussion}

We defined operational taxonomic units (= clades consisting of identical or closely related sequences) as those with $\geq 99.5 \% \mathrm{SSU}$-sequence similarity. The most psychrotolerant strains of Chlorella and Stichococcus are conspecific (or closely related) with strains from the temperate zone. Including our new sequences and the sequences published in GenBank (De Wever et al. 2009), we recognized seven phylogenetic species of polar Chlorellaceae. Five of these species are the psychrotolerant Chlorellaceae isolated by Shukla et al. (2011). Such a low phylogenetic diversity of Chlorella is also known from arid regions in which six species have been reported so far (Lewis and Lewis 2005; Flechtner et al. 2013; Fučíková et al. 2014). Our results suggest that particularly two species of the Chlorellaceae, Chlorella vulgaris (Bock et al. 2011) and Muriella terrestris (Hanagata 1998), might be distributed in both polar and arid desert environments. Terrestrial Stichococcus-like microalgae might be even more widely dispersed than members of the Chlorellaceae, because in addition to the polar and temperate regions, they were detected in the tropics as well. However, six Stichococcus clades with a temperate-polar distribution do not include any tropical relatives. And vice versa, Stichococcus clades with a temperate-tropical distribution do not contain any psychrotolerant species.

\section{Chlorella-like psychrotolerant strains have allies in temperate zones and hot deserts}

The strains morphologically resembling Muriella (Petersen 1935) are phylogenetically nested in Muriella terrestris (Hanagata 1998). We detected a considerably low genetic distance-a difference of three nucleotides within ITS2-among the $M$. terrestris strains sampled in Antarctica (L20; Shukla et al. 2011; Kochkina et al. 2014) and Germany (LH08SG3009). Their putative conspecifity is supported by the absence of compensatory base changes (CBCs; Müller et al. 2007b; Wolf et al. 2013). Further, SSU-based evidence suggests a widespread distribution of $M$. terrestris in streams of high $\mathrm{CO}_{2}$-pressure (Hodač et al. 2015), freshwaters (Fawley et al. 2004) and even in hot deserts (Flechtner et al. 2013; Fučíková et al. 2014). Another Nannochloris-like clade includes the Antarctic isolates L13/L14/L15/L23/L32 (Shukla et al. 2011), which are weakly related to Marvania geminata (Henley et al. 2004; Eliáš and Neustupa 2009). These strains cluster together with another Antarctic Nannochloris sp. Ant-1 (Gilichinsky et al. 2007), with Chlorella sp. 193-GA188 from the Siberian permafrost (Vishnivetskaya 2009) and with multiple isolates from German soils (e.g., LH08SG2053) and from freshwater (Nannochloris sp. JL 4-6; Fawley et al. 2004). The relatives of the tropical species Hindakia fallax (Bock et al. 2010) exhibited low genetic distance (three nucleotides in ITS2) between the isolate N9 from 
Svalbard (Shukla et al. 2011) and the Antarctic isolate KNUA034 (Hong et al. 2015). The Arctic isolate N9 is phylogenetically nested in a clade containing other accessions provisionally denominated as Chlorella spp., a putative sister lineage to the tropical Hindakia fallax. Hindakia spp. was already recorded multiple times from Antarctica (Hu et al. 2008; De Wever et al. 2009; Hong et al. 2015) and includes freshwater strains, which were screened for application in biotechnology, e.g., CCAP 211/79 (Germond et al. 2013; Osundeko et al. 2013; Driver et al. 2015). High ITS2 similarity between isolates from Nova Scotia (KP204587; Park et al. 2015) and the South China Sea (GU942201), which are both relatives of the Antarctic isolate L3 (Shukla et al. 2011), additionally supports the long-distance dispersal of Chlorella spp.

Chlorella vulgaris, the epitome of spherical green microalgae (Krienitz et al. 2015), was isolated from Antarctica and represented by the intensively investigated psychrotolerant strain NJ-7 (Li et al. 2009; Lu et al. 2009; Lu et al. 2010). The SSU sequences of our Antarctic (L1/L4) and Arctic (L5/L6) isolates (Shukla et al. 2011) clustered between $C$. vulgaris SAG 211-11b and its closest named relative C. pituita ACOI 311 (Bock et al. 2011). The Antarctic strains are more different from the temperate $C$. vulgaris SAG 211-11b than the Arctic strains; the most similar strain to the Antarctic strains L1/L4 was isolated from a hot desert (JX446471; Flechtner et al. 2013) and a German soil (LH10HG2081; this study). ITS2 analysis of multiple $C$. vulgaris strains revealed considerable intraspecific diversity; the particular ITS2 ribotypes do not cluster according to their original habitat (freshwater vs. terrestrial). Remarkably, the ITS2 of the Antarctic strain L4 differs from all $C$. vulgaris strains, but is almost identical to the isolate LH10HG2081. Taking the ITS2 difference between $C$. vulgaris SAG 211-11b and C. pituita ACOI 311 (32 nucleotides and 3-4 CBCs) into account, then the psychrotolerant (L4) and $C$. vulgaris SAG 211-11b might be conspecifics (differing in 14 nucleotides; 0 CBC). In comparison, another trebouxiophycean genus Coccomyxa exhibits intraspecific variability of 26 nucleotide positions in ITS2 (Darienko et al. 2015). Evidence of identical ITS2 ribotypes from Europe (SAG 211-11b) and North America (CCAP 254/5) confirms the long-distance dispersal of C. vulgaris. Chlorella sorokiniana (Bock et al. 2011) is another remarkable Chlorella, so far known from both cold and hot environments, e.g., the Antarctic (De Wever et al. 2009) and North-American deserts (Flechtner et al. 2013). In contrast to the terrestrial Chlorella-like species mentioned above, $C$. sorokiniana might be dispersed even in the humid tropics and was recorded (based on DNA data) from Central America (de-Bashan et al. 2008), South America (de-Bashan et al. 2008; Bashan et al. 2015) and Southeast Asia (identified by S. Marimuthu; http://studentsrepo.um.edu.my/3562/). The species is thermotolerant (de-Bashan et al. 2008; Zheng et al. 2013). Other thermotolerant Chlorellaceae recorded from hot deserts have close 
relatives from phytoplankton, e.g., Meyerella (Fučíková et al. 2014), Micractinium (Flechtner et al. 2013); the distribution of the recently described Chlorella thermophila (Ma et al. 2015) remains unknown. Apart from the psychrotolerant strains described above, another two polar strains, i.e., L24 (Shukla et al. 2011) and VPL1-3 (FJ946890; De Wever et al. 2009) are incertae sedis in the Chlorellaceae.

\section{Stichococcus-like clades including psychrotolerant species and species from hot deserts}

The psychrotolerant strains of Stichococcus which have been sequenced so far (Hughes 2006; Chen et al. 2012) cluster within three different lineages of the Stichococcus clade sensu Sluiman and Guihal (2008). We provisionally denominated these clades as Pseudostichococcus (SAG 2067; SAG 380-1; CCAP 379/3), Stichococcus clade2 (SAG 2482) and S. clade7 (SAG 2060; SAG 2119). The Pseudostichococcus clade (including the strain P. monallantoides SAG 380-1; Moewus 1951) is an early diverging lineage consisting of species morphologically resembling Stichococcus (Nägeli 1849) and Desmococcus (Brandt and Stockmayer 1925; Gärtner and Ingolić 2003). Two Antarctic strains are nested within the Pseudostichococcus clade: Trebouxiophyceae EO7-4 (De Wever et al. 2009) and Stichococcus minutus NJ-17 (Chen et al. 2012). The freshwater strain EO7-4 is genetically similar to German isolates from soil (LH08SW8044; this study) and a karst-water stream (WB69; Hodač et al. 2015). The allies of the subaerial isolate $\mathrm{NJ}-17$ were detected as environmental clones from German soils and isolated from a lichen (Thüs et al. 2011). Further Antarctic strains-phylogenetically distant from the Pseudostichococcus clade-were isolated from permafrost (Stichococcus bacillaris s3; Hughes 2006) and wet rocks (S. bacillaris NJ-10; Chen et al. 2012) and belong to a poorly resolved clade provisionally denominated as Stichococcus clade7. As a result of the SSU-based phylogeny, the species within the Stichococcus clade7 might be allies of S. deasonii (Neustupa et al. 2007). The ITS2-based inference, however, did not unambiguously support the monophyletic origin of both clades, suggesting a considerable evolutionary distance between $S$. clade7 (including the above mentioned polar species) and the $S$. deasonii lineage (including tropical species). Notably, the Stichococcus clade7 further consists of species from soils and subaerial habitats (Karsten et al. 2005), but, in contrast to most Stichococcus-like clades, no aquatic species are known so far. The only psychrotolerant Stichococcus-like strain obtained from the Arctic permafrost is Stichococcus sp. 594-GA18 (Vishnivetskaya 2009) isolated from a $4.65 \mathrm{~m}$ depth in Siberia. This strain clusters within the Stichococcus clade2, an unnamed monophyletic lineage closely related to the above mentioned S. clade7. Stichococcus clade7 contains German isolates from a karst-water stream (D4-2A; Hodač et al. 2015) and soils (e.g., SAG 2482). Remarkably, S. clade7 is the only known 
Stichococcus-like clade which might have a bipolar distribution; it includes environmental clones (HM490287, HM490288; Khan et al. 2011) from the extreme McMurdo Dry Valleys, Antarctica, which are highly similar to the above mentioned soil strain SAG 2482 (6-9 nucleotide differences in ITS2, no CBCs).

The phylodiversity of Chlorellaceae in hot deserts might be higher than that of Stichococcus, which is limited to a single known clade. This clade including desert species we denominated Diplosphaera, based on the species D. mucosa (Broady 1983) and D. sphaerica (Karbovska and Kostikov 2012b). Diplosphaera mucosa SAG 48.86 is the only polar strain within the Diplosphaera clade, however, the clade contains another putatively psychrotolerant species, represented by the environmental clone QE28 detected in the Tibetan tundra (FJ790654; Wong and Lacap 2010). The members of the Diplosphaera clade inhabit not only extremely cold and hot deserts (Lewis and Lewis 2005; Flechtner et al. 2013), but also acidic freshwater (Aguilera et al. 2007), oceanic sediment (AB183601) and occur as common lichen photobionts (Thüs et al. 2011; Fontaine et al. 2012; Fontaine et al. 2013). Diplosphaera ITS2 sequences, which were retrieved from leaf surfaces in an Ecuadorian tropical rainforest (e.g., KS120SM6L), were almost identical (i.e., differing in only three nucleotides; no CBC) to the isolate LH08HW8075 (this study) from a German forest soil. The SSU data suggest close relatedness of both temperatetropical strains, the Antarctic Diplosphaera mucosa SAG 48.86 and lichen photobiont Diplosphaera sp. J4028B (Thüs et al. 2011). In Stichococcus clade7, we also observed genetic relatedness of a lichen photobiont (Diplosphaera sp. W1118; Thüs et al. 2011), tropical clone KSK870SM6T and polar isolates (e.g., Stichococcus bacillaris NJ-10; Chen et al. 2012), however, this evidence is based on lower genetic similarities as compared to the Diplosphaera clade.

\section{Biogeography of terrestrial Chlorella and Stichococcus}

Patterns in species distribution have been accepted for freshwater microalgae (De Wever et al. 2009; Naselli-Flores and Padisák 2015). Some freshwater species of "true" Chlorella, which were described from the tropics (e.g., C. pulchelloides, C. rotunda, C. singularis, C. volutis; Bock et al. 2011), have not yet been uncovered in polar regions. Furthermore, the Chlorella-like species we detected in Antarctica and the Arctic (C. vulgaris, C. spp., Muriella terrestris, Marvaniarelative) belong to clades with temperate-polar distributions; they are genetically divergent from their tropical relatives (e.g., Hindakia fallax or Chlorella sorokiniana). However, concerning terrestrial green microalgae, Earth's biodiversity hotspots such as tropical rainforests still remain largely unexplored. Morphology-based observations suggest that true Chlorella (and other 
Chlorellaceae) might be less common in tropical rainforests than Stichococcus (Neustupa and Škaloud 2008; 2010). Accordingly, the most Chlorella-like species, which were described from tropical rainforests using molecular data, belong either to the trebouxiophycean Watanabea clade (Zhang et al. 2008; Neustupa et al. 2009; Suutari et al. 2010; Song et al. 2015) or to the class Chlorophyceae (e.g., Jenufa; Němcová et al. 2011; Hodač et al. 2015). Preliminary cloning studies (unpublished data of the coauthors of this study) on the diversity of terrestrial green microalgae in Ecuadorian rainforests revealed only Chlorella sequences related to C. sorokiniana, a putatively pantropic and thermotolerant species. Remarkably, common terrestrial species such as Chlorella vulgaris or Muriella terrestris were not detected, although the approach did uncover common species of Stichococcus, which were almost identical to isolates from European soils and freshwaters. The Stichococcus species with temperate-tropical distribution were provisionally denominated as Stichococcus clade1, S. clade3 and S. clade5, and do not contain any psychrotolerant strains. The same is true for the first Stichococcus described from the tropics, S. jenerensis SAG 2138 (Southeast Asia; Neustupa et al. 2007). The discovery of S. jenerensis as well in Ecuador (KS126SM6L) confirmed the pantropic-temperate distribution of the $S$. jenerensis clade.

The deepest divergence within the Stichococcus clade (Sluiman and Guihal 2008), or Stichococcus/Prasiola clade (Neustupa et al. 2007) occurred in the Mesozoic, during the transition from the Lower Jurassic to Middle Cretaceous (De Wever et al. 2009). According to the same study, the deepest splits within the Chlorellaceae would be dated much earlier, i.e., approximately in the Lower Devonian to Lower Jurassic. The earliest divergence of the Antarctic versus non-Antarctic species within the Chlorella clade would thus have happened during the Cenozoic (Upper Paleocene to Upper Oligocene). Our accordingly calibrated phylogeny suggests a remarkable coincidence of (1) species divergence in the Chlorella clade and (2) species divergence in the Stichococcus clade. During the same period in the Triassic, the following splits resulting in new lineages might have appeared: split 1) Stichococcus jenerensis (rod-shapes; temperate-pantropic distribution) and Diplosphera (rod-shapes/cell packages; temperate-tropicarid-polar distribution); split 2) Stichococcus bacillaris (rod-shapes; temperate distribution) and Desmococcus (cell packages/filaments; temperate-polar distribution); split 3) Stichococcus clade3 (rod-shapes; temperate-tropical distribution) and S. clade4+clade5 (rod-shapes/cell packages; temperate-tropical-polar(?) distribution). In summary, Stichococcus- and Diplosphaera/Desmococcus-like morphologies evolved multiple times within the Stichococcus clade, possibly in successive order. The morphological plasticity of the Stichococcus lineages could explain its success in colonizing almost all terrestrial substrates (soil, epiphytic, epixylic, 
epilithic, endolithic, lichens). In contrast to Stichococcus, the lineages of terrestrial Chlorellaceae are morphologically uniform, without any signs of multicellularity. The strikingly poor phylogenetic diversity of the Chlorellaceae found in humid tropical rainforests might be, apart from a simple sampling effect, due to low competitiveness. Instead, Chlorellaceae successfully colonized low-competitive microbial communities of extreme cold and hot terrestrial environments. Nonetheless, most regions on Earth have not been sampled yet. Although polar research undoubtedly contributes valuable biodiversity data, the picture of algal biogeography should be completed by much more samplings in rapidly disappearing biodiversity hotspots such as tropical rainforests.

\section{Funding}

The work was supported by the Priority Program 1374 "Infrastructure-BiodiversityExploratories" of the German Research Foundation (DFG) [grant number DFG-FR 905/16-1, FR 905/17-1] extended to T.F.; by the German Federal Ministry of Education and Research [grant number BMBF UKR 08/038] extended to T.F.; by the German Academic Exchange Service (DAAD; doctoral scholarship extended to L.H.) and by Ministry of Education, Youth and Sports of the Czech Republic [grant number: LM2010009, RVO67985939] extended to J.E.

\section{Acknowledgements}

We thank the managers of the three Exploratories, Kirsten Reichel-Jung, Swen Renner, Katrin Hartwich, Sonja Gockel, Kerstin Wiesner, and Martin Gorke for their work in maintaining the plot and project infrastructure; Christiane Fischer and Simone Pfeiffer for giving support through the central office, Michael Owonibi for managing the central data base, and Markus Fischer, Eduard Linsenmair, Dominik Hessenmöller, Jens Nieschulze, Daniel Prati, Ingo Schöning, François Buscot, Ernst-Detlef Schulze, Wolfgang W. Weisser and the late Elisabeth Kalko for their role in setting up the Biodiversity Exploratories project. Field work permits were issued by the responsible state environmental offices of Baden-Württemberg, Thüringen, and Brandenburg (according to $§ 72$ BbgNatSchG). We thank Alena Lukešová and Klára Řeháková for providing us with the cultures of polar Chlorella. We further thank Kathrin I. Mohr and Kristin C. Pahlmann for their sequencing efforts and Maike Lorenz for depositing new algal strains in the Culture Collection of Algae at the University of Göttingen (SAG). 


\title{
Chapter 4
}

\section{Molecular evidence for the wide distribution of two lineages of terrestrial green algae (Chlorophyta) from tropics to temperate}

\author{
zone \\ Ladislav Hodač ${ }^{1}$, Christine Hallmann ${ }^{1}$, Helen Rosenkranz ${ }^{2}$, Fabian Faßhauer ${ }^{1}$ and Thomas Friedl ${ }^{1}$ \\ ${ }^{1}$ University of Göttingen, Experimental Phycology and Culture Collection of Algae (SAG), Göttingen, Germany \\ ${ }^{2}$ School of Biological Sciences, University of Bristol, Bristol, United Kingdom
}

(Published in ISRN Ecology; doi: 10.5402/2012/795924)

\begin{abstract}
Phylogenetic analyses of $18 \mathrm{~S}$ rDNA sequences from environmental clones and culture strains revealed a widespread distribution of two subaerial green algal lineages, Jenufa and Xylochloris, recently described from rainforests in Southeast Asia. A new lineage of Jenufa (Chlorophyceae), most closely related to or even conspecific with J. minuta, was formed by sequences of European origin. Two more lineages of Jenufa were formed by three additional sequences from Ecuador and Panama. The other lineage was a close relative of Xylochloris irregularis (Trebouxiophyceae), probably representing a new species of the genus and distinct from the only so far described species, $X$. irregularis. It comprised two distinct clades each containing almost identical sequences from Germany and Ecuador. Analyses of the new sequences for both genera allowed to presume a preference of $J$. minuta to subaerial growth on rock or artificial hard substrates combined with a remarkable adaptation to extended periods of darkness, whereas Xylochloris may preferably occur on tree bark or in the soil.
\end{abstract}

Keywords: green algae; Jenufa; Xylochloris; molecular phylogeny; 18S rDNA; biogeography; substrate specificity

Abbreviations: BioNJ, Bio-neighbor-joining; CS, chlorophyte superclades including Chlamydomonadales and Sphaeropleales; MB, Bayesian inference; ML, Maximum likelihood; MP, Maximum parsimony; NMDS, Non-metric multidimensional scaling 


\section{Introduction}

The neutral dispersal model (Fenchel and Finlay 2004) suggests that microorganisms do not exhibit biogeography, i.e. they are so small that no distributions barriers exist for them. This has been subject of debates by several authors, i.e. geographic distribution was found in ciliates

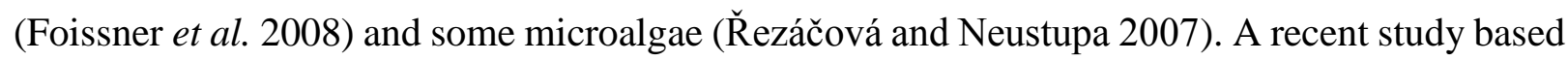
on 18S rDNA sequence comparisons provided evidence for endemism in an Antarctic habitat, i.e. several new independent lineages of green algae were found in the benthos of an Antarctic lake (Lawley et al. 2004; De Wever et al. 2009). However, caution needs to be applied because there may still be an insufficient number of sequences available for many groups within the green algae. A lineage may appear "endemic" only as long as no other close relatives to it are known and this may easily change with the availability of new sequences from close relatives. In contrast to benthic freshwater algae, subaerial algae may be widely dispersed due to their remarkable adaptation to fast changing and adverse environmental conditions. Their resting cysts may easily survive transportation over long distances in the air being resistant to drought, high as well as low light intensities, and high UV radiation, e.g. due to the presence of thickened cell walls or light protection pigments ( ̌̌ezanka et al. 2004; Karsten et al. 2005; Häubner et al. 2006; Gustavs et al. 2010).

Recently, subaerial microalgae from Southeast Asian tropical rainforest habitats gained increased interest by a series of studies (Eliáš et al. 2008; Neustupa et al. 2009; Eliáš et al. 2010; Němcová et al. 2011; Neustupa et al. 2011) which lead to the establishment of two new genera of green algae, Jenufa (Němcová et al. 2011) and Xylochloris (Neustupa et al. 2011). Both genera appeared as somehow distant to any known genera of green algae, despite a taxon sampling as large as possible has been applied in the 18S rDNA sequence analyses of both studies. The environmental sequencing approach extends the data pool available for diversity comparisons and may enable us to assess distribution patterns of protists (Šlapeta et al. 2005; Edgcomb et al. 2011). In the present study we took the advantage of an extended taxon sampling available through new 18S rDNA environmental sequences we obtained from our own ongoing studies which focus on changes in the algal diversity of certain terrestrial habitats along gradients of abiotic parameters in Europe (Germany, Ukraine) and South America (Ecuador). In addition, a variety of closely related environmental sequences have become available from GenBank. Here we demonstrate the presence of new lineages for both recently described genera, Jenufa (Chlorophyceae) and Xylochloris (Trebouxiophyceae), their wide distribution over long geographic distances and that both differ in their preferences towards certain substrates. 


\section{Materials and methods}

\section{Origin of analyzed sequences}

The analyzed sequences and culture strains were provided by several our own on-going studies which focus on the algal diversities of various terrestrial habitats, see Table 1. They were derived from soil, tree bark or stone surfaces samples at four temperate habitats and one tropical habitat (Table 1). The majority of sequences were from environmental 18S rDNA clone libraries (3341761 base pairs long), three from cultured strains which were accessioned by the SAG culture collection as strains SAG 2379, SAG 2382, and SAG 2383. Soil and tree bark samples originating from Germany were collected within the frame of the German Biodiversity Exploratories project (http://www.biodiversity-exploratories.de), for project description see Fischer et al. (2010) (Fig. 1a). Samples of biofilms dominated by green algae were investigated from the surface of sandstone samples within the framework of another study, focusing on wall sections of the castle "Burg Gleichen", near Gotha (Thuringia, Germany) (Hallmann et al. 2011a). The biofilm samples were either from a sun-exposed wall area (Fig. 1b) or from the inner face of a scale from a dark basement vault (Fig. 1c). Samples from an even less light-exposed locality were from the concrete walls inside a World War II bunker monument on the North Sea offshore island Helgoland, Germany (Fig. 1d-e). Here the algal biofilms were exposed to extended periods of darkness because light is available from fluorescent tubes only during guided tours for tourists, i.e., 1.5 hours per day and around 8 months per years. The soil sample from Ukraine was from the arid steppe zone (Chernomorskiy Biosphere Reserve). The soil and tree bark samples from South America originated from the tropical mountain rain forest in Southern Ecuador along an elevation transect at $1000 \mathrm{~m}, 2000 \mathrm{~m}$ and $3000 \mathrm{~m}$ above sea level (a.s.l.) corresponding to three different regions (Table 1; Fig. 1f-g).

\section{Microscopy and rDNA sequence determination}

Microscopic observations of cultures were conducted using an Olympus BX60 microscope (Tokyo, Japan) with Nomarski DIC optics and an attached ColorView III camera (Soft Imaging System, Münster, Germany). Micrographs were processed using Cell^D image software (Soft Imaging System, Münster, Germany). The detailed experimental procedures on how the $18 \mathrm{~S}$ rDNA clone libraries and cultures were established will be described elsewhere. Briefly, DNA was extracted from environmental samples using MoBio PowerSoil DNA isolation Kit (MoBio Laboratories Inc. Carlsbad, CA, USA) and from mechanically disrupted cultured algal cells using Invisorb® Spin Plant Mini Kit (Stratec, Berlin, Germany). Almost full length 18S rDNA 
sequences were amplified from the cultures and European environmental samples with primers preferentially binding to green algal rDNAs, for the samples from Ecuador a region from about 350 base pairs upstream of the 3' end of $18 \mathrm{~S}$ rDNA downstream to the $26 \mathrm{~S}$ rDNA which included the ITS2 rDNA for DNA barcoding purposes was amplified. Either almost full 18S rDNA sequences were obtained or partial sequences comprising either the hypervariable regions $\mathrm{V} 2, \mathrm{~V} 3$ and V4 (European environmental samples) or hypervariable regions V8 and V9 regions (Ecuador environmental samples) of 18S rDNA (Neefs and De Wachter 1990; Lee and Gutell 2012). The newly determined sequences are available from GenBank under the accession numbers JQ988923-JQ988943.

Table 1. List of new sequences obtained for this study.

\begin{tabular}{|c|c|c|c|c|c|c|c|}
\hline Taxon & $\begin{array}{l}\text { Clone identifier } \\
\text { ('cultured strains) }\end{array}$ & $\begin{array}{l}\text { Accession } \\
\text { number }\end{array}$ & $\begin{array}{l}\text { Length } \\
\text { (base pairs) }\end{array}$ & Region of origin & GPS-coordinates & Habitat & Substrate \\
\hline \multirow[t]{12}{*}{ Xylochloris } & AEG1B-K1547 & JQ988937 & 748 & $\begin{array}{l}\text { Germany, } \\
\text { Swabian Alb }\end{array}$ & $48^{\circ} 23^{\prime} \mathrm{N}, 9^{\circ} 20^{\prime} \mathrm{E}$ & Grassland & Soil \\
\hline & AEW2R-K255 & JQ988938 & 1734 & $\begin{array}{l}\text { Germany, } \\
\text { Swabian Alb }\end{array}$ & $48^{\circ} 37^{\prime} \mathrm{N}, 9^{\circ} 35^{\prime} \mathrm{E}$ & Spruce forest & $\begin{array}{l}\text { Tree bark, } \\
\text { Picea abies }\end{array}$ \\
\hline & AEW2R-K265 & JQ988934 & 842 & $\begin{array}{l}\text { Germany, } \\
\text { Swabian Alb }\end{array}$ & $48^{\circ} 37^{\prime} \mathrm{N}, 9^{\circ} 35^{\prime} \mathrm{E}$ & Spruce forest & $\begin{array}{l}\text { Tree bark, } \\
\text { Picea abies }\end{array}$ \\
\hline & BOAEW3R1-K03 & JQ988939 & 716 & $\begin{array}{l}\text { Germany, } \\
\text { Swabian Alb }\end{array}$ & $48^{\circ} 41^{\prime} \mathrm{N}, 9^{\circ} 35^{\prime} \mathrm{E}$ & Spruce forest & $\begin{array}{l}\text { Tree bark, } \\
\text { Picea abies }\end{array}$ \\
\hline & SAG $2382^{*}$ & JQ988942 & 1719 & $\begin{array}{l}\text { Germany, } \\
\text { Swabian Alb }\end{array}$ & $48^{\circ} 39^{\prime} \mathrm{N}, 9^{\circ} 26^{\prime} \mathrm{E}$ & Beech forest & Soil \\
\hline & HEG9B-K2617 & JQ988940 & 1702 & Germany, Hainich & $51^{\circ} 13^{\prime} \mathrm{N}, 10^{\circ} 22^{\prime} \mathrm{E}$ & Grassland & Soil \\
\hline & HEW10SB-K5621 & JQ988935 & 854 & Germany, Hainich & $51^{\circ} 05^{\prime} \mathrm{N}, 10^{\circ} 27^{\prime} \mathrm{E}$ & Beech forest & Soil \\
\hline & HEW4SB-K5577 & JQ988936 & 854 & Germany, Hainich & $51^{\circ} 24^{\prime} \mathrm{N}, 10^{\circ} 32^{\prime} \mathrm{E}$ & Beech forest & Soil \\
\hline & HEW4SB-K5606 & JQ988941 & 733 & Germany, Hainich & $51^{\circ} 24^{\prime} \mathrm{N}, 10^{\circ} 32^{\prime} \mathrm{E}$ & Beech forest & Soil \\
\hline & U33B-K2708 & JQ988943 & 628 & $\begin{array}{l}\text { Ukraine, Ascania } \\
\text { Nova }\end{array}$ & $46^{\circ} 27^{\prime} \mathrm{N}, 33^{\circ} 54^{\prime} \mathrm{E}$ & Steppe & Soil \\
\hline & C32U6-13 & JQ988925 & 334 & $\begin{array}{l}\text { Ecuador, } \\
\text { Cajanuma }\end{array}$ & $04^{\circ} 06^{\prime} \mathrm{S}, 079^{\circ} 10^{\prime} \mathrm{W}$ & Rainforest 3000 m a.s.l. & Soil \\
\hline & S30L6-32 & JQ988926 & 334 & $\begin{array}{l}\text { Ecuador, San } \\
\text { Francisco }\end{array}$ & $03^{\circ} 58^{\prime} \mathrm{S}, 079^{\circ} 04^{\prime} \mathrm{W}$ & Rainforest 2000 m a.s.l. & Tree bark \\
\hline \multirow[t]{8}{*}{ Jenufa } & 3GA1K3658 & JQ988931 & 851 & $\begin{array}{l}\text { Germany, Drei } \\
\text { Gleichen }\end{array}$ & $50^{\circ} 52^{\prime} \mathrm{N}, 10^{\circ} 50^{\prime} \mathrm{E}$ & Castle Gleichen & $\begin{array}{l}\text { Sandstone, } \\
\text { surface }\end{array}$ \\
\hline & GS1K32 & JQ988932 & 1761 & $\begin{array}{l}\text { Germany, Drei } \\
\text { Gleichen }\end{array}$ & $50^{\circ} 52^{\prime} \mathrm{N}, 10^{\circ} 50^{\prime} \mathrm{E}$ & Castle Gleichen & $\begin{array}{l}\text { Sandstone, } \\
\text { scale } \\
\text { (endolithic) }\end{array}$ \\
\hline & KB2B11-06 & JQ988928 & 1596 & $\begin{array}{l}\text { Germany, } \\
\text { Helgoland }\end{array}$ & $54^{\circ} 11^{\prime} \mathrm{N}, 7^{\circ} 53^{\prime} \mathrm{E}$ & Bunker & Concrete \\
\hline & KB4B5-06 & JQ988929 & 1570 & $\begin{array}{l}\text { Germany, } \\
\text { Helgoland }\end{array}$ & $54^{\circ} 11^{\prime} \mathrm{N}, 7^{\circ} 53^{\prime} \mathrm{E}$ & Bunker & Concrete \\
\hline & SAG 2379* & JQ988927 & 1563 & $\begin{array}{l}\text { Germany, } \\
\text { Helgoland }\end{array}$ & $54^{\circ} 11^{\prime} \mathrm{N}, 7^{\circ} 53^{\prime} \mathrm{E}$ & Bunker & Concrete \\
\hline & SAG 2383* & JQ988933 & 1700 & $\begin{array}{l}\text { Germany, } \\
\text { Swabian Alb }\end{array}$ & $48^{\circ} 38^{\prime} \mathrm{N}, 9^{\circ} 38^{\prime} \mathrm{E}$ & Beech forest & Soil \\
\hline & FLUL-B69M5-34 & JQ988923 & 335 & $\begin{array}{l}\text { Ecuador, } \\
\text { Bombuscaro }\end{array}$ & $04^{\circ} 07^{\prime} \mathrm{S}, 078^{\circ} 58^{\prime} \mathrm{W}$ & Rainforest 1000 m a.s.l. & Tree bark \\
\hline & S46L1-7 & JQ988924 & 338 & $\begin{array}{l}\text { Ecuador, San } \\
\text { Francisco }\end{array}$ & $03^{\circ} 58^{\prime} \mathrm{S}, 079^{\circ} 04^{\prime} \mathrm{W}$ & Rainforest 2000 m a.s.l. & Soil \\
\hline
\end{tabular}



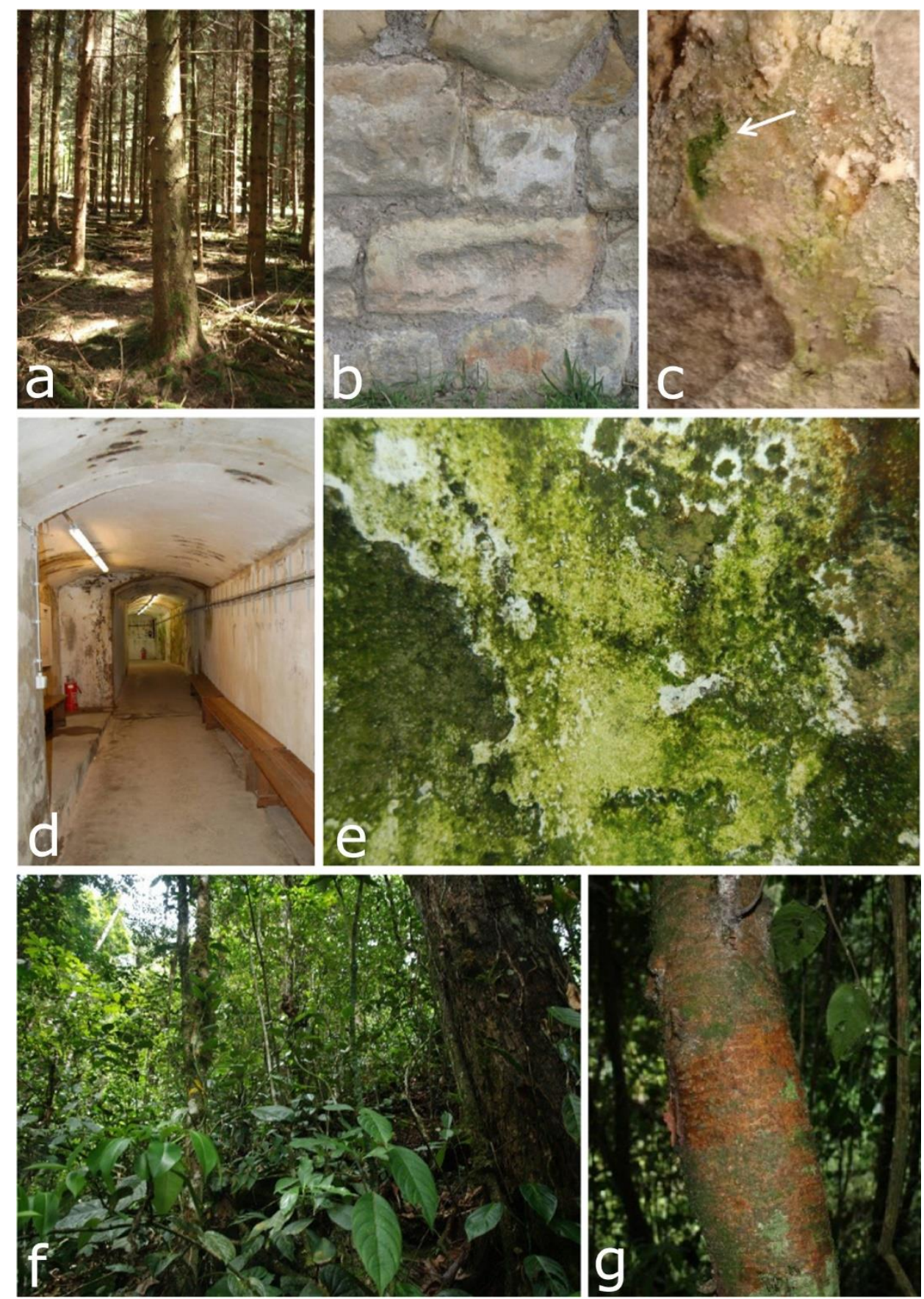

Figure 1. Sampling sites. (a) Young spruce forest in the Swabian Alb, (b-c) wall sections of castle Gleichen (Germany), (b) sunexposed wall area, (c) inner face of a scale from a dark basement vault (the white arrow shows the sampled green biofilm), (d-e) biofilm samples from concrete walls in a World War II bunker on the North Sea offshore island Helgoland, (e) sampled green biofilm in detail, (f-g) tropical sub-mountain rainforest in Ecuador (the study site in Bombuscaro, $1000 \mathrm{~m}$ a.s.l.), and (g) green biofilms on tree bark in detail. 


\section{Phylogenetic analyses}

The newly determined sequences were used in database queries using BLAST (Altschul et al. 1997) at NCBI (http://www.ncbi.nlm.nih.gov/) to retrieve their closest neighboring sequences available from public sequence data bases (state in March 2012). In addition, the newly determined and their retrieved closest relative sequences were compared to a broad selection of green algal sequences which was available in the internal 18S rDNA sequence database of our laboratory and is maintained in the ARB program (version 05.05.26, 2004, www.arb-home.de) (Ludwig et al. 2004). This database was updated with all currently available $18 \mathrm{~S}$ rDNA sequences from green algae. The comparisons identified sequences of both genera, Jenufa and Xylochloris, as well as their next relatives. Potential chimeras were identified by Bellerophon (Huber et al. 2004) and excluded from the analyses. The almost full 18S rDNA sequences of Jenufa, Xylochloris and their close relatives were aligned in two separated data sets, i.e. together with representatives of all known lineages from the two green algal classes, Chlorophyceae and Trebouxiophyceae. First the sequences were aligned with MAFFT ver. 6 (Katoh and Toh 2008) online (http://mafft.cbrc.jp/alignment/server/index.html) in order to identify and exclude intron positions. Then the sequences from each class were aligned with MUSCLE online (http://www.ebi.ac.uk/Tools/msa/muscle). The alignments were carefully checked for possible misaligned positions by eye in BioEdit (Hall 1999). The final alignment of Chlorophyceae comprised 97 sequences and 1790 positions (742 variable/535 parsimony informative sites), the alignment of Trebouxiophyceae included 99 sequences and 1807 positions (710 variable/497 parsimony informative sites). Based on the AIC criterion in jModelTest 0.1.1 (Posada 2008), the $\mathrm{GTR}+\Gamma+\mathrm{I}$ nucleotide substitution model was selected as to fit best both alignments. ML phylogenies were obtained from RAxML 7.0.4 (Stamatakis et al. 2008). Confidence values for the obtained groups (edge support) were inferred from rapid bootstrapping algorithm (1000 replicates) as implemented in RAxML. Bayesian posterior probabilities for internal nodes were obtained using MrBayes 3.2 (Ronquist et al. 2012). Two MCMC runs for four million generations each with one cold and three heated chains using the GTR $+\Gamma+\mathrm{I}+\mathrm{COV}$ evolutionary model (parameters were estimated from the data) were performed with trees sampled every 100 generations. For comparisons of European with Ecuadorian environmental clones for which different regions of the $18 \mathrm{~S}$ rDNA were determined (see above), only the hypervariable regions V8/V9 of the 18S rDNA could be used because they represent an overlapping sequence region available in both data sets (European data set comprised the full sequences whereas the Ecuadorian one the partial ones). Then only sequences of closest relatives were used for the 
phylogenetic analyses, i.e. the investigated datasets comprised less than 10 sequences and were only about 300 base pairs long. Bio-Neighbor-Joining distance phylogenies based on JukesCantor genetic distances and 1000 bootstrap replicates were computed in the program SeaView ver. 4 (Gouy et al. 2010). The parsimony analysis was conducted using DNAPARS as implemented in SeaView with 10 times randomization of the sequence order and 1000 bootstrap replicates. Evolutionary distances among selected sequences were computed as p-distances in MEGA5 (Tamura et al. 2011) with ambiguous alignment positions removed for each sequence pair.

\section{Multivariate statistical analysis}

In order to compare the clones originating from the different substrates within Europe (tree bark, soil, stone surfaces) Non-metric Multidimensional Scaling (NMDS) analysis based on genetic distances was computed. The analysis was conducted on the matrix of Jukes-Cantor genetic distances (within alignments comprising hypervariable regions V2, V3 and V4 of the 18S rDNA) as implemented in the program package PAST ver. 2.12 (Hammer et al. 2001). The reliability of the resulting ordination was examined by checking the stress values from Shepard-plot. The alignment comprising the new sequences of Jenufa minuta relatives included 8 sequences and 658 positions (Fig. 2d), whereas the other comprising the new sequences of Xylochloris-relatives included 10 sequences and 595 positions (Fig. 3d).

\section{Results and Discussion}

\section{Jenufa lineage}

The 18S rDNA phylogeny revealed the position of several of the obtained new environmental sequences and the three cultured isolates into the immediate vicinity of the genera Jenufa (Chlorophyceae, Fig. 2a, 2b, 2d; Fig. 1 in Appendix) and Xylochloris (Trebouxiophyceae, Fig. 3a, 3b, 3d; Fig. 2 in Appendix). We recovered sequences assigned to Jenufa from environmental clone libraries from Germany (soil, hard substrates), Ukraine (soil) and Ecuador (soil, tree bark) (Table 1). The full European environmental sequences and the culture strains formed a wellsupported monophyletic cluster (p-distances 0.001-0.004) closely related to Jenufa minuta. The p-distances among European sequences and J. minuta (0.016-0.019) were about two times lower than between $J$. minuta and $J$. perforata (0.038). The partial environmental sequences from Ecuador constitute a lineage by its own positioned between J. minuta and J. perforata, but without significant bootstrap support (Fig. 2d). As already discussed previously in Němcová et al. (2011), 
Jenufa may represent a distinct probably new lineage of Chlorophyceae of still unresolved relationship to the orders Sphaeropleales and Chlamydomonadales. Within the 18S rDNA phylogeny presented here, Jenufa is placed together with Golenkinia on the very basis of Chlamydomonadales, although without statistical support (ML/MB: 69/0.83 in Fig. 2a; Fig. 1 in supplement). In contrast, in Bayesian tree topology, Jenufa- and Golenkinia clades formed a lineage independent of the Chlamydomonadales and Sphaeropleales. The light-microscopic observations on the two available culture strains SAG 2383 and SAG 2379 were in agreement with their assignment to Jenufa inferred from the $18 \mathrm{~S}$ rDNA phylogenetic analyses. Both strains exhibited morphological characters as previously described for J. minuta and J. perforata (Fig. 4a). Bot species were found indistinguishable from each other without the use of a confocal microscope and the analysis of cell wall structures (Němcová et al. 2011).

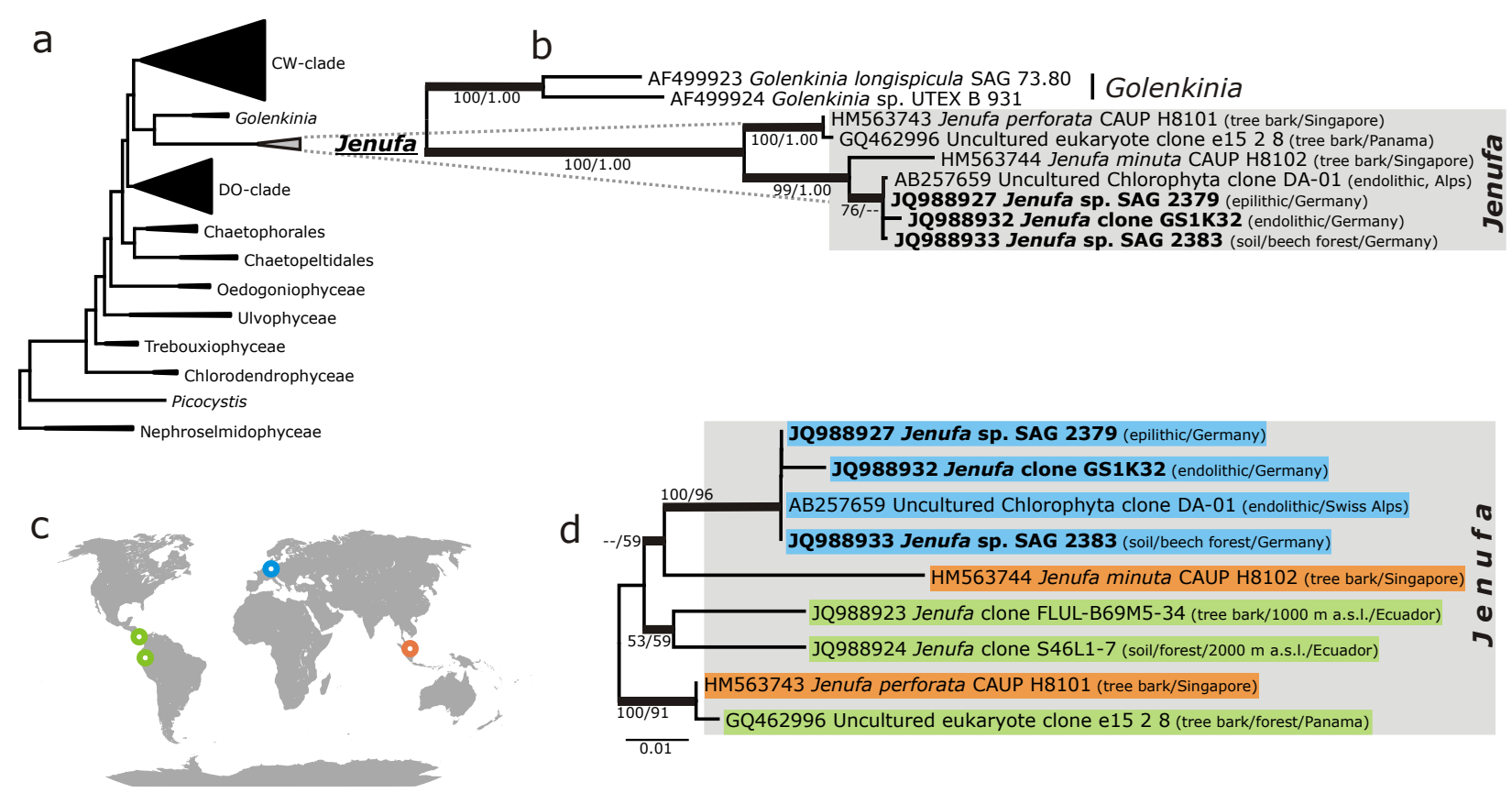

Figure 2. (a) Schematic 18S-based phylogenetic tree of the Chlorophyceae showing the position of sequences assigned to Jenufa minuta, (b) Jenufa/Golenkinia clades in detail shown as a section of the full $18 \mathrm{~S}$ rDNA ML-phylogenetic tree (Fig. S1 in Appendix), (c) world map with dots representing regions where Jenufa sequences were detected, and (d) BioNJ-tree based on partial 18S sequences (hypervariable regions V8 and V9). Colors in figures (c) and (d) indicate three geographical regions where Jenufa occurs (orange-southeast Asia, green-south and Latin America, blue-Europe). Names in bold represent specimens analyzed as both partial- and full-length $18 \mathrm{~S}$ rDNA sequences.

\section{Xylochloris lineage}

Other new environmental sequences and the sequence of strain SAG 2382 were closely neighboring to Xylochloris irregularis (Trebouxiophyceae) in the $18 \mathrm{~S}$ rDNA phylogenetic analyses, but without significant bootstrap support (Fig. 3b). The p-distances among the full European sequences and $X$. irregularis $(0.031-0.035)$ was slightly smaller than those between 
the two previously described species of Jenufa (Chlorophyceae) which may imply that the European sequences and SAG 2382 represent a species independent of $X$. irregularis. The Xylochloris clade comprised environmental clones from soils and tree bark from Germany, Singapore and Ecuador (Fig. 3d). The addition of new sequences supported a sister-group relationship of Xylochloris with a clade comprising of environmental clones from cold-fumarole soils of the Andes (Costello et al. 2009) and two culture strains from terrestrial habitats in Germany assigned to Dictyochloropsis (Fig. 3b, Fig. 2 in Appendix). The latter clade was well supported in bootstrap tests, whereas its sister-group relationship with Xylochloris was only supported in MB analyses. Strain SAG 2382 shared several morphological characters with $X$. irregularis (Neustupa et al. 2011), i.e. the presence of a pyrenoid, the lobed chloroplast in vegetative cells, similar cell size, and the irregular cell shape. However, the strain also exhibited distinguishing characters of its own, namely the presence cylindrical as well as spherical autospores at the same time (Fig. 4 b-d).

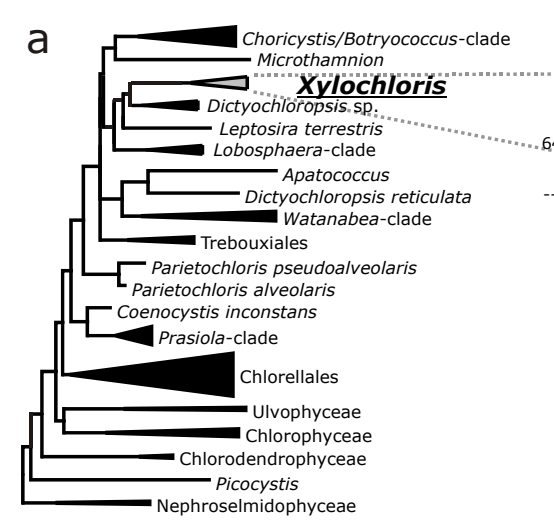

C

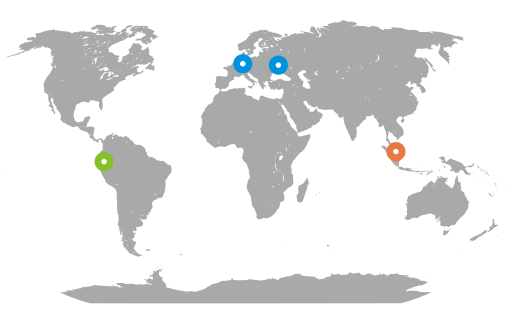

b
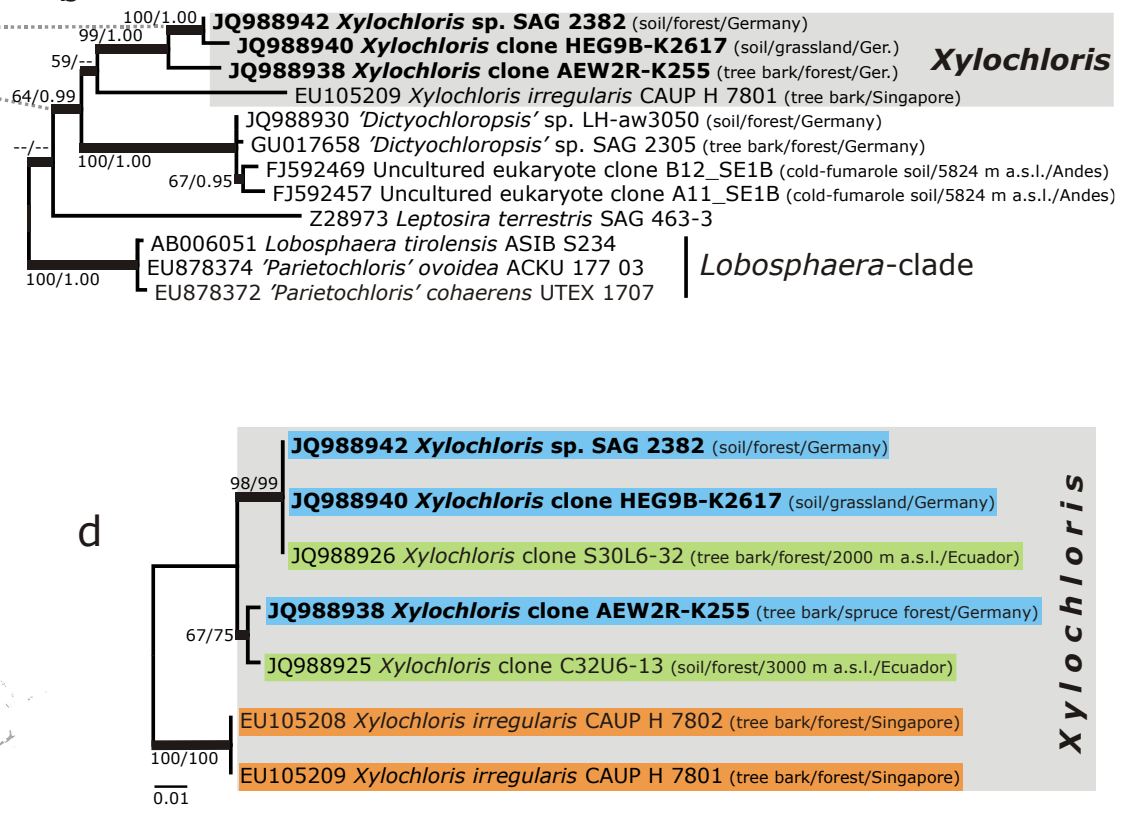

Figure 3. (a) Schematic 18S-based phylogenetic tree of the Trebouxiophyceae showing the placement of sequences assigned to Xylochloris, (b) the clade including Xylochloris irregularis and its closest relatives from Europe shown as a section of the full $18 \mathrm{~S}$ rDNA ML-phylogenetic tree of the Trebouxiophyceae (Fig. S2), (c) world map with dots representing regions where Xylochloris sequences were detected, and (d) BioNJ-tree based on partial 18S sequences (hypervariable regions V8 and V9). Colors in Figures (c) and (d) indicate three geographical regions where Xylochloris occurs (orange-southeast Asia, greenSouth and Latin America, blue-Europe). Names in bold represent specimens analyzed as both partial- and full-length 18S rDNA sequences. 

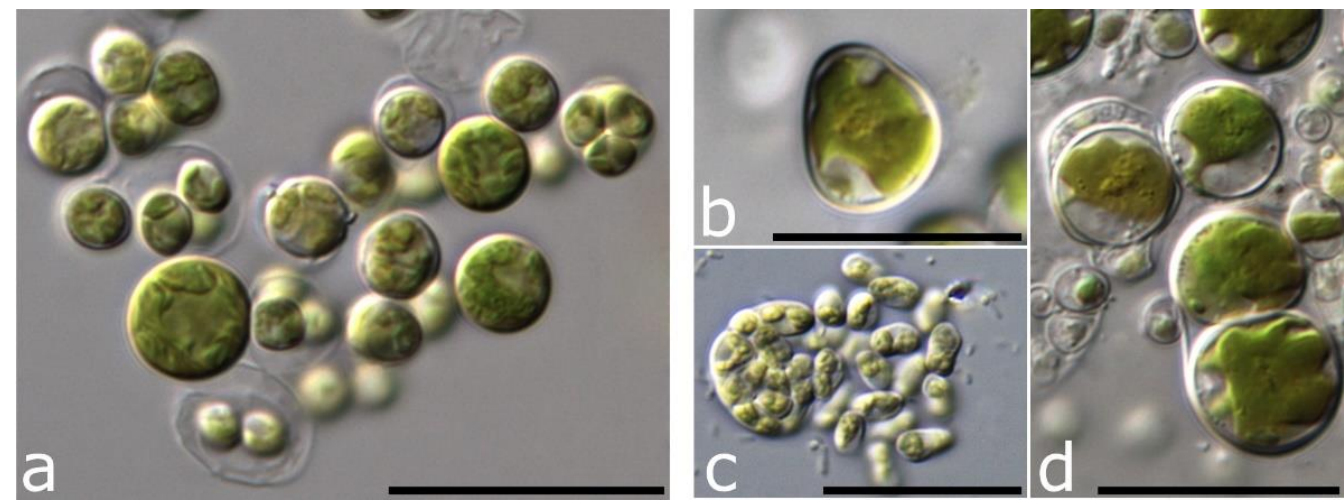

Figure 4. Microphotographs of the strains SAG 2383 (a) and SAG 2382 (b-d), (a) vegetative cells and autospores of the European Jenufa strain SAG 2383 isolated from a soil sample from Swabian Alb (Germany), (b) vegetative cell of Xylochloris strain SAG 2382 exhibiting the presence of one pyrenoid, (c) irregular cylindrical to oval autospores typical for the strain, and (d) vegetative cells with lobed margins of the chloroplast. Scale bar represents $20 \mu \mathrm{m}$.

\section{Substrate preferences of Jenufa and Xylochloris}

Jenufa (Chlorophyceae) and Xylochloris (Trebouxiophyceae) represent distinct lineages of terrestrial green algae without any known members from freshwater habitats. Despite Jenufa and Xylochloris were detected in both habitats, soils and tree barks, in the tropics, the sequence analysis of their closest relatives from Europe suggests a pattern reflecting different substrate preferences for both genera. In our study Jenufa was recovered from hard substrates, except for one isolate (SAG 2383) established from soil. The hard substrates were epilithic biofilms (surface of bunker walls on Helgoland, outer surface of walls of castle Gleichen, walls of Altamira cave in Spain) as well as endolithic biofilms (rocks in Piora Valley/1,965 m a.s.1./Swiss Alps; Horath and Bachofen 2009), or and the upper side of wall scales on castle Gleichen) Although the NMDS analysis (Fig. 5) of European partial sequences did not point out any clear groupings due to substrate type (endolithic versus epilithic/soil), the second ordination axis indicated variation among sequences due to different light intensities at the sampling sites. This may be seen among Jenufa sequences from the Helgoland bunker monument. Two sequences, (KB2B11-06 and SAG 2379 , originating from two sites of low irradiance within the bunker were genetically closer than both to another Jenufa sequence, KB4B5-06, from a site of three times higher irradiance (HR unpublished results). In addition, the genetic differences between two Jenufa sequences, 3GA1K3658 from epilithic and GS1K32 from endolithic habitats of the Gleichen castle sandstone walls, may be explained by adaptation to different light intensities as well, as pointed out by the NMDS-plot (Fig. 5). The newly determined Xylochloris sequences were all recovered from tree bark as well as from soils. The NMDS-analysis pointed out a separation of two groups of very closely related sequences from tree bark against from those from soils (Fig. 6). These two groups may represent even two separate taxa at the level of species or below, as already discussed 
above. Members of both groups were recovered also from Ecuador, hence both may be widespread as well.

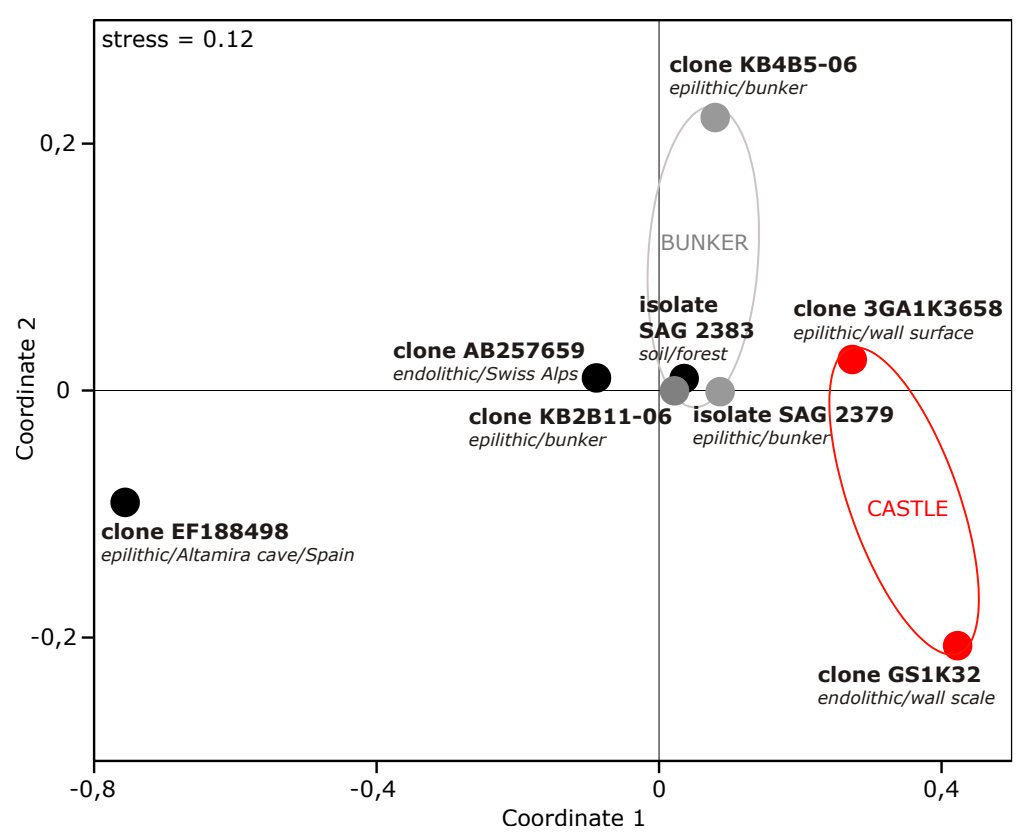

Figure 5. Nonmetric multidimensional Scaling (NMDS) diagram. The ordination analysis based on the Jukes-Cantor genetic distances among six environmental clones and two isolates representing sequences of Jenufa minuta detected in Europe on various hard substrates. For the analysis, 18S partial sequences comprising hypervariable regions $\mathrm{V} 2, \mathrm{~V} 3$, and $\mathrm{V} 4$ were used. The red-colored points represent sequences from the walls of castle Gleichen/Germany, the blue ones are from the bunker on Helgoland, an offshore island in the North Sea. For the corresponding accession numbers of the sequences see Table 1.

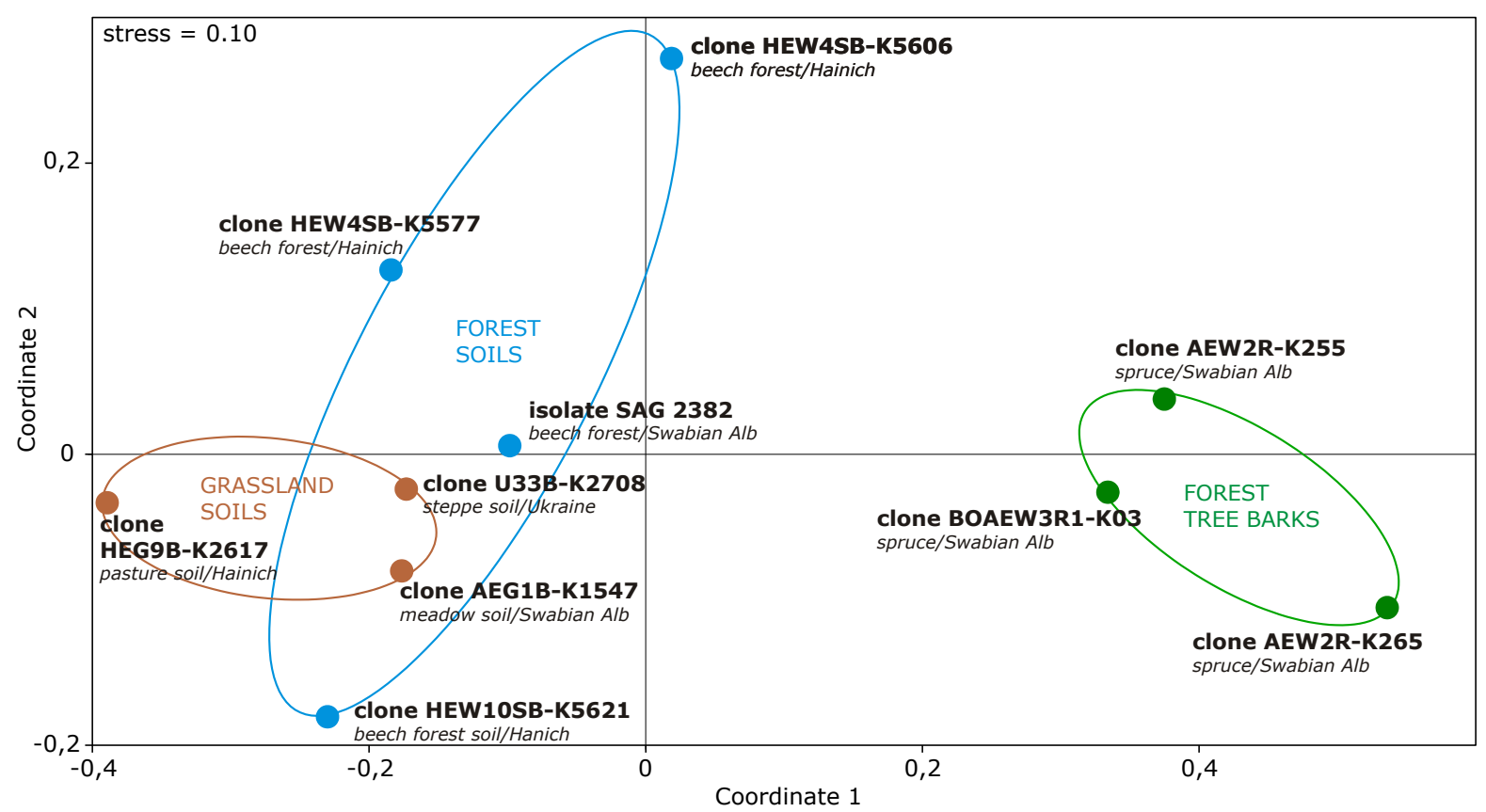

Figure 6. Nonmetric multidimensional scaling (NMDS) diagram. The ordination based on Jukes-Cantor genetic distances among nine environmental clones and one 1 isolate representing a yet undescribed European species of the genus Xylochloris. For the analysis $18 \mathrm{~S}$ partial sequences comprising hypervariable regions $\mathrm{V} 2, \mathrm{~V} 3$, and $\mathrm{V} 4$ were used. The colors encode for three different habitats: green-tree bark, blue-forest soil, brown—grassland soil. For the corresponding accession numbers of the sequences see Table 1. 


\section{Identification of environmental rDNA clones using new cultured isolates}

A high 18S rDNA sequence similarity between strains of closely related terrestrial green algae from tropics and temperate habitats has already been presented recently (Němcová et al. 2011). Two cultured isolates of J. minuta from the tropics were found closely related to hitherto unidentified environmental clones from the Swiss Alps. This example shows that unidentified environmental clones may become identified as soon as cultures of close relatives become available, at least at the generic level if a morphology-based species or generic concept is applied. In our study we revealed two new isolates, SAG 2379 and SAG 2383, whose sequences were almost identical (p-distances 0.001-0.004) to the environmental clones from the Swiss Alps. Another case where environmental clones became identified due to the availability of culture strains was found in the "Dictyochloropsis" clade, sister with Xylochloris in the Trebouxiophyceae (Fig. 3d). Due to the very low genetic distances (p-distances $0.001-0.005$ ) present within the clade, the strains of Dictyochloropsis from tree barks in Germany and the environmental clones from the high Andes Mountains (Chile) may be regarded as member of a single species which again exhibits a wide geographic distribution. The genetic distances between the Andes clones/Dictyochloropsis pairs were even shorter (0.003-0.004) than between both Andes environmental clones (0.005). In conclusion, for a better understanding of the biogeography of terrestrial microalgae it seems to be of crucial importance not only to sequence environmental clones from somehow exotic or extreme habitats, but also to establish and sequence cultures from seemingly well investigated regions like Central Europe. For the studies on biogeography of terrestrial algae we are still faced with the lack of sufficient molecular data to compare new findings to, because the most diversity evidences from either Central Europe or many other geographical regions relies on morphological observations only (Hoffmann et al. 2007; Neustupa and Škaloud 2008; Büdel et al. 2009; Khaybullina et al. 2010). We expect the situation to change in the future through extended application of the next generation sequencing (e.g. 454-pyrosequencing) concentrated on hypervariable regions of the 18S rDNA (AmaralZettler et al. 2009).

\section{Acknowledgements}

This study would not have been possible without the stipend of the DAAD extended to L.H. This work was supported by two grants from the German Research Foundation (DFG) extended to T.F. (contract no. FR 905/16-1 and FR 905/17-1) and a grant from the German Federal Ministry of Education and Research (BMBF) extended to T.F. (contract no. UKR 08/038). Parts of the 
work were supported by the USA National Science Foundation (DEB-0529737) to Louise A. Lewis, University of Connecticut, in collaboration with T.F. T.F. and H.R. thank Kathrin I. Mohr for initiating and supervising the study of algal biofilms of the Helgoland bunker, T.F. thanks Igor Y. Kostikov for provision of the opportunity to study samples from the steppe zone of Ukraine within the project BMBF UKR 08/038. C.H. acknowledges the financial support provided by the Deutsche Bundesstiftung Umwelt (DBU) extended to M. Hoppert, Göttingen. We acknowledge provision of environmental sequences determined by a student summer course in 2011 (project FoLL of Göttingen University). We also thank two anonymous reviewers for their critical comments on the manuscript. 


\section{General Summary}

Green microalgae (Chlorophyta) dominate soils in the northern temperate climate zone, however, their biodiversity is still poorly understood. Available diversity inventories rely on light microscopy and morphospecies, which are hardly distinguishable without molecular markers. Whereas particular attention was paid to molecular diversity of terrestrial microalgae in extreme regions, temperate climate zones remain almost unexplored. We aim to uncover the phylogenetic diversity of green microlagae isolated from Central European soils and from periodically desiccating freshwater creek biofilms. Such terrestrial and semi-aquatic habitats are inhabited by green microalgae, which are presumably able of long-distance dispersal. Their cosmopolitan distribution is presumed, however, supporting molecular evidence is almost missing. By assembling newly obtained green algal sequences together with accessions from remote geographic regions, we further aim to address a question of biogeography of terrestrial microalgae.

Soil samples were taken from grassland and forest plots within the German Biodiversity Exploratories. Freshwater biofilms dominated by green microalgae were sampled in two karstwater creeks in Germany. In total, 280 new monoclonal cultures of green microalgae were examined by molecular phylogenetic methods and by light microscopy. By using ribosomal $18 \mathrm{~S}$ and ITS2 sequences, we recognized about 100 monophyletic species of green microalgae. The newly obtained sequences were blasted against public databases in order to infer taxonomy, distribution and ecology of the detected species. Further monoclonal cultures and environmental clones originated from additional samplings in Germany, Ecuador, the Arctic and Antarctic.

Most Chlorophyta isolated from German soils were highly similar ( $\geq 99.5 \%$ threshold) to cultured relatives already known from Europe, predominantly from soils and further terrestrial substrates such as tree barks and rocks. Considering a lower similarity threshold ( $\geq 99 \%)$, about $90 \%$ of our cultures matched environmental clones inferred from various terrestrial and aquatic habitats. Similarly, Chlorophyta detected in creek biofilms partly represent terrestrial species, but some are known also from planktonic communities. In soils, we detected novel species mostly within established lineages. Only one soil isolate, provisionally named Navichloris fusiformis, was recognized as a member of a novel monophyletic lineage, comprising accessions from South and North American deserts. In multiple cases, molecular data supported close relatedness between the European soil isolates and species from tropics and polar regions. We show, that well supported monophyletic clades of Stichococcus exhibit either temperate-polar or temperate- 
tropical distributions. In contrast, some monophyletic clades of Chlorella-like microalgae were so far evidenced only from polar regions and hot deserts. The long-distance dispersal was finally confirmed for particular species of Stichococcus, Chlorella and Klebsormidum.

Forest and grassland soils in Central Europe host species of green microalgae otherwise known from a broad spectrum of terrestrial as well as aquatic habitats. New species and even novel lineages can still be uncovered by using standard culturing techniques. Despite the striking similarity between some European and exotic species, unambiguous molecular evidence of the intraspecific long-distance dispersal is still scant. At the present state of knowledge, our data suggest the existence of biogeography of airborne microalgae. 


\section{References}

Achtman M, Wagner M. 2008. Microbial diversity and the genetic nature of microbial species. Nature Reviews Microbiology 6(6):431-440.

Adl SM, Simpson AG, Farmer MA, Andersen RA, Anderson OR, Barta JR, Bowser SS, Brugerolle G, Fensome RA, Fredericq S, James TY, Karpov S, Kugrens P, Krug J, Lane CE, Lewis LA, Lodge J, Lynn DH, Mann DG, McCourt RM, Mendoza L, Moestrup O, Mozley-Standridge SE, Nerad TA, Shearer CA, Smirnov AV, Spiegel FW, Taylor MF. 2005. The new higher level classification of eukaryotes with emphasis on the taxonomy of protists. Journal of Eukaryotic Microbiology 52(5):399-451.

Aguilera A, Zettler E, Gomez F, Amaral-Zettler L, Rodriguez N, Amils R. 2007. Distribution and seasonal variability in the benthic eukaryotic community of Rio Tinto (SW, Spain), an acidic, high metal extreme environment. Systematic and Applied Microbiology 30(7):531-546.

Aguilera Á, Souza-Egipsy V, González-Toril E, Rendueles O, Amils R. 2010. Eukaryotic microbial diversity of phototrophic microbial mats in two Icelandic geothermal hot springs. International Microbiology 13(1):21-32.

Al-Fredan M, Fathi A. 2007. Preliminary survey of edaphic algae in Al-Hasa region, Saudi Arabia. Pakistan Journal of Biological Sciences 10(18):3210-3214.

Altschul SF, Madden TL, Schaffer AA, Zhang J, Zhang Z, Miller W, Lipman DJ. 1997. Gapped BLAST and PSI-BLAST: a new generation of protein database search programs. Nucleic Acids Research 25(17):3389-3402.

Amaral-Zettler LA, McCliment EA, Ducklow HW, Huse SM. 2009. A method for studying protistan diversity using massively parallel sequencing of V9 hypervariable regions of small-subunit ribosomal RNA genes. PLoS ONE 4(7):e6372.

Andreyeva V. 1973. Novye vidy Chlorella Beijer. Botanicheskij Zhurnal 58:1735-1741.

Andreyeva VM. 1998. Pochvennye i aerophilnye zelenye vodorosli: Chlorophyta (Tetrasporales, Chlorococcales, Chlorosarcinales). Nauka. 315 pp.

Androsova EJ. 1964. O sostave vodoroslej pochv g. Novosibirska i ego okrestnostej. Aktualnye problemy sovremennoj algologii. Tscherkassy. p. 23-25.

Archibald PA, Bold HC. 1970. Phycological Studies XI. The Genus Chlorococcum Meneghini. University of Texas Publication No. 7015. 115 pp.

Archibald PA. 1972. A preliminary survey of the edaphic algae of Costa Rica and San Andreas Isle. Soil Science 113:63-64.

Archibald PA, Bold HC. 1975. Notes on the edaphic algae of the Galapagos. Soil Science 120:400-402.

Arp G, Bissett A, Brinkmann N, Cousin S, Reimer A, Shiraishi F, Stackebrandt E, Zippel B. 2010. Tufa-forming biofilms of German karstwater streams: microorganisms, exopolymers, hydrochemistry and calcification. Geological Society, London, Special Publications. p. 83-118.

Arvik JH, Willson DL. 1974. Soil algae of Eniwetok Atoll, The Marshall Islands. Pacific Science 28(2):189-190.

Asfandijarova LZ. 2008. Analiz pochvennoj algoflory posevov mnogoletnikh trav Preduralja respubliki Bashkortostan. Fundamentalnye i prikladnye problemy botaniki v nachale XXI veka. Algologija. p. 10-12. 
Ashley J, Rushforth SR, Johansen JR. 1985. Soil algae of cryptogamic crusts from the Uintah Basin, Utah, USA. Western North American Naturalist 45(3):432-442.

Bachura JM, Khramchenkova OM. 2008. Osobennosti vosstanovlenija algogruppirovok pochv na kostrishchakh. Fundamentalnye i prikladnye problemy botaniki v nachale XXI veka. Algologija. p. 13-16.

Bailly J, Fraissinet-Tachet L, Verner M-C, Debaud J-C, Lemaire M, Wésolowski-Louvel M, Marmeisse R. 2007. Soil eukaryotic functional diversity, a metatranscriptomic approach. The ISME Journal 1(7):632-642.

Baker BJ, Tyson GW, Goosherst L, Banfield JF. 2009. Insights into the diversity of eukaryotes in acid mine drainage biofilm communities. Applied and Environmental Microbiology 75(7):2192-2199.

Balezina L. 1974. Effect of concentrated mineral fertilizers on the growth of soil algae. Soviet Soil Science: 550-552.

Barreiro DL, Zamalloa C, Boon N, Vyverman W, Ronsse F, Brilman W, Prins W. 2013. Influence of strain-specific parameters on hydrothermal liquefaction of microalgae. Bioresource Technology 146:463-471.

Barsanti L, Frassanito AM, Passarelli V, Evangelista V, Etebari M, Paccagnini E, Lupetti P, Lenzi P, Verni F, Gualtieri P. 2013. Tetraflagellochloris mauritanica gen. et sp. nov. (Chlorophyceae), a new flagellated alga from the Mauritanian Desert: morphology, ultrastructure, and phylogenetic framing. Journal of Phycology 49(1):178-193.

Bashan Y, Lopez BR, Huss VA, Amavizca E, de-Bashan LE. 2015. Chlorella sorokiniana (formerly $C$. vulgaris) UTEX 2714, a non-thermotolerant microalga useful for biotechnological applications and as a reference strain. Journal of Applied Phycology:doi:10.1007/s10811-015-0571-z.

Bates ST, Clemente JC, Flores GE, Walters WA, Parfrey LW, Knight R, Fierer N. 2013. Global biogeography of highly diverse protistan communities in soil. The ISME Journal 7(3):652-659.

Bazin P, Jouenne F, Friedl T, Deton-Cabanillas A-F, Le Roy B, Véron B. 2014. Phytoplankton diversity and community composition along the estuarine gradient of a temperate macrotidal ecosystem: combined morphological and molecular approaches. PLoS ONE 9(4):e94110.

Becker B, Marin B. 2009. Streptophyte algae and the origin of embryophytes. Annals of Botany 103(7):999-1004.

Becker B. 2013. Snow ball earth and the split of Streptophyta and Chlorophyta. Trends in Plant Science 18(4):180-183.

Behnke A, Bunge J, Barger K, Breiner H-W, Alla V, Stoeck T. 2006. Microeukaryote community patterns along an $\mathrm{O}_{2} / \mathrm{H}_{2} \mathrm{~S}$ gradient in a supersulfidic anoxic fjord (Framvaren, Norway). Applied and Environmental Microbiology 72(5):3626-3636.

Beijerinck M. 1893. Bericht über meine Kulturen niederer Algen auf Nährgelatine. Zentralblatt Für Bakteriologie 13:781-786.

Benson D, Karsch-Mizrachi I, Clark K, Lipman D, Ostell J, Sayers E. 2012. GenBank. Nucleic Acids Research 40:D48-D53.

Bérard A, Rimet F, Capowiez Y, Leboulanger C. 2004. Procedures for determining the pesticide sensitivity of indigenous soil algae - a possible bioindicator of soil contamination? Archives of Environmental Contamination and Toxicology 46:24-31. 
Bérard A, Dorigo U, Humbert JF, Martin-Laurent F. 2005. Microalgae community structure analysis based on $18 \mathrm{~S}$ rDNA amplification from DNA extracted directly from soil as a potential soil bioindicator. Agronomie 25:1-7.

Bhatnagar A, Makandar MB, Garg MK, Bhatnagar M. 2008. Community structure and diversity of cyanobacteria and green algae in the soils of Thar Desert (India). Journal of Arid Environments 72(2):73-83.

Bilan M, Usov A. 2001. Polysaccharides of calcareous algae and their effect on the calcification process. Russian Journal of Bioorganic Chemistry 27(1):2-16.

Bischoff HW, Bold HC. 1963. Phycological Studies IV. Some soil algae from enchanted rock and related algal species. The University of Texas Press No. 6318. 95 pp.

Bock C, Pröschold T, Krienitz L. 2010. Two new Dictyosphaerium-morphotype lineages of the Chlorellaceae (Trebouxiophyceae): Heynigia gen. nov. and Hindakia gen. nov. European Journal of Phycology 45(3):267-277.

Bock C, Krienitz L, Pröschold T. 2011. Taxonomic reassessment of the genus Chlorella (Trebouxiophyceae) using molecular signatures (barcodes), including description of seven new species. Fottea 11(2):293-312.

Boenigk J, Pfandl K, Stadler P, Chatzinotas A. 2005. High diversity of the 'Spumella-like' flagellates: an investigation based on the SSU rRNA gene sequences of isolates from habitats located in six different geographic regions. Environmental Microbiology 7(5):685-697.

Borzì A. 1895. Studi algologici: saggio di ricerche sulla biologia delle alghe. Fasc. 1-2. G. Capara. $476 \mathrm{pp}$.

Brandt F, Stockmayer S. 1925. Analyse der aerophilen Grünalgenanflüge, insbesondere der proto-pleurococcoiden Formen. Archiv für Protistenkunde 52:265-355.

Bråte J, Klaveness D, Rygh T, Jakobsen KS, Shalchian-Tabrizi K. 2010. Telonemia-specific environmental $18 \mathrm{~S}$ rDNA PCR reveals unknown diversity and multiple marinefreshwater colonizations. BMC Microbiology 10(1):168.

Brinkmann N, Hodač L, Mohr KI, Hodačová A, Jahn R, Ramm J, Hallmann C, Arp G, Friedl T. 2015. Diversity of Cyanobacteria and Diatom communities along $\mathrm{pCO}_{2}$ and calcite saturation gradients in calcifying biofilms covering karst-water stromatolites in Germany. Geomicrobiology Journal 32(3-4):255-274.

Bristol BM. 1920. On the alga-flora of some desiccated English soils: an important factor in soil biology. Annals of Botany 34(1):35-80.

Bristol BM. 1927. On the algae of some normal English soils. The Journal of Agricultural Science 17(4):563-588.

Broady P. 1983. New records of Chlorophycean micro-algae cultured from antartic terrestrial habitats. Nova Hedwigia 36(2-4):445-84.

Broady PA. 1978. The terrestrial algae of Glerardalur Akureyri, Iceland. Acta Botanica Islandica 5:3-60.

Broady PA. 1979a. A preliminary survey of the terrestrial algae of the Antarctic Peninsula and South Georgia. British Antarctic Survey Bulletin 48:47-70.

Broady PA. 1979b. Qualitative and quantitative observations on green and yellow-green algae in some English soils. British Phycological Journal 14:151-160.

Broady PA. 1984. Taxonomic and ecological investigations of algae on steam-warmed soil on Mt. Erebus, Ross Island, Antarctica. Phycologia 23(3):257-271.

Broady PA. 1989. Survey of algae and other terrestrial biota at Edward VII Penninsula, Mary Bird Land. Antarctic Science 1:215-224. 
Broady PA. 1996. Diversity, distribution and dispersal of Antarctic terrestrial algae. Biodiversity and Conservation 5:1307-1335.

Brown MV, Philip GK, Bunge JA, Smith MC, Bissett A, Lauro FM, Fuhrman JA, Donachie SP. 2009. Microbial community structure in the North Pacific ocean. The ISME Journal 3(12):1374-1386.

Buchheim MA, Keller A, Koetschan C, Förster F, Merget B, Wolf M. 2011. Internal transcribed spacer 2 (nu ITS2 rRNA) sequence-structure phylogenetics: towards an automated reconstruction of the green algal tree of life. PLoS ONE 6(2):e16931.

Büdel B. 2003. Biological soil crusts of South America. In: Belnap J, Lange OL, editors. Biological soil crusts: Structure, function, and management. Springer. p. 51-55.

Büdel B, Darienko T, Deutschewitz K, Dojani S, Friedl T, Mohr KI, Salisch M, Reisser W, Weber B. 2009. Southern African biological soil crusts are ubiquitous and highly diverse in drylands, being restricted by rainfall frequency. Microbial Ecology 57(2):229-247.

Buzer J, Dohmeier R, Du Toit D. 1985. The survival of algae in dry soils exposed to high temperatures for extended time periods. Phycologia 24(2):249-251.

Cadoret J-P, Garnier M, Saint-Jean B. 2012. Microalgae, functional genomics and biotechnology. Advances in Botanical Research 64:285-341.

Caisová L, Marin B, Melkonian M. 2013. A consensus secondary structure of ITS2 in the Chlorophyta Identified by phylogenetic reconstruction. Protist 164:482-496.

Cantino PD, de Queiroz K. International Code of Phylogenetic Nomenclature, Version 4b. https://www.ohio.edu/phylocode/index.html.

Caron DA, Countway PD, Savai P, Gast RJ, Schnetzer A, Moorthi SD, Dennett MR, Moran DM, Jones AC. 2009. Defining DNA-based operational taxonomic units for microbialeukaryote ecology. Applied and Environmental Microbiology 75(18):5797-5808.

Carson JL, Brown RM. 1976. The correlation of soil algae, airborne algae, and fern spores with meteorological conditions on the Island of Hawaii. Pacific Science 30(2):197-205.

Carson JL, Brown RM. 1978. Studies on Hawaiian freshwater and soil algae II. Algal colonization and succession on a dated volcanic substrate. Journal of Phycology 14(2):171-178.

Cary SC, Fierer N. 2014. The importance of sample archiving in microbial ecology. Nature Reviews Microbiology 12(12):789-790.

Cavacini P. 2001. Soil algae from northern Victoria Land (Antarctica). Polar Bioscience 14:4560.

Chariton AA, Court LN, Hartley DM, Colloff MJ, Hardy CM. 2010. Ecological assessment of estuarine sediments by pyrosequencing eukaryotic ribosomal DNA. Frontiers in Ecology and the Environment 8(5):233-238.

Chen Z, He C, Hu H. 2012. Temperature responses of growth, photosynthesis, fatty acid and nitrate reductase in Antarctic and temperate Stichococcus. Extremophiles 16(1):127-133.

Cheung MK, Au CH, Chu KH, Kwan HS, Wong CK. 2010. Composition and genetic diversity of picoeukaryotes in subtropical coastal waters as revealed by 454 pyrosequencing. The ISME Journal 4(8):1053-1059.

Chornevych TM, Maltseva IA, Nikorych VA. 2008. Soil algaes of the coniferous forests of Ukraine. Ekologia ta noosferologija 18(3-4):77-82.

Chunxiang H, Yongding L. 2003. Primary succession of algal community structure in desert soil. Acta Botanica Sinica 45(8):917-924.

Clement M, Posada D, Crandall KA. 2000. TCS: a computer program to estimate gene genealogies. Molecular Ecology 9(10):1657-1659. 
Coleman AW. 2009. Is there a molecular key to the level of "biological species" in eukaryotes? A DNA guide. Molecular Phylogenetics and Evolution 50(1):197-203.

Costello EK, Halloy SRP, Reed SC, Sowell P, Schmidt SK. 2009. Fumarole-supported islands of biodiversity within a hyperarid, high-elevation landscape on Socompa volcano, Puna de Atacama, Andes. Applied and Environmental Microbiology 75(3):735-747.

Cox E, Deason T. 1969. Heterochlamydomonas, a new alga from Tennessee. Journal of the Tennessee Academy of Sciences 44:105-107.

Dahlin S, Witter E, Mårtensson A, Turner A, Bååth E. 1997. Where's the limit? Changes in the microbiological properties of agricultural soils at low levels of metal contamination. Soil Biology and Biochemistry 29(9-10):1405-1415.

Dal Grande F, Beck A, Cornejo C, Singh G, Cheenacharoen S, Nelsen MP, Scheidegger C. 2014. Molecular phylogeny and symbiotic selectivity of the green algal genus Dictyochloropsis s.l. (Trebouxiophyceae): a polyphyletic and widespread group forming photobiontmediated guilds in the lichen family Lobariaceae. New Phytologist 202(2):455-470.

Daniel R. 2005. The metagenomics of soil. Nature Reviews Microbiology 3(6):470-478.

Darienko T, Friedl T, Pröschold T. 2009. Desmochloris mollenhaueri - a new terrestrial ulvophycean alga from south-west African soils. Algological Studies 129(1):25-40.

Darienko T, Gustavs L, Mudimu O, Menendez CR, Schumann R, Karsten U, Friedl T, Pröschold T. 2010. Chloroidium, a common terrestrial coccoid green alga previously assigned to Chlorella (Trebouxiophyceae, Chlorophyta). European Journal of Phycology 45(1):7995.

Darienko T, Gustavs L, Eggert A, Wolf W, Pröschold T. 2015. Evaluating the species boundaries of Green microalgae (Coccomyxa, Trebouxiophyceae, Chlorophyta) using integrative taxonomy and DNA barcoding with further implications for the species identification in environmental samples. PLoS ONE 10(6):e0127838.

Darienko T, Pröschold T. 2015. Genetic variability and taxonomic revision of the genus Auxenochlorella (Shihira et Krauss) Kalina et Punčochářová (Trebouxiophyceae, Chlorophyta). Journal of Phycology 51(2):394-400.

Darienko TM. 2012. Terrestrial algae of Zmiinyj island (Black sea): general characteristic and peculiarities of species composition. Ukrajinskij Botanichnij Zhurnal 69(1):111-124.

Darty K, Denise A, Ponty Y. 2009. VARNA: Interactive drawing and editing of the RNA secondary structure. Bioinformatics 25(15):1974-1975.

Day JG, Pröschold T, Friedl T, Lorenz M, Silva PC. 2010. Conservation of microalgal type material: approaches needed for $21^{\text {st }}$ century science. Taxon 59(1):3-6.

de-Bashan LE, Trejo A, Huss VA, Hernandez J-P, Bashan Y. 2008. Chlorella sorokiniana UTEX 2805 , a heat and intense, sunlight-tolerant microalga with potential for removing ammonium from wastewater. Bioresource Technology 99(11):4980-4989.

De Wever A, Leliaert F, Verleyen E, Vanormelingen P, Van der Gucht K, Hodgson DA, Sabbe K, Vyverman W. 2009. Hidden levels of phylodiversity in Antarctic green algae: further evidence for the existence of glacial refugia. Proceedings of the Royal Society B: Biological Sciences 276(1673):3591-3599.

Deason T, Bold H. 1960. Phycological studies 1. Exploratory studies of Texas soil algae. The University of Texas Press No. 6022. 71 pp.

Deason TR, Floyd GL. 1987. Comparative ultrastructure of three species of Chlorosarcina (Chlorosarcinaceae, Chlorophyta). Journal of Phycology 23(1):187-195. 
del Campo J, Sieracki ME, Molestina R, Keeling P, Massana R, Ruiz-Trillo I. 2014. The others: our biased perspective of eukaryotic genomes. Trends in Ecology and Evolution 29(5):252-259.

Dickie IA. 2010. Insidious effects of sequencing errors on perceived diversity in molecular surveys. New Phytologist 188(4):916-918.

Doherty M, Costas BA, McManus GB, Katz LA. 2007. Culture-independent assessment of planktonic ciliate diversity in coastal northwest Atlantic waters. Aquatic Microbial Ecology 48(2):141-154.

Dorigo U, Bérard A, Humbert J. 2002. Comparison of eukaryotic phytobenthic community composition in a polluted river by partial 18S rRNA gene cloning and sequencing. Microbial Ecology 44(4):372-380.

Dorokhova MF, Kosheleva NE, Terskaya EV. 2015. Algae and Cyanobacteria in soils of Moscow. American Journal of Plant Sciences 6(15):2461-2471.

Dotsenko KA. 2008. Study of soil algae as bioindicators in areas of petroleum production. Nauchnyj Zhurnal of the Kuban State Agrarian University 41(7):1-6.

Driver T, Bajhaiya AK, Allwood JW, Goodacre R, Pittman JK, Dean AP. 2015. Metabolic responses of eukaryotic microalgae to environmental stress limit the ability of FT-IR spectroscopy for species identification. Algal Research 11:148-155.

Drummond AJ, Rambaut A. 2007. BEAST: Bayesian evolutionary analysis by sampling trees. BMC Evolutionary Biology 7(214):doi:10.1186/1471-2148-7-214.

Drummond AJ, Suchard MA, Xie D, Rambaut A. 2012. Bayesian phylogenetics with BEAUti and the BEAST 1.7. Molecular Biology and Evolution 29(8):1969-1973.

Edgcomb V, Orsi W, Bunge J, Jeon S, Christen R, Leslin C, Holder M, Taylor GT, Suarez P, Varela R. 2011. Protistan microbial observatory in the Cariaco Basin, Caribbean. I. Pyrosequencing vs Sanger insights into species richness. The ISME Journal 5(8):13441356.

Ehrenberg CG. 1833. Dritter Beitrag zur Erkenntniss grosser Organisation in der Richtung des kleinsten Raumes. Berlin: Königliche Akademie der Wissenschaften. p. 145-336

Eliáš M, Neustupa J, Škaloud P. 2008. Elliptochloris bilobata var. corticola var. nov. (Trebouxiophyceae, Chlorophyta), a novel subaerial coccal green alga. Biologia 63(6):791-798.

Eliáš M, Neustupa J. 2009. Pseudomarvania, gen. nov. (Chlorophyta, Trebouxiophyceae), a new genus for "budding" subaerial green algae Marvania aerophytica Neustupa et Šejnohová and Stichococcus ampulliformis Handa. Fottea 9(2):169-177.

Eliáš M, Němcová Y, Škaloud P, Neustupa J, Kaufnerová V, Šejnohová L. 2010. Hylodesmus singaporensis gen. et sp. nov., a new autosporic subaerial green alga (Scenedesmaceae, Chlorophyta) from Singapore. International Journal of Systematic and Evolutionary Microbiology 60:1224-1235.

Eliáš M, Neustupa J, Pažoutová M, Škaloud P. 2013. A case of taxonomic inflation in coccoid algae: Ellipsoidion parvum and Neocystis vischeri are conspecific with Neocystis (=Nephrodiella) brevis (Chlorophyta, Trebouxiophyceae). Phytotaxa 76(2):15-27.

Elster J. 1999. Algal versatility in various extreme environments. Enigmatic microorganisms and life in extreme environments. Springer. p. 215-227.

Elster J, Lukešová A, Svoboda J, Kopecký J, Kanda H. 1999. Diversity and abundance of soil algae in the polar desert Sverdrup Pass, central Ellesmere Island. Polar Record 35:231254. 
Elster J, Benson E. 2004. Life in the polar terrestrial environment with a focus on algae and cyanobacteria. In: Fuller BJ, Lane N, Benson EE, editors. Life in the frozen state. CRC Press. p. 111-150.

Ettl H. 1963. Massenhaftes Auftreten von Chloromonas-Arten in Regenpfützen. Nova Hedwigia Beiheft 6:395-400.

Ettl H, Gärtner G. 1995. Syllabus der Boden-, Luft-und Flechtenalgen. Springer-Verlag. 721. pp.

Ettl H, Gärtner G. 2014. Syllabus der Boden-, Luft-und Flechtenalgen. Springer-Verlag. 773. pp.

Fairchild ED, Willson DL. 1967. The algal flora of two Washington soils. Ecology 48(6):10531055.

Faßhauer F, Beck A, Hodač L, Kaufholz F, Probst P, Sasonowa M, Weber A, Friedl T. 2011. Biodiversity of terrestrial algae in tropical mountain habitats in Podocarpus National Park, Ecuador. Posters, European Journal of Phycology 46(Supp1ement). p. 166.

Faßhauer F. in prep. Biodiversity of terrestrial algae in tropical mountain habitats in Podocarpus National Park, Ecuador. PhD thesis, Georg August University of Göttingen.

Fawley MW, Fawley KP, Buchheim MA. 2004. Molecular diversity among communities of freshwater microchlorophytes. Microbial Ecology 48:489-499.

Feher D. 1948. Researches on the geographical distribution of soil microflora. II. The geographical distribution of soil algae. Communications of the Botanical Institute of the Hungarian University of Technical and Economic Sciences, Sopron (Hungary) 21:1-35.

Fenchel T, Finlay BJ. 2004. The ubiquity of small species: patterns of local and global diversity. BioScience 54(8):777-784.

Fermani P, Mataloni G, Van de Vijver B. 2007. Soil microalgal communities on an antarctic active volcano (Deception Island, South Shetlands). Polar Biology 30(11):1381-1393.

Fierer N, Cary C. 2014. Archiving: Don't let microbial samples perish. Nature 512(7514):253253.

Filker S, Gimmler A, Dunthorn M, Mahé F, Stoeck T. 2015. Deep sequencing uncovers protistan plankton diversity in the Portuguese Ria Formosa solar saltern ponds. Extremophiles 19(2):283-295.

Finlay BJ, Clarke KJ. 1999. Ubiquitous dispersal of microbial species. Nature 400(6747):828828.

Finlay BJ. 2002. Global dispersal of free-living microbial eukaryote species. Science 296(5570):1061-1063.

Fischer M, Bossdorf O, Gockel S, Hänsel F, Hemp A, Hessenmöller D, Korte G, Nieschulze J, Pfeiffer S, Prati D, Renner S, Schöning I, Schumacher U, Wells K, Buscot F, Kalko EKV, Linsenmair KE, Schulze E-D, Weisser WW. 2010. Implementing large-scale and longterm functional biodiversity research: The Biodiversity Exploratories. Basic and Applied Ecology 11(6):473-485.

Flechtner VR, Johansen JR, Clark WH. 1998. Algal composition of microbiotic crusts from the central desert of Baja California, Mexico. The Great Basin Naturalist 58(4):295-311.

Flechtner VR, Ng RA, Johansen JR, Antionio S. 2005. Algal diversity in North American desert soils. Proc. SPIE 5906, Astrobiology and Planetary Missions. p. 1-9.

Flechtner VR, Johansen JR, Belnap J. 2008. The biological soil crusts of the San Nicolas island. Western North American Naturalist 68(4):405-436.

Flechtner VR, Pietrasiak N, Lewis LA. 2013. Newly revealed diversity of green microalgae from wilderness areas of Joshua tree national park (JTNP). Monographs of the Western North American Naturalist 6:43-63. 
Foissner W, Chao A, Katz L. 2008. Diversity and geographic distribution of ciliates (Protista: Ciliophora). Biodiversity and Conservation 17(2):345-363.

Fontaine KM, Beck A, Stocker-Wörgötter E, Piercey-Normore MD. 2012. Photobiont relationships and phylogenetic history of Dermatocarpon luridum var. luridum and related Dermatocarpon species. Plants 1(2):39-60.

Fontaine KM, Stocker-Woergoetter E, Booth T, Piercey-Normore MD. 2013. Genetic diversity of the lichen-forming alga, Diplosphaera chodatii, in North America and Europe. The Lichenologist 45(6):799-813.

Ford T, Pedley H. 1996. A review of tufa and travertine deposits of the world. Earth-Science Reviews 41(3):117-175.

Forney LJ, Zhou X, Brown CJ. 2004. Molecular microbial ecology: land of the one-eyed king. Current Opinion in Microbiology 7(3):210-220.

Freeman KR, Pescador MY, Reed SC, Costello EK, Robeson MS, Schmidt SK. 2009. Soil CO 2 flux and photoautotrophic community composition in high-elevation,'barren'soil. Environmental Microbiology 11(3):674-686.

Frey B, Bühler L, Schmutz S, Zumsteg A, Furrer G. 2013. Molecular characterization of phototrophic microorganisms in the forefield of a receding glacier in the Swiss Alps. Environmental Research Letters 8(1):doi:10.1088/1748-9326/8/1/015033.

Freytet P, Plet A. 1991. The modern stromatolitic formations (calcareous-tufa) of the Tournus area (Burgundy, France). Geobios 24(2):123-139.

Freytet P, Plet A. 1996. Modern freshwater microbial carbonates: the Phormidium stromatolites (tufa-travertine) of southeastern Burgundy (Paris Basin, France). Facies 34(1):219-237.

Freytet P, Verrecchia EP. 1998. Freshwater organisms that build stromatolites: a synopsis of biocrystallization by prokaryotic and eukaryotic algae. Sedimentology 45(3):535-563.

Friedl T. 1996. Evolution of the polyphyletic genus Pleurastrum (Chlorophyta): inferences from nuclear-encoded ribosomal DNA sequences and motile cell ultrastructure. Phycologia 35(5):456-469.

Friedl T, Rokitta C. 1997. Species relationships in the lichen alga Trebouxia (Chlorophyta, Trebouxiophyceae): molecular phylogenetic analyses of nuclear-encoded large subunit rRNA gene sequences. Symbiosis 23:125-148.

Friedl T, O'Kelly CJ. 2002. Phylogenetic relationships of green algae assigned to the genus Planophila (Chlorophyta): evidence from 18S rDNA sequence data and ultrastructure. European Journal of Phycology 37(3):373-384.

Friedl T, Rybalka N. 2012. Systematics of the Green Algae: A Brief Introduction to the current status. In: Lüttge U, editor. Progress in Botany. p. 259-280.

Friedmann EI, Lipkin Y, Ocampo-Paus R. 1967. Desert algae of the Negev (Israel). Phycologia 6(4):185-200.

Friedrich J, Dandekar T, Wolf M, Müller T. 2005. ProfDist: a tool for the construction of large phylogenetic trees based on profile distances. Bioinformatics 21(9):2108-2109.

Frouz J, Keplin B, Pižl V, Tajovský K, Starý J, Lukešová A, Nováková A, Balík Vr, Háněl L, Materna J. 2001. Soil biota and upper soil layer development in two contrasting postmining chronosequences. Ecological Engineering 17(2):275-284.

Fučíková K, Rada JC, Lewis LA. 2011a. The tangled taxonomic history of Dictyococcus, Bracteacoccus and Pseudomuriella (Chlorophyceae, Chlorophyta) and their distinction based on a phylogenetic perspective. Phycologia 50(4):422-429. 
Fučíková K, Rada JC, Lukešová A, Lewis LA. 2011b. Cryptic diversity within the genus Pseudomuriella Hanagata (Chlorophyta, Chlorophyceae, Sphaeropleales) assessed using four barcode markers. Nova Hedwigia 93(1-2):29-46.

Fučíková K, Flechtner VR, Lewis LA. 2012. Revision of the genus Bracteacoccus Tereg (Chlorophyceae, Chlorophyta) based on a phylogenetic approach. Nova Hedwegia 96(12):15-59.

Fučíková K, Lewis LA. 2012. Intersection of Chlorella, Muriella and Bracteacoccus: Resurrecting the genus Chromochloris Kol et Chodat (Chlorophyceae, Chlorophyta). Fottea 12(1):83-93.

Fučíková K, Lewis PO, Lewis LA. 2013. Putting incertae sedis taxa in their place: a proposal for ten new families and three new genera in Sphaeropleales (Chlorophyceae). Journal of Phycology 50(1):14-25.

Fučíková K, Lewis PO, Lewis LA. 2014. Widespread desert affiliation of trebouxiophycean algae (Trebouxiophyceae, Chlorophyta) including discovery of three new desert genera. Phycological Research 62(4):294-305.

Garcia-Pichel F, Al-Horani FA, Farmer JD, Ludwig R, Wade BD. 2004. Balance between microbial calcification and metazoan bioerosion in modern stromatolitic oncolites. Geobiology 2:49-57.

Gärtner G. 1996. ASIB - the Culture Collection of Algae at the Botanical Institute of the University at Innsbruck (Austria) Catalogue of Strains. p. 45-69.

Gärtner G, Ingolić E. 2003. Further studies on Desmococcus Brand emend. Vischer (Chlorophyta, Trebouxiophyceae) and a new species Desmococcus spinocystis sp. nov. from soil. Biologia 58(4):517-523.

Gaysina L, Němcová Y, Škaloud P, Ševčíková T, Eliáš M. 2013. Chloropyrula uraliensis gen. et sp. nov. (Trebouxiophyceae, Chlorophyta), a new green coccoid alga with a unique ultrastructure, isolated from soil in South Urals. Journal of Systematics and Evolution 51(4):476-484.

Genitsaris S, Moustaka-Gouni M, Kormas KA. 2011. Airborne microeukaryote colonists in experimental water containers: diversity, succession, life histories and established food webs. Aquatic Microbial Ecology 62(2):139-152.

Germond A, Hata H, Fujikawa Y, Nakajima T. 2013. The phylogenetic position and phenotypic changes of a Chlorella-like alga during 5-year microcosm culture. European Journal of Phycology 48(4):485-496.

Gilichinsky D, Wilson G, Friedmann E, McKay C, Sletten R, Rivkina E, Vishnivetskaya T, Erokhina L, Ivanushkina N, Kochkina G. 2007. Microbial populations in Antarctic permafrost: biodiversity, state, age, and implication for astrobiology. Astrobiology 7(2):275-311.

Goiris K, Muylaert K, Voorspoels S, Noten B, De Paepe D, Baart E, Gino J, De Cooman L. 2014. Detection of flavonoids in microalgae from different evolutionary lineages. Journal of Phycology 50(3):483-492.

Gollerbach MM, Shtina EA. 1969. Soil algae. Nauka. 228 pp.

Gouy M, Guindon S, Gascuel O. 2010. SeaView version 4: a multiplatform graphical user interface for sequence alignment and phylogenetic tree building. Molecular Biology and Evolution 27(2):221-224.

Grattepanche J-D, Santoferrara LF, McManus GB, Katz LA. 2014. Diversity of diversity: conceptual and methodological differences in biodiversity estimates of eukaryotic microbes as compared to bacteria. Trends in Microbiology 22(8):432-437. 
Guiry MD. 2012. How many species of algae are there? Journal of Phycology 48(5):1057-1063.

Guiry MD, Guiry GM. 2015. AlgaeBase. World-wide electronic publication, National University of Ireland, Galway. http://www.algaebase.org.

Gustavs L, Eggert A, Michalik D, Karsten U. 2010. Physiological and biochemical responses of green microalgae from different habitats to osmotic and matric stress. Protoplasma 243(14):3-14.

Hahn MW, Koll U, Jezberová J, Camacho A. 2015. Global phylogeography of pelagic Polynucleobacter bacteria: Restricted geographic distribution of subgroups, isolation by distance and influence of climate. Environmental Microbiology 17(3):829-840.

Hall JD, Fucikova K, Lo C, Lewis LA. 2010. An assessment of proposed DNA barcodes in freshwater green algae. Cryptogamie, Algologie 31(4):529-555.

Hall TA. 1999. BioEdit: a user-friendly biological sequence alignment editor and analysis program for Windows 95/98/NT. Nucleic Acids Symposium Series. Oxford University Press. p. 95-98.

Hallmann C, Hodač L, Pahlmann KC, Faßhauer F, Friedl T. 2009. Changes in the biodiversity of algae in soils along gradients of land use intensities. Posters, Phycologia 48 (Supplement). p. 32.

Hallmann C, Fritzlar D, Stannek L, Hoppert M. 2011a. Ascomycete fungi on dimension stone of the "Burg Gleichen", Thuringia. Environmental Earth Sciences 63(7-8):1713-1722.

Hallmann C, Rüdrich J, Enseleit M, Friedl T, Hoppert M. 2011b. Microbial diversity on a marble monument: a case study. Environmental Earth Sciences 63:1701-1711.

Hallmann C, Stannek L, Fritzlar D, Hause-Reitner D, Friedl T, Hoppert M. 2013a. Molecular diversity of phototrophic biofilms on building stone. FEMS Microbiology Ecology 84(2):355-372.

Hallmann C, Wedekind W, Hause-Reitner D, Hoppert M. 2013b. Cryptogam covers on sepulchral monuments and re-colonization of a marble surface after cleaning. Environmental Earth Sciences 69:1149-1160.

Hallmann C, Hodač L, Faßhauer F, Hoppert M, Friedl T. in prep.-a. Green algal communities from soil and tree bark in middle European forests: a molecular comparison.

Hallmann C, Hodač L, Friedl T. in prep.-b. Green algae in grassland and forest soils under different management types - a culture-independent approach.

Hamby KR, Sims L, Issel L, Zimmer E. 1988. Direct ribosomal RNA sequencing: Optimization of extraction and sequencing methods for work with higher plants. Plant Molecular Biology Reporter 6(3):175-192.

Hammer Ø, Harper DAT, Ryan PD. 2001. PAST: Paleontological Statistics software package for education and data analysis. Palaeontologia Electronica 4(1):1-9.

Hanagata N. 1998. Phylogeny of the subfamily Scotiellocystoideae (Chlorophyceae, Chlorophyta) and related taxa inferred from $18 \mathrm{~S}$ ribosomal RNA gene sequence data. Journal of Phycology 34(6):1049-1054.

Handa S, Nakahara M, Tsubota H, Deguchi H, Nakano T. 2003. A new aerial alga, Stichococcus ampulliformis sp. nov. (Trebouxiophyceae, Chlorophyta) from Japan. Phycological Research 51(3):203-210.

Hansgirg A. 1888. Prodromus der Algenflora von Böhmen. Commissions-Verlag von Fr. Řivnáč. Prague. 282 pp.

Hastings KL, Smith LE, Lindsey ML, Blotsky LC, Downing GR, Zellars DQ, Downing JK, Corena-McLeod M. 2014. Effect of microalgae application on soil algal species diversity, 
cation exchange capacity and organic matter after herbicide treatments. F1000Research 3(281):doi:10.12688/f1000research.4016.1

Häubner N, Schumann R, Karsten U. 2006. Aeroterrestrial microalgae growing in biofilms on facades - response to temperature and water stress. Microbial Ecology 51(3):285-293.

Heath CR, Leadbeater BC, Callow ME. 1995. Effect of inhibitors on calcium carbonate deposition mediated by freshwater algae. Journal of Applied Phycology 7(4):367-380.

Heeg JS, Wolf M. 2015. ITS2 and 18S rDNA sequence-structure phylogeny of Chlorella and allies (Chlorophyta, Trebouxiophyceae, Chlorellaceae). Plant Gene 4:20-28.

Hegewald E, Hanagata N. 2000. Phylogenetic studies on Scenedesmaceae (Chlorophyta). Archiv für Hydrobiologie. Supplementband, Algological Studies 136:29-49.

Hegewald E, Bock C, Krienitz L. 2013. A phylogenetic study on Scenedesmaceae with the description of a new species of Pectinodesmus and the new genera Verrucodesmus and Chodatodesmus. Fottea 13:149-164.

Henley WJ, Hironaka JL, Guillou L, Buchheim MA, Buchheim JA, Fawley MW, Fawley KP. 2004. Phylogenetic analysis of the 'Nannochloris-like' algae and diagnoses of Picochlorum oklahomensis gen. et sp. nov. (Trebouxiophyceae, Chlorophyta). Phycologia 43(6):641-652.

Hepperle D, Krienitz L. 1996. The extracellular calcification of zoospores of Phacotus lenticularis (Chlorophyta, Chlamydomonadales). European Journal of Phycology 31(1):11-21.

Hill MO. 1979. TWINSPAN - A FORTRAN programme for arranging multivariate data in an ordered two-way table by classification of individuals and attributes. Section of Ecology and Systematics. 90. pp.

Hirsch PR, Mauchline TH, Clark IM. 2010. Culture-independent molecular techniques for soil microbial ecology. Soil Biology and Biochemistry 42(6):878-887.

Hodač L, Hallmann C, Rosenkranz H, Faßhauer F, Friedl T. 2012. Molecular evidence for the wide distribution of two lineages of terrestrial Green algae (Chlorophyta) over tropics to temperate zone. ISRN Ecology:doi:10.5402/2012/795924.

Hodač L, Brinkmann N, Mohr KI, Arp G, Hallmann C, Ramm J, Spitzer K, Friedl T. 2015. Diversity of microscopic green algae (Chlorophyta) in calcifying biofilms of two karstic streams in Germany. Geomicrobiology Journal 32(3-4):275-290.

Hodač L, Hallmann C, Spitzer K, Elster J, Faßhauer F, Brinkmann N, Lepka D, Diwan V, Friedl T. subm. Phylogenetic analysis of polar Chlorella and Stichococcus suggests biogeography of airborne microalgae. FEMS Microbiology Ecology.

Hoffmann L. 1989. Algae of terrestrial habitats. The Botanical Review 55(2):77-105.

Hoffmann L, Ector L, Kostikov I. 2007. Algal flora from limed and unlimed forest soils in the Ardenne (Belgium). Systematics and Geography of Plants:15-90.

Hong JW, Jo SW, Cho HW, Nam SW, Shin W, Park KM, Lee KI, Yoon HS. 2015. Phylogeny, morphology, and physiology of Micractinium strains isolated from shallow ephemeral freshwater in Antarctica. Phycological Research 63(3):212-218.

Hope ACA. 1968. A simplified Monte Carlo significance test procedure. Journal of the Royal Statistical Society: Series B 30:581-598.

Hoppert M, Reimer R, Kemmling A, Schröder A, Günzl B, Heinken T. 2004. Structure and reactivity of a biological soil crust from a xeric sandy soil in Central Europe. Geomicrobiology Journal 21(3):183-191.

Horath T, Bachofen R. 2009. Molecular characterization of an endolithic microbial community in dolomite rock in the Central Alps (Switzerland). Microbial Ecology 58:290-306. 
Hu C, Liu Y, Song L, Zhang D. 2002. Effect of desert soil algae on the stabilization of fine sands. Journal of Applied Phycology 14:281-292.

Hu C, Liu Y, Paulsen BS, Petersen D, Klaveness D. 2003a. Extracellular carbohydrate polymers from five desert soil algae with different cohesion in the stabilization of fine sand grain. Carbohydrate Polymers 54(1):33-42.

Hu C, Zhang D, Huang Z, Liu Y. 2003b. The vertical microdistribution of cyanobacteria and green algae within desert crusts and the development of the algal crusts. Plant and Soil 257(1):97-111.

$\mathrm{Hu} \mathrm{H}, \mathrm{Li} \mathrm{H}, \mathrm{Xu} \mathrm{X}$. 2008. Alternative cold response modes in Chlorella (Chlorophyta, Trebouxiophyceae) from Antarctica. Phycologia 47(1):28-34.

Huber-Pestalozzi G, Fott B, Komárek J, Förster K. 1983. Das Phytoplankton des Süsswassers: Systematik und Biologie. E. Schweizerbart. 606 pp.

Huber T, Faulkner G, Hugenholtz P. 2004. Bellerophon: a program to detect chimeric sequences in multiple sequence alignments. Bioinformatics 20(14):2317-2319.

Hughes KA. 2006. Solar UV-B radiation, associated with ozone depletion, inhibits the Antarctic terrestrial microalga, Stichococcus bacillaris. Polar Biology 29:327-336.

Hunt ME, Floyd GL, Stout BB. 1979. Soil algae in field and forest environments. Ecology 60(2):362-375.

Huson DH, Bryant D. 2006. Application of phylogenetic networks in evolutionary studies. Molecular Biology and Evolution 23(2):254-267.

Huss VAR, Ciniglia C, Cennamo P, Cozzolino S, Pinto G, Pollio A. 2002. Phylogenetic relationships and taxonomic position of Chlorella-like isolates from low $\mathrm{pH}$ environments $(\mathrm{pH}<3.0)$. BMC Evolutionary Biology 2:doi:10.1186/1471-2148-2-13.

Iljushenko AE. 2008. Algokompleksy pochvennykh vodoroslej bora-brusnichnika. Fundamentalnye i prikladnye problemy botaniki v nachale XXI veka. Algologija. p. 3739.

Islam N. 1960. Some subaerial green algae from East Pakistan. Transactions of the American Microscopical Society 10(4):471-479.

Jaarsma CF, van Langevelde F, Botma H. 2006. Flattened fauna and mitigation: Traffic victims related to road, traffic, vehicle, and species characteristics. Transportation Research Part D: Transport and Environment 11(4):264-276.

Jobb G, Von Haeseler A, Strimmer K. 2004. TREEFINDER: a powerful graphical analysis environment for molecular phylogenetics. BMC Evolutionary Biology 4(1):doi: 10.1186/1471-2148-4-18.

Johansen JR, Casamatta DA. 2005. Recognizing cyanobacterial diversity through adoption of a new species paradigm. Algological Studies 117(1):71-93.

Johnson LR, John DM. 1992. Taxonomic observations on some uncommon and new Gongrosira species (Chaetophorales sensu stricto, division Chlorophyta). European Journal of Phycology 27(2):153-163.

Jurdíková K, Kulichová J, Bestová H, Leliaert F, Škaloud P. 2014. Exploration of nuclear DNA markers for population structure assessment in the desmid Micrasterias rotata (Zygnematophyceae, Streptophyta). Journal of Eukaryotic Microbiology 61(5):509-519.

Kabirov R, Gaisina L, Safiullina L. 2010. Universal criteria for assessing the ecological state of soil algocenoses. Russian Journal of Ecology 41(4):302-306.

Kabirov RR. 1993. Algoindications with using soil algae (methodological aspects). Algologia $3(3): 73-83$. 
Kalinowska R, Trzcinska M, Pawlik-Skowronska B. 2008. Glony glebowe terenow pogorniczych skazonych metalami ciezkimi. Wiadomości Botaniczne 52(3-4):63-79.

Kalinowska R, Pawlik-Skowrońska B. 2010. Response of two terrestrial green microalgae (Chlorophyta, Trebouxiophyceae) isolated from $\mathrm{Cu}$-rich and unpolluted soils to copper stress. Environmental Pollution 158(8):2778-2785.

Karbovska VM, Kostikov IY. 2012a. New morphological features of the members of genus Stichococcus Nägeli (Trebouxiophyceae, Chlorophyta). Modern Phytomorphology 1:111-114.

Karbovska VM, Kostikov IY. 2012b. Chlorella sphaerica and its position in the genus Diplosphaera (Chlorophyta, Trebouxiophyceae). Visnyk of Dnipropetrovsk University. Biology, Ecology 20(1):34-42.

Karsten U, Friedl T, Schumann R, Hoyer K, Lembcke S. 2005. Mycosporine-like amino acids and phylogenies in green algae: Prasiola and its relationships from the Trebouxiophyceae (Chlorophyta). Journal of Phycology 41(3):557-566.

Kaštovská K, Elster J, Stibal M, Šantrůčková H. 2005. Microbial assemblages in soil microbial succession after glacial retreat in Svalbard (High Arctic). Microbial Ecology 50(3):396407.

Kaštovská K, Stibal M, Šabacká M, Černá B, Šantrůčková H, Elster J. 2007. Microbial community structure and ecology of subglacial sediments in two polythermal Svalbard glaciers characterized by epifluorescence microscopy and PLFA. Polar Biology 30(3):277-287.

Katoh K, Toh H. 2008. Recent developments in the MAFFT multiple sequence alignment program. Briefings in Bioinformatics 9(4):286-298.

Kaufnerová V, Eliáš M. 2013. The demise of the genus Scotiellopsis Vinatzer (Chlorophyta). Nova Hedwigia 97(3-4):415-428.

Keller A, Schleicher T, Schultz J, Müller T, Dandekar T, Wolf M. 2009. 5.8S-28S rRNA interaction and HMM-based ITS2 annotation. Gene 430(1-2):50-57.

Keller A. 2011. Secondary (and tertiary) structure of the ITS2 and its application for phylogenetic tree reconstructions and species identification. $\mathrm{PhD}$ thesis. Universität Würzburg.

Khan N, Tuffin M, Stafford W, Cary C, Lacap DC, Pointing SB, Cowan D. 2011. Hypolithic microbial communities of quartz rocks from Miers Valley, McMurdo Dry Valleys, Antarctica. Polar Biology 34(11):1657-1668.

Khaybullina LS, Gaysina L, Johansen JR, Krautová M. 2010. Examination of the terrestrial algae of the Great Smoky Mountains National Park, USA. Fottea 10(2):201-215.

Kim GH, Klochkova TA, Kang SH. 2008. Notes on freshwater and terrestrial algae from NyAlesund, Svalbard (high Arctic sea area). Journal of Environmental Biology 29(4):485491.

King JM, Ward C. 1977. Distribution of edaphic algae as related to land usage. Phycologia 16(1):23-30.

Kochkina G, Ozerskaya S, Ivanushkina N, Chigineva N, Vasilenko O, Spirina E, Gilichinskii D. 2014. Fungal diversity in the Antarctic active layer. Microbiology 83(1-2):94-101.

Koetschan C, Forster F, Keller A, Schleicher T, Ruderisch B, Schwarz R, Müller T, Wolf M, Schultz J. 2010. The ITS2 Database III - sequences and structures for phylogeny. Nucleic Acids Research 38:275-279.

Koetschan C, Hackl T, Müller T, Wolf M, Forster F, Schultz J. 2012. ITS2 database IV: interactive taxon sampling for internal transcribed spacer 2 based phylogenies. Molecular Biology and Evolution 63(3):585-588. 
Kol E. 1967. Algal growth experiments in the Baradla cave at Aggletek. International Journal of Speleology 2:457-474.

Komárek J, Anagnostidis K. 1998. Cyanoprokaryota $-1^{\text {st }}$ Part: Chroococcales. Stuttgart, Germany: Gustav Fischer Verlag. 548 pp.

Komárek J, Anagnostidis K. 2005. Cyanoprokaryota $-2^{\text {nd }}$ Part: Oscillatoriales. Stuttgart, Germany: Gustav Fischer Verlag. 759 pp.

Komárek J. 2013. Cyanoprokaryota $-3^{\text {rd }}$ Part: Nostocales. Stuttgart, Germany: Gustav Fischer Verlag. 1131 pp.

Komaromy ZP. 1976. Soil algal growth types as edaphyc adaptation in Hungarian forest and grassland ecosystems. Acta Botanica Hungarica 22:373-379.

Komáromy ZP. 1983. A comparative study on the algal synusia of Hungarian grasslands and deciduous forests. Annales Historico-Naturales Musei Nationalis Hungarici 75:47-53.

Kostikov I, Carnol M, Dulière J, Hoffman L. 2001a. Effects of limiting on forest soil algal communities. Algological Studies 102:161-178.

Kostikov I, Romanenko PO, Demchenko EM, Darienko TM, Mikhailyuk TI, Rybchnnskiy OV, Solonenko AM. 2001b. Soil algae of Ukraine: methods of study, system, checklist. In: Kondratiuk SJ, Massjiuk NP, editors. Phytosociocenter. 300 pp.

Krienitz L, Hegewald E, Hepperle D, Wolf M. 2003. The systematics of coccoid green algae: 188 rRNA gene sequence data versus morphology. Biologia 58(4):437-446.

Krienitz L, Hegewald EH, Hepperle D, Huss VA, Rohr T, Wolf M. 2004. Phylogenetic relationship of Chlorella and Parachlorella gen. nov. (Chlorophyta, Trebouxiophyceae). Phycologia 43(5):529-542.

Krienitz L, Bock C, Dadheech P, Pröschold T. 2011. Taxonomic reassessment of the genus Mychonastes (Chlorophyceae, Chlorophyta) including the description of eight new species. Phycologia 50(1):89-106.

Krienitz L, Bock C. 2012. Present state of the systematics of planktonic coccoid green algae of inland waters. Hydrobiologia 698(1):295-326.

Krienitz L, Huss VAR, Bock C. 2015. Chlorella: 125 years of the green survivalist. Trends in Plant Science 20(2):67-69.

Krivorotov SB, Bukareva OV. 2005. Soil algae as biological indicators of soil pollution of protected areas of the north-western Caucasus with heavy metals. Uspechi Sovremennogo Estestvovania 11:12-15.

Kroken S, Taylor JW. 2000. Phylogenetic species, reproductive mode, and specificity of the green alga Trebouxia forming lichens with the fungal genus Letharia. The Bryologist 103(4):645-660.

Kulichová J, Škaloud P, Neustupa J. 2014. Molecular diversity of green corticolous microalgae from two sub-Mediterranean European localities. European Journal of Phycology 49(3):345-355.

Kutovaya O, Vasilenko E, Lebedeva M. 2012. Microbiological and micromorphological characteristics of extremely arid desert soils in the Ili Depression (Kazakhstan). Eurasian Soil Science 45(12):1147-1158.

Kützing FT. 1843. Phycologia generalis: oder Anatomie, Physiologie und Systemkunde der Tange. Leipzig: Brockhaus. 458 pp.

Kuzyakhmetov GG. 1998. Productivity of algocenoses in zonal arable soils of steppe and foreststeppe. Eurasian Soil Science 31(4):406-410. 
Kvíderová J, Lukavský J. 2005. The comparison of ecological characteristics of Stichococcus (Chlorophyta) strains isolated from polar and temperate regions. Algological Studies 118(1):127-40.

Lang I, Hodač L, Friedl T, Feussner I. 2011. Fatty acid profiles and their distribution patterns in microalgae: a comprehensive analysis of more than 2000 strains from the SAG culture collection. BMC Plant Biology 11(124):doi:10.1186/1471-2229-11-124.

Langhans TM, Storm C, Schwabe A. 2009. Community assembly of biological soil crusts of different successional stages in a temperate sand ecosystem, as assessed by direct determination and enrichment techniques. Microbial Ecology 58(2):394-407.

Lara E, Berney C, Harms H, Chatzinotas A. 2007. Cultivation-independent analysis reveals a shift in ciliate 18S rRNA gene diversity in a polycyclic aromatic hydrocarbon-polluted soil. FEMS Microbiology Ecology 62(3):365-373.

Lauber CL, Hamady M, Knight R, Fierer N. 2009. Pyrosequencing-based assessment of soil pH as a predictor of soil bacterial community structure at the continental scale. Applied and Environmental Microbiology 75(15):5111-5120.

Lawley B, Ripley S, Bridge P, Convey P. 2004. Molecular analysis of geographic patterns of eukaryotic diversity in Antarctic soils. Applied and Environmental Microbiology 70(10):5963-5672.

Lee JC, Gutell RR. 2012. A comparison of the crystal structures of eukaryotic and bacterial SSU ribosomal RNAs reveals common structural features in the hypervariable regions. PLoS ONE 7(5):e38203.

Leff JW, Jones SE, Prober SM, Barberán A, Borer ET, Firn JL, Harpole WS, Hobbie SE, Hofmockel KS, Knops JM. 2015. Consistent responses of soil microbial communities to elevated nutrient inputs in grasslands across the globe. Proceedings of the National Academy of Sciences:doi:10.1073/pnas.1508382112.

Leliaert F, Verbruggen H, Wysor B, De Clerck O. 2009. DNA taxonomy in morphologically plastic taxa: algorithmic species delimitation in the Boodlea complex (Chlorophyta: Cladophorales). Molecular Phylogenetics and Evolution 53(1):122-133.

Leliaert F, Smith DR, Herron MD, Verbruggen H, Delwiche CF, Clerck OD. 2012. Phylogeny and molecular evolution of the Green algae. Critical Reviews in Plant Sciences 31(1):146.

Leliaert F, Verbruggen H, Vanormelingen P, Steen F, Lopez-Bautista JM, Zuccarello GC, De Clerck O. 2014. DNA-based species delimitation in algae. European Journal of Phycology 49(2):179-196.

Leliaert F, Lopez-Bautista JM. 2015. The chloroplast genomes of Bryopsis plumosa and Tydemania expeditiones (Bryopsidales, Chlorophyta): compact genomes and genes of bacterial origin. BMC Genomics 16(1):doi:10.1186/s12864-015-1418-3.

Lemieux C, Otis C, Turmel M. 2014. Chloroplast phylogenomic analysis resolves deep-level relationships within the green algal class Trebouxiophyceae. BMC Evolutionary Biology 14(1):doi:10.1186/s12862-014-0211-2.

Lepka D. 2007. Phylogenie Chlorella-ähnlicher Grünalgen von rDNA-Sequenzanalysen. MSc thesis, Georg-August-Universität Göttingen. 94 pp.

Lesaulnier C, Papamichail D, McCorkle S, Ollivier B, Skiena S, Taghavi S, Zak D, Van Der Lelie D. 2008. Elevated atmospheric $\mathrm{CO}_{2}$ affects soil microbial diversity associated with trembling aspen. Environmental Microbiology 10(4):926-941.

Lewis LA, Flechtner VR. 2002. Green algae (Chlorophyta) of desert microbiotic crusts: diversity of North American taxa. Taxon 41:443-451. 
Lewis LA, Flechtner VR. 2004. Cryptic species of Scenedesmus (Chlorophyta) from desert soil communities of Western North America. Journal of Phycology 40:1127-1137.

Lewis LA, Lewis PO. 2005. Unearthing the molecular phylodiversity of desert soil green algae (Chlorophyta). Systematic Biology 54(6):936-947.

Li H, Liu X, Wang Y, Hu H, Xu X. 2009. Enhanced expression of antifreeze protein genes drives the development of freeze tolerance in an Antarctica isolate of Chlorella vulgaris. Progress in Natural Science 19(9):1059-1062.

Librado P, Rozas J. 2009. DnaSP v5: a software for comprehensive analysis of DNA polymorphism data. Bioinformatics 25(11):1451-1452.

Lie AA, Liu Z, Hu SK, Jones AC, Kim DY, Countway PD, Amaral-Zettler LA, Cary SC, Sherr EB, Sherr BF. 2014. Investigating microbial eukaryotic diversity from a global census: insights from a comparison of pyrotag and full-length sequences of $18 \mathrm{~S}$ rRNA genes. Applied and Environmental Microbiology 80(14):4363-4373.

Lin C-s, Lin Y-h, Wu J-t. 2012. Biodiversity of the epiphyllous algae in a Chamaecyparis forest of northern Taiwan. Botanical Studies 53:489-499.

Lin C-S, Chou T-L, Wu J-T. 2013. Biodiversity of soil algae in the farmlands of mid-Taiwan. Botanical Studies 54(41):doi:10.1186/1999-3110-54-41.

Lin C-S, Wu J-T. 2014. Environmental factors affecting the diversity and abundance of soil photomicrobes in arid lands of subtropical Taiwan. Geomicrobiology Journal 31(4):350359.

Lindstrøm E-A, Johansen SW, Saloranta T. 2004. Periphyton in running waters - long-term studies of natural variation. The Atna River: Studies in an Alpine-Boreal Watershed. Springer. p. 63-86.

López-Bautista JM, Chapman RL. 2003. Phylogenetic affinities of the Trentepohliales inferred from small-subunit rDNA. International Journal of Systematic and Evolutionary Microbiology 53(6):2099-2106.

López-Bautista JM, Rindi F, Casamatta D. 2007. The systematics of subaerial algae. In: Seckbach J, editor. Algae and cyanobacteria in extreme environments. Springer. p. 599-617.

Lu Y, Chi X, Yang Q, Li Z, Liu S, Gan Q, Qin S. 2009. Molecular cloning and stress-dependent expression of a gene encoding $\Delta 12$-fatty acid desaturase in the Antarctic microalga Chlorella vulgaris NJ-7. Extremophiles 13(6):875-84.

Lu Y, Chi X, Li Z, Yang Q, Li F, Liu S, Gan Q, Qin S. 2010. Isolation and characterization of a stress-dependent plastidial $\Delta 12$ fatty acid desaturase from the Antarctic microalga Chlorella vulgaris NJ-7. Lipids 45(2):179-187.

Ludwig W, Strunk O, Westram R. 2004. ARB: a software environment for sequence data. Nucleic Acids Research 32(4):1363-1371.

Lukešová A, Komárek J. 1987. Succession of soil algae on dumps from strip coal-mining in the Most region (Czechoslovakia). Folia Geobotanica et Phytotaxonomica 22(4):355-362.

Lukešová A. 1993. Soil algae in four secondary succesional stages on abandoned fields. Algological Studies 71:81-102.

Lukešová A, Hoffmann L. 1996. Soil algae from acid rain impacted forest areas of the Krušné hory Mts. 1. Algal communities. Vegetatio 125(2):123-136.

Lukešová A. 2001. Soil algae in brown coal and lignite post-mining areas in Central Europe (Czech Republic and Germany). Restoration Ecology 9(4):341-350.

Lukešová A, Kociánová M, Váňa J, Štursová H, Elster J, Harčarik J, Halda J, Kocourková J, Jankovská V. 2010. Mud boils of the Giant Mts and Abisko Mts tundra - preliminary comparative study. Opera Corcontica 47:55-82. 
Lund JW. 1945. Observations on soil algae. New Phytologist 44(2):196-196.

Lund JW. 1947. Observations on soil algae. II. Notes on groups other than diatoms. New Phytologist 46(1):35-60.

Luo W, Pröschold T, Bock C, Krienitz L. 2010. Generic concept in Chlorella-related coccoid green algae (Chlorophyta, Trebouxiophyceae). Plant Biology 12:545-553.

Lutz S, Anesio AM, Edwards A, Benning LG. 2015. Microbial diversity on Icelandic glaciers and ice caps. Frontiers in Microbiology 6:doi:10.3389/fmicb.2015.00307.

Ma S, Han B, Huss VA, Hu X, Sun X, Zhang J. 2015. Chlorella thermophila (Trebouxiophyceae, Chlorophyta), a novel thermo-tolerant Chlorella species isolated from an occupied rooftop incubator. Hydrobiologia 760:81-89.

MacEntee FJ, Bold HC, Archibald PA. 1977. Notes on some edaphic algae of the South Pacific and Malaysian areas, with special reference to Pseudotetraedron polymorphum gen. et spec. nov. Soil Science 124(3):161-166.

Maistro S, Broady PA, Andreoli C, Negrisolo E. 2009. Phylogeny and Taxonomy of Xanthophyceae (Stramenopiles, Chromalveolata). Protist 160(3):412-426.

Mallet J. 2005. Hybridization as an invasion of the genome. Trends in Ecology and Evolution 20(5):229-237.

Maltsev EI. 2013. Ecological features of algae communities in forest floor of floodplain oak woods in steppe area of Ukraine. Gruntoznavstvo 14(3):330-339.

Maltsev EI, Negrulja DM. 2013. Algal flora of forest floor in plantings of park-monument landscape art "forest nursery". Gruntoznavstvo 14(3):98-105.

Maltseva IA. 2005a. Soil algae of flooded forests of the steppe sone of Ukraine. Melitopol: Liuks. p. 44-53.

Maltseva IA. 2005b. Soil algae from the forests of gorges of the steppe zone of Ukraine. Ekologija ta Noosferologija 16(3):150-156.

Maltseva IA. 2007. Algae groups of pine-woods of sandy terracing of river valleys in steppe area of Ukraine. Visnik Charkivskogo natsiolnogo agrarnogo universitetu, serija biologija. p. 106-112.

Maltseva IA, Baranova OO, Maltsev EI. 2009. Algogrouppings of biogeocenoses landscapetechnogenic systems of the Kryvoy Rog region. Visnik Zaporizkogo Nacionalnogo Universitetu. p. 20-23.

Mansour HA, Shaaban AS. 2010. Algae of soil surface layer of Wadi Al-Hitan Protective Area (World Heritage Site), El-Fayum Depression, Egypt. Journal of American Science 6(8):243-255.

Marande W, López-García P, Moreira D. 2009. Eukaryotic diversity and phylogeny using smalland large-subunit ribosomal RNA genes from environmental samples. Environmental Microbiology 11(12):3179-3188.

Marschner P, Kandeler E, Marschner B. 2003. Structure and function of the soil microbial community in a long-term fertilizer experiment. Soil Biology and Biochemistry 35(3):453-461.

Martiny JBH, Bohannan BJ, Brown JH, Colwell RK, Fuhrman JA, Green JL, Horner-Devine MC, Kane M, Krumins JA, Kuske CR. 2006. Microbial biogeography: putting microorganisms on the map. Nature Reviews Microbiology 4(2):102-112.

Mataloni G, Tell G, Wynn-Williams D. 2000. Structure and diversity of soil algal communities from Cierva Point (Antarctic Peninsula). Polar Biology 23(3):205-211.

Matula J, Pietryka M, Richter D, Wojtun B. 2007. Cyanoprokaryota and algae of Arctic terrestrial ecosystems in the Hornsund area, Spitsbergen. Polish Polar Research 28(4):283-315. 
Maxwell CD. 1991. Floristic changes in soil algae and cyanobacteria in reclaimed metalcontaminated land at Sudbury, Canada. Water, Air, and Soil Pollution 60:381-393.

McNeill J, Barrie F, Buck W, Demoulin V, Greuter W, Hawksworth D, Herendeen P, Knapp S, Marhold K, Prado J. 2012. International code of nomenclature for algae, fungi, and plants (Melbourne Code). Koeltz Scientific Books. ISBN 978-3-87429-425-6. 232 pp.

Medinger R, Nolte V, Pandey RV, Jost S, Ottenwaelder B, Schloetterer C, Boenigk J. 2010. Diversity in a hidden world: potential and limitation of next-generation sequencing for surveys of molecular diversity of eukaryotic microorganisms. Molecular Ecology 19:3240.

Megharaj M, Venkateswarlu K, Rao AS. 1986. Growth response of four species of soil algae to monocrotophos and quinalphos. Environmental Pollution Series A, Ecological and Biological 42(1):15-22.

Megharaj M, Kantachote D, Singleton I, Naidu R. 2000. Effects of long-term contamination of DDT on soil microflora with special reference to soil algae and algal transformation of DDT. Environmental Pollution 109(1):35-42.

Metting B, Rayburn W. 1979. Algal communities and soil microenvironments in an eastern Washington silt loam. Soil Science 127(2):doi:10.1097/00010694-197902000-00003.

Metting B. 1981. The systematics and ecology of soil algae. The Botanical Review 47(2):195312.

Meyen FJF. 1829. Beobachtungen über einige niedere Algenformen. Weber. p. 769-778.

Mikhailyuk TI, Sluiman HJ, Massalski A, Mudimu O, Demchenko EM, Kondratyuk SY, Friedl T. 2008. New streptophyte green algae from terrestrial habitats and an assessment of the genus Interfilum (Klebsormidiophyceae, Streptophyta). Journal of Phycology 44(6):1586-1603.

Mocali S, Benedetti A. 2010. Exploring research frontiers in microbiology: the challenge of metagenomics in soil microbiology. Research in Microbiology 161(6):497-505.

Moewus L. 1951. Systematische Bestimmung einzelliger grüner Algen auf Grund von Kulturversuchen. Botaniska Notiser 4:287-318.

Mondav R, Woodcroft BJ, Kim E-H, McCalley CK, Hodgkins SB, Crill PM, Chanton J, Hurst GB, VerBerkmoes NC, Saleska SR, Hugenholtz P, Rich VI, Tyson GW. 2014. Discovery of a novel methanogen prevalent in thawing permafrost. Nature Communications 5: doi:10.1038/ncomms4212.

Moon-van der Staay S, De Wachter R, Vaulot D. 2001. Oceanic 18S rRNA gene sequences from picoplankton reveal unsuspected eukaryotic diversity. Nature 409:607-610.

Moon-van der Staay SY, Tzeneva VA, van Der Staay GW, De Vos WM, Smidt H, Hackstein JH. 2006. Eukaryotic diversity in historical soil samples. FEMS Microbiology Ecology 57(3):420-428.

Moreira D, Lopez-Garcia P. 2002. The molecular ecology of microbial eukaryotes unveils a hidden world. Trends in Microbiology 10(1):31-38.

Mudimu O, Rybalka N, Bauersachs T, Born J, Friedl T, Schulz R. 2014. Biotechnological screening of microalgal and cyanobacterial strains for biogas production and antibacterial and antifungal effects. Metabolites 4(2):373-393.

Müller J, Day JG, Harding K, Hepperle D, Lorenz M, Friedl T. 2007a. Assessing genetic stability of a range of terrestrial microalgae after cryopreservation using amplified fragment length polymorphism (AFLP). American Journal of Botany 94(5):799-808. 
Müller T, Rahmann S, Dandekar T, Wolf M. 2004. Accurate and robust phylogeny estimation based on profile distances: a study of the Chlorophyceae (Chlorophyta). BMC Evolutionary Biology 4(1):doi:10.1186/1471-2148-4-20.

Müller T, Philippi N, Dandekar T, Schultz J, Wolf M. 2007b. Distinguishing species. RNA 13(9):1469-1472.

Musabaeva IA. Osobennosti organizacii gruppirovok pochvennykh vodoroslej stepnogo fitocenoza. http://iesen.nspu.ru/images/sborn/nr2011/kaf_botaniki/ch2.html.

Nacke H, Thurmer A, Wollherr A, Will C, Hodac L, Herold N, Schoning I, Schrumpf M, Daniel R. 2011. Pyrosequencing-based assessment of bacterial community structure along different management types in German forest and grassland soils. PLoS ONE 6(2):doi:10.1371/journal.pone.0017000.

Nägeli C. 1849. Gattungen einzelliger Algen. Schulthess. 139 pp.

Nagy ML, Johansen JR, Clair LLS, Webb BL. 2005. Recovery patterns of microbiotic soils crusts 70 years after arsenic contamination. Journal of Arid Environments 63:304-323.

Nakada T, Misawa K, Nozaki H. 2008. Molecular systematics of Volvocales (Chlorophyceae, Chlorophyta) based on exhaustive 18S rRNA phylogenetic analyses. Molecular Phylogenetics and Evolution 48(1):281-291.

Naselli-Flores L, Padisák J. 2015. Blowing in the wind: how many roads can a phytoplanktont walk down? A synthesis on phytoplankton biogeography and spatial processes. Hydrobiologia:doi:10.1007/s10750-015-2519-3.

Neefs J-M, De Wachter R. 1990. A proposal for the secondary structure of a variable area of eukaryotic small ribosomal subunit RNA involving the existence of a pseudoknot. Nucleic Acids Research 18(19):5695-5704.

Němcová Y, Eliáš M, Škaloud P, Hodač L, Neustupa J. 2011. Jenufa gen. nov.: a new genus of coccoid green algae (Chlorophyceae, incertae sedis) previously recorded by environmental sequencing. Journal of Phycology 47(4):928-938.

Néron B, Ménager H, Maufrais C, Joly N, Maupetit J, Letort S, Carrere S, Tuffery P, Letondal C. 2009. Mobyle: a new full web bioinformatics framework. Bioinformatics 25(22):30053011.

Neustupa J. 2001. Soil algae from marlstone-substratum based biotopes in the Nature park Džbán (Central Bohemia, Czech Republic) with special attention to the natural treeless localities. Archiv für Hydrobiologie. Supplementband, Algological Studies 137:109-120.

Neustupa J, Škaloud P. 2004. Contribution to the knowledge of soil algae of two abandoned industrial sedimentation basins in Eastern Bohemia. In: Kovár P, editor. Natural recovery of human-made deposits in landscape (Biotic interactions and ore/ash-slag artificial ecosystems). Prague: Academia. p. 194-199.

Neustupa J, Eliáš M, Šejnohová L. 2007. A taxonomic study of two Stichococcus species (Trebouxiophyceae, Chlorophyta) with a starch-enveloped pyrenoid. Nova Hedwigia 84(1-2):51-63.

Neustupa J, Škaloud P. 2008. Diversity of subaerial algae and cyanobacteria on tree bark in tropical mountain habitats. Biologia 63(6):806-812.

Neustupa J, Němcová Y, Eliáš M, Škaloud P. 2009. Kalinella bambusicola gen. et sp. nov. (Trebouxiophyceae, Chlorophyta), a novel coccoid Chlorella-like subaerial alga from Southeast Asia. Phycological Research 57(3):159-169.

Neustupa J, Škaloud P. 2010. Diversity of subaerial algae and cyanobacteria growing on bark and wood in the lowland tropical forests of Singapore. Plant Ecology and Evolution 143(1):51-62. 
Neustupa J, Eliáš M, Škaloud P, Němcová Y, Šejnohová L. 2011. Xylochloris irregularis gen. et sp. nov. (Trebouxiophyceae, Chlorophyta), a novel subaerial coccoid green alga. Phycologia 50(1):57-66.

Neustupa J, Němcová Y, Veselá J, Steinova J, Škaloud P. 2013a. Leptochlorella corticola gen. et sp. nov. and Kalinella apyrenoidosa sp. nov.: two novel Chlorella-like green microalgae (Trebouxiophyceae, Chlorophyta) from subaerial habitats. International Journal of Systematic and Evolutionary Microbiology 63:377-387.

Neustupa J, Němcová Y, Veselá J, Steinová J, Škaloud P. 2013b. Parachloroidium gen. nov. (Trebouxiophyceae, Chlorophyta), a novel genus of coccoid green algae from subaerial corticolous biofilms. Phycologia 52(5):411-421.

Nikorych V, Chornevych T. 2012. Ecobiomorph structure of algocenoses organization of brownish-podzolic gleied soils of Ukrainian Ciscarpathia. Soil Forming Factors and Processes from the Temperate Zone 10:141-151.

Novakovskaya IV, Patova EN. 2007. Changes in soil algal communities in spruce phytocenoses under the influence of aerotechnogenic pollution. Eurasian Soil Science 40(5):576-582.

Novakovskaya IV, Patova EN. 2008. Green algae in spruce forests in the north-east of European Russia. Biologia 63(6):836-842.

Novichkova-Ivanova LN. 1972. Soil algae of middle Asia deserts. In: Rodin LE, editor. Ecophysiology foundation ecosystems productivity in arid zones. Nauka. p. 180-182.

Novis PM, Beer T, Vallance J. 2008. New records of microalgae from the New Zealand alpine zone, and their distribution and dispersal. New Zealand Journal of Botany 46(3):347-366.

Novis PM, Aislabie J, Turner S, McLeod M. 2015. Chlorophyta, Xanthophyceae and Cyanobacteria in Wright Valley, Antarctica. Antarctic Science 27(5):439-454.

Olivieri G, Marzocchella A, Andreozzi R, Pinto G, Pollio A. 2011. Biodiesel production from Stichococcus strains at laboratory scale. Journal of Chemical Technology and Biotechnology 86(6):776-783.

Olivieri G, Gargano I, Andreozzi R, Marotta R, Marzocchella A, Pinto G, Pollio A. 2013. Effects of photobioreactors design and operating conditions on Stichococcus bacillaris biomass and biodiesel production. Biochemical Engineering Journal 74:8-14.

Osundeko O, Davies H, Pittman JK. 2013. Oxidative stress-tolerant microalgae strains are highly efficient for biofuel feedstock production on wastewater. Biomass Bioenergy 56:284-294.

Palacios C, Zettler E, Amils R, Amaral-Zettler L. 2008. Contrasting microbial community assembly hypotheses: a reconciling tale from the Rio Tinto. PLoS ONE 3(12):e3853.

Park KC, Whitney C, Kozera C, O'Leary SJ, McGinn PJ. 2015. Seasonal isolation of microalgae from municipal wastewater for remediation and biofuel applications. Journal of Applied Microbiology 119:76-87.

Patova EN, Dorokhova MF. 2008. Green algae in tundra soils affected by coal mine pollutions. Biologia 63(6):831-835.

Patterson D. 1989. Stramenopiles: chromophytes from a protistan perspective. In: Green J, Leadbeater B, Diver W, editors. The chromophyte algae: problems and perspectives.: Oxford Science Publications. p. 357-379.

Paulsen BS, Vieira AAH, Klaveness D. 1992. Structure of extracellular polysaccharides produced by a soil Cryptomonas sp. (Cryptophyceae). Journal of Phycology 28(1):61-63.

Peer T, Türk R, Gruber J, A T. 2010. Species composition and pedological characteristics of biological soil crusts in a high alpine ecosystem, Hohe Tauern, Austria. Eco.mont 2(1):2330 . 
Pentecost A. 1988. Observations on growth rates and calcium carbonate deposition in the green alga Gongrosira. New Phytologist 110(2):249-253.

Pentecost A, Spiro B. 1990. Stable carbon and oxygen isotope composition of calcites associated with modern freshwater cyanobacteria and algae. Geomicrobiology Journal 8(1):17-26.

Perútková K. 2014. Diverzita terestrických řas urbánních habitatů ve střední Evropě. MSc thesis, Masaryk University (Brno). 70 pp.

Petersen JB. 1935. Studies on the biology and taxonomy of soil algae. Dansk Botanisk Arkiv 8. $183 \mathrm{pp}$.

Pia J. 1926. Pflanzen als Gesteinsbildner. Berlin: Verlag Gebrüder Bornträger. 355 pp.

Pimm SL, Raven P. 2000. Biodiversity: extinction by numbers. Nature 403(6772):843-845.

Posada D. 2008. jModelTest: phylogenetic model averaging. Molecular Biology and Evolution 25(7):1253-1256.

Posrednikova AV, Pakhomov OY, Kulbachko YL. 2009. Soil algae and mesofauna communities in biotopes of forest rehabilitation in Zhovti Vody (Dnipropetrovsk region). Visnyk of Dnipropetrovsk University, Biology, Ecology 17(1):172-177.

Preston-Mafham J, Boddy L, Randerson PF. 2002. Analysis of microbial community functional diversity using sole-carbon-source utilisation profiles - a critique. FEMS Microbiology Ecology 42(1):1-14.

Prober SM, Leff JW, Bates ST, Borer ET, Firn J, Harpole WS, Lind EM, Seabloom EW, Adler PB, Bakker JD. 2015. Plant diversity predicts beta but not alpha diversity of soil microbes across grasslands worldwide. Ecology Letters 18(1):85-95.

Pröschold T, Marin B, Gert U, Melkonian M. 2001. Molecular phylogeny and taxonomic revision of Chlamydomonas (Chlorophyta). I. Emendation of Chlamydomonas Ehrenberg and Chloromonas Gobi, and description of Oogamochlamys gen. nov. and Lobochlamys gen. nov. Protist 152:265-300.

Pröschold T, Bock C, Luo W, Krienitz L. 2010. Polyphyletic distribution of bristle formation in Chlorellaceae: Micractinium, Diacanthos, Didymogenes and Hegewaldia gen. nov. (Trebouxiophyceae, Chlorophyta). Phycological Research 58(1):doi:10.1111/j.14401835.2009.00552.x.

Prosser JI. 2002. Molecular and functional diversity in soil micro-organisms. Plant and Soil 244(1-2):9-17.

Ragon M, Fontaine MC, Moreira D, López-García P. 2012. Different biogeographic patterns of prokaryotes and microbial eukaryotes in epilithic biofilms. Molecular Ecology 21(15):3852-3868.

Rahmann S, Müller T, Dandekar T, Wolf M. 2006. Efficient and robust analysis of large phylogenetic datasets. In: Hsu H-H, editor. Advanced Data Mining Technologies in Bioinformatics. Hershey, PA, USA: Idea Group, Inc. p. 104-117.

Rahmonov O, Piątek J. 2007. Sand colonization and initiation of soil development by Cyanobacteria and algae. Ekológia 26(1):52-63.

Rambaut A. FigTree, a graphical viewer of phylogenetic trees. http://tree.bio.ed.ac.uk/software/ figtree.

Rastogi G, Sani RK. 2011. Molecular techniques to assess microbial community structure, function, and dynamics in the environment. Microbes and Microbial Technology. Springer. p. 29-57.

Raven JA. 2012. Algal biogeography: metagenomics shows distribution of a picoplanktonic pelagophyte. Current Biology 22(17):R682-R683. 
Ray JG, Thomas BT. 2012. Fertility characteristics of oxic dystrustepts under natural forest, rubber, and teak plantations in different seasons, Kerala, South India. Communications in Soil Science and Plant Analysis 43(17):2247-2261.

Reisigl H. 1958. Über die Verteilung der Bodenalgen in der Gipfelstufe der Ötztaler Alpen. Berichte des Naturwissenschaftlich-medizinischen Vereins in Innsbruck 53:163-172.

Reisigl H. 1964. Zur Systematik und Ökologie alpiner Bodenalgen. Österreichische botanische Zeitschrift 111 (4): 402-499.

Reuter JS, Mathews DH. 2010. RNAstructure: software for RNA secondary structure prediction and analysis. BMC Bioinformatics 11:doi:10.1186/1471-2105-11-129.

Reynaud PA, Lumpkin TA. 1988. Microalgae of the Lanzhou (China) cryptogamic crust. Arid Land Research and Management 2(3):145-155.

Řezáčová M, Neustupa J. 2007. Distribution of the genus Mallomonas (Synurophyceae) ubiquitous dispersal in microorganisms evaluated. Protist 158(1):29-37.

Řezanka T, Temina M, Tolstikov A, Dembitsky V. 2004. Natural microbial UV radiation filtersmycosporine-like amino acids. Folia Microbiologica 49(4):339-352.

Richards TA, Bass D. 2005. Molecular screening of free-living microbial eukaryotes: diversity and distribution using a meta-analysis. Current Opinion in Microbiology 8(3):240-252.

Richards TA, Vepritskiy AA, Gouliamova DE, Nierzwicki-Bauer SA. 2005. The molecular diversity of freshwater picoeukaryotes from an oligotrophic lake reveals diverse, distinctive and globally dispersed lineages. Environmental Microbiology 7(9):1413-1425.

Riding R. 1991. Cambrian calcareous cyanobacteria and algae. In: Riding R, editor. Calcareous algae and stromatolites. Berlin: Springer-Verlag. 571 pp.

Ř́dká T, Peksa O, Rai H, Upreti DK, Škaloud P. 2014. Photobiont diversity in Indian Cladonia lichens, with special emphasis on the geographical patterns. In: Rai H, Upreti DK, editors. Terricolous lichens in India. Springer. p. 53-71.

Rindi F, McIvor L, Guiry GM. 2004. The Prasiolales (Chlorophyta) of Atlantic Europe: An assessment based on morphological, molecular, and ecological data, including the characterization of Rosenvingiella radicans (Kützing) comb. nov. Journal of Phycology 40(5):977-997.

Rindi F, McIvor L, Sherwood A, Friedl T, Guiry M, Sheath R. 2007. Molecular phylogeny of the green algal order Prasiolales (Trebouxiophyceae, Chlorophyta). Journal of Phycology 43:811-822.

Rindi F, Guiry MD, Lo JM. 2008. Distribution, morphology, and phylogeny of Klebsormidium (Klebsormidiales, Charophyceae) in urban environments in Europe. Journal of Phycology 44:1529-1540.

Rindi F, Allali HA, Lam DW, López-Bautista JM. 2009. An overview of the biodiversity and biogeography of terrestrial green algae. In: Rescigno V, editor. Biodiversity Hotspots. Nova Science Publishers. 25 pp.

Rindi F, Hodkinson T, Jones M, Waldren S, Parnell J. 2011. Terrestrial green algae: systematics, biogeography and expected responses to climate change. In: Hodkinson TR, editor. Climate Change, Ecology and Systematics. Cambridge University Press. p. 201-227.

Rodó JDC-P, Molinari-Novoa EA. 2015. A nomenclatural note and cultural note on Chlorella peruviana G. Chacón and other species of the genus Chlorella Beij. (Chlorellaceae, Chlorellales). The Biologist (Lima) 13(1):71-74.

Roger P-A, Reynaud P-A. 1982. Free-living blue-green algae in tropical soils. In: Dommergues Y, Diem H, editors. Microbiology of tropical soils and plant productivity. La Hague: Martinus Nijhoff Publisher. p. 147-168. 
Rondon MR, August PR, Bettermann AD, Brady SF, Grossman TH, Liles MR, Loiacono KA, Lynch BA, MacNeil IA, Minor C. 2000. Cloning the soil metagenome: a strategy for accessing the genetic and functional diversity of uncultured microorganisms. Applied and Environmental Microbiology 66(6):2541-2547.

Ronquist F, Teslenko M, van der Mark P, Ayres DL, Darling A, Hohna S, Larget B, Liu L, Suchard MA, Huelsenbeck JP. 2012. MrBayes 3.2: Efficient Bayesian phylogenetic inference and model choice across a large model space. Systematic Biology 61(3):539542 .

Rosa K, Lhotský O. 1971. Edaphische Algen und Protozoen im Isergebirge, Tschechoslowakei. Oikos 22:21-29.

Rybalka N, Andersen RA, Kostikov I, Mohr KI, Massalski A, Olech M, Friedl T. 2009. Testing for endemism, genotypic diversity and species concepts in Antarctic terrestrial microalgae of the Tribonemataceae (Stramenopiles, Xanthophyceae). Environmental Microbiology 11(3):554-565.

Rybalka N, Wolf M, Andersen RA, Friedl T. 2013. Congruence of chloroplast- and nuclearencoded DNA sequence variations used to assess species boundaries in the soil microalga Heterococcus (Stramenopiles, Xanthophyceae). BMC Evolutionary Biology 13(39): doi:10.1186/1471-2148-13-39.

Ryšánek D, Hrčková K, Škaloud P. 2014. Global ubiquity and local endemism of free-living terrestrial protists: phylogeographic assessment of the streptophyte alga Klebsormidium. Environmental Microbiology 17(3):689-698.

Safi C, Zebib B, Merah O, Pontalier P-Y, Vaca-Garcia C. 2014. Morphology, composition, production, processing and applications of Chlorella vulgaris: A review. Renewable \& Sustainable Energy Reviews 35:265-278.

Şalaru V, Trofim A, Melniciuc C, Dontu N. 2008. Taxonomic and ecologic structure of communities of edaphic algae from the agrophytocenoses of the northern districts of Moldova. Journal of Plant Development 15:3-6.

Santoferrara LF, Grattepanche JD, Katz LA, McManus GB. 2014. Pyrosequencing for assessing diversity of eukaryotic microbes: analysis of data on marine planktonic ciliates and comparison with traditional methods. Environmental Microbiology 16(9):2752-2763.

Scalzi G, Selbmann L, Zucconi L, Rabbow E, Horneck G, Albertano P, Onofri S. 2012. LIFE Experiment: isolation of cryptoendolithic organisms from Antarctic colonized sandstone exposed to space and simulated Mars conditions on the International Space Station. Origins of Life and Evolution of Biospheres 42:253-262.

Scherbina V, Maltseva I, Solonenko A. 2014. Peculiarities of postpyrogene development of algae in steppe biocenoses at Askania Nova Biospheric National Park. Contemporary Problems of Ecology 7(2):187-191.

Schloss PD, Westcott SL, Ryabin T, Hall JR, Hartmann M, Hollister EB, Lesniewski RA, Oakley BB, Parks DH, Robinson CJ. 2009. Introducing mothur: open-source, platformindependent, community-supported software for describing and comparing microbial communities. Applied and Environmental Microbiology 75(23):7537-7541.

Schmidle W. 1901. Ueber drei Algengenera. Berichte der Deutschen Botanischen Gesellschaft 19:149-158.

Schmidt SK, Lynch RC, King AJ, Karki D, Robeson MS, Nagy L, Williams MW, Mitter MS, Freeman KR, Schmidt SK, Lynch RC, King AJ, Karki D. 2011. Phylogeography of microbial phototrophs in the dry valleys of the high Himalayas and Antarctica. Proceedings of the Royal Society B: Biological Sciences 278:702-708. 
Schmidt SK, Darcy JL. 2014. Phylogeny of ulotrichalean algae from extreme high-altitude and high-latitude ecosystems. Polar Biology:doi:10.1007/s00300-014-1631-6.

Schneider CA, Rasband WS, Eliceiri KW. 2012. NIH Image to ImageJ: 25 years of image analysis. Nature Methods 9(7):671-675.

Schultz J, Müller T, Achtziger M, Seibel PN, Dandekar T, Wolf M. 2006. The internal transcribed spacer 2 database - a web server for (not only) low level phylogenetic analyses. Nucleic Acids Research 34:704-707.

Sciuto K, Lewis LA, Verleyen E, Moro I, La Rocca N. 2015. Chodatodesmus australis sp. nov. (Scenedesmaceae, Chlorophyta) from Antarctica, with the emended description of the genus Chodatodesmus, and circumscription of Flechtneria rotunda gen. et sp. nov. Journal of Phycology:doi:10.1111/jpy.12355-14-227.

Seibel PN, Muller T, Dandekar T, Schultz J, Wolf M. 2006. 4SALE - a tool for synchronous RNA sequence and secondary structure alignment and editing. BMC Bioinformatics 7(498):doi:10.1186/1471-2105-7-498.

Seibel PN, Muller T, Dandekar T, Wolf M. 2008. Synchronous visual analysis and editing of RNA sequence and secondary structure alignments using 4SALE. BMC Research Notes 1(91):doi:10.1186/1756-0500-1-91.

Selig C, Wolf M, Müller T, Dandekar T, Schultz J. 2008. The ITS2 Database II: homology modelling RNA structure for molecular systematics. Nucleic Acids Research 36:377-380.

Shade A, Hogan CS, Klimowicz AK, Linske M, McManus PS, Handelsman J. 2012. Culturing captures members of the soil rare biosphere. Environmental Microbiology 14(9):22472252.

Sharma NK, Rai AK, Singh S, Brown RMJ. 2007. Airborne algae: their present status and relevance. Journal of Phycology 43(4):615-627.

Sharma NK, Rai AK. 2010. Biodiversity and biogeography of microalgae: progress and pitfalls. Environmental Reviews 19:doi:10.1139/A10-020.

Shekhovtseva OG. 2014. Soil algae in urban ecosystems of the city of Mariupol. Gruntoznavstvo 15(1-2):88-92.

Shi XL, Marie D, Jardillier L, Scanlan DJ, Vaulot D. 2009. Groups without cultured representatives dominate eukaryotic picophytoplankton in the oligotrophic South East Pacific Ocean. PLoS ONE 4(10):e7657.

Shields LM, Durrell LW. 1964. Algae in relation to soil fertility. The Botanical Review 30(1):92128.

Shiraishi F, Bissett A, de Beer D, Reimer A, Arp G. 2008a. Photosynthesis, respiration and exopolymer calcium-binding in biofilm calcification (Westerhöfer and Deinschwanger Creek, Germany). Geomicrobiology Journal 25(2):83-94.

Shiraishi F, Reimer A, Bissett A, de Beer D, Arp G. 2008b. Microbial effects on biofilm calcification, ambient water chemistry and stable isotope records in a highly supersaturated setting (Westerhöfer Bach, Germany). Palaeogeography, Palaeoclimatology, Palaeoecology 262(1):91-106.

Shtina EA, Gollerbach MM. 1976. Ecology of soil algae. Nauka. 143 pp.

Shtina EA. 1990. Soil algae as ecological indicators. Botanical Journal(4):441-452.

Shukla SP, Kvíderová J, Elster J. 2011. Nutrient requirements of polar Chlorella-like species. Czech Polar Reports 1(1):1-10.

Sivakumar G, Jeong K, Lay JO. 2014. Bioprocessing of Stichococcus bacillaris strain siva2011. Biotechnology for Biofuels 7(62):doi:10.1186/1754-6834-7-62. 
Škaloud P, Jiří N, Radochová B, Kubínová L. 2005. Confocal microscopy of chloroplast morphology and ontogeny in three strains of Dictyochloropsis (Trebouxiophyceae, Chlorophyta). Phycologia 44(3):261-269.

Škaloud P. 2009. Species composition and diversity of aero-terrestrial algae and cyanobacteria of the Boreč Hill ventaroles. Fottea 9(1):65-80.

Škaloud P, Rindi F. 2013. Ecological differentiation of cryptic species within an asexual protist morphospecies: a case study of filamentous green alga Klebsormidium (Streptophyta). Journal of Eukaryotic Microbiology 60(4):350-362.

Škaloud P, Lukešová A, Malavasi V, Ryšánek D, Hrčková K, Rindi F. 2014a. Molecular evidence for the polyphyletic origin of low $\mathrm{pH}$ adaptation in the genus Klebsormidium (Klebsormidiophyceae, Streptophyta). Plant Ecology and Evolution 147(3):333-345.

Škaloud P, Škaloudová M, Procházková A, Němcová Y. 2014b. Morphological delineation and distribution patterns of four newly described species within the Synura petersenii species complex (Chrysophyceae, Stramenopiles). European Journal of Phycology 49(2):213229.

Škaloud P, Steinová J, Řídká T, Vančurová L, Peksa O. 2015. Assembling the challenging puzzle of algal biodiversity: species delimitation within the genus Asterochloris (Trebouxiophyceae, Chlorophyta). Journal of Phycology 51:507-527.

Šlapeta J, Moreira D, López-García P. 2005. The extent of protist diversity: insights from molecular ecology of freshwater eukaryotes. Proceedings of the Royal Society B: Biological Sciences 272(1576):2073-2081.

Šlapeta J, López-García P, Moreira D. 2006. Global dispersal and ancient cryptic species in the smallest marine eukaryotes. Molecular Biology and Evolution 23(1):23-29.

Slocombe SP, Zhang Q, Ross M, Anderson A, Thomas NJ, Lapresa Á, Rad-Menéndez C, Campbell CN, Black KD, Stanley MS, Day JG. 2015. Unlocking nature's treasure-chest: screening for oleaginous algae. Scientific Reports 5:doi:10.1038/srep09844.

Sluiman HJ, Guihal C. 2008. Assessing phylogenetic affinities and species delimitations in Klebsormidiales (Streptophyta): Nuclear-encoded rDNA phylogenies and its secondary structure models in Klebsormidium, Hormidiella and Entransia. Journal of Phycology 44:183-195.

Smith H, Hardy P, Leith I, Spaull V, Twelves E. 1974. A biological survey of St. Paul's Rocks in the equatorial Atlantic Ocean. Biological Journal of the Linnean Society 6(2):89-96.

Smith S, Abed R, Garcia-Pichel F. 2004. Biological soil crusts of sand dunes in Cape Cod National Seashore, Massachusetts, USA. Microbial Ecology 48(2):200-208.

Soare LC, Dobrescu CM. 2010. Preliminary data on edaphic algae in the city of Piteşti (Romania). Analele Universităţii din Oradea - Fascicula Biologie. p. 186-189.

Song H, Zhang Q, Liu G, Hu Z. 2015. Polulichloris henanensis gen. et sp. nov. (Trebouxiophyceae, Chlorophyta), a novel subaerial coccoid green alga. Phytotaxa 218(2):137-146.

Spitzer K. 2013. Biodiversity of terrestrial green algae in tropical mountain rain forest in Podocarpus National Park (Ecuador) using a culture dependent approach. MSc thesis, University of Duisburg-Essen. 122 pp.

Spitzer K, Faßhauer F, Hodač L, Friedl T. 2014. Biodiversity of terrestrial green algae in a tropical mountain rain forest of Ecuador (Podocarpus National Park). Poster. Book of Abstracts of the $44^{\text {th }}$ Annual Meeting of the Ecological Society of Germany, Austria and Switzerland. Hildesheim (Germany). p. 240. 
Stamatakis A, Hoover P, Rougemont J. 2008. A rapid bootstrap algorithm for the RAxML Web servers. Systematic Biology 57(5):758-771.

Stanek-Tarkowska J, Noga T. 2012. Diversity of diatoms (Bacillariophyceae) in the soil under traditional tillage and reduced tillage. Inżynieria Ekologiczna 30:287-296.

Starks TL, Shubert LE, Trainor FR. 1981. Ecology of soil algae: a review. Phycologia 20:65-80.

Stibal M, Šabacká M, Kaštovská K. 2006. Microbial communities on glacier surfaces in Svalbard: impact of physical and chemical properties on abundance and structure of cyanobacteria and algae. Microbial Ecology 52(4):644-654.

Strimmer K, Von Haeseler A. 1996. Quartet puzzling: a quartet maximum-likelihood method for reconstructing tree topologies. Molecular Biology and Evolution 13(7):964-969.

Sumbali G, Mehrotra R. 2009. Principles of Microbiology. Tata McGraw-Hill. 926 pp.

Sun Z, Li G, Wang C, Jing Y, Zhu Y, Zhang S, Liu Y. 2014. Community dynamics of prokaryotic and eukaryotic microbes in an estuary reservoir. Scientific Reports 4(6966):doi:10.1038/srep06966.

Suutari M, Majaneva M, Fewer D, Voirin B, Aiello A, Friedl T, Chiarello A, Blomster J. 2010. Molecular evidence for a diverse green algal community growing in the hair of sloths and a specific association with Trichophilus welckeri (Chlorophyta, Ulvophyceae). BMC Evolutionary Biology 10(1):86.

Taib N, Mangot J-F, Domaizon I, Bronner G, Debroas D. 2013. Phylogenetic affiliation of SSU rRNA genes generated by massively parallel sequencing: new insights into the freshwater protist diversity. PLoS ONE 8(3):e58950.

Tamura K, Peterson D, Peterson N, Stecher G, Nei M, Kumar S. 2011. MEGA5: molecular evolutionary genetics analysis using maximum likelihood, evolutionary distance, and maximum parsimony methods. Molecular Biology and Evolution 28(10):2731-2739.

Tamura K, Stecher G, Peterson D, Filipski A, Kumar S. 2013. MEGA6: molecular evolutionary genetics analysis version 6.0. Molecular Biology and Evolution 30(12):2725-2729.

Tell G. 1976. Algas del suelo de los alrededores de Buenos Aires (República Argentina). Darwiniana 20(3-4):491-548.

Temraleeva A, Pinskii D, Patova E, Spirina E. 2011. The use of algae-cyanobacterial communities for the assessment of lead pollution of gray forest soils. Eurasian Soil Science 44(3):326-331.

ter Braak CJF, Šmilauer P. 2002. CANOCO reference manual and CanoDraw for Windows user's guide: software for canonical community ordination (version 4.5). Microcomputer Power. $500 \mathrm{pp}$.

Terlizzi DE, Karlander EP. 1979. Soil algae from a Maryland serpentine formation. Soil Biology and Biochemistry 11(2):205-207.

Thüs H, Muggia L, Pérez-Ortega S, Favero-Longo SE, Joneson S, O’Brien H, Nelsen MP, Duque-Thüs R, Grube M, Friedl T. 2011. Revisiting photobiont diversity in the lichen family Verrucariaceae (Ascomycota). European Journal of Phycology 46(4):399-415.

Trainor FR. 1970. Survival of algae in a desiccated soil. Phycologia 9(2):111-113.

Trainor FR. 1985. Survival of algae in a desiccated soil: a 25 year study. Phycologia 24(1):7982.

Trainor FR, Gladych R. 1995. Survival of algae in a desiccated soil: a 35-year study. Phycologia 34(3):191-192.

Trenkwalder H. 1975. Neue Bodenalgen aus Föhrenwäldern im Raum von Brixen (Südtirol, Italien). Berichte des naturwissenschaftlich-medizinischen Vereins in Innsbruck 62:7-19. 
Treves H, Raanan H, Finkel OM, Berkowicz SM, Keren N, Shotland Y, Kaplan A. 2013. A newly isolated Chlorella sp. from desert sand crusts exhibits a unique resistance to excess light intensity. FEMS Microbiology Ecology 86(3):373-380.

Trzcińska M, Pawlik-Skowrońska B. 2008. Soil algal communities inhabiting zinc and lead mine spoils. Journal of Applied Phycology 20(4):341-348.

Tschermak-Woess E. 1988. New and known taxa of Chlorella (Chlorophyceae): Occurrence as lichen phycobionts and observations on living dictyosomes. Plant Systematics and Evolution 159(1-2):123-139.

Türk R, Gärtner G. 2001. Biological soil crusts of the subalpine, alpine and nival areas in the Alps. Ecological Studies 150:67-73.

Turmel M, Otis C, Lemieux C. 2015. Dynamic evolution of the chloroplast genome in the Green algal classes Pedinophyceae and Trebouxiophyceae. Genome Biology and Evolution 7:2062-2082.

Veselá J. 2006. Benthic algal communities and their ecology in sandstone periodically desiccated brook in National Park Bohemian Switzerland (Czech Republic). Czech Phycology 6:99110.

Veselá J, Urbánková P, Cerná K, Neustupa J. 2012. Ecological variation within traditional diatom morphospecies: diversity of Frustulia rhomboides sensu lato (Bacillariophyceae) in European freshwater habitats. Phycologia 51(5):552-561.

Viles HA. 2012. Microbial geomorphology: a neglected link between life and landscape. Geomorphology 157-158:6-16.

Vinogradova OM, Darienko TM. 2008. Terrestrial algae of hypersaline environments of the Central Syvash islands (Kherson Region, Ukraine). Biologia 63(6):813-823.

Viprey M, Guillou L, Ferréol M, Vaulot D. 2008. Wide genetic diversity of picoplanktonic green algae (Chloroplastida) in the Mediterranean Sea uncovered by a phylum-biased PCR approach. Environmental Microbiology 10(7):1804-1822.

Vishnivetskaya TA. 2009. Viable Cyanobacteria and Green algae from the permafrost darkness. In: Margesin R, editor. Permafrost Soils. Berlin, Heidelberg: Springer-Verlag. p. 73-84.

Vyverman W, Verleyen E, Wilmotte A, Hodgson DA, Willems A, Peeters K, Van de Vijver B, De Wever A, Leliaert F, Sabbe K. 2010. Evidence for widespread endemism among Antarctic micro-organisms. Polar Science 4:103-113.

Wallner J. 1934. Über die Beteiligung kalkablagernder Pflanzen bei der Bildung südbayerischer Tuffe. Bibliotheca Botanica 110:1-30.

Watanabe S. 1983. New and Interesting Green Algae from Soils of some Asian and Oceanian Regions. Archiv für Protistenkunde 127(3):223-270.

Watanabe S, Nakayama T. 2007. Ultrastructure and phylogenetic relationships of the unicellular green algae Ignatius tetrasporus and Pseudocharacium americanum (Chlorophyta). Phycological Research 55:doi:10.1111/j.1440-1835.2006.00439.x.

Wheeler DL, Barrett T, Benson DA, Bryant SH, Canese K, Chetvernin V, Church DM, DiCuccio M, Edgar R, Federhen S. 2007. Database resources of the National Center for Biotechnology Information. Nucleic Acids Research 35:D5-D12.

Winsborough BM, Golubić S. 1987. The role of diatoms in stromatolite growth: two examples from modern freshwater settings. Journal of Phycology 23(2):195-201.

Wojtuń B, Samecka-Cymerman A, Kolon K, Kempers A, Skrzypek G. 2013. Metals in some dominant vascular plants, mosses, lichens, algae, and the biological soil crust in various types of terrestrial tundra, SW Spitsbergen, Norway. Polar Biology 36(12):1799-1809. 
Wolf M, Achtziger M, Schultz J, Dandekar T, Müller T. 2005. Homology modeling revealed more than 20,000 rRNA internal transcribed spacer 2 (ITS2) secondary structures. RNA 11:1616-1623.

Wolf M, Ruderisch B, Dandekar T, Schultz J, Müller T. 2008. ProfDistS: (profile-) distance based phylogeny on sequence - structure alignments. Bioinformatics 24(20):2401-2402.

Wolf M, Chen S, Song J, Ankenbrand M, Muller T. 2013. Compensatory base changes in ITS2 secondary structures correlate with the biological species concept despite intragenomic variability in ITS2 sequences-a proof of concept. PLoS ONE 8(6):e66726.

Wong FKY, Lacap DC. 2010. Hypolithic microbial community of quartz pavement in the highaltitude tundra of Central Tibet. Environmental Microbiology 60:730-739.

Xiao X, Sogge H, Lagesen K, Tooming-Klunderud A, Jakobsen KS, Rohrlack T. 2014. Use of high throughput sequencing and light microscopy show contrasting results in a study of phytoplankton occurrence in a freshwater environment. PLoS ONE 9(8):e106510.

Yamamoto M, Nishikawa T, Kajitani H, Kawano S. 2007. Patterns of asexual reproduction in Nannochloris bacillaris and Marvania geminata (Chlorophyta, Trebouxiophyceae). Planta 226(4):917-927.

Zancan S, Trevisan R, Paoletti MG. 2006. Soil algae composition under different agroecosystems in North-Eastern Italy. Agriculture, Ecosystems and Environment 112:1-12.

Zenova G, Shtina E. 1990. Soil Algae: Tutorial. Moscow State University. 80 pp.

Zenova G, Shtina E, Dedysh S, Glagoleva O, Likhacheva A, Gracheva T. 1995. Ecological relations of algae in biocenoses. Microbiology 64(2):121-133.

Zhan A, Hulak M, Sylvester F, Huang X, Adebayo AA, Abbott CL, Adamowicz SJ, Heath DD, Cristescu ME, MacIsaac HJ. 2013. High sensitivity of 454 pyrosequencing for detection of rare species in aquatic communities. Methods in Ecology and Evolution 4(6):558-565.

Zhang J, Huss VA, Sun X, Chang K, Pang D. 2008. Morphology and phylogenetic position of a trebouxiophycean green alga (Chlorophyta) growing on the rubber tree, Hevea brasiliensis, with the description of a new genus and species. European Journal of Phycology 43(2):185-193.

Zheng Y, Li T, Yu X, Bates PD, Dong T, Chen S. 2013. High-density fed-batch culture of a thermotolerant microalga Chlorella sorokiniana for biofuel production. Applied Energy 108:281-287.

Zuker M. 2003. Mfold web server for nucleic acid folding and hybridization prediction. Nucleic Acids Research 31(13):3406-3415. 


\section{Appendix | Chapter 1}

\section{Supporting Figures}

Figure S1. Microphotographs of mixed cultures (Archaeplastida).

Figure S2. Microphotographs of mixed cultures (Archaeplastida, Stramenopiles, Cryptophyta).

Figure S3. Diatoms in mixed cultures.

Figure S4. Cyanobacteria in mixed cultures.

Figure S5. Diversity in mixed cultures as inferred by light-microscopy.

Figure S6. Geographic evidence of 25 most common Green algal morphotypes.

\section{Supporting Tables}

Table S1. List of all analyzed isolates.

Table S2a. List of all detected species belonging to Trebouxiophyceae.

Table S2b. List of all detected species belonging to Chlorophyceae, other green algae and Stramenopiles.

Table S3. List of the closest GenBank-relatives of our detected species.

Table S4a. List of all analyzed full and partial 18S rDNA sequences.

Table S4b. Distribution of the green algal species across the sampling sites.

Table S5. General morphological characteristics of the new isolates.

Table S6a. Diversity of morphospecies in soil drill cores.

Table S6b. Diversity of morphospecies in topsoil samples.

Table S7. Frequency of the morphospecies in soil drill cores. 

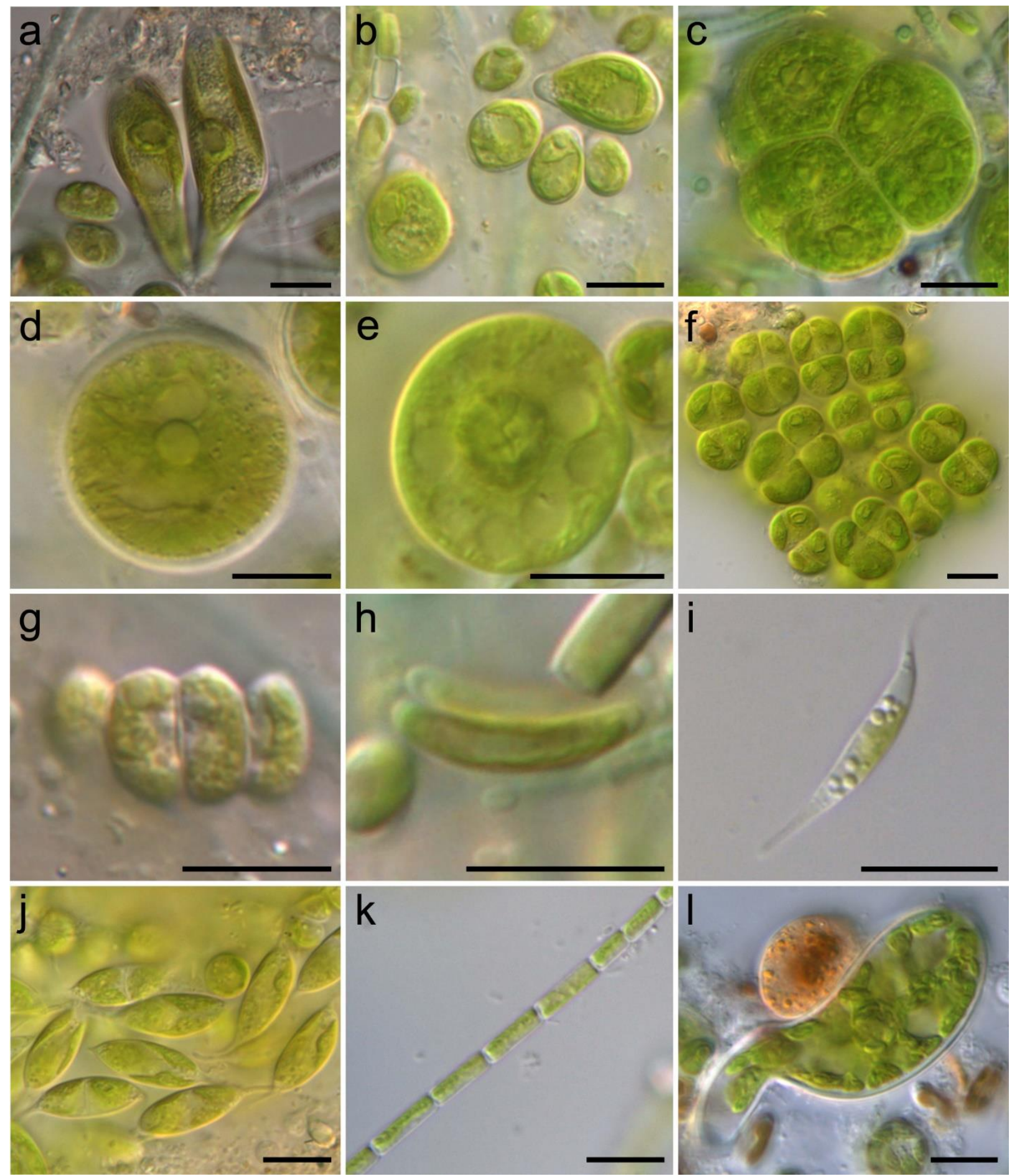

Figure S1. Microphotographs of mixed cultures (Archaeplastida). (a) Characiochloridaceae, Chlamydopodium (HEG2); (b) Characiochloridaceae, Chlamydopodium (HEG9); (c) cf. Tetracystis (HEG9); (d) Actinochloridaceae (HEW1); (e) Actinochloridaceae (HEW1); (f) Chlorosarcinaceae, cf. Desmotetra (HEG6); (g) Scenedesmaceae, Scenedesmus cf. soil (HEG7); (h) Selenastraceae, Monoraphidium terrestre (HEG9); (i) cf. Keratococcus (HEG7); (j) Podohedra (HEG7); (k) cf. Geminella (HEW3); (I) Scotinosphaera (HEG9). Scale bars $=10 \mu \mathrm{m}$. 

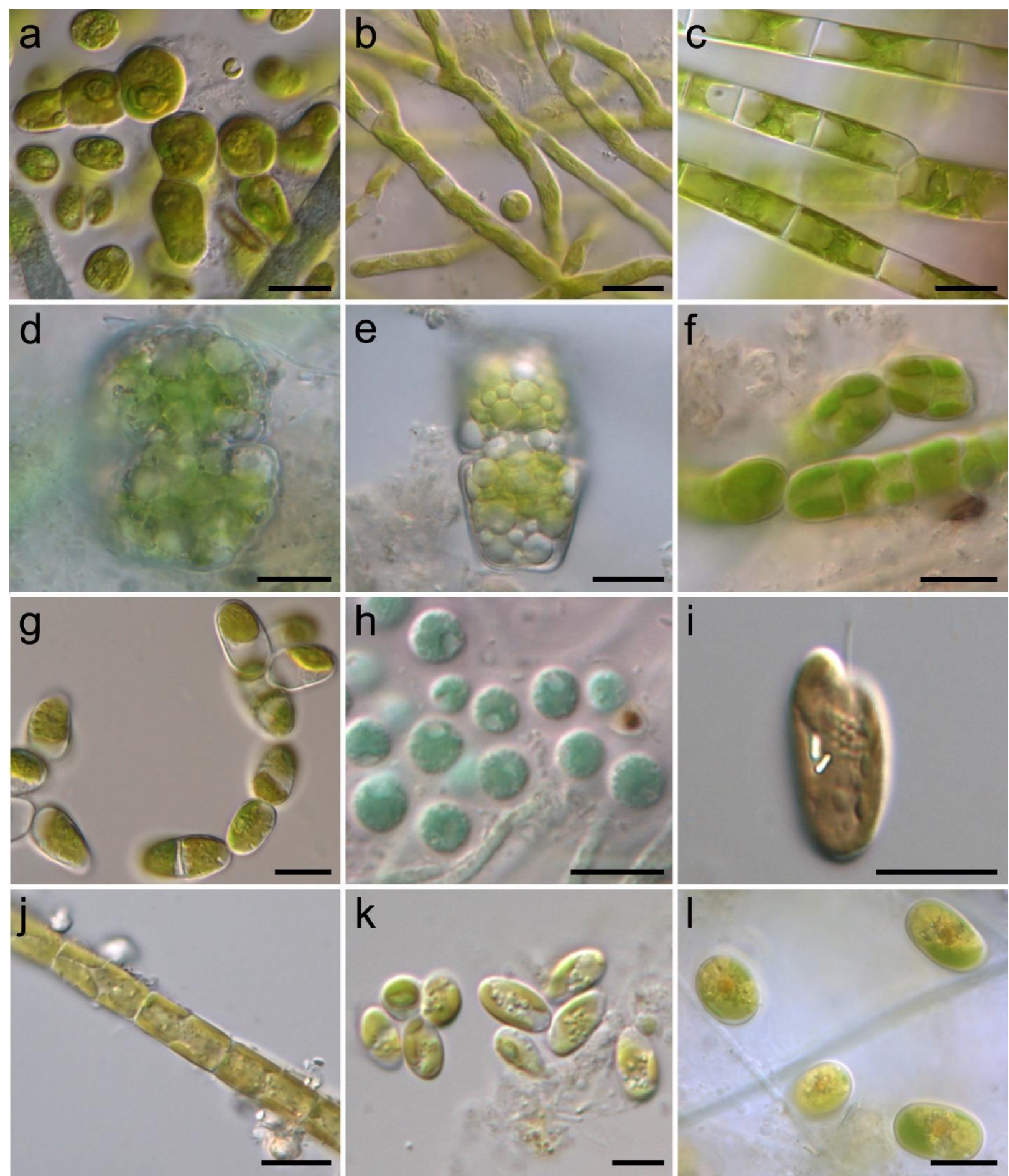

Figure S2. Microphotographs of mixed cultures (Archaeplastida, Stramenopiles, Cryptophyta). (a) cf. Pseudendoclonium (HEG7); (b) cf. Dilabifilum (SEG8); (c) cf. Chaetophora (HEG9); (d) Cosmarium (HEG9); (e) Cosmarium (HEG9); (f) cf. Klebsormidium (HEG9); (g) Interfilum cf. terricola (HEW); (h) Rhodophyta, Porphyridium (HEG9); (i) Cryptomonas (HEG9); (j) Tribonema (HEG9); (k) cf. Ellipsoidion (HEG6); (I) cf. Monallantus (HEW4). Scale bars $=10 \mu \mathrm{m}$. 

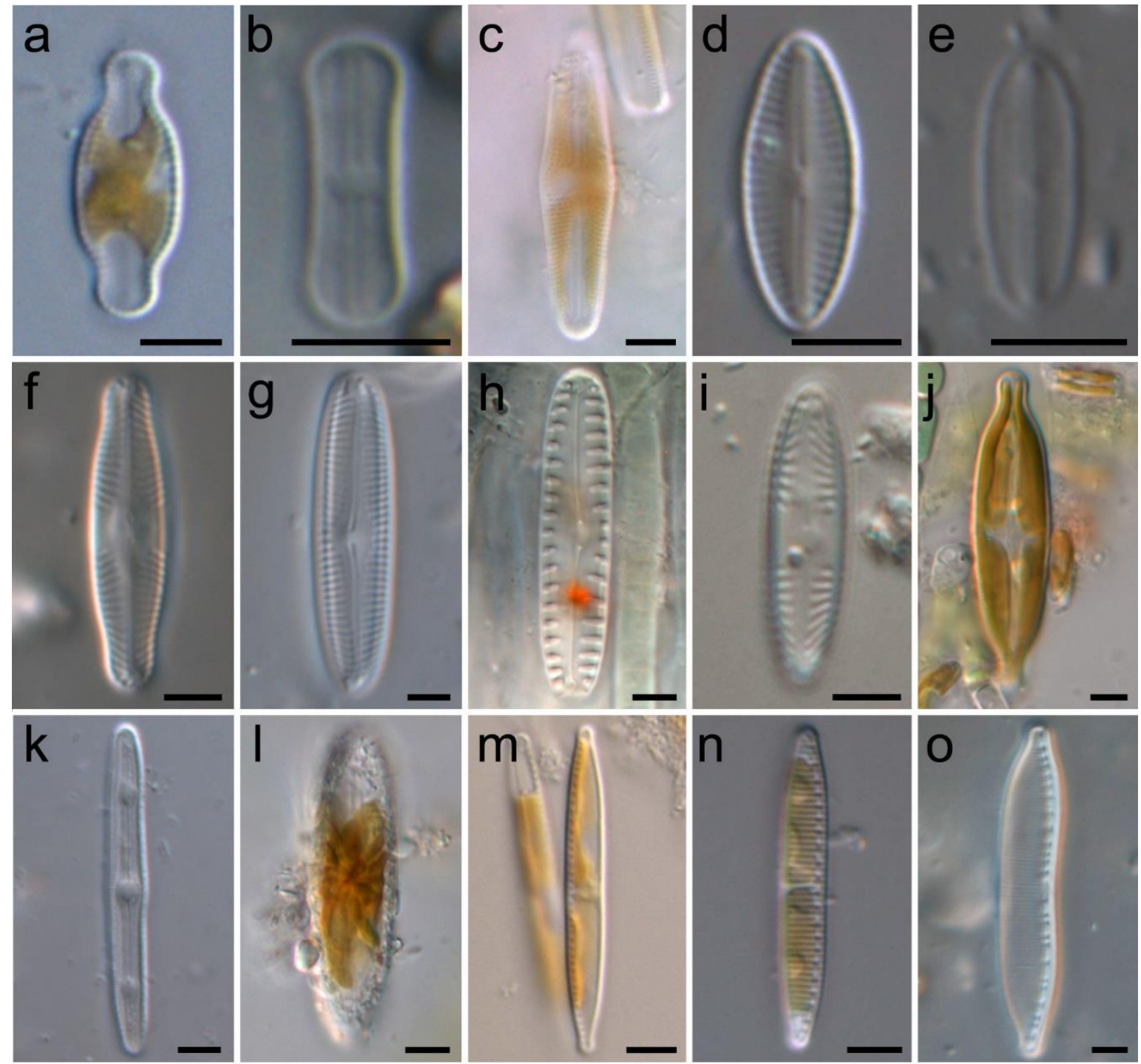

Figure S3. Diatoms in mixed cultures. (a) Monoraphid diatoms, cf. Planothidium (HEG9); (b)-(k) Symmetrical biraphid diatoms; (b) Humidophila (HEG6); (c) Luticola (HEG9); (d) cf. Eolimna (HEW3); (e) cf. Mayamaea (HEW3); (f) Pinnularia (HEW6); (g) Pinnularia (HEW9); (h) Pinnularia (HEW); (i) Pinnularia (HEG8); (j) Stauroneis (HEG9); (k) cf. Neidium (HEW4); (I) Surirelloid diatoms, Surirella (HEW); (m)-(o) Nitzschioid diatoms; (m) Nitzschia (HEG4); (n) Nitzschia (HEG9); (o) Hantzschia (HEG6). Scale bars $=5 \mu \mathrm{m}$. 

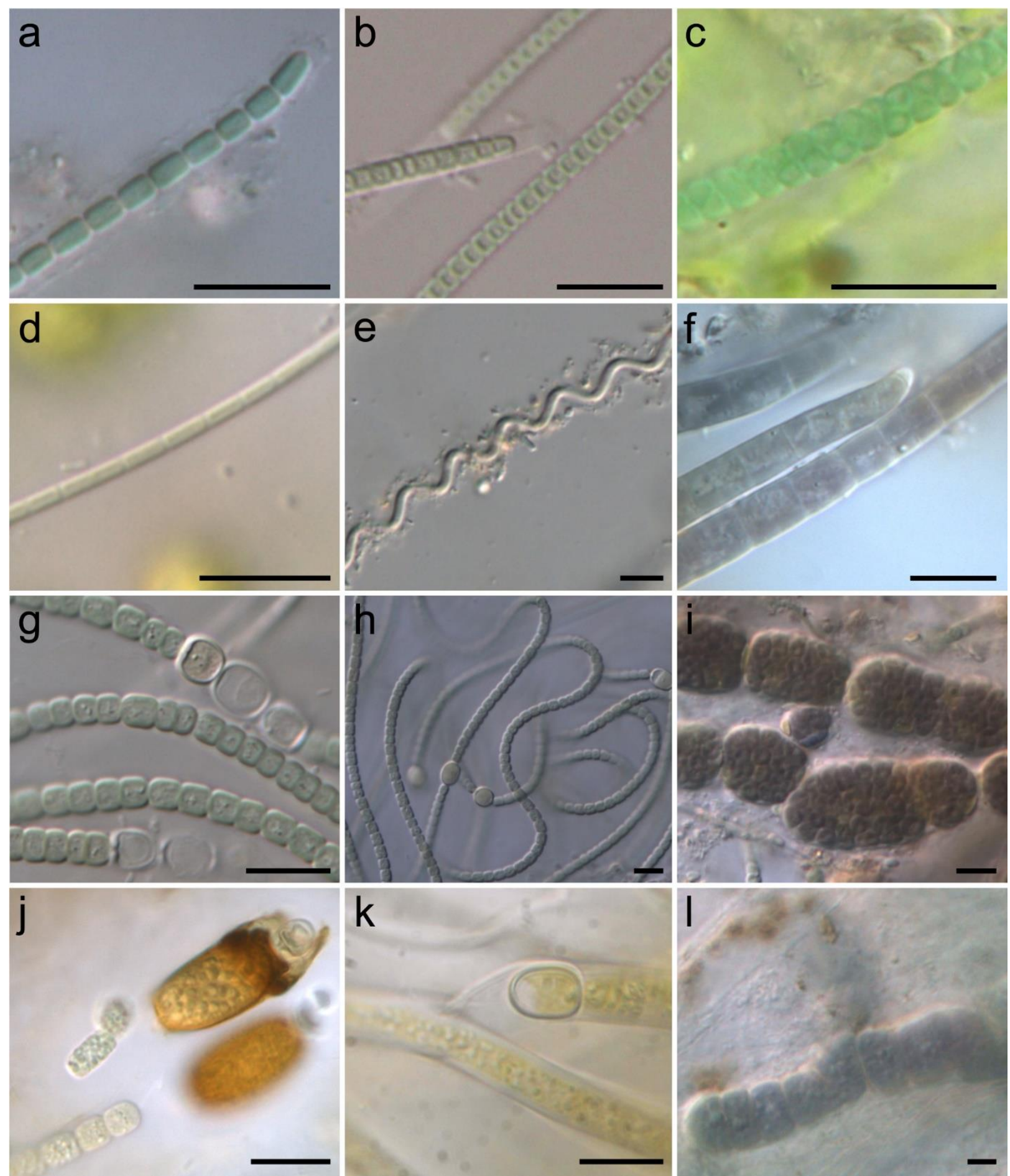

Figure S4. Cyanobacteria in mixed cultures. (a) cf. Pseudanabaena (HEG9); (b) cf. Leptolynbgya (HEW4); (c) cf. Leptolyngbya (HEG9); (d) cf. Limnothrix (HEG6); (e) Pseudanabaenales (HEG7); (f) Phormidium (HEG7); (g) Nostoc (HEG7); (h) Nostoc (HEG7); (i) Nostoc (HEG7); (j) Cylindrospermum (HEG6); (k) cf. Tolypothrix (HEG2); (I) cf. Stigonema (HEG9). Scale bars = 10 $\mu \mathrm{m}$. 


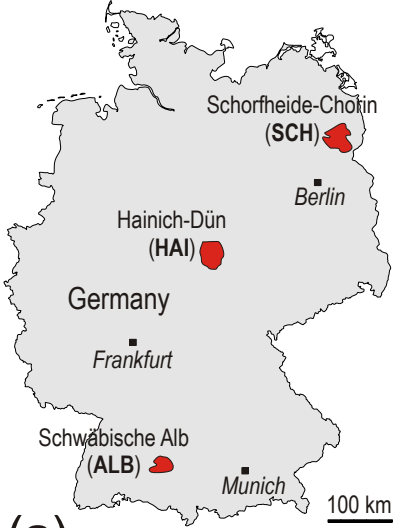

(a)

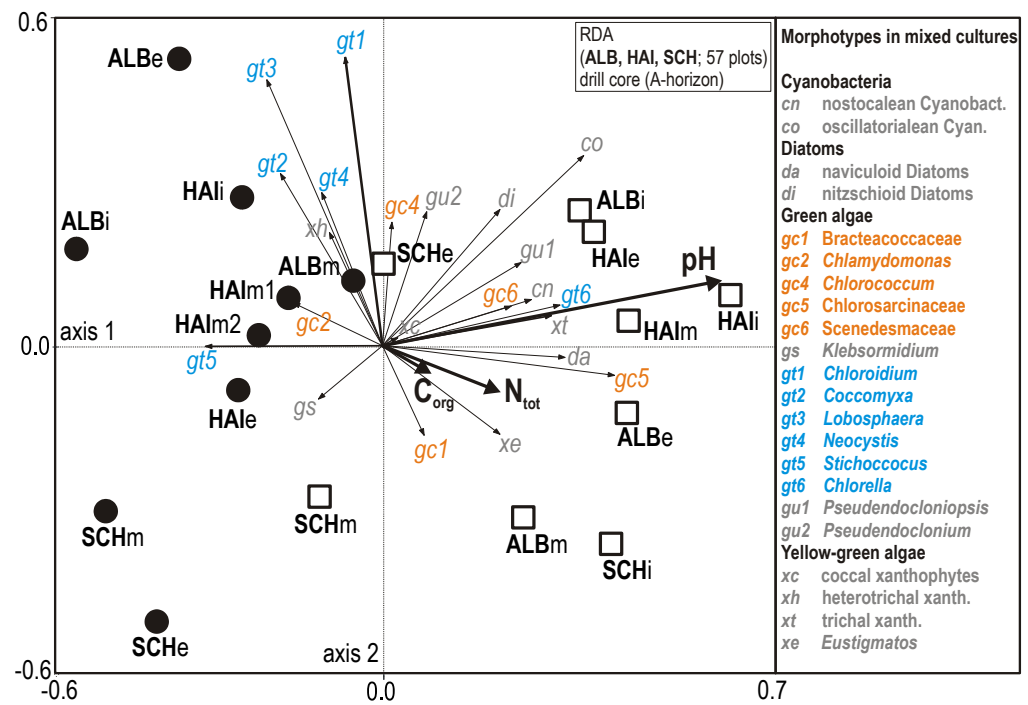

(d)

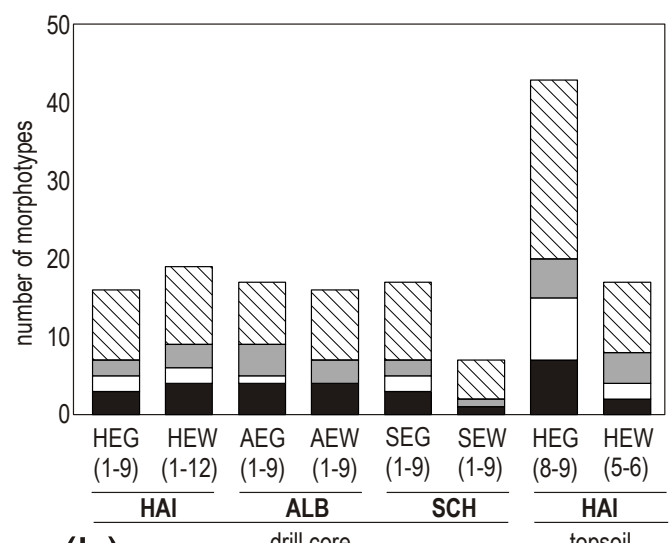

(b)
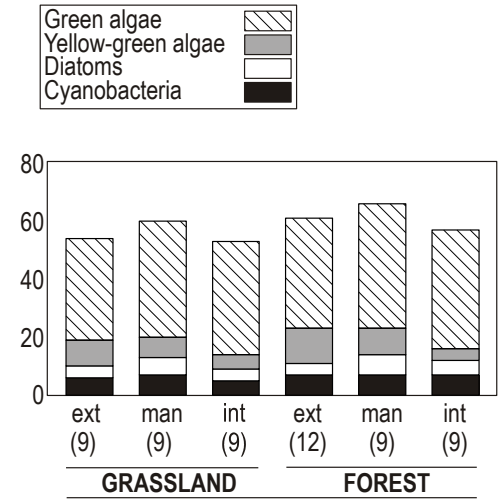

(c)

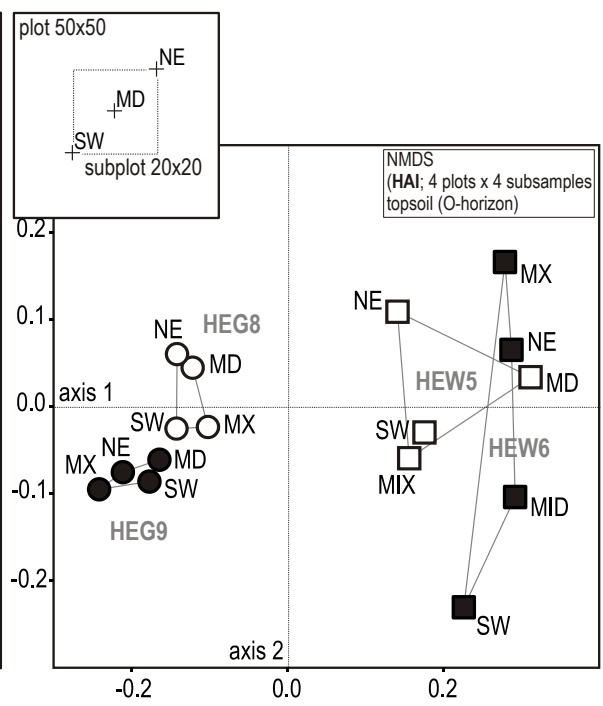

(e)

Figure S5. Diversity in mixed cultures as inferred by light-microscopy. (a) Localization of the three German Biodiversity Exploratories. (b) Comparison of alpha-diversities (= numbers of morphotypes) of soil algae in grasslands and forests. Each bar represents pooled data from nine to twelve plots (e.g., HEG(1-9) includes observations from all Hainich grassland plots; (c) Comparison of alpha-diversities among the land-uses (ext=extensive, man=managed, int=intensive). Each bar includes pooled data from multiple plots (the number is given in parentheses). (d) Redundancy analysis (RDA) of algal communities changing along environmental gradients ( $\mathrm{pH}, \mathrm{Corg}=0$ rganic carbon, $\mathrm{N}_{\text {tot }}=$ total nitrogen). Dots (forests) and squares (grasslands) represent algal communities which were pooled within each land-use category (e.g., ALBe includes observations from plots AEW7-AEW9; $\mathrm{e}=$ extensively used plots). A subset of 22 most frequently observed morphotypes are shown (black vectors). (e) Non-metric multidimensional scaling (NMDS) of algal communities in multiple subsamples within a plot. Each dot (forest subsample) and square (grassland subsample) represents data recorded during three seasons. Within each plot (HEG8, HEG9, HEW5, HEW6) three subsamples were analyzed ( $\mathrm{NE}=$ =north-east plot edge, $\mathrm{MID}=$ middle point, $\mathrm{SW}=$ =south-west plot edge, $\mathrm{MIX}=$ pooled sample combining all three subsamples within a plot; sampling scheme is shown above). 


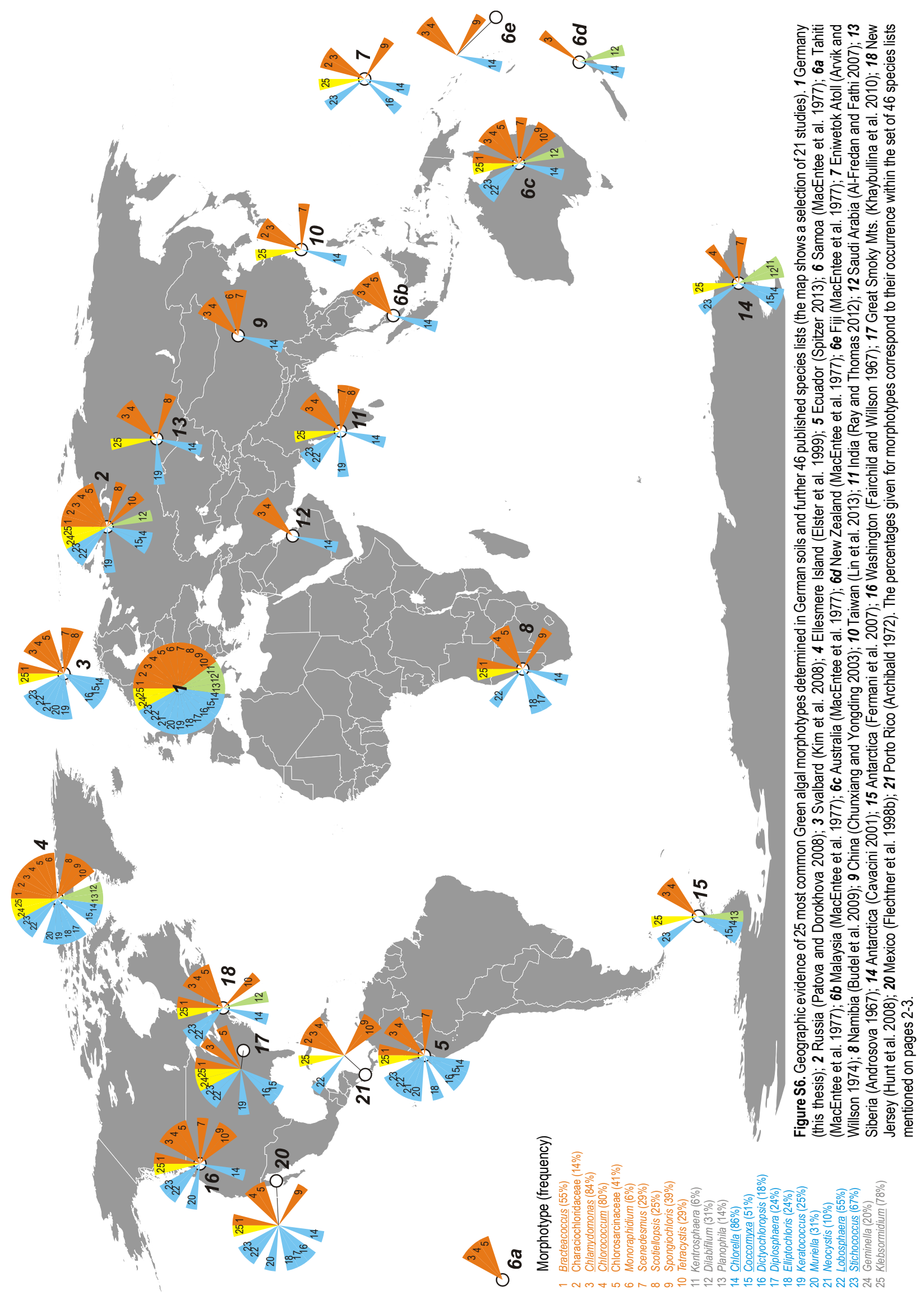


Table S1. List of all analyzed isolates.

\begin{tabular}{|c|c|c|c|c|c|c|c|c|}
\hline Species & Identifier & GPS & Exploratory & Plot & Habitat & Sampling & $18 \mathrm{~S}$ & ITS2 \\
\hline Acutodesmus rubescens & LH08SG8041 & $\begin{array}{l}\mathrm{N}^{\circ} 3^{\circ} 6^{\prime} 50.294^{\prime \prime} \\
\text { E14 }{ }^{\circ} 1^{\prime} 1.559^{\prime \prime}\end{array}$ & $\mathrm{SCH}$ & SEG8 & grassland & drill core & 1656 & - \\
\hline Bracteacoccus cohaerens & LH10HG9034 & $\begin{array}{l}\text { N51 }{ }^{\circ} 13^{\prime} 26.031 " \\
\text { E10 }\end{array}$ & $\mathrm{HAl}$ & HEG9 & grassland & soil surface & 1722 & - \\
\hline Bracteacoccus cohaerens (cf.) & LH08SG2015 & $\begin{array}{l}\mathrm{N}^{5} 3^{\circ} 5^{\prime} 21.505^{\prime \prime} \\
\mathrm{E} 13^{\circ} 58^{\prime} 48.169^{\prime \prime}\end{array}$ & $\mathrm{SCH}$ & SEG2 & grassland & drill core & 2056 & - \\
\hline Chlamydomonas gerloffii (cf.) & LH08SW5031 & $\begin{array}{l}{\mathrm{N} 53^{\circ}}^{\prime} 3^{\prime} 25.321^{\prime \prime} \\
\mathrm{E}^{\circ} 3^{\circ} 53^{\prime} 7.318^{\prime \prime}\end{array}$ & $\mathrm{SCH}$ & SEW5 & forest & drill core & 1711 & - \\
\hline Chlamydomonas rapa & LH08SG1077 & 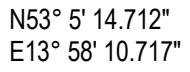 & $\mathrm{SCH}$ & SEG1 & grassland & drill core & 1694 & - \\
\hline Chlamydomonas rapa & LH08SG9055 & $\begin{array}{l}{\mathrm{N} 53^{\circ}}^{\circ} 5^{\prime} 53.455^{\prime \prime} \\
\mathrm{E}^{\circ} 3^{\circ} 36^{\prime} 45.241^{\prime \prime}\end{array}$ & $\mathrm{SCH}$ & SEG9 & grassland & drill core & 1660 & - \\
\hline Chlamydomonas rapa (cf.) & LH10HG1027 & $\begin{array}{l}\text { N50 } 58^{\prime} 17.934^{\prime \prime} \\
\text { E10 } 24^{\prime} 19.306^{\prime \prime}\end{array}$ & $\mathrm{HAl}$ & HEG1 & grassland & soil surface & 1710 & 290 \\
\hline Chlamydomonas typica (cf.) & LH08SG9022 & $\begin{array}{l}{\mathrm{N} 53^{\circ}}^{\circ} 5^{\prime} 53.455^{\prime \prime} \\
\mathrm{E}^{\circ} 3^{\circ} 36^{\prime} 45.241^{\prime \prime}\end{array}$ & $\mathrm{SCH}$ & SEG9 & grassland & drill core & 1262 & - \\
\hline Chlamydopodium vacuolatum & LH10HG1013 & $\begin{array}{l}\text { N50 } 58^{\prime} 17.934^{\prime \prime} \\
\text { E10 } 24^{\prime} 19.306^{\prime \prime}\end{array}$ & HAl & HEG1 & grassland & soil surface & 1728 & 287 \\
\hline Chlorococcum minutum (cf.) & LH08AG701K & $\begin{array}{l}\text { N48 } 28^{\circ} 29.116^{\prime \prime} \\
\mathrm{E} 9^{\circ} 22^{\prime} 36.65^{\prime \prime}\end{array}$ & ALB & AEG7 & grassland & drill core & 793 & - \\
\hline Chlorococcum minutum (cf.) & $\begin{array}{l}\text { LH08AW5056 } \\
(=S A G ~ 2479)\end{array}$ & $\begin{array}{l}\text { N48 } 28^{\circ} 5^{\prime} 10.626^{\prime \prime} \\
\mathrm{E}^{\circ} 24^{\prime} 52.854^{\prime \prime}\end{array}$ & ALB & AEW5 & forest & drill core & 1707 & - \\
\hline Chlorococcum minutum (cf.) & LH08AW5107 & $\begin{array}{l}\text { N48 }{ }^{\circ} 25^{\prime} 10.626^{\prime \prime} \\
\mathrm{E} 9^{\circ} 24^{\prime} 52.854^{\prime \prime}\end{array}$ & ALB & AEW5 & forest & drill core & 1662 & - \\
\hline Chlorococcum minutum (cf.) & LH08AW5111 & $\begin{array}{l}\text { N48 } 28^{\circ} 5^{\prime} 10.626^{\prime \prime} \\
\mathrm{E}^{\circ} 24^{\prime} 52.854^{\prime \prime}\end{array}$ & ALB & AEW5 & forest & drill core & 1346 & - \\
\hline Chlorococcum sphacosum & LH10HG3113 & $\begin{array}{l}\text { N50 } 59^{\prime} 53.129^{\prime \prime} \\
\text { E10 } 25^{\prime} 58.616^{\prime \prime}\end{array}$ & $\mathrm{HAl}$ & HEG3 & grassland & soil surface & 1713 & - \\
\hline Coelastrella multistriata & LH08AG2003 & $\begin{array}{l}\text { N48 } 28^{\circ} 36.686^{\prime \prime} \\
\mathrm{E}^{\circ} 28^{\prime} 22.023^{\prime \prime}\end{array}$ & ALB & AEG2 & grassland & drill core & 535 & - \\
\hline Coelastrella multistriata & LH08AW4118 & $\begin{array}{l}\text { N48 }{ }^{\circ} 23^{\prime} 56.755^{\prime \prime} \\
\text { E9 } 14^{\circ} 41.378^{\prime \prime}\end{array}$ & ALB & AEW4 & forest & drill core & 1208 & - \\
\hline Coelastrella multistriata & LH10HG7083 & $\begin{array}{l}\text { N51 }{ }^{\circ} 16^{\prime} 24.897^{\prime \prime} \\
\text { E10 } 24^{\circ} 37.485^{\prime \prime}\end{array}$ & $\mathrm{HAl}$ & HEG7 & grassland & soil surface & 2117 & - \\
\hline Coelastrella multistriata & LH10HG7097 & $\begin{array}{l}\text { N51 }{ }^{\circ} 16^{\prime} 24.897^{\prime \prime} \\
\text { E10 } 24^{\circ} 37.485^{\prime \prime}\end{array}$ & $\mathrm{HAl}$ & HEG7 & grassland & soil surface & 512 & - \\
\hline Coelastrella multistriata & LH10HG7100 & $\begin{array}{l}\text { N51 }{ }^{\circ} 16^{\prime} 24.897^{\prime \prime} \\
\text { E10 } 24^{\circ} 37.485^{\prime \prime}\end{array}$ & $\mathrm{HAl}$ & HEG7 & grassland & soil surface & 700 & - \\
\hline Coelastrella multistriata & LH10HG7102 & $\begin{array}{l}\text { N51 }{ }^{\circ} 16^{\prime} 24.897^{\prime \prime} \\
\text { E10 } 24^{\circ} 37.485^{\prime \prime}\end{array}$ & $\mathrm{HAl}$ & HEG7 & grassland & soil surface & 411 & - \\
\hline Coelastrella multistriata & LH10HG8109 & $\begin{array}{l}\text { N51 }{ }^{\circ} 16^{\prime} 16.527^{\prime \prime} \\
\text { E10 } 25^{\circ} 4.6^{\prime \prime}\end{array}$ & $\mathrm{HAl}$ & HEG8 & grassland & soil surface & 454 & - \\
\hline Coelastrella sp. & LH10HG1009 & $\begin{array}{l}\text { N50 } 58^{\prime} 17.934^{\prime \prime} \\
\text { E10 } 24^{\prime} 19.306^{\prime \prime}\end{array}$ & $\mathrm{HAl}$ & HEG1 & grassland & soil surface & 1770 & - \\
\hline Coelastrella sp. & LH10HG1033 & $\begin{array}{l}\text { N50 } 58^{\circ} 17.934^{\prime \prime} \\
\text { E10 } 24^{\prime} 19.306^{\prime \prime}\end{array}$ & HAI & HEG1 & grassland & soil surface & 1622 & - \\
\hline Coelastrella sp. & LH10HG2087 & $\begin{array}{l}\mathrm{N} 51^{\circ} 0^{\prime} 2.696 " \\
\mathrm{E} 10^{\circ} 25^{\prime \prime} 48.036 "\end{array}$ & $\mathrm{HAl}$ & HEG2 & grassland & soil surface & 470 & - \\
\hline Coelastrella sp. & LH10HG2098 & 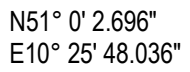 & $\mathrm{HAl}$ & HEG2 & grassland & soil surface & 2206 & - \\
\hline Coelastrella sp. & LH10HG2P01 & $\begin{array}{l}\text { N51 }{ }^{\circ} 0^{\prime} 2.696 " \\
\text { E10² } 25^{\prime} 48.036^{\prime \prime}\end{array}$ & $\mathrm{HAl}$ & HEG2 & grassland & soil surface & 589 & - \\
\hline Coelastrella sp. & LH10HG2P12 & $\begin{array}{l}\text { N51 }{ }^{\circ} 0^{\prime} 2.696 " \\
\text { E10 } 20^{\circ} 25^{\prime} 48.036 "\end{array}$ & $\mathrm{HAl}$ & HEG2 & grassland & soil surface & 599 & - \\
\hline Coelastrella sp. & LH10HG5136 & $\begin{array}{l}\text { N51 } 11^{\circ} 57.22 " \\
\text { E10 } 10^{\prime} 21.096^{\prime \prime}\end{array}$ & $\mathrm{HAl}$ & HEG5 & grassland & soil surface & 1313 & - \\
\hline Coelastrella sp. & LH10HG6035 & $\begin{array}{l}\text { N51 }{ }^{\circ} 12^{\prime} 53.766^{\prime \prime} \\
\text { E10 } 23^{\prime} 28.395^{\prime \prime}\end{array}$ & $\mathrm{HAl}$ & HEG6 & grassland & soil surface & 1511 & - \\
\hline Coelastrella sp. & LH10HG6060 & $\begin{array}{l}\text { N51 }{ }^{\circ} 12^{\prime} 53.766^{\prime \prime} \\
\text { E10 } 23^{\circ} 28.395^{\prime \prime}\end{array}$ & $\mathrm{HAl}$ & HEG6 & grassland & soil surface & 1616 & 266 \\
\hline Coelastrella sp. & LH10HG7017 & $\begin{array}{l}\text { N51 }{ }^{\circ} 16^{\prime} 24.897^{\prime \prime} \\
\text { E10 } 24^{\circ} 37.485^{\prime \prime}\end{array}$ & $\mathrm{HAl}$ & HEG7 & grassland & soil surface & 1683 & - \\
\hline Coelastrella sp. & LH10HG7018 & $\begin{array}{l}\text { N51 } 11^{\circ} 24.897^{\prime \prime} \\
\text { E10 } 24^{\circ} 37.485^{\prime \prime}\end{array}$ & $\mathrm{HAl}$ & HEG7 & grassland & soil surface & 1429 & 297 \\
\hline Coelastrella sp. & LH10HG7023 & $\begin{array}{l}\text { N51 }{ }^{\circ} 16^{\prime} 24.897^{\prime \prime} \\
\text { E10 } 24^{\circ} 37.485^{\prime \prime}\end{array}$ & $\mathrm{HAl}$ & HEG7 & grassland & soil surface & 1637 & - \\
\hline Coelastrella sp. & LH10HG7030 & $\begin{array}{l}\text { N51 }{ }^{\circ} 16^{\prime} 24.897^{\prime \prime} \\
\text { E10 } 24^{\circ} 37.485^{\prime \prime}\end{array}$ & $\mathrm{HAl}$ & HEG7 & grassland & soil surface & 1506 & 298 \\
\hline Coelastrella sp. & LH10HG9130 & $\begin{array}{l}\text { N51 }{ }^{\circ} 13^{\prime} 26.031^{\prime \prime} \\
\mathrm{E} 10^{\circ} 22^{\prime} 50.834^{\prime \prime}\end{array}$ & $\mathrm{HAl}$ & HEG9 & grassland & soil surface & 1053 & - \\
\hline Desmotetra stigmatica & LH08SG2049 & $\begin{array}{l}\mathrm{N} 53^{\circ} 5^{\prime} 21.505^{\prime \prime} \\
\mathrm{E} 13^{\circ} 58^{\prime} 48.169^{\prime \prime}\end{array}$ & $\mathrm{SCH}$ & SEG2 & grassland & drill core & 1667 & - \\
\hline
\end{tabular}


Table S1. (continuation)

\begin{tabular}{|c|c|c|c|c|c|c|c|c|}
\hline Species & Identifier & GPS & Exploratory & Plot & Habitat & Sampling & $18 S$ & ITS2 \\
\hline Desmotetra stigmatica & LH10HG6P18 & $\begin{array}{l}\text { N51ํ } 12^{\prime} 53.766^{\prime \prime} \\
\text { E102 } 23^{\prime} 28.395^{\prime \prime}\end{array}$ & $\mathrm{HAl}$ & HEG6 & grassland & soil surface & 494 & - \\
\hline Heterochlamydomonas sp. & LH08AG2004 & $\begin{array}{l}\text { N48 }{ }^{\circ} 22^{\prime} 36.686^{\prime \prime} \\
\text { E9 }^{\circ} 28^{\prime} 22.023 "\end{array}$ & ALB & AEG2 & grassland & drill core & 1699 & - \\
\hline Jenufa sp. & $\begin{array}{l}\text { LH08AW8035 } \\
\text { (=SAG 2383) }\end{array}$ & $\begin{array}{l}\text { N48 } 22^{\prime} 57.322^{\prime \prime} \\
\text { E9 } 22^{\prime} 56.584^{\prime \prime}\end{array}$ & ALB & AEW8 & forest & drill core & 1700 & - \\
\hline Jenufa sp. & LH08AW8098 & $\begin{array}{l}\text { N48 }{ }^{\circ} 22^{\prime} 57.322^{\prime \prime} \\
\text { E9 }^{\circ} 22^{\prime} 56.584^{\prime \prime}\end{array}$ & ALB & AEW8 & forest & drill core & 1277 & - \\
\hline Oogamochlamys sp.(I) & LH08SG8047 & $\begin{array}{l}\text { N53 }{ }^{\circ} 6^{\prime} 50.294^{\prime \prime} \\
\text { E14 }{ }^{\circ} 1^{\prime} 1.559^{\prime \prime}\end{array}$ & $\mathrm{SCH}$ & SEG8 & grassland & drill core & 1654 & - \\
\hline Oogamochlamys sp.(II) & $\begin{array}{l}\text { LH08AW1069 } \\
\text { (=SAG 2476) }\end{array}$ & $\begin{array}{l}\text { N48 }{ }^{\circ} 28^{\prime} 41.063^{\prime \prime} \\
E 9^{\circ} 20^{\prime} 3.877^{\prime \prime}\end{array}$ & ALB & AEW1 & forest & drill core & 1279 & - \\
\hline Pseudomuriella aurantiaca & LH10HG2039 & $\begin{array}{l}\mathrm{N} 51^{\circ} 0^{\prime} 2.696 " 1 \\
\mathrm{E} 10^{\circ} 25^{\prime} 48.036 "\end{array}$ & $\mathrm{HAl}$ & HEG2 & grassland & soil surface & 1656 & - \\
\hline Pseudomuriella aurantiaca & LH10HG9038 & $\begin{array}{l}N 51^{\circ} 13^{\prime} 26.031^{\prime \prime} \\
\mathrm{E} 10^{\circ} 22^{\prime} 50.834^{\prime \prime}\end{array}$ & $\mathrm{HAl}$ & HEG9 & grassland & soil surface & 2081 & - \\
\hline Stephanosphaerinia sp. & LH10HG6108 & $\begin{array}{l}\text { N51 }{ }^{\circ} 12^{\prime} 53.766^{\prime \prime} \\
\text { E10 } 23^{\prime} 28.395^{\prime \prime}\end{array}$ & $\mathrm{HAl}$ & HEG6 & grassland & soil surface & 1719 & - \\
\hline Tatrensinia sp.(l) & LH08SW7115 & $\begin{array}{l}\text { N53 }{ }^{\circ} 6^{\prime} 26.453^{\prime \prime} \\
\mathrm{E}^{\circ} 3^{\circ} 41^{\prime} 39.908^{\prime \prime}\end{array}$ & $\mathrm{SCH}$ & SEW7 & forest & drill core & 1716 & - \\
\hline Tatrensinia sp.(II) & LH10HG7016 & $\begin{array}{l}\text { N51 }{ }^{\circ} 16^{\prime} 24.897^{\prime \prime} \\
\text { E10 } 24^{\prime} 37.485^{\prime \prime}\end{array}$ & $\mathrm{HAl}$ & HEG7 & grassland & soil surface & 1733 & - \\
\hline Tatrensinia sp.(II) & LH10HG9131 & 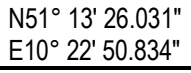 & $\mathrm{HAl}$ & HEG9 & grassland & soil surface & 527 & - \\
\hline Pedinomonas minor (cf.) & LH08SG2033 & $\begin{array}{l}\mathrm{N} 53^{\circ} 5^{\prime} 21.505^{\prime \prime} \\
\mathrm{E} 13^{\circ} 58^{\prime} 48.169^{\prime \prime}\end{array}$ & $\mathrm{SCH}$ & SEG2 & grassland & drill core & 1489 & - \\
\hline Auxenochlorella protothecoides & LH10HG5119 & $\begin{array}{l}\text { N51 }{ }^{\circ} 12^{\prime} 57.22^{\prime \prime} \\
\text { E10 } 19^{\prime} 21.096 "\end{array}$ & $\mathrm{HAl}$ & HEG5 & grassland & soil surface & 1711 & - \\
\hline Auxenochlorella protothecoides & LH10HG6096 & $\begin{array}{l}\mathrm{N} 51^{\circ} 12^{\prime} 53.766^{\prime \prime} \\
\mathrm{E} 10^{\circ} 23^{\prime} 28.395^{\prime \prime}\end{array}$ & $\mathrm{HAl}$ & HEG6 & grassland & soil surface & 1725 & - \\
\hline Auxenochlorella protothecoides & LH10HG7124 & $\begin{array}{l}\text { N51 }{ }^{\circ} 16^{\prime} 24.897^{\prime \prime} \\
\text { E10² } 24^{\prime} 37.485^{\prime \prime}\end{array}$ & $\mathrm{HAl}$ & HEG7 & grassland & soil surface & 1720 & - \\
\hline Auxenochlorella sp. & $\begin{array}{l}\text { LH08AW4103 } \\
\text { (=SAG 2478) }\end{array}$ & $\begin{array}{l}\text { N48 }{ }^{\circ} 23^{\prime} 56.755^{\prime \prime} \\
\text { E9 }^{\circ} 14^{\prime} 41.378^{\prime \prime}\end{array}$ & ALB & AEW4 & forest & drill core & 1655 & 321 \\
\hline Chlorella mirabilis (cf.) & LH08AG9040 & $\begin{array}{l}\mathrm{N} 48^{\circ} 23^{\prime} 40.815^{\prime \prime} \\
\mathrm{Eg}^{\circ} 30^{\prime} 10.053^{\prime \prime}\end{array}$ & ALB & AEG9 & grassland & drill core & 1648 & 314 \\
\hline Chlorella mirabilis (cf.) & LH10HG6139 & $\begin{array}{l}\text { N51 }{ }^{\circ} 12^{\prime} 53.766^{\prime \prime} \\
\text { E10² } 23^{\prime} 28.395^{\prime \prime}\end{array}$ & $\mathrm{HAl}$ & HEG6 & grassland & soil surface & 1683 & - \\
\hline Chlorella vulgaris & LH08HG1081 & $\begin{array}{l}N 50^{\circ} 58^{\prime} 17.934 " \\
\text { E10 } 20^{\circ} 24^{\prime} 19.306^{\prime \prime}\end{array}$ & $\mathrm{HAl}$ & HEG1 & grassland & drill core & 1687 & 300 \\
\hline Chlorella vulgaris & LH08HG2013 & $\begin{array}{l}\mathrm{N} 51^{\circ} 0^{\prime} 2.696 " 1 \\
\mathrm{E} 10^{\circ} 25^{\prime} 48.036 "\end{array}$ & $\mathrm{HAl}$ & HEG2 & grassland & drill core & 1692 & - \\
\hline Chlorella vulgaris & LH08HG2065 & $\begin{array}{l}\text { N51 }{ }^{\circ} 0^{\prime} 2.696^{\prime \prime} \\
\text { E10 } 20^{\circ} 48.036 "\end{array}$ & $\mathrm{HAl}$ & HEG2 & grassland & drill core & 1695 & - \\
\hline Chlorella vulgaris & LH08HG2083 & $\begin{array}{l}\mathrm{N} 51^{\circ} 0^{\prime} 2.696^{\prime \prime} \\
\mathrm{E} 10^{\circ} 25^{\prime} 48.036^{\prime \prime}\end{array}$ & $\mathrm{HAl}$ & HEG2 & grassland & drill core & 1676 & - \\
\hline Chlorella vulgaris & LH08HG2091 & $\begin{array}{l}\text { N51 }{ }^{\circ} 0^{\prime} 2.696^{\prime \prime} \\
\text { E10 } 20^{\circ} 48.036 "\end{array}$ & $\mathrm{HAl}$ & HEG2 & grassland & drill core & 1695 & - \\
\hline Chlorella vulgaris & LH08HG2096 & $\begin{array}{l}\mathrm{N} 51^{\circ} 0^{\prime} 2.696^{\prime \prime} \\
\mathrm{E} 10^{\circ} 25^{\prime} 48.036^{\prime \prime}\end{array}$ & $\mathrm{HAl}$ & HEG2 & grassland & drill core & 1678 & - \\
\hline Chlorella vulgaris & LH08HG4032 & $\begin{array}{l}\mathrm{N} 51^{\circ} 6^{\prime} 48.104^{\prime \prime} \\
\mathrm{E} 10^{\circ} 26^{\prime} 10.249^{\prime \prime}\end{array}$ & $\mathrm{HAl}$ & HEG4 & grassland & drill core & 1671 & 300 \\
\hline Chlorella vulgaris & LH08HG4088 & $\begin{array}{l}\mathrm{N} 51^{\circ} 6^{\prime} 48.104^{\prime \prime} \\
\mathrm{E} 10^{\circ} 26^{\prime} 10.249^{\prime \prime}\end{array}$ & $\mathrm{HAl}$ & HEG4 & grassland & drill core & 1692 & 300 \\
\hline Chlorella vulgaris & LH08HG5074 & $\begin{array}{l}\text { N51 }{ }^{\circ} 12^{\prime} 57.22 " \\
\text { E10 } 10^{\circ} 19^{\prime} 21.096^{\prime \prime}\end{array}$ & $\mathrm{HAl}$ & HEG5 & grassland & drill core & 1695 & 300 \\
\hline Chlorella vulgaris & LH08HG5082 & $\begin{array}{l}\text { N51ํ' } 12^{\prime} 57.22^{\prime \prime} \\
\text { E10 } 19^{\prime} 21.096^{\prime \prime}\end{array}$ & $\mathrm{HAl}$ & HEG5 & grassland & drill core & 1692 & 300 \\
\hline Chlorella vulgaris & LH08HW6087 & $\begin{array}{l}\mathrm{N} 51^{\circ} 16^{\prime} 3.791^{\prime \prime} \\
\mathrm{E} 10^{\circ} 14^{\prime} 21.762^{\prime \prime}\end{array}$ & $\mathrm{HAl}$ & HEW6 & forest & drill core & 303 & - \\
\hline Chlorella vulgaris & LH08HW9060 & $\begin{array}{l}\mathrm{N} 51^{\circ} 7^{\prime} 48.871^{\prime \prime} \\
\mathrm{E} 10^{\circ} 22^{\prime} 52.139^{\prime \prime}\end{array}$ & $\mathrm{HAl}$ & HEW9 & forest & drill core & 1686 & - \\
\hline Chlorella vulgaris & LH08HW9094 & $\begin{array}{l}\text { N51 }{ }^{\circ} 7^{\prime} 48.871 " \\
\text { E10 } 22^{\circ} 52.139^{\prime \prime}\end{array}$ & $\mathrm{HAl}$ & HEW9 & forest & drill core & 1686 & 300 \\
\hline Chlorella vulgaris & LH08SG1071 & 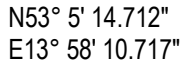 & $\mathrm{SCH}$ & SEG1 & grassland & drill core & 1695 & 300 \\
\hline Chlorella vulgaris & LH08SG3006 & $\begin{array}{l}\mathrm{N} 53^{\circ} 6^{\prime} 10.204^{\prime \prime} \\
\mathrm{E} 13^{\circ} 59^{\prime} 8.519^{\prime \prime}\end{array}$ & $\mathrm{SCH}$ & SEG3 & grassland & drill core & 1695 & 300 \\
\hline Chlorella vulgaris & LH10HG2049 & $\begin{array}{l}\text { N51 }{ }^{\circ} 0^{\prime} 2.696^{\prime \prime} \\
\text { E10 } 25^{\circ} 48.036 "\end{array}$ & $\mathrm{HAl}$ & HEG2 & grassland & soil surface & 1720 & 300 \\
\hline Chlorella vulgaris & LH10HG3088 & $\begin{array}{l}\text { N50 } 59^{\prime} 53.129^{\prime \prime} \\
\text { E10² } 25^{\prime} 58.616^{\prime \prime}\end{array}$ & $\mathrm{HAl}$ & HEG3 & grassland & soil surface & 1674 & - \\
\hline
\end{tabular}


Table S1. (continuation)

\begin{tabular}{|c|c|c|c|c|c|c|c|c|}
\hline Species & Identifier & GPS & Exploratory & Plot & Habitat & Sampling & $18 S$ & ITS2 \\
\hline Chlorella vulgaris & LH10HG4093 & $\begin{array}{l}\mathrm{N} 51^{\circ} 6^{\prime} 48.104^{\prime \prime} \\
\mathrm{E} 10^{\circ} 26^{\prime} 10.249^{\prime \prime}\end{array}$ & $\mathrm{HAl}$ & HEG4 & grassland & soil surface & 1724 & - \\
\hline Chlorella vulgaris & LH10HG6014 & $\begin{array}{l}\text { N51 }{ }^{\circ} 12^{\prime} 53.766^{\prime \prime} \\
\text { E10 } 23^{\prime} 28.395^{\prime \prime}\end{array}$ & $\mathrm{HAl}$ & HEG6 & grassland & soil surface & 1719 & 300 \\
\hline Chlorella vulgaris & LH10HG6052 & $\begin{array}{l}\text { N51 }{ }^{\circ} 12^{\prime} 53.766^{\prime \prime} \\
\text { E10 } 23^{\prime} 28.395^{\prime \prime}\end{array}$ & $\mathrm{HAl}$ & HEG6 & grassland & soil surface & 1735 & 300 \\
\hline Chlorella vulgaris & LH10HG6099 & $\begin{array}{l}\text { N51 }{ }^{\circ} 12^{\prime} 53.766^{\prime \prime} \\
\text { E10 } 23^{\circ} 28.395^{\prime \prime}\end{array}$ & $\mathrm{HAl}$ & HEG6 & grassland & soil surface & 1665 & - \\
\hline Chlorella vulgaris & LH10HG9075 & $\begin{array}{l}\mathrm{N} 51^{\circ} 13^{\prime} 26.031^{\prime \prime} \\
\mathrm{E} 10^{\circ} 22^{\prime} 50.834^{\prime \prime}\end{array}$ & $\mathrm{HAl}$ & HEG9 & grassland & soil surface & 1720 & 300 \\
\hline Chlorella vulgaris (cf.) & LH10HG2081 & $\begin{array}{l}\mathrm{N} 51^{\circ} 0^{\prime} 2.696^{\prime \prime} \\
\mathrm{E} 10^{\circ} 25^{\prime} 48.036^{\prime \prime}\end{array}$ & $\mathrm{HAl}$ & HEG2 & grassland & soil surface & 1690 & 305 \\
\hline Chlorella vulgaris (cf.) & LH10HG7072 & $\begin{array}{l}\text { N51 }{ }^{\circ} 16^{\prime} 24.897^{\prime \prime} \\
\text { E10 } 24^{\circ} 37.485^{\prime \prime}\end{array}$ & $\mathrm{HAl}$ & HEG7 & grassland & soil surface & 1448 & - \\
\hline Chloroidium ellipsoideum (cf.) & LH08AG8046 & $\begin{array}{l}\mathrm{N} 48^{\circ} 25^{\prime} 21.505^{\prime \prime} \\
\mathrm{E} 9^{\circ} 29^{\prime} 31.649^{\prime \prime}\end{array}$ & ALB & AEG8 & grassland & drill core & 1658 & - \\
\hline Chloroidium ellipsoideum (cf.) & LH08AG9062 & $\begin{array}{l}\text { N48 }{ }^{\circ} 23^{\prime} 40.815^{\prime \prime} \\
\text { E9 }^{\circ} 30^{\prime} 10.053^{\prime \prime}\end{array}$ & ALB & AEG9 & grassland & drill core & 1706 & - \\
\hline Chloroidium ellipsoideum (cf.) & LH10HG6086 & $\begin{array}{l}\text { N51 }{ }^{\circ} 12^{\prime} 53.766^{\prime \prime} \\
\mathrm{E} 10^{\circ} 23^{\prime} 28.395^{\prime \prime}\end{array}$ & $\mathrm{HAl}$ & HEG6 & grassland & soil surface & 1677 & - \\
\hline Chloroidium ellipsoideum (cf.) & LH10HG7132 & $\begin{array}{l}\text { N51 }{ }^{\circ} 16^{\prime} 24.897^{\prime \prime} \\
\mathrm{E} 10^{\circ} 24^{\prime} 37.485^{\prime \prime}\end{array}$ & $\mathrm{HAl}$ & HEG7 & grassland & soil surface & 1632 & - \\
\hline Chloroidium ellipsoideum (cf.) & LH10HG9105 & $\begin{array}{l}\mathrm{N} 51^{\circ} 13^{\prime} 26.031^{\prime \prime} \\
\mathrm{E} 10^{\circ} 22^{\prime} 50.834^{\prime \prime}\end{array}$ & $\mathrm{HAl}$ & HEG9 & grassland & soil surface & 1724 & - \\
\hline Chloroidium saccharophilum & LH08AW8042 & $\begin{array}{l}\text { N48 } 22^{\prime} 57.322^{\prime \prime} \\
\text { E9 }^{\circ} 22^{\prime} 56.584 "\end{array}$ & ALB & AEW8 & forest & drill core & 1694 & - \\
\hline Chloroidium saccharophilum & LH10HG5089 & $\begin{array}{l}\text { N51 }{ }^{\circ} 12^{\prime} 57.22^{\prime \prime} \\
\mathrm{E} 10^{\circ} 19^{\prime} 21.096^{\prime \prime}\end{array}$ & $\mathrm{HAl}$ & HEG5 & grassland & soil surface & 568 & - \\
\hline Chloroidium saccharophilum & LH10HG7062 & $\begin{array}{l}\text { N51 }{ }^{\circ} 16^{\prime} 24.897^{\prime \prime} \\
\text { E10 } 24^{\prime} 37.485^{\prime \prime}\end{array}$ & $\mathrm{HAl}$ & HEG7 & grassland & soil surface & 1719 & - \\
\hline Coccomyxa viridis & $\begin{array}{l}\text { LH08AW8039 } \\
\text { (=SAG 2483) }\end{array}$ & $\begin{array}{l}\text { N48 }{ }^{\circ} 22^{\prime} 57.322^{\prime \prime} \\
\text { E9 }^{\circ} 22^{\prime} 56.584^{\prime \prime}\end{array}$ & ALB & AEW8 & forest & drill core & 1731 & - \\
\hline Coccomyxa simplex & LH08SG9051 & $\begin{array}{l}\text { N53 }{ }^{\circ} 5^{\prime} 53.455^{\prime \prime} \\
\text { E13 } 36^{\circ} 45.241^{\prime \prime}\end{array}$ & $\mathrm{SCH}$ & SEG9 & grassland & drill core & 1409 & - \\
\hline Coccomyxa sp. & LH08AW1017 & $\begin{array}{l}\text { N48 }{ }^{\circ} 28^{\prime} 41.063^{\prime \prime} \\
E^{\circ} 20^{\prime} 3.877^{\prime \prime}\end{array}$ & ALB & AEW1 & forest & drill core & 1732 & - \\
\hline Dictyochloropsis splendida & LH08AW3050 & $\begin{array}{l}\mathrm{N} 48^{\circ} 24^{\prime} 44.145^{\prime \prime} \\
\mathrm{E} 9^{\circ} 21^{\prime} 20.127^{\prime \prime}\end{array}$ & ALB & AEW3 & forest & drill core & 1722 & - \\
\hline Diplosphaera sp.(I) & LH08HW8075 & $\begin{array}{l}\mathrm{N} 51^{\circ} 21^{\prime} 20.852^{\prime \prime} \\
\mathrm{E} 10^{\circ} 31^{\prime} 1.083^{\prime \prime}\end{array}$ & $\mathrm{HAl}$ & HEW8 & forest & drill core & 1824 & 326 \\
\hline Diplosphaera sp.(II) & LH08AG9089 & $\begin{array}{l}N 48^{\circ} 23^{\prime} 40.815^{\prime \prime} \\
E 9^{\circ} 30^{\prime} 10.053^{\prime \prime}\end{array}$ & ALB & AEG9 & grassland & drill core & 1660 & 334 \\
\hline Lobosphaera bisecta (cf.) & LH10HG3P15 & $\begin{array}{l}\text { N50 } 59^{\prime} 53.129^{\prime \prime} \\
\mathrm{E} 10^{\circ} 25^{\prime} 58.616^{\prime \prime}\end{array}$ & $\mathrm{HAl}$ & HEG3 & grassland & soil surface & 601 & - \\
\hline Lobosphaera irregularis (cf.) & LH08AW3064 & $\begin{array}{l}N 48^{\circ} 24^{\prime} 44.145^{\prime \prime} \\
\text { E9 }^{\circ} 21^{\prime} 20.127^{\prime \prime}\end{array}$ & ALB & AEW3 & forest & drill core & 1731 & - \\
\hline Lobosphaera irregularis (cf.) & LH08SW5063 & $\begin{array}{l}\mathrm{N}^{\prime} 3^{\circ} 3^{\prime} 25.321^{\prime \prime} \\
\mathrm{E} 13^{\circ} 53^{\prime} 7.318^{\prime \prime}\end{array}$ & $\mathrm{SCH}$ & SEW5 & forest & drill core & 705 & - \\
\hline Muriella terrestris (cf.) & LH08SG3009 & $\begin{array}{l}\mathrm{N} 53^{\circ} 6^{\prime} 10.204^{\prime \prime} \\
\mathrm{E} 13^{\circ} 59^{\prime} 8.519^{\prime \prime}\end{array}$ & $\mathrm{SCH}$ & SEG3 & grassland & drill core & 1692 & 312 \\
\hline Muriella terrestris (cf.) & LH10HG1070 & $\begin{array}{l}\text { N50 } 58^{\prime} 17.934^{\prime \prime} \\
\text { E10 } 24^{\circ} 19.306^{\prime \prime}\end{array}$ & $\mathrm{HAl}$ & HEG1 & grassland & soil surface & 1767 & - \\
\hline Muriella terrestris (cf.) & LH10HG1076 & $\begin{array}{l}\text { N50 } 58^{\prime} 17.934^{\prime \prime} \\
\text { E10 } 24^{\prime} 19.306^{\prime \prime}\end{array}$ & $\mathrm{HAl}$ & HEG1 & grassland & soil surface & 1732 & 312 \\
\hline Muriella terrestris (cf.) & LH10HG7118 & $\begin{array}{l}\text { N51 }{ }^{\circ} 16^{\prime} 24.897^{\prime \prime} \\
\mathrm{E} 10^{\circ} 24^{\prime} 37.485^{\prime \prime}\end{array}$ & $\mathrm{HAl}$ & HEG7 & grassland & soil surface & 1675 & - \\
\hline Muriella terrestris (cf.) & LH10HG8050 & $\begin{array}{l}\mathrm{N} 51^{\circ} 16^{\prime} 16.527^{\prime \prime} \\
\mathrm{E} 10^{\circ} 25^{\prime} 4.6^{\prime \prime}\end{array}$ & $\mathrm{HAl}$ & HEG8 & grassland & soil surface & 1733 & 311 \\
\hline Muriella terrestris (cf.) & LH10HG9077 & $\begin{array}{l}\text { N51 }{ }^{\circ} 13^{\prime} 26.031^{\prime \prime} \\
\mathrm{E} 10^{\circ} 22^{\prime} 50.834^{\prime \prime}\end{array}$ & $\mathrm{HAl}$ & HEG9 & grassland & soil surface & 1729 & - \\
\hline Nannochloris sp. & LH08SG3102 & $\begin{array}{l}\mathrm{N}^{3} 3^{\circ} 6^{\prime} 10.204^{\prime \prime} \\
\mathrm{E} 13^{\circ} 59^{\prime} 8.519^{\prime \prime}\end{array}$ & $\mathrm{SCH}$ & SEG3 & grassland & drill core & 802 & - \\
\hline Nannochloris sp. & LH08SG8030 & $\begin{array}{l}\text { N53 }{ }^{\circ} 6^{\prime} 50.294^{\prime \prime} \\
\text { E14 } 1^{\prime} 1.559^{\prime \prime}\end{array}$ & $\mathrm{SCH}$ & SEG8 & grassland & drill core & 1648 & - \\
\hline Nannochloris sp. & LH10HG6095 & $\begin{array}{l}\text { N51 }{ }^{\circ} 12^{\prime} 53.766^{\prime \prime} \\
\text { E10 } 23^{\prime} 28.395^{\prime \prime}\end{array}$ & $\mathrm{HAl}$ & HEG6 & grassland & soil surface & 1721 & - \\
\hline $\begin{array}{l}\text { Navichloris fusiformis } \\
\text { (provisional denomination) }\end{array}$ & $\begin{array}{l}\text { LH08AW3007 } \\
\text { (=SAG 2477) }\end{array}$ & $\begin{array}{l}\text { N48 }{ }^{\circ} 24^{\prime} 44.145^{\prime \prime} \\
\mathrm{E} 9^{\circ} 21^{\prime} 20.127^{\prime \prime}\end{array}$ & ALB & AEW3 & forest & drill core & 1694 & - \\
\hline Neocystis brevis & LH08AG9012 & $\begin{array}{l}\mathrm{N} 48^{\circ} 23^{\prime} 40.815^{\prime \prime} \\
\mathrm{E} 9^{\circ} 30^{\prime} 10.053^{\prime \prime}\end{array}$ & ALB & AEG9 & grassland & drill core & 1673 & - \\
\hline Neocystis brevis & $\begin{array}{l}\text { LH08AW8001 } \\
\text { (=SAG 2480) }\end{array}$ & $\begin{array}{l}\text { N48 } 22^{\prime} 57.322^{\prime \prime} \\
\mathrm{E} 9^{\circ} 22^{\prime} 56.584^{\prime \prime}\end{array}$ & ALB & AEW8 & forest & drill core & 1735 & - \\
\hline
\end{tabular}


Table S1. (continuation)

\begin{tabular}{|c|c|c|c|c|c|c|c|c|}
\hline Species & Identifier & GPS & Exploratory & Plot & Habitat & Sampling & $18 \mathrm{~S}$ & ITS2 \\
\hline Neocystis brevis & LH08HW6059 & $\begin{array}{l}\text { N51ํ16' 3.791" } \\
\text { E10 } 14^{\prime} 21.762 "\end{array}$ & $\mathrm{HAl}$ & HEW6 & forest & drill core & 1713 & - \\
\hline Neocystis brevis & LH08HW6108 & 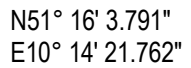 & $\mathrm{HAl}$ & HEW6 & forest & drill core & 1687 & - \\
\hline Neocystis brevis & LH08SG2072 & $\begin{array}{l}\text { N53 } 5^{\prime} 21.505^{\prime \prime} \\
\text { E13 } 58^{\circ} 48.169^{\prime \prime}\end{array}$ & $\mathrm{SCH}$ & SEG2 & grassland & drill core & 1696 & - \\
\hline Neocystis brevis & LH10HG4082 & $\begin{array}{l}\text { N51 }{ }^{\circ} 6^{\prime} 48.104^{\prime \prime} \\
\text { E10 } 26^{\circ} 10.249^{\prime \prime}\end{array}$ & $\mathrm{HAl}$ & HEG4 & grassland & soil surface & 357 & - \\
\hline Neocystis brevis & LH10HG9054 & $\begin{array}{l}\text { N51 }{ }^{\circ} 13^{\prime} 26.031^{\prime \prime} \\
\mathrm{E} 10^{\circ} 22^{\prime} 50.834^{\prime \prime}\end{array}$ & $\mathrm{HAl}$ & HEG9 & grassland & soil surface & 1731 & - \\
\hline Neocystis brevis & LH10HG9080 & $\begin{array}{l}\mathrm{N} 51^{\circ} 13^{\prime} 26.031^{\prime \prime} \\
\mathrm{E} 10^{\circ} 22^{\prime} 50.834^{\prime \prime}\end{array}$ & $\mathrm{HAl}$ & HEG9 & grassland & soil surface & 1736 & 295 \\
\hline Neocystis brevis & LH10HG9P02 & $\begin{array}{l}\text { N51ํ1' 26.031" } \\
\text { E10 } 22^{\prime} 50.834^{\prime \prime}\end{array}$ & $\mathrm{HAl}$ & HEG9 & grassland & soil surface & 543 & - \\
\hline $\begin{array}{l}\text { Pseudostichococcus } \\
\text { monallantoides }\end{array}$ & LH08AG7097 & $\begin{array}{l}\mathrm{N} 48^{\circ} 23^{\prime} 29.116^{\prime \prime} \\
\mathrm{E} 9^{\circ} 22^{\prime} 36.65^{\prime \prime}\end{array}$ & ALB & AEG7 & grassland & drill core & 392 & - \\
\hline $\begin{array}{l}\text { Pseudostichococcus } \\
\text { monallantoides }\end{array}$ & LH10HG2066 & $\begin{array}{l}\mathrm{N} 51^{\circ} 0^{\prime} 2.696 " \\
\mathrm{E} 10^{\circ} 25^{\prime} 48.036 "\end{array}$ & $\mathrm{HAl}$ & HEG2 & grassland & soil surface & 1709 & - \\
\hline $\begin{array}{l}\text { Pseudostichococcus } \\
\text { monallantoides }\end{array}$ & LH10HG3045 & $\begin{array}{l}\text { N50 } 59^{\prime} 53.129^{\prime \prime} \\
\text { E10 } 25^{\circ} 58.616^{\prime \prime}\end{array}$ & $\mathrm{HAl}$ & HEG3 & grassland & soil surface & 1737 & 277 \\
\hline Pseudostichococcus sp. & LH08SW8044 & $\begin{array}{l}\text { N53 }{ }^{\circ} 11^{\prime} 30.47^{\prime \prime} \\
\mathrm{E} 13^{\circ} 55^{\prime} 49.216^{\prime \prime}\end{array}$ & $\mathrm{SCH}$ & SEW8 & forest & drill core & 1661 & 285 \\
\hline Stichococcus sp.(I) & LH08AG9028 & $\begin{array}{l}\mathrm{N} 48^{\circ} 23^{\prime} 40.815^{\prime \prime} \\
\mathrm{E} 9^{\circ} 30^{\prime} 10.053^{\prime \prime}\end{array}$ & ALB & AEG9 & grassland & drill core & 1245 & - \\
\hline Stichococcus sp.(I) & LH08AW8025 & $\begin{array}{l}\mathrm{N} 48^{\circ} 22^{\prime} 57.322^{\prime \prime} \\
\mathrm{E}^{\circ} 22^{\prime} 56.584^{\prime \prime}\end{array}$ & ALB & AEW8 & forest & drill core & 1712 & - \\
\hline Stichococcus sp.(II) & LH08AG1100 & $\begin{array}{l}\text { N48 } 23^{\prime} 52.818^{\prime \prime} \\
\mathrm{E} 9^{\circ} 20^{\prime} 31.152^{\prime \prime}\end{array}$ & ALB & AEG1 & grassland & drill core & 573 & - \\
\hline Stichococcus sp.(II) & LH08SG5057 & 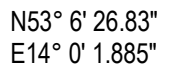 & $\mathrm{SCH}$ & SEG5 & grassland & drill core & 1695 & 302 \\
\hline Stichococcus sp.(II) & LH08SG5067 & $\begin{array}{l}\text { N53 }{ }^{\circ} 6^{\prime} 26.83^{\prime \prime} \\
\mathrm{E} 14^{\circ} 0^{\prime} 1.885^{\prime \prime}\end{array}$ & $\mathrm{SCH}$ & SEG5 & grassland & drill core & 1659 & - \\
\hline Stichococcus sp.(II) & LH08SG5079 & $\begin{array}{l}\text { N53 }{ }^{\circ} 6^{\prime} 26.83^{\prime \prime} \\
E 14^{\circ} 0^{\prime} 1.885^{\prime \prime}\end{array}$ & $\mathrm{SCH}$ & SEG5 & grassland & drill core & 1692 & 302 \\
\hline Stichococcus sp.(II) & LH08SG5090 & $\begin{array}{l}\mathrm{N} 53^{\circ} 6^{\prime} 26.83^{\prime \prime} \\
\mathrm{E} 14^{\circ} 0^{\prime} 1.885^{\prime \prime}\end{array}$ & $\mathrm{SCH}$ & SEG5 & grassland & drill core & 1683 & 302 \\
\hline Stichococcus sp.(II) & LH08SG5092 & 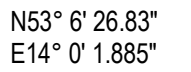 & $\mathrm{SCH}$ & SEG5 & grassland & drill core & 785 & - \\
\hline Stichococcus sp.(II) & LH10HG2063 & $\begin{array}{l}\mathrm{N} 51^{\circ} 0^{\prime} 2.696^{\prime \prime} \\
\mathrm{E} 10^{\circ} 25^{\prime} 48.036^{\prime \prime}\end{array}$ & $\mathrm{HAl}$ & HEG2 & grassland & soil surface & 1721 & 302 \\
\hline Stichococcus sp.(II) & LH10HG2067 & $\begin{array}{l}\mathrm{N} 51^{\circ} 0^{\prime} 2.696^{\prime \prime} \\
\mathrm{E} 10^{\circ} 25^{\prime} 48.036 "\end{array}$ & $\mathrm{HAl}$ & HEG2 & grassland & soil surface & 1721 & - \\
\hline Stichococcus sp.(II) & LH10HG3128 & $\begin{array}{l}\text { N50 } 50^{\circ} 53.129^{\prime \prime} \\
\text { E10 } 25^{\prime} 58.616^{\prime \prime}\end{array}$ & $\mathrm{HAl}$ & HEG3 & grassland & soil surface & 1721 & - \\
\hline Stichococcus sp.(II) & LH10HG7090 & $\begin{array}{l}N 51^{\circ} 16^{\prime} 24.897^{\prime \prime} \\
\text { E10 } 24^{\circ} 37.485^{\prime \prime}\end{array}$ & $\mathrm{HAl}$ & HEG7 & grassland & soil surface & 1719 & 302 \\
\hline Stichococcus sp.(II) & LH10HG7092 & $\begin{array}{l}\text { N51 }{ }^{\circ} 16^{\prime} 24.897^{\prime \prime} \\
\text { E10 } 24^{\prime} 37.485^{\prime \prime}\end{array}$ & $\mathrm{HAl}$ & HEG7 & grassland & soil surface & 1710 & - \\
\hline Stichococcus sp.(II) & LH10HG9068 & $\begin{array}{l}\text { N51 }{ }^{\circ} 13^{\prime} 26.031^{\prime \prime} \\
\text { E10 } 22^{\circ} 50.834^{\prime \prime}\end{array}$ & $\mathrm{HAl}$ & HEG9 & grassland & soil surface & 1722 & - \\
\hline Stichococcus sp.(III) & LH08AG7010 & $\begin{array}{l}\mathrm{N} 48^{\circ} 23^{\prime} 29.116^{\prime \prime} \\
\mathrm{E} 9^{\circ} 22^{\prime} 36.65^{\prime \prime}\end{array}$ & ALB & AEG7 & grassland & drill core & 1660 & 274 \\
\hline Stichococcus sp.(III) & LH10HG6110 & $\begin{array}{l}\text { N51ํ12' } 53.766^{\prime \prime} \\
\text { E10 } 23^{\prime} 28.395^{\prime \prime}\end{array}$ & $\mathrm{HAl}$ & HEG6 & grassland & soil surface & 1724 & - \\
\hline Stichococcus sp.(IV) & LH08SG1073 & 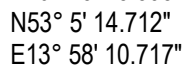 & $\mathrm{SCH}$ & SEG1 & grassland & drill core & 1696 & 325 \\
\hline Stichococcus sp.(V) & LH08SW1099 & $\begin{array}{l}\text { N52 }{ }^{\circ} 54^{\prime} 3.05^{\prime \prime} \\
\text { E13 } 50^{\circ} 46.921 "\end{array}$ & $\mathrm{SCH}$ & SEW1 & forest & drill core & 1653 & 310 \\
\hline Stichococcus sp.(VI) & $\begin{array}{l}\text { LH08AW8023 } \\
\text { (=SAG 2482) }\end{array}$ & $\begin{array}{l}\mathrm{N} 48^{\circ} 22^{\prime} 57.322^{\prime \prime} \\
\mathrm{Eg}^{\circ} 22^{\prime} 56.584^{\prime \prime}\end{array}$ & ALB & AEW8 & forest & drill core & 1722 & 311 \\
\hline Stichococcus sp.(VI) & LH08AW8104 & $\begin{array}{l}\text { N48 } 22^{\prime} 57.322 " \\
\mathrm{E}^{\circ} 22^{\prime} 56.584^{\prime \prime}\end{array}$ & ALB & AEW8 & forest & drill core & 1687 & 311 \\
\hline Stichococcus sp.(VII) & $\begin{array}{l}\text { LH08AW8002 } \\
\text { (=SAG 2481) }\end{array}$ & $\begin{array}{l}\text { N48 } 22^{\prime} 57.322^{\prime \prime} \\
\mathrm{E} 9^{\circ} 22^{\prime} 56.584^{\prime \prime}\end{array}$ & ALB & AEW8 & forest & drill core & 1730 & - \\
\hline Unidentified Chlorellaceae (I) & LH08AG1034 & $\begin{array}{l}\mathrm{N} 48^{\circ} 23^{\prime} 52.818^{\prime \prime} \\
\mathrm{E}^{\circ} 20^{\prime} 31.152^{\prime \prime}\end{array}$ & ALB & AEG1 & grassland & drill core & 1681 & 274 \\
\hline Unidentified Chlorellaceae (I) & LH10HG7073 & $\begin{array}{l}\text { N51 }{ }^{\circ} 16^{\prime} 24.897^{\prime \prime} \\
\text { E10 } 24^{\prime} 37.485^{\prime \prime}\end{array}$ & $\mathrm{HAl}$ & HEG7 & grassland & soil surface & 1409 & - \\
\hline Unidentified Chlorellaceae (II) & LH08SG2053 & 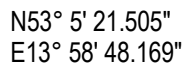 & $\mathrm{SCH}$ & SEG2 & grassland & drill core & 1672 & - \\
\hline Unidentified Chlorellaceae (II) & LH08SG3029 & $\begin{array}{l}N 53^{\circ} 6^{\prime} 10.204^{\prime \prime} \\
E 13^{\circ} 59^{\prime} 8.519^{\prime \prime}\end{array}$ & $\mathrm{SCH}$ & SEG3 & grassland & drill core & 798 & - \\
\hline
\end{tabular}


Table S1. (continuation)

\begin{tabular}{|c|c|c|c|c|c|c|c|c|}
\hline Species & Identifier & GPS & Exploratory & Plot & Habitat & Sampling & $18 \mathrm{~S}$ & ITS2 \\
\hline Unidentified Chlorellaceae (II) & LH10HG2094 & $\begin{array}{l}\mathrm{N} 51^{\circ} 0^{\prime} 2.696 " \\
\mathrm{E} 10^{\circ} 25^{\prime} 48.036^{\prime \prime}\end{array}$ & $\mathrm{HAl}$ & HEG2 & grassland & soil surface & 1719 & - \\
\hline Unidentified Chlorellaceae (II) & LH10HG3135 & $\begin{array}{l}\mathrm{N} 50^{\circ} 59^{\prime} 53.129^{\prime \prime} \\
\mathrm{E} 10^{\circ} 25^{\prime} 58.616^{\prime \prime}\end{array}$ & $\mathrm{HAl}$ & HEG3 & grassland & soil surface & 1718 & - \\
\hline Unidentified Chlorellaceae (II) & LH10HG7071 & $\begin{array}{l}\mathrm{N} 51^{\circ} 16^{\prime} 24.897^{\prime \prime} \\
\mathrm{E} 10^{\circ} 24^{\prime} 37.485^{\prime \prime}\end{array}$ & $\mathrm{HAl}$ & HEG7 & grassland & soil surface & 1732 & - \\
\hline Unidentified Chlorellaceae (II) & LH10HG9020 & $\begin{array}{l}\mathrm{N} 51^{\circ} 13^{\prime} 26.031^{\prime \prime} \\
\mathrm{E} 10^{\circ} 22^{\prime} 50.834^{\prime \prime}\end{array}$ & $\mathrm{HAl}$ & HEG9 & grassland & soil surface & 1617 & 299 \\
\hline Unidentified Chlorellaceae (II) & LH10HG9026 & $\begin{array}{l}\mathrm{N} 51^{\circ} 13^{\prime} 26.031^{\prime \prime} \\
\mathrm{E} 10^{\circ} 22^{\prime} 50.834^{\prime \prime}\end{array}$ & $\mathrm{HAl}$ & HEG9 & grassland & soil surface & 1716 & 274 \\
\hline Unidentified Chlorellaceae (III) & LH08SG3078 & $\begin{array}{l}\mathrm{N} 53^{\circ} 6^{\prime} 10.204^{\prime \prime} \\
\mathrm{E} 13^{\circ} 59^{\prime} 8.519^{\prime \prime}\end{array}$ & $\mathrm{SCH}$ & SEG3 & grassland & drill core & 1611 & 299 \\
\hline Unidentified Chlorellaceae (III) & LH08SG3093 & $\begin{array}{l}\mathrm{N} 53^{\circ} 6^{\prime} 10.204^{\prime \prime} \\
\mathrm{E} 13^{\circ} 59^{\prime} 8.519^{\prime \prime}\end{array}$ & $\mathrm{SCH}$ & SEG3 & grassland & drill core & 1678 & 299 \\
\hline Unidentified Chlorellaceae (IV) & LH10HG709K & $\begin{array}{l}\text { N51 }{ }^{\circ} 16^{\prime} 24.897^{\prime \prime} \\
\text { E10 } 24^{\prime} 37.485^{\prime \prime}\end{array}$ & $\mathrm{HAl}$ & HEG7 & grassland & soil surface & 1685 & - \\
\hline Xylochloris sp. & $\begin{array}{l}\text { LH08AG7024 } \\
(=S A G ~ 2382)\end{array}$ & $\begin{array}{l}\mathrm{N} 48^{\circ} 23^{\prime} 29.116^{\prime \prime} \\
\mathrm{E}^{\circ} 22^{\prime} 36.65^{\prime \prime}\end{array}$ & ALB & AEG7 & grassland & drill core & 1719 & - \\
\hline Pseudendocloniopsis botryoides & LH08HW9058 & $\begin{array}{l}\text { N51 }{ }^{\circ} 7^{\prime} 48.871 " \\
\mathrm{E} 10^{\circ} 22^{\prime} 52.139^{\prime \prime}\end{array}$ & $\mathrm{HAl}$ & HEW9 & forest & drill core & 2058 & - \\
\hline $\begin{array}{l}\text { Klebsormidium } \\
\text { dissectum/elegans (cf.) }\end{array}$ & LH08AG1113 & $\begin{array}{l}\text { N48 } 23^{\circ} 52.818^{\prime \prime} \\
\text { E9 }^{\circ} 20^{\prime} 31.152^{\prime \prime}\end{array}$ & ALB & AEG1 & grassland & drill core & 2038 & - \\
\hline $\begin{array}{l}\text { Klebsormidium } \\
\text { dissectum/elegans (cf.) }\end{array}$ & LH08AG7011 & $\begin{array}{l}\text { N48 } 28^{\circ} 29.116^{\prime \prime} \\
\mathrm{E}^{\circ} 22^{\prime} 36.65^{\prime \prime}\end{array}$ & ALB & AEG7 & grassland & drill core & 2236 & - \\
\hline $\begin{array}{l}\text { Klebsormidium } \\
\text { dissectum/elegans (cf.) }\end{array}$ & LH08AG9045 & $\begin{array}{l}\text { N48 } 28^{\circ} 23^{\prime} 40.815^{\prime \prime} \\
E^{\circ} 30^{\prime} 10.053^{\prime \prime}\end{array}$ & ALB & AEG9 & grassland & drill core & 2093 & - \\
\hline $\begin{array}{l}\text { Klebsormidium } \\
\text { dissectum/elegans (cf.) }\end{array}$ & LH08HW1038 & $\begin{array}{l}\text { N51 }{ }^{\circ} 11^{\prime} 7.278^{\prime \prime} \\
\text { E10 } 19^{\prime} 25.036^{\prime \prime}\end{array}$ & $\mathrm{HAl}$ & HEW1 & forest & drill core & 1882 & 274 \\
\hline $\begin{array}{l}\text { Klebsormidium } \\
\text { dissectum/elegans (cf.) }\end{array}$ & LH08HW4109 & $\begin{array}{l}\mathrm{N} 51^{\circ} 22^{\prime} 10.134^{\prime \prime} \\
\mathrm{E} 10^{\circ} 31^{\prime} 59.979^{\prime \prime}\end{array}$ & $\mathrm{HAl}$ & HEW4 & forest & drill core & 2234 & - \\
\hline $\begin{array}{l}\text { Klebsormidium } \\
\text { dissectum/elegans (cf.) }\end{array}$ & LH08HW5021 & $\begin{array}{l}\mathrm{N} 51^{\circ} 15^{\prime} 49.961^{\prime \prime} \\
\mathrm{E} 10^{\circ} 14^{\prime} 27.448^{\prime \prime}\end{array}$ & $\mathrm{HAl}$ & HEW5 & forest & drill core & 1827 & - \\
\hline $\begin{array}{l}\text { Klebsormidium } \\
\text { dissectum/elegans (cf.) }\end{array}$ & LH08HW9005 & $\begin{array}{l}\text { N51 } 1^{\circ} 7^{\prime} 48.871^{\prime \prime} \\
\text { E10 } 22^{\prime} 52.139^{\prime \prime}\end{array}$ & $\mathrm{HAl}$ & HEW9 & forest & drill core & 1736 & - \\
\hline $\begin{array}{l}\text { Klebsormidium } \\
\text { dissectum/elegans (cf.) }\end{array}$ & LH08HW9106 & $\begin{array}{l}\mathrm{N} 51^{\circ} 7^{\prime} 48.871^{\prime \prime} \\
\mathrm{E} 10^{\circ} 22^{\prime} 52.139^{\prime \prime}\end{array}$ & $\mathrm{HAl}$ & HEW9 & forest & drill core & 2211 & 274 \\
\hline Klebsormidium flaccidum (cf.) & LH08HW5061 & $\begin{array}{l}\mathrm{N} 51^{\circ} 15^{\prime} 49.961^{\prime \prime} \\
\mathrm{E} 10^{\circ} 14^{\prime} 27.448^{\prime \prime}\end{array}$ & $\mathrm{HAl}$ & HEW5 & forest & drill core & 2064 & 274 \\
\hline Klebsormidium flaccidum (cf.) & LH08SG3027 & $\begin{array}{l}\mathrm{N} 53^{\circ} 6^{\prime} 10.204^{\prime \prime} \\
\mathrm{E} 13^{\circ} 59^{\prime} 8.519^{\prime \prime}\end{array}$ & $\mathrm{SCH}$ & SEG3 & grassland & drill core & 1817 & - \\
\hline Klebsormidium flaccidum (cf.) & LH10HG2056 & $\begin{array}{l}\mathrm{N} 51^{\circ} 0^{\prime} 2.696 " \\
\mathrm{E} 10^{\circ} 25^{\prime} 48.036^{\prime \prime}\end{array}$ & $\mathrm{HAl}$ & HEG2 & grassland & soil surface & 1777 & 274 \\
\hline Klebsormidium flaccidum (cf.) & LH10HG7028 & $\begin{array}{l}\mathrm{N} 51^{\circ} 16^{\prime} 24.897^{\prime \prime} \\
\mathrm{E} 10^{\circ} 24^{\prime} 37.485^{\prime \prime}\end{array}$ & $\mathrm{HAl}$ & HEG7 & grassland & soil surface & 1794 & 275 \\
\hline Asterosiphon sp. & LH10HG3064 & $\begin{array}{l}\text { N50 } 59^{\prime} 53.129^{\prime \prime} \\
\text { E10 } 25^{\prime} 58.616^{\prime \prime}\end{array}$ & $\mathrm{HAl}$ & HEG3 & grassland & drill core & 1780 & - \\
\hline Botrydiopsalean sp. & LH08AW1076 & $\begin{array}{l}\mathrm{N} 48^{\circ} 28^{\prime} 41.063^{\prime \prime} \\
\mathrm{E} 9^{\circ} 20^{\prime} 3.877^{\prime \prime}\end{array}$ & ALB & AEW1 & forest & soil surface & 1763 & - \\
\hline Botrydiopsalean sp. & LH08SG2K53 & $\begin{array}{l}\mathrm{N} 53^{\circ} 5^{\prime} 21.505^{\prime \prime} \\
\mathrm{E} 13^{\circ} 58^{\prime} 48.169^{\prime \prime}\end{array}$ & $\mathrm{SCH}$ & SEG2 & grassland & soil surface & 619 & - \\
\hline Botrydiopsis callosa (cf.) & LH08AW4043 & $\begin{array}{l}\mathrm{N} 48^{\circ} 23^{\prime} 56.755^{\prime \prime} \\
\mathrm{E} 9^{\circ} 14^{\prime} 41.378^{\prime \prime}\end{array}$ & ALB & AEW4 & forest & soil surface & 1753 & - \\
\hline Heterococcus sp. & LH10HG2140 & $\begin{array}{l}\mathrm{N} 51^{\circ} 0^{\prime} 2.696 " \\
\mathrm{E} 10^{\circ} 25^{\prime} 48.036^{\prime \prime}\end{array}$ & $\mathrm{HAl}$ & HEG2 & grassland & drill core & 1734 & - \\
\hline Heterococcus sp. & LH10HG9085 & $\begin{array}{l}\mathrm{N} 51^{\circ} 13^{\prime} 26.031^{\prime \prime} \\
\mathrm{E} 10^{\circ} 22^{\prime} 50.834^{\prime \prime}\end{array}$ & $\mathrm{HAl}$ & HEG9 & grassland & drill core & 1734 & - \\
\hline Heterococcus chodatii (cf.) & LH10HG9111 & $\begin{array}{l}\mathrm{N} 51^{\circ} 13^{\prime} 26.031^{\prime \prime} \\
\mathrm{E} 10^{\circ} 22^{\prime} 50.834^{\prime \prime}\end{array}$ & $\mathrm{HAl}$ & HEG9 & grassland & drill core & 1693 & - \\
\hline Heterococcus chodatii (cf.) & LH10HG9126 & $\begin{array}{l}\mathrm{N} 51^{\circ} 13^{\prime} 26.031^{\prime \prime} \\
\mathrm{E} 10^{\circ} 22^{\prime} 50.834^{\prime \prime}\end{array}$ & HAI & HEG9 & grassland & drill core & 627 & - \\
\hline Heterococcus caespitosus (cf.) & LH08AG2020 & $\begin{array}{l}\mathrm{N} 48^{\circ} 22^{\prime} 36.686^{\prime \prime} \\
\mathrm{E} 9^{\circ} 28^{\prime} 22.023^{\prime \prime}\end{array}$ & ALB & AEG2 & grassland & soil surface & 1690 & - \\
\hline Heterothrix sessile & LH10HG5079 & $\begin{array}{l}\text { N51 }{ }^{\circ} 12^{\prime} 57.22^{\prime \prime} \\
\text { E10 } 19^{\prime} 21.096^{\prime \prime}\end{array}$ & $\mathrm{HAl}$ & HEG5 & grassland & drill core & 1791 & - \\
\hline Heterothrix sessile & LH10HG9037 & $\begin{array}{l}\mathrm{N} 51^{\circ} 13^{\prime} 26.031^{\prime \prime} \\
\mathrm{E} 10^{\circ} 22^{\prime} 50.834^{\prime \prime}\end{array}$ & $\mathrm{HAl}$ & HEG9 & grassland & drill core & 1568 & - \\
\hline Heterothrix sp. & LH08SG5052 & $\begin{array}{l}\mathrm{N} 53^{\circ} 6^{\prime} 26.83^{\prime \prime} \\
\mathrm{E} 14^{\circ} 0^{\prime} 1.885^{\prime \prime}\end{array}$ & $\mathrm{SCH}$ & SEG5 & grassland & soil surface & 1779 & - \\
\hline Heterothrix sp. & LH10HG7061 & $\begin{array}{l}\mathrm{N} 51^{\circ} 16^{\prime} 24.897^{\prime \prime} \\
\mathrm{E} 10^{\circ} 24^{\prime} 37.485^{\prime \prime}\end{array}$ & $\mathrm{HAl}$ & HEG7 & grassland & drill core & 1806 & - \\
\hline
\end{tabular}


Table S1. (continuation)

\begin{tabular}{|c|c|c|c|c|c|c|c|c|}
\hline Species & Identifier & GPS & Exploratory & Plot & Habitat & Sampling & $18 \mathrm{~S}$ & ITS2 \\
\hline Xanthonema bristolianum (cf.) & LH08HW9018 & $\begin{array}{l}\text { N51 }{ }^{\circ} 7^{\prime} 48.871 " \\
\mathrm{E} 10^{\circ} 222^{\prime} 52.139^{\prime \prime}\end{array}$ & $\mathrm{HAl}$ & HEW9 & forest & soil surface & 1752 & - \\
\hline Xanthonema bristolianum (cf.) & LH10HG6059 & $\begin{array}{l}\text { N51 }{ }^{\circ} 12^{\prime} 53.766^{\prime \prime} \\
\mathrm{E} 10^{\circ} 23^{\prime} 28.395^{\prime \prime}\end{array}$ & $\mathrm{HAl}$ & HEG6 & grassland & drill core & 1780 & - \\
\hline Xanthonema exile (cf.) & LH10HG7078 & $\begin{array}{l}\text { N51ํ 16' 24.897" } \\
\text { E10 } 24^{\prime} 37.485^{\prime \prime}\end{array}$ & $\mathrm{HAl}$ & HEG7 & grassland & drill core & 1783 & - \\
\hline Xanthonema sp. & LH10HG1K69 & $\begin{array}{l}\text { N50 } 58^{\circ} 17.934 " \\
\text { E10 } 24^{\prime} 19.306^{\prime \prime}\end{array}$ & $\mathrm{HAl}$ & HEG1 & grassland & drill core & 1716 & - \\
\hline Xanthonema sp. & LH10HG3065 & $\begin{array}{l}\mathrm{N} 50^{\circ} 59^{\prime} 53.129^{\prime \prime} \\
\mathrm{E} 10^{\circ} 25^{\prime} 58.616^{\prime \prime}\end{array}$ & $\mathrm{HAl}$ & HEG3 & grassland & drill core & 1783 & - \\
\hline Xanthonema sp. & LH10HG7029 & $\begin{array}{l}\text { N51 }{ }^{\circ} 16^{\prime} 24.897^{\prime \prime} \\
\mathrm{E} 10^{\circ} 24^{\prime} 37.485^{\prime \prime}\end{array}$ & $\mathrm{HAl}$ & HEG7 & grassland & drill core & 1782 & - \\
\hline Xanthonema sp. & LH10HG8112 & $\begin{array}{l}\mathrm{N} 51^{\circ} 16^{\prime} 16.527^{\prime \prime} \\
\mathrm{E} 10^{\circ} 25^{\prime} 4.6^{\prime \prime}\end{array}$ & $\mathrm{HAl}$ & HEG8 & grassland & drill core & 374 & - \\
\hline Xanthonema sp. & LH10HG9031 & $\begin{array}{l}\text { N51 }{ }^{\circ} 13^{\prime} 26.031^{\prime \prime} \\
\text { E10 } 22 \text { ' } 50.834^{\prime \prime}\end{array}$ & $\mathrm{HAl}$ & HEG9 & grassland & drill core & 1782 & - \\
\hline Xanthonema sp. & LH10HG9058 & $\begin{array}{l}\text { N51 }{ }^{\circ} 13^{\prime} 26.031^{\prime \prime} \\
\mathrm{E} 10^{\circ} 22^{\prime} 50.834^{\prime \prime}\end{array}$ & $\mathrm{HAl}$ & HEG9 & grassland & drill core & 1782 & - \\
\hline Xanthonema sp. & LH10HW9129 & $\begin{array}{l}\mathrm{N} 51^{\circ} 7^{\prime} 48.871 " \\
\mathrm{E} 10^{\circ} 22^{\prime} 52.139^{\prime \prime}\end{array}$ & $\mathrm{HAl}$ & HEW9 & forest & drill core & 1575 & - \\
\hline Eustigmatos sp. & LH10HG5036 & $\begin{array}{l}\mathrm{N} 51^{\circ} 12^{\prime} 57.22^{\prime \prime} \\
\mathrm{E} 10^{\circ} 19^{\prime} 21.096^{\prime \prime}\end{array}$ & $\mathrm{HAl}$ & HEG5 & grassland & drill core & 1764 & - \\
\hline Eustigmatos sp. & LH10HG9133 & $\begin{array}{l}\mathrm{N} 51^{\circ} 13^{\prime} 26.031^{\prime \prime} \\
\mathrm{E} 10^{\circ} 22^{\prime} 50.834^{\prime \prime}\end{array}$ & $\mathrm{HAl}$ & HEG9 & grassland & drill core & 1691 & - \\
\hline
\end{tabular}

Legend. ALB=Schwäbische Alb, HAl=Hainich-Dün, $\mathrm{SCH}=$ Schorfheide-Chorin. 
Table S2a. List of all detected species belonging to Trebouxiophyceae.

\begin{tabular}{|c|c|c|c|}
\hline Species & Clade & Representative isolates and SAG-strains & GenBank \\
\hline Auxenochlorella protothecoides & Chlorellaceae & LH10HG6096 & - \\
\hline Auxenochlorella sp. & Chlorellaceae & LH08AW4103 (=SAG 2478) & KP081390 \\
\hline Chlorella mirabilis (cf.) & Prasiola & LH08AG9040 & - \\
\hline Chlorella mirabilis (cf.) & Prasiola & LH10HG6139 & - \\
\hline Chlorella vulgaris & Chlorellaceae & LH08HG1081 & - \\
\hline Chlorella vulgaris & Chlorellaceae & LH08SG3006 & - \\
\hline Chlorella vulgaris (cf.) & Chlorellaceae & LH10HG2081 (partial 18S) & - \\
\hline Chlorella vulgaris (cf.) & Chlorellaceae & LH10HG7072 (partial 18S) & - \\
\hline Chloroidium ellipsoideum (cf.) & Watanabea & LH10HG9105 & - \\
\hline Chloroidium saccharophilum & Watanabea & LH10HG7062 & - \\
\hline Coccomyxa viridis & Botryococcus & LH08AW8039 (=SAG 2483) & KP081391 \\
\hline Coccomyxa simplex & Botryococcus & LH08SG9051 (partial 18S) & - \\
\hline Coccomyxa sp. & Botryococcus & LH08AW1017 & KP081392 \\
\hline Dictyochloropsis splendida & incertae sedis & LH08AW3050 (= LH-aw3050) & JQ988930 \\
\hline Diplosphaera sp.(I) & Prasiola & LH08HW8075 & - \\
\hline Diplosphaera sp.(II) & Prasiola & LH08AG9089 & - \\
\hline Lobosphaera bisecta (cf.) & Lobosphaera & LH10HG3P15 (partial 18S) & - \\
\hline Lobosphaera irregularis (cf.) & Lobosphaera & LH08AW3064 & KP081398 \\
\hline Muriella terrestris (cf.) & Chlorellaceae & LH08SG3009 & - \\
\hline Nannochloris sp. & Chlorellaceae & LH08SG8030 & - \\
\hline Navichloris fusiformis (proposed taxon) & incertae sedis & LH08AW3007 (=SAG 2477) & KP081399 \\
\hline Neocystis brevis & Neocystis & LH10HG9080 & - \\
\hline Pseudostichococcus monallantoides & Prasiola & LH10HG3045 & - \\
\hline Pseudostichococcus sp. & Prasiola & LH08SW8044 & - \\
\hline Stichococcus sp.(I) & Prasiola & LH08AW8025 & KP081397 \\
\hline Stichococcus sp.(II) & Prasiola & LH08SG5057 & - \\
\hline Stichococcus sp.(III) & Prasiola & LH10HG6110, LH08AG7010 & - \\
\hline Stichococcus sp.(IV) & Prasiola & LH08SG1073 & - \\
\hline Stichococcus sp.(V) & Prasiola & LH08SW1099 & - \\
\hline Stichococcus sp.(VI) & Prasiola & LH08AW8023 (=SAG 2482) & KP081395 \\
\hline Stichococcus sp.(VII) & Prasiola & LH08AW8002 (=SAG 2481) & KP081394 \\
\hline Unidentified Chlorellaceae (I) & Chlorellaceae & LH08AG1034 & - \\
\hline Unidentified Chlorellaceae (II) & Chlorellaceae & LH10HG9020 & - \\
\hline Unidentified Chlorellaceae (III) & Chlorellaceae & LH08SG3093 & - \\
\hline Unidentified Chlorellaceae (IV) & Chlorellaceae & LH10HG709K & - \\
\hline Xylochloris sp. & incertae sedis & LH08AG7024 (= SAG 2382) & JQ988942 \\
\hline
\end{tabular}


Table S2b. List of all detected species belonging to Chlorophyceae, other green algae and Stramenopiles.

\begin{tabular}{|c|c|c|c|}
\hline Species & Clade & Representative isolates and SAG-strains & GenBank \\
\hline Acutodesmus rubescens & Sphaeropleales & LH08SG8041 & - \\
\hline Bracteacoccus cohaerens & Sphaeropleales & LH10HG9034 & - \\
\hline Bracteacoccus cohaerens (cf.) & Sphaeropleales & LH08SG2015 & - \\
\hline Chlamydomonas gerloffii (cf.) & Chlamydomonadales & LH08SW5031 & - \\
\hline Chlamydomonas rapa & Chlamydomonadales & LH08SG1077 & - \\
\hline Chlamydomonas rapa (cf.) & Chlamydomonadales & LH10HG1027 & - \\
\hline Chlamydomonas typica (cf.) & Chlamydomonadales & LH08SG9022 (partial 18S) & - \\
\hline Chlamydopodium vacuolatum & Chlamydomonadales & LH10HG1013 & - \\
\hline Chlorococcum minutum (cf.) & Chlamydomonadales & LH08AW5056 (=SAG 2479) & KP081402 \\
\hline Chlorococcum sphacosum & Chlamydomonadales & LH10HG3113 & - \\
\hline Coelastrella multistriata & Sphaeropleales & LH10HG7083 & - \\
\hline Coelastrella sp. & Sphaeropleales & LH10HG2098 (+LH10HG7018) & - \\
\hline Desmotetra stigmatica & Chlamydomonadales & LH08SG2049 & - \\
\hline Heterochlamydomonas sp. & Chlamydomonadales & LH08AG2004 & - \\
\hline Jenufa sp. & incertae sedis & LH08AW8035 (= SAG 2383) & JQ988933 \\
\hline Oogamochlamys sp.(I) & Chlamydomonadales & LH08SG8047 & - \\
\hline Oogamochlamys sp.(II) & Chlamydomonadales & LH08AW1069 (= SAG 2476; partial 18S) & KP081401 \\
\hline Pseudomuriella aurantiaca & Sphaeropleales & LH10HG2039 & - \\
\hline Stephanosphaerinia sp. & Chlamydomonadales & LH10HG6108 & - \\
\hline Tatrensinia sp.(I) & Chlamydomonadales & LH08SW7115 & - \\
\hline Tatrensinia sp.(II) & Chlamydomonadales & LH10HG7016 (+LH10HG9131 ) & - \\
\hline Pseudendocloniopsis botryoides (cf.) & Ulotrichales & LH08HW9058 & - \\
\hline Pedinomonas minor (cf.) & Pedinophyceae & LH08SG2033 (partial 18S) & - \\
\hline Klebsormidium dissectum/elegans (cf.) & Streptophyta & LH08HW9106 & - \\
\hline Klebsormidium flaccidum (cf.) & Streptophyta & LH10HG2056 & - \\
\hline Asterosiphon sp. & incertae sedis & LH10HG3064 & - \\
\hline Botrydiopsalean sp. & Botrydiopsalean & LH08AW1076 & - \\
\hline Botrydiopsis callosa (cf.) & Botrydiopsalean & LH08AW4043 & - \\
\hline Eustigmatos sp. & Eustigmatophyceae & LH10HG9133, LH10HG5036 & - \\
\hline Heterococcus caespitosus (cf.) & Chlorellidialean & LH08AG2020 & - \\
\hline Heterococcus chodatii (cf.) & Chlorellidialean & LH10HG9111 & - \\
\hline Heterococcus sp. & Chlorellidialean & LH10HG9085, LH10HG2140 & - \\
\hline Heterothrix sessile & Tribonematalean & LH10HG5079 & - \\
\hline Heterothrix sp. & Tribonematalean & LH10HG7061, LH08SG5052 & - \\
\hline Xanthonema bristolianum (cf.) & Tribonematalean & LH08HW9018 & - \\
\hline Xanthonema exile (cf.) & Tribonematalean & LH10HG7078 & - \\
\hline Xanthonema sp. & Tribonematalean & LH10HG9058, LH10HG7029 & - \\
\hline
\end{tabular}


Table S3. List of the closest GenBank-relatives of our detected species.

\begin{tabular}{|c|c|c|c|c|c|}
\hline $\begin{array}{l}\text { Representative isolate(s) } \\
\text { in } 18 \mathrm{~S} \text { tree }\end{array}$ & Closest GenBank relatives; authentic strain* & GenBank & Similarity \% & Habitat of origin & Land \\
\hline LH10HG6096 & Auxenochlorella protothecoides SAG 211-7a* & $X 56101$ & 100.00 & terrestrial/sap & $\mathrm{DE}$ \\
\hline SAG 2478) & Auxenochlorella protothecoides SAG 211-7a* & X56101 & 97.46 & terrestrial/sap & DE \\
\hline LH08AG9040 & Chlorella mirabilis SAG $38.88^{\star}$ & X74000 & 99.88 & terrestrial/soil & RU \\
\hline LH10HG6139 & Chlorella mirabilis SAG $38.88^{*}$ & X74000 & 99.82 & terrestrial/soil & RU \\
\hline LH08HG1081 & Chlorella vulgaris SAG 211-11 b* & FM205832 & 99.94 & freshwater & $\mathrm{NL}$ \\
\hline LH08SG3006 & Chlorella vulgaris SAG 211-11b* & FM205832 & 100.00 & freshwater & NL \\
\hline LH10HG2081 (PS) & Chlorella vulgaris SAG 211-11 b* & FM205832 & 99.75 & freshwater & $\mathrm{NL}$ \\
\hline LH10HG7072 (PS) & Chlorella vulgaris SAG 211-11 $\mathrm{b}^{*}$ & FM205832 & 99.82 & freshwater & $\mathrm{NL}$ \\
\hline LH10HG9105 & Chloroidium ellipsoideum SAG $3.95^{\star}$ & FM946012 & 99.88 & $\mathrm{n} / \mathrm{a}$ & $\mathrm{n} / \mathrm{a}$ \\
\hline LH10HG7062 & Chloroidium saccharophilum SAG 211-9a* & FM946000 & 100.00 & terrestrial/sap & $\mathrm{DE}$ \\
\hline SAG 2483 & Coccomyxa viridis CCALA 306 & AM167525 & 100.00 & terrestrial/lichen & $\mathrm{CZ}$ \\
\hline LH08SG9051 (PS) & Coccomyxa simplex SAG 216-8* & HQ317304 & 100.00 & freshwater & RO \\
\hline LH08AW1017 & Coccomyxa sp. KN-2011-T3 & HE586515 & 99.94 & terrestrial/lichen & ID \\
\hline LH08AW3050 & Dictyochloropsis splendida CAUP H8601 & GU017662 & 100.00 & terrestrial/soil/fumarole & $\mathrm{CZ}$ \\
\hline LH08HW8075 & Chlorella sphaerica SAG $11.88^{*}$ & AJ416105 & 99.94 & terrestrial/lichen & $\mathrm{NZ}$ \\
\hline LH08AG9089 & Chlorella sphaerica SAG $11.88^{*}$ & AJ416105 & 99.82 & terrestrial/lichen & $\mathrm{NZ}$ \\
\hline LH10HG3P15 (PS) & Myrmecia bisecta SAG $2043^{*}$ & Z47209 & 100.00 & terrestrial/soil & IT \\
\hline LH08AW3064 & Myrmecia irregularis CCAP 221/8 & HQ902935 & 100.00 & $\mathrm{n} / \mathrm{a}$ & $\mathrm{n} / \mathrm{a}$ \\
\hline LH08SG3009 & Muriella terrestris ASIB V38 & AB012845 & 99.94 & terrestrial/soil & IT \\
\hline LH08SG8030 & Nannochloris bacillaris & AB080300 & 99.94 & $\mathrm{n} / \mathrm{a}$ & $\mathrm{n} / \mathrm{a}$ \\
\hline SAG 2477) & Leptochlorella sp. clone QE17 & FJ790649 & 95.54 & terrestrial/epilithic & $\mathrm{CN}$ \\
\hline LH10HG9080 & Neocystis brevis CAUP D $802^{*}$ & JQ920360 & 100.00 & terrestrial/soil & $\mathrm{CH}$ \\
\hline LH10HG3045 & Stichococcus mirabilis CCAP $379 / 3$ & AJ311638 & 99.82 & $\mathrm{n} / \mathrm{a}$ & $\mathrm{n} / \mathrm{a}$ \\
\hline LH08SW8044 & Stichococcus mirabilis CCAP $379 / 3$ & AJ311638 & 100.00 & $\mathrm{n} / \mathrm{a}$ & $\mathrm{n} / \mathrm{a}$ \\
\hline LH08AW8025 & Trebouxiophyte sp. UR47/4 & AY762604 & 100.00 & terrestrial/facade & $\mathrm{DE}$ \\
\hline LH08SG5057 & Trebouxiophyte sp. UR55/3 & AY762606 & 100.00 & terrestrial/facade & $\mathrm{DE}$ \\
\hline LH10HG6110, LH08AG7010 & Stichococcus deasonii UTEX $1706^{*}$ & DQ275460 & 99.70 & terrestrial/soil & USA \\
\hline LH08SG1073 & Stichococcus sp.4 WB47 & KF144240 & 100.00 & freshwater/biofilm & $\mathrm{DE}$ \\
\hline LH08SW1099 & Stichococcus deasonii UTEX $1706^{*}$ & DQ275460 & 98.90 & terrestrial/soil & USA \\
\hline SAG 2482 & Stichococcus sp.2 D4-2A & KF144238 & 100.00 & freshwater/biofilm & $\mathrm{DE}$ \\
\hline SAG 2481 & Stichococcus K4-4 & AB055866 & 100.00 & $\mathrm{n} / \mathrm{a}$ & $\mathrm{n} / \mathrm{a}$ \\
\hline LH08AG1034 & Marvania sp. WB67 & KF144207 & 100.00 & freshwater/biofilm & $\mathrm{DE}$ \\
\hline LH10HG9020 & Nannochloris sp. JL-4-6 & AY195983 & 100.00 & freshwater & USA \\
\hline LH08SG3093 & Nannochloris sp. Ant-1 & EF440182 & 100.00 & terrestrial/permafrost & $A Q$ \\
\hline LH10HG709K & Nannochloris sp. AS 2-10 & AY195968 & 97.76 & freshwater & USA \\
\hline SAG 2382 & Xylochloris irregularis CAUP H7801* & EU105209 & 96.47 & terrestrial/epixylic & SG \\
\hline LH08SG8041 & Scenedesmus rubescens CCAP 232/1 & $\mathrm{X} 74002$ & 100.00 & $\mathrm{n} / \mathrm{a}$ & $\mathrm{n} / \mathrm{a}$ \\
\hline LH10HG9034 & Bracteacoccus cohaerens UTEX $1272^{*}$ & GQ985406 & 100.00 & terrestrial/soil & USA \\
\hline LH08SG2015 & Bracteacoccus cohaerens UTEX $1272^{*}$ & GQ985406 & 99.94 & terrestrial/soil & USA \\
\hline LH08SW5031 & Chlamydomonas gerloffii CCAP $11 / 72^{*}$ & FR865610 & 99.82 & freshwater & $\mathrm{CZ}$ \\
\hline LH08SG1077 & Chlamydomonas rapa SAG $48.72^{*}$ & U70790 & 100.00 & freshwater/plankton & SK \\
\hline LH10HG1027 & Chlamydomonas rapa SAG $48.72^{*}$ & U70790 & 99.94 & freshwater/plankton & SK \\
\hline LH08SG9022 (PS) & Chlamydomonas typica SAG $61.72^{*}$ & AB701557 & 99.92 & terrestrial/soil & USA \\
\hline LH10HG1013 & Chlorococcum robustum $\mathrm{Kr} 8630$ & AY122332 & 100.00 & n/a & $\mathrm{n} / \mathrm{a}$ \\
\hline
\end{tabular}


Table S3. (continuation)

\begin{tabular}{|c|c|c|c|c|c|}
\hline $\begin{array}{l}\text { Representative isolate(s) } \\
\text { in } 18 \mathrm{~S} \text { tree }\end{array}$ & Closest GenBank relatives; authentic strain* & GenBank & Similarity \% & Habitat of origin & Land \\
\hline SAG 2479 & Chlorococcum minutum SAG 21.95 & JN968585 & 99.77 & terrestril/soil & $\mathrm{IT}$ \\
\hline LH10HG3113 & Chlorococcum sphacosum SAG $66.80^{*}$ & JN968580 & 100.00 & terrestrial/soil & USA \\
\hline LH10HG7083 & Coelastrella multistriata Hanagata C6-2 & AB012846 & 100.00 & terrestrial/epixylic & JP? \\
\hline $\begin{array}{l}\text { LH10HG2098 } \\
(+\mathrm{LH} 10 \mathrm{HG} 7018)\end{array}$ & Scenedesmus sp. KGU Y002 & AB742453 & 100.00 & $\mathrm{n} / \mathrm{a}$ & $\mathrm{n} / \mathrm{a}$ \\
\hline LH08SG2049 & Desmotetra stigmatica UTEX $962^{*}$ & DQ009760 & 100.00 & $\mathrm{n} / \mathrm{a}$ & $\mathrm{n} / \mathrm{a}$ \\
\hline LH08AG2004 & Heterochlamydomonas rugosa SAG 45.86 & AF367859 & 99.76 & freshwater & UK \\
\hline SAG 2383) & Jenufa minuta CAUP H $8102^{*}$ & HM563744 & 98.21 & terrestrial/epixylic & SG \\
\hline LH08SG8047 & Oogamochlamys gigantea SAG $21.72^{*}$ & AJ410468 & 98.41 & terrestrial/soil & USA \\
\hline SAG 2476 (PS) & Chlamydomonas sp. CCAP 11/159 & FR865553 & 97.69 & freshwater & USA \\
\hline LH10HG2039 & Pseudomuriella aurantiaca SAG 249-1* & X91268 & 100.00 & terrestrial/soil & $\mathrm{CH}$ \\
\hline LH10HG6108 & Chlorococcales sp. VII3 & FJ946904 & 99.59 & freshwater & $A Q$ \\
\hline LH08SW7115 & $\begin{array}{l}\text { Uncultured Haematococcaceae clone } \\
\text { Amb } 18 \mathrm{~S} 582\end{array}$ & EF023273 & 99.77 & terrestrial/soil & NL \\
\hline $\begin{array}{l}\text { LH10HG7016 } \\
(+\mathrm{LH} 10 \mathrm{HG} 9131)\end{array}$ & Chlorococcum cf. tatrense CCCryo 101-99 & AF514407 & 99.71 & $\mathrm{n} / \mathrm{a}$ & $\mathrm{n} / \mathrm{a}$ \\
\hline LH08HW9058 & Pseudendocloniopsis botryoides SAG 465-1* & AJ416103 & 99.88 & freshwater & $\mathrm{CH}$ \\
\hline LH08SG2033 (PS) & Pedinomonas minor SAG 1965-3 & HE610132 & 99.80 & freshwater/plankton & SK \\
\hline LH08HW9106 & Klebsormidium dissectum SAG 2155* & EF372518 & 100.00 & terrestrial/soil & FR \\
\hline LH10HG2056 & Klebsormidium flaccidum SAG 7.91 & EU434019 & 100.00 & freshwater & RU \\
\hline LH10HG3064 & Asterosiphon dichotomus UTEX LB 2066 & AM490829 & 98.54 & $\mathrm{n} / \mathrm{a}$ & $\mathrm{n} / \mathrm{a}$ \\
\hline LH08AW1076 & Chlorellidium pyrenoidosum PAB 785 & AJ579338 & 99.31 & soil(?) & $A Q$ \\
\hline LH08AW4043 & Botrydiopsis callosa SAG 30.83 & AJ579340 & 99.29 & soil & IT \\
\hline LH10HG9133, LH10HG5036 & Eustigmatos magna CCMP 387 & U41051 & 99.82 & terrestrial/soil & NZ \\
\hline LH08AG2020 & Heterococcus protonematoides SAG 835-9 & AJ579334 & 99.94 & terrestrial/soil & $\mathrm{CH}$ \\
\hline LH10HG9111 & Heterococcus chodatii SAG 835- $3^{*}$ & AM490822 & 100.00 & terrestrial/subaerial & $\mathrm{CH}$ \\
\hline LH10HG9085, LH10HG2140 & Xanthophyceae sp. IX3 & FJ946906 & 99.59 & freshwater & $A Q$ \\
\hline LH10HG5079 & Heterothrix sessile IBSG-V28 & AM490818 & 100.00 & $\mathrm{n} / \mathrm{a}$ & $\mathrm{n} / \mathrm{a}$ \\
\hline LH10HG7061, LH08SG5052 & Heterothrix sp.1 ACOL A1 & AM491612 & $99.26-99.31$ & $\mathrm{n} / \mathrm{a}$ & $\mathrm{n} / \mathrm{a}$ \\
\hline LH08HW9018 & Xanthonema bristolianum CCALA 516 & AM490819 & 100.00 & terrestrial/snow & SK \\
\hline LH10HG7078 & Xanthonema exile PAB 395 & AM491615 & 99.94 & $\mathrm{n} / \mathrm{a}$ & $\mathrm{n} / \mathrm{a}$ \\
\hline LH10HG9058, LH10HG7029 & Xanthonema exile PAB 395 & AM491615 & $99.89+99.77$ & $\mathrm{n} / \mathrm{a}$ & $\mathrm{n} / \mathrm{a}$ \\
\hline
\end{tabular}

Legend. $\mathrm{AQ}=$ Antarctica, $\mathrm{CH}=$ Switzerland, $\mathrm{CN}=\mathrm{China}, \mathrm{CZ}=\mathrm{Czech}$ Republic, $\mathrm{DE}=$ Germany, $\mathrm{FR}=\mathrm{France}, \mathrm{ID}=$ Indonesia, IT=Italy, $\mathrm{JP}=\mathrm{Japan}, \mathrm{NL}=$ Netherlands, NZ=New Zealand, RO=Romania, RU=Russia, SG=Singapore, SK=Slovakia, UK=United Kingdom, USA=United States of America. 
Table S4a. List of all analyzed full and partial (=PS) 18S rDNA sequences.

\begin{tabular}{|c|c|c|c|}
\hline Species & $\begin{array}{l}\text { Representative isolates } \\
\text { (in phylogenetic trees) }\end{array}$ & $\begin{array}{l}\text { Similar full 18S } \\
\text { (OTU/0.00-level) }\end{array}$ & $\begin{array}{l}\text { Similar partial } 18 \mathrm{~S} \\
\text { (OTU/0.00-level ) }\end{array}$ \\
\hline Auxenochlorella protothecoides & LH10HG6096 & LH10HG7124, LH10HG5119 & \\
\hline Auxenochlorella sp. & LH08AW4103 & & \\
\hline Chlorella mirabilis (cf.) & LH08AG9040 & & \\
\hline Chlorella mirabilis (cf.) & LH10HG6139 & & \\
\hline Chlorella vulgaris & LH08HG1081 & & \\
\hline Chlorella vulgaris & LH08SG3006 & $\begin{array}{l}\text { LH10HG6014, LH08HG2065, } \\
\text { LH08HG2013, LH08HG5074, } \\
\text { LH08HW9060, LH10HG2049, } \\
\text { LH08HG4088, LH10HG6052, } \\
\text { LH10HG9075, LH08HW9094, } \\
\text { LH08SG1071, LH08HG5082, } \\
\text { LH08HG2083, LH08HG2091, } \\
\text { LH08HG2096, LH08HG4032, } \\
\text { LH10HG4093, LH10HG6099, } \\
\text { LH10HG3088 }\end{array}$ & LH08HW6087 \\
\hline Chlorella vulgaris (cf.) & LH10HG7072 (PS) & & \\
\hline Chlorella vulgaris (cf.) & LH10HG2081 (PS) & & \\
\hline Chloroidium ellipsoideum (cf.) & LH10HG9105 & $\begin{array}{l}\text { LH10HG6086, LH10HG7132, } \\
\text { LH08AG8046, LH08AG9062 }\end{array}$ & \\
\hline Chloroidium saccharophilum & LH10HG7062 & LH08AW8042 & LH10HG5089 \\
\hline Coccomyxa viridis & SAG 2483 & & \\
\hline Coccomyxa simplex & LH08SG9051 (PS) & & \\
\hline Coccomyxa sp. & LH08AW1017 & & \\
\hline Dictyochloropsis splendida & LH08AW3050 & & \\
\hline Diplosphaera sp.(I) & LH08HW8075 & & \\
\hline Diplosphaera sp.(II) & LH08AG9089 & & \\
\hline Lobosphaera bisecta (cf.) & LH10HG3P15 (PS) & & \\
\hline Lobosphaera irregularis (cf.) & LH08AW3064 & & LH08SW5063 \\
\hline Muriella terrestris (cf.) & LH08SG3009 & $\begin{array}{l}\text { LH10HG1070, LH10HG8050, } \\
\text { LH10HG1076, LH10HG7118, } \\
\text { LH10HG9077 }\end{array}$ & \\
\hline Nannochloris sp. & LH08SG8030 & LH10HG6095 & LH08SG3102 \\
\hline Navichloris fusiformis (proposed taxon) & SAG 2477 & & \\
\hline Neocystis brevis & LH10HG9080 & $\begin{array}{l}\text { LH08SG2072, LH08AG9012, } \\
\text { LH08AW8001, LH08HW6108, } \\
\text { LH08HW6059, LH10HG4082, } \\
\text { LH10HG9054 }\end{array}$ & LH10HG9P02 \\
\hline Pseudostichococcus monallantoides & LH10HG3045 & LH10HG2066 & LH08AG7097 \\
\hline Pseudostichococcus sp. & LH08SW8044 & & \\
\hline Stichococcus sp.(I) & LH08AW8025 & & LH08AG9028 \\
\hline Stichococcus sp.(II) & LH08SG5057 & $\begin{array}{l}\text { LH10HG7092, LH08SG5067, } \\
\text { LH08SG5079, LH08SG5090, } \\
\text { LH10HG2067, LH10HG2063, } \\
\text { LH10HG9068, LH10HG3128, } \\
\text { LH10HG7090 }\end{array}$ & LH08AG1100, LH08SG5092 \\
\hline Stichococcus sp.(III) & LH10HG6110, LH08AG7010 & & \\
\hline Stichococcus sp.(IV) & LH08SG1073 & & \\
\hline Stichococcus sp.(V) & LH08SW1099 & & \\
\hline Stichococcus sp.(VI) & SAG 2482 & LH08AW8104 & \\
\hline Stichococcus sp.(VII) & SAG 2481 & & \\
\hline Unidentified Chlorellaceae (I) & LH08AG1034 & & LH10HG7073 \\
\hline Unidentified Chlorellaceae (II) & LH10HG9020 & $\begin{array}{l}\text { LH08SG2053, LH10HG7071, } \\
\text { LH10HG9026, LH10HG2094, } \\
\text { LH10HG3135 }\end{array}$ & LH08SG3029 \\
\hline Unidentified Chlorellaceae (III) & LH08SG3093 & LH08SG3078 & \\
\hline Unidentified Chlorellaceae (IV) & LH10HG709K & & \\
\hline Xylochloris sp. & SAG 2382 & & \\
\hline Acutodesmus rubescens & LH08SG8041 & & \\
\hline Bracteacoccus cohaerens & LH10HG9034 & & \\
\hline Bracteacoccus cohaerens (cf.) & LH08SG2015 & & \\
\hline Chlamydomonas gerloffii (cf.) & LH08SW5031 & & \\
\hline Chlamydomonas rapa & LH08SG1077 & LH08SG9055 & \\
\hline
\end{tabular}


Table S4a. (continuation)

\begin{tabular}{|c|c|c|c|}
\hline Species & $\begin{array}{l}\text { Representative isolates } \\
\text { (in phylogenetic trees) }\end{array}$ & $\begin{array}{l}\text { Similar full 18S } \\
\text { (OTU/0.00-level) }\end{array}$ & $\begin{array}{l}\text { Similar partial } 18 \mathrm{~S} \\
\text { (OTU/0.00-level ) }\end{array}$ \\
\hline Chlamydomonas rapa (cf.) & LH10HG1027 & & \\
\hline Chlamydomonas typica (cf.) & LH08SG9022 (PS) & & \\
\hline Chlamydopodium vacuolatum & LH10HG1013 & & \\
\hline Chlorococcum minutum (cf.) & SAG 2479 & LH08AW5107 & LH08AG701K, LH08AW5111 \\
\hline Chlorococcum sphacosum & LH10HG3113 & & \\
\hline Coelastrella multistriata & LH10HG7083 & & $\begin{array}{l}\text { LH10HG7102, LH10HG7100, } \\
\text { LH10HG8109, LH10HG7097, } \\
\text { LH08AG2003, LH08AW4118 }\end{array}$ \\
\hline Coelastrella sp. & LH10HG2098 & $\begin{array}{l}\text { LH10HG1009, LH10HG1033, } \\
\text { LH10HG6060, LH10HG7017, } \\
\text { LH10HG7023 }\end{array}$ & $\begin{array}{l}\text { LH10HG2087, LH10HG7018, } \\
\text { LH10HG6035, LH10HG7030, } \\
\text { LH10HG9130, LH10HG2P12, } \\
\text { LH10HG2P01, LH10HG5136 }\end{array}$ \\
\hline Desmotetra stigmatica & LH08SG2049 & & LH10HG6P18 \\
\hline Heterochlamydomonas sp. & LH08AG2004 & & \\
\hline Jenufa sp. & SAG 2383 & & LH08AW8098 \\
\hline Oogamochlamys sp.(I) & LH08SG8047 & & \\
\hline Oogamochlamyssp.(II) & SAG 2476 & & \\
\hline Pseudomuriella aurantiaca & LH10HG2039 & LH10HG9038 & \\
\hline Stephanosphaerinia sp. & LH10HG6108 & & \\
\hline Tatrensinia sp.(I) & LH08SW7115 & & \\
\hline Tatrensinia sp.(II) & LH10HG7016 & & LH10HG9131 \\
\hline Pseudendocloniopsis botryoides (cf.) & LH08HW9058 & & \\
\hline Pedinomonas minor (cf.) & LH08SG2033 (PS) & & \\
\hline Klebsormidium dissectum/elegans (cf.) & LH08HW9106 & $\begin{array}{l}\text { LH08AG9045, LH08HW9005, } \\
\text { LH08AG1113, LH08HW1038, } \\
\text { LH08AG7011, LH08HW4109, } \\
\text { LH08HW5021 }\end{array}$ & \\
\hline Klebsormidium flaccidum (cf.) & LH10HG2056 & $\begin{array}{l}\text { LH08HW5061, LH10HG7028, } \\
\text { LH08SG3027 }\end{array}$ & \\
\hline Asterosiphon sp. & LH10HG3064 & & \\
\hline Botrydiopsalean sp. & LH08AW1076 & & LH08SG2K53 \\
\hline Botrydiopsis callosa (cf.) & LH08AW4043 & & \\
\hline Heterococcus caespitosus (cf.) & LH08AG2020 & & \\
\hline Heterococcus chodatii (cf.) & LH10HG9111 & & LH10HG9126 \\
\hline Heterococcus sp. & LH10HG9085, LH10HG2140 & & \\
\hline Heterothrix sessile & LH10HG5079 & LH10HG9037 & \\
\hline Heterothrix sp. & LH10HG7061, LH08SG5052 & & \\
\hline Xanthonema bristolianum (cf.) & LH08HW9018 & LH10HG6059 & \\
\hline Xanthonema exile (cf.) & LH10HG7078 & & \\
\hline Xanthonema sp. & LH10HG9058, LH10HG7029 & $\begin{array}{l}\text { LH10HG3065, LH10HG9031, } \\
\text { LH10HG1K69 }\end{array}$ & LH10HG8112, LH10HW9129 \\
\hline Eustigmatos sp. & LH10HG9133, LH10HG5036 & & \\
\hline
\end{tabular}


Table S4b. Distribution of the green algal species across the sampling sites.

\begin{tabular}{|c|c|c|c|c|c|c|c|c|}
\hline Species & $\begin{array}{l}\text { Representative } \\
\text { isolates } \\
\text { (in phylogenetic } \\
\text { trees) }\end{array}$ & $\begin{array}{l}\text { Drill core } \\
(2008) \\
\mathrm{SCH}\end{array}$ & $\begin{array}{l}\text { Drill core } \\
(2008) \\
\text { ALB }\end{array}$ & $\begin{array}{l}\text { Drill core } \\
(2008) \\
\text { HAI }\end{array}$ & $\begin{array}{l}\text { Topsoil } \\
(2010) \\
\text { HAI }\end{array}$ & $\mathrm{T}$ & $\bar{M}$ & $\bar{E}$ \\
\hline Auxenochlorella protothecoides & LH10HG6096 & & & & HEG5/6/7 & & 1 & 1 \\
\hline Auxenochlorella sp. & LH08AW4103 & & AEW4 & & & & 1 & \\
\hline Chlorella mirabilis (cf.) & LH08AG9040 & & AEG9 & & & & & 1 \\
\hline Chlorella mirabilis (cf.) & LH10HG6139 & & & & HEG6 & & 1 & \\
\hline Chlorella vulgaris & LH08HG1081 & & & HEG1 & & 1 & & \\
\hline Chlorella vulgaris & LH08SG3006 & SEG $1 / 3$ & & $\begin{array}{l}\text { HEG } 2 / 4 / 5 \text {, } \\
\text { HEW6/9 }\end{array}$ & HEG2/3/4/6/9 & 1 & 1 & 1 \\
\hline Chlorella vulgaris (cf.) & LH10HG7072 (PS) & & & & HEG7 & & & 1 \\
\hline Chlorella vulgaris (cf.) & LH10HG2081 (PS) & & & & HEG2 & 1 & & \\
\hline Chloroidium ellipsoideum (cf.) & LH10HG9105 & & AEG8/9 & & HEG $6 / 7 / 9$ & & & 1 \\
\hline Chloroidium saccharophilum & LH10HG7062 & & AEW8 & & HEG5/7 & & 1 & 1 \\
\hline Coccomyxa viridis & SAG 2483 & & AEW8 & & & & & 1 \\
\hline Coccomyxa simplex & LH08SG9051 (PS) & SEG9 & & & & & & 1 \\
\hline Coccomyxa sp. & LH08AW1017 & & AEW1 & & & 1 & & \\
\hline Dictyochloropsis splendida & LH08AW3050 & & AEW3 & & & 1 & & \\
\hline Diplosphaera sp.(I) & LH08HW8075 & & & HEW8 & & & & 1 \\
\hline Diplosphaera sp.(II) & LH08AG9089 & & AEG9 & & & & & 1 \\
\hline Lobosphaera bisecta (cf.) & LH10HG3P15 (PS) & & & & HEG3 & 1 & & \\
\hline Lobosphaera irregularis (cf.) & LH08AW3064 & SEW5 & AEW3 & & & 1 & 1 & \\
\hline Muriella terrestris (cf.) & LH08SG3009 & SEG3 & & & HEG1/7/8/9 & 1 & & 1 \\
\hline Nannochloris sp. & LH08SG8030 & SEG3/8 & & & HEG6 & 1 & 1 & 1 \\
\hline Navichloris fusiformis (proposed taxon) & SAG 2477 & & AEW3 & & & 1 & & \\
\hline Neocystis brevis & LH10HG9080 & SEG2 & AEG9, AEW8 & HEW6 & HEG $4 / 9$ & 1 & 1 & 1 \\
\hline Pseudostichococcus monallantoides & LH10HG3045 & & AEG7 & & $\mathrm{HEG} 2 / 3$ & 1 & & 1 \\
\hline Pseudostichococcus sp. & LH08SW8044 & SEW8 & & & & & & 1 \\
\hline Stichococcus sp.(I) & LH08AW8025 & & AEG9/AEW8 & & & & & 1 \\
\hline Stichococcus sp.(II) & LH08SG5057 & SEG5 & AEG1 & & HEG2/3/7/9 & 1 & 1 & 1 \\
\hline Stichococcus sp.(III) & $\begin{array}{l}\text { LH10HG6110, } \\
\text { LH08AG7010 }\end{array}$ & & AEG7 & & HEG6 & & 1 & 1 \\
\hline Stichococcus sp.(IV) & LH08SG1073 & SEG1 & & & & 1 & & \\
\hline Stichococcus sp.(V) & LH08SW1099 & SEW1 & & & & 1 & & \\
\hline Stichococcus sp.(VI) & SAG 2482 & & AEW8 & & & & & 1 \\
\hline Stichococcus sp.(VII) & SAG 2481 & & AEW8 & & & & & 1 \\
\hline Unidentified Chlorellaceae (I) & LH08AG1034 & & AEG1 & & HEG7 & 1 & & 1 \\
\hline Unidentified Chlorellaceae (II) & LH10HG9020 & SEG $2 / 3$ & & & HEG2/3/7/9 & 1 & & 1 \\
\hline Unidentified Chlorellaceae (III) & LH08SG3093 & SEG3 & & & & 1 & & \\
\hline Unidentified Chlorellaceae (IV) & LH10HG709K & & & & HEG7 & & & 1 \\
\hline Xylochloris sp. & SAG 2382 & & AEG7 & & & & & 1 \\
\hline Acutodesmus rubescens & LH08SG8041 & SEG8 & & & & & & 1 \\
\hline Bracteacoccus cohaerens & LH10HG9034 & & & & HEG9 & & & 1 \\
\hline Bracteacoccus cohaerens (cf.) & LH08SG2015 & SEG2 & & & & 1 & & \\
\hline Chlamydomonas gerloffii (cf.) & LH08SW5031 & SEW5 & & & & & 1 & \\
\hline Chlamydomonas rapa & LH08SG1077 & SEG $1 / 9$ & & & & 1 & & 1 \\
\hline Chlamydomonas rapa (cf.) & LH10HG1027 & & & & HEG1 & 1 & & \\
\hline Chlamydomonas typica (cf.) & LH08SG9022 (PS) & SEG9 & & & & & & 1 \\
\hline Chlamydopodium vacuolatum & LH10HG1013 & & & & HEG1 & 1 & & \\
\hline Chlorococcum minutum (cf.) & SAG 2479 & & AEG7/AEW5 & & & & 1 & 1 \\
\hline Chlorococcum sphacosum & LH10HG3113 & & & & HEG3 & 1 & & \\
\hline Coelastrella multistriata & LH10HG7083 & & AEG2/AEW4 & & $\mathrm{HEG} 7 / 8$ & 1 & 1 & 1 \\
\hline Coelastrella sp. & LH1OHG2098 & & & & HEG1/2/5/6/7/9 & 1 & 1 & 1 \\
\hline Desmotetra stigmatica & LH08SG2049 & SEG2 & & & HEG6 & 1 & 1 & \\
\hline Heterochlamydomonas sp. & LH08AG2004 & & AEG2 & & & 1 & & \\
\hline Jenufa sp. & SAG 2383 & & AEW8 & & & & & 1 \\
\hline Oogamochlamys sp.(I) & LH08SG8047 & SEG8 & & & & & & 1 \\
\hline Oogamochlamys sp.(II) & SAG 2476 & & AEW1 & & & 1 & & \\
\hline
\end{tabular}


Table S4b. (continuation)

\begin{tabular}{|c|c|c|c|c|c|c|c|c|}
\hline Species & $\begin{array}{l}\text { Representative } \\
\text { isolates } \\
\text { (in phylogenetic } \\
\text { trees) }\end{array}$ & $\begin{array}{l}\text { Drill core } \\
(2008) \\
\text { SCH }\end{array}$ & $\begin{array}{l}\text { Drill core } \\
(2008) \\
\text { ALB }\end{array}$ & $\begin{array}{l}\text { Drill core } \\
(2008) \\
\mathrm{HAl}\end{array}$ & $\begin{array}{l}\text { Topsoil } \\
(2010) \\
\mathrm{HAl}\end{array}$ & I & M & $E$ \\
\hline Pseudomuriella aurantiaca & LH10HG2039 & & & & HEG2/9 & 1 & 0 & 1 \\
\hline Stephanosphaerinia sp. & LH10HG6108 & & & & HEG6 & & 1 & \\
\hline Tatrensinia sp.(I) & LH08SW7115 & SEW7 & & & & & & 1 \\
\hline Tatrensinia sp.(II) & LH10HG7016 & & & & HEG7/9 & & & 1 \\
\hline Pseudendocloniopsis botryoides (cf.) & LH08HW9058 & & & HEW9 & & & & 1 \\
\hline Pedinomonas minor (cf.) & LH08SG2033 (PS) & SEG2 & & & & 1 & & \\
\hline Klebsormidium dissectum/elegans (cf.) & LH08HW9106 & & AEG1/7/9 & HEW1/4/5/9 & & 1 & 1 & 1 \\
\hline Klebsormidium flaccidum (cf.) & LH10HG2056 & SEG3 & & HEW5 & HEG2/7 & 1 & 1 & 1 \\
\hline Asterosiphon sp. & LH10HG3064 & & & & HEG3 & 1 & & \\
\hline Botrydiopsalean sp. & LH08AW1076 & SEG2 & AEW1 & & & 1 & & \\
\hline Botrydiopsis callosa (cf.) & LH08AW4043 & & AEW4 & & & & 1 & \\
\hline Heterococcus caespitosus (cf.) & LH08AG2020 & & AEG2 & & & 1 & & \\
\hline Heterococcus chodatii (cf.) & LH10HG9111 & & & & HEG9 & & & 1 \\
\hline Heterococcus sp. & $\begin{array}{l}\text { LH10HG9085, } \\
\text { LH10HG2140 }\end{array}$ & & & & HEG2/9 & 1 & & 1 \\
\hline Heterothrix sessile & LH10HG5079 & & & & HEG5/9 & & 1 & 1 \\
\hline Heterothrix sp. & $\begin{array}{l}\text { LH10HG7061, } \\
\text { LH08SG5052 }\end{array}$ & SEG5 & & & HEG7 & & & 1 \\
\hline Xanthonema bristolianum (cf.) & LH08HW9018 & & & HEW9 & HEG6 & & 1 & \\
\hline Xanthonema exile (cf.) & LH10HG7078 & & & & HEG7 & & & 1 \\
\hline Xanthonema sp. & $\begin{array}{l}\text { LH10HG9058, } \\
\text { LH10HG7029 }\end{array}$ & & & & $\begin{array}{l}\text { HEG1/3/7/8/9, } \\
\text { HEW9 }\end{array}$ & 1 & & 1 \\
\hline Eustigmatos sp. & $\begin{array}{l}\text { LH10HG9133, } \\
\text { LH10HG5036 }\end{array}$ & & & & HEG5/9 & & 1 & 1 \\
\hline
\end{tabular}

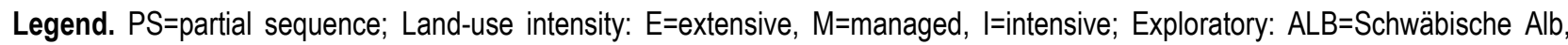
$\mathrm{HAl}=$ Hainich-Dün, $\mathrm{SCH}=\mathrm{Schorfheide-Chorin.}$ 
Table S5. General morphological characteristics of the new isolates.

\begin{tabular}{|c|c|c|c|c|}
\hline Representative isolates & $\begin{array}{l}\text { Morphotype } \\
\text { assignation }\end{array}$ & Cell shape & Cell size & Figure \\
\hline LH10HG6096 & Chlorella & spherical & $\varnothing=2.9-6.5 \mu \mathrm{m}$ & Figure $7 \mathrm{k}$ \\
\hline SAG 2478 & Chlorella & spherical & $\varnothing=2.2-5.2 \mu \mathrm{m}$ & Figure 71 \\
\hline LH08AG9040 & Chlorella-like & spherical & $\varnothing=3.0-6.4 \mu \mathrm{m}$ & Figure $7 a$ \\
\hline LH10HG6139 & Chlorella-like & spherical & $\mathrm{n} / \mathrm{a}$ & n/a \\
\hline LH08HG1081 & Chlorella & spherical/ellipsoidal & $\varnothing=2.3-5.2 \mu \mathrm{m}$ & Figure $7 \mathrm{~b}$ \\
\hline LH08SG3006 & Chlorella & spherical/ellipsoidal & $\mathrm{n} / \mathrm{a}$ & $\mathrm{n} / \mathrm{a}$ \\
\hline LH10HG2081 (PS) & Chlorella & spherical/ellipsoidal & $\varnothing=2.5-6.2 \mu \mathrm{m}$ & Figure $7 c$ \\
\hline LH10HG7072 (PS) & Chlorella & spherical/ellipsoidal & $\mathrm{n} / \mathrm{a}$ & $\mathrm{n} / \mathrm{a}$ \\
\hline LH10HG9105 & Chloroidium & ellitpical & $\mathrm{I}=4.8-7.8 \mu \mathrm{m}, \mathrm{w}=3.3-5.3 \mu \mathrm{m}$ & Figure $6 \mathrm{i}$ \\
\hline LH10HG7062 & Chloroidium & ellitpical & $\mathrm{I}=5.6-9.1 \mu \mathrm{m}, \mathrm{w}=4.0-6.2 \mu \mathrm{m}$ & Figure $6 \mathrm{~h}$ \\
\hline SAG 2483 & Coccomyxa & fusiform (asymmetrical) & $\mathrm{I}=4.7-8.4 \mu \mathrm{m}, \mathrm{w}=1.8-3.6 \mu \mathrm{m}$ & Figure $6 f$ \\
\hline LH08SG9051 (PS) & Coccomyxa & fusiform (asymmetrical) & $\mathrm{I}=4.4-7.8 \mu \mathrm{m}, \mathrm{w}=2.0-4.3 \mu \mathrm{m}$ & Figure $6 \mathrm{e}$ \\
\hline LH08AW1017 & Coccomyxa & fusiform (asymmetrical) & $\mathrm{n} / \mathrm{a}$ & $\mathrm{n} / \mathrm{a}$ \\
\hline LH08AW3050 & Dictyochloropsis & spherical & $\varnothing=7.7-39.4 \mu \mathrm{m}$ & Figure 61 \\
\hline LH08HW8075 & Diplosphaera & broadly oval/cylindric & $\mathrm{I}=3.2-6.2 \mu \mathrm{m}, \mathrm{w}=1.9-4.4 \mu \mathrm{m}$ & Figure 8b (Chapter 3) \\
\hline LH08AG9089 & Diplosphaera & broadly oval/cylindric & $\mathrm{n} / \mathrm{a}$ & $\mathrm{n} / \mathrm{a}$ \\
\hline LH10HG3P15 (PS) & Lobosphaera & spherical & $\varnothing=6.2-9.6 \mu \mathrm{m}$ & Figure $6 \mathrm{j}$ \\
\hline LH08AW3064 & Lobosphaera & spherical & $\mathrm{n} / \mathrm{a}$ & $\mathrm{n} / \mathrm{a}$ \\
\hline LH08SG3009 & Chlorella & spherical & $\varnothing=1.7-4.2 \mu \mathrm{m}$ & Figure 3b (Chapter 3) \\
\hline LH08SG8030 & Chlorella & spherical & $\varnothing=2.2-3.9 \mu \mathrm{m}$ & Figure 7e (Chapter 3) \\
\hline SAG 2477 & Coccomyxa-like & fusiform (symmetrical) & $\mathrm{I}=8.3-15.3 \mu \mathrm{m}, \mathrm{w}=3.6-7.8 \mu \mathrm{m}$ & Figure $6 \mathrm{~g}$ \\
\hline LH10HG9080 & Neocystis & broadly oval & $\mathrm{I}=4.8-8.7 \mu \mathrm{m}, \mathrm{w}=2.7-6.6 \mu \mathrm{m}$ & Figure $6 \mathrm{~d}$ \\
\hline LH10HG3045 & Stichococcus & cylindrical & $\mathrm{n} / \mathrm{a}$ & $\mathrm{n} / \mathrm{a}$ \\
\hline LH08SW8044 & Stichococcus & cylindrical & $\mathrm{I}=3.4-9.8 \mu \mathrm{m} ; \mathrm{w}=1.6-3.3 \mu \mathrm{m}$ & Figure 8d (Chapter 3) \\
\hline LH08AW8025 & Stichococcus & cylindrical & $\mathrm{I}=1.7-19.8 \mu \mathrm{m}, \mathrm{w}=1.4-3.6 \mu \mathrm{m}$ & Figure $6 a$ \\
\hline LH08SG5057 & Stichococcus & cylindrical & $\mathrm{I}=4.5-9.8 \mu \mathrm{m}, \mathrm{w}=1.9-3.3 \mu \mathrm{m}$ & Figure $6 \mathrm{~b}$ \\
\hline LH10HG6110, LH08AG7010 & Stichococcus & cylindrical & $\mathrm{n} / \mathrm{a}$ & $\mathrm{n} / \mathrm{a}$ \\
\hline LH08SG1073 & Stichococcus & cylindrical & $\mathrm{n} / \mathrm{a}$ & $\mathrm{n} / \mathrm{a}$ \\
\hline LH08SW1099 & $\begin{array}{l}\text { Stichococcus/ } \\
\text { Diplosphaera }\end{array}$ & cylindrical/packages & $\mathrm{I}=3.0-6.3 \mu \mathrm{m} ; \mathrm{w}=1.0-4.2 \mu \mathrm{m}$ & Figure 8j (Chapter 3) \\
\hline SAG 2482 & Stichococcus & cylindrical & $\mathrm{I}=3.8-7.2 \mu \mathrm{m}, \mathrm{w}=1.9-6.3$ & Figure $6 c$ \\
\hline SAG 2481 & Stichococcus & cylindrical & $\mathrm{I}=3.1-8.7 \mu \mathrm{m} ; \mathrm{w}=1.6-2.9 \mu \mathrm{m}$ & Figure 8e (Chapter 3) \\
\hline LH08AG1034 & Chlorella & spherical & $\varnothing=2.4-4.5 \mu \mathrm{m}$ & Figure $7 \mathrm{~g}$ \\
\hline LH10HG9020 & Chlorella & spherical & $\varnothing=2.4-4.8 \mu \mathrm{m}$ & Figure $7 \mathrm{~h}$ \\
\hline LH08SG3093 & Chlorella & spherical & $\varnothing=2.3-3.8 \mu \mathrm{m}$ & Figure 3d (Chapter 3) \\
\hline LH10HG709K & Chlorella & spherical/ellipsoidal & $\mathrm{I}=3.1-5.1 \mu \mathrm{m}, \mathrm{w}=2.0-3.7 \mu \mathrm{m}$ & Figure $7 \mathrm{j}$ \\
\hline SAG 2382 & Trebouxia-like & spherical & $\mathrm{I}=4.7-12.9 \mu \mathrm{m}, \mathrm{w}=3.1-10.6 \mu \mathrm{m}$ & Figure $6 \mathrm{k}$ \\
\hline LH08SG8041 & Scenedesmus-like & $\begin{array}{l}\text { ellipsoidal, obtuse cell } \\
\text { poles }\end{array}$ & $\varnothing=3.9-7.4 \mu \mathrm{m}$ & Figure $3 g$ \\
\hline LH10HG9034 & Bracteacoccus-like & spherical & $\varnothing=5.4-12.3 \mu \mathrm{m}$ & Figure $3 i$ \\
\hline LH08SG2015 & Bracteacoccus-like & spherical & $\mathrm{n} / \mathrm{a}$ & n/a \\
\hline LH08SW5031 & Chlamydomonas & oval flagellated & $\mathrm{I}=10.9-16.4 \mu \mathrm{m}, \mathrm{w}=4.3-10.1 \mu \mathrm{m}$ & Figure $4 \mathrm{c}$ \\
\hline LH08SG1077 & Chlamydomonas & oval flagellated & $n / a$ & $\mathrm{n} / \mathrm{a}$ \\
\hline LH10HG1027 & Chlamydomonas & oval flagellated & $\mathrm{I}=6.1-9.9 \mu \mathrm{m}, \mathrm{w}=2.7-5.1 \mu \mathrm{m}$ & Figure 4d \\
\hline LH08SG9022 (PS) & Chlamydomonas & oval flagellated & $\mathrm{I}=6.7-12.2 \mu \mathrm{m}, \mathrm{w}=4.0-8.0 \mu \mathrm{m}$ & Figure $4 f$ \\
\hline
\end{tabular}


Table S5. (continuation)

\begin{tabular}{|c|c|c|c|c|}
\hline Representative isolates & $\begin{array}{l}\text { Morphotype } \\
\text { assignation }\end{array}$ & Cell shape & Cell size & Figure \\
\hline LH10HG1013 & Chlorococcum & spherical/oval & $\mathrm{n} / \mathrm{a}$ & $\mathrm{n} / \mathrm{a}$ \\
\hline SAG 2479 & Chlorococcum & spherial/oval & $\mathrm{I}=5.6-9.5 \mu \mathrm{m}, \mathrm{w}=5.6-9.5 \mu \mathrm{m}$ & Figure $4 \mathrm{k}$ \\
\hline LH10HG3113 & Chlorococcum & spherial/oval & $\mathrm{I}=5.8-9.3 \mu \mathrm{m}, \mathrm{w}=2.6-6.3 \mu \mathrm{m}$ & Figure $4 \mathrm{j}$ \\
\hline LH10HG7083 & Scenedesmus-like & $\begin{array}{l}\text { ellipsoidal, obtuse cell } \\
\text { poles }\end{array}$ & $\varnothing=3.5-7.0 \mu \mathrm{m}$ & Figure $3 e$ \\
\hline LH10HG2098 LH10HG7018 & Scenedesmus-like & $\begin{array}{l}\text { ellipsoidal, obtuse cell } \\
\text { poles }\end{array}$ & $\mathrm{I}=6.8-12.5 \mu \mathrm{m}, \mathrm{w}=7.0-10.9 \mu \mathrm{m}$ & $\begin{array}{l}\mathrm{n} / \mathrm{a} \\
\text { Figure } 3 \mathrm{f}\end{array}$ \\
\hline LH08SG2049 & Chlorosarcina-like & spherial & $\varnothing=3.5-6.6 \mu \mathrm{m}$ & Figure $4 \mathrm{~g}$ \\
\hline LH08AG2004 & Chlamydomonas & oval flagellated & $\mathrm{I}=6.0-10.5 \mu \mathrm{m}, \mathrm{w}=3.1-7.5 \mu \mathrm{m}$ & Figure $4 \mathrm{e}$ \\
\hline LH08AW8035 (= SAG 2383) & Bracteacoccus-like & spherical & $\varnothing=2.9-7.3 \mu \mathrm{m}$ & Figure $4 \mathrm{I}$ \\
\hline LH08SG8047 & Chlamydomonas & oval flagellated & $\varnothing=5.1-8.9 \mu \mathrm{m}$ & Figure $4 a$ \\
\hline SAG 2476 (PS) & Chlamydomonas & oval flagellated & $\mathrm{I}=9.1-14.5 \mu \mathrm{m}, \mathrm{w}=5.1-10.0 \mu \mathrm{m}$ & Figure $4 b$ \\
\hline LH10HG2039 & Bracteacoccus-like & spherical & $\varnothing=3.6-8.2 \mu \mathrm{m}$ & Figure $3 \mathrm{~h}$ \\
\hline LH10HG6108 & Chlorococcum & spherical/oval & n/a & n/a \\
\hline LH08SW7115 & Chlorococcum & spherical/oval & $\mathrm{I}=8.3-17.8 \mu \mathrm{m}, \mathrm{w}=5.2-13.3 \mu \mathrm{m}$ & Figure $4 \mathrm{~h}$ \\
\hline LH10HG7016 LH10HG913 & Chlorococcum & spherical/oval & $\varnothing=7.9-13.1 \mu \mathrm{m}$ & $\begin{array}{l}\text { n/a } \\
\text { Figure } 4 \mathrm{i}\end{array}$ \\
\hline LH08HW9058 & Pseudendocloniopsis & $\begin{array}{l}\text { sphaerical, obtuse cell } \\
\text { pole }\end{array}$ & $\varnothing=4.7-9.5 \mu \mathrm{m}$ & Figure $3 d$ \\
\hline LH08SG2033 (PS) & Chlamydomonas-like & oval flagellated & $\mathrm{I}=2.9-5.6 \mu \mathrm{m}, \mathrm{w}=2.5-4.8 \mu \mathrm{m}$ & Figure $3 c$ \\
\hline LH08HW9106 & Klebsormidium & trichal & $\mathrm{I}=4.2-12.8 \mu \mathrm{m}, \mathrm{w}=4.1-6.2 \mu \mathrm{m}$ & Figure $3 b$ \\
\hline LH10HG2056 & Klebsormidium & trichal & $\mathrm{I}=6.4-19.7 \mu \mathrm{m}, \mathrm{w}=4.5-7.0 \mu \mathrm{m}$ & Figure $3 a$ \\
\hline LH10HG3064 & Asterosiphon & spherical/siphonal & $\varnothing=5.4-12.1 \mu \mathrm{m}$ & Figure $11 \mathrm{k}$ \\
\hline LH08AW1076 & Botrydiopsis & sphaerical & $\varnothing=4.9-10.2 \mu \mathrm{m}$ & Figure 11i \\
\hline LH08AW4043 & Botrydiopsis & sphaerical & $\varnothing=5.1-8.8 \mu \mathrm{m}$ & Figure $11 \mathrm{j}$ \\
\hline LH10HG9133, LH10HG5036 & Eustigmatos & spherical & $\varnothing=6.1-13.1 \mu \mathrm{m}$ & $\begin{array}{l}\text { Figure 11l } \\
\mathrm{n} / \mathrm{a}\end{array}$ \\
\hline LH08AG2020 & Heterococcus & heterotrichal & $\mathrm{n} / \mathrm{a}$ & $\mathrm{n} / \mathrm{a}$ \\
\hline LH10HG9111 & Heterococcus & heterotrichal & $\mathrm{I}=5.2-12.4 \mu \mathrm{m}, \mathrm{w}=4.1-13.2 \mu \mathrm{m}$ & Figure $11 \mathrm{~g}, \mathrm{~h}$ \\
\hline LH10HG9085, LH10HG2140 & Heterococcus & heterotrichal & $n / a$ & Figure $11 \mathrm{f}$ \\
\hline LH10HG5079 & Xanthonema & trichal & $\mathrm{n} / \mathrm{a}$ & $\mathrm{n} / \mathrm{a}$ \\
\hline LH10HG7061, LH08SG5052 & Xanthonema & trichal & $\mathrm{I}=10.2-25.4 \mu \mathrm{m}, \mathrm{w}=2.7-10.7 \mu \mathrm{m}$ & $\begin{array}{l}\text { Figure 11d } \\
\text { Figure 11e }\end{array}$ \\
\hline LH08HW9018 & Xanthonema & trichal & $\mathrm{I}=3.3-15.3 \mu \mathrm{m}, \mathrm{w}=2.9-5.7 \mu \mathrm{m}$ & Figure 11c \\
\hline LH10HG7078 & Xanthonema & trichal & $\mathrm{I}=4.2-11.2 \mu \mathrm{m}, \mathrm{w}=3.3-6.0 \mu \mathrm{m}$ & Figure $11 \mathrm{~b}$ \\
\hline LH10HG9058, LH10HG7029 & Xanthonema & trichal & $\mathrm{I}=5.7-12.1 \mu \mathrm{m}, \mathrm{w}=3.4-6.5 \mu \mathrm{m}$ & Figure 11a, n/a \\
\hline
\end{tabular}

Legend. PS=partial sequence. 
Table S6a. Diversity of morphospecies in soil drill cores.

\begin{tabular}{|c|c|c|c|c|c|c|c|c|c|c|c|c|c|c|c|c|c|c|}
\hline Soil horizon & $\frac{\Gamma}{\dot{\alpha}}$ & $\frac{c}{\dot{1}}$ & $\frac{\Gamma}{\dot{1}}$ & $\frac{\Gamma}{\dot{1}}$ & 安 & $\frac{5}{\dot{\alpha}}$ & $\frac{5}{\dot{\alpha}}$ & $\frac{5}{\dot{\alpha}}$ & $\frac{5}{\dot{\alpha}}$ & $\frac{c}{\dot{1}}$ & $\frac{c}{\dot{\alpha}}$ & $\frac{c}{\dot{c}}$ & $\frac{c}{\dot{\alpha}}$ & $\frac{c}{\dot{1}}$ & $\frac{\sigma}{\dot{\alpha}}$ & $\frac{\sigma}{\dot{\alpha}}$ & $\frac{\sigma}{\dot{\alpha}}$ & $\frac{c}{\dot{L}}$ \\
\hline Exploratory & I & 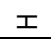 & $I$ & $エ$ & I & I & $\varangle$ & $\varangle$ & $\varangle$ & $\varangle$ & $\varangle$ & $\varangle$ & $\infty$ & $\infty$ & $\infty$ & $\infty$ & $\infty$ & $\infty$ \\
\hline Landscape/plot Nr. & $\frac{m}{0}$ & 足 & $\frac{9}{1}$ & $\stackrel{m}{\frac{m}{3}}$ & $\stackrel{0}{+}$ & $\frac{3}{\frac{1}{3}}$ & $\frac{m}{0}$ & $\frac{0}{1}$ & $\frac{9}{1}$ & $\stackrel{m}{\stackrel{m}{3}}$ & $\stackrel{0}{+}$ & $\stackrel{9}{\stackrel{1}{3}}$ & $\frac{m}{0}$ & $\frac{0}{1}$ & $\frac{9}{10}$ & $\stackrel{m}{\frac{m}{3}}$ & $\stackrel{0}{+}$ & 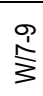 \\
\hline Land-use & ш & $\Sigma$ & - & ш & $\Sigma$ & - & ш & $\Sigma$ & - & ш & $\Sigma$ & - & ш & $\Sigma$ & - & ш & $\Sigma$ & - \\
\hline Subsample & $\stackrel{\times}{\Sigma}$ & $\stackrel{x}{\Sigma}$ & $\stackrel{x}{\Sigma}$ & $\stackrel{x}{\Sigma}$ & $\stackrel{\times}{\Sigma}$ & $\stackrel{\times}{\Sigma}$ & $\stackrel{x}{\Sigma}$ & $\stackrel{x}{\Sigma}$ & $\stackrel{x}{\Sigma}$ & $\stackrel{x}{\Sigma}$ & $\stackrel{x}{\Sigma}$ & $\stackrel{x}{\Sigma}$ & $\stackrel{\times}{\Sigma}$ & $\stackrel{x}{\Sigma}$ & $\stackrel{\times}{\Sigma}$ & $\stackrel{x}{\Sigma}$ & $\stackrel{x}{\Sigma}$ & $\stackrel{x}{\Sigma}$ \\
\hline Bracteacoccus & - & - & - & 1 & 1 & - & 1 & 1 & - & - & - & - & 1 & 1 & - & - & - & - \\
\hline Characiochloridaceae & - & - & - & - & - & - & - & - & - & - & - & - & - & - & - & - & - & - \\
\hline Chlamydomonas & 1 & 1 & - & - & 1 & 1 & - & - & 1 & 1 & - & 1 & 1 & 1 & 1 & - & 1 & 1 \\
\hline Chlorococcum & 1 & 1 & 1 & - & 1 & 1 & - & 1 & - & 1 & 1 & 1 & 1 & 1 & 1 & - & 1 & - \\
\hline Chlorosarcinaceae & - & - & 1 & - & - & - & 1 & - & 1 & - & - & - & - & 1 & - & - & - & - \\
\hline $\begin{array}{l}\text { Monoraphidium } \\
\text { terrestre }\end{array}$ & - & - & - & - & - & - & - & - & - & - & - & - & - & - & - & - & - & - \\
\hline Radiococcaceae & - & - & - & - & - & - & - & - & - & - & - & - & - & - & - & - & - & - \\
\hline Scenedesmaceae & - & 1 & - & - & 1 & - & 1 & - & 1 & - & 1 & - & 1 & 1 & 1 & - & - & - \\
\hline Chlorella & 1 & 1 & 1 & 1 & 1 & 1 & 1 & 1 & 1 & 1 & 1 & - & 1 & 1 & 1 & 1 & 1 & - \\
\hline Chloroidium & - & - & - & - & - & 1 & - & - & - & - & 1 & - & - & 1 & - & - & - & - \\
\hline Coccomyxa & - & 1 & - & 1 & 1 & 1 & - & - & - & 1 & - & 1 & 1 & 1 & 1 & - & - & - \\
\hline Dictyochloropsis & - & - & - & - & - & - & - & - & - & - & - & - & - & - & - & - & - & - \\
\hline Keratococcus & - & - & - & - & - & - & - & - & - & - & - & - & - & - & - & - & - & - \\
\hline Lobosphaera & - & - & 1 & - & 1 & 1 & 1 & - & - & 1 & - & 1 & 1 & - & 1 & - & - & 1 \\
\hline Muriella & - & - & - & - & - & - & - & - & - & - & - & - & - & - & - & - & - & - \\
\hline Neocystis & 1 & 1 & - & - & - & - & - & - & - & - & - & - & - & - & - & - & - & - \\
\hline Stichococcus & - & 1 & - & 1 & 1 & 1 & 1 & - & 1 & 1 & 1 & 1 & 1 & 1 & 1 & 1 & 1 & 1 \\
\hline Dilabifilum & - & - & - & - & - & - & - & - & - & - & - & - & - & - & - & - & - & - \\
\hline Kentrosphaera & - & - & - & - & - & - & - & - & - & - & - & - & - & - & - & - & - & - \\
\hline Pseudendocloniopsis & 1 & 1 & - & - & - & 1 & - & - & - & - & - & - & 1 & - & - & - & - & - \\
\hline Pseudendoclonium & - & - & - & - & - & 1 & - & - & 1 & - & 1 & 1 & - & - & - & - & - & - \\
\hline Cosmarium & - & - & - & - & - & - & - & - & - & - & - & - & - & - & - & - & - & - \\
\hline Klebsormidium & - & - & - & - & 1 & 1 & 1 & - & 1 & - & - & 1 & 1 & 1 & - & 1 & - & 1 \\
\hline Porphyridium & - & - & - & - & - & - & - & - & - & - & - & - & - & - & - & - & - & - \\
\hline Diadesmis & - & - & - & - & - & - & - & - & - & - & - & - & - & - & - & - & - & - \\
\hline Hantzschia & 1 & 1 & 1 & - & 1 & - & - & - & - & - & - & - & 1 & - & 1 & - & - & - \\
\hline Mayamaea & - & - & - & - & - & - & - & - & - & - & - & - & - & - & - & - & - & - \\
\hline Navicula & 1 & 1 & 1 & - & 1 & - & - & 1 & - & - & - & - & 1 & 1 & 1 & - & - & - \\
\hline Nitzschia & - & - & - & - & - & - & - & - & - & - & - & - & - & - & - & - & - & - \\
\hline Pinnularia & - & - & - & - & - & - & - & - & - & - & - & - & - & - & - & - & - & - \\
\hline Stauroneis & - & - & - & - & - & - & - & - & - & - & - & - & - & - & - & - & - & - \\
\hline Surirella & - & - & - & - & - & - & - & - & - & - & - & - & - & - & - & - & - & - \\
\hline Eustigmatos & - & 1 & - & - & - & - & - & 1 & - & - & - & - & - & - & - & - & - & - \\
\hline Heterococcus & - & - & - & - & 1 & - & 1 & - & - & 1 & - & 1 & 1 & 1 & - & - & - & - \\
\hline Tribonema & - & - & - & - & - & - & - & - & - & - & - & - & - & - & - & - & - & - \\
\hline Xanthonema & - & - & - & - & - & 1 & 1 & - & - & 1 & - & - & - & - & - & - & - & - \\
\hline Xanth. coccal & 1 & 1 & 1 & - & 1 & - & 1 & - & 1 & - & - & 1 & 1 & 1 & - & 1 & - & - \\
\hline Cryptomonas & - & - & - & - & - & - & - & - & - & - & - & - & - & - & - & - & - & - \\
\hline Cylindrospermum & - & - & - & - & - & - & - & - & - & - & - & - & - & - & - & - & - & - \\
\hline Leptolyngbya & - & - & - & - & - & - & - & - & - & - & - & - & - & - & - & - & - & - \\
\hline Nostoc & 1 & 1 & - & - & 1 & - & - & 1 & 1 & 1 & - & - & - & 1 & 1 & - & - & - \\
\hline Phormidium & 1 & 1 & 1 & - & 1 & - & 1 & - & 1 & 1 & - & - & 1 & 1 & 1 & - & - & - \\
\hline Plectonema & - & - & - & - & - & - & - & - & - & - & - & - & - & - & - & - & - & - \\
\hline Pseudanabaena & - & - & - & - & - & - & - & - & - & - & - & - & - & - & - & - & - & - \\
\hline Stigonema & - & - & - & - & - & - & - & - & - & - & - & - & - & - & - & - & - & - \\
\hline
\end{tabular}

Legend. $A-h=$ drill core; $\mathrm{O}-\mathrm{h}=$ topsoil. $\mathrm{H}=$ Hainich-Dün; $\mathrm{A}=\mathrm{Schwäbische} \mathrm{Alb}$; $\mathrm{S}=$ Schorfheide-Chorin. $\mathrm{G}=$ grassland; W=forest. $\mathrm{E}=$ extensive; $\mathrm{M}=$ managed; I=intensive. $M X=$ mixed. 
Table S6b. Diversity of morphospecies in topsoil samples.

\begin{tabular}{|c|c|c|c|c|c|c|c|c|c|c|c|c|c|c|c|c|c|c|c|c|}
\hline Soil horizon & ơ & ơ & ํํ & ํํ & ธุ́ & ơ & ণิ & ธૃ & ธَ' & ธૃ่ & ธ่ & ઠิ & ô & ণૃ & ণิ & ণิ & ธิ & ธุ' & ธิ & ธุ́ \\
\hline Exploratory & I & I & I & I & I & I & $I$ & $I$ & I & 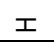 & I & I & I & I & I & エ & I & I & I & I \\
\hline Landscape/plot Nr. & S & $\widehat{\widehat{O}}$ & $\frac{\widehat{N}}{0}$ & No & $\frac{\infty}{0}$ & $\frac{\infty}{0}$ & $\frac{\infty}{0}$ & $\frac{\infty}{0}$ & $\frac{9}{10}$ & $\frac{9}{10}$ & $\frac{9}{10}$ & $\frac{9}{10}$ & $\stackrel{20}{3}$ & $\stackrel{20}{3}$ & $\frac{10}{3}$ & $\stackrel{100}{3}$ & $\stackrel{0}{3}$ & $\stackrel{0}{3}$ & $\stackrel{0}{3}$ & $\stackrel{0}{3}$ \\
\hline Land-use & ш & ш & ш & ш & ш & ш & ш & ш & ш & ш & ш & ш & $\Sigma$ & $\Sigma$ & $\Sigma$ & $\Sigma$ & $\Sigma$ & $\Sigma$ & $\Sigma$ & $\Sigma$ \\
\hline Subsample & $\stackrel{\rho}{\Sigma}$ & 㞱 & 交 & $\stackrel{x}{\Sigma}$ & 足 & ए & 令 & $\stackrel{x}{\Sigma}$ & $\stackrel{\rho}{\Sigma}$ & 㞱 & $\sum_{\infty}$ & $\stackrel{x}{\Sigma}$ & $\stackrel{\rho}{\Sigma}$ & 㞱 & 命 & $\stackrel{x}{\Sigma}$ & 足 & 㞱 & $\sum_{\infty}$ & $\stackrel{x}{\Sigma}$ \\
\hline Bracteacoccus & - & 1 & - & 1 & - & - & 1 & - & 1 & - & 1 & 1 & - & - & - & - & - & - & - & - \\
\hline Characiochloridaceae & - & 1 & - & - & - & - & - & - & 1 & 1 & 1 & 1 & - & - & - & - & - & - & 1 & - \\
\hline Chlamydomonas & 1 & 1 & 1 & 1 & 1 & 1 & 1 & 1 & 1 & 1 & 1 & 1 & 1 & 1 & 1 & 1 & 1 & 1 & 1 & 1 \\
\hline Chlorococcum & 1 & 1 & 1 & 1 & 1 & 1 & 1 & 1 & 1 & 1 & 1 & 1 & - & 1 & 1 & 1 & - & 1 & 1 & 1 \\
\hline Chlorosarcinaceae & - & - & - & 1 & - & - & - & - & 1 & 1 & - & 1 & - & - & - & - & - & - & - & - \\
\hline $\begin{array}{l}\text { Monoraphidium } \\
\text { terrestre }\end{array}$ & - & - & - & - & - & - & - & - & - & 1 & 1 & - & - & - & - & - & - & - & - & - \\
\hline Radiococcaceae & 1 & - & - & - & - & - & - & - & - & - & - & - & - & - & - & - & - & - & - & - \\
\hline Scenedesmaceae & 1 & 1 & 1 & 1 & 1 & 1 & 1 & 1 & 1 & 1 & 1 & 1 & - & 1 & - & - & - & - & - & - \\
\hline Chlorella & - & 1 & 1 & - & 1 & 1 & - & 1 & 1 & 1 & 1 & - & - & - & - & - & - & - & - & - \\
\hline Chloroidium & - & - & - & - & 1 & - & 1 & - & - & - & 1 & - & - & - & - & - & - & - & - & - \\
\hline Coccomyxa & - & - & - & 1 & 1 & 1 & 1 & 1 & 1 & 1 & 1 & 1 & 1 & 1 & 1 & 1 & - & 1 & 1 & - \\
\hline Dictyochloropsis & - & - & - & - & 1 & - & - & - & - & - & - & - & - & - & - & - & - & - & - & - \\
\hline Keratococcus & 1 & 1 & - & 1 & 1 & 1 & 1 & 1 & 1 & 1 & 1 & 1 & - & - & - & - & - & - & - & - \\
\hline Lobosphaera & - & 1 & - & 1 & 1 & 1 & 1 & 1 & 1 & 1 & 1 & 1 & - & - & - & - & - & - & - & 1 \\
\hline Muriella & 1 & - & - & 1 & - & 1 & 1 & - & 1 & 1 & 1 & 1 & - & 1 & - & - & - & - & - & - \\
\hline Neocystis & - & - & - & - & 1 & - & - & - & 1 & - & 1 & - & - & - & - & - & - & - & 1 & - \\
\hline Stichococcus & 1 & 1 & - & 1 & 1 & 1 & 1 & 1 & 1 & 1 & 1 & 1 & - & - & 1 & 1 & 1 & - & 1 & - \\
\hline Dilabifilum & - & - & - & - & - & - & - & - & - & - & - & 1 & - & - & - & - & - & - & - & - \\
\hline Kentrosphaera & - & - & - & - & - & - & - & 1 & - & 1 & 1 & 1 & - & - & - & - & - & - & - & - \\
\hline Pseudendocloniopsis & 1 & 1 & 1 & 1 & 1 & 1 & 1 & - & 1 & 1 & 1 & 1 & - & - & - & - & - & - & - & - \\
\hline Pseudendoclonium & - & - & - & - & - & - & 1 & - & 1 & 1 & 1 & 1 & - & - & - & - & - & - & - & - \\
\hline Cosmarium & - & - & - & - & - & - & - & - & - & - & - & 1 & - & - & - & - & - & - & - & - \\
\hline Klebsormidium & - & 1 & 1 & 1 & - & 1 & 1 & 1 & 1 & 1 & 1 & 1 & 1 & 1 & 1 & 1 & - & - & - & - \\
\hline Porphyridium & - & - & - & - & - & - & - & - & - & 1 & 1 & 1 & - & - & - & - & - & - & - & - \\
\hline Diadesmis & - & 1 & - & 1 & - & - & 1 & - & 1 & - & 1 & - & - & - & - & - & - & - & - & - \\
\hline Hantzschia & 1 & 1 & 1 & 1 & 1 & 1 & 1 & 1 & 1 & 1 & 1 & 1 & - & - & - & - & - & - & - & - \\
\hline Mayamaea & 1 & 1 & 1 & 1 & 1 & 1 & 1 & 1 & 1 & 1 & 1 & 1 & - & - & - & - & - & - & - & 1 \\
\hline Navicula & - & - & 1 & - & - & 1 & 1 & 1 & 1 & 1 & 1 & 1 & 1 & 1 & 1 & 1 & 1 & 1 & 1 & 1 \\
\hline Nitzschia & - & - & - & - & 1 & 1 & - & 1 & - & 1 & 1 & 1 & - & - & - & - & - & - & - & - \\
\hline Pinnularia & - & - & - & - & - & - & - & - & 1 & 1 & 1 & 1 & - & - & - & - & - & - & - & - \\
\hline Stauroneis & - & - & - & - & - & - & - & - & - & - & - & 1 & - & - & - & - & - & - & - & - \\
\hline Surirella & - & - & - & - & - & - & - & - & - & 1 & - & 1 & - & - & - & - & - & - & - & - \\
\hline Eustigmatos & - & 1 & - & - & - & - & 1 & 1 & 1 & 1 & 1 & 1 & - & - & - & 1 & - & - & - & - \\
\hline Heterococcus & 1 & 1 & - & 1 & 1 & - & 1 & 1 & 1 & 1 & 1 & 1 & 1 & 1 & 1 & 1 & 1 & 1 & 1 & 1 \\
\hline Tribonema & 1 & - & - & - & 1 & - & - & - & - & 1 & - & 1 & - & - & - & - & - & - & - & - \\
\hline Xanthonema & 1 & 1 & - & 1 & 1 & 1 & 1 & 1 & 1 & 1 & 1 & 1 & 1 & 1 & 1 & 1 & 1 & - & - & 1 \\
\hline Xanth. coccal & 1 & 1 & - & 1 & 1 & - & 1 & 1 & 1 & 1 & 1 & 1 & - & - & - & 1 & 1 & - & - & - \\
\hline Cryptomonas & - & - & - & - & - & - & - & - & - & - & - & 1 & - & - & - & - & - & - & - & - \\
\hline Cylindrospermum & - & - & - & 1 & - & - & - & 1 & - & - & - & - & - & - & - & - & - & - & - & - \\
\hline Leptolyngbya & 1 & 1 & 1 & 1 & 1 & 1 & 1 & 1 & 1 & 1 & 1 & 1 & - & - & - & - & - & - & - & - \\
\hline Nostoc & 1 & 1 & 1 & 1 & 1 & 1 & 1 & 1 & 1 & 1 & 1 & 1 & - & - & - & - & - & - & - & - \\
\hline Phormidium & 1 & - & 1 & 1 & 1 & 1 & 1 & 1 & 1 & 1 & 1 & 1 & - & - & - & - & - & - & - & - \\
\hline Plectonema & - & - & - & - & - & - & - & - & - & - & - & 1 & - & - & - & - & - & - & - & - \\
\hline Pseudanabaena & - & - & - & - & - & - & - & - & - & - & - & 1 & - & - & - & - & - & - & - & - \\
\hline Stigonema & - & - & - & - & - & - & - & - & - & - & - & 1 & - & - & - & - & - & - & - & - \\
\hline
\end{tabular}

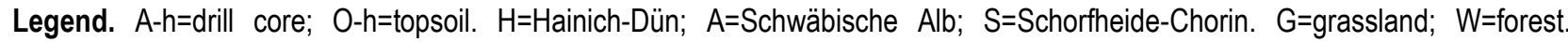
$\mathrm{E}=$ extensive; $\mathrm{M}=$ managed; I=intensive. $\mathrm{MX}=$ mixed; $M D=$ middle; $N E=$ NorthEast; $S W=S o u t h W e s t$. 
Table S7. Frequency of the morphospecies in soil drill cores.

\begin{tabular}{|c|c|c|c|c|}
\hline Morphotype and the authority & Nr. obs & $\%$ of samples & Microphotographs & Isolate/mixed culture \\
\hline Bracteacoccus Tereg & 12 & 32 & Figure $3 \mathrm{i}$ & isolate \\
\hline Characiochloridaceae Skuja & 6 & 16 & Supplementary Figure 1a-b & mixed culture \\
\hline Chlamydomonas Ehrenberg & 32 & 84 & Figure 4a-f & isolate \\
\hline Chlorococcum Meneghini & 31 & 82 & Figure $4 h-k$ & isolate \\
\hline Chlorosarcinaceae Bourrelly ex Groover \& Bold & 8 & 21 & Supplementary Figure $1 f$ & isolate \\
\hline Monoraphidium terrestre (Bristol) Krienitz & 2 & 5 & Supplementary Figure $1 \mathrm{~h}$ & mixed culture \\
\hline Radiococcaceae Fott \& Komárek & 1 & 3 & $\mathrm{n} / \mathrm{a}$ & mixed culture \\
\hline Scenedesmaceae Oltmanns & 21 & 55 & Supplementary Figure $1 \mathrm{~g}$ & isolate \\
\hline Chlorella Beijerinck & 24 & 63 & Figure 7a-c & isolate \\
\hline Chloroidium Nadson & 6 & 16 & Figure $6 h-i$ & isolate \\
\hline Coccomyxa Schmidle & 24 & 63 & Figure 6e-g & isolate \\
\hline Dictyochloropsis Geitler em. Tschermak-Woess & 1 & 3 & Figure 61 & isolate \\
\hline Keratococcus Pascher & 11 & 29 & Supplementary Figure $1 \mathrm{i}-\mathrm{j}$ & mixed culture \\
\hline Lobosphaera Reisigl & 20 & 53 & Figure $6 \mathrm{j}$ & isolate \\
\hline Muriella J. B. Petersen & 9 & 24 & Figure $7 \mathrm{~d}-\mathrm{i}$ & isolate \\
\hline Neocystis F. Hindák & 6 & 16 & Figure $6 \mathrm{~d}$ & isolate \\
\hline Stichococcus Nägeli & 30 & 79 & Figure 6a-c & isolate \\
\hline Dilabifilum Tschermak-Woess & 1 & 3 & Supplementary Figure $2 b$ & mixed culture \\
\hline Kentrosphaera Borzì & 4 & 11 & Supplementary Figure 1 l & mixed culture \\
\hline Pseudendocloniopsis Vischer & 15 & 39 & Figure $3 d$ & isolate \\
\hline Pseudendoclonium Wille & 9 & 24 & Supplementary Figure 2a & isolate \\
\hline Cosmarium Corda & 1 & 3 & Supplementary Figure $2 \mathrm{~d}-\mathrm{e}$ & mixed culture \\
\hline Klebsormidium Silva, Mattox 8c Blackwell & 23 & 61 & Figure 3a-b & isolate \\
\hline Porphyridium Nägeli & 3 & 8 & Supplementary Figure $2 \mathrm{~h}$ & mixed culture \\
\hline Diadesmis contenta (Grunow) D. G. Mann & 5 & 13 & Supplementary Figure 3b & mixed culture \\
\hline Hantzschia amphioxys (Ehrenberg) Grunow & 18 & 47 & Supplementary Figure 30 & mixed culture \\
\hline Mayamaea H. Lange-Bertalot & 13 & 34 & Supplementary Figure $3 e$ & mixed culture \\
\hline Navicula Bory de Saint-Vincent & 24 & 63 & Supplementary Figure 3a,c-d & mixed culture \\
\hline Nitzschia Hassall & 6 & 16 & Supplementary Figure 3m-n & mixed culture \\
\hline Pinnularia Ehrenberg & 4 & 11 & Supplementary Figure 3f-i & mixed culture \\
\hline Stauroneis Ehrenberg & 1 & 3 & Supplementary Figure $3 \mathrm{j}$ & mixed culture \\
\hline Surirella Turpin & 2 & 5 & Supplementary Figure 3l & mixed culture \\
\hline Eustigmatos D. J. Hibberd & 10 & 26 & Figure 11l & isolate \\
\hline Heterococcus Chodat & 24 & 63 & Figure 11f-h & isolate \\
\hline Tribonema Derbès \& Solier & 4 & 11 & Supplementary Figure $2 \mathrm{j}$ & mixed culture \\
\hline Xanthonema Silva & 20 & 53 & Figure 11a-e & isolate \\
\hline Xanth. coccal & 22 & 58 & Figure 11i-j; Suppl. Figure 2k-I & Isolate; mix.cul. \\
\hline Cryptomonas Ehrenberg & 1 & 3 & Supplementary Figure $2 \mathrm{i}$ & mixed culture \\
\hline $\begin{array}{l}\text { Cylindrospermum F. T. Kützing ex É. Bornet \& C. } \\
\text { Flahault }\end{array}$ & 2 & 5 & Supplementary Figure $4 \mathrm{j}$ & mixed culture \\
\hline Leptolyngbya Anagnostidis \& Komárek & 12 & 32 & Supplementary Figure 4b-e & mixed culture \\
\hline Nostoc Vaucher ex Bornet \& Flahault & 20 & 53 & Supplementary Figure 4g-i & mixed culture \\
\hline Phormidium Kützing ex Gomont & 21 & 55 & Supplementary Figure $4 f$ & mixed culture \\
\hline Plectonema Thuret ex Gomont & 1 & 3 & $\mathrm{n} / \mathrm{a}$ & mixed culture \\
\hline Pseudanabaena Lauterborn & 1 & 3 & Supplementary Figure 4a & mixed culture \\
\hline Stigonema Agardh ex Bornet et Flahault & 1 & 3 & Supplementary Figure $4 \mathrm{I}$ & mixed culture \\
\hline
\end{tabular}




\section{Appendix | Chapter 2}

\section{Supporting Tables}

Table S1. Species of green algae recovered from creek biofilms.

Table S2. Green algal species recovered from creek biofilms and their main morphological features.

Table S3. Percentage identities (PID) to closest GenBank-relatives.

Table S4. Sequences representing additional groups and lineages shown as collapsed triangles in Figures 1-3. 
Table S1. Species of green algae recovered from creek biofilms.

\begin{tabular}{|c|c|c|c|c|}
\hline Species & $\begin{array}{l}\text { Isolate(s) studied } \\
\text { in detail }\end{array}$ & $\begin{array}{l}\text { Sequence accession } \\
\text { numbers (original } \\
\text { identifiers) }\end{array}$ & Additional isolates & Sequence accession numbers \\
\hline Chlorella sp. & $\begin{array}{l}\text { RK52 } \\
\text { D11-2 }\end{array}$ & $\begin{array}{l}\text { KF144182 } \\
\text { KF144172 }\end{array}$ & $\begin{array}{l}\text { DB1-5, DB1-10 } \\
\text { DB2-4, DB2-7 } \\
\text { DB3-2, DB6-28 } \\
\text { DB6-33, DB14-17 } \\
\text { DB14-21, DB14-23 }\end{array}$ & $\begin{array}{l}\text { KF144171, KF144173 } \\
\text { KF144177-KF144181 } \\
\text { KF144174-KF144176 }\end{array}$ \\
\hline Coccomyxa cf. pringsheimii & $\begin{array}{l}\text { WB28 } \\
\text { WB32 }\end{array}$ & KF144225 & DB14-12, SAG 2391 & $\begin{array}{l}\text { KF144223 } \\
\text { KF144224 (DB14-14) }\end{array}$ \\
\hline Coccomyxa cf. simplex & WB40 & KF144226 & & \\
\hline Elliptochloris subsphaerica & WB5-D1e & KF144205 & WB27 & KF144204 \\
\hline Marvania sp. & WB67 & KF144207 & & \\
\hline Muriella terrestris & D6-DB2 & KF144209 & SAG 2390 & \\
\hline Neocystis cf. mucosa & SAG 2405 & KF144212 (WB21) & & \\
\hline $\begin{array}{l}\text { Stichococcus bacillaris } \\
\text { Stichococcus cf deasonii }\end{array}$ & $\begin{array}{l}\text { WB13 } \\
\text { WB74 } \\
\text { WB38 }\end{array}$ & $\begin{array}{l}\text { KF144231 } \\
\text { KF144232 } \\
\text { KF144233 }\end{array}$ & & \\
\hline Stichococcus mirabilis & WB69 & KF144234 & & \\
\hline Stichococcus sp.2 & $\begin{array}{l}\text { DB6-27 } \\
\text { WB65 } \\
\text { SAG 2407 } \\
\text { D4-2A }\end{array}$ & $\begin{array}{l}\text { KF144235 } \\
\text { KF144236 } \\
\text { KF144237 (WB68) } \\
\text { KF144238 }\end{array}$ & & \\
\hline Stichococcus sp.3 & SAG 2408 & KF144239 (WB8) & & \\
\hline Stichococcus sp.4 & SAG 2406 & KF144240 (WB47) & & \\
\hline Acutodesmus obliquus & D22-6-2B & KF144164 & & \\
\hline Bracteacoccus aerius-relative & SAG 2403 & KF144165 (WB18) & & \\
\hline Bracteacoccus sp. & DB9-3 & KF144166 & & \\
\hline Chlamydomonas sp. & RK68 & KF144168 & DB6-21 & KF144167 \\
\hline Chlamydopodium sp. & $\begin{array}{l}\text { SAG } 2402 \\
\text { RK50 }\end{array}$ & $\begin{array}{l}\text { KF144169 (RK41) } \\
\text { KF144170 }\end{array}$ & & \\
\hline Chlorococcum sphacosum & SAG 2398 & KF144183 (GRK6-DB1) & & \\
\hline Chlorococcum ellipsoideum-relatives 1 & GRK6-DB5 & KF144184 & & \\
\hline Chlorococcum ellipsoideum-relatives2 & $\begin{array}{l}\text { SAG } 2400 \\
\text { GRK6-DB6 } \\
\text { SAG } 2401\end{array}$ & $\begin{array}{l}\text { KF144186 (GRK7-WB4) } \\
\text { KF144185 } \\
\text { KF144187 (GRK7-WB5) }\end{array}$ & & \\
\hline Chlorococcum minutum-relative & SAG 2399 & KF144188 (GRK6-DB3) & & \\
\hline Desmodesmus cf. armatus & $\begin{array}{l}\text { RK43 } \\
\text { SAG } 2395\end{array}$ & $\begin{array}{l}\text { KF144195 } \\
\text { KF144191 (D7-6-1B) }\end{array}$ & $\begin{array}{l}\text { DB6-23, DB6-26 } \\
\text { DB6-30, SAG } 2389\end{array}$ & KF144192-KF144194 \\
\hline Monoraphidium terrestre cf. dybowskii & SAG 2393 & KF144208 (D21-6-5B) & & \\
\hline Mychonastes cf. homosphaera & RK48 & KF144210 & & \\
\hline Mychonastes sp. & DB6-29 & KF144211 & & \\
\hline Pseudomuriella cf. schumacherensis & RK3 & KF144227 & & \\
\hline Scenedesmaceae sp. & RK49 & KF144228 & & \\
\hline Desmochloris cf. halophila & SAG 2397 & KF144190 (DB1-9) & & \\
\hline Dilabifilum printzii & WB41 & KF144201 & $\begin{array}{l}\text { DB15-6, WB3 } \\
\text { WB24, WB31 } \\
\text { WB43, WB59 }\end{array}$ & $\begin{array}{l}\text { KF144197, KF144200 } \\
\text { KF144199, KF144196 } \\
\text { KF144202, KF144203 }\end{array}$ \\
\hline Hazenia mirabilis & SAG 2396 & KF144206 (D9-14B) & & \\
\hline Pseudendocloniopsis botryoides & $\begin{array}{l}\text { SAG } 2394 \\
\text { DB6-19 }\end{array}$ & $\begin{array}{l}\text { KF144213 (D2-6-1A) } \\
\text { KF144219 }\end{array}$ & $\begin{array}{l}\text { DB14-6 } \\
\text { DB14-11 } \\
\text { DB14-24 } \\
\text { DB14-28 } \\
\text { DB14-45 } \\
\text { WB4 } \\
\text { WB45 }\end{array}$ & $\begin{array}{l}\text { KF144218 } \\
\text { KF144214-KF144217 } \\
\text { KF144221 } \\
\text { KF144220 }\end{array}$ \\
\hline Pseudendoclonium akinetum & SAG 2404 & KF144222 (WB20) & & \\
\hline
\end{tabular}

Legend. The 74 unialgal isolates, their affiliation to a certain class, their strain designations and NCBI accession numbers of their $18 \mathrm{~S}$ rRNA gene sequences are listed. The 45 strains used as exemplar for detailed examination are listed separately from those additional strains representing the same species. When an isolate was accessioned by the SAG culture collection, the corresponding SAG strain number is given and the original strain designation is in brackets next to its sequence accession number. 
Table S2. Green algal species recovered from creek biofilms and their main morphological features.

\begin{tabular}{|c|c|c|}
\hline Species & Figure & Morphological features \\
\hline Chlorella sp. & Figure $5 c, 5 d$ & $\begin{array}{l}\text { Cells globose or ellipsoidal with smooth cell walls. Single parietal chloroplast with single pyrenoid. } \\
\text { Reproduction by } 2-8 \text { autospores per cell, released by rupture of parental cell wall. Cell diameter } 4.5- \\
8.0 \mu \mathrm{m} \text {. }\end{array}$ \\
\hline Coccomyxa cf. pringsheimii & Figure $5 i$ & $\begin{array}{l}\text { Heteropolar cells oval, fusiform or egg-shaped with smooth cell walls. Single parietal chloroplast } \\
\text { without pyrenoids. Cell diameter: } 4.4-8.0 \mu \mathrm{m} \text {. }\end{array}$ \\
\hline Coccomyxa cf. simplex & $\mathrm{n} / \mathrm{a}$ & $\begin{array}{l}\text { Heteropolar cells oval, fusiform or egg-shaped with smooth cell walls. Single parietal chloroplast } \\
\text { without pyrenoids. Cell length: } 4.6-8.3 \mu \mathrm{m} \text {. }\end{array}$ \\
\hline Elliptochloris subsphaerica & Figure $5 \mathrm{~h}$ & $\begin{array}{l}\text { Young cells cylindric, slightly inflexed, mature cells ellipsoidal to globose. Single parietal chloroplast } \\
\text { with single pyrenoid. Autosporangia ellipsoidal with several cylindric autospores. Cell diameter } 7.0 \text { - } \\
10.5 \mu \mathrm{m} \text {. }\end{array}$ \\
\hline Marvania sp. & Figure $5 f$ & Cells spherical, with 2 parietal chloroplasts, pyrenoids absent. Cell diameter 2.5-4.2 $\mu \mathrm{m}$. \\
\hline Muriella terrestris & Figure $5 \mathrm{e}$ & $\begin{array}{l}\text { Cells spherical, with } 2 \text { or more parietal chloroplasts without a pyrenoid. Asexual reproduction by } \\
\text { autospores, released by rupture of parental cell wall. Cell diameter: } 2.4-4.4 \mu \mathrm{m} \text {. }\end{array}$ \\
\hline Neocystis cf. mucosa & Figure $5 g$ & $\begin{array}{l}\text { Cells broadly oval with single parietal chloroplast without a pyrenoid. Asexual reproduction by } \\
\text { autospores, } 2-4 \text { per autosporangium, released by rupture of the cell wall, remaining visible in the } \\
\text { colony. Cell diameter: } 2.9-5.7 \mu \mathrm{m} \text {. }\end{array}$ \\
\hline Stichococcus bacillaris & Figure $5 \mathrm{a}$ & $\begin{array}{l}\text { Few-celled fragmenting filaments or individual cells. Cells cylindrical or elongate, sometimes slightly } \\
\text { oval with single parietal chloroplast without pyrenoids. Cell length: } 4.1-17.4 \mu \mathrm{m} \text {. }\end{array}$ \\
\hline Stichococcus cf. deasonii & $\mathrm{n} / \mathrm{a}$ & $\begin{array}{l}\text { Cylindrical elongate cells with a single parietal chloroplast containing a single starch-enveloped } \\
\text { pyrenoid, cell length: } 4.1-8.9 \mu \mathrm{m} \text {. }\end{array}$ \\
\hline Stichococcus mirabilis & $\mathrm{n} / \mathrm{a}$ & For description see Stichococcus bacillaris above, cell length: 3.2-9.5 $\mu \mathrm{m}$. \\
\hline Stichococcus sp.1 & $\mathrm{n} / \mathrm{a}$ & For description see Stichococcus bacillaris above, cell length: 2.9-11.9 $\mu \mathrm{m}$. \\
\hline Stichococcus sp.2 & $\mathrm{n} / \mathrm{a}$ & For description see Stichococcus cf. deasonii above, cell length: 6.2-11.4 $\mu \mathrm{m}$. \\
\hline Stichococcus sp.3 & $\mathrm{n} / \mathrm{a}$ & For description see Stichococcus cf. deasonii above, cells sometimes flexed. Cell length: 6.2-11.4 $\mu \mathrm{m}$. \\
\hline Stichococcus sp.4 & Figure $5 b$ & For description see Stichococcus cf. deasonii above, cell length: 3.7-9.5 $\mu \mathrm{m}$. \\
\hline Acutodesmus obliquus & Figure $6 \mathrm{~h}$ & Fusiform cells with acute cell poles, without bristles. Cell length: $6.2-10.9 \mu \mathrm{m}$. \\
\hline Bracteacoccus sp. & Figure $7 \mathrm{~b}$ & $\begin{array}{l}\text { Sphaerical cells with several to many parietal lens-shaped chloroplasts without pyrenoids. Cell } \\
\text { diameter: } 4.9-10.0 \mu \mathrm{m} \text {. }\end{array}$ \\
\hline Chlamydomonas sp. & Figure $6 f$ & $\begin{array}{l}\text { Spherical cells with anterior contractile vacuole and two isokont anterior flagella. Cells contain single } \\
\text { cup-shaped chloroplast with one basal pyrenoid and an eyespot at the cell anterior. Cell length: } 7.2- \\
11.1 \mu \mathrm{m} \text {. }\end{array}$ \\
\hline Chlorococcum cf. sphacosum & Figure $6 a$ & $\begin{array}{l}\text { Cells spherical to ellipsoidal with smooth cell walls. One parietal chloroplast per cell containing single } \\
\text { pyrenoid. Biflagellated zoospores remain ellipsoidal after motility ceases (Chlamydomonas-type). Cell } \\
\text { diameter: } 7.0-10.5 \mu \mathrm{m} \text {. }\end{array}$ \\
\hline $\begin{array}{l}\text { Chlorococcum ellipsoideum- } \\
\text { relatives } 1\end{array}$ & Figure $6 \mathrm{c}$ & $\begin{array}{l}\text { Cells spherical to ellipsoidal with smooth cell walls. One parietal chloroplast per cell containing single } \\
\text { pyrenoid. Cell diameter: } 8.5-11.7 \mu \mathrm{m} \text {. }\end{array}$ \\
\hline $\begin{array}{l}\text { Chlorococcum ellipsoideum- } \\
\text { relatives } 2\end{array}$ & Figure $6 \mathrm{~d}, 6 \mathrm{e}$ & $\begin{array}{l}\text { Spherical cells with anterior contractile vacuole, possessing two isokont anterior flagella. Single band- } \\
\text { shaped chloroplast per cell with a single lateral pyrenoid and an eyespot at the cell anterior. Cell length: } \\
5.8-11.8 \mu \mathrm{m} \text {. }\end{array}$ \\
\hline Desmodesmus cf. armatus & Figure $6 \mathrm{~g}$ & $\begin{array}{l}\text { Ellipsoidal cells with obtuse cell poles and bristles. Cells form 4-8-celled coenobia. Cell length: } 6.1- \\
17.6 \mu \mathrm{m} \text {. }\end{array}$ \\
\hline Mychonastes cf. homosphaera & Figure 7c & Spherical Chlorella-like cells with single chloroplasts without a pyrenoid. Cell diameter: $1.8-3.3 \mu \mathrm{m}$. \\
\hline Mychonastes sp. & Figure $7 \mathrm{~b}$ & For description see Mychonastes cf. homosphaera above, cell diameter: 1.8-3.3 $\mu \mathrm{m}$. \\
\hline $\begin{array}{l}\text { Pseudomuriella cf. } \\
\text { schumacherensis }\end{array}$ & Figure $7 a$ & $\begin{array}{l}\text { Spherical cells containing chloroplast (parietal saucer-shaped in young cells or divided into several } \\
\text { segments in mature cells. Pyrenoids are absent. Cell diameter: } 3.7-8.1 \mu \mathrm{m} .\end{array}$ \\
\hline Scenedesmaceae sp. & Figure $6 \mathrm{i}$ & For description see Desmodesmus cf. armatus above, cell length: 5.3-8.4 $\mu \mathrm{m}$. \\
\hline Dilabifilum printzii & Figure $7 \mathrm{i}$ & For description see Desmodesmus cf. armatus above, cell length: 5.0-10.1 $\mu \mathrm{m}$. \\
\hline Hazenia mirabilis & Figure $7 e$ & $\begin{array}{l}\text { Individual cells contain single parietal chloroplast with a pyrenoid. Thalli heterotrichous, consisting of } \\
\text { uniseriate, irregularly branched filaments. Cell length: } 7.6-21.4 \mu \mathrm{m} \text {. }\end{array}$ \\
\hline Pseudendocloniopsis botryoides & Figure $7 f, 7 g$ & $\begin{array}{l}\text { Globose cells form roundish, irregular packets or heterotrichous thalli. Cells with single parietal } \\
\text { chloroplast and a pyrenoid. Cell diameter: } 4.0-11.1 \mu \mathrm{m} \text {. }\end{array}$ \\
\hline Pseudendoclonium akinetum & Figure $7 \mathrm{~h}$ & $\begin{array}{l}\text { Globose cells forming uniseriate filaments, irregularly branched. Cells containing one parietal } \\
\text { chloroplast with one or two pyrenoids. Cell length: } 5.6-10.2 \mu \mathrm{m} \text {. }\end{array}$ \\
\hline
\end{tabular}


Table S3. Percentage identities (PID) to closest GenBank-relatives.

\begin{tabular}{|c|c|c|c|c|}
\hline Identification of taxa based on $18 \mathrm{~S}$ rDNA & Strain(s) & $\begin{array}{l}\text { Closest relative(s) } \\
\text { accession number(s) }\end{array}$ & $\begin{array}{l}\text { Percentage } \\
\text { identity }\end{array}$ & Habitat \\
\hline Acutodesmus obliquus & D22-6-2B & FR865726 & 100 & freshwater \\
\hline Bracteacoccus & DB9-3 & U63103 & 99.59 & air-borne dust \\
\hline Bracteacoccus aerius-relative & SAG 2403 & U63101 & 98.59 & air-borne dust \\
\hline Chlamydomonas sp. & RK68 & X53904 & 98.65 & freshwater \\
\hline Chlamydomonas sp. & RK68 & JN903974 & 99.13 & freshwater \\
\hline Chlamydomonas sp. & RK68 & AY781664 & 99.18 & freshwater \\
\hline Chlamydopodium vacuolatum & RK50+SAG 2402 & M63001 & 98.77 & $\mathrm{n} / \mathrm{a}$ \\
\hline Chlamydopodium vacuolatum & RK50+SAG 2402 & GQ122367 & 99.82 & freshwater \\
\hline Chlorella sp. & RK52, D11-2 & GQ487223 & 99.71 & freshwater \\
\hline Chlorella sp. & RK52/D11-2 & $\begin{array}{l}\text { FR865683, AY197628, } \\
\text { FJ946888, DQ377324 }\end{array}$ & 99.90 & freshwater \\
\hline Chlorococcum cf. sphacosum & SAG 2398 & JN968580/JN968584 & 99.94 & freshwater \\
\hline Chlorococcum ellipsoideum-relative1 & GRK6-DB5 & U70586 & 98.45 & terrestrial (soil) \\
\hline Chlorococcum ellipsoideum-relative2 & $\begin{array}{l}\text { SAG 2401/SAG } 2400 \\
\text { /GRK6-DB6 }\end{array}$ & U70586 & 98.33 & terrestrial (soil) \\
\hline Chlorococcum minutum-relative & SAG 2399 & JN968585 & 97.65 & terrestrial (soil) \\
\hline Coccomyxa cf. pringsheimii & WB28 & AY762603 & 99.61 & terrestrial (phycobiont) \\
\hline Cooccomyxa cf. simplex & WB40 & FJ648514 & 99.83 & freshwater \\
\hline Desmochloris sp. & SAG 2397 & FM882216/AB049416 & 99.30 & freshwater \\
\hline Desmodesmus cf. armatus & SAG 2395/RK43 & FR865727 & 99.71 & freshwater \\
\hline Dilabifilum printzii & WB41 (PS) & KF144198 & 100 & freshwater \\
\hline Elliptochloris cf. subsphaerica & WB5-D1e & FJ648515, FJ648518 & 99.94 & terrestrial (roof tile) \\
\hline Hazenia sp. & SAG 2396 & AF387156 & 99.94 & freshwater \\
\hline Marvania & WB67 & AF124336 & 97.83 & freshwater \\
\hline Marvania & WB67 & EF440182 & 99.30 & terrestrial (permafrost) \\
\hline Marvania & WB67 & AY195983 & 99.60 & freshwater \\
\hline Monoraphidium terrestre cf. dybowskii & SAG 2393 & Y16939 & 99.54 & freshwater \\
\hline Muriella terrestris & D6-DB2 & AB012845 & 99.94 & terrestrial (soil) \\
\hline Muriella terrestris & D6-DB2 & AY195969 & 100 & freshwater \\
\hline Mychonastes cf. homosphaera & RK48 & GU799581 & 99.42 & freshwater \\
\hline Mychonastes sp. & DB6-29 & AY543066 & 99.94 & freshwater \\
\hline Neocystis cf. mucosa & SAG 2405 & HQ287929 & 99.56 & terrestrial (soil) \\
\hline Neocystis cf. mucosa & SAG 2405 & HM565928 & 99.71 & $\mathrm{n} / \mathrm{a}$ \\
\hline Pseudendocloniopsis botryoides & SAG 2394 & AJ416102 & 99.76 & terrestrial (soil) \\
\hline Pseudendocloniopsis botryoides & SAG 2394 & FR865755 & 99.94 & freshwater \\
\hline Pseudendoclonium akinetum & SAG 2404 & AM087962 & 99.81 & freshwater \\
\hline Pseudendoclonium akinetum & SAG 2404 & AM109906 & 99.75 & freshwater \\
\hline Pseudendoclonium akinetum & SAG 2404 & DQ011230 & 99.94 & freshwater \\
\hline Pseudendoclonium basiliense & D9-14B & Z47996 & 99.82 & freshwater \\
\hline Pseudomuriella cf. schumacherensis & RK3 & FR865690/X91268 & 99.01 & freshwater/terrestrial (soil) \\
\hline Pseudomuriella cf. schumacherensis & RK3 & HQ292768 & 99.54 & terrestrial (epiphytic) \\
\hline Stichococcus bacillaris & WB74 & AJ311637 & 99.88 & freshwater \\
\hline Stichococcus bacillaris & WB13 & AJ311637 & 100 & freshwater \\
\hline Stichococcus cf. deasonii & WB38 & DQ275460 & 99.74 & terrestrial (soil) \\
\hline Stichococcus mirabilis & WB69 & AJ311638 & 100 & $\mathrm{n} / \mathrm{a}$ \\
\hline Stichococcus sp.1 & DB6-27 & AB055866 & 99.71 & $\mathrm{n} / \mathrm{a}$ \\
\hline Stichococcus sp.1 & DB6-27, WB65 & AB055866 & 100 & $\mathrm{n} / \mathrm{a}$ \\
\hline Stichococcus sp.2 & $\mathrm{D} 4-2 \mathrm{~A}$ & DQ275460 & 99.48 & terrestrial (soil) \\
\hline Stichococcus sp. 3 & SAG 2408 & DQ275460 & 98.60 & terrestrial (soil) \\
\hline Stichococcus sp.4 & SAG 2406 & DQ275460 & 99.01 & terrestrial (soil) \\
\hline
\end{tabular}

Legend. For each species, their closest available neighbouring sequences and pairwise sequence similarity (isolate/neighbouring sequence; in percentage calculated from $p$-distances) and the habitat from which the closest neighbours have been isolated are listed. The species are in two groups, i.e. one with high simililarities with neighbouring sequences (99.9 and $100.0 \%)$ so that they represent the same species as the isolate, the other with lower sequence similarities so that the neighbouring sequences do not represent the same species as the isolate. "n.a." represents for "not applicable" where origin is not known. 
Table S4. Sequences representing additional groups and lineages shown as collapsed triangles in Figures 1-3.

\begin{tabular}{|c|c|c|}
\hline Figure & Taxon & GenBank accession number(s) \\
\hline \multirow[t]{14}{*}{ Figure 1} & Chlorodendrophyceae & $\mathrm{X} 68484, \mathrm{X70802}$ \\
\hline & Chlorophyceae & DQ078295, AY779858, M62861, M32703, COU83125 \\
\hline & Choricystis/Botryococcus & AY195970, AY762605, AY197640, AJ581912 \\
\hline & Desmococcus & AJ431571, EU434017 \\
\hline & Hegewaldia & FM205843, FM205844 \\
\hline & Lobosphaera clade & AB006051, EU878372, EU878374 \\
\hline & Oocystaceae & AF387154, AF228686, AF228691, AF228690, AF228689 \\
\hline & Parachlorella-clade & AB176664, AB037085, Y17470, X56105, FM205845 \\
\hline & Prasiola/Prasiolopsis/Trichophilus & AJ416106, EF200524, AY762600, AY762601 \\
\hline & Pseudochlorella/Koliella & AB006050, X63520, U18520, AF514410 \\
\hline & Pseudomarvania & AB087559, FJ896222 \\
\hline & Trebouxiales clade & Z28971, M62995, AB080310, Z21551 \\
\hline & Ulvophyceae & AB183610, AY278217 \\
\hline & Watanabea clade & AJ439401, X73991, FM946000, EU346910, X73998, EF595524 \\
\hline \multirow[t]{25}{*}{ Figure 2} & Arenicolinia & AB218701, AY271673, AF513371 \\
\hline & Chaetopeltidales & U83126, U83125, U83124 \\
\hline & Chaetophorales & AF182820, AF182816, AF182821, D86499 \\
\hline & Characiosiphonia & AF395437, AF395435 \\
\hline & Chlorodendrophyceae & $X 68484, X 70802$ \\
\hline & Chlorogonia & AJ410443, U70589 \\
\hline & Chloromonadinia & U57696, AJ410448, U57697 \\
\hline & Crucicarteria & D86501, U70595 \\
\hline & Dunaliellinia & EU589200, DQ009743 \\
\hline & Golenkinia & AF499924, AF499923 \\
\hline & Hafniomonas & AB248251, D86496 \\
\hline & Jenufa & HM563743, HM563744, AB257660, AB257659 \\
\hline & Moewusinia & U70782, AY220571 \\
\hline & Monadinia & AF514404, U57694 \\
\hline & Nephroselmidophyceae & $X 75565, X 74754$ \\
\hline & Oedogoniales & DQ018735, U83135, U83132 \\
\hline & Oogamochlamydinia & AJ410461, AJ410464, AJ410466, AB175836, AB175837 \\
\hline & Phacotinia & AY009898, AF395438 \\
\hline & Polytominia & U22941, AJ781310, U13984, U13985 \\
\hline & Radicarteria & AF182817, AF182819 \\
\hline & Sphaeropleales & see Fig. 3 - Chlorophyceae which are not included in collapsed triangles \\
\hline & Tatrensinia & AF514407, EF023280, AF514411 \\
\hline & Trebouxiophyceae & AY762602, AB017435 \\
\hline & Treubarinia & AY008844, U73471, U73474, AF277651 \\
\hline & Ulvophyceae & AY278217, AB183610 \\
\hline \multirow[t]{13}{*}{ Figure 3} & Chaetopeltidales & U83126, U83125, U83124 \\
\hline & Chaetophorales & AF182820, AF182816, AF182821, D86499 \\
\hline & Chlamyodomonadales & see Fig.2 - Chlorophyceae which are not included in collapsed triangles \\
\hline & Chlorodendrophyceae & X68484, X70802 \\
\hline & Golenkinia & AF499924, AF499923 \\
\hline & Hydrodictyaceae & AY663044, AY663041, AY779858, M62997 \\
\hline & Jenufa & HM563743, HM563744, AB257660, AB257659 \\
\hline & Neochloridaceae & AF288361, AF288364, M62861, AJ581914, M63000 \\
\hline & Nephroselmidophyceae & $\mathrm{X} 75565, \mathrm{X} 74754$ \\
\hline & Oedogoniales & DQ018735, U83135, U83132 \\
\hline & Sphaeropleaceae & AF302770, AJU73469 \\
\hline & Trebouxiophyceae & AB017435, AY762602 \\
\hline & Ulvophyceae & AY278217, AB183610 \\
\hline
\end{tabular}




\section{Appendix | Chapter 3}

\section{Supporting Figures}

Figure S1. ITS2 secondary structure analysis of Chlorella vulgaris and C. pituita.

Figure S2. Analysis of compensatory base changes (CBCs) in ITS2 sequences of Chlorella-like species.

Figure S3. 18S ML phylogeny of Stichococcus-like species.

Figure S4. ITS2 secondary structure analysis of Stichococcus-like species.

Figure S5. Neighbor-joining tree of Stichococcus-like species based on ITS2 sequences/secondary structures.

Figure S6. Microphotographs of representatives of the Prasiola clade.

\section{Supporting Tables}

Table S1. List of all analyzed accessions (isolates, strains and clones) of the polar Chlorella-like species and relatives.

Table S2. List of all analyzed accessions (isolates, strains and clones) of the Stichococcus-like species and relatives.

Table S3. List of the analyzed Stichococcus-like clades and their representative strains. 


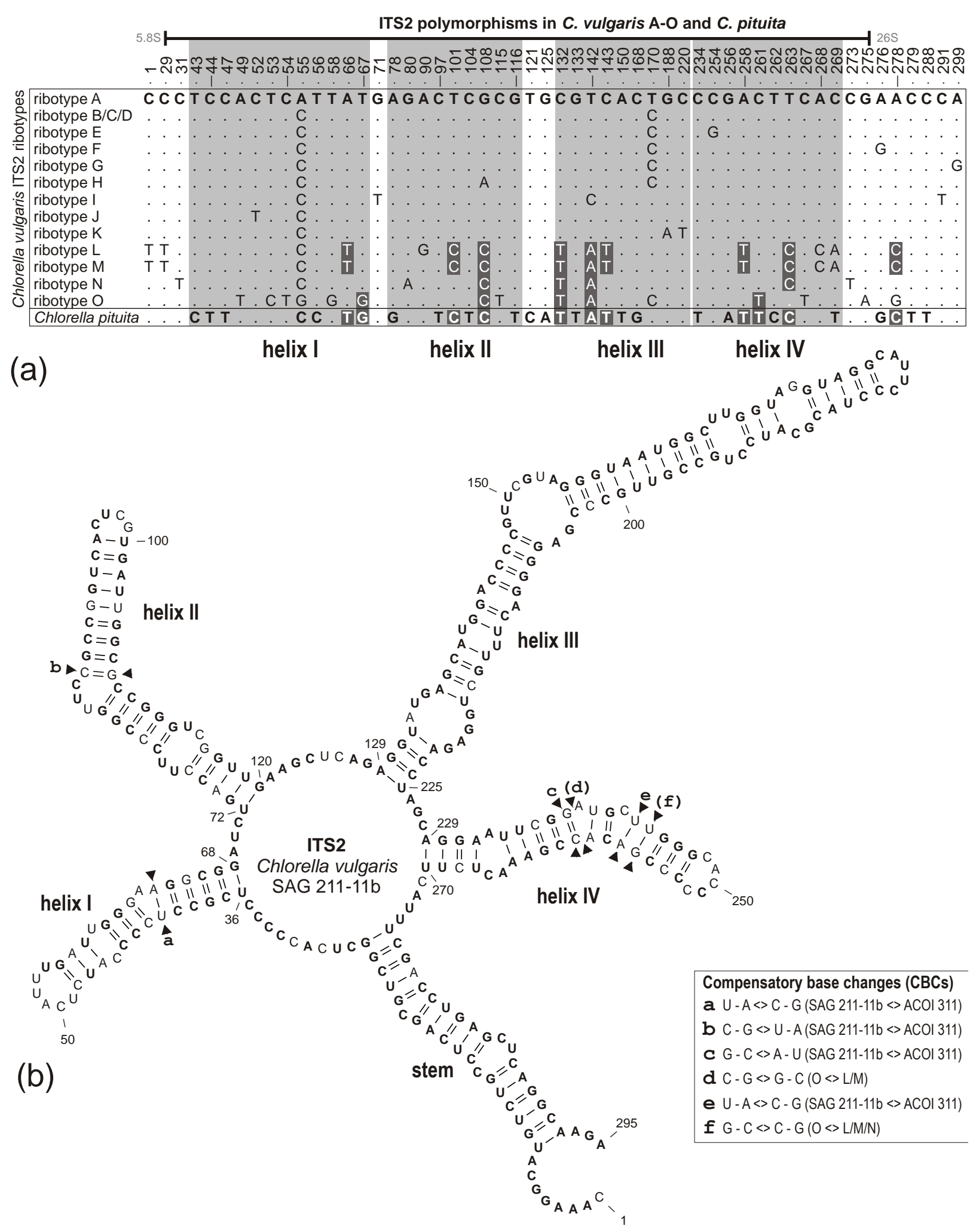

Figure S1. ITS2 secondary structure analysis of Chlorella vulgaris and C. pituita. (a) Polymorphic nucleotide sites detected in 13 ribotypes of $C$. vulgaris (ribotype $A=S A G$ 211-11b) and in C. pituita. (b) ITS2 secondary structure model of $C$. vulgaris SAG 21111b. Compensatory base changes (CBCs) between $C$. vulgaris and $C$. pituita (a, b, c,e) are marked by black triangles. Additional CBCs (d, f) among intraspecific ribotypes of $C$. vulgaris are shown as well. Bold letters represent nucleotide sites conserved in C. vulgaris and C. pituita. 


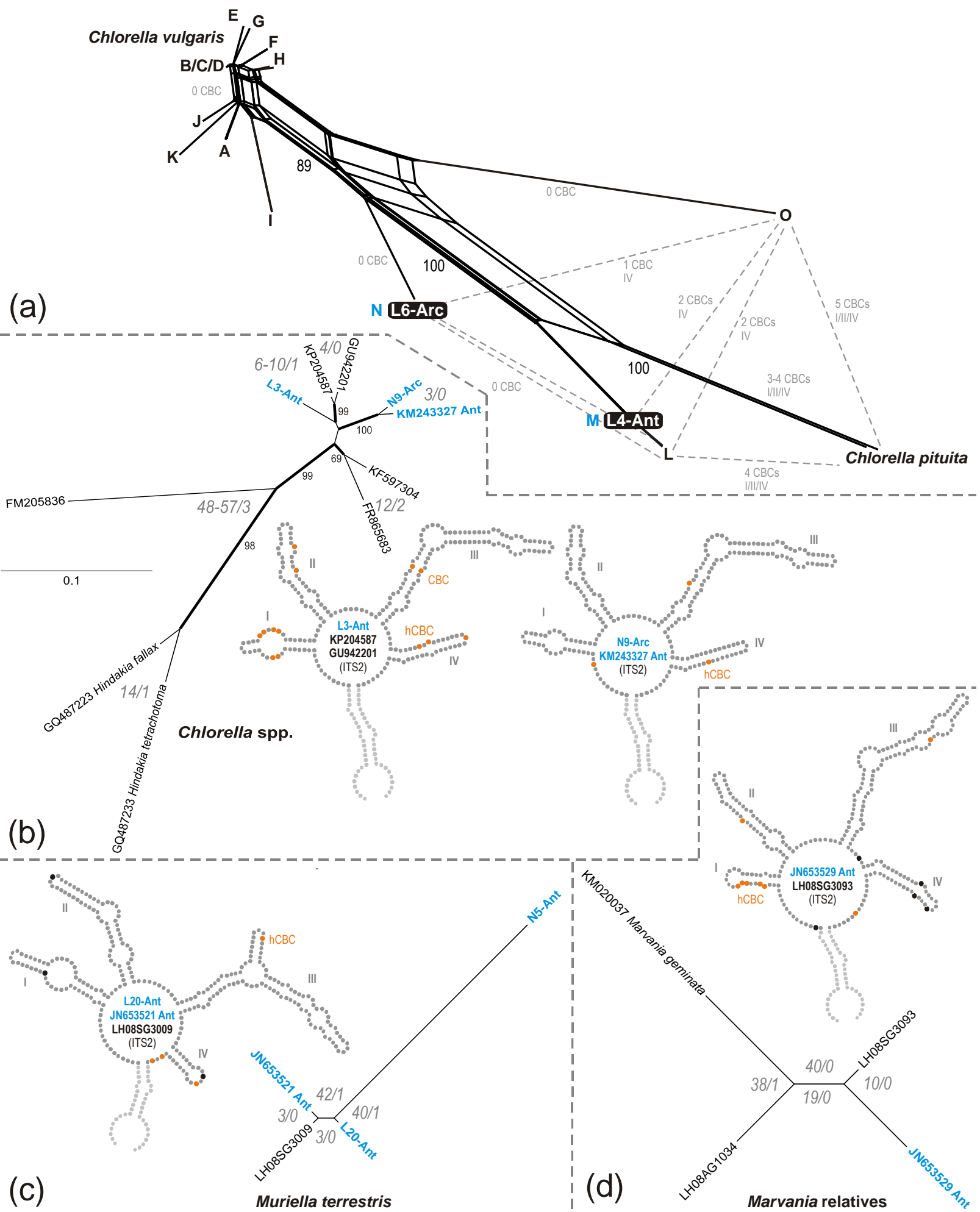

Figure S2. Analysis of compensatory and hemi-compensatory base changes (CBCs, hCBCs) in ITS2 sequences of Chlorellalike species. (a) Neighbor-net analysis of Chlorella vulgaris SAG 211-11b (ribotype A), other C. vulgaris ribotypes (B-O) and C. pituita ACOI 311 (Fig. S1a). The numbers close to the solid lines represent bootstrap support values; the numbers close to the dashed lines represent CBCs between two sequences (the respective helices (Fig. S1b) are given by Roman numerals). (b)-(d) Consensus secondary structures of closely related accessions of the Chlorellaceae: (b) Chlorella sp.1 and C. sp.2; neighborjoining tree (based on ITS2 sequences and secondary structures) and consensus ITS2 secondary structure of accessions closely related to the polar strains. (c) Muriella terrestris. (d) Marvania relatives. Nucleotide positions (represented as dots) within the ITS2 secondary structures are colored as follows: grey dots=conserved positions, orange dots=nucleotide substitutions and black dots=nucleotide deletions. Grey numbers between sequences in the NJ-trees give nucleotide differences and compensatory base changes (CBCs), respectively. 


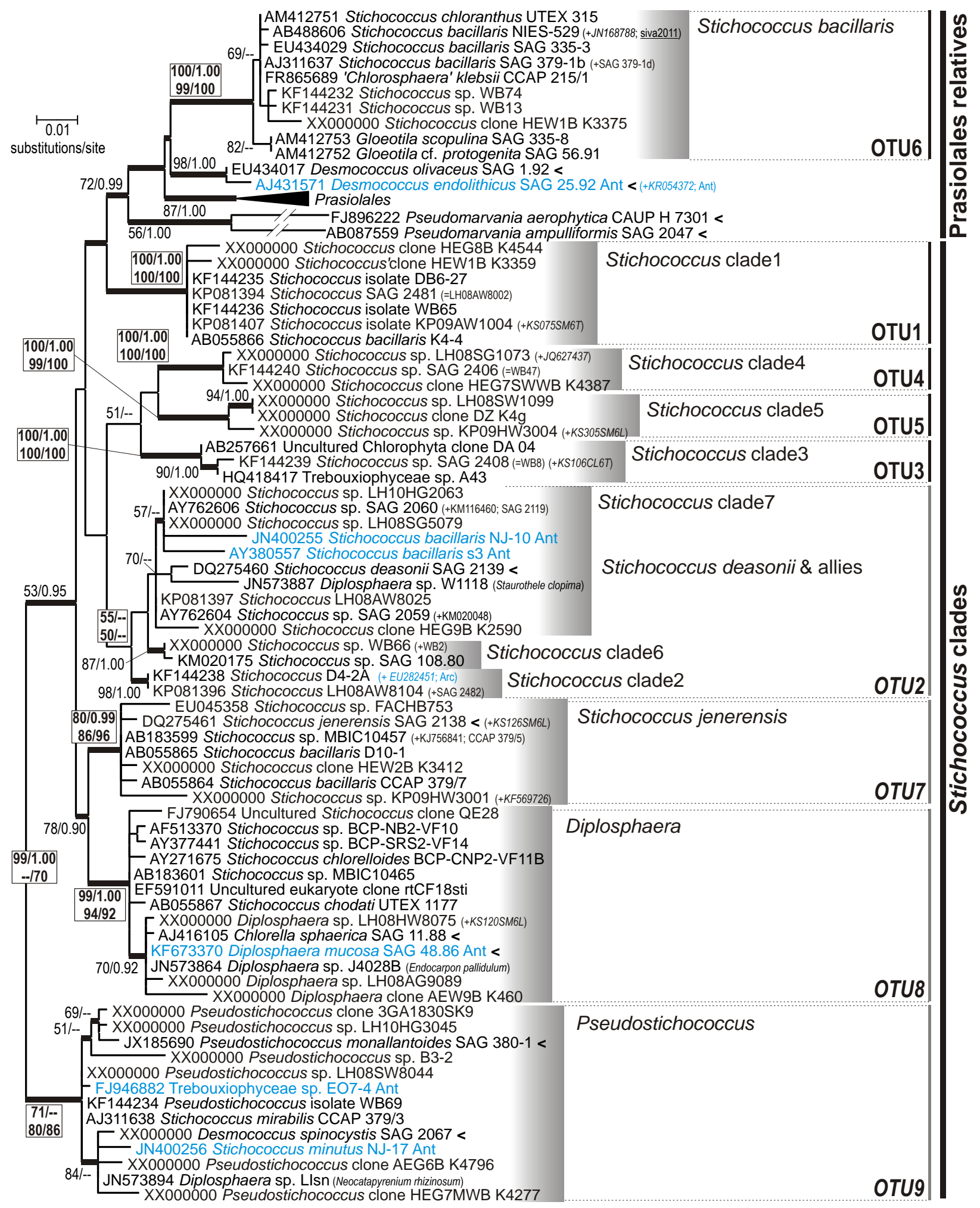

Figure S3. 18S ML phylogeny of Stichococcus-like species. Sequences of authentic strains are marked by a '<' sign. The numbers next to branches indicate statistical support values (maximum-likelihood bootstraps (ML)/Bayesian posterior probabilities $(\mathrm{BI})$ ); the clades of particular interest were additionally tested via maximum parsimony (MP) and bio-neighbor-joining $(\mathrm{NJ})$ and the statistic support values are given in the following order: ML/BI/MP/NJ. Assignations into operational taxonomic units are based on sequence similarities $\geq 99.5 \%$ (= OTU) and $\geq 99.0 \%$ (= OTU). 


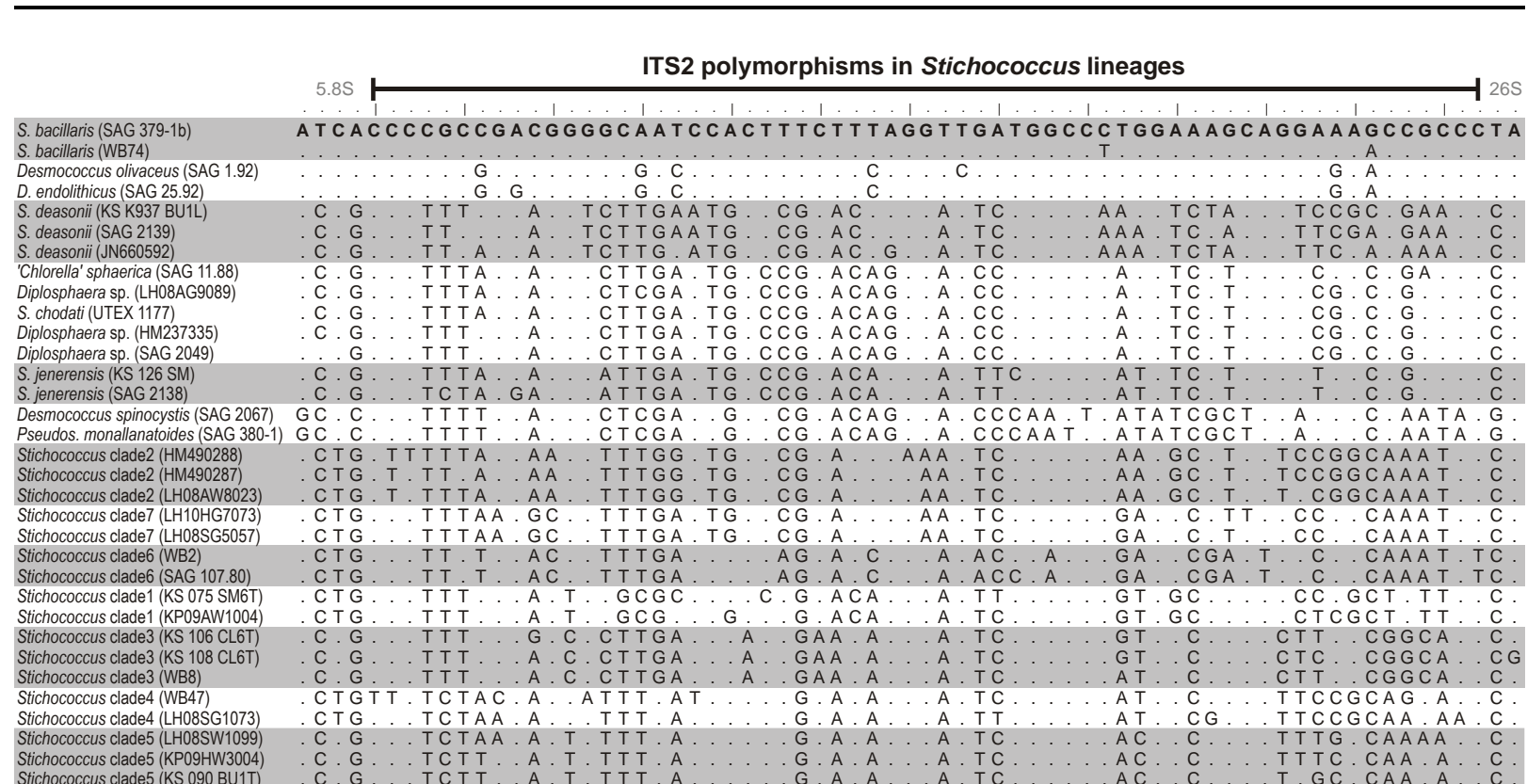

(a)

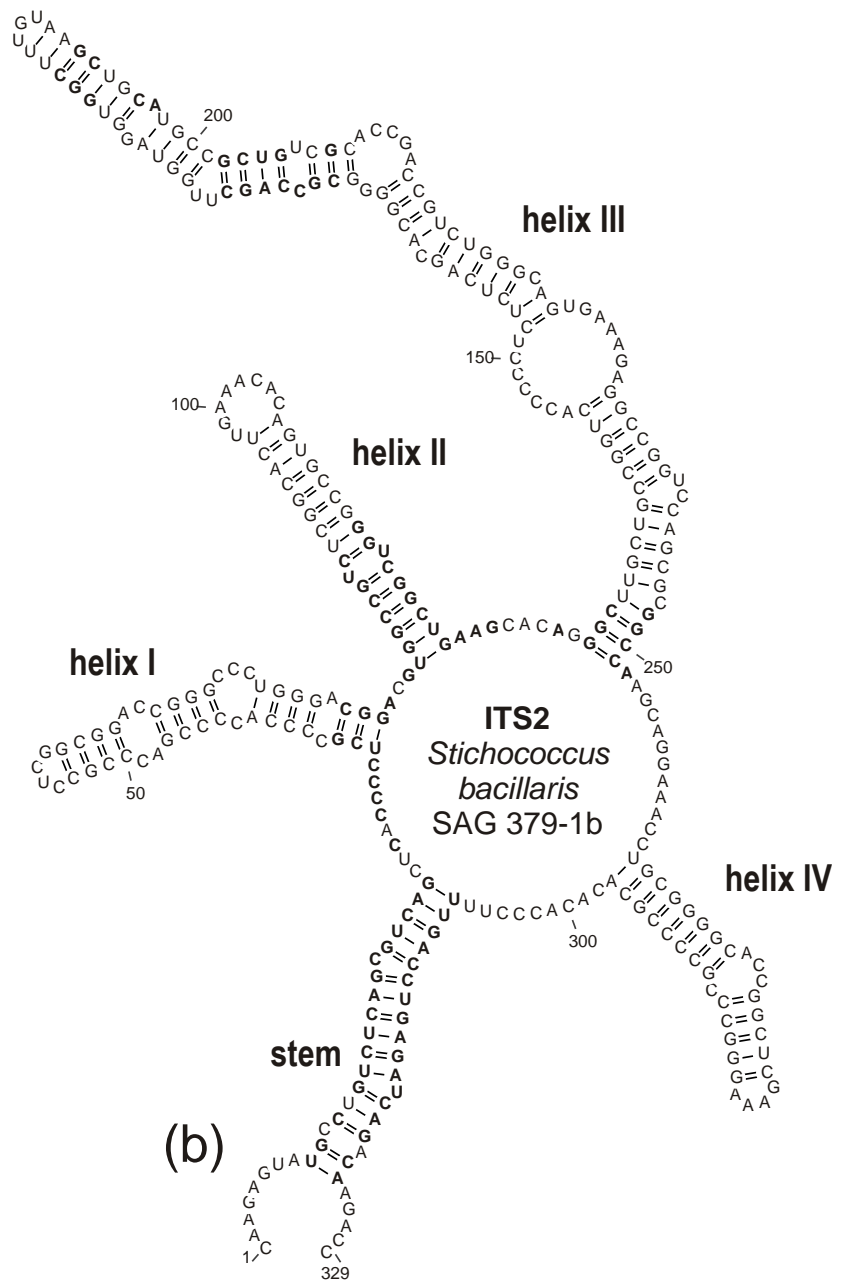

Figure S4. ITS2 secondary structure analysis of Stichococcus-like species. (a) Polymorphic nucleotide sites detected in 33 ribotypes of Stichococcus-like species and allies. (b) ITS2 secondary structure model of S. bacillaris SAG 379-1b. Bold letters represent nucleotide sites which are conserved across all 56 analyzed ITS2 accessions. 


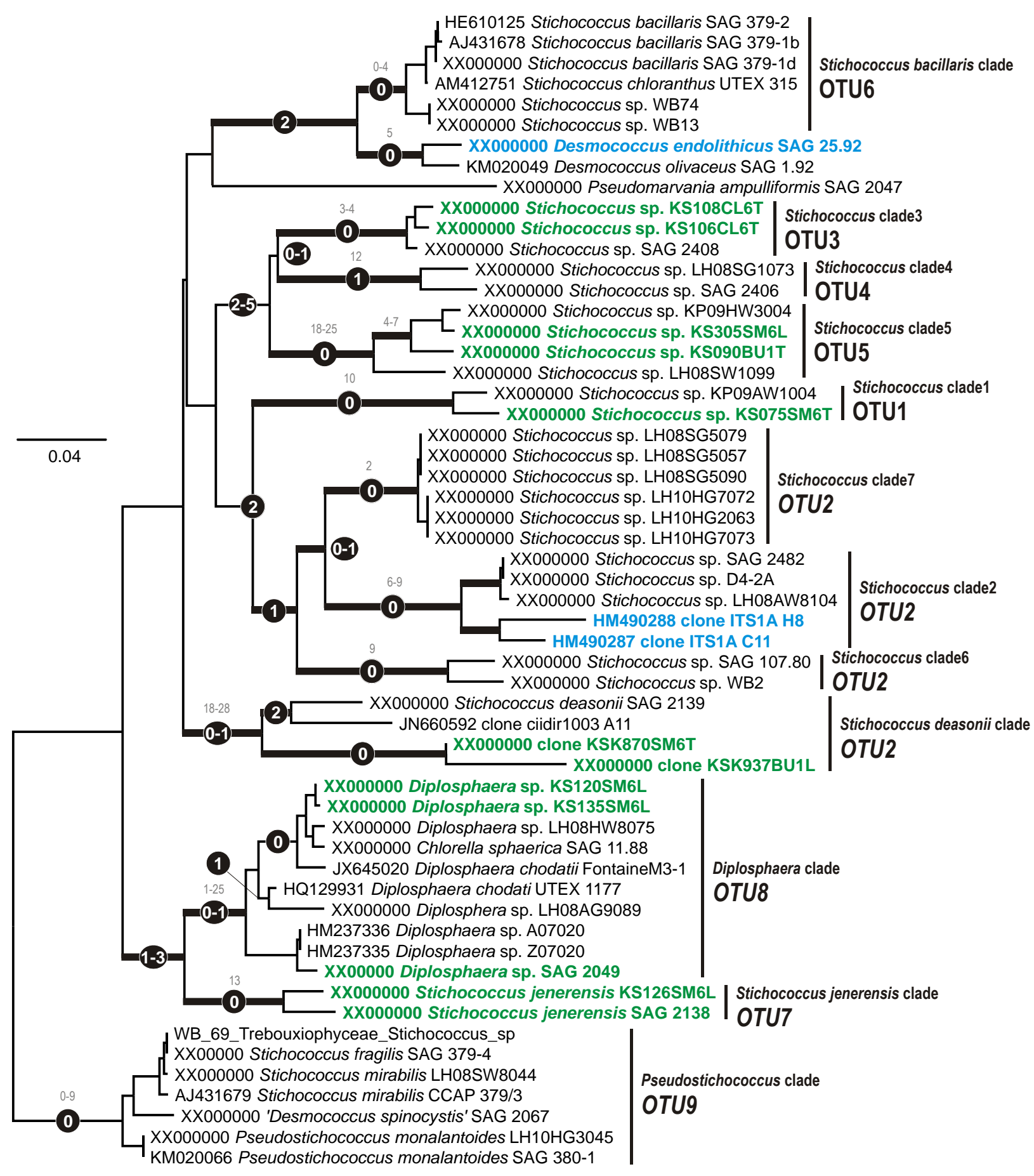

Figure S5. Neighbor-joining tree of Stichococcus-like species based on ITS2 sequences/secondary structures. Thick lines indicate bootstrap support values $\geq 80$. Compensatory base changes (CBCs) detected within clades are given on branches as white numbers in black circles; the grey numbers above CBCs give nucleotide differences detected within clades. The polar and tropical accessions are blue and green colored, respectively. 

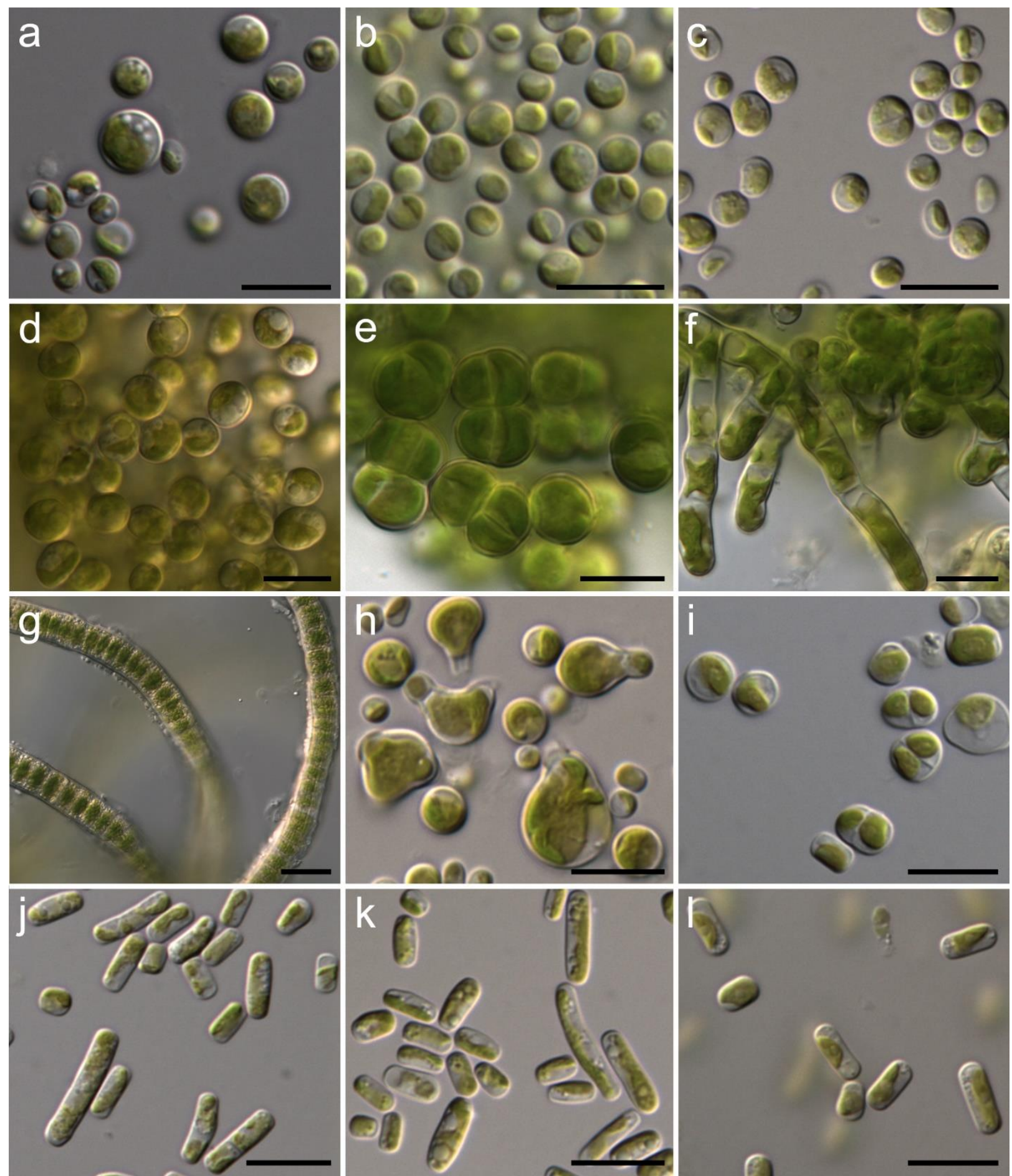

Figure S6. Microphotographs of representatives of the Prasiola clade. (a) 'Chlorella' mirabilis SAG 38.88; (b) 'Chlorella' sphaerica SAG 11.88; (c) Diplosphaera sp. SAG 2049; (d) 'Desmococcus' spinocystis SAG 2067; (e) Desmococcus olivaceus SAG 1.92; (f) Prasiolopsis ramosa SAG 26.82; (g) Prasola crispa SAG 43.96; (h) Pseudomarvania ampulliformis SAG 2047; (i) Stichococcus jenerensis SAG 2138; (j) Pseudostichococcus monallantoides SAG 380-1; (k) Stichococcus fragilis SAG 379-4; (I) Stichococcus deasonii SAG 2139. 
Table S1. List of all analyzed accessions (isolates, strains and clones) of the polar Chlorella-like species and relatives.

\begin{tabular}{|c|c|c|c|c|c|c|c|}
\hline Clade & Species & Identifier & $18 \mathrm{~S}$ & ITS2 & GPS & Land (region) & Habitat \\
\hline Chlorella s.str. & Chlorella pituita & ACOI 311 & GQ176852 & GQ176853 & - & PT (Mira) & $\begin{array}{l}\text { freshwater (trout } \\
\text { nursery) }\end{array}$ \\
\hline Chlorella s.str. & Chlorella pituita & ACOI 856 & FM205855 & FM205856 & - & PT (Manteigas) & freshwater \\
\hline Chlorella s.str. & Chlorella vulgaris & 3GSG1R K22 & this study & $\mathrm{n} / \mathrm{a}$ & $\begin{array}{l}\text { N5052'0.000" } \\
\text { E10 } 50^{\circ} 0.000^{\prime \prime}\end{array}$ & $\begin{array}{l}\text { DE (Castle } \\
\text { Gleichen) }\end{array}$ & $\begin{array}{l}\text { subaerial } \\
\text { (epilithic; } \\
\text { sandstone) }\end{array}$ \\
\hline Chlorella s.str. & Chlorella vulgaris & CCAP 211/79 & FR865683 & FR865683 & - & UK (Edinburgh) & $\begin{array}{l}\text { waste solvent } \\
\text { biofilter }\end{array}$ \\
\hline Chlorella s.str. & Chlorella vulgaris & DB1-10 & KF144171 & this study & & $\begin{array}{l}\text { DE (Deinschwanger } \\
\text { Bach) }\end{array}$ & $\begin{array}{l}\text { semi-terrestrial } \\
\text { (creek biofilm) }\end{array}$ \\
\hline Chlorella s.str. & Chlorella vulgaris & $\begin{array}{l}\text { HEG7SWWB } \\
\text { K4396 }\end{array}$ & this study & $\mathrm{n} / \mathrm{a}$ & $\begin{array}{l}\text { N51 }{ }^{\circ} 16^{\prime} 24.897^{\prime \prime} \\
\text { E10²4'37.485" }\end{array}$ & DE (Hainich-Dün) & soil (grassland) \\
\hline Chlorella s.str. & Chlorella vulgaris & L1 & this study & $\mathrm{n} / \mathrm{a}$ & $\begin{array}{l}S 62^{\circ} 10^{\prime} 0.000^{\prime \prime} \\
\text { W58 } \\
{ }^{\circ} 30^{\prime} 0.000^{\prime \prime}\end{array}$ & $\begin{array}{l}\mathrm{AQ} \text { (King George } \\
\text { Island) }\end{array}$ & soil (deglaciated) \\
\hline Chlorella s.str. & Chlorella vulgaris & L5 & this study & $\mathrm{n} / \mathrm{a}$ & $\begin{array}{l}\text { N79 } 08^{\prime} 0.000^{\prime \prime} \\
\text { W80 } 30^{\prime} 0.000^{\prime \prime}\end{array}$ & $\begin{array}{l}\text { CA (Ellesmere } \\
\text { Island) }\end{array}$ & $\begin{array}{l}\text { soil (close to } \\
\text { river) }\end{array}$ \\
\hline Chlorella s.str. & Chlorella vulgaris & NJ-7 & DQ377323 & $\mathrm{n} / \mathrm{a}$ & - & $\begin{array}{l}\mathrm{AQ} \text { (Zhongshan } \\
\text { Station) }\end{array}$ & $\begin{array}{l}\text { terrestrial (wet } \\
\text { rocks) }\end{array}$ \\
\hline Chlorella s.str. & Chlorella vulgaris A & $\begin{array}{l}\text { CCAP } \\
211 / 11 \mathrm{~B}\end{array}$ & $\mathrm{n} / \mathrm{a}$ & AY591507 & - & NL (Delft) & freshwater \\
\hline Chlorella s.str. & Chlorella vulgaris A & CCAP 254/5 & FR865696 & FR865697 & - & $\begin{array}{l}\text { US (Bloomington, } \\
\text { Indiana) }\end{array}$ & $\begin{array}{l}\text { freshwater } \\
\text { (aquarium) }\end{array}$ \\
\hline Chlorella s.str. & Chlorella vulgaris A & SAG 211-11b & FM205832 & FM205832 & - & NL (Delft) & freshwater \\
\hline Chlorella s.str. & Chlorella vulgaris B & CCAP 211/110 & FN298918 & FN298918 & - & US (n/a) & $\begin{array}{l}\text { freshwater } \\
\text { (Paramecium } \\
\text { symbiont) }\end{array}$ \\
\hline Chlorella s.str. & Chlorella vulgaris B & CCAP 211/74 & FR865682 & FR865682 & - & UK (Cumbria) & $\begin{array}{l}\text { freshwater } \\
\text { (Esthwaite Water) }\end{array}$ \\
\hline Chlorella s.str. & Chlorella vulgaris B & CCAP 211/80 & FM205853 & FM205853 & - & DE (Elsnigk) & $\begin{array}{l}\text { freshwater } \\
\text { (Molkerteich) }\end{array}$ \\
\hline Chlorella s.str. & Chlorella vulgaris B & CCAP 211/81 & FM205854 & FM205854 & - & DE (Trebbichau) & $\begin{array}{l}\text { freshwater } \\
\text { (Salzteich) }\end{array}$ \\
\hline Chlorella s.str. & Chlorella vulgaris B & LH08HG4032 & this study & this study & $\begin{array}{l}\text { N51 }{ }^{\circ} 6^{\prime} 48.104^{\prime \prime} \\
\text { E10²6'10.249" }\end{array}$ & DE (Hainich-Dün) & soil (grassland) \\
\hline Chlorella s.str. & Chlorella vulgaris B & LH08HG4088 & this study & this study & $\begin{array}{l}\text { N51 }{ }^{\circ} 6^{\prime} 48.104^{\prime \prime} \\
\text { E10² } 26^{\prime} 10.249^{\prime \prime}\end{array}$ & DE (Hainich-Dün) & soil (grassland) \\
\hline Chlorella s.str. & Chlorella vulgaris B & LH08HG5074 & this study & this study & $\begin{array}{l}\text { N51 }{ }^{\circ} 12^{\prime} 57.220^{\prime \prime} \\
\text { E10¹9'21.096" }\end{array}$ & DE (Hainich-Dün) & soil (grassland) \\
\hline Chlorella s.str. & Chlorella vulgaris B & LH08HW9094 & this study & this study & $\begin{array}{l}\text { N51 }{ }^{\circ} 7 ' 48.871^{\prime \prime} \\
\text { E10²2'52.139" }\end{array}$ & DE (Hainich-Dün) & soil (forest) \\
\hline Chlorella s.str. & Chlorella vulgaris $\mathrm{B}$ & LH08SG1071 & this study & this study & $\begin{array}{l}\text { N53 }{ }^{\circ} 5^{\prime} 14.712^{\prime \prime} \\
\text { E135ㅇ'10.717" }\end{array}$ & $\begin{array}{l}\text { DE (Schorfheide- } \\
\text { Chorin) }\end{array}$ & soil (grassland) \\
\hline Chlorella s.str. & Chlorella vulgaris B & LH08SG3006 & this study & this study & $\begin{array}{l}\text { N53 }{ }^{\circ} 6^{\prime} 10.204^{\prime \prime} \\
\text { E13 } 59^{\prime} 8.519^{\prime \prime}\end{array}$ & $\begin{array}{l}\text { DE (Schorfheide- } \\
\text { Chorin) }\end{array}$ & soil (grassland) \\
\hline Chlorella s.str. & Chlorella vulgaris B & LH10HG2049 & this study & this study & $\begin{array}{l}\text { N51 }{ }^{\circ} 0^{\prime} 2.696 " 1 \\
\text { E10 } 25^{\prime} 48.036^{\prime \prime}\end{array}$ & DE (Hainich-Dün) & soil (grassland) \\
\hline Chlorella s.str. & Chlorella vulgaris B & LH10HG6014 & this study & this study & $\begin{array}{l}\text { N51 }{ }^{\circ} 12^{\prime} 53.766^{\prime \prime} \\
\text { E10²3'28.395" }\end{array}$ & DE (Hainich-Dün) & soil (grassland) \\
\hline Chlorella s.str. & Chlorella vulgaris B & LH10HG6019 & this study & this study & $\begin{array}{l}\text { N51 }{ }^{\circ} 12^{\prime} 53.766^{\prime \prime} \\
\text { E10²3'28.395" }\end{array}$ & DE (Hainich-Dün) & soil (grassland) \\
\hline Chlorella s.str. & Chlorella vulgaris $\mathrm{B}$ & LH10HG9075 & this study & this study & $\begin{array}{l}\text { N51 }{ }^{\circ} 13^{\prime} 26.031^{\prime \prime} \\
\text { E10²2'50.834" }\end{array}$ & DE (Hainich-Dün) & soil (grassland) \\
\hline Chlorella s.str. & Chlorella vulgaris B & NB-1 & $n / a$ & KC840685 & - & $\mathrm{CN} ?$ & $\mathrm{n} / \mathrm{a}$ \\
\hline Chlorella s.str. & Chlorella vulgaris $\mathrm{E}$ & SAG 9.88 & $\mathrm{n} / \mathrm{a}$ & AY591500 & - & ES (Madrid) & $\begin{array}{l}\text { freshwater } \\
\text { (waste-water) }\end{array}$ \\
\hline Chlorella s.str. & Chlorella vulgaris $\mathrm{F}$ & $\begin{array}{l}\text { CCAP } \\
211 / 11 P\end{array}$ & FR865658 & FR865658 & - & SE (Lund) & $\begin{array}{l}\text { freshwater (pond } \\
\text { in park) }\end{array}$ \\
\hline Chlorella s.str. & Chlorella vulgaris $\mathrm{F}$ & SAG 211-11p & $\mathrm{n} / \mathrm{a}$ & AY591505 & - & SE (Lund) & $\begin{array}{l}\text { freshwater (pond } \\
\text { in park) }\end{array}$ \\
\hline Chlorella s.str. & Chlorella vulgaris $\mathrm{G}$ & TW-1 & $\mathrm{n} / \mathrm{a}$ & KC840684 & - & $\mathrm{CN}(?)$ & $\mathrm{n} / \mathrm{a}$ \\
\hline Chlorella s.str. & Chlorella vulgaris $\mathrm{H}$ & CCAP 211/82 & FM205855 & FM205855 & - & DE (Micheln) & freshwater \\
\hline Chlorella s.str. & Chlorella vulgaris $\mathrm{H}$ & LH08HG5082 & this study & this study & $\begin{array}{l}\text { N51 }{ }^{\circ} 12^{\prime} 57.220^{\prime \prime} \\
\text { E10¹9'21.096" }\end{array}$ & DE (Hainich-Dün) & soil (grassland) \\
\hline Chlorella s.str. & Chlorella vulgaris $\mathrm{H}$ & LH10HG6052 & this study & this study & $\begin{array}{l}\text { N51 }{ }^{\circ} 12^{\prime} 53.766^{\prime \prime} \\
\text { E10²3'28.395" }\end{array}$ & DE (Hainich-Dün) & soil (grassland) \\
\hline Chlorella s.str. & Chlorella vulgaris I & CCAP 211/63 & FR865681 & FR865681 & - & UK (Cambridge) & $\begin{array}{l}\text { freshwater } \\
\text { (Peterhouse } \\
\text { Ditch) }\end{array}$ \\
\hline Chlorella s.str. & Chlorella vulgaris I & LH08HG1081 & this study & this study & $\begin{array}{l}\text { N5058'17.934" } \\
\text { E10²4'19.306" }\end{array}$ & DE (Hainich-Dün) & soil (grassland) \\
\hline Chlorella s.str. & Chlorella vulgaris J & CCAP 211/109 & FN298917 & FN298917 & & US & $\begin{array}{l}\text { freshwater } \\
\text { (Paramecium } \\
\text { symbiont) }\end{array}$ \\
\hline
\end{tabular}


Table S1. (continuation)

\begin{tabular}{|c|c|c|c|c|c|c|c|}
\hline Clade & Species & Identifier & $18 \mathrm{~S}$ & ITS2 & GPS & Land (region) & Habitat \\
\hline Chlorella s.str. & Chlorella vulgaris $\mathrm{K}$ & D2 & JX185297 & JX185298 & - & $\mathrm{CN}(?)$ & $\mathrm{n} / \mathrm{a}$ \\
\hline Chlorella s.str. & Chlorella vulgaris $\mathrm{L}$ & LH10HG2067 & this study & this study & 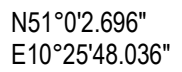 & DE (Hainich-Dün) & soil (grassland) \\
\hline Chlorella s.str. & Chlorella vulgaris L & LH10HG2081 & this study & this study & $\begin{array}{l}\mathrm{N} 51^{\circ} 0^{\prime} 2.696^{\prime \prime} \\
\mathrm{E} 10^{\circ} 25^{\prime} 48.036 "\end{array}$ & DE (Hainich-Dün) & soil (grassland) \\
\hline Chlorella s.str. & Chlorella vulgaris M & L4 & this study & this study & $\begin{array}{l}\mathrm{S} 62^{\circ} 10^{\prime} 0.000^{\prime \prime} \\
\text { W58 } 30^{\circ} 0.000^{\prime \prime}\end{array}$ & $\begin{array}{l}\text { AQ (King George } \\
\text { Island) }\end{array}$ & soil (deglaciated) \\
\hline Chlorella s.str. & Chlorella vulgaris $\mathrm{N}$ & L6 & this study & this study & $\begin{array}{l}\text { N7908'0.000" } \\
\text { W80 } 30^{\circ} 0.000^{\prime \prime}\end{array}$ & $\begin{array}{l}\text { CA (Ellesmere } \\
\text { Island) }\end{array}$ & soil (moraine) \\
\hline Chlorella s.str. & Chlorella vulgaris 0 & LH10HG1069 & this study & this study & $\begin{array}{l}\text { N5058'17.934" } \\
\text { E10²4'19.306" }\end{array}$ & DE (Hainich-Dün) & soil (grassland) \\
\hline Chlorella sp. & Chlorella sp. & KNUA034 & KM243327 & KM243327 & - & $\mathrm{AQ}$ & freshwater \\
\hline Chlorella sp. & Chlorella sp.2 & L3 & this study & this study & $\begin{array}{l}S 62^{\circ} 10^{\prime} 0.000^{\prime \prime} \\
\text { W5 } 58^{\circ} 30^{\prime} 0.000^{\prime \prime}\end{array}$ & $\begin{array}{l}\text { AQ (King George } \\
\text { Island) }\end{array}$ & soil (deglaciated) \\
\hline Chlorella sp. & Chlorella sp.1 & N9 & this study & this study & $\begin{array}{l}\mathrm{N} 79^{\circ} 58^{\prime} 0.000^{\prime \prime} \\
\mathrm{E} 11^{\circ} 21^{\prime} 0.000^{\prime \prime}\end{array}$ & NO (Svalbard) & soil (deglaciated) \\
\hline Chlorella sp. & Chlorella sp. & NDem 9/21 & AY197628 & $\mathrm{n} / \mathrm{a}$ & - & US & freshwater \\
\hline Chlorella sp. & Chlorella sp. & NJ-18 & DQ377324 & $\mathrm{n} / \mathrm{a}$ & - & $\begin{array}{l}\mathrm{AQ} \text { (Zhongshan } \\
\text { Station) }\end{array}$ & $\begin{array}{l}\text { terrestrial (wet } \\
\text { rocks) }\end{array}$ \\
\hline Chlorella sp. & Chlorella sp. & NMX37N & JF767012 & $\mathrm{n} / \mathrm{a}$ & $\begin{array}{l}N 41.48 \\
\text { E112.63 }\end{array}$ & $\mathrm{CN} ?$ & freshwater \\
\hline Chlorella sp. & Chlorella sp. & RK52 & KF144182 & $\mathrm{n} / \mathrm{a}$ & - & $\begin{array}{l}\text { DE (Deinschwanger } \\
\text { Bach) }\end{array}$ & freshwater biofilm \\
\hline Chlorella sp. & Chlorella sp. & VPL9-6 & FJ946888 & $\mathrm{n} / \mathrm{a}$ & - & $\begin{array}{l}A Q \text { (Trinity } \\
\text { Peninsula) }\end{array}$ & freshwater \\
\hline Chlorella sp. & Chlorellaceae & MCWWW9 & KP204587 & KP204587 & - & CA (Nova Scotia) & $\begin{array}{l}\text { freshwater } \\
\text { (wastewater) }\end{array}$ \\
\hline Chlorella sp. & Chlorellaceae & N505T04 & GU942201 & GU942201 & - & $\begin{array}{l}\text { CN (South China } \\
\text { Sea) }\end{array}$ & marine \\
\hline Chlorella sp. & $\begin{array}{l}\text { Micractinium } \\
\text { inerimum }\end{array}$ & NLP-F014 & KF597304 & KF597304 & 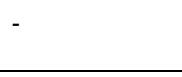 & $\mathrm{KR}$ & $\begin{array}{l}\text { freshwater } \\
\text { (wastewater) }\end{array}$ \\
\hline Chlorellaceae & Chlorellaceae & L24 & this study & $\mathrm{n} / \mathrm{a}$ & $\begin{array}{l}\text { S62 }{ }^{\circ} 10^{\prime} 0.000^{\prime \prime} \\
\text { W5 } 8^{\circ} 30^{\prime} 0.000^{\prime \prime}\end{array}$ & $\begin{array}{l}\text { AQ (King George } \\
\text { Island) }\end{array}$ & soil (deglaciated) \\
\hline Hindakia & Hindakia fallax & CCAP 222/29 & GQ487223 & GQ487223 & - & - & - \\
\hline Hindakia & $\begin{array}{l}\text { Hindakia } \\
\text { tetrachotoma }\end{array}$ & CCAP 222/80 & GQ487233 & GQ487233 & - & - & - \\
\hline Micractinium & $\begin{array}{l}\text { Micractinium } \\
\text { pusillum }\end{array}$ & CCAP 248/5 & FM205836 & FM205836 & - & - & - \\
\hline Muriella & Chlorophyta & clone WIM107 & AM114820 & $\mathrm{n} / \mathrm{a}$ & - & - & - \\
\hline Muriella & Muriella sp. & AS 2-4 & AY195969 & $\mathrm{n} / \mathrm{a}$ & - & - & - \\
\hline Muriella & Muriella terrestris & AEG6B K4797 & this study & $\mathrm{n} / \mathrm{a}$ & $\begin{array}{l}\text { N48 } 23^{\circ} 52.818^{\prime \prime} \\
E 9^{\circ} 20^{\prime} 31.152^{\prime \prime}\end{array}$ & $\begin{array}{l}\text { DE (Schwäbische } \\
\text { Alb) }\end{array}$ & soil (grassland) \\
\hline Muriella & Muriella terrestris & ASIB V38 & AB012845 & $\mathrm{n} / \mathrm{a}$ & - & - & - \\
\hline Muriella & Muriella terrestris & $\begin{array}{l}\text { clone Ant } \\
8 / 104\end{array}$ & $\mathrm{n} / \mathrm{a}$ & JN653521 & - & $A Q$ & soil (permafrost) \\
\hline Muriella & Muriella terrestris & D6-DB2 & KF144209 & $\mathrm{n} / \mathrm{a}$ & - & - & - \\
\hline Muriella & Muriella terrestris & L20 & this study & this study & $\begin{array}{l}S 62^{\circ} 10^{\prime} 0.000^{\prime \prime} \\
\text { W58 } 30^{\circ} 0.000^{\prime \prime}\end{array}$ & $\begin{array}{l}A Q \text { (King George } \\
\text { Island) }\end{array}$ & soil (deglaciated) \\
\hline Muriella & Muriella terrestris & LH08SG3009 & this study & this study & 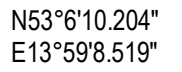 & $\begin{array}{l}\text { DE (Schorfheide- } \\
\text { Chorin) }\end{array}$ & soil (grassland) \\
\hline Muriella & Muriella terrestris & LH10HG9077 & this study & $\mathrm{n} / \mathrm{a}$ & $\begin{array}{l}\text { N51 }{ }^{\circ} 13^{\prime} 26.031^{\prime \prime} \\
\text { E10²2'50.834" }\end{array}$ & DE (Hainich-Dün) & soil (grassland) \\
\hline Muriella & Muriella terrestris & N5 & this study & this study & $\begin{array}{l}S 67^{\circ} 34^{\prime} 0.000^{\prime \prime} \\
\text { W68 } 08^{\circ} 0.000^{\prime \prime}\end{array}$ & $\begin{array}{l}\mathrm{AQ} \text { (Adelaide } \\
\text { Island) }\end{array}$ & soil (rookeries) \\
\hline Nannochloris-like & Marvania geminata & SAG 12.88 & AF124336 & KM020037 & - & - & - \\
\hline Nannochloris-like & Chlorella sp. & 193-GA188 & EU282456 & $\mathrm{n} / \mathrm{a}$ & - & $\begin{array}{l}\text { RU (Siberia, } \\
\text { Kolyma) }\end{array}$ & soil (permafrost) \\
\hline Nannochloris-like & Marvania relative1 & $\begin{array}{l}\text { clone Ant } \\
8 / 117\end{array}$ & $\mathrm{n} / \mathrm{a}$ & JN653529 & 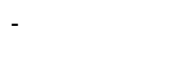 & $A Q$ & soil (permafrost) \\
\hline Nannochloris-like & Marvania relative & $\begin{array}{l}\text { HEG9 B } \\
\text { K25502 }\end{array}$ & this study & $\mathrm{n} / \mathrm{a}$ & $\begin{array}{l}\text { N51 } 13^{\circ} 26.031^{\prime \prime} \\
\text { E10²2'50.834" }\end{array}$ & DE (Hainich-Dün) & soil (grassland) \\
\hline Nannochloris-like & Marvania relative1 & L13 & this study & $\mathrm{n} / \mathrm{a}$ & $\begin{array}{l}\mathrm{S} 62^{\circ} 10^{\prime} 0.000^{\prime \prime} \\
\text { W58 } 30^{\circ} 0.000^{\prime \prime}\end{array}$ & $\begin{array}{l}\text { AQ (King George } \\
\text { Island) }\end{array}$ & soil (deglaciated) \\
\hline Nannochloris-like & Marvania relative1 & L14 & this study & $\mathrm{n} / \mathrm{a}$ & $\begin{array}{l}S 62^{\circ} 10^{\prime} 0.000^{\prime \prime} \\
\text { W5 } 8^{\circ} 30^{\prime} 0.000^{\prime \prime}\end{array}$ & $\begin{array}{l}\text { AQ (King George } \\
\text { Island) }\end{array}$ & soil (deglaciated) \\
\hline Nannochloris-like & Marvania relative1 & L15 & this study & $\mathrm{n} / \mathrm{a}$ & $\begin{array}{l}S 67^{\circ} 36^{\prime} 0.000^{\prime \prime} \\
\text { W68 } 68^{\circ} 15^{\prime} 0.000^{\prime \prime}\end{array}$ & $\begin{array}{l}\mathrm{AQ} \text { (Anchorage } \\
\text { Island) }\end{array}$ & soil (bare ground) \\
\hline Nannochloris-like & Marvania relative1 & L23 & this study & $\mathrm{n} / \mathrm{a}$ & $\begin{array}{l}S 67^{\circ} 32^{\prime} 0.000^{\prime \prime} \\
\text { W68 } 07^{\circ} 0.000^{\prime \prime}\end{array}$ & $\begin{array}{l}A Q \text { (Killingbeck } \\
\text { Island) }\end{array}$ & $\begin{array}{l}\text { endozoic (gut } \\
\text { content; } \\
\text { Cryptopygus) }\end{array}$ \\
\hline Nannochloris-like & Marvania relative1 & L32 & this study & $\mathrm{n} / \mathrm{a}$ & $\begin{array}{l}S 62^{\circ} 10^{\prime} 0.000^{\prime \prime} \\
\text { W58 } 38^{\circ} 0.000^{\prime \prime}\end{array}$ & $\begin{array}{l}\text { AQ (King George } \\
\text { Island) }\end{array}$ & soil (deglaciated) \\
\hline
\end{tabular}


Table S1. (continuation)

\begin{tabular}{|c|c|c|c|c|c|c|c|}
\hline Clade & Species & Identifier & $18 \mathrm{~S}$ & ITS2 & GPS & Land (region) & Habitat \\
\hline Nannochloris-like & Marvania relative2 & LH08AG1034 & this study & this study & $\begin{array}{l}\text { N48ㅇ2'ㄴ.818" } \\
E 9^{\circ} 20^{\prime} 31.152^{\prime \prime}\end{array}$ & $\begin{array}{l}\text { DE (Schwäbische } \\
\text { Alb) }\end{array}$ & soil (grassland) \\
\hline Nannochloris-like & Marvania relative1 & LH08SG2053 & this study & $\mathrm{n} / \mathrm{a}$ & $\begin{array}{l}\text { N53ํ5'21.505" } \\
\text { E135ㅇ'48.169" }\end{array}$ & $\begin{array}{l}\text { DE (Schorfheide- } \\
\text { Chorin) }\end{array}$ & soil (grassland) \\
\hline Nannochloris-like & Marvania relative1 & LH08SG3078 & this study & this study & $\begin{array}{l}\mathrm{N} 53^{\circ} 6^{\prime} 10.204^{\prime \prime} \\
\mathrm{E} 13^{\circ} 59^{\prime} 8.519^{\prime \prime}\end{array}$ & $\begin{array}{l}\text { DE (Schorfheide- } \\
\text { Chorin) }\end{array}$ & soil (grassland) \\
\hline Nannochloris-like & Marvania relative1 & LH08SG3093 & this study & this study & $\begin{array}{l}\text { N53 }{ }^{\circ} 6^{\prime} 10.204^{\prime \prime} \\
\text { E13 } 59^{\prime} 8.519^{\prime \prime}\end{array}$ & $\begin{array}{l}\text { DE (Schorfheide- } \\
\text { Chorin) }\end{array}$ & soil (grassland) \\
\hline Nannochloris-like & Marvania relative1 & LH10HG2094 & this study & $\mathrm{n} / \mathrm{a}$ & $\begin{array}{l}\mathrm{N} 51^{\circ} 0^{\prime} 2.696^{\prime \prime} \\
\mathrm{E} 10^{\circ} 25^{\prime} 48.036^{\prime \prime}\end{array}$ & DE (Hainich-Dün) & soil (grassland) \\
\hline Nannochloris-like & Marvania sp. & WB67 & KF144207 & $\mathrm{n} / \mathrm{a}$ & - & - & - \\
\hline Nannochloris-like & $\begin{array}{l}\text { Nannochloris } \\
\text { coccoides }\end{array}$ & CCAP 251/1b & AB080301 & $\mathrm{n} / \mathrm{a}$ & - & - & - \\
\hline Nannochloris-like & Nannochloris sp. & $A n-1$ & EF440182 & $n / a$ & - & - & - \\
\hline Nannochloris-like & Nannochloris sp. & JL 4-6 & AY195983 & $\mathrm{n} / \mathrm{a}$ & - & - & - \\
\hline
\end{tabular}

Legend. $A Q=A n t a r c t i c a, C A=C a n a d a, C N=C h i n a, D E=G e r m a n y, E S=S p a i n, K R=S o u t h$ Korea, $N L=N e t h e r l a n d s, N O=N o r w a y$, PT=Portugal, RU=Russia, SE=Sweden, UK=United Kingdom, US=United States of America. 
Table S2. List of all analyzed accessions (isolates, strains and clones) of the Stichococcus-like species and relatives.

\begin{tabular}{|c|c|c|c|c|c|c|c|}
\hline Clade & Species & Identifier & $18 \mathrm{~S}$ & ITS2 & GPS & Land (region) & Habitat \\
\hline Chlorella mirabilis & Chlorella mirabilis & KFFB12-1 & this study & $\begin{array}{l}\text { this } \\
\text { study }\end{array}$ & $\begin{array}{l}\mathrm{S} 4^{\circ} 6^{\prime} 49.852^{\prime \prime} \\
\text { W78 } 58^{\circ} 58^{\prime} 1.012^{\prime \prime}\end{array}$ & EC (Bombuscaro) & subaerial (litter) \\
\hline Chlorella mirabilis & Chlorella mirabilis & L10 & this study & $\mathrm{n} / \mathrm{a}$ & $\begin{array}{l}S 62^{\circ} 10^{\prime} 0.000^{\prime \prime} \\
W 58^{\circ} 30^{\prime} 0.000^{\prime \prime}\end{array}$ & $\begin{array}{l}\text { AQ (King George } \\
\text { Island) }\end{array}$ & $\begin{array}{l}\text { soil } \\
\text { (deglaciated) }\end{array}$ \\
\hline Chlorella mirabilis & Chlorella mirabilis & LH10HG6139 & this study & $\mathrm{n} / \mathrm{a}$ & $\begin{array}{l}\text { N5111'⒊766" } \\
\text { E1023'28.395" }\end{array}$ & DE (Hainich-Dün) & soil (grassland) \\
\hline Chlorella mirabilis & Chlorella mirabilis & $\begin{array}{l}\text { Andreyeva } \\
748-1\end{array}$ & X74000 & $\mathrm{n} / \mathrm{a}$ & - & RU & tundra \\
\hline Chlorella mirabilis & Chlorella mirabilis & LH8AG9040 & this study & $\begin{array}{l}\text { this } \\
\text { study }\end{array}$ & 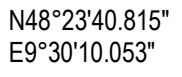 & $\begin{array}{l}\text { DE (Schwäbische } \\
\text { Alb) }\end{array}$ & soil (grassland) \\
\hline Chlorella mirabilis & Chlorella mirabilis & SEG5B K5318 & this study & $\mathrm{n} / \mathrm{a}$ & $\begin{array}{l}\mathrm{N} 53^{\circ} 6^{\prime} 26.830^{\prime \prime} \\
\mathrm{E} 14^{\circ} 0^{\prime} 1.885^{\prime \prime}\end{array}$ & $\begin{array}{l}\text { DE (Schorfheide- } \\
\text { Chorin) }\end{array}$ & soil (grassland) \\
\hline Desmococcus & $\begin{array}{l}\text { Desmococcus } \\
\text { endolithicus }\end{array}$ & clone EN5JG & KR054372 & $\mathrm{n} / \mathrm{a}$ & - & - & - \\
\hline Desmococcus & $\begin{array}{l}\text { Desmococcus } \\
\text { endolithicus }\end{array}$ & SAG 25.92 & EU434026 & $\begin{array}{l}\text { this } \\
\text { study }\end{array}$ & - & $\begin{array}{l}\text { AQ (Marie Bird } \\
\text { Land) }\end{array}$ & $\begin{array}{l}\text { subaerial } \\
\text { (chasmoendolit } \\
\text { hic) }\end{array}$ \\
\hline Desmococcus & $\begin{array}{l}\text { Desmococcus } \\
\text { olivaceus }\end{array}$ & SAG 1.92 & EU434017 & $\begin{array}{l}\text { KM0200 } \\
49\end{array}$ & & AT (Vienna) & $\begin{array}{l}\text { subaerial } \\
\text { (epixylic; tree } \\
\text { bark) }\end{array}$ \\
\hline Diplosphaera & Stichococcus sp. & $\begin{array}{l}\text { BCP-NB2- } \\
\text { VF10 }\end{array}$ & AF513370 & $\mathrm{n} / \mathrm{a}$ & - & - & - \\
\hline Diplosphaera & Stichococcus sp. & $\begin{array}{l}\text { BCP-SRS2- } \\
\text { VF14 }\end{array}$ & AY377441 & $\mathrm{n} / \mathrm{a}$ & - & - & - \\
\hline Diplosphaera & Diplosphaera & clone rtCF18sti & EF591011 & $\mathrm{n} / \mathrm{a}$ & - & - & freshwater \\
\hline Diplosphaera & Diplosphaera & MBIC10465 & AB183601 & $\mathrm{n} / \mathrm{a}$ & - & - & - \\
\hline Diplosphaera & $\begin{array}{l}\text { Diplosphaera } \\
\text { chodatii }\end{array}$ & FontaineM3-1 & $\mathrm{n} / \mathrm{a}$ & $\begin{array}{l}J X 6450 \\
20\end{array}$ & - & AT (Waldaist) & $\begin{array}{l}\text { subaerial } \\
\text { (Dermatocarpo } \\
n \text { symboint) }\end{array}$ \\
\hline Diplosphaera & Diplosphaera sp. & A07020 & $\mathrm{n} / \mathrm{a}$ & $\begin{array}{l}\text { HM237 } \\
336\end{array}$ & - & $\begin{array}{l}\text { CN (Shapotou } \\
\text { desert) }\end{array}$ & $\begin{array}{l}\text { subaerial } \\
\text { (Endocarpon } \\
\text { symbiont) }\end{array}$ \\
\hline Diplosphaera & Diplosphaera sp. & AEW9B K460 & this study & $\mathrm{n} / \mathrm{a}$ & 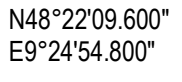 & $\begin{array}{l}\text { DE (Schwäbische } \\
\text { Alb) }\end{array}$ & soil (forest) \\
\hline Diplosphaera & Diplosphaera sp. & Z07020 & $\mathrm{n} / \mathrm{a}$ & $\begin{array}{l}\mathrm{HM} 237 \\
335\end{array}$ & - & $\begin{array}{l}\text { CN (Shapotou } \\
\text { desert) }\end{array}$ & $\begin{array}{l}\text { subaerial } \\
\text { (Endocarpon } \\
\text { symbiont) }\end{array}$ \\
\hline Diplosphaera & $\begin{array}{l}\text { Stichococcus } \\
\text { chlorelloides }\end{array}$ & $\begin{array}{l}\text { BCP-CNP2- } \\
\text { VF11B }\end{array}$ & AY271675 & $\mathrm{n} / \mathrm{a}$ & - & - & - \\
\hline Diplosphaera & Stichococcus sp. & clone QE28 & FJ790654 & $\mathrm{n} / \mathrm{a}$ & - & - & - \\
\hline Diplosphaera & Chlorella sphaerica & SAG 11.88 & AJ416105 & $\begin{array}{l}\text { this } \\
\text { study }\end{array}$ & - & $\begin{array}{l}\text { NZ (Waweira } \\
\text { Scenic Reserve) }\end{array}$ & $\begin{array}{l}\text { subaerial } \\
\text { (phycobiont) }\end{array}$ \\
\hline Diplosphaera & $\begin{array}{l}\text { Stichococcus } \\
\text { chodati }\end{array}$ & UTEX 1177 & AB055867 & $\mathrm{n} / \mathrm{a}$ & - & - & - \\
\hline Diplosphaera & $\begin{array}{l}\text { Diplosphaera } \\
\text { chodati }\end{array}$ & UTEX 1177 & AB055867 & $\begin{array}{l}\text { HQ1299 } \\
31\end{array}$ & - & US (Texas) & $\begin{array}{l}\text { soil (blackland } \\
\text { prairie soil) }\end{array}$ \\
\hline Diplosphaera & $\begin{array}{l}\text { Diplosphaera } \\
\text { mucosa }\end{array}$ & SAG 48.86 & KF673370 & $\mathrm{n} / \mathrm{a}$ & - & $\begin{array}{l}\mathrm{AQ} \text { (Princess } \\
\text { Elizabeth Land) }\end{array}$ & $\begin{array}{l}\text { subaerial } \\
\text { (moss) }\end{array}$ \\
\hline Diplosphaera & Diplosphaera sp. & $\mathrm{J} 4028 \mathrm{~B}$ & JN573864 & $\mathrm{n} / \mathrm{a}$ & - & - & - \\
\hline Diplosphaera & Diplosphaera sp. & KS120SM6L & this study & $\begin{array}{l}\text { this } \\
\text { study }\end{array}$ & $\begin{array}{l}\mathrm{S} 3^{\circ} 58^{\prime} 31.436 " \\
\text { W79 } 74^{\prime} 17.341^{\prime \prime}\end{array}$ & $\begin{array}{l}\text { EC (San } \\
\text { Francisco) }\end{array}$ & $\begin{array}{l}\text { subaerial } \\
\text { (epiphytic; } \\
\text { leaves) }\end{array}$ \\
\hline Diplosphaera & Diplosphaera sp. & KS135SM6L & $\mathrm{n} / \mathrm{a}$ & $\begin{array}{l}\text { this } \\
\text { study }\end{array}$ & $\begin{array}{l}\mathrm{S} 3^{\circ} 58^{\prime} 31.436 " \\
\text { W79² } 4^{\prime} 17.341^{\prime \prime}\end{array}$ & $\begin{array}{l}\text { EC (San } \\
\text { Francisco) }\end{array}$ & $\begin{array}{l}\text { subaerial } \\
\text { (epiphytic; } \\
\text { leaves) }\end{array}$ \\
\hline
\end{tabular}


Table S2. (continuation)

\begin{tabular}{|c|c|c|c|c|c|c|c|}
\hline Clade & Species & Identifier & $18 \mathrm{~S}$ & ITS2 & GPS & Land (region) & Habitat \\
\hline Diplosphaera & Diplosphaera sp. & LH08AG9089 & this study & $\begin{array}{l}\text { this } \\
\text { study }\end{array}$ & $\begin{array}{l}\mathrm{N} 48^{\circ} 23^{\prime} 40.815^{\prime \prime} \\
\mathrm{E} 9^{\circ} 30^{\prime} 10.053^{\prime \prime}\end{array}$ & $\begin{array}{l}\text { DE (Schwäbische } \\
\text { Alb) }\end{array}$ & soil (grassland) \\
\hline Diplosphaera & Diplosphaera sp. & LH08HW8075 & this study & $\begin{array}{l}\text { this } \\
\text { study }\end{array}$ & $\begin{array}{l}\text { N51ํ21'20.852" } \\
\text { E1031'1.083" }\end{array}$ & DE (Hainich-Dün) & soil (forest) \\
\hline Diplosphaera & Diplosphaera sp. & SAG 2049 & $\mathrm{n} / \mathrm{a}$ & $\begin{array}{l}\text { this } \\
\text { study }\end{array}$ & $\begin{array}{l}\mathrm{S} 8^{\circ} 16^{\prime} 25.200^{\prime \prime} \\
\mathrm{E} 115^{\circ} 09^{\prime} 57.900^{\prime \prime}\end{array}$ & $\begin{array}{l}\text { ID (Bali, Lake } \\
\text { Bratan) }\end{array}$ & freshwater \\
\hline Pseudomarvania & $\begin{array}{l}\text { Pseudomarvania } \\
\text { aerophytica }\end{array}$ & CAUP H 7301 & FJ896222 & $\mathrm{n} / \mathrm{a}$ & - & - & - \\
\hline Pseudomarvania & $\begin{array}{l}\text { Pseudomarvania } \\
\text { ampulliformis }\end{array}$ & SAG 2047 & AB087559 & $\begin{array}{l}\text { this } \\
\text { study }\end{array}$ & - & $\begin{array}{l}\text { JP (Taishaku-kyo } \\
\text { Gorge) }\end{array}$ & $\begin{array}{l}\text { subaerial } \\
\text { (epixylic; tree } \\
\text { bark) }\end{array}$ \\
\hline Pseudostichococcus & $\begin{array}{l}\text { Pseudostichococcus } \\
\text { sp. }\end{array}$ & LH08SW8044 & this study & $\begin{array}{l}\text { this } \\
\text { study }\end{array}$ & 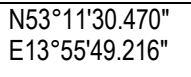 & $\begin{array}{l}\text { DE (Schorfheide- } \\
\text { Chorin) }\end{array}$ & soil (forest) \\
\hline Pseudostichococcus & $\begin{array}{l}\text { Pseudostichococcus } \\
\text { sp. }\end{array}$ & WB69 & KF144234 & $\begin{array}{l}\text { this } \\
\text { study }\end{array}$ & - & $\begin{array}{l}\text { DE (Westerhöfer } \\
\text { Bach) }\end{array}$ & $\begin{array}{l}\text { semi-terrestrial } \\
\text { (creek biofilm) }\end{array}$ \\
\hline Pseudostichococcus & $\begin{array}{l}\text { Stichococcus } \\
\text { mirabilis }\end{array}$ & CCAP $379 / 3$ & AJ311638 & $\begin{array}{l}\text { AJ4316 } \\
79\end{array}$ & - & $\mathrm{n} / \mathrm{a}$ & $\mathrm{n} / \mathrm{a}$ \\
\hline Pseudostichococcus & $\begin{array}{l}\text { Trebouxiophyceae } \\
\text { sp. }\end{array}$ & E07-4 & FJ946882 & $\mathrm{n} / \mathrm{a}$ & - & AQ (East Ongul) & freshwater \\
\hline Pseudostichococcus & $\begin{array}{l}\text { Pseudostichococcus } \\
\text { monalantoides }\end{array}$ & SAG 380-1 & JX185690 & $\begin{array}{l}\text { KM0200 } \\
66\end{array}$ & $\mathrm{n} / \mathrm{a}$ & DE & $\begin{array}{l}\text { freshwater } \\
\text { (culture; } \\
\text { Enteromorpha) }\end{array}$ \\
\hline Pseudostichococcus & $\begin{array}{l}\text { Pseudostichococcus } \\
\text { sp. }\end{array}$ & 3GA1830SK9 & this study & $\mathrm{n} / \mathrm{a}$ & $\begin{array}{l}\mathrm{N} 50^{\circ} 52^{\prime} 0.000^{\prime \prime} \\
\mathrm{E} 10^{\circ} 50^{\prime} 0.000^{\prime \prime}\end{array}$ & $\begin{array}{l}\text { DE (Castle } \\
\text { Gleichen) }\end{array}$ & $\begin{array}{l}\text { subaerial } \\
\text { (epilithic; } \\
\text { sandstone) }\end{array}$ \\
\hline Pseudostichococcus & $\begin{array}{l}\text { Pseudostichococcus } \\
\text { sp. }\end{array}$ & B3-2 & this study & $\mathrm{n} / \mathrm{a}$ & $\begin{array}{l}\mathrm{N} 54^{\circ} 11^{\prime} 0.000^{\prime \prime} \\
\mathrm{E} 7^{\circ} 53^{\prime} 0.000^{\prime \prime}\end{array}$ & DE (Helgoland) & $\begin{array}{l}\text { subaerial } \\
\text { (epilithic; } \\
\text { bunker wall) }\end{array}$ \\
\hline Pseudostichococcus & $\begin{array}{l}\text { Pseudostichococcus } \\
\text { sp. }\end{array}$ & LH10HG3045 & this study & $\begin{array}{l}\text { this } \\
\text { study }\end{array}$ & $\begin{array}{l}\mathrm{N} 50^{\circ} 59^{\prime} 53.129^{\prime \prime} \\
\mathrm{E} 10^{\circ} 25^{\prime} 58.616^{\prime \prime}\end{array}$ & DE (Hainich-Dün) & soil (grassland) \\
\hline Pseudostichococcus & Stichococcus fragilis & SAG 379-4 & $\mathrm{n} / \mathrm{a}$ & $\begin{array}{l}\text { this } \\
\text { study }\end{array}$ & $\begin{array}{l}\text { N41 }{ }^{\circ} 35^{\prime} 58.300^{\prime \prime} \\
\text { W70 } 34^{\prime} 51.900^{\prime \prime}\end{array}$ & $\begin{array}{l}\text { US } \\
\text { (Massachusetts) }\end{array}$ & $\begin{array}{l}\text { freshwater } \\
\text { (aquarium) }\end{array}$ \\
\hline Pseudostichococcus & $\begin{array}{l}\text { Desmococcus } \\
\text { spinocystis }\end{array}$ & SAG 2067 & this study & $\begin{array}{l}\text { this } \\
\text { study }\end{array}$ & $\begin{array}{l}\mathrm{N} 43^{\circ} 45^{\prime} 07.700^{\prime \prime} \\
\mathrm{E} 15^{\circ} 22^{\prime} 10.300^{\prime \prime}\end{array}$ & $\begin{array}{l}\text { HR (Island of } \\
\text { Lavsa) }\end{array}$ & soil \\
\hline Pseudostichococcus & Diplosphaera sp. & Lsn & JN573894 & $\mathrm{n} / \mathrm{a}$ & - & - & - \\
\hline Pseudostichococcus & $\begin{array}{l}\text { Pseudostichococcus } \\
\text { sp. }\end{array}$ & AEG6B K4796 & this study & $\mathrm{n} / \mathrm{a}$ & $\begin{array}{l}\text { N48 } 24 ' 4.600^{\prime \prime} \\
E 9^{\circ} 26^{\prime} 30.100^{\prime \prime}\end{array}$ & $\begin{array}{l}\text { DE (Schwäbische } \\
\text { Alb) }\end{array}$ & soil (grassland) \\
\hline Pseudostichococcus & $\begin{array}{l}\text { Pseudostichococcus } \\
\text { sp. }\end{array}$ & $\begin{array}{l}\text { HEG7MW B } \\
\text { K4277 }\end{array}$ & this study & $\mathrm{n} / \mathrm{a}$ & $\begin{array}{l}\text { N51ํ16'24.897" } \\
\text { E10²4'37.485" }\end{array}$ & DE (Hainich-Dün) & soil (grassland) \\
\hline Pseudostichococcus & $\begin{array}{l}\text { Stichococcus } \\
\text { minutus }\end{array}$ & $\mathrm{NJ}-17$ & JN400256 & $\mathrm{n} / \mathrm{a}$ & - & $\begin{array}{l}\text { AQ (Zhongshan } \\
\text { Station) }\end{array}$ & $\begin{array}{l}\text { terrestrial (wet } \\
\text { rocks) }\end{array}$ \\
\hline $\begin{array}{l}\text { Stichococcus } \\
\text { bacillaris }\end{array}$ & $\begin{array}{l}\text { Chlorosphaera } \\
\text { klebsii }\end{array}$ & CCAP 215/1 & FR865689 & $\begin{array}{l}\text { FR8656 } \\
89\end{array}$ & - & - & - \\
\hline $\begin{array}{l}\text { Stichococcus } \\
\text { bacillaris }\end{array}$ & $\begin{array}{l}\text { Gloeotila cf. } \\
\text { protogenita }\end{array}$ & SAG 56.91 & AM412752 & $\begin{array}{l}\text { AM4127 } \\
52\end{array}$ & - & - & - \\
\hline $\begin{array}{l}\text { Stichococcus } \\
\text { bacillaris }\end{array}$ & Gloeotila scopulina & SAG 335-8 & AM412753 & $\begin{array}{l}\text { AM4127 } \\
53\end{array}$ & - & - & - \\
\hline $\begin{array}{l}\text { Stichococcus } \\
\text { bacillaris }\end{array}$ & $\begin{array}{l}\text { Stichococcus } \\
\text { bacillaris }\end{array}$ & HEW1B K3375 & this study & $\mathrm{n} / \mathrm{a}$ & $\begin{array}{l}\text { N5111'7.278" } \\
\text { E10¹9'25.036" }\end{array}$ & DE (Hainich-Dün) & soil (forest) \\
\hline $\begin{array}{l}\text { Stichococcus } \\
\text { bacillaris }\end{array}$ & $\begin{array}{l}\text { Stichococcus } \\
\text { bacillaris }\end{array}$ & NIES-529 & AB488606 & $\mathrm{n} / \mathrm{a}$ & - & - & - \\
\hline $\begin{array}{l}\text { Stichococcus } \\
\text { bacillaris }\end{array}$ & $\begin{array}{l}\text { Stichococcus } \\
\text { bacillaris }\end{array}$ & RCC 1054 & KF899844 & $\mathrm{n} / \mathrm{a}$ & - & - & - \\
\hline $\begin{array}{l}\text { Stichococcus } \\
\text { bacillaris }\end{array}$ & $\begin{array}{l}\text { Stichococcus } \\
\text { bacillaris }\end{array}$ & SAG 335-3 & EU434029 & $\mathrm{n} / \mathrm{a}$ & - & - & - \\
\hline $\begin{array}{l}\text { Stichococcus } \\
\text { bacillaris }\end{array}$ & $\begin{array}{l}\text { Stichococcus } \\
\text { bacillaris }\end{array}$ & SAG 379-1b & AJ311637 & $\begin{array}{l}\text { AJ4316 } \\
78\end{array}$ & - & $\mathrm{CH}$ (Basel) & $\begin{array}{l}\text { freshwater } \\
\text { (water dish) }\end{array}$ \\
\hline
\end{tabular}


Table S2. (continuation)

\begin{tabular}{|c|c|c|c|c|c|c|c|}
\hline Clade & Species & Identifier & $18 \mathrm{~S}$ & ITS2 & GPS & Land (region) & Habitat \\
\hline $\begin{array}{l}\text { Stichococcus } \\
\text { bacillaris }\end{array}$ & $\begin{array}{l}\text { Stichococcus } \\
\text { bacillaris }\end{array}$ & SAG 379-1d & $\mathrm{n} / \mathrm{a}$ & $\begin{array}{l}\text { this } \\
\text { study }\end{array}$ & $\mathrm{n} / \mathrm{a}$ & US (Alaska) & $\mathrm{n} / \mathrm{a}$ \\
\hline $\begin{array}{l}\text { Stichococcus } \\
\text { bacillaris }\end{array}$ & $\begin{array}{l}\text { Stichococcus } \\
\text { bacillaris }\end{array}$ & SAG 379-2 & HE610125 & $\begin{array}{l}\text { HE6101 } \\
25\end{array}$ & - & DE (Bernburg) & $\mathrm{n} / \mathrm{a}$ \\
\hline $\begin{array}{l}\text { Stichococcus } \\
\text { bacillaris }\end{array}$ & $\begin{array}{l}\text { Stichococcus } \\
\text { bacillaris }\end{array}$ & siva2011 & JN168788 & $\mathrm{n} / \mathrm{a}$ & - & - & - \\
\hline $\begin{array}{l}\text { Stichococcus } \\
\text { bacillaris }\end{array}$ & $\begin{array}{l}\text { Stichococcus } \\
\text { bacillaris }\end{array}$ & UTEX 315 & AM412751 & $\begin{array}{l}\text { AM4127 } \\
51\end{array}$ & - & DE (Bernburg) & $\mathrm{n} / \mathrm{a}$ \\
\hline $\begin{array}{l}\text { Stichococcus } \\
\text { bacillaris }\end{array}$ & $\begin{array}{l}\text { Stichococcus } \\
\text { bacillaris }\end{array}$ & WB13 & KF144231 & $\begin{array}{l}\text { this } \\
\text { study }\end{array}$ & & $\begin{array}{l}\text { DE (Westerhöfer } \\
\text { Bach) }\end{array}$ & $\begin{array}{l}\text { semi-terrestrial } \\
\text { (creek biofilm) }\end{array}$ \\
\hline $\begin{array}{l}\text { Stichococcus } \\
\text { bacillaris }\end{array}$ & $\begin{array}{l}\text { Stichococcus } \\
\text { bacillaris }\end{array}$ & WB74 & KF144232 & $\begin{array}{l}\text { this } \\
\text { study }\end{array}$ & & $\begin{array}{l}\text { DE (Westerhöfer } \\
\text { Bach) }\end{array}$ & $\begin{array}{l}\text { semi-terrestrial } \\
\text { (creek biofilm) }\end{array}$ \\
\hline Stichococcus clade1 & $\begin{array}{l}\text { Stichococcus } \\
\text { bacillaris }\end{array}$ & K4-4 & AB055866 & $\mathrm{n} / \mathrm{a}$ & - & - & - \\
\hline Stichococcus clade1 & Stichococcus sp. & DB6-27 & KF144235 & $\mathrm{n} / \mathrm{a}$ & - & - & - \\
\hline Stichococcus clade1 & Stichococcus sp. & HEG8B K4544 & this study & $\mathrm{n} / \mathrm{a}$ & $\begin{array}{l}\text { N51ํ16'16.527" } \\
\text { E102ㄴ' } 4.6^{\prime \prime}\end{array}$ & DE (Hainich-Dün) & soil (grassland) \\
\hline Stichococcus clade1 & Stichococcus sp. & HEW1B K3359 & this study & $\mathrm{n} / \mathrm{a}$ & $\begin{array}{l}\text { N51ำ11'7.278" } \\
\text { E1019'25.036" }\end{array}$ & DE (Hainich-Dün) & soil (forest) \\
\hline Stichococcus clade1 & Stichococcus sp. & KP09AW1004 & KP081407 & $\begin{array}{l}\text { this } \\
\text { study }\end{array}$ & & $\begin{array}{l}\text { DE (Schwäbische } \\
\text { Alb) }\end{array}$ & $\begin{array}{l}\text { subaerial } \\
\text { (epixylic; tree } \\
\text { bark) }\end{array}$ \\
\hline Stichococcus clade1 & Stichococcus sp. & KS075SM6T & this study & $\begin{array}{l}\text { this } \\
\text { study }\end{array}$ & $\begin{array}{l}\mathrm{S} 3^{\circ} 58^{\prime} 31.436^{\prime \prime} \\
\text { W79²'17.341" }\end{array}$ & $\begin{array}{l}\text { EC (San } \\
\text { Francisco) }\end{array}$ & $\begin{array}{l}\text { subaerial } \\
\text { (epixylic; tree } \\
\text { bark) }\end{array}$ \\
\hline Stichococcus clade1 & Stichococcus sp. & $\begin{array}{l}\text { SAG } 2481 \\
\text { (=LH08AW800 } \\
\text { 2) }\end{array}$ & KP081394 & $\mathrm{n} / \mathrm{a}$ & - & $\begin{array}{l}\text { DE (Schwäbische } \\
\text { Alb) }\end{array}$ & soil (forest) \\
\hline Stichococcus clade1 & Stichococcus sp. & WB65 & KF144236 & $\mathrm{n} / \mathrm{a}$ & - & - & - \\
\hline Stichococcus clade2 & Stichococcus sp. & 594-GA18 & EU282451 & $\mathrm{n} / \mathrm{a}$ & - & $\begin{array}{l}\text { RU (Siberia, } \\
\text { Kolyma) }\end{array}$ & $\begin{array}{l}\text { soil } \\
\text { (permafrost) }\end{array}$ \\
\hline Stichococcus clade2 & Stichococcus sp. & D4-2A & KF144238 & $\begin{array}{l}\text { this } \\
\text { study }\end{array}$ & - & $\begin{array}{l}\text { DE } \\
\text { (Deinschwanger } \\
\text { Bach) }\end{array}$ & $\begin{array}{l}\text { semi-terrestrial } \\
\text { (creek biofilm) }\end{array}$ \\
\hline Stichococcus clade2 & Stichococcus sp. & ITS1A C11 & $\mathrm{n} / \mathrm{a}$ & $\begin{array}{l}\text { HM490 } \\
287\end{array}$ & - & AQ (Miers Valley) & $\begin{array}{l}\text { subaerial } \\
\text { (hypolithic) }\end{array}$ \\
\hline Stichococcus clade2 & Stichococcus sp. & ITS1A H8 & $\mathrm{n} / \mathrm{a}$ & $\begin{array}{l}\text { HM490 } \\
288\end{array}$ & - & AQ (Miers Valley) & $\begin{array}{l}\text { subaerial } \\
\text { (hypolithic) }\end{array}$ \\
\hline Stichococcus clade2 & Stichococcus sp. & LH08AW8104 & KP081396 & $\begin{array}{l}\text { this } \\
\text { study }\end{array}$ & - & $\begin{array}{l}\text { DE (Schwäbische } \\
\text { Alb) }\end{array}$ & soil (forest) \\
\hline Stichococcus clade2 & Stichococcus sp. & $\begin{array}{l}\text { SAG } 2482 \\
\text { (=LH08AW802 } \\
\text { 3) }\end{array}$ & KP081395 & $\begin{array}{l}\text { this } \\
\text { study }\end{array}$ & - & $\begin{array}{l}\text { DE (Schwäbische } \\
\text { Alb) }\end{array}$ & soil (forest) \\
\hline Stichococcus clade3 & Stichococcus sp. & A43 & HQ418417 & $\mathrm{n} / \mathrm{a}$ & - & - & $\begin{array}{l}\text { soil; } \\
\text { Yellowstone } \\
\text { NP }\end{array}$ \\
\hline Stichococcus clade3 & Stichococcus sp. & clone: DA-04 & AB257661 & $\mathrm{n} / \mathrm{a}$ & 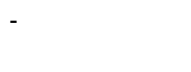 & 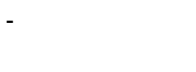 & $\begin{array}{l}\text { rock surface, } \\
\text { Swiss Alps }\end{array}$ \\
\hline Stichococcus clade3 & Stichococcus sp. & KS106CL6T & this study & $\begin{array}{l}\text { this } \\
\text { study }\end{array}$ & $\begin{array}{l}\mathrm{S} 4^{\circ} 6^{\prime} 52.681^{\prime \prime} \\
\mathrm{W}^{\circ} 9^{\circ} 10^{\prime} 30.940^{\prime \prime}\end{array}$ & EC (Cajanuma) & $\begin{array}{l}\text { subaerial } \\
\text { (epixylic; tree } \\
\text { bark) }\end{array}$ \\
\hline Stichococcus clade3 & Stichococcus sp. & KS108CL6T & $\mathrm{n} / \mathrm{a}$ & $\begin{array}{l}\text { this } \\
\text { study }\end{array}$ & $\begin{array}{l}\mathrm{S} 4^{\circ} 6^{\prime} 52.681^{\prime \prime} \\
\mathrm{W}^{\circ} 9^{\circ} 10^{\prime} 30.940^{\prime \prime}\end{array}$ & EC (Cajanuma) & $\begin{array}{l}\text { subaerial } \\
\text { (epixylic; tree } \\
\text { bark) }\end{array}$ \\
\hline Stichococcus clade3 & Stichococcus sp. & $\begin{array}{l}\text { SAG } 2408 \\
\text { (=WB8) }\end{array}$ & KF144239 & $\begin{array}{l}\text { this } \\
\text { study }\end{array}$ & - & $\begin{array}{l}\text { DE (Westerhöfer } \\
\text { Bach) }\end{array}$ & $\begin{array}{l}\text { semi-terrestrial } \\
\text { (creek biofilm) }\end{array}$ \\
\hline Stichococcus clade4 & Stichococcus sp. & $\begin{array}{l}\text { clone } \\
\text { B1_3_1E_88 }\end{array}$ & JQ627437 & $\mathrm{n} / \mathrm{a}$ & - & - & - \\
\hline Stichococcus clade4 & Stichococcus sp. & $\begin{array}{l}\text { HEG7SWWB } \\
\text { K4387 }\end{array}$ & this study & $\mathrm{n} / \mathrm{a}$ & 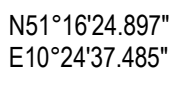 & DE (Hainich-Dün) & soil (grassland) \\
\hline Stichococcus clade4 & Stichococcus sp. & LH08SG1073 & this study & $\begin{array}{l}\text { this } \\
\text { study }\end{array}$ & $\begin{array}{l}N 53^{\circ} 5^{\prime} 14.712^{\prime \prime} \\
E 13^{\circ} 58^{\prime} 10.717^{\prime \prime}\end{array}$ & $\begin{array}{l}\text { DE (Schorfheide- } \\
\text { Chorin) }\end{array}$ & soil (grassland) \\
\hline Stichococcus clade4 & Stichococcus sp. & $\begin{array}{l}\text { SAG } 2406 \\
\text { (=WB47) }\end{array}$ & KF144240 & $\begin{array}{l}\text { this } \\
\text { study }\end{array}$ & 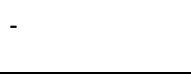 & $\begin{array}{l}\text { DE (Westerhöfer } \\
\text { Bach) }\end{array}$ & $\begin{array}{l}\text { semi-terrestrial } \\
\text { (creek biofilm) }\end{array}$ \\
\hline Stichococcus clade5 & Stichococcus sp. & DZ K4g & this study & $\mathrm{n} / \mathrm{a}$ & 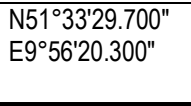 & DE (Göttingen) & $\begin{array}{l}\text { subaerial } \\
\text { (epilithic; roof } \\
\text { tile) }\end{array}$ \\
\hline
\end{tabular}


Table S2. (continuation)

\begin{tabular}{|c|c|c|c|c|c|c|c|}
\hline Clade & Species & Identifier & $18 \mathrm{~S}$ & ITS2 & GPS & Land (region) & Habitat \\
\hline Stichococcus clade5 & Stichococcus sp. & KP09HW3004 & this study & $\begin{array}{l}\text { this } \\
\text { study }\end{array}$ & $\begin{array}{l}\text { N51 }{ }^{\circ} 16^{\prime} 17.900^{\prime \prime} \\
\text { E10 } 10^{\prime} 38.700^{\prime \prime}\end{array}$ & DE (Hainich-Dün) & $\begin{array}{l}\text { subaerial } \\
\text { (epixylic; tree } \\
\text { bark) }\end{array}$ \\
\hline Stichococcus clade5 & Stichococcus sp. & KS090BU1T & $\mathrm{n} / \mathrm{a}$ & $\begin{array}{l}\text { this } \\
\text { study }\end{array}$ & $\begin{array}{l}\mathrm{S} 4^{\circ} 6^{\prime} 49.852^{\prime \prime} \\
\text { W78 } 58^{\circ} 1.012^{\prime \prime}\end{array}$ & EC (Bombuscaro) & $\begin{array}{l}\text { subaerial } \\
\text { (epixylic; tree } \\
\text { bark) }\end{array}$ \\
\hline Stichococcus clade5 & Stichococcus sp. & KS305SM6L & this study & $\begin{array}{l}\text { this } \\
\text { study }\end{array}$ & $\begin{array}{l}\text { S35ㅇ'31.436" } \\
\text { W79² } 4^{\prime} 17.341^{\prime \prime}\end{array}$ & $\begin{array}{l}\text { EC (San } \\
\text { Francisco) }\end{array}$ & $\begin{array}{l}\text { subaerial } \\
\text { (epiphytic; } \\
\text { leaves) }\end{array}$ \\
\hline Stichococcus clade5 & Stichococcus sp. & LH08SW1099 & this study & $\begin{array}{l}\text { this } \\
\text { study }\end{array}$ & $\begin{array}{l}\mathrm{N} 52^{\circ} 54^{\prime} 3.050^{\prime \prime} \\
\mathrm{E} 13^{\circ} 50^{\prime} 46.921^{\prime \prime}\end{array}$ & $\begin{array}{l}\text { DE (Schorfheide- } \\
\text { Chorin) }\end{array}$ & soil (forest) \\
\hline Stichococcus clade6 & Stichococcus sp. & SAG 107.80 & $\mathrm{n} / \mathrm{a}$ & $\begin{array}{l}\text { this } \\
\text { study }\end{array}$ & $\begin{array}{l}\text { N4515'33.900" } \\
\text { W62 } 56^{\prime} 0.100^{\prime \prime}\end{array}$ & CA (Nova Scotia) & $\mathrm{n} / \mathrm{a}$ \\
\hline Stichococcus clade6 & Stichococcus sp. & SAG 108.80 & KM020175 & $\mathrm{n} / \mathrm{a}$ & - & - & - \\
\hline Stichococcus clade6 & Stichococcus sp. & WB2 & $\mathrm{n} / \mathrm{a}$ & $\begin{array}{l}\text { this } \\
\text { study }\end{array}$ & $\begin{array}{l}\mathrm{N} 51^{\circ} 45^{\prime} 0.000^{\prime \prime} \\
\mathrm{E} 10^{\circ} 5^{\prime} 0.000^{\prime \prime}\end{array}$ & $\begin{array}{l}\text { DE (Westerhöfer } \\
\text { Bach) }\end{array}$ & $\begin{array}{l}\text { semi-terrestrial } \\
\text { (creek biofilm) }\end{array}$ \\
\hline Stichococcus clade6 & Stichococcus sp. & WB66 & this study & $\mathrm{n} / \mathrm{a}$ & $\begin{array}{l}\mathrm{N} 51^{\circ} 45^{\prime} 0.000^{\prime \prime} \\
\mathrm{E} 10^{\circ} 5 ' 0.000^{\prime \prime}\end{array}$ & $\begin{array}{l}\text { DE (Westerhöfer } \\
\text { Bach) }\end{array}$ & $\begin{array}{l}\text { semi-terrestrial } \\
\text { (creek biofilm) }\end{array}$ \\
\hline Stichococcus clade7 & Stichococcus sp. & W1118 & JN573887 & $\mathrm{n} / \mathrm{a}$ & - & - & - \\
\hline Stichococcus clade7 & $\begin{array}{l}\text { Stichococcus } \\
\text { bacillaris }\end{array}$ & NJ-10 & JN400255 & $\mathrm{n} / \mathrm{a}$ & - & $\begin{array}{l}A Q \text { (Zhongshan } \\
\text { Station) }\end{array}$ & $\begin{array}{l}\text { terrestrial (wet } \\
\text { rocks) }\end{array}$ \\
\hline Stichococcus clade7 & $\begin{array}{l}\text { Stichococcus } \\
\text { bacillaris }\end{array}$ & s3 & AY380557 & $\mathrm{n} / \mathrm{a}$ & - & $A Q$ & soil \\
\hline Stichococcus clade7 & Stichococcus sp. & FG2/4.2 & KM020048 & $\begin{array}{l}\text { KM0200 } \\
48\end{array}$ & - & - & - \\
\hline Stichococcus clade7 & Stichococcus sp. & HEG9B K2590 & this study & $\mathrm{n} / \mathrm{a}$ & $\begin{array}{l}\text { N51ํ1'26.031" } \\
\text { E10²2'50.834" }\end{array}$ & DE (Hainich-Dün) & soil (grassland) \\
\hline Stichococcus clade7 & Stichococcus sp. & LH08AW8025 & KP081397 & $\mathrm{n} / \mathrm{a}$ & - & $\begin{array}{l}\text { DE (Schwäbische } \\
\text { Alb) }\end{array}$ & soil (forest) \\
\hline Stichococcus clade7 & Stichococcus sp. & LH08SG5057 & this study & $\begin{array}{l}\text { this } \\
\text { study }\end{array}$ & $\begin{array}{l}\mathrm{N} 53^{\circ} 6^{\prime} 26.830^{\prime \prime} \\
\mathrm{E} 14^{\circ} 0^{\prime} 1.885^{\prime \prime}\end{array}$ & $\begin{array}{l}\text { DE (Schorfheide- } \\
\text { Chorin) }\end{array}$ & soil (grassland) \\
\hline Stichococcus clade7 & Stichococcus sp. & LH08SG5079 & this study & $\begin{array}{l}\text { this } \\
\text { study }\end{array}$ & $\begin{array}{l}\mathrm{N} 53^{\circ}{ }^{\prime} 26.830^{\prime \prime} \\
\mathrm{E} 14^{\circ} 0^{\prime} 1.885^{\prime \prime}\end{array}$ & $\begin{array}{l}\text { DE (Schorfheide- } \\
\text { Chorin) }\end{array}$ & soil (grassland) \\
\hline Stichococcus clade7 & Stichococcus sp. & LH08SG5090 & this study & $\begin{array}{l}\text { this } \\
\text { study }\end{array}$ & $\begin{array}{l}\mathrm{N} 53^{\circ}{ }^{\circ} 26.830^{\prime \prime} \\
\mathrm{E} 14^{\circ} 0 ' 1.885^{\prime \prime}\end{array}$ & $\begin{array}{l}\text { DE (Schorfheide- } \\
\text { Chorin) }\end{array}$ & soil (grassland) \\
\hline Stichococcus clade7 & Stichococcus sp. & LH10HG2063 & this study & $\begin{array}{l}\text { this } \\
\text { study }\end{array}$ & $\begin{array}{l}\mathrm{N} 51^{\circ} 0^{\prime} 2.696 " \\
\mathrm{E} 10^{\circ} 25^{\prime} 48.036^{\prime \prime}\end{array}$ & DE (Hainich-Dün) & soil (grassland) \\
\hline Stichococcus clade7 & Stichococcus sp. & LH10HG7072 & this study & $\begin{array}{l}\text { this } \\
\text { study }\end{array}$ & $\begin{array}{l}\text { N51ํ16'24.897" } \\
\text { E1024'37.485" }\end{array}$ & DE (Hainich-Dün) & soil (grassland) \\
\hline Stichococcus clade7 & Stichococcus sp. & LH10HG7073 & this study & $\begin{array}{l}\text { this } \\
\text { study }\end{array}$ & 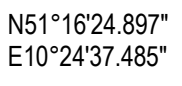 & DE (Hainich-Dün) & soil (grassland) \\
\hline Stichococcus clade7 & Stichococcus sp. & SAG 2059 & AY762604 & $\mathrm{n} / \mathrm{a}$ & - & - & - \\
\hline Stichococcus clade7 & Stichococcus sp. & SAG 2060 & AY762606 & $\mathrm{n} / \mathrm{a}$ & - & - & - \\
\hline Stichococcus clade7 & Stichococcus sp. & SAG 2119 & KM116460 & $\mathrm{n} / \mathrm{a}$ & - & - & - \\
\hline $\begin{array}{l}\text { Stichococcus } \\
\text { deasonii }\end{array}$ & $\begin{array}{l}\text { Stichococcus } \\
\text { deasonii }\end{array}$ & SAG 2139 & DQ275460 & $\begin{array}{l}\text { this } \\
\text { study }\end{array}$ & - & $\begin{array}{l}\text { US (Dauphin } \\
\text { Island) }\end{array}$ & soil \\
\hline $\begin{array}{l}\text { Stichococcus } \\
\text { deasonii }\end{array}$ & Stichococcus sp. & ciidir1003 A11 & $\mathrm{n} / \mathrm{a}$ & $\begin{array}{l}\text { JN6605 } \\
92\end{array}$ & - & $\mathrm{MX}$ & $\begin{array}{l}\text { soil } \\
\text { (rhizosphere } \\
\text { from } \\
\text { commercial } \\
\text { field) }\end{array}$ \\
\hline $\begin{array}{l}\text { Stichococcus } \\
\text { deasonii }\end{array}$ & Stichococcus sp. & KSK870SM6T & $\mathrm{n} / \mathrm{a}$ & $\begin{array}{l}\text { this } \\
\text { study }\end{array}$ & 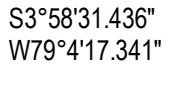 & $\begin{array}{l}\text { EC (San } \\
\text { Francisco) }\end{array}$ & $\begin{array}{l}\text { subaerial } \\
\text { (epixylic; tree } \\
\text { bark) }\end{array}$ \\
\hline $\begin{array}{l}\text { Stichococcus } \\
\text { deasonii }\end{array}$ & Stichococcus sp. & KSK937BU1L & $\mathrm{n} / \mathrm{a}$ & $\begin{array}{l}\text { this } \\
\text { study }\end{array}$ & $\begin{array}{l}\mathrm{S} 4^{\circ} 6^{\prime} 49.852^{\prime \prime} \\
\text { W78 } 58^{\circ} 1.012^{\prime \prime}\end{array}$ & EC (Bombuscaro) & $\begin{array}{l}\text { subaerial } \\
\text { (epiphytic; } \\
\text { leaves) }\end{array}$ \\
\hline $\begin{array}{l}\text { Stichococcus } \\
\text { jenerensis }\end{array}$ & $\begin{array}{l}\text { Stichococcus } \\
\text { bacillaris }\end{array}$ & CCAP 379/7 & AB055864 & $\mathrm{n} / \mathrm{a}$ & - & - & acid springs \\
\hline
\end{tabular}


Table S2. (continuation)

\begin{tabular}{|c|c|c|c|c|c|c|c|}
\hline Clade & Species & Identifier & $18 \mathrm{~S}$ & ITS2 & GPS & Land (region) & Habitat \\
\hline $\begin{array}{l}\text { Stichococcus } \\
\text { jenerensis }\end{array}$ & $\begin{array}{l}\text { Stichococcus } \\
\text { bacillaris }\end{array}$ & D10-1 & AB055865 & $\mathrm{n} / \mathrm{a}$ & - & - & - \\
\hline $\begin{array}{l}\text { Stichococcus } \\
\text { jenerensis }\end{array}$ & $\begin{array}{l}\text { Stichococcus } \\
\text { jenerensis }\end{array}$ & CCAP $379 / 5$ & KJ756841 & $\mathrm{n} / \mathrm{a}$ & - & - & marine \\
\hline $\begin{array}{l}\text { Stichococcus } \\
\text { jenerensis }\end{array}$ & $\begin{array}{l}\text { Stichococcus } \\
\text { jenerensis }\end{array}$ & LU1 & KF569726 & $\mathrm{n} / \mathrm{a}$ & - & - & - \\
\hline $\begin{array}{l}\text { Stichococcus } \\
\text { jenerensis }\end{array}$ & $\begin{array}{l}\text { Stichococcus } \\
\text { jenerensis }\end{array}$ & SAG 2138 & DQ275461 & $\begin{array}{l}\text { this } \\
\text { study }\end{array}$ & - & $\begin{array}{l}\text { MY (Kampong } \\
\text { Kuala Jenera) }\end{array}$ & $\begin{array}{l}\text { soil (crust on } \\
\text { tree base) }\end{array}$ \\
\hline $\begin{array}{l}\text { Stichococcus } \\
\text { jenerensis }\end{array}$ & Stichococcus sp. & FACHB753 & EU045358 & $\mathrm{n} / \mathrm{a}$ & - & - & - \\
\hline $\begin{array}{l}\text { Stichococcus } \\
\text { jenerensis }\end{array}$ & Stichococcus sp. & HEW2B K3412 & this study & $\mathrm{n} / \mathrm{a}$ & $\begin{array}{l}\text { N511'⒍000" } \\
\text { E10²2'11.800" }\end{array}$ & DE (Hainich-Dün) & soil (forest) \\
\hline $\begin{array}{l}\text { Stichococcus } \\
\text { jenerensis }\end{array}$ & Stichococcus sp. & KP09HW3001 & this study & $\mathrm{n} / \mathrm{a}$ & $\begin{array}{l}\text { N511'17.900" } \\
\text { E10¹8'38.700" }\end{array}$ & DE (Hainich-Dün) & $\begin{array}{l}\text { subaerial } \\
\text { (epixylic; tree } \\
\text { bark) }\end{array}$ \\
\hline $\begin{array}{l}\text { Stichococcus } \\
\text { jenerensis }\end{array}$ & Stichococcus sp. & KS126SM6L & this study & $\begin{array}{l}\text { this } \\
\text { study }\end{array}$ & $\begin{array}{l}\mathrm{S} 3^{\circ} 58^{\prime} 31.436 " \\
\text { W79 }\end{array}$ & $\begin{array}{l}\text { EC (San } \\
\text { Francisco) }\end{array}$ & $\begin{array}{l}\text { subaerial } \\
\text { (epiphytic; } \\
\text { leaves) }\end{array}$ \\
\hline $\begin{array}{l}\text { Stichococcus } \\
\text { jenerensis }\end{array}$ & Stichococcus sp. & MBIC10457 & AB183599 & $\mathrm{n} / \mathrm{a}$ & - & - & - \\
\hline
\end{tabular}

Legend. $A Q=A$ ntarctica, $A T=A u s t r i a, C A=C a n a d a, C H=S$ witzerland, $C N=C h i n a, D E=G e r m a n y, E C=E c u a d o r, H R=C r o a t i a$, $\mathrm{ID}=$ Indonesia, JP=Japan, MX=Mexico, MY=Malaysia, NZ=New Zealand, RU=Russia, US=United States of America.

Table S3. List of the analyzed Stichococcus-like clades and their representative strains.

\begin{tabular}{|c|c|c|c|c|}
\hline $\begin{array}{l}\text { OTU inferred from } 18 \mathrm{~S} \text { rDNA } \\
\text { similarity } \geq 99.5 \% ; \geq 99.0 \%{ }^{*}\end{array}$ & Representative strain & $\begin{array}{l}\text { Author of } \\
\text { denomination }\end{array}$ & Isolator/year & $\begin{array}{l}\text { Synonymic GenBank } \\
\text { accessions 18S/ITS2* }\end{array}$ \\
\hline OTU8* Diplosphaera & SAG 48.86 & Broady 1983 & Broady/1979 & $\begin{array}{l}\text { Stichococcus chodati } \\
\text { Stichococcus chlorelloides } \\
\text { Chlorella sphaerica* }^{*}\end{array}$ \\
\hline OTU2* Stichococcus deasonii & SAG 2139 & Neustupa et al. 2007 & Deason/1969 & $\begin{array}{l}\text { Stichococcus bacillaris } \\
\text { Diplosphaera sp. }\end{array}$ \\
\hline OTU7* Stichococcus jenerensis* & SAG 2138 & Neustupa et al. 2007 & Neustupa/2000 & Stichococcus bacillaris \\
\hline OTU6 Stichococcus bacillaris & SAG 379-1b & Vischer(?) & Vischer/1923 & $\begin{array}{l}\text { Stichococcus chloranthus } \\
\text { Chlorosphaera klebsii } \\
\text { Gloeotila cf. protogenita } \\
\text { Gloeotila scopulina }\end{array}$ \\
\hline OTU3 Stichococcus clade3 & SAG 2408 (=WB8) & Hodač 2015 & Mohr/2005 & $\mathrm{n} / \mathrm{a}$ \\
\hline OTU4 Stichococcus clade4 & SAG 2406 (=WB47) & Hodač 2015 & Mohr/2005 & $\mathrm{n} / \mathrm{a}$ \\
\hline OTU5 Stichococcus clade5 & LH08SW1099 & Hodač 2015 & Hodač/2008 & $\mathrm{n} / \mathrm{a}$ \\
\hline OTU2* Stichococcus clade6 & SAG 107.80 & Lewin(?) & Lewin/1952 & $\mathrm{n} / \mathrm{a}$ \\
\hline OTU2* Stichococcus clade7 & LH10HG2063 & Hodač 2015 & Hodač/2010 & $\mathrm{n} / \mathrm{a}$ \\
\hline
\end{tabular}




\section{Appendix | Chapter 4}

\section{Supporting Figures}

Figure S1. ML phylogeny based on 18S rDNA sequences showing the phylogenetic position of Jenufa (Fig. 2).

Figure S2. ML phylogeny based on $18 \mathrm{~S}$ rDNA sequences showing the phylogenetic position Xylochloris (Fig. 3). 
Figure S1. Maximum likelihood phylogeny based on 18S rDNA sequences showing the phylogenetic position of Jenufa (Fig. 2).

(1)

(1)

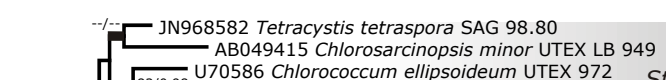
AB049415 Chlorosarcinopsis minor UTEX
82/0.98 U70586 Chlorococcum ellipsoideum UTEX 972

949 JN968584 Neospongiococcum gelatinosum SAG 64.80 100/1.00

U70797 Haematococcus zimbabwiensis UTEX LB 1758 U70798 Stephanosphaera sp. UTEX 2409

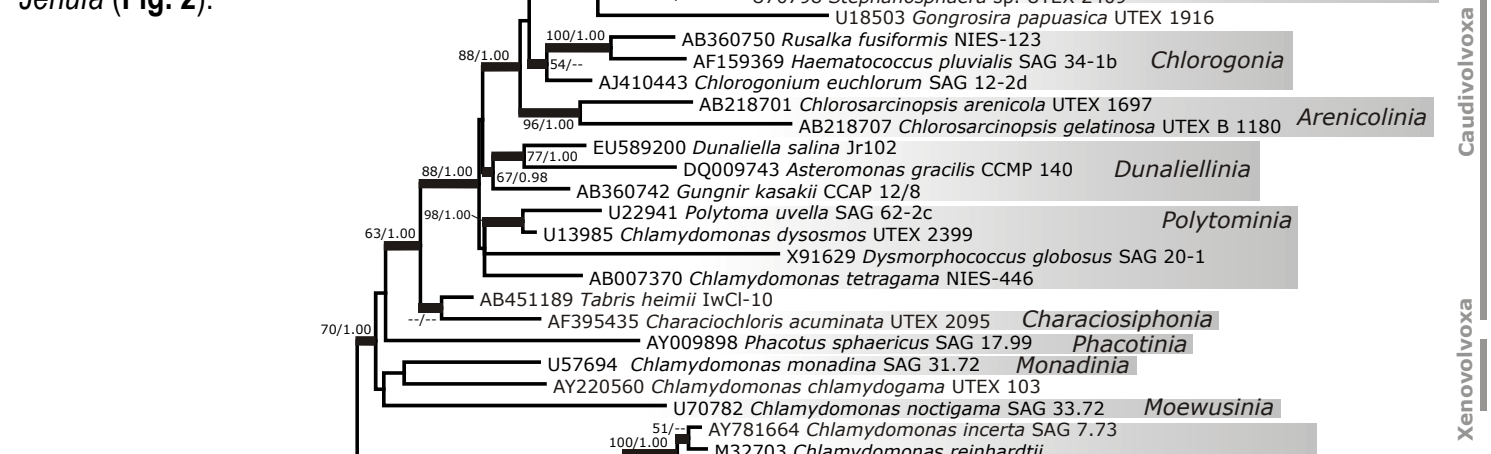

100/1.00-1- AY781664 Chlamydomonas incerta SAG 7.73 - M32703 Chlamydomonas reinhardtil

100/1.00 X53904 Volvox carteri UTEX LB 1885 - U83120 Paulschulzia pseudovolvox AF367857 Heterochlamydomonas inaequalis UTEX 1705

Reinhardtinia AB248251 Hafniomonas conica NIES-1714

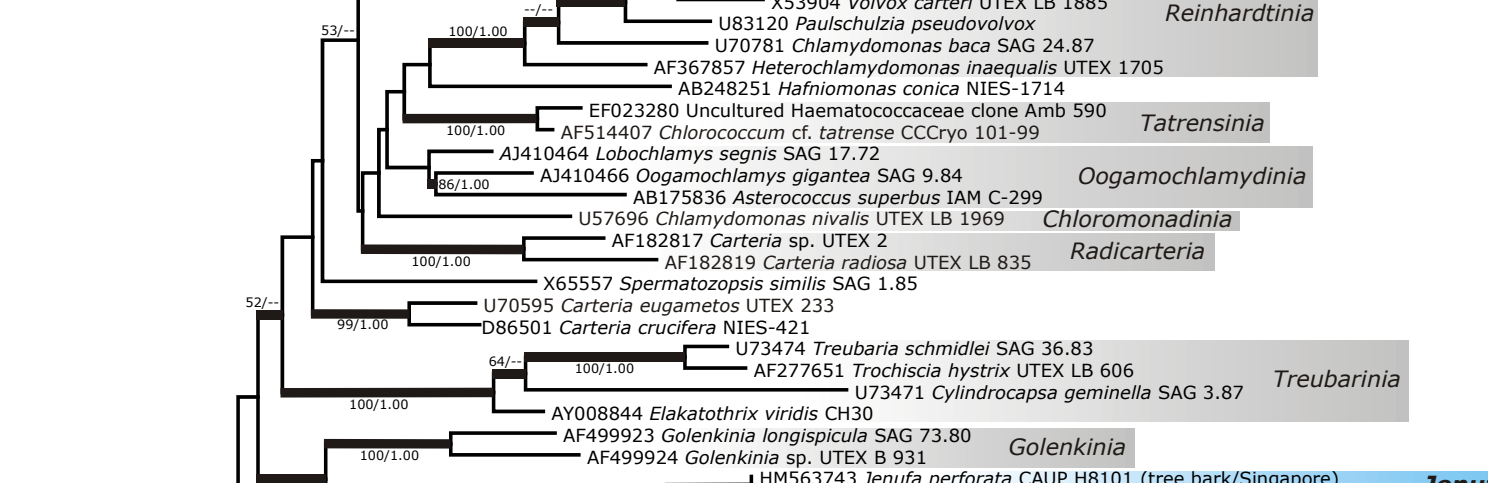

y

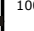

HM563743 Jenufa perforata CAUP H8101 (tree bark/Singapore) Jenufa Uncultured eukaryote clone e15_2_8 (tree bark/Panama) HM563744 Jenufa minuta CAUP H8102 (tree bark/Singapore) AB257659 Uncultured Chlorophyta clone DA-01 (endolithic,
JQ988927 Jenufa sp. SAG 2379 (epilithic/Germany) - JQ988932 Jenufa clone GS1K32 (endolithic/Germany)

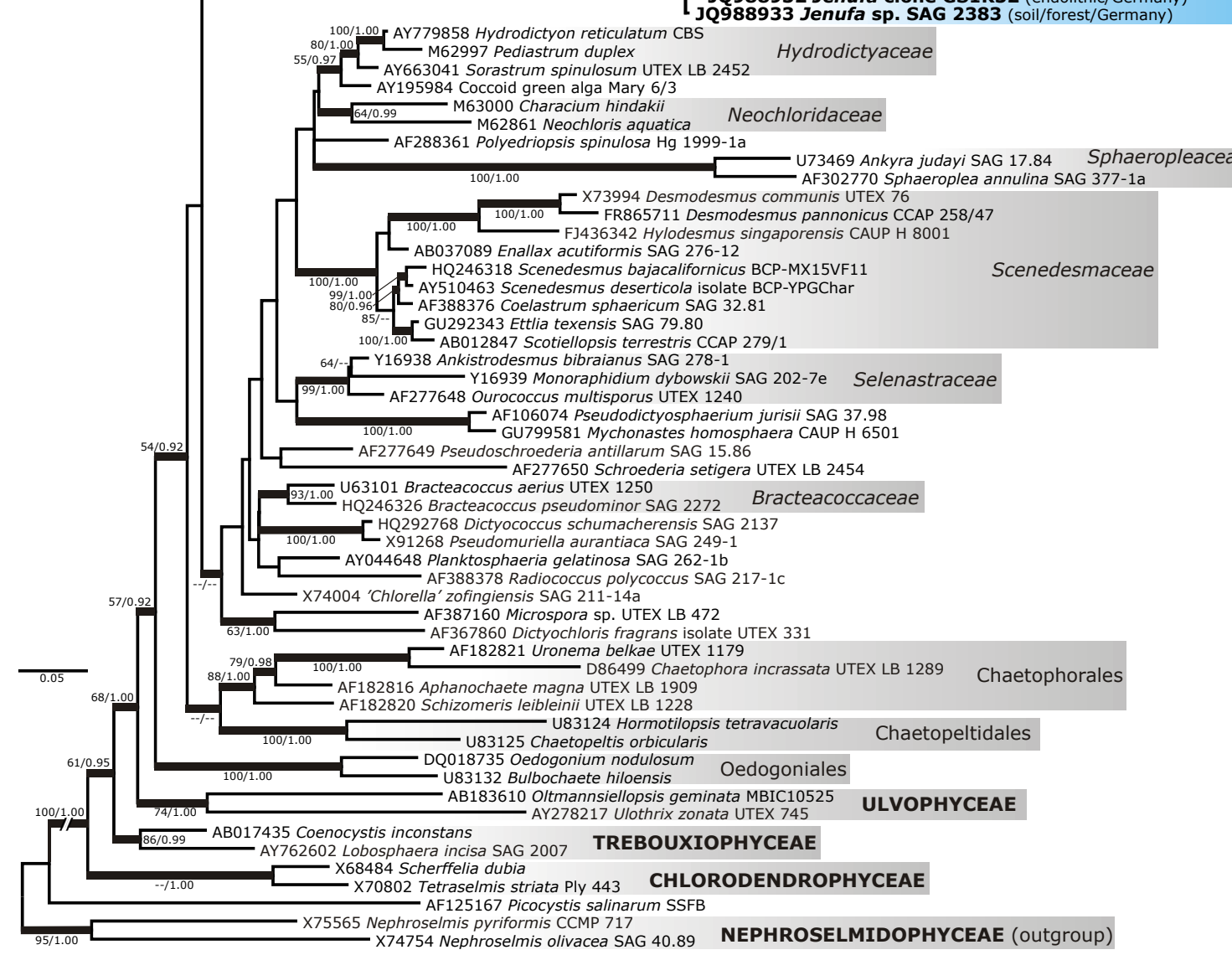


Figure S2. Maximum likelihood phylogeny based on 18S rDNA sequences showing the phylogenetic position Xylochloris (Fig. 3 ).

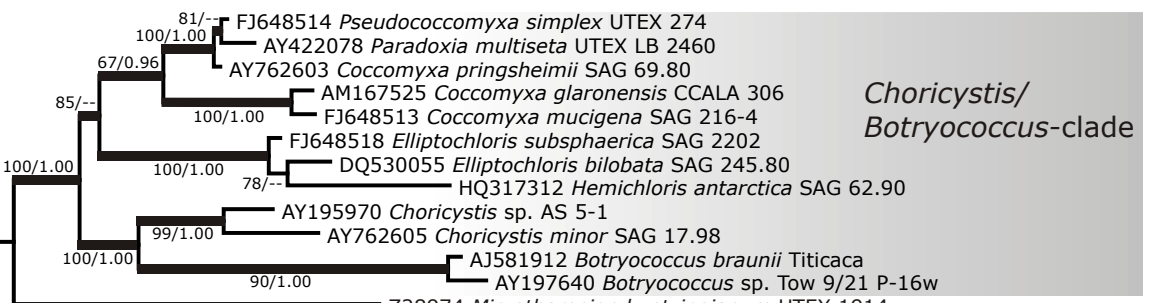

81- [ FJ648514 Pseudococcomyxa simplex UTEX 274 L AY422078 Paradoxia multiseta UTEX LB 2460 AY762603 Coccomyxa pringsheimii SAG 69.80

Z21551 Trebouxia impressa U U
EU878373 Parietochloris alveolaris UTEX B 836
M63002 Parietochloris pseudoalveolaris 99/1.00 UQ988940 Xylochloris clone HEG9B-K2617 (grassland soil/Germany) Xylochloris 64/0.99 $\begin{aligned} & \text { JQ988 } \\ & \text { EU105209 Xylochloris irregularis CAUP H } 7801 \text { (tree bark/Singapore) }\end{aligned}$ JQ988930 'Dictyochloropsis' sp. LH-aw3050 (forest soil, Germany) GU017658 'Dictyochloropsis' sp. SAG 2305 (tree bark, Germany) FJ52469 Uncultured eukaryote clone B12 SE1B (cold-fumarole soil/5824 m a.s.//Andes) 592457 Uncultured eukaryote clone A11_SE1B (cold-fumarole soil/5824 m a.s.I./Andes) 728973 Leptosira terrestris SAG 463-3

AB006051 Lobosphaera tirolensis ASIB S234 Lobosphaera-clade Z47207 Dictyochloropsis reticulata CCHU 5616 71/-- X73991 Watanabea reniformis SAG 211-9b 9b 237 X73998 Heterochlorella luteoviridis SAG 211-2a EU346910 Kalinella bambusicola CAUP H 7901

96/1.00 FM946000 Chloroidium saccharophilum SAG 211-9a $97 / 1.00$
Z Z28971 Myrmecia biatorellae UTEX 907

\section{is erici IAM C-593 Trebouxiales} 100/1.00 AJ311637 Stichococcus bacillaris SAG 379-1b 55/-- LM412753 Gloeotila scopulina SAG 335-8

68/0.99 LU434017 Desmococcus olivaceus SAG 1.92

EF200524 Prasiola stipitata Psti1

81/0.97 AB087559 Stichococcus ampulliformis Handa-299(f)

FJ896222 Pseudomarvania aer

- AB055866 Stichococcus bacillaris K4-4

AY762604 Trebouxiophyte sp. UR47/4

62/0.96 AY762604 Trebouxiophyte sp. UR47/4

87/1.00-1 AB055864 Stichococcus bacillaris CCAP 379/7

82/0.99 [- DQ275461 Stichococcus jenerensis D 4

94/1.00.92/. 2 AJ416105 Chlorella sphaerica SAG 11.88

52/-- FJ946882 Tr Stichococcus chodati UTEX 1177

FJ946882 Trebouxiophyceae sp. EO7-4

100/1.00 12900 AJ311638 Stichococcus mirabilis CCAP 379/3

00/100 AB006050 Pseudochlora longiseta UTEX 339 X74000 Chlorella mirabilis Andreyeva 748-I

AB017435 Coenocystis inconstans

95/1.00 FM205832 Chlorella vulgaris SAG 211-11b

86/1.00 GQ176853 Dictyosphaerium tetrachotomu

59/--- FM205859 Chlorella sorokiniana CCAP 211/8K
72/1.00

FM205882 Actinastrum hantzschii CCAP 200/1

98/1.00
FM205840 Didymogenes palatina

- AB037085 Dicloster acuatus SAG 41.98

- X56105 Chlorella kessleri SAG 211-11g

AY195969 Muriella sp. AS 2-4

AB012845 Muriella terrestris ASIB V38

- Fm205844 Hegewaldia sp. CCAP 283/3

99/1.00 AY195983 Nannochloris Sp. JL 4-6

63/- EF440182 Nannochloris sp. Ant-1

AJ439399 Chlorella protothecoides var.

Y14950 Chlorella sp. Yanaqocha RA1

[ GU592792 Catena viridis KR 1991/4

89/0.98 AJ306534 Koliella spiculiformis SAG 14.91

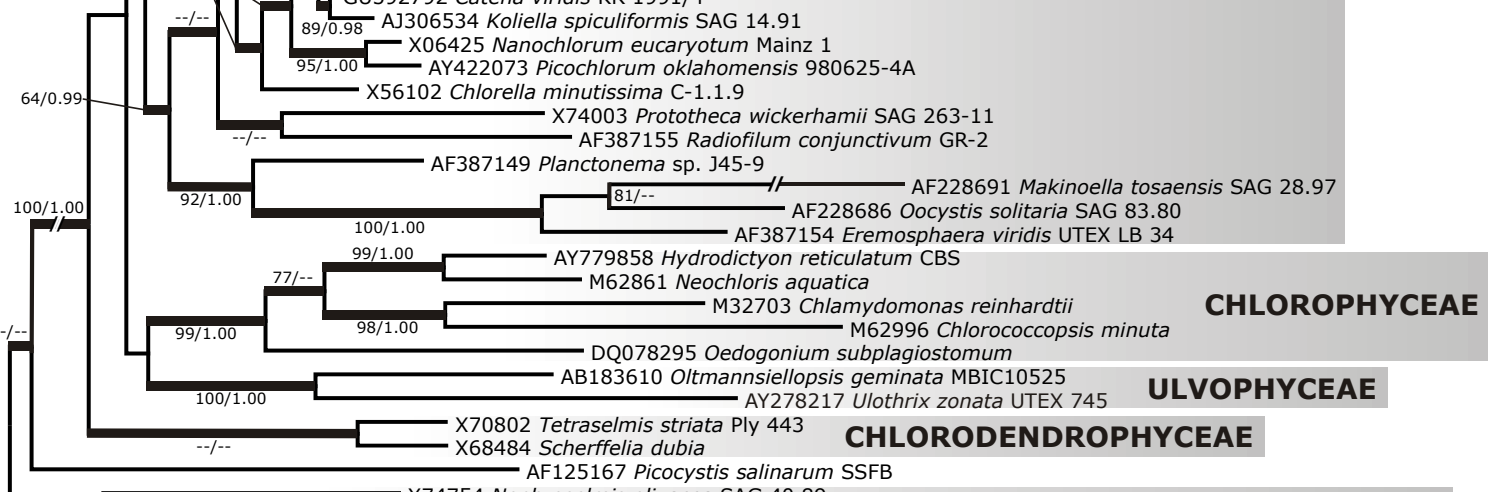

Chlorellales

\section{Prasiola-clade}

(1)

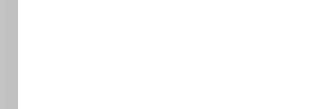




\section{Publications}

\section{Contributions to the papers and manuscript drafts included in this thesis}

\section{Chapter 1 | Molecular diversity of microscopic Green algae isolated from German soils}

(Manuscript draft)

Ladislav Hodač, Christine Hallmann, Karolin Spitzer and Thomas Friedl

University of Göttingen, Experimental Phycology and Culture Collection of Algae (SAG), Göttingen, Germany

Conceived and designed the experiments: LH CH TF

Performed the experiments: LH KS

Analyzed the data: LH, KS

Wrote the paper: $\mathrm{LH}$

Chapter 2 | Diversity of microscopic green algae (Chlorophyta) in calcifying biofilms of two karstic streams in Germany

(Published in Geomicrobiology Journal; doi: 10.1080/01490451.2013.878418)

Ladislav Hodač ${ }^{1}$, Nicole Brinkmann ${ }^{2}$, Kathrin I. Mohr ${ }^{3}$, Gernot Arp ${ }^{2}$, Christine Hallmann ${ }^{1}$, Jessica Ramm ${ }^{4}$, Karolin Spitzer ${ }^{1}$, Thomas Friedl ${ }^{1}$

${ }^{1}$ University of Göttingen, Experimental Phycology and Culture Collection of Algae (SAG), Göttingen, Germany, ${ }^{2}$ University of Göttingen, Geoscience Centre, Göttingen, Germany, ${ }^{3}$ Helmholtz Centre for Infection Research, Braunschweig, Germany, ${ }^{4}$ Brandenburg University of Technology, Department of Freshwater Conservation, Bad Saarow, Germany

Conceived and designed the experiments: KM NB TF LH

Performed the experiments: KM NB LH JR KS CH

Analyzed the data: LH KM TF

Wrote the paper: LH TF GA KI NB 
Chapter 3 | Phylogenetic analysis of polar Chlorella and Stichococcus suggests biogeography of airborne microalgae

(Manuscript draft submitted to FEMS Microbiology Ecology; FEMSEC-15-11-0606)

Ladislav Hodač ${ }^{1}$, Christine Hallmann ${ }^{1}$, Karolin Spitzer ${ }^{1}$, Josef Elster ${ }^{2,3}$, Fabian Faßhauer ${ }^{1}$, Nicole Brinkmann $^{4}$, Daniela Lepka ${ }^{1}$, Vaibhav Diwan ${ }^{1}$ and Thomas Fried ${ }^{1}$

${ }^{1}$ Experimental Phycology and Culture Collection of Algae (SAG), University of Göttingen, Germany, ${ }^{2}$ Centre for Polar Ecology, University of South Bohemia, České Budějovice, Czech Republic, ${ }^{3}$ Institute of Botany, Phycology Centrum, Academy of Sciences of the Czech Republic, Třeboň, Czech Republic, ${ }^{4}$ Department of Forest Botany, University of Göttingen, Germany

Conceived and designed the experiments: LH TF JE

Performed the experiments: LH KS CH NB JE FF DL VD

Analyzed the data: LH, KS, TF

Wrote the paper: $\mathrm{LH}$

Chapter 4 | Molecular evidence for the wide distribution of two lineages of terrestrial green algae (Chlorophyta) from tropics to temperate zone

(Published in ISRN Ecology; doi: 10.5402/2012/795924)

Ladislav Hodač ${ }^{1}$, Christine Hallmann ${ }^{1}$, Helen Rosenkranz ${ }^{2}$, Fabian Faßhauer ${ }^{1}$ and Thomas Fried $^{1}$

${ }^{1}$ University of Göttingen, Experimental Phycology and Culture Collection of Algae (SAG), Göttingen, Germany, ${ }^{2}$ School of Biological Sciences, University of Bristol, Bristol, United Kingdom

Conceived and designed the experiments: LH TF

Performed the experiments: LH CH FF HR

Analyzed the data: LH

Wrote the paper: LH TF CH HR FF 


\section{Comprehensive publication list (2009-2015)}

Hodač L, Brinkmann N, Mohr K, Arp G, Hallmann C, Ramm J, Spitzer K, Friedl T. 2015. Diversity of microscopic green algae (Chlorophyta) in calcifying biofilms of two karstic streams in Germany. Geomicrobiology Journal 32(3-4): doi:10.1080/01490451.2013. 878418.

Brinkmann N, Hodač L, Mohr KI, Hodačová A, Jahn R, Ramm J, Hallmann C, Arp G, Friedl T. 2015. Cyanobacteria and diatoms in biofilms of two karstic streams in Germany and changes of their communities along calcite saturation gradients. Geomicrobiology Journal 32(3-4): doi:10.1080/01490451.2014.901438.

Hodač L, Scheben, AP, Hojsgaard D, Paun O, Hörandl E. 2014. ITS polymorphisms shed light on hybrid evolution in apomictic plants: a case study from the Ranunculus auricomus complex. PLoS ONE 9(7): doi:10.1371/journal.pone.0103003.

Patzelt DJ, Hodač L, Friedl T, Pietrasiak N, Johansen JR. 2014. Biodiversity of soil cyanobacteria in the hyper-arid Atacama Desert, Chile, assessed by culture dependent and independent approaches. Journal of Phycology 50: doi:10.1111/jpy.12196.

Allan E, Bossdorf O, Dormann CF, Prati D, Gossner M, Tscharntke T, Blüthgen N, Barto K, Bellach M, Birkhofer K, Boch S, Böhm S, Börschig C, Chatzinotas A, Christ S, Daniel R, Diekoetter T, Fischer C, Friedl T, Glaser K, Hallmann C, Hodač L, et al. 2013. Interannual variation in land-use intensity enhances grassland multidiversity. PNAS 111(1): doi:10.1073/pnas.1312213111.

Hodač L, Hallmann C, Rosenkranz H, Faßhauer F, Friedl T. 2012. Molecular evidence for the wide distribution of two lineages of terrestrial Green algae (Chlorophyta) over tropics to temperate zone. ISRN Ecology 2012: doi:10.5402/2012/795924.

Němcová Y, Eliáš M, Škaloud P, Hodač L, Neustupa J. 2011. Jenufa gen. nov.: a new genus of coccoid green algae (Chlorophyceae, incertae sedis) previously recorded by environmental sequencing. Journal of Phycology 47(4): doi:10.1111/j.1529-8817.2011.01009.x.

Nacke H, Thürmer A, Wollherr A, Will C, Hodač L, Herold N, Schöning I, Schrumpf M, Daniel R. 2011. Pyrosequencing-based assessment of bacterial community structure along different management types in german forest and grassland soils. PLOS ONE 6(2): doi:10.1371/journal.pone.0017000.

Lang I, Hodač L, Friedl T, Feussner I. 2011. Fatty acid profiles and their distribution patterns in microalgae: a comprehensive analysis of more than 2000 strains from the SAG culture collection. BMC Plant Biology 11(124): doi:10.1186/1471-2229-11-124. 


\section{Danksagung}

Meinem Betreuer, Herrn Prof. Dr. Thomas Friedl, verdanke ich die Möglichkeit, meine Doktorarbeit in der Sammlung von Algenkulturen Göttingen, einem ganz besonderen und traditionsreichen Standort der Phykologie, abfassen zu dürfen. Anfänglich konnte er mich für die terrestrischen Mikroalgen vollkommen begeistern und seine anregenden Denkanstöße waren für meine Arbeit wegweisend. Herzlichen Dank! Bei meinem Korreferenten, Herrn Prof. Dr. Rolf Daniel, bedanke ich mich für die kritische Begutachtung der vorliegenden Arbeit!

Mein ganz besonderer Dank gilt Frau Dr. Maike Lorenz; ihr kompetenter Rat und ihre Hilfe kamen mir in vielen Angelegenheiten immer sehr zugute. An dieser Stelle möchte ich mich auch bei Frau Prof. Dr. Elvira Hörandl herzlichst für ihr unschätzbares Entgegenkommen bedanken! Für die vielseitige Unterstützung, den regen Austausch und unzählige Diskussionen bedanke ich mich recht herzlich bei meiner Mitdoktorandin Christine Hallmann. Dankbar bin ich auch für die immer freundliche und geduldige Hilfsbereitschaft meines Mitdoktoranden Fabian Faßhauer sowie aller Mitarbeiterinnen und Mitarbeiter der EPSAG, insbesondere Ilse Kunkel, Marlis Heinemann, Gabrielle Curdt-Hollmann, Hella Timmermann, Reiner Freter, Kathrin I. Mohr, Nicole Brinkmann, Anastasija Kryvenda und Nataliya Rybalka. Mein Dank geht ebenso an die Mitglieder der Verwaltung des Instituts, insbesondere Herrn Dr. Hans Depta, der mir mehrfach hilfreich zur Seite stand.

Großer Dank gebührt meiner Familie, insbesondere meinen Eltern, die mich während des gesamten Promotionsstudiums hilfsbereit und unterstützend aufgemuntert haben. Auch meinem werten Freund David danke ich dafür, dass er mir stets zur Seite stand. Meiner Lebensgefährtin Karolin danke ich aus ganzem Herzen für alles. Ihre stetige und unermüdliche sowohl fachliche als auch private Unterstützung, oder kurz-ihr Dasein in meinem Leben, haben wesentlich zum Gelingen der vorliegenden Arbeit beigetragen. 